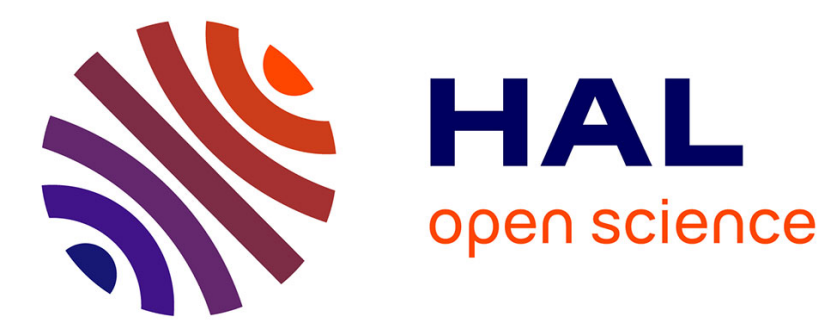

\title{
Thermodynamics of ultracold Fermi gases
}

Sylvain Nascimbène

\section{To cite this version:}

Sylvain Nascimbène. Thermodynamics of ultracold Fermi gases. Condensed Matter [cond-mat]. Université Pierre et Marie Curie - Paris VI, 2010. English. NNT : . tel-00491711v2

\section{HAL Id: tel-00491711 \\ https://theses.hal.science/tel-00491711v2}

Submitted on 11 Jul 2010

HAL is a multi-disciplinary open access archive for the deposit and dissemination of scientific research documents, whether they are published or not. The documents may come from teaching and research institutions in France or abroad, or from public or private research centers.
L'archive ouverte pluridisciplinaire HAL, est destinée au dépôt et à la diffusion de documents scientifiques de niveau recherche, publiés ou non, émanant des établissements d'enseignement et de recherche français ou étrangers, des laboratoires publics ou privés. 
Département de physique

Laboratoire Kastler Brossel

École Normale Supérieure

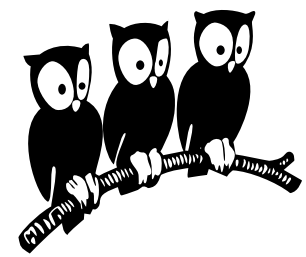

THÈSE de DOCTORAT de I'UNIVERSITÉ PARIS 6

Spécialité : Physique Quantique

présentée par

\section{Sylvain Nascimbène}

pour obtenir le grade de DOCTEUR de l'UNIVERSITÉ PARIS 6

\section{Thermodynamique des gaz de fermions ultrafroids Thermodynamics of ultracold Fermi gases}

Soutenue le 11 Juin 2010

devant le jury composé de :

M. Immanuel Bloch ............. Examinateur

M. Philippe Bouyer .............. Rapporteur

M. Frédéric Chevy .............. Membre invité

M. Roland Combescot ........... Examinateur

M. Stefano Giorgini ............. Rapporteur

M. Christophe Salomon ........... Directeur de thèse 





\section{Contents}

Remerciements 99

$\begin{array}{lll}1 & \text { Introduction } & 11\end{array}$

1.1 Ultracold Fermi Gases: State of the Art . . . . . . . . . . . . . . . . . . . . . 12

1.1 .1 BEC-BCS Crossover . . . . . . . . . . . . . . . . . . . . . . . . . . . 12

1.1 .2 'High- $T_{c}$ ' Superfluidity of a Fermi Gas with Resonant Interactions $\ldots \ldots \ldots$. . . 13

1.1 .3 Spin-Imbalanced Fermi Gases $\ldots \ldots \ldots \ldots$. . . . . . . . . . . . . . . 14

1.2 Issues and Perspectives Addressed in this Thesis $\ldots \ldots \ldots \ldots \ldots \ldots . \ldots \ldots$

1.2 .1 Universal Thermodynamics of an Ultracold Fermi Gas . . . . . . . . . . . . . . . 14

1.2 .2 Previous Thermodynamic Studies . . . . . . . . . . . . . . . . 15

1.2 .3 Measurement of the Local Pressure Inside a Trapped Gas . . . . . . . . . . . . . 16

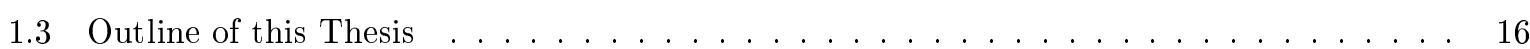

$1.3 .1 \quad$ Chapter 2: Experimental Setup . . . . . . . . . . . . . . . . . . . . 16

1.3.2 Chapter 3: Measuring the Equation of State of a Homogeneous Ultracold Gas . . 16

1.3 .3 Chapter 4: Thermodynamics of a Strongly-Interacting Fermi Gas . . . . . . . . . 17

1.3.4 Chapter 5: Ground State of an Attractive Fermi Gas: Phase Diagram and Equation

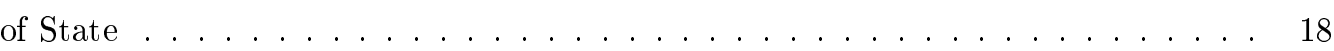

1.3.5 Chapter 6: Axial Breathing Modes of a Spin-Imbalanced Fermi Gas . . . . . . . . 18

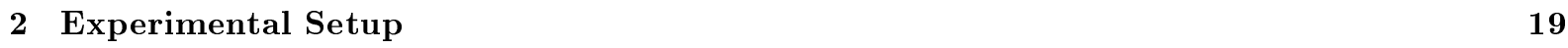

$2.1 \quad$ Global Description of the Experimental Setup . . . . . . . . . . . . . . . . . . . . . 19

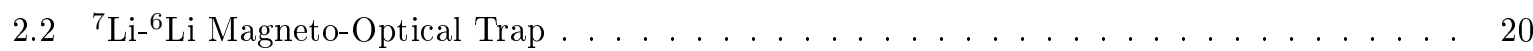

2.2 .1 Laser System . . . . . . . . . . . . . . . . . . . . . . . 20

2.2 .2 Zeeman Slower . . . . . . . . . . . . . . . . . . . . . . . 21

2.2 .3 Dual Species Magneto-Optical Trap _. . . . . . . . . . . . . . . . . . . . 21

2.3 Sympathetic Cooling in a Magnetic Trap . . . . . . . . . . . . . . . . . . . . . 21

2.3 .1 Transfer into an Ioffe-Pritchard Trap . . . . . . . . . . . . . . . . . . . . 22

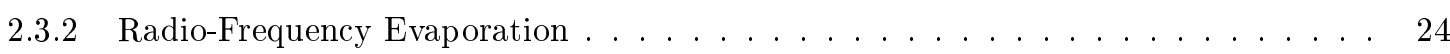

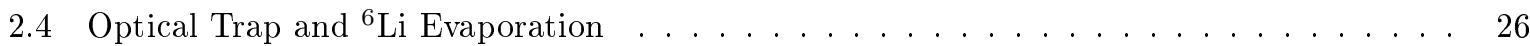

2.4 .1 Geometry of the Hybrid Optical and Magnetic Trap . . . . . . . . . . . . 26

2.4 .2 Optical System for the Dipole Trap. . . . . . . . . . . . . . . . . . 28

2.4 .3 Preparation of a Strongly-Interacting ${ }^{6} \mathrm{Li}$ Mixture $\ldots \ldots \ldots \ldots$. . . . . . . . 30

2.4 .4 Evaporation of $\mathrm{a}^{6} \mathrm{Li}$ Gas With Resonant Interactions . . . . . . . . . . . . . 32

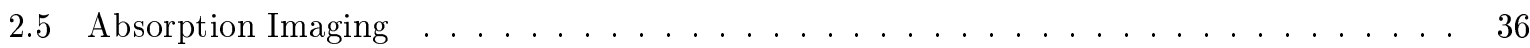

2.5 .1 Generation of High-Field Resonant Probes . . . . . . . . . . . . . . . . . 36 
$2.5 .2 \quad$ Imaging Optical System $\ldots \ldots \ldots \ldots \ldots \ldots \ldots \ldots \ldots \ldots$

2.5 .3 Imaging System Resolution $\ldots \ldots \ldots \ldots \ldots$. . . . . . . . . . . 38

2.5 .4 Double in Situ Images $\ldots \ldots \ldots \ldots$. . . . . . . . . . . . . . . . . 38

\begin{tabular}{|lll}
3 & Measuring the Equation of State of a Homogeneous Ultracold Gas & 41
\end{tabular}

$3.1 \quad$ Equation of State of an Ultracold Gas: State of the Art $\ldots \ldots \ldots \ldots \ldots$. . . . . . . . . . . . . . . .

3.1 .1 Equation of State of a Trapped Gas . . . . . . . . . . . . . . . . 42

3.1 .2 Direct Measurement of the Equation of State of a Homogeneous Gas . . . . . . . . 44

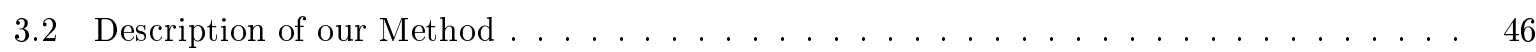

3.2 .1 Measurement of the Local Pressure inside a Trapped Gas . . . . . . . . . . . . . 46

3.2 .2 Determination of a Grand-Canonical Equation of State $\ldots \ldots \ldots$. . . . . . . 47

$3.3 \quad$ Equation of State of a Weakly-Interacting Bose Gas $\ldots \ldots \ldots \ldots \ldots$

3.4 Mott Insulator Behavior of a Bose Gas in an Optical Lattice $\ldots \ldots \ldots \ldots$

3.4 .1 Realization of a Bose-Hubbard Model . . . . . . . . . . . . . . . . . . . 49

3.4 .2 The Mott-Insulator Regime $\ldots \ldots \ldots \ldots \ldots \ldots \ldots$. . . . . . . . . . . . . . . . . . . . . 50

3.4 .3 Extraction of the Equation of State $\ldots \ldots \ldots \ldots \ldots \ldots$. . . . . . . . . 51

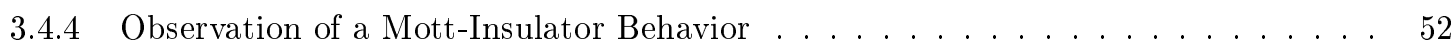

3.4 .5 Estimation of Finite-Temperature Effects $\ldots \ldots \ldots \ldots \ldots \ldots \ldots$

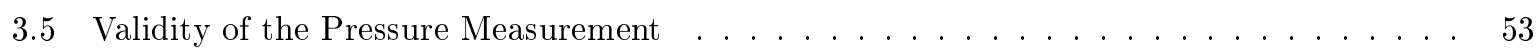

3.5 .1 Calibration of the Pressure $\ldots \ldots \ldots \ldots \ldots \ldots \ldots$

3.5 .2 Deviation from Local Density Approximation . . . . . . . . . . . . . . . 54

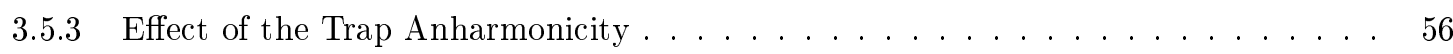

3.5 .4 Effect of the Imaging System Resolution $\ldots \ldots \ldots \ldots$. . . . . . . . . 57

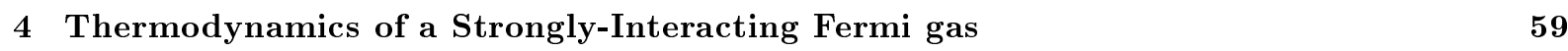

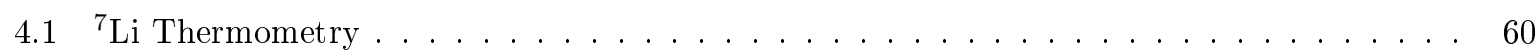

4.1 .1 Preparation of a Three-Component ${ }^{6} \mathrm{Li}^{7} \mathrm{Li}$ Mixture $\ldots \ldots \ldots \ldots$. . . . . 60

4.1 .2 Two-Species Evaporation and Thermalization . . . . . . . . . . . . . . . . 61

4.1 .3 Temperature Measurement . . . . . . . . . . . . . . . . . . . . 62

4.1 .4 Limitation of our Thermometer at Low Temperature . . . . . . . . . . . . . . . . . . . . 62

4.2 Extraction of $h_{T}(\zeta)$ from In Situ Images $\ldots \ldots \ldots \ldots \ldots \ldots \ldots$

4.2 .1 Direct Measurement of the High-Temperature Equation of State . . . . . . . . . 63

$4.2 .2 \quad$ Construction of the Low-Temperature Equation of State . . . . . . . . . . . . . . . 64

4.2 .3 Systematic Error on the Equation of State Determined from our Data . . . . . . . 65

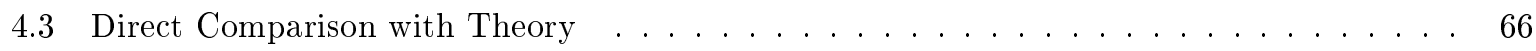

4.4 Comparison with the Tokyo Group Measurements $\ldots \ldots \ldots \ldots \ldots$

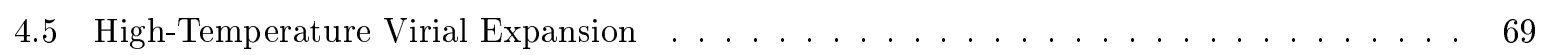

$4.5 .1 \quad$ Virial Expansion of a Unitary Fermi Gas: Generalities . . . . . . . . . . . . . 70

4.5 .2 Virial Coefficients Extracted from our Data . . . . . . . . . . . . . . . . . . 71

4.6 Fermi-Liquid Behavior in the Normal Phase . . . . . . . . . . . . . . . . 73

$4.6 .1 \quad$ Low-Temperature Normal Phases in Strongly-Interacting Systems . . . . . . . . 73

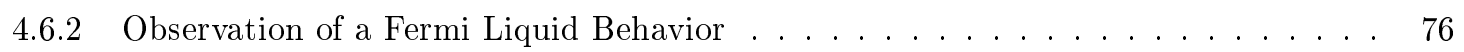

$4.6 .3 \quad$ Estimation of the Maximum Pseudogap Amplitude . . . . . . . . . . . . . 78

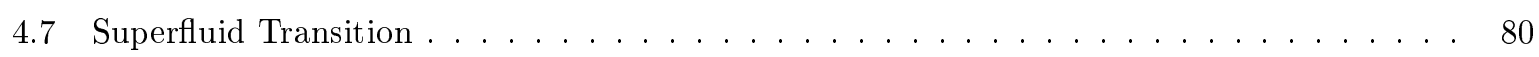

4.7 .1 Deviation from the Fermi Liquid Equation of State . . . . . . . . . . . . . . 80

4.7 .2 Low-Temperature Excitations in the Superfluid Phase . . . . . . . . . . . . . . . 81

4.7 .3 Critical Temperature for Superfluidity . . . . . . . . . . . . . . . . 82

4.7 .4 Validity of Local Density Approximation in the Critical Region . . . . . . . . . . . 84 
4.8 Equation of State of a Trapped Gas $\ldots \ldots \ldots \ldots \ldots \ldots \ldots \ldots$

$\begin{array}{lll}5 & \text { Ground State of an Attractive Fermi Gas: Phase Diagram and Equation of State } & 87\end{array}$

5.1 Sketch of the Phase Diagram $\ldots \ldots \ldots \ldots \ldots \ldots \ldots \ldots \ldots$

$5.1 .1 \quad$ Superfluid to Normal Quantum Phase Transition . . . . . . . . . . . . . 88

5.1 .2 The Impurity Problem . . . . . . . . . . . . . . . . . . . . . . . . . . . . 89

5.1 .3 Beyond the Impurity Problem . . . . . . . . . . . . . . . . . . . . . . 90

5.2 Equation of State Measurement Scheme $\ldots \ldots \ldots \ldots \ldots \ldots \ldots$

5.2 .1 Experimental Sequence $\ldots \ldots \ldots \ldots \ldots \ldots$

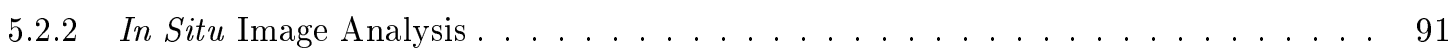

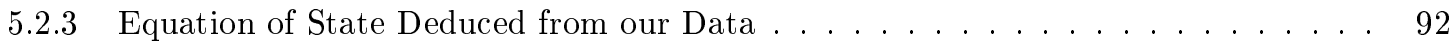

5.2 .4 Systematic Error of our Data $\ldots \ldots \ldots \ldots$. . . . . . . . . . . . 93

5.3 Superfluid to Normal Phase Transition $\ldots \ldots \ldots \ldots \ldots \ldots$. . . . . . . . . . . . 93

5.3 .1 Critical Chemical Potential Ratio . . . . . . . . . . . . . . . . . . . . . . . 93

5.3 .2 Critical Impurity Concentration $\ldots \ldots \ldots \ldots \ldots \ldots$

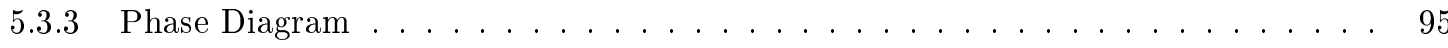

5.3 .4 Comparison with the Single-Particle Excitation Gap . . . . . . . . . . . . . . . 96

5.4 Fermi Liquid Behavior in the Partially Polarized Phase. . . . . . . . . . . . . . . . . . . 97

5.4 .1 Observation of a Fermi Liquid Behavior . . . . . . . . . . . . . . . . 97

5.4 .2 Measurement of the Polaron Effective Mass . . . . . . . . . . . . . . . . . . 98

$5.4 .3 \quad$ Fermi Liquid Equation of State in the Unitary Limit . . . . . . . . . . . . . . . . . 99

5.4 .4 Canonical Equation of State. . . . . . . . . . . . . . . . . . . . . 100

5.4 .5 Magnetic Susceptibility of a Spin-Unpolarized Fermi Gas . . . . . . . . . . . . 102

5.5 Superfluid Equation of State in the BEC-BCS Crossover . . . . . . . . . . . . . . . . . . 104

5.5 .1 Parametrization of the Superfluid Equation of State . . . . . . . . . . . . . . 104

5.5 .2 Direct Comparison with Theory _.. . . . . . . . . . . . . . . . . 105

5.5 .3 Extracting Asymptotic Behaviors of the Equation of State . . . . . . . . . . . . 108

5.5 .4 Superfluid Equation of State Around the Unitary Limit . . . . . . . . . . . . . . . 109

5.5.5 Equation of State of a Fermionic Superfluid from the Weakly-Interacting Regime . 111

5.5 .6 Equation of State of a Bosonic Superfluid . . . . . . . . . . . . . . . . 113

5.6 Ground State of a Trapped Fermi Gas . . . . . . . . . . . . . . . . . . 115

5.6 .1 Equation of State of a Trapped Spin-Imbalanced Fermi Gas . . . . . . . . . . . . 115

5.6 .2 Collective Modes of a Balanced Fermi Gas . . . . . . . . . . . . . . . . . 117

5.7 Molecular Physics Beyond the Scope of this Work . . . . . . . . . . . . . . . . . . . 118

5.7 .1 Polarized Superfluid . . . . . . . . . . . . . . . . . . . . . . . . . . . . 118

5.7 .2 Polaron to Molecule Transition in the Impurity Problem . . . . . . . . . . . . . . . 119

5.7 .3 Thermodynamic Instability of the Impurity Problem . . . . . . . . . . . . . . . 120

$6 \quad$ Axial Breathing Modes of a Spin-Imbalanced Fermi Gas $\quad 121$

6.1 Hydrodynamic Behavior of a Balanced Fermi Gas . . . . . . . . . . . . . . . . . . . 122

$6.1 .1 \quad$ Scaling Ansatz Solution of the Hydrodynamic Equations . . . . . . . . . . . . . . 122

6.1 .2 Hydrodynamic Expansion . . . . . . . . . . . . . . . . . . . . . . . 122

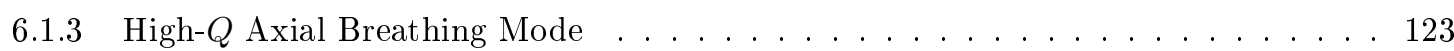

6.2 In-Phase Axial Breathing Mode . . . . . . . . . . . . . . . . . . . . . . . 124

6.2 .1 Observation of the In-Phase Mode . . . . . . . . . . . . . . . . . . . 124

6.2 .2 Frequency in the Hydrodynamic and Collisionless Regimes. . . . . . . . . . . . 125

6.2 .3 Crossover from a Hydrodynamic to a Collisionless Behavior . . . . . . . . . . . 126

$6.2 .4 \quad$ Oscillation Amplitude and Phase . . . . . . . . . . . . . . . . . . . 126 
6.2 .5 Relaxation of a Two-Component Fermi Gas . . . . . . . . . . . . . . . . . . 127

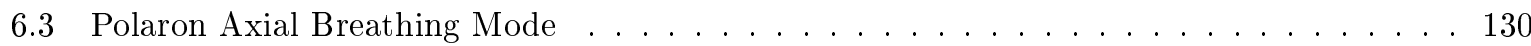

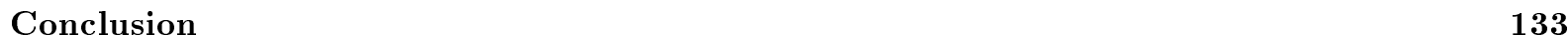

\begin{tabular}{|lr|}
\hline A Simple Thermodynamic Relations & 137
\end{tabular}

A.1 Thermodynamics of an Ideal Fermi Gas . . . . . . . . . . . . . . . . . . . . . 137

A.1.1 Equation of State of an Ideal Fermi Gas . . . . . . . . . . . . . . . . . . 137

A.1.2 High- and Low-Temperature Expansions . . . . . . . . . . . . . . . . . . . . . 138

A.2 Conversion between the Canonical and Grand-Canonical Equations of State of a Unitary

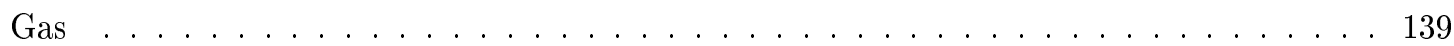

A.2.1 Conversion $g(\theta) \rightarrow h(\zeta) \ldots \ldots \ldots \ldots \ldots \ldots$

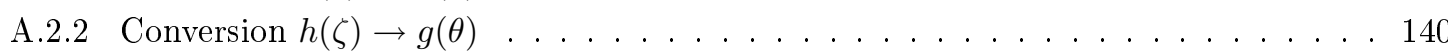

A.3 Calculation of the Second-Order Virial Coefficient . . . . . . . . . . . . . . . . . . 140

A.4 Clogston-Chandrasekhar Limit in a BCS Mean-Field Model . . . . . . . . . . . . . . . . 140

B Technical Details in Chapter 5

B.1 Construction of the Equation of State $\ldots \ldots \ldots \ldots$. . . . . . . . . . . 143

B.1.1 Determination of $\mu_{2}^{0} \ldots \ldots \ldots \ldots$. . . . . . . . . . . . . . 143

B.1.2 Equation of State in the BEC-BCS Crossover . . . . . . . . . . . . . . . 144

B.2 Amplitude of Finite-Temperature Effects . . . . . . . . . . . . . . . . . . . . 145

B.2.1 Upper Bound on the Cloud Temperature . . . . . . . . . . . . . . . . 145

B.2.2 $\quad$ Systematic Error in the Normal Phase . . . . . . . . . . . . . . . . . . . . . . 146

B.2.3 Systematic Error in the Superfluid Phase . . . . . . . . . . . . . . . . . . . . 147

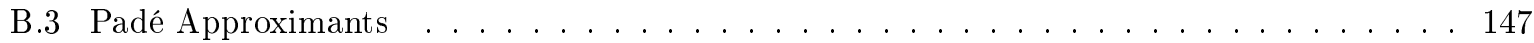

B.3.1 Padé Approximant on the BCS side of the Resonance . . . . . . . . . . . . . 147

B.3.2 Padé Approximants on the BEC side of the Resonance. . . . . . . . . . . . . . . 147

B.3.3 $\quad$ Fit Function of the Complete Data . . . . . . . . . . . . . . . . . . . . 148

B.4 Surface Tension at the Superfluid/Normal Boundary of a Trapped Spin-Imbalanced Fermi

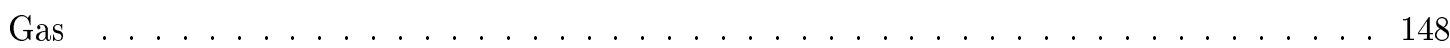

$\begin{array}{ll}\text { C Relaxation of the Axial Breathing Mode } & 151\end{array}$

\begin{tabular}{ll}
\hline Publications & 155
\end{tabular}

D.1 Collective Oscillations of an Imbalanced Fermi Gas... . . . . . . . . . . . . . . . . . 156

D.2 Exploring the Phase Diagram of a Universal Fermi Gas . . . . . . . . . . . . . . 160

D.3 The Equation of State of a Low-Temperature Fermi Gas with Tunable Interactions . . . . 165

$\begin{array}{lr}\text { References } & 169\end{array}$

\begin{tabular}{ll}
\hline Resumé - Abstract & 186
\end{tabular} 


\section{Remerciements}

Je souhaiterais remercier l'ensemble des personnes qui m'ont aidé dans la réalisation de cette thèse. J'ai effectué ce travail au sein du laboratoire Kastler Brossel, à l'École Normale Supérieure, de septembre 2006 à juin 2010. Je remercie toutes les personnes qui rendent cet environnement scientifique exceptionnel, notamment Paul Indelicato, directeur du laboratoire et Jean-Michel Raimond, directeur du département.

Je suis très reconnaissant à Christophe Salomon de m'avoir donné la chance de rejoindre l'équipe Lithium. La passion contagieuse de Christophe pour la physique expérimentale a été une source de motivation tout au long de ma thèse. Durant les années de construction de l'expérience, son optimisme et ses talents d'expérimentateur nous ont permis de rebondir dans les moments difficiles : nombreuses sont les soirées où Christophe ne pouvait se résoudre à quitter la salle de manip, jusqu'à trouver l'idée qui permet de progresser. J'ai par ailleurs beaucoup apprécié la confiance qu'il a montrée à notre égard en nous laissant beaucoup de libertés, tant sur le plan du montage expérimental que sur les expériences que nous avons menées.

Je remercie aussi tout particulièrement Frédéric Chevy pour son encadrement en tant que second directeur de thèse officieux. Frédéric a toujours été disponible pour de longues discussions, nous apportant un précieux recul sur la conduite de l'expérience. Il nous a par ailleurs souvent éclairé sur la physique complexe des gaz de fermions. J'ai beaucoup apprécié son souci de comprendre en profondeur la physique que nous découvrions, et de nous faire partager ses réflexions théoriques. Enfin, je le remercie de nous avoir fait bénéficier, en plus de ses qualités de physicien, de sa grande expérience gastronomique!

Je remercie aussi l'ensemble des gens avec qui j'ai travaillé. De manière générale j'ai beaucoup appris de discussions avec l'ensemble des membres du groupe atomes froids, ainsi que des autres groupes et laboratoires de l'ENS.

Le travail présenté dans ce mémoire étant celui d'une équipe, je tiens à exprimer ma gratitude envers les thésards et postdocs de l'expérience Lithium.

J'ai travaillé durant la majeure partie de ma thèse avec Nir, souvent quasiment en tête à tête. Nous avons eu la chance de nous entendre particulièrement bien, et travailler avec lui a vraiment été un plaisir. Le tuning de la manip aux couleurs de l'Equipe ou de films mythiques a rendu l'étroite pièce S22 quasiment conviviale. Ensemble, nous avons bravé les catastrophes expérimentales - inondations, incendie ou autres, et vécu ensemble des moments « énormes », que Nir a toujours fait en sorte de célébrer dignement. Je pense que notre première image de «plateau superfluide » restera longtemps dans nos mémoires... Je le remercie aussi de m'avoir fait découvrir des choses aussi différentes que le footing et les bières belges. Je souhaite à Nir et à Canh une bonne continuation sur la manip pour les années à venir.

Je remercie aussi les autres personnes avec qui j'ai travaillé durant ma thèse. Je suis particulièrement reconnaissant du travail des anciens, Martin, Jason et Leticia. Je les remercie notamment de m'avoir patiemment appris tous les rouages de l'expérience lors sa construction. Grainne nous a ensuite rejoints durant une année supplémentaire de construction, et son humour nous a permis de travailler dans une ambiance excellente. Je remercie enfin Kai-Jun, qui nous a ensuite aidé pendant plus d'un an.

Je souhaite remercier Immanuel Bloch, Philippe Bouyer, Roland Combescot et Stefano Giorgini de 
m'avoir fait l'honneur d'accepter d'être membres du jury de ma thèse. Je les remercie en particulier pour leur lecture attentive de ce mémoire, leurs remarques sur le manuscript et plus généralement pour l'intérêt qu'ils ont porté à ce travail.

Je suis reconnaissant à l'équipe enseignante de la formation interuniversitaire de physique de m'avoir donné la chance d'enseigner dans le cadre particulier de l'ENS. Je remercie plus particulièrement JeanMichel Raimond et Adrien Mahé, avec qui j'ai travaillé pendant les quatres années de thèse, ainsi que Claude Aslangul, Christophe Mora, Amir-Kian Kashani-Poor, et Frédéric.

Je remercie aussi tous ceux qui nous ont permis de travailler dans d'excellentes conditions. Je tiens à exprimer ma reconnaissance envers l'ensemble du personnel des services techniques pour leur aide précieuse et leur disponibilité lors de la construction de l'expérience. Je remercie aussi l'équipe du secrétariat du laboratoire et de l'IFRAF, pour leur efficacité quotidienne, ainsi que pour avoir patiemment résolu de nombreux problèmes administratifs. Enfin je remercie les autres services généraux du département de physique pour leur assistance.

Je remercie aussi les étudiants que j'ai rencontré à l'ENS, et grâce à qui j'ai travaillé dans une ambiance chaleureuse, et qui sont devenus de très bons amis. Je remercie en particulier Armix pour son enthousiasme et sa créativité en matière de surnoms, Félix pour toutes nos discussions de physique sans fin ainsi que pour son hospitalité, le groupe du DEA, Antoine, Samuel et Benoît, ainsi que Thomix, Camille, Olivier, Marie-Béatrice, Élise, Mathieu et Arnaud.

Je souhaite également remercier vivement mes amis de toujours, Adrien, Maïté, Marie et Pascal. J'espère qu'il savent à quel point leur présence compte pour moi. Je tiens à exprimer ma gratitude envers mes parents, Lucie et Didier pour leur aide très proche et leurs conseils, malgré mon mauvais caractère... Enfin, j'ai une pensée particulière pour Laura, qui m'a beaucoup apporté en m'accompagnant durant ces années. 


\section{Chapter 1}

\section{Introduction}

The achievement of Bose-Einstein condensation in 1995 [1, 2,3 paved the way for the realization of new states of matter using ultracold gases. At very low temperature, as soon as the quantum coherence length becomes comparable to the inter-particle distance, quantum statistics plays an essential role in their description. Despite their extremely low atom density, these gases exhibit many-body correlations that affect both their microscopic and macroscopic properties. As a spectacular example, the first quantum gases produced in the laboratory, namely weakly-interacting Bose gases, become superfluid when cooled below the Bose-Einstein condensation temperature. Until 1995, the only superfluid made of bosons observed in nature was liquid ${ }^{4} \mathrm{He}[4]$; due to the small inter-particle distance, interactions between ${ }^{4} \mathrm{He}$ atoms are rather complex to model and it is extremely hard to describe liquid ${ }^{4} \mathrm{He}$ from the first principles of quantum mechanics. The intimate link between superfluidity and Bose-Einstein condensation thus remains rather complex to understand [5]. The extreme diluteness of ultracold gases prepared in the laboratory allows us to describe interactions between atoms in a very simple manner. One can then make a clear connection between the Hamiltonian describing the system and the physical behavior of the latter.

Most properties of weakly-interacting Bose-Einstein condensates in three dimensions were investigated in detail in recent years. For example, long-range phase coherence was directly observed by making spatially separated regions of a trapped gas interfere [6], and made the observation of Anderson localization of matter waves in a disordered potential possible [7,8]. Superfluidity of Bose-Einstein condensates was demonstrated through the observation of quantized vortices in a rotating cloud [9,10,11]. While most observed phenomena in weakly-interacting Bose gases are well accounted for by a mean-field approach developed in the 1950's 12,13, complex many-body theories are required to describe ultracold gases in the strongly correlated regime [14]. Such gases were produced more recently, using different approaches:

- The interaction strength can be varied using the phenomenon of Feshbach resonance by applying an external magnetic field. While the realization of stable strongly-interacting Bose gases is prevented by the large inelastic losses encountered when approaching a Feshbach resonance [15], Pauli exclusion principle strongly inhibits inelastic losses in Fermi gases even for large interaction strengths [16. This enabled one to produce ultracold Fermi gases in the strongly-interacting regime [17, 18.

- Using periodic potentials in three directions created by off-resonant standing waves of laser light, it is possible to pin atoms into the wells of a periodic lattice 19,20 . The gas is then described by a Hubbard Hamiltonian, a fundamental model introduced in solid state physics in order to describe a transition between conducting and insulating systems. On-site interactions, than can be larger than the atom tunneling amplitude, induce strong many-body correlations. By varying the optical lattice depth, a quantum phase transition between a superfluid state to a Mott insulator state was observed using dilute Bose gases in 20]. The recent realization of ultracold Fermi gases 
in the Hubbard regime 21,22 offers a unique opportunity to measure the phase diagram of the Fermi-Hubbard model, and possibly understand its connection to high- $T_{c}$ superconductivity.

- By freezing the atom motion along one or two directions using tightly confining potentials, it is possible to create ultracold gases in an effective reduced dimensionality, a situation in which manybody correlations are enhanced 14. A Bose gas in one dimension, described by Luttinger liquid theory, was first obtained in [23,24], while the Berezinskii-Kosterlitz-Thouless transition of a twodimensional Bose gas was observed in $[25,26]$. Very recently spin-imbalanced Fermi gases in one dimension were produced at Rice 27 .

The viewpoint can be reversed by considering ultracold gases as unique tools to investigate open problems from condensed matter. Thanks to their extreme purity, the good control of the trapping potentials used to hold the gas, and the simple description of interactions, it is possible to write down the system's Hamiltonian from first principles of quantum mechanics. Reference Hamiltonians, such as the Fermi-Hubbard model, or spin chain/ladder Hamiltonians, were proposed and extensively studied in the field of condensed matter, due to their analogy with (much more complex) real condensed matter materials. However, most of these Hamiltonians are unsolved. In addition, their numerical simulation is practically impossible due to the exponential growth of the size of quantum systems' Hilbert space with atom number. Using an adequate ultracold gas system, it is possible to realize these Hamiltonians in the laboratory and directly observe their solutions. Ultracold gases thus realize an analog simulation of physical problems, an idea initially proposed by R. Feynman in 1982 [28].

\subsection{Ultracold Fermi Gases: State of the Art}

Let us now introduce the field of ultracold Fermi gases that will be addressed in this thesis. Following the achievement of Bose-Einstein condensation, ultracold Fermi gases were first produced in the regime of degeneracy and weak interactions, and the effect of Fermi-Dirac statistics was identified through the observation of Fermi pressure 29, 30. 31. While $s$-wave interactions are forbidden between fermionic atoms in the same internal state due to Pauli exclusion principle, interactions are allowed in a twocomponent Fermi gas. In the ultracold regime, they are described by a single parameter, the scattering length $a$. Using the phenomenon of Feshbach resonance, $a$ can be tuned using an external magnetic field (see Fig 1.1 a), making it possible to reach the strongly-interacting regime and hope to observe a BCS-type superfluid [32]. Strongly-interacting Fermi gases were first produced in $2002[17,18$ and, contrary to Bose gases, were found to be particularly stable even for very large interaction strengths, as first explained in [16. The production of degenerate and strongly interacting Fermi gases was then achieved in a several

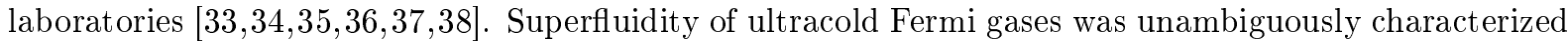
through the gas response to a rotation of the confining potential, more precisely a resistance to rotation in the low rotation speed regime $[39]$ and the formation of a vortex lattice for a larger rotation speed [40] (see Fig 1.1p). Other evidence for superfluidity was provided by the observation of a critical velocity for energy dissipation of a moving object immersed into the gas [41.

\subsubsection{BEC-BCS Crossover}

By varying the scattering length value across a Feshbach resonance, one observes a smooth crossover between two limiting situations $33|34,35| 36$ :

- The two-body problem admits a molecular bound state when the scattering length $a$ is positive, with a binding energy $E_{b}=-\hbar^{2} / m a^{2}$. When $\left|E_{b}\right|$ is much larger than the gas temperature $k_{B} T$ and Fermi energy $E_{F}$, the gas can be viewed as a mixture of strongly bound molecules, possibly 

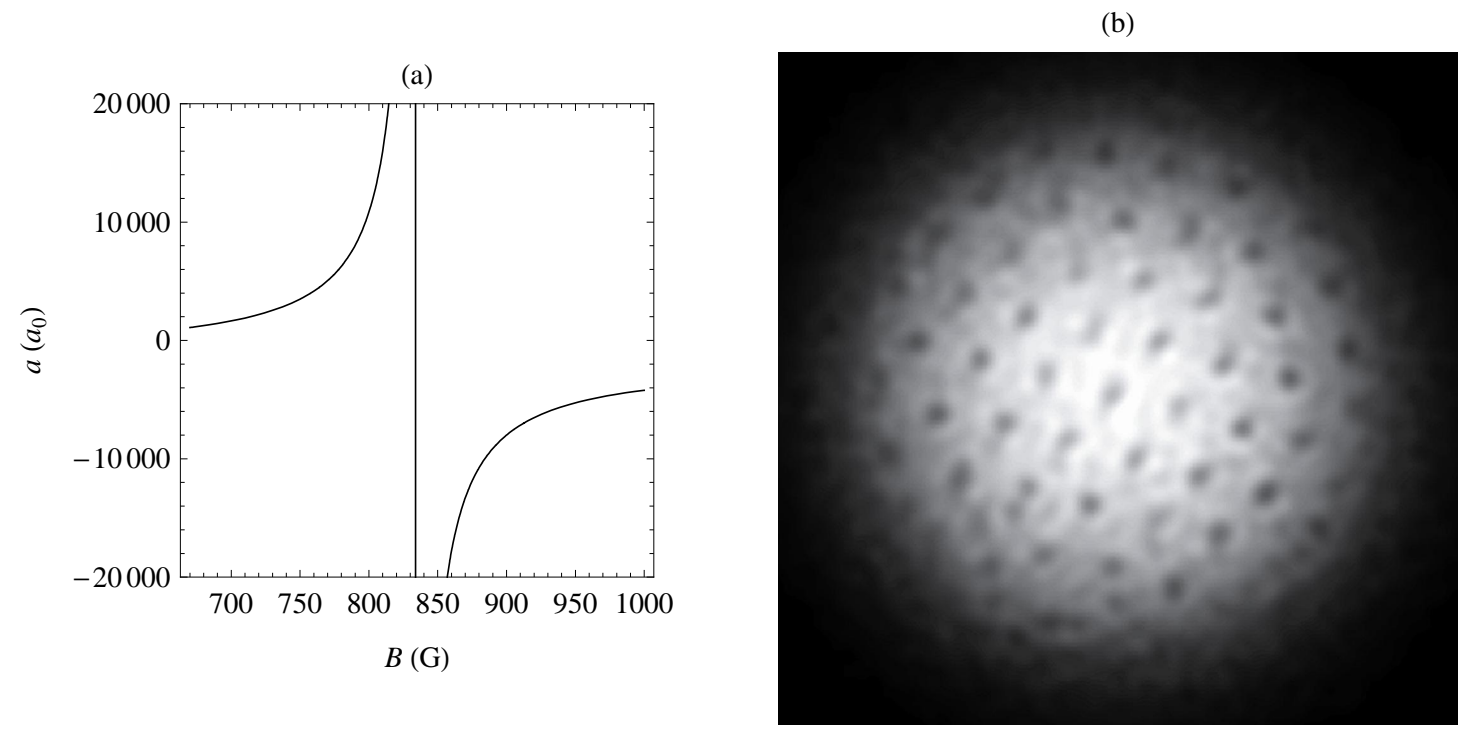

Figure 1.1: (a) $s$-wave scattering length $a$ (in units of the Bohr radius $a_{0}$ ) describing ultracold collisions between the two lowest internal states of ${ }^{6} \mathrm{Li}$, as a function of magnetic field. (b) Absorption image of a rotating strongly-interacting Fermi gas, from [40]. The observation of a vortex lattice demonstrates the superfluid character of degenerate strongly-interacting Fermi gases.

mixed with unbound majority atoms in the case of spin population imbalance. Molecules made of two fermionic atoms behave in this limit as points-like bosons and form in the degenerate regime a molecular Bose-Einstein condensate [42, $43[36]$.

- In the limit of small negative values of $a$, the amplitude of interactions is small. Although the two-body problem does not admit a bound state, interactions between atoms are strongly modified by Pauli exclusion principle which forbids scattering towards states already occupied by other atoms. Effective bound states, the so-called Cooper pairs, become stabilized by this many-body behavior [44], and the gas forms a Bardeen-Cooper-Schrieffer superfluid at very low temperature [45. However, this pairing is not very robust and the critical temperature for superfluidity $T_{c} \sim$ $T_{F} \exp \left(-\pi / 2 k_{F}|a|\right)$ is exponentially small for weak interactions $\left(k_{F}\right.$ is the Fermi momentum, $T_{F}$ is the Fermi temperature).

The strongly-interacting regime $1 / k_{F}|a| \lesssim 1$ smoothly interpolates between the bosonic and fermionic regimes. The strength of interactions then makes the theoretical understanding difficult.

\subsection{2 'High- $T_{c}$ ' Superfluidity of a Fermi Gas with Resonant Interactions}

By applying a magnetic field right at the center of a Feshbach resonance, it is possible to reach the regime $a=\infty$ where the interaction strength takes the maximum value allowed by quantum mechanics, the so-called unitary limit. In this situation superfluidity is found to be particularly robust.

The transition temperature $T_{c}$ for superfluidity was first measured for a trapped gas in $4346 \mid 39$ | 47 . In this thesis we describe the first measurement of the transition temperature for a homogeneous gas [47,48]:

$$
T_{c}=0.16 T_{F}, \quad T_{F}=\frac{\hbar^{2}}{2 m}\left(3 \pi^{2} n\right)^{2 / 3},
$$

where $n$ is the total atom density. The critical temperature $T_{c}$ being on the order of the Fermi temperature 
$T_{F}$, ultracold Fermi gases constitute in a sense a high- $T_{c}$ system ${ }^{\text {* }}$. The exceptional robustness of the superfluid state was also demonstrated through the large critical velocity measured by the MIT group [41]:

$$
v_{c}=0.3 v_{F}, \quad v_{F}=\sqrt{\frac{2 E_{F}}{m}},
$$

as well as the chemical potential difference between the two spin components $\mu_{1}-\mu_{2}$ required to break superfluidity 49 48] (see Chapter 5 of this thesis):

$$
\left|\mu_{1}-\mu_{2}\right|_{c}=0.18 E_{F} .
$$

The connection with Bose-Einstein condensation was characterized by a direct measurement of the condensate fraction $35,36,47$, and the importance of many-body effects was established through the measurement of the single-particle excitation energy gap [50,51] and closed-channel fraction [38].

\subsubsection{Spin-Imbalanced Fermi Gases}

Superfluidity of spin-balanced Fermi gases is intimately related to pairing between atoms with opposite spins. A new degree of freedom is provided by the possibility to prepare different atom numbers in both spin states. The first open question is then to understand whether superfluidity survives to spin imbalance. This issue was addressed in the context of solid-state superconductors by Clogston [52] and Chandrasekhar [53] in the 1960's. They predicted that superfluidity resists to a magnetic field (lifting the degeneracy between the two electronic spin states) up to a critical value.

These old issues of solid state physics were first addressed using ultracold gases in 2006, by pioneering works from the MIT and Rice groups $[54,55]$. Both groups revealed that a spin-imbalanced trapped gas exhibits a phase separation between a superfluid core where atoms are paired and densities are thus equal for both species, and an external normal phase. Surprisingly, both groups' observations were not in agreement concerning the normal phase: in Rice experiment, the normal phase was found to be fully polarized with majority atoms, while in the MIT experiment the normal phase is split into an intermediate shell with atoms from both species mixed together, and a fully polarized outer rim. This discrepancy has remained unexplained up to now.

The phase diagram of spin-imbalanced Fermi gases is very rich. Exotic phases with spin-asymmetric pairing are predicted to be stable, the most famous one being the Fulde-Ferrell-Larkin-Ovchinnikov state in which the order parameter is modulated in space [56, 57. Among other proposals, we mention a gapless ('breached pair') superfluid state [58, or a state with deformed Fermi surfaces [59]. However these states are expected to occupy a very small part of the phase diagram and their observation may require a substantial experimental effort.

\subsection{Issues and Perspectives Addressed in this Thesis}

\subsubsection{Universal Thermodynamics of an Ultracold Fermi Gas}

The thermodynamic equation of state is a key quantity for the macroscopic description of ultracold Fermi gases, and its determination from experiment would constitute a benchmark for many-body theories. We will see that expressing the equation of state in the grand-canonical ensemble is more convenient for its investigation in the laboratory. In this ensemble, the equation of state is written as a relationship $\Omega\left(V, \mu_{1}, \mu_{2}, T, a\right)$ between the grand-potential $\Omega$, the volume of the system $V$, the chemical potentials $\mu_{1}, \mu_{2}$ of both spin states, the gas temperature $T$, and the scattering length $a$ describing low-energy

* The mechanism for superfluidity in high- $T_{c}$ materials from condensed matter, for which interactions are expected to be repulsive, is probably very different from the one in ultracold Fermi gases, where interactions are effectively attractive. 
collisions. The grand-potential being related to the pressure $P$ through, $\Omega=-P V$, the equation of state can also be written using intensive variables only, as:

$$
P\left(\mu_{1}, \mu_{2}, T, a\right)
$$

The correspondence with equations of states expressed in other statistical ensembles is provided by Legendre transforms.

It is believed that for Fermi gases with short-range interactions, such as the ${ }^{6} \mathrm{Li}$ gases addressed in this thesis, the scattering length $a$ is sufficient to account for all interaction effects. Therefore the precise nature of the fermionic species plays no role in the equation of state. Hence the relation $P\left(\mu_{1}, \mu_{2}, T, a\right)$ is expected to be universal, in the sense that it is identical for all systems of fermions with short-range interactions. Its determination using ultracold gases is thus directly relevant to describe another similar system, namely neutron matter in the outer crust of neutron stars. Indeed, neutron matter is made of a mixture of the two spin states of neutrons, whose interactions are also short-range and in the cold-collision regime, captured by a scattering length $a=-18.6 \mathrm{fm}$ (at least in the low-density regime $\left.n \ll 10^{41} \mathrm{~m}^{-3}\right]\left[60\right.$. As the Fermi temperature $T_{F}>10^{11} \mathrm{~K}$ is much larger than the temperature $T \sim 10^{6} \mathrm{~K}$, temperature effects are negligible in neutron matter. The measurement of the equation of state $P\left(\mu_{1}, \mu_{2}, T \simeq 0, a\right)$ that we describe in Chapter 5 is thus directly relevant to the description of the crust of neutron stars. We also mention analogies with other quantum many-body systems such as heavy nuclei and dense QCD matter [61.

\subsubsection{Previous Thermodynamic Studies}

The measurement of the equation of state of an ultracold gas aims to provide a benchmark for manybody theories. However, most calculations are made on homogeneous systems while ultracold gases prepared in the laboratory are held in a trapping potential and are thus inhomogeneous. However, the comparison can still me made in most situations using the local density approximation: The cloud size, imposed by the trap stiffness, is usually much larger than the characteristic length scale over which the cloud feels a variation of the trapping potential $V(\mathbf{r})$. Therefore the gas can be locally described by the equation of state of a homogeneous gas. While the temperature $T$ is uniform over the cloud, the trapping potential induces a slow pressure variation $\nabla P=-\left(n_{1}+n_{2}\right) \nabla V$, according to the laws of hydrostatics. Equivalently, the mechanical equilibrium can be written as:

$$
\mu_{i}(\mathbf{r})=\mu_{i}^{0}-V(\mathbf{r})
$$

$\mu_{i}^{0}$ being the global chemical potential for species $i(i=1,2)$.

In the first thermodynamic studies of ultracold Fermi gases, the equation of state of the whole trapped gas was measured. For example, an equation of state of a trapped Fermi gas with resonant interactions was obtained in 62, 63, relating the total energy to the total entropy. In 64 was performed a precision measurement of the frequency of collective modes of a trapped gas in the BEC-BCS crossover. In both cases the comparison with theory requires to integrate theoretical equations of state over the trap. This makes the comparison indirect, and tends to hide possibly small differences between theories. Discriminating between a mean-field BCS theory of the BEC-BCS crossover and advanced Monte-Carlo calculations $65,66,67$ thus required to reach an excellent accuracy in the measurement of collective mode frequencies 64 . Finally, the trap averaging smears sharp features in the equation of state, and the superfluid/normal phase transition is almost invisible in 63 .

${ }^{\dagger}$ Since free neutrons are unstable, neutron matter must be viewed as a model system in the large dilution limit. 


\subsubsection{Measurement of the Local Pressure Inside a Trapped Gas}

In this thesis we developed a new method for directly measuring the equation of state of a homogeneous gas. Since the gas is locally homogeneous in the local density framework, measuring local properties inside a trapped gas directly gives access to the equation of state of the homogeneous gas. This idea was first exploited in 68]: an Abel transform of in situ images of a trapped gas was used to probe the local atom density. Due to the dramatic decrease of the signal-to-noise ratio induced by the Abel transform, the equation of state deduced from this procedure is very noisy, despite the large atom numbers reached in the MIT experiment. This shows the apparent difficulty to probe local quantities inside a trapped gas.

During my PhD, I established, simultaneously to [69, a simple relation between the local pressure inside a trapped gas and the optical density of an in situ absorption image (see Fig 1.2 ):

$P\left(\mu_{1 z}, \mu_{2 z}, T\right)=\frac{m \omega_{x} \omega_{y}}{2 \pi}\left(\bar{n}_{1}(z)+\bar{n}_{2}(z)\right), \quad$ where $\quad \mu_{i z}=\mu_{i}^{0}-\frac{1}{2} m \omega_{z}^{2} z^{2} \quad$ and $\quad \bar{n}_{i}(z)=\int \mathrm{d} x \mathrm{~d} y n(x, y, z)$.

We mention that the idea of this relation can also be found in an earlier work 70. Equation (1.2) states that the pressure on the $z$ axis is obtained by integrating along $x$ the cloud absorption image, taken along $y$. Here $\omega_{u}(u=x, y, z)$ is the trapping frequency along $u$. Contrary to the Abel transform process, integrating along $x$ increases the signal-to-noise ratio and the pressure profile along $z$ is thus obtained with a low noise. As soon as the gas temperature $T$ and global chemical potentials $\mu_{i}^{0}$ are determined, each pixel row $z$ provides an experimental value $P\left(\mu_{1 z}, \mu_{2 z}, T\right)$ of the equation of state of the homogeneous gas. The analysis of a single image of a trapped gas thus leads to a large number of independent determinations of the equation of state. By collecting the data from all pixel rows of several images, one obtains after proper averaging a very-low-noise equation of state 48,71 . This is a great improvement over the studies of the thermodynamics of trapped gases, for which one experimental run is required to obtain one point.

The main line of this thesis is the implementation of a new method for determining the grand-canonical equation of state of a homogeneous Fermi gas, making use of equation (1.2).

\subsection{Outline of this Thesis}

\subsubsection{Chapter 2: Experimental Setup}

We first describe the experimental setup producing ultracold ${ }^{6} \mathrm{Li}$ Fermi gases. Our setup is based on the combined use of ${ }^{7} \mathrm{Li}$ and ${ }^{6} \mathrm{Li}$ species. ${ }^{6} \mathrm{Li}$ atoms are sympathetically cooled by forced evaporation of the ${ }^{7} \mathrm{Li}$ component in a magnetic trap. This allows us to transfer a large number of ${ }^{6} \mathrm{Li}$ atoms in an optical dipole trap, where a very efficient evaporation is performed on a mixture of the two lowest internal states, with resonant interactions. Thermometry is performed by keeping a small amount of ${ }^{7} \mathrm{Li}$ atoms.

\subsubsection{Chapter 3: Measuring the Equation of State of a Homogeneous Ultracold Gas}

The method for determining the equation of state of a homogeneous ultracold gas is presented in Chapter 3. We demonstrate equation 1.2 and discuss its validity range. We then illustrate the power of our method by applying it to Bose gases in well understood regimes.

Using a single in situ image from [31], we obtain the equation of state of a weakly-interacting Bose gas, from the classical to the condensed regime. It reveals the characteristic features of a weakly-interacting Bose gas, namely a bosonic bunching behavior in the normal phase, a Thomas-Fermi pressure in the Bose-Einstein condensate, and a condensation threshold $\mu=0$. 


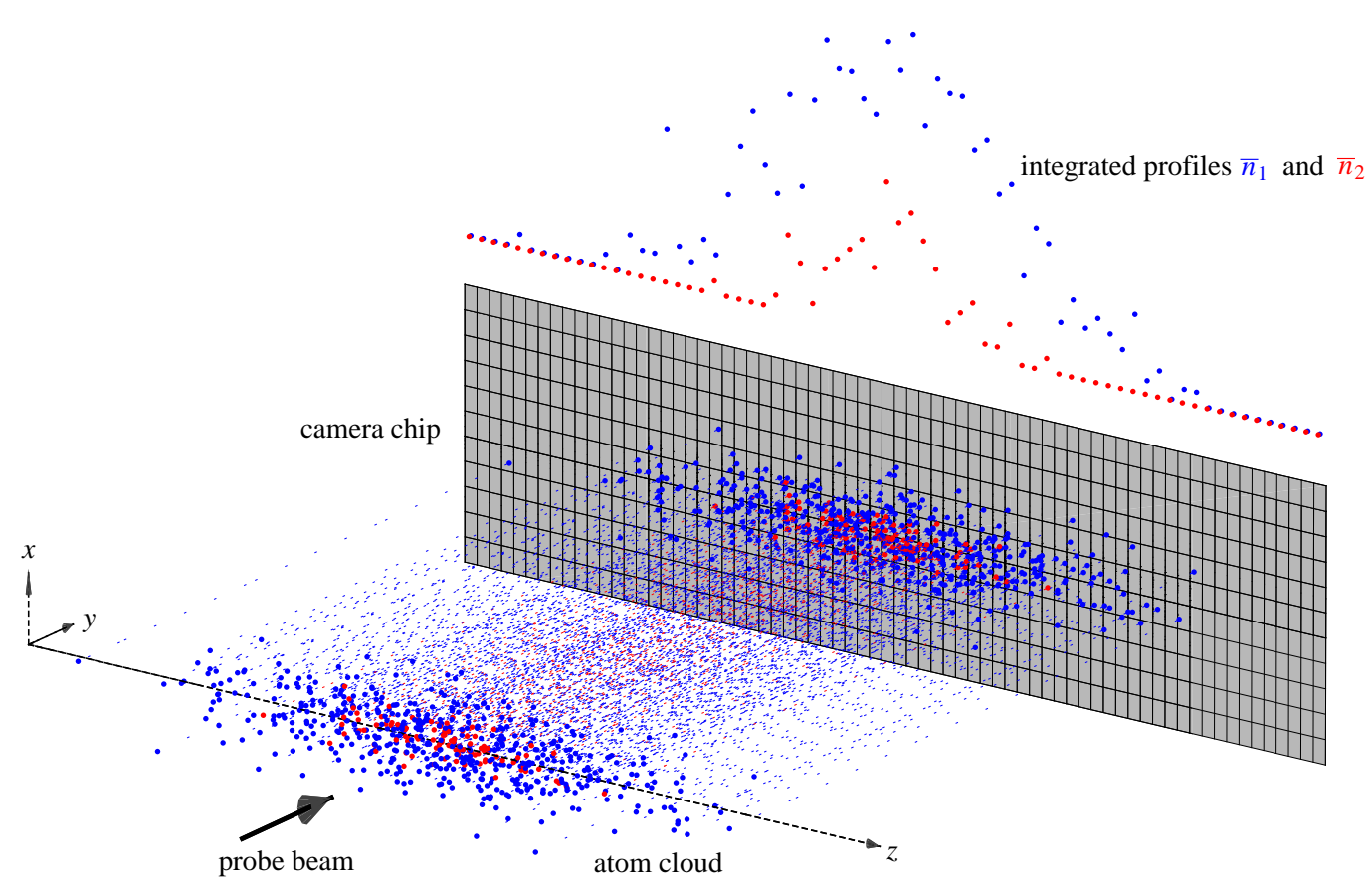

Figure 1.2: Local pressure measurement scheme: a two-component ultracold gas is imaged in situ along $y$. Further integration along $x$ provides the doubly-integrated profiles for both components, $\bar{n}_{1}(z)$ and $\bar{n}_{2}(z)$. The gas pressure along the $z$ axis is then obtained using equation 1.2 .

We also apply the method to Bose gases in an optical lattice, in the limit of large lattice depth, using experimental data from 72 . The grand-canonical equation of state deduced from this analysis directly reveals a Mott-insulator behavior: sites are occupied by an integer number of atoms, whose value depends on the chemical potential value. We show that representing the experimental data in terms of an equation of state is suited for investigating finite-temperature effects.

\subsubsection{Chapter 4: Thermodynamics of a Strongly-Interacting Fermi Gas}

In Chapter 4 we study the thermodynamics of a Fermi gas with resonant interactions $[48$. For simplicity reasons we restrict our study to a spin-symmetric configuration. Applying our method requires to independently measure the gas temperature, which is notoriously difficult in the case of strong interactions. Inspired by the Innsbruck group [73], we implemented a new thermometry for strongly-interacting gases: the temperature is measured on a small amount of weakly-interacting ${ }^{7} \mathrm{Li}$ atoms, immersed in the ${ }^{6} \mathrm{Li}$ cloud and at thermal equilibrium with it.

The equation of state deduced from our data enables us to make the first direct comparison with many-body theories. Surprisingly, none of them accounts for our observations over their full temperature range. In the high-temperature regime, we extract several coefficients of the virial expansion. They agree with the exact resolution of the three-body problem 74,75 and provide a benchmark for a future resolution of the four-body problem. In the low-temperature regime, which is the most difficult to handle from theory, our data reveal an unexpected feature: above the critical temperature for superfluidity, the normal phase is accurately described as a Landau Fermi liquid. This observation is in disagreement with the expectations of several many-body theories, which predict that pair correlations should significantly 
modify the Fermi liquid picture. Finally, we observe a clear thermodynamic signature of the superfluid transition, occurring at a critical temperature:

$$
\frac{T_{c}}{\mu}=0.32(3),
$$

quantitatively confirming the 'high- $T_{c}$ ' character of Fermi gases with resonant interactions.

\subsubsection{Chapter 5: Ground State of an Attractive Fermi Gas: Phase Diagram and Equation of State}

In Chapter 5 we describe the measurement of the equation of state $P\left(\mu_{1}, \mu_{2}, T \simeq 0, a\right)$ of a two-component Fermi gas in the BEC-BCS crossover, in the limit of very low temperatures 71 .

In particular we obtain the equation of state in the spin-symmetric configuration, a situation where the gas is superfluid. As explained above, this equation of state is directly relevant to the description of the outer crust of neutron stars. Our observations validate Fixed-Node Monte-Carlo simulations in the entire BEC-BCS crossover 65, 66 67, and are in agreement with the Lee-Huang-Yang corrections to mean-field for low-density bosonic and fermionic superfluids. These exact results, obtained 60 years ago $76,77,78]$, had only been indirectly observed up to now [64 79$]$.

The equation of state $P\left(\mu_{1}, \mu_{2}, T \simeq 0, a\right)$ also addresses the physics of spin-imbalanced Fermi gases. Before our work, it was believed that the difference between the observations in Rice and MIT experiments (see above) was due to different atom numbers and trap aspect ratios. We prepared spin-imbalanced Fermi gases in a configuration close to Rice experiment and our observations unambiguously confirm the ones of the MIT group, namely the existence of a normal phase where atoms of both spin components are mixed, with different densities. We show that the normal phase can be accurately described as a Landau Fermi liquid, despite strong interactions: minority atoms are dressed in quasi-particles, the so-called Fermi polarons, with renormalized characteristics such as an effective mass 80, 81, 82. The normal phase is then merely described, in most of the parameter range, as a mixture of ideal Fermi gases of majority atoms and Fermi polarons. The polaron effective masses extracted from our data are in agreement with the most advanced theories $83,84,85,86$.

Finally we characterize the quantum phase transition between the superfluid and normal states, in good agreement with Fixed-Node Monte-Carlo calculations [81,67.

\subsubsection{Chapter 6: Axial Breathing Modes of a Spin-Imbalanced Fermi Gas}

In Chapter 6 the effect of spin imbalance is addressed using a different technique, namely the study of axial breathing modes. When the two spin components oscillate in phase, we observe a smooth crossover between a hydrodynamic oscillation when the superfluid core is large, and a collisionless behavior for highly polarized gases. This reveals the amplitude of relaxation phenomena occurring in Fermi gases, in the different polarization regimes. For very large polarizations, we also observe an out-of-phase collective mode, associated with the oscillation of Fermi polarons inside the majority Fermi sea. We extract from the frequency of this oscillation the polaron effective mass, in agreement with the value deduced from the equation of state.

In conclusion, we present the most important open questions that could be answered by extending the equation of state measurement to other parameter domains, such as finite-temperature effects in the BEC-BCS crossover and with spin imbalance. This method will also be applied in our experiment to strongly-interacting Bose gases using Feshbach resonances with the ${ }^{7} \mathrm{Li}$ isotope. 


\section{Chapter 2}

\section{Experimental Setup}

In this chapter we describe the experimental setup used for our study of ultracold Fermi gases. After a short explanation of the global strategy used for producing degenerate Fermi gases, all stages will be depicted one by one. The first stages of the experiment were already described in detail in L. Tarruell's thesis [87]; therefore I will focus on the performances of our setup reached in these stages, and then give a more extensive description of the optical trap and atom imaging system.

\subsection{Global Description of the Experimental Setup}

A first version of the ${ }^{6} \mathrm{Li}^{7} \mathrm{Li}$ experiment was constructed and operated between 1997 and 2006, from the first realization of degenerate Fermi gases to the first studies of the BEC-BCS crossover. At the beginning of this $\mathrm{PhD}$ (in the fall of 2006), the construction of a second-generation setup was under way, in order to realize more complex experiments. The general experimental scheme, based on the combined use of ${ }^{6} \mathrm{Li}$ and ${ }^{7} \mathrm{Li}$, was preserved, and the improvement of several stages in the experiment led to a 10-times improvement of the atom number in the quantum-degenerate regime, as well as a 4-times increase of the experiment cycling rate. In addition, the geometry of the final trap was completely modified, allowing the implementation a new image analysis technique specific to elongated traps.

Our experiment is based on an intermediate stage where atoms are transfered from a magneto-optical to a magnetic Ioffe-Pritchard trap, and cooled using radio-frequency-induced forced evaporation (see Fig 2.1. Evaporation is performed on a spin-polarized ${ }^{7} \mathrm{Li}$ cloud with a much smaller amount of ${ }^{6} \mathrm{Li}$, the latter being progressively cooled by contact with the ${ }^{7} \mathrm{Li}$ component. This stage allows us to prepare up to $5 \times 10^{6}{ }^{6} \mathrm{Li}$ atoms at a temperature low enough to yield a $100 \%$-efficient transfer into an optical dipole trap. This number is several times larger than the ones obtained in experiments where ${ }^{6} \mathrm{Li}$ atoms are directly transfered from a magneto-optical trap to an optical dipole trap. We then make a ${ }^{6} \mathrm{Li}$ mixture in the two lowest internal states, in a bias magnetic field corresponding to resonant interactions. Thanks to the very large collision rate, an efficient evaporation is performed by lowering the optical trap power. The superfluid transition is reached with more than $2 \times 10^{5}$ atoms in each spin state, and we obtain, after a deeper evaporation, clouds containing up to $10^{5}$ atoms in each spin state at a temperature $T / T_{F}=0.03(3)$. Our setup also enables us to produce spin-imbalanced Fermi gases in the degenerate regime.

In addition, our setup gives us the possibility to use ${ }^{7} \mathrm{Li}$ in the final experiments on degenerate Fermi gases. In Chapter 4 we thus use a small amount of ${ }^{7} \mathrm{Li}$, left inside the ${ }^{6} \mathrm{Li}$ cloud, as a thermometer, solving the notoriously difficult issue of strongly-interacting gases thermometry. Furthermore, broad Feshbach resonances in ${ }^{7} \mathrm{Li}$ makes it a versatile tool for future experiments, such as the study of Bose-Einstein condensates in the strongly-interacting regime or Bose-Fermi mixtures. 

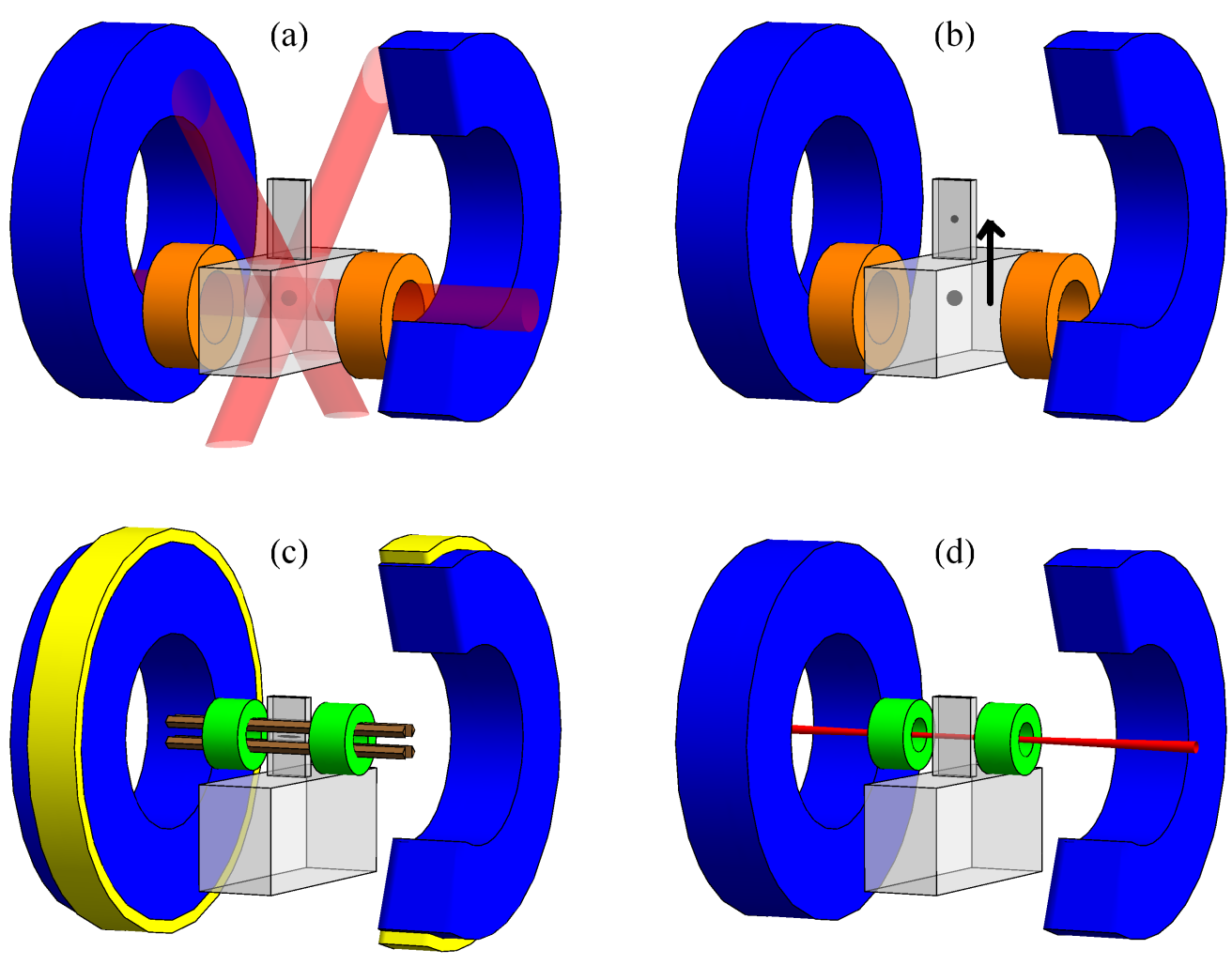

Figure 2.1: Scheme of the main steps for producing degenerate Fermi gases. (a) In the MOT stage, ${ }^{6} \mathrm{Li}$ and ${ }^{7} \mathrm{Li}$ atoms are trapped using a combination of a quadrupole trap and three pairs of light beams. (b) They are then transfered into a quadrupole trap and moved into a small appendage of the cell, using two pairs of coils in anti-Helmoltz configuration. (c) In a tight Ioffe trap, ${ }^{7} \mathrm{Li}$ is evaporated up to $100 \mu \mathrm{K}$. (d) ${ }^{6} \mathrm{Li}$ atoms are then transfered into a hybrid magnetic-optical trap (bottom-right), and further evaporation is performed on a mixture of the two lowest internal states at $834 \mathrm{G}$. A small quantity of ${ }^{7} \mathrm{Li}$ atoms can be kept for thermometry.

\section{$2.2{ }^{7} \mathrm{Li}-{ }^{6} \mathrm{Li}$ Magneto-Optical Trap}

The first stage of the experiment is the simultaneous trapping of ${ }^{7} \mathrm{Li}$ and ${ }^{6} \mathrm{Li}$ clouds with large atom numbers in a magneto-optical trap (MOT). We first describe the specificities of our laser system, and the performance of the Zeeman slower and MOT.

\subsubsection{Laser System}

Compared with the previous setup, we completely changed the laser system. Due to the very small Zeeman structure in the $2^{2} P_{3 / 2}$ excited states, Lithium MOTs require large laser powers for both cooling and repumping transitions. Instead of concentrating all frequencies emitted by Master lasers into a single tapered amplifier [88], we attributed one slave diode laser per frequency, delivering an intermediate power. We use low-cost laser diodes manufactured for DVD players (HITACHI HL6545MG), delivering up to 180 $\mathrm{mW}$ of laser power. These diodes are heated up to $70{ }^{\circ} \mathrm{C}$ in order to bring the laser wavelength at $671 \mathrm{~nm}$. This tends to increase temperature fluctuations and a new design of well thermally isolated boxes was 
made. The power available for each frequency allows us to use optical fibers as spatial filters between the laser diodes and the MOT. This strongly increases the long-term stability of the MOT alignment, and enables one to maintain all optical elements before the fibers optimally aligned without having to realign the other components. In addition, since the replacement of a single diode does not require to realign the MOT stage, we compensate the loss of laser power, due to the $\sim 70 \%$ fiber coupling efficiency, by increasing the current driving the diodes much higher that the constructor's specifications, paying the price of a lifetime reduced to $\sim 3$ months. In the end, we typically obtain about $50 \mathrm{~mW}$ of laser power directly available for the MOT, in a nearly perfect gaussian mode. These diodes are also used as Master lasers, emitting $40 \mathrm{~mW}$ of laser power scannable over $1 \mathrm{GHz}$, as well as for Zeeman slowing. The large number of lasers (15 lasers) makes the run-to-run laser stability relatively poor. However, using a single type of diode strongly simplifies the laser maintenance and makes our setup very flexible.

\subsubsection{Zeeman Slower}

We use a spin-flip Zeeman slower for the MOT loading from an atomic beam. The atomic beam is emitted by an oven containing natural liquid $\mathrm{Li}$ (with a $7.5 \%{ }^{6} \mathrm{Li}$ fraction). The Zeeman slower is characterized by a large capture velocity $\sim 1100 \mathrm{~m} / \mathrm{s}$, and final magnetic field of $200 \mathrm{G}$ in the MOT region, so that slowing laser beams do not affect the magneto-optical trap. The large laser power available allows us to use beams with a large diameter $\left(3 \mathrm{~cm}\right.$ in the MOT region), and an intensity $\sim 2 I_{s}$, where $I_{s} \simeq 2.5 \mathrm{~mW} / \mathrm{cm}^{2}$ is the saturation intensity for the $S-P$ lines. With these favorable characteristics, we obtain a large flux of about $10^{9}{ }^{7} \mathrm{Li}$ atoms $/ \mathrm{s}$ (and $10^{8}{ }^{6} \mathrm{Li}$ atoms $/ \mathrm{s}$ ), loaded into the magneto-optical trap.

\subsubsection{Dual Species Magneto-Optical Trap}

Slowed atoms are then captured into a magneto-optical trap, represented in Fig 2.2 . The characteristics of the magneto-optical trap are listed in Table 2.1. As the atoms are the transfered into a deep magnetic trap, the MOT optimization aims to increase the atom number, regardless of the MOT temperature or maximum density. Therefore we use a relatively small quadrupole field gradient, of amplitude $24 \mathrm{G} / \mathrm{cm}$ in the strong direction, in order to minimize inelastic losses. Laser beams are also far detuned to the red in order to increase the capture velocity. The laser beams have a $2.5 \mathrm{~cm}$ diameter and a laser intensity $I \simeq I_{s}$, values for which the trapped atom number is maximum. We typically trap $10^{10}{ }^{7} \mathrm{Li}$ atoms at a temperature of $4 \mathrm{mK}$. The ${ }^{6} \mathrm{Li}$ MOT is strongly affected by the presence of ${ }^{7} \mathrm{Li}$ due to light-assisted inelastic ${ }^{6} \mathrm{Li}-{ }^{7} \mathrm{Li}$ collisions. We thus only trap up to $4 \times 10^{8}{ }^{6} \mathrm{Li}$ atoms, while up to $8 \times 10^{8}$ atoms can be trapped when ${ }^{7} \mathrm{Li}$ laser light is switched off. We also mention that the ${ }^{6} \mathrm{Li}$ cooling light induces a $25 \%$ loss of ${ }^{7} \mathrm{Li}$ atoms, due to the proximity of several hundred of $\mathrm{MHz}$ with ${ }^{7} \mathrm{Li} D_{1}$ transitions, inducing light-assisted inelastic collisions.

Full atom loading is achieved in about $30 \mathrm{~s}$. Before transferring the atoms into a magnetic trap, we perform a dynamical compressed-MOT phase: the repumping light intensity is ramped to zero in $8 \mathrm{~ms}$ while the cooling light is brought closer to resonance. The cloud's temperature is decreased by a factor 4 , down to $1 \mathrm{mK}$, at the price of a $35 \%$ atom loss. At the end of this stage, atoms are spread in all Zeeman states of the lowest hyperfine manifold.

In the next section, we describe the transfer into a purely magnetic trap and the radio-frequency forced evaporation.

\subsection{Sympathetic Cooling in a Magnetic Trap}

The magnetic trap stage aims at producing relatively large ${ }^{6} \mathrm{Li}$ clouds at a temperature low enough to ensure a complete transfer into an optical dipole trap. A more efficient cooling scheme can then be used, 


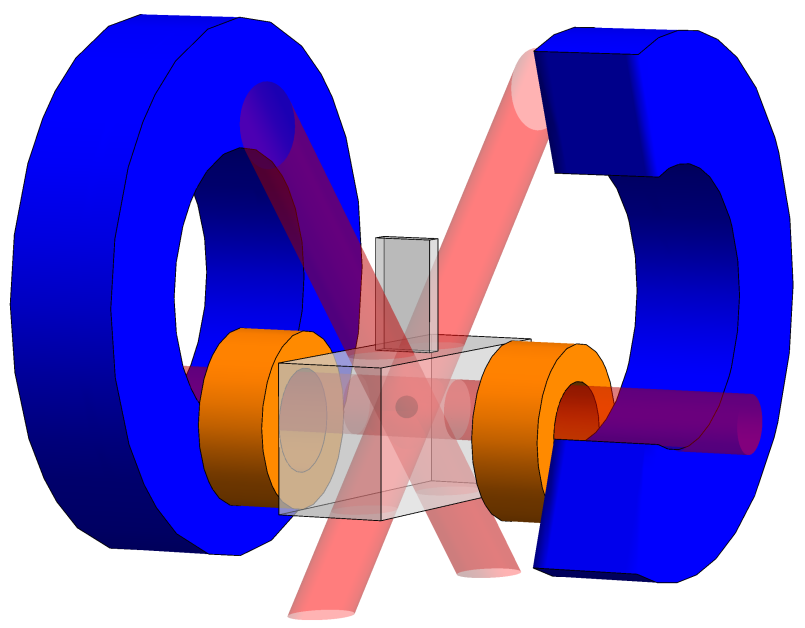

Figure 2.2: Scheme of the magneto-optical trap. The quadrupole field is created by two pairs of coils in anti-Helmoltz configuration, in order to position the zero-field point in the middle of the laser beams (in red).

\begin{tabular}{lcccc}
\hline \hline & ${ }^{7} \mathrm{Li} \mathrm{MOT}$ & ${ }^{7} \mathrm{Li} \mathrm{CMOT}$ & ${ }^{6} \mathrm{Li} \mathrm{MOT}$ & ${ }^{6} \mathrm{Li} \mathrm{CMOT}$ \\
\hline Cooling beam intensity $\left(I_{s} \simeq 2.54 \mathrm{~mW} / \mathrm{cm}^{2}\right)$ & 1.0 & 1.0 & 1.0 & 1.0 \\
Cooling beam detuning $(\Gamma \simeq 5.87 \mathrm{MHz})$ & -6.5 & -3.3 & -5.5 & -1.7 \\
Repumping beam intensity $\left(I_{s}\right)$ & 1.0 & 0 & 1.0 & 0 \\
Repumping beam detuning $(\Gamma)$ & -5.5 & -4.7 & -2.5 & -1.7 \\
Atom number & $10^{10}$ & $6 \times 10^{9}$ & $4 \times 10^{8}$ & $2.5 \times 10^{8}$ \\
Temperature $(\mathrm{mK})$ & 4.2 & 1.0 & 4.2 & 1.0 \\
\hline \hline
\end{tabular}

Table 2.1: Characteristics of the ${ }^{7} \mathrm{Li}^{6}{ }^{6} \mathrm{Li}$ magneto-optical trap, in steady state and at the end of the dynamic CMOT phase.

taking advantage of the Feshbach resonance relative to ${ }^{6} \mathrm{Li}$ atoms in the lowest internal states. In this section we describe the sympathetic cooling of ${ }^{6} \mathrm{Li}$ atoms with ${ }^{7} \mathrm{Li}$, the latter being cooled using forced evaporation.

\subsubsection{Transfer into an loffe-Pritchard Trap}

After the compressed MOT phase, we optically pump the atoms into the stretched states $\left|F=2, m_{F}=2\right\rangle$ for ${ }^{7} \mathrm{Li}$ and $\left|F=\frac{3}{2}, m_{F}=\frac{3}{2}\right\rangle$ for ${ }^{6} \mathrm{Li}$. The ${ }^{7} \mathrm{Li}-{ }^{6} \mathrm{Li}$ mixture is loaded into a magnetic trap created by the quadrupole field previously used for the MOT stage, and ramped up to much larger magnetic field gradients. In these internal states, the ${ }^{7} \mathrm{Li}^{6}{ }^{6} \mathrm{Li}$ mixture is stable against spin-exchange inelastic collisions. Due to the high MOT temperature and the small scattering length value $a_{77}=-27 a_{0}$ describing collisions between ${ }^{7} \mathrm{Li}$ atoms, the achievement of an efficient evaporative cooling requires the transfer of the atoms into a tight Ioffe-Pritchard trap, as well as an additional in situ Doppler cooling stage.

\section{Optical Pumping and Transfer into a Quadrupole Magnetic Trap}

After the compressed MOT stage, atoms are spread into all levels of the lowest hyperfine manifold. We rapidly switch off the MOT quadrupole field and create a 10-G bias magnetic field. After waiting 
$100 \mu \mathrm{s}$ for the magnetic fields to stabilize, we send optical pumping beams onto the atoms along the bias field direction, and during $300 \mu \mathrm{s} .{ }^{7} \mathrm{Li}$ atoms are mostly pumped in the $\left|F=2, m_{F}=2\right\rangle$ state using a combination of a hyperfine optical pumping beam on the $F=1 \rightarrow F^{\prime}=2 D_{2}$ line, and a Zeeman optical pumping beam on the $F=2 \rightarrow F^{\prime}=2 D_{1}$ line, both beams being circularly $\sigma_{+}$polarized. The Zeeman optical pumping beam is far detuned from the absorption lines in order to address all atoms despite the large cloud's optical density for resonant beams. The MOT quadrupole magnetic field is then ramped up to a maximum gradient of $335 \mathrm{G} / \mathrm{cm}$. After waiting for inelastic collisions with atoms in other spin states, we are left with about $50 \%$ of the ${ }^{7} \mathrm{Li}$ atoms initially held in the compressed MOT. For ${ }^{6} \mathrm{Li}$ atoms we only use a hyperfine optical pumping beam, which is actually the same than the Zeeman optical pumping beam for ${ }^{7} \mathrm{Li}$ due to proximity of ${ }^{7} \mathrm{Li} D_{1}$ lines and ${ }^{6} \mathrm{Li} D_{2}$ lines. The transfer efficiency from the compressed MOT to the magnetic trap is about $35 \%$ for ${ }^{6} \mathrm{Li}$ atoms.

\section{Magnetic Transport into the Science Region}

The vacuum cell was designed with a small appendage in order to be surrounded with a small IoffePritchard trap, providing a very tight confinement (see Fig 2.4. Atoms are transfered from the MOT region to the appendage using two pairs of coils in anti-Helmoltz configuration (see Fig 2.3). The quadrupole zero-field point is displaced in $100 \mathrm{~ms}$ by ramping down the current in the lower coil pair, while ramping up the current in the upper coil pair. In the previous version of the experiment the appendage size was too small and $90 \%$ of the atoms collided with the appendage walls and were lost during the transfer. By increasing the appendage inner size from $3 \mathrm{~mm}$ to $5 \mathrm{~mm}$, the transfer efficiency was brought to $50 \%$. The choice of the appendage size results from a compromise between the increase in transfer efficiency and the decrease in the Ioffe trap confinement when the appendage size is increased.

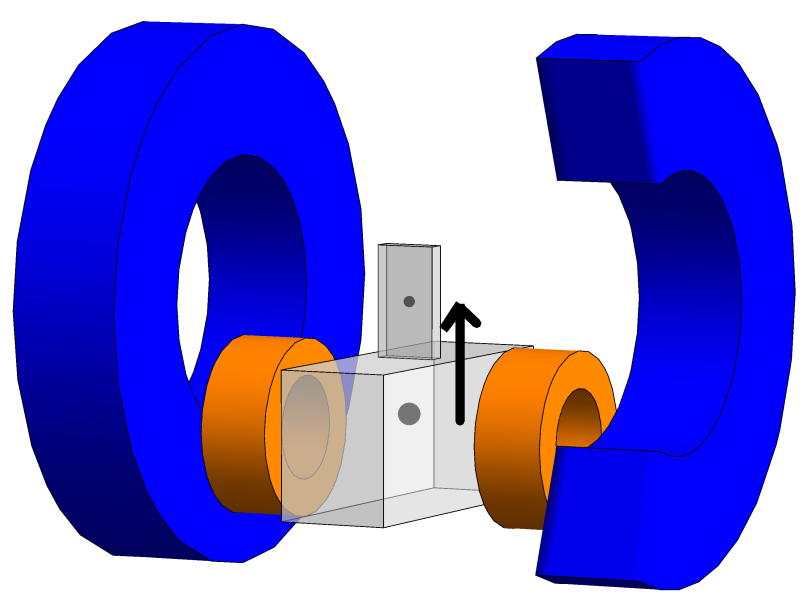

Figure 2.3: Scheme of the magnetic transport. The orange and blue pairs of coils are in anti-Helmoltz configuration.

\section{loffe Trap Loading}

Atoms are then transfered from a quadrupole trap to an Ioffe-Pritchard trap (see Fig 2.4). Due to the very different magnetic field configurations for each trap, it is not possible to make an adiabatic transfer from the quadrupole to the Ioffe trap. We minimize atom losses during the transfer by keeping as much as possible a tight confinement in the direction where the cell walls are close to the atomic cloud. In the end, we end up with up to $3 \times 10^{8}{ }^{7} \mathrm{Li}$ atoms and $2 \times 10^{7}{ }^{6} \mathrm{Li}$ atoms in the Ioffe trap. 


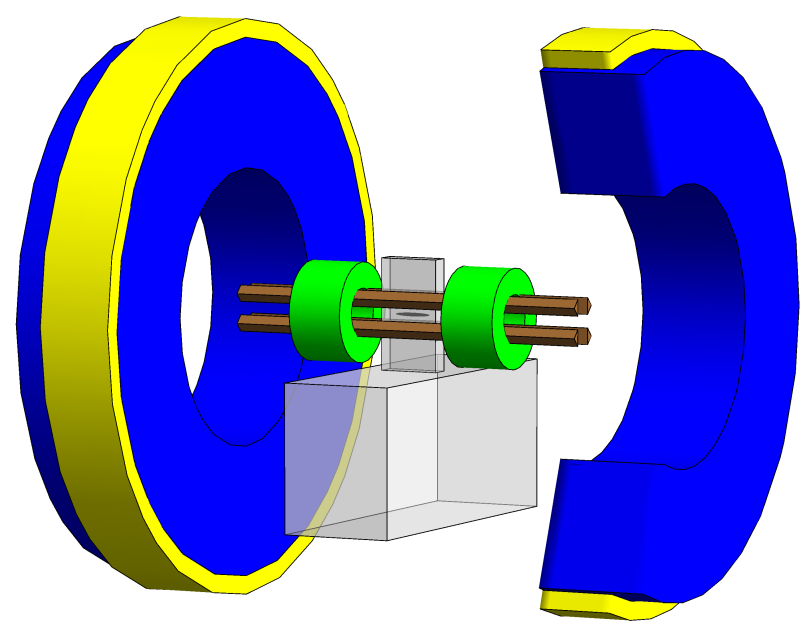

Figure 2.4: Scheme of the Ioffe-Pritchard trap. The radial confinement is provided by Ioffe bars in quadrupole configuration (in brown), the axial curvature by the small green coils, and the bias field is compensated by the large blue coils in Helmoltz configuration. The yellow coils are used for a fine control of the bias magnetic field.

\subsubsection{Radio-Frequency Evaporation}

In this section we describe the sympathetic cooling of ${ }^{6} \mathrm{Li}$ atoms using forced evaporation of the ${ }^{7} \mathrm{Li}$ component.

\section{Decrease of the Scattering Length at Finite Momenta}

The atom cloud is initially held, at a temperature $T \simeq 0.9 \mathrm{mK}$, in a decompressed Ioffe trap with a large bias field $B_{0} \simeq 500 \mathrm{G}$, the trap frequencies being $\omega_{r} / 2 \pi=198 \mathrm{~Hz}$ in the radial direction and $\omega_{z} / 2 \pi=66 \mathrm{~Hz}$ in the axial direction. The collision rate, $\Gamma_{c} \sim 0.5 \mathrm{~s}^{-1}$, is too small for initiating the evaporation due the small scattering length value $a_{77}=-27 a_{0}$.

By decreasing the bias magnetic field, the trap frequencies can be strongly increased. However, the collision rate is not increased due to particular scattering properties of ${ }^{7} \mathrm{Li}$ in the $\mathrm{mK}$ temperature range. The scattering cross-section between two colliding atoms has a dependence on the relative momentum $k$ given by 89 :

$$
\sigma(k)=8 \pi a_{77}^{2} \frac{1+a_{77} r_{e} k^{2}}{1+\left(k a_{77}\right)^{2}},
$$

in the low- $k$ limit. $r_{e}$ is the potential effective range, on the order of the Van-der-Waals range $a_{c}=$ $\left(2 m_{r} C_{6} / \hbar^{2}\right)^{1 / 4} \simeq 2.6 a_{77}[90]$. Due to the negative sign of the scattering length, the factor $1+a_{77} r_{e} k^{2}$ decreases with $k$. The exact calculation of $\sigma(k)$ reveals that the cross section actually cancels for a relative energy of $6.6 \mathrm{mK}[90$. This leads to a strong decrease of the mean collision rate inside a trapped gas at thermal equilibrium: at $2 \mathrm{mK}$, the scattering rate is decreased by a factor $\simeq 4$ with respect to the collision rate corresponding to $\sigma=8 \pi a_{77}^{2}$. The temperature increase induced by an adiabatic trap compression accentuates this effect and makes the compression inefficient for increasing the collision rate. Therefore it was necessary to implement an in situ Doppler cooling stage before compressing the trap. 


\section{In Situ Doppler Cooling}

We send during $2 \mathrm{~s}$ a weak $\left(\simeq 10 \mu \mathrm{W} / \mathrm{cm}^{2}\right)$ and circularly polarized light beam along the bias magnetic field direction, detuned to the red of the closed $\left|F=2, m_{F}=2\right\rangle \rightarrow\left|F^{\prime}=3, m_{F}^{\prime}=3\right\rangle$ transition for ${ }^{7} \mathrm{Li}$. The large magnetic field value provides a small magnetic field direction dependence with atom position, and brings atomic transitions towards other states, induced by slight laser polarization imperfections, far off resonance. Therefore we do not excite other states than $\left|F^{\prime}=3, m_{F}^{\prime}=3\right\rangle$. Doppler cooling, after optimization of the cooling beam intensity and detuning on the final collision rate, reduces the cloud's temperature by a factor 5 , with a moderate $30 \%$ atom loss. After a trap compression, the collision rate is increased by a factor $\sim 20$ compared with the collision rate without Doppler cooling, thanks to the temperature reduction and the subsequent scattering cross-section increase. In situ Doppler cooling thus appears to be a very efficient cooling stage, whose use is now generalized to other atomic species with high-temperature magneto-optical traps, such as $\mathrm{Cr}$ [91], Ne [92] or He [93].

\section{${ }^{7}$ Li Evaporation}

We first describe the evaporation of a pure ${ }^{7} \mathrm{Li}$ cloud. ${ }^{7} \mathrm{Li}$ atoms are held in a tight Ioffe trap whose frequencies are $\omega_{r} / 2 \pi=3.5 \mathrm{kHz}$ and $\omega_{z} / 2 \pi=80 \mathrm{~Hz}$. They are cooled using radio-frequency evaporation on the $\left|F=2, m_{F}=2\right\rangle \rightarrow\left|F=1, m_{F}=1\right\rangle$ hyperfine transition. The mean collision rate remains almost constant, at a value $\Gamma_{c} \simeq 40 s^{-1 \dagger}$. This shows that the evaporation is at the runaway threshold. The evaporation trajectory, plotted in Fig.2.5 reveals an efficient evaporation up to Bose-Einstein condensation, where the cloud becomes unstable because of the negative scattering length value $a_{77}=-27 a_{0}$. Thanks to better starting conditions, the new version of the experiment enables us to produce larger clouds at a given phase-space density $n \lambda_{d B}^{3}(T)$. We were thus able to reach the Bose-Einstein condensation threshold with $\simeq 5 \times 10^{4}$ atoms, without having specifically optimized the evaporation ramp.
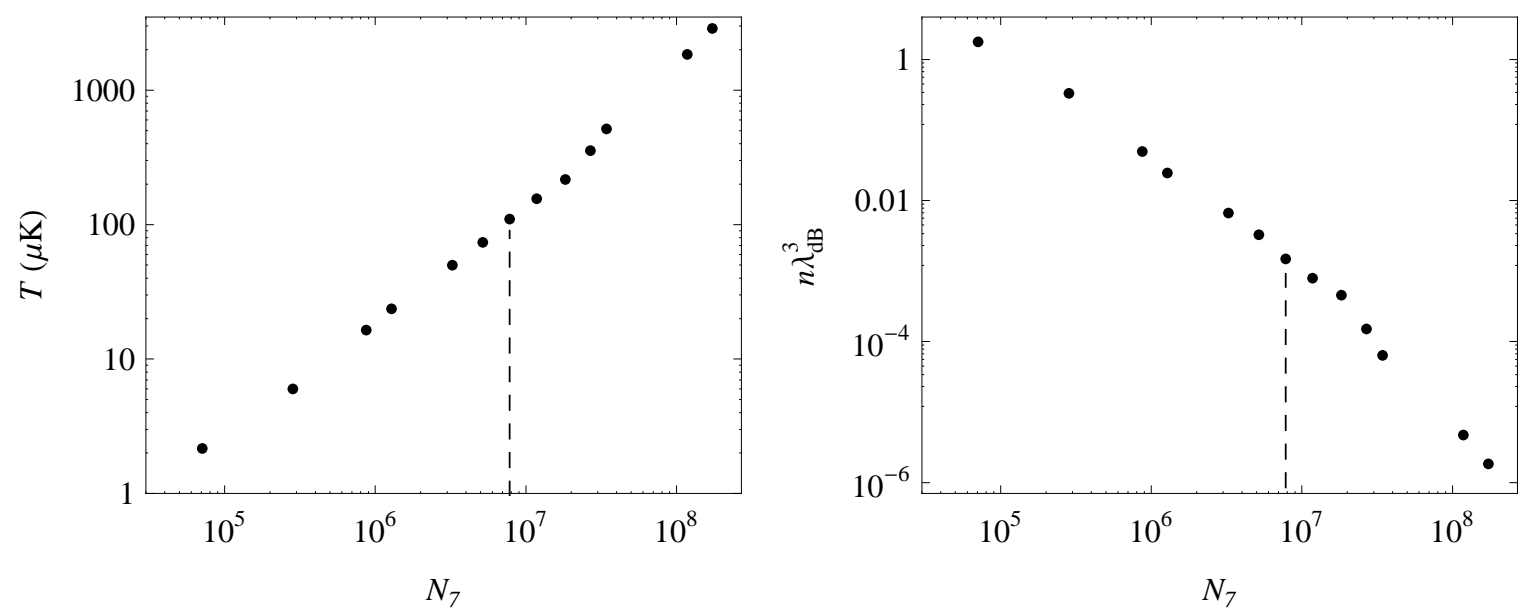

Figure 2.5: Trajectory of ${ }^{7} \mathrm{Li}$ forced evaporation: temperature $T$ as a function of atom number $N_{7}$, or peak phase space density $n \lambda_{d B}^{3}(T)$ as a function of $N_{7}$. The dashed line indicates the evaporation point reached with a maximum ${ }^{6} \mathrm{Li}$ atom number $N_{6}=5 \times 10^{6}$ (see section 2.3.2).

${ }^{*}$ By monitoring the Doppler cooling beam absorption, we obtain a convenient diagnosis of the ${ }^{7} \mathrm{Li}$ atom number before radio-frequency evaporation, allowing a control of the stability of the first stages of the experiment.

† at least up to very low temperatures close to degeneracy, a regime which is not addressed when we use ${ }^{7} \mathrm{Li}$ for ${ }^{6} \mathrm{Li}$ cooling. 


\section{${ }^{6}$ Li Sympathetic Cooling}

Until the end of evaporation, the ${ }^{6} \mathrm{Li}$ atom number $N_{6}$ is much smaller than the ${ }^{7} \mathrm{Li}$ atom number $N_{7}$. While spin-polarized ${ }^{6} \mathrm{Li}$ atoms do not collide with each other, the interspecies collision rate (per ${ }^{6} \mathrm{Li}$ atom) is comparable to the ${ }^{7} \mathrm{Li}^{7}{ }^{7} \mathrm{Li}$ collision rate (per ${ }^{7} \mathrm{Li}$ atom), since the scattering length describing ${ }^{6} \mathrm{Li}-{ }^{7} \mathrm{Li}$ collisions, $a_{67}=41 a_{0}$, is on the order of $a_{77}=-27 a_{0}$. The ${ }^{6} \mathrm{Li}$ component's thermalization with ${ }^{7} \mathrm{Li}$ is thus as efficient as the ${ }^{7} \mathrm{Li}$ self-thermalization. Therefore ${ }^{6} \mathrm{Li}$ atoms remains at thermal equilibrium with ${ }^{7} \mathrm{Li}$ atoms during evaporation.

Sympathetic cooling becomes inefficient when $N_{7}$ becomes comparable to $N_{6}$, and the final temperature reached after full ${ }^{7} \mathrm{Li}$ evaporation thus depends on the ${ }^{6} \mathrm{Li}$ atom number. With the maximum ${ }^{6} \mathrm{Li}$ atom number $N_{6}=5 \times 10^{6}$ we are able to bring at this stage, the final temperature is typically equal to $100 \mu \mathrm{K}$, a temperature low enough to ensure an efficient loading into the optical dipole trap. The corresponding phase-space density, $n \lambda_{d B}^{3} \simeq 5 \times 10^{-4}$, is already relatively large, and we will see in section 2.4.3 that it remains constant during the atom loading into the optical trap. At this stage we can compare more quantitatively the performances of our setup with the ones of all-optical experiments: at Duke university, the same phase space density is obtained for $N_{6} \simeq 10^{6}$, i.e. several times smaller than in our experiment $[94]$.

Let us mention that by reducing $N_{6}$ to much lower values, it is possible to push the evaporation further, and we obtained a degenerate Bose-Fermi mixture with $N_{7}=7 \times 10^{4}, N_{6}=3 \times 10^{4}$, and $T \simeq T_{\mathrm{BEC}} \simeq 0.3 T_{F}$, where $T_{\mathrm{BEC}}$ is the ${ }^{7} \mathrm{Li}$ Bose-Einstein condensation temperature and $T_{F}$ is the ${ }^{6} \mathrm{Li}$ Fermi temperature.

\subsection{Optical Trap and ${ }^{6} \mathrm{Li}$ Evaporation}

The internal states involved in the strongly-interacting ${ }^{6} \mathrm{Li}$ mixture have a positive magnetic moment and cannot be held in a magnetic trap. After sympathetic cooling in the magnetic trap, ${ }^{6} \mathrm{Li}$ atoms are thus transferred into an optical dipole trap before making the spin mixture in the two lowest internal states, labeled $|1\rangle$ and $|2\rangle$. In this section we first describe the trap geometry and motivate our choice. We then present the performance of our setup for obtaining degenerate ${ }^{6} \mathrm{Li}$ gases in the strongly-interacting regime.

\subsubsection{Geometry of the Hybrid Optical and Magnetic Trap}

By contrast with the previous setup, we chose a single-beam optical dipole trap configuration, with an additional magnetic curvature for a precise control of the atom confinement in the weak direction of the optical trap, labeled $z$ (see Fig 2.6). This choice is suitable for measuring the equation of state of ultracold Fermi gases from the analysis of in situ absorption images. Indeed, in this elongated potential, the typical cloud size along $z$ is about $500 \mu \mathrm{m}$, allowing a high resolution imaging in this direction. The image analysis presented in the next chapters, does not require a good resolution of the radial cloud dimension. By strongly confining the gas along $x$ and $y$, we concentrate the atom signal on a few camera pixel and increase the signal-to-noise ratio, which is determined by the camera background pixel noise. In addition, the magnetic axial confinement is very robust and reproducible, allowing the study of axial collective modes using experimental data taken over several days (see Chapter 6). 


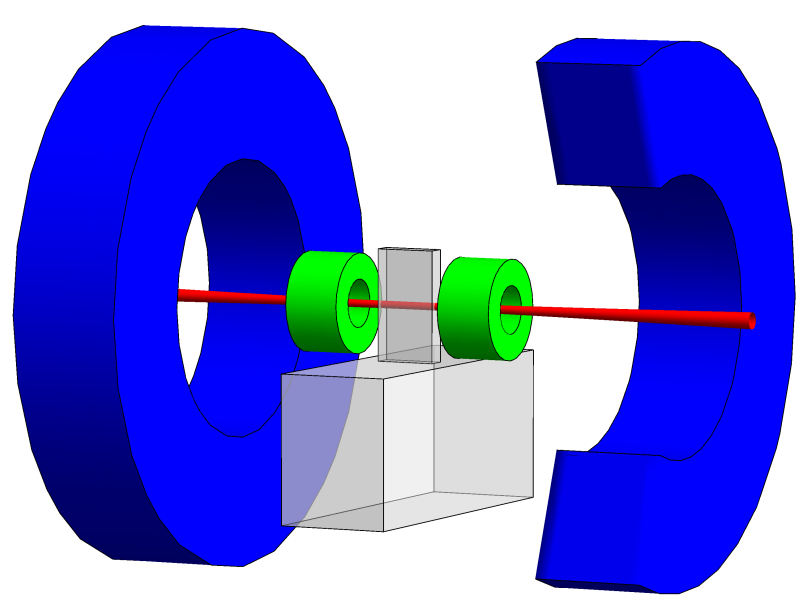

Figure 2.6: Scheme of the hybrid optical and magnetic trap. The optical dipole trap is created using a high-power red-detuned laser beam focused down to a waist $w_{0}=35 \mu \mathrm{m}$. An axial curvature, produced by the green coils, provides the axial confinement. The blue coils, in Helmoltz configuration, create a bias magnetic field up to $1000 \mathrm{G}$.

\section{The Single Beam Optical Dipole Trap}

We use for the optical dipole trap a 120-W IPG fiber laser emitting at $\lambda_{O T}=1071 \mathrm{~nm}$. Atoms experience an AC-Stark shift proportional to the intensity $I(\mathrm{r}) \mid 95$ :

$$
V(\mathrm{r})=\frac{\hbar \Gamma^{2}}{8 \delta} \frac{I(\mathrm{r})}{I_{s}},
$$

where $\Gamma=2 \pi \times 5.9 \mathrm{MHz}$ is the natural width of the $S-P$ absorption line at $671 \mathrm{~nm}, I_{s}=2.5 \mathrm{~mW} / \mathrm{cm}^{2}$ is its saturation intensity, and $\delta=-2 \pi \times 1.67 \times 10^{14} \mathrm{~Hz}$ is the dipole laser frequency detuning from the $671 \mathrm{~nm}$ line. The intensity variation with position leads to an effective trapping potential proportional to the local AC-Stark shift, attracting atoms towards the regions of high intensity.

We focus the laser beam at the bottom of the Ioffe magnetic trap potential, with a gaussian TEM T0 $_{00}$ intensity profile:

$$
I(x, y, z)=\frac{2 P}{\pi w_{0}^{2}\left(1+z^{2} / z_{R}^{2}\right)} \exp \left(-\frac{2\left(x^{2}+y^{2}\right)}{w_{0}^{2}\left(1+z^{2} / z_{R}^{2}\right)}\right),
$$

where $P$ is the total laser power, $w_{0}=35 \mu \mathrm{m}$ is the beam waist and $z_{R}=\pi w_{0}^{2} / \lambda_{O T}=3.6 \mathrm{~mm}$ is the Rayleigh length. Combining (2.1) and (2.2), we obtain the trapping potential:

$$
V(x, y, z)=\frac{-U_{0}}{1+z^{2} / z_{R}^{2}} \exp \left(-\frac{2\left(x^{2}+y^{2}\right)}{w_{0}^{2}\left(1+z^{2} / z_{R}^{2}\right)}\right), \quad \text { where } \quad U_{0}=\frac{\hbar \Gamma^{2} P}{4 \pi \delta I_{s} w_{0}^{2}}
$$

is the optical dipole trap depth. Close to the trap bottom, atoms experience a harmonic potential given by the quadratic expansion of (2.3) around $\mathbf{0}$ :

$$
V(x, y, z)=-U_{0}+\frac{1}{2} m \omega_{r}^{2}\left(x^{2}+y^{2}\right)+\frac{1}{2} m \omega_{z}^{2} z^{2}, \quad \text { where } \quad \omega_{r}=\sqrt{\frac{4 U_{0}}{m w_{0}^{2}}}, \omega_{z}=\frac{1}{\sqrt{2} \pi} \frac{\lambda_{O T}}{w_{0}} \omega_{r} .
$$

For atom loading into the optical dipole trap, the laser power is ramped up to its maximum value $P \simeq 65 \mathrm{~W}$, corresponding to a trap depth $U_{0} \simeq k_{B} \times 1.6 \mathrm{mK}$, the trapping frequencies being $\omega_{r} / 2 \pi \simeq$ $13 \mathrm{kHz}$ and $\omega_{z} / 2 \pi \simeq 95 \mathrm{~Hz}$. The trap depth is large enough to ensure a transfer of the ${ }^{6} \mathrm{Li}$ cloud with unit efficiency, despite the large increase in trapping frequencies. 
The choice of a $35 \mu \mathrm{m}$ waist was made empirically: we did not succeed to make an efficient atom loading into an optical trap designed with a larger $\left(w_{0} \simeq 100 \mu \mathrm{m}\right)$ waist. This behavior is not merely due to atom spilling due to a smaller trap depth. Indeed, the cloud temperature after loading is increased from the magnetic trap value by the ratio of the geometrical mean frequency in the optical trap over the one in the magnetic trap, and thus scales with $w_{0}^{-7 / 3}$. On the other hand, the optical trap depth scales with $w_{0}^{2}$. The amplitude of atom spilling is a priori driven by the ratio $k_{B} T / U_{0}$ which has a small dependence $\propto w_{0}^{-1 / 3}$ on the waist value. The inefficient transfer observed for a large trap waist may be due to the very low axial trapping frequency $\omega_{z} \simeq 4 \mathrm{~Hz}$ in that case, which makes the transfer from the magnetic to the optical trap non adiabatic. The cloud size along $z$ then become comparable to the cell size $\simeq 1 \mathrm{~cm}$ along $z$, and atoms may collide with the cell walls.

Smaller waist values are not preferable for the final stage of the experiment. The radial size of the cloud would then become comparable to the imaging system resolution, and the peak optical density would be relatively large. The combination of these two phenomena would lead to a strong distortion of in situ absorption images, as explained in section 3.5.4. It would also imply a very large final trap aspect ratio, casting doubt on the validity of local density approximation, which is at the heart of our data analysis (see Appendix B.4). The $35-\mu \mathrm{m}$ waist was thus chosen as the maximum waist value allowing an efficient loading.

\section{Axial Magnetic Trapping}

The optical dipole trap aspect ratio $\lambda=\omega_{r} / \omega_{z}=\sqrt{2} \pi w_{0} / \lambda_{O T} \simeq 150$ is very large. While the axial confinement provided by the optical trap $\omega_{z} / 2 \pi \simeq 95 \mathrm{~Hz}$ is sufficient at maximum laser power, it becomes very small $\left(\omega_{z} / 2 \pi \simeq 3 \mathrm{~Hz}\right)$ when the ${ }^{6} \mathrm{Li}$ cloud is evaporated by lowering the optical trap up to $1 / 1000$ of the initial value. Thermalization then becomes very slow and may result in heating due to non-adiabatic trap modification ${ }^{\text {f }}$

We use a magnetic curvature for an independent control of the axial confinement at low laser power. We have at our disposal the pair of coils which provide the Ioffe trap magnetic curvature along $z$ (see Fig. 2.6. They create a magnetic curvature of $C=1.0 \mathrm{G} / \mathrm{cm}^{2} / \mathrm{A}$, as well as a bias magnetic field $B_{0}=2.28 \mathrm{G} / \mathrm{A}$. The magnetic curvature, being positive in the direction of the bias field, expels ${ }^{6} \mathrm{Li}$ atoms in the $|1\rangle$ or $|2\rangle$ states (see Fig $2.7 \mathrm{a}$ ). We use an extra pair of coils to control the bias magnetic field. They create a bias magnetic field $B_{0}=-2.28 \mathrm{G} / \mathrm{A}$ in the opposite direction and a much smaller curvature $-0.080 \mathrm{G} / \mathrm{cm}^{2} / \mathrm{A}$ (see Fig. 2.6). The total bias magnetic field is reversed by imposing a larger current through the second pair of coils, and the total curvature thus becomes negative with respect to the reversed bias field direction (see Fig 2.7p). Using this magnetic field configuration we create a confining potential along $z$, up to $40 \mathrm{~Hz}$.

\subsubsection{Optical System for the Dipole Trap}

In this section we give more technical details relative to the optical system for the dipole trap. Special care had to be taken for the design of a stable optical system adapted to the large laser power provided by the 120-W IPG laser.

Strong heating rates were observed in the first version of the optical system, where water-cooled acousto-optic modulators (AOM) were used for the trap depth control. These AOM are designed to handle large laser powers, but the water flow induces strong mechanical vibrations. Using a quadrant photodiode, we measured the beam pointing position noise power spectrum $S_{x}(\nu)$ (see Fig 2.8 $\mathrm{a}$ ). Gas heating

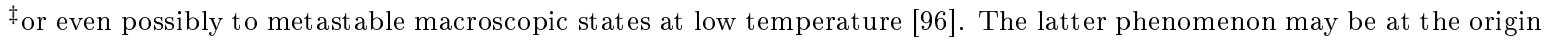
of the discrepancy between the observations in Rice university [55 97] and in other groups [98 49 99] on spin-imbalanced Fermi gases (see Chapter 5 , as suggested in 100 .
} 
(a)

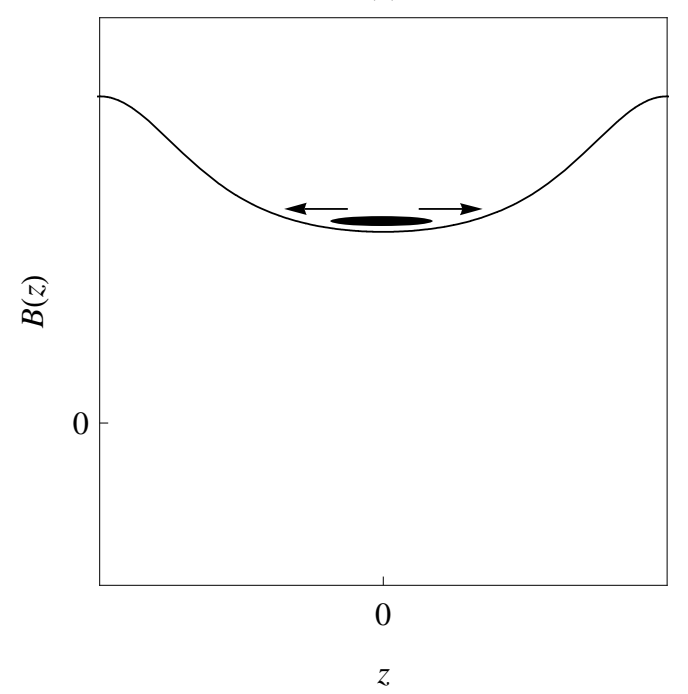

(b)

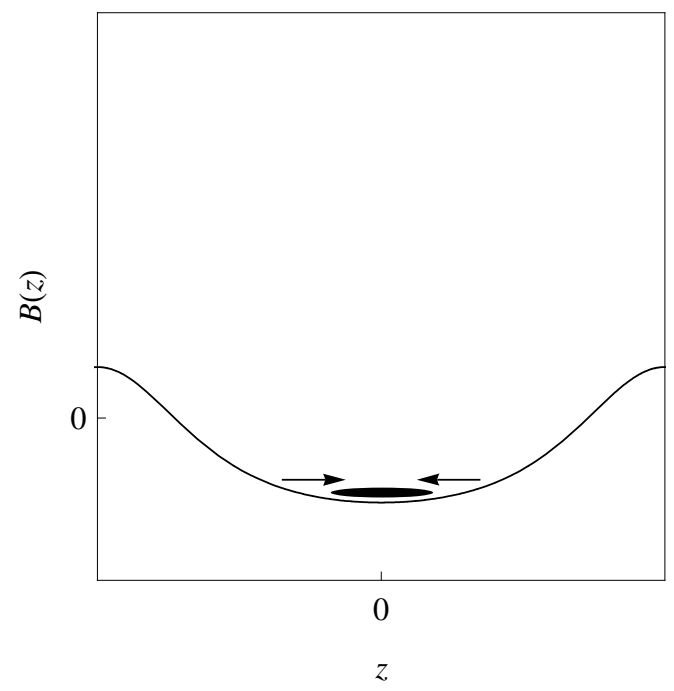

Figure 2.7: Magnetic field created by the curvature coils only (a), or by the combination of curvature coils and extra coils providing an opposite bias field (b). High-field seeker states are expelled along $z$ in the first configuration, and confined in the other configuration.

is associated with the trap shaking spectral component at the radial trapping frequency $\omega_{r} / 2 \pi$ [101]:

$$
\dot{E}=\frac{1}{4} m \omega_{r}^{4} S_{x}\left(\frac{\omega_{r}}{2 \pi}\right) .
$$

When water cooling is used, we estimate the heating rate to be about $500 \mu \mathrm{K} / \mathrm{s}$ at full laser power, to be compared with $10 \mu \mathrm{K} / \mathrm{s}$ without water cooling.

(a)

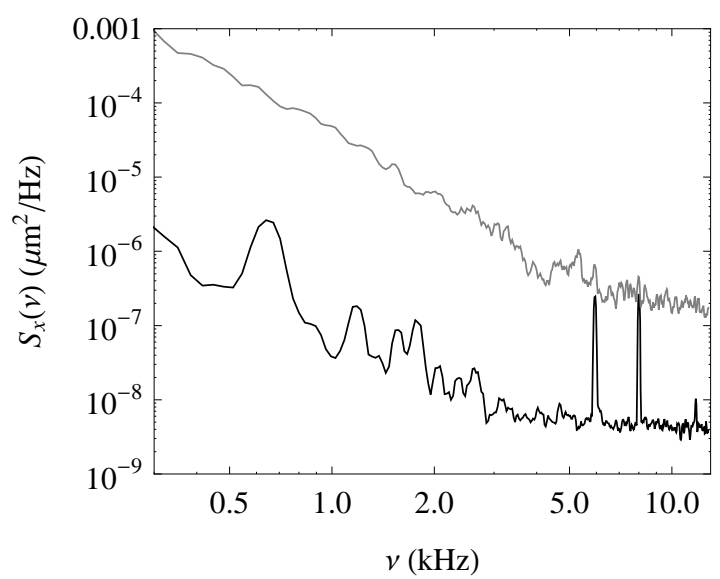

(b)

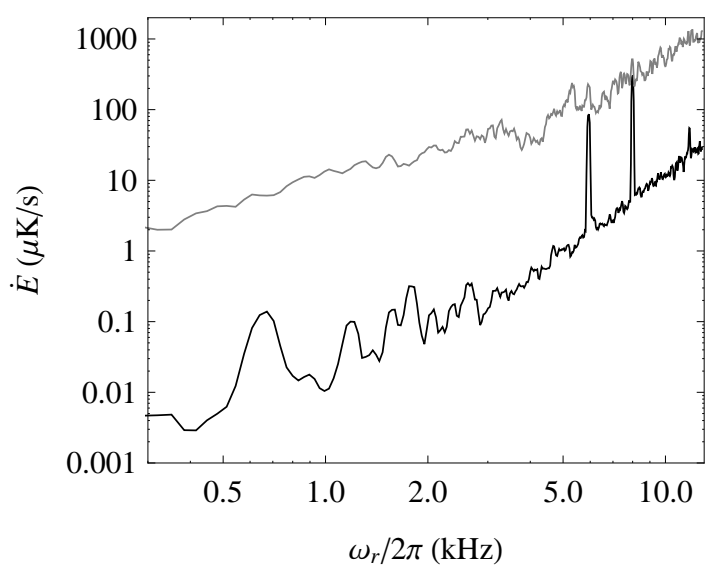

Figure 2.8: (a) Position noise power spectrum $S_{x}(\nu)$, as a function of frequency $\nu$, with a water-cooled AOM (gray line) and without water cooling (black line). (b) Associated heating rate $\dot{E}$ as a function of radial trapping frequency $\omega_{r} / 2 \pi$.

The strong influence of beam pointing fluctuations encouraged us to improve the mechanical stability of the optical system:

- We use a single acousto-optic modulator without water cooling for the trap depth contro $\$$. Since $\S$ From Crystal Technology Inc. 
laser light absorption by the AOM causes strong heating, we send an air flow through a hole in the hood of the modulator. This air flow is stopped during the dipole trapping stage using an electronic air valve. In order to avoid trap shaking associated with AOM frequency fluctuations, we use an ultra-stable DDS function generator for the radio-frequency generation.

- The optical system is made of 'cage system' elements from Thorlabs company. All elements are linked together with stainless steel rods and aluminum tubes, and the laser output coupler is glued to this system, in order to minimize beam pointing fluctuations. The tubes also isolate the optical path from ambient air flow and impurities. Day-to-day mechanical drifts are very small as well, and the optical trap rarely needs to be realigned.

The complete optical setup scheme is shown in Fig 2.9

\subsubsection{Preparation of a Strongly-Interacting ${ }^{6} \mathrm{Li}$ Mixture}

\section{Atom Loading into the Optical Dipole Trap}

After full evaporation of ${ }^{7} \mathrm{Li}$, we load pre-cooled ${ }^{6} \mathrm{Li}$ atoms in the optical dipole trap. The ${ }^{6} \mathrm{Li}$ cloud typically contains 2 to $5 \times 10^{6}$ atoms at $100 \mu \mathrm{K}$, and is held in magnetic trap whose frequencies are $\omega_{r} / 2 \pi \simeq 3.5 \mathrm{kHz}, \omega_{z} / 2 \pi \simeq 80 \mathrm{~Hz}$. We first strongly decompress the magnetic trap by slowly increasing the bias magnetic field from $3.5 \mathrm{G}$ to $30 \mathrm{G}$ and decreasing the curvature coils current from $500 \mathrm{~A}$ to $10 \mathrm{~A}$. The trap frequencies are, after decompression, $\omega_{r} / 2 \pi \simeq 400 \mathrm{~Hz}$ and $\omega_{z} / 2 \pi \simeq 10 \mathrm{~Hz}$. The subsequent cloud size increase makes the transfer into the optical trap less sensitive to the alignment of the dipole laser on the Ioffe trap bottom. Fine adjustments of the infrared beam pointing are made using a mirror whose orientation is controlled with a step-motor system (see Fig 2.9.

The transfer is performed by ramping down the Ioffe bars current while increasing the optical trap power in $200 \mathrm{~ms}$. Atoms are adiabatically attracted into the dimple made by the optical trap. No significant atom loss is observed in the loading process. The temperature after transfer, $T \simeq 250 \mu \mathrm{K}$, is 3 times larger than the temperature reached at the end of sympathetic cooling. Since the mean trapping frequency is increased, from the magnetic to the optical trap, by a comparable factor, this temperature is consistent with an adiabatic loading

\section{Heating and Loss Rates}

We first tried to characterize the trap heating and loss rates in the dipole trap using a ${ }^{7} \mathrm{Li}$ cloud, and observed large loss rates, with a $1 / e$ time constant $\tau \sim 300 \mathrm{~ms}$. Since ${ }^{7} \mathrm{Li}$ atoms collide with each other, one expects atoms to escape from the trap because of collision-induced evaporation. However, we did not observe a temperature reduction while atoms were lost. The atom loss is rather attributed to inelastic dipolar losses, enhanced with respect to the Ioffe trap by the increase in trapping frequencies.

Loss rates were found to be much smaller for ${ }^{6} \mathrm{Li}$ spin-polarized clouds, where both elastic and inelastic collisions are absent, and are consistent with $\tau>20 \mathrm{~s}$ at full laser power, and no significant temperature increase on a timescale of several seconds. This is consistent with the following heating rate estimates:

- The heating rate associated with beam-pointing fluctuations was estimated earlier to $\dot{E} \simeq 10 \mu \mathrm{K} / \mathrm{s}$ at the maximum trap depth (see Fig.2.8).

- Dipole laser intensity noise gives rise to parametric heating, whose amplitude is proportional to the intensity noise power spectrum $S_{I}(\nu)$ at twice the radial trapping frequency $\omega_{r} / 2 \pi$ 101:

$$
\dot{E}=-\frac{E}{\tau_{I}}, \quad \text { where } \quad \frac{1}{\tau_{I}}=\frac{\omega_{r}^{2}}{4} S_{I}\left(\frac{\omega_{r}}{\pi}\right) .
$$

T The ${ }^{6} \mathrm{Li}$ cloud being fully polarized, no collision occurs during the trap modifications, and adiabaticity is a single-particle process. 


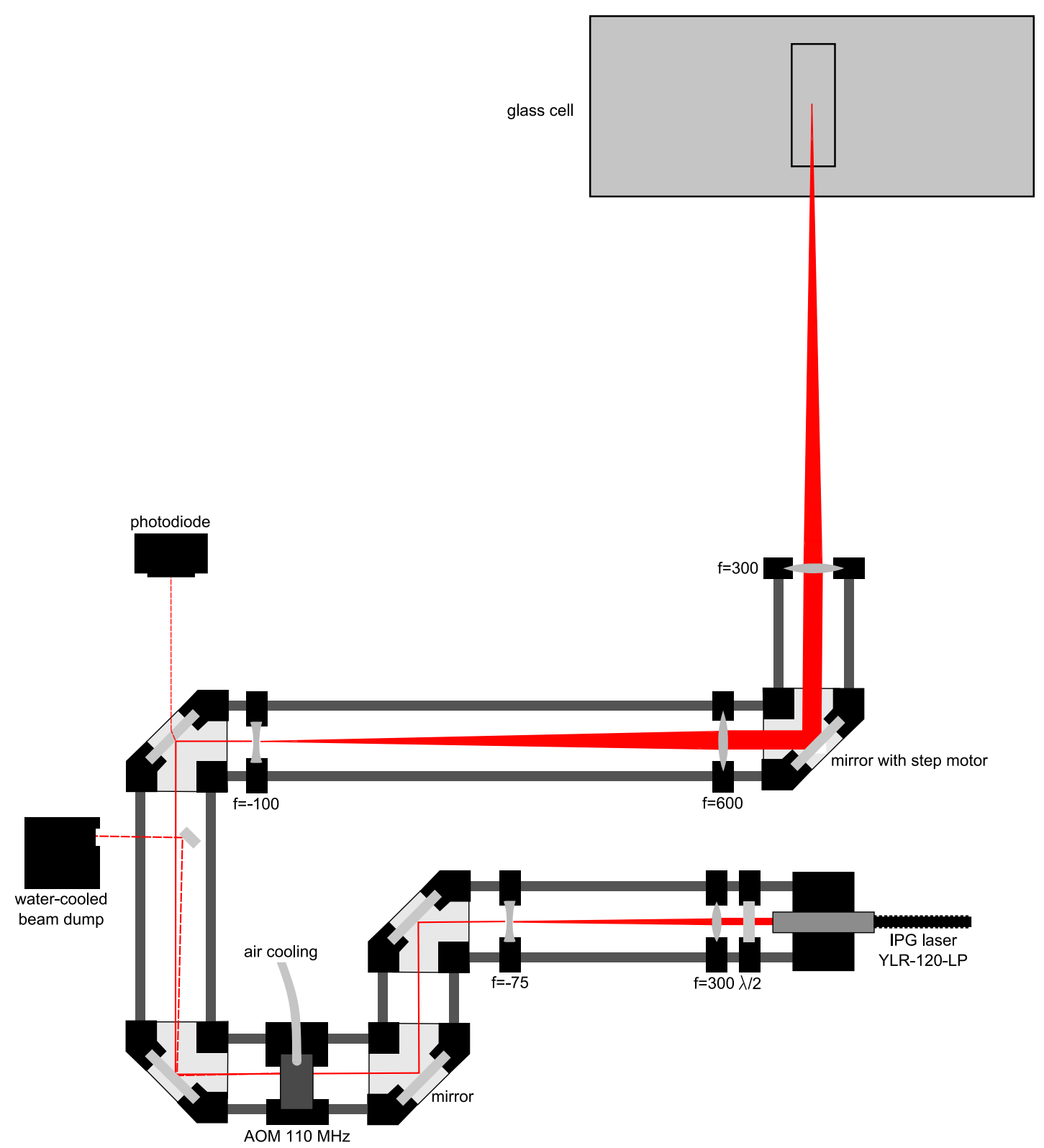

Figure 2.9: Scheme of the optical system for the dipole trap. The infrared beam is emitted by a $120-\mathrm{W}$ IPG fiber laser, in a gaussian $\mathrm{TEM}_{00}$ mode with a $5 \mathrm{~mm} \mathrm{1/e} \mathrm{diameter.} \mathrm{After} \mathrm{reducing} \mathrm{its} \mathrm{diameter} \mathrm{with}$ a first telescope, the beam is diffracted by an air-cooled acousto-optic modulator. It is then enlarged by a second telescope, and focused into the vacuum cell down to a 35- $\mu$ m waist. A step-motor-driven mirror provides a fine adjustment of the laser pointing.

From the intensity noise $S_{I}(\nu)$ measured experimentally, and shown in Fig 2.10a, we deduce a heating time constant $\tau_{I} \simeq 100 \mathrm{~s}$ at full dipole laser power (see Fig $2.10 \mathrm{~b}$ ). Therefore we did not need to install an intensity stabilization lock, and the optical trap depth is controlled in open loop.

- Because of the very high intensity at the trap bottom, light absorption is not completely negligible despite the very large frequency detuning, and occurs with a rate 95 :

$$
\Gamma_{a b s}=\frac{\Gamma}{\hbar \delta} U_{0} \simeq 7 \mathrm{~s}^{-1} .
$$

Each absorbed photon leads to an energy gain of about one recoil energy $E_{r}$, and the heating rate 
(a)

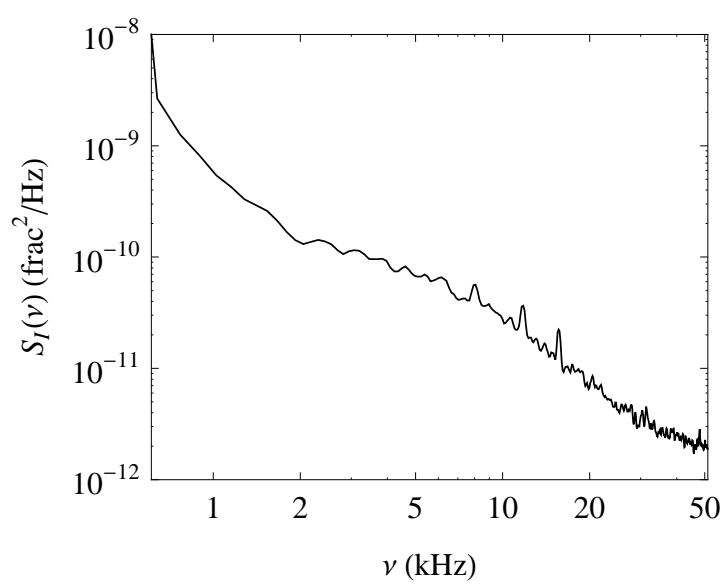

(b)

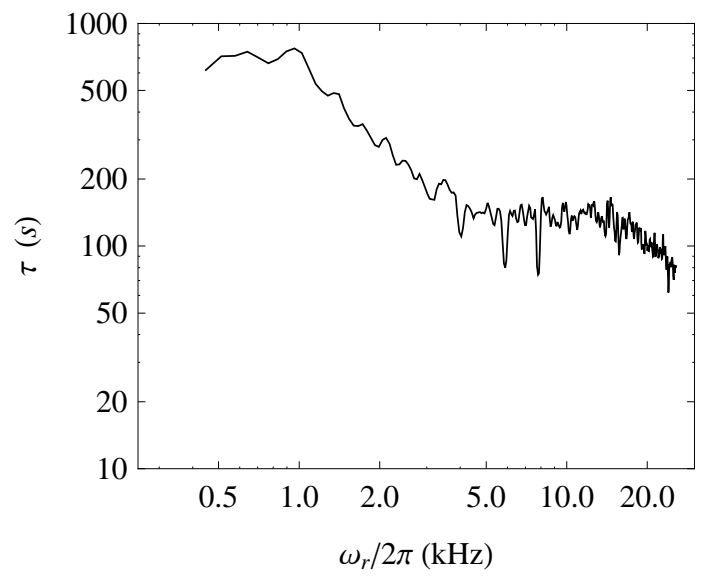

Figure 2.10: (a) Intensity noise power spectrum $S_{I}(\nu)$, as a function of frequency $\nu$ (b) $1 / e$ time constant $\tau$ of the associated heating rate, as a function of radial trapping frequency $\omega_{r} / 2 \pi$.

associated with off-resonant photon scattering reads 95 :

$$
\dot{E}=\frac{2}{3} \Gamma_{a b s} E_{r} \simeq 7 \mu \mathrm{K} / \mathrm{s}, \quad \text { where } \quad E_{r}=\frac{\hbar^{2}}{2 m}\left(\frac{2 \pi}{\lambda_{O T}}\right)^{2} \simeq k_{B} \times 1.4 \mu \mathrm{K} .
$$

\section{Preparation of a Strongly-Interacting ${ }^{6} \mathrm{Li}$ Mixture}

The ${ }^{6} \mathrm{Li}$ cloud loaded from the magnetic trap into the optical dipole trap is polarized in the low-field seeking state $|6\rangle=\left|F=3 / 2, m_{F}=3 / 2\right\rangle$. The states involved in the strongly-interacting mixture are the two lowest internal states $|1\rangle$ and $|2\rangle$, which exhibit a wide Feshbach resonance centered at a magnetic field $B_{0}=834 \mathrm{G}$ (see Fig 2.11). As these states have a positive magnetic moment, their magnetic confinement along $z$ requires an opposite curvature than the one used for the Ioffe trap (see Fig 2.7). Therefore during the transfer $|6\rangle \rightarrow|1\rangle$ we need to switch off the magnetic curvature, the axial confinement being solely produced by the optical trap. A small bias magnetic field is kept on, and the $|6\rangle \rightarrow|1\rangle$ transfer is performed using an adiabatic passage across the hyperfine transition. In order to produce a reversed magnetic curvature with the same coils, we suddenly reverse the bias magnetic field, and then slowly ramp the bias up to $B_{0}=834 \mathrm{G}$, and the curvature fields up to $\omega_{z} / 2 \pi=40 \mathrm{~Hz}$ (see Fig, 2.7). About 20\% of the atoms are lost in the process, and no significant atom loss is observed when crossing the $p$-wave Feshbach resonance at $B_{0}=159 \mathrm{G}[102,103]$. Finally, a $|1\rangle-|2\rangle$ mixture is created using a non-adiabatic Landau Zener passage around the nuclear spin-flip transition $|1\rangle \rightarrow|2\rangle$ at $76,4 \mathrm{MHz}$.

\subsubsection{Evaporation of a ${ }^{6} \mathrm{Li}$ Gas With Resonant Interactions}

\section{Evaporation of a Spin-Balanced Gas}

The evaporation efficiency was characterized using spin-balanced gases. Right after the preparation of a $|1\rangle-|2\rangle$ mixture at the center of the Feshbach resonance, the collision rate is particularly large [104]:

$$
\Gamma_{c}=\frac{2 N \hbar^{2} \omega_{r}^{2} \omega_{z}}{\pi\left(k_{B} T\right)^{2}} \simeq 4000 \mathrm{~s}^{-1}
$$

where $N=2 \times 10^{6}$ is the atom number per spin state. This enables us to perform a very efficient evaporation.

No noticeable evaporation is observed as long as the trap depth is kept at the maximum value, despite the large collision rate. This confirms that the atom transfer from the Ioffe trap to the optical dipole 

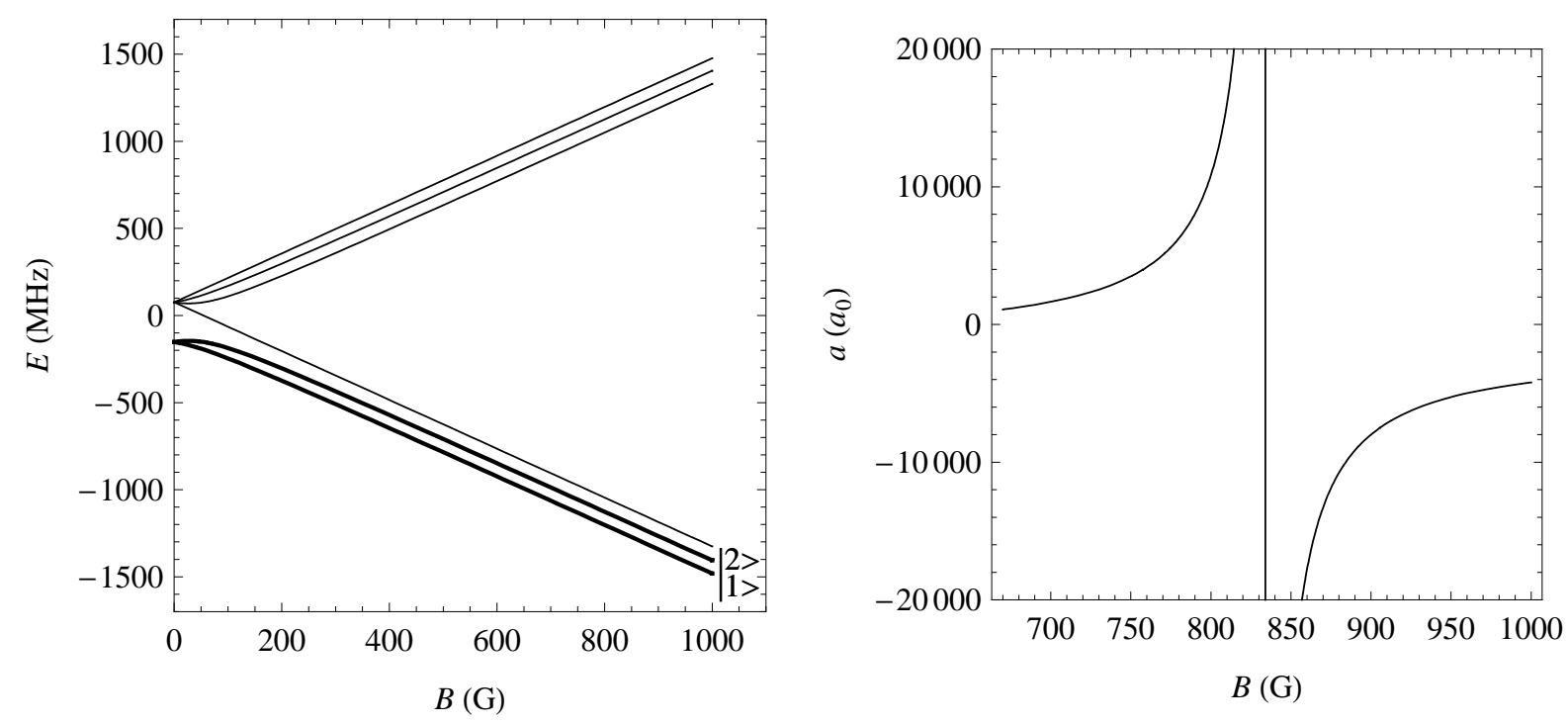

Figure 2.11: Left: Energy $E$ of the states $2^{2} S_{1 / 2}$ as a function of the magnetic field $B$. In the last stage of the experiment we use a mixture of the two lowest internal states, $|1\rangle$ and $|2\rangle$. Right: $s$-wave scattering length describing ultracold collisions between states $|1\rangle$ and $|2\rangle$, as a function of magnetic field.

trap does not induce significant heating. Evaporative cooling is forced by lowering the optical trap depth, using an acousto-optic modulator for the laser power adjustment. As mentioned above, the laser power is controlled in open loop; the AOM radio-frequency power is controlled using a 16-bit computer analog output, providing a fine control of the laser power even in the range $P \sim 10^{-3} P_{0}$ addressed at the end of evaporation.

In Fig 2.12 we show a typical evaporation trajectory. The dipole laser power is decreased in $5 \mathrm{~s}$ from the maximum power $P_{0}$ up to a final power $P_{\infty}$, using an exponential function with a $1 / e$ time constant of $500 \mathrm{~ms}$. Fermi degeneracy is reached with more than $2.5 \times 10^{5}$ atoms per spin state at $T / T_{F}=0.5$, and by pushing the evaporation further the gas is cooled to the lowest temperatures $\left(T / T_{F}=0.03(3)\right.$, see Chapter 5), with $10^{5}$ atoms per spin state. At the end of evaporation we measure a $1 /$ e lifetime of about $5 \mathrm{~s}$, the optical laser power being kept to the minimum value. Since residual evaporation is not suppressed, this provides a lower bound to the intrinsic lifetime of the ${ }^{6} \mathrm{Li}$ gas. This value is much larger than the one observed for ${ }^{40} \mathrm{~K}$ gases in the strongly-interacting regime. This difference is related to the very different Feshbach resonance widths for ${ }^{6} \mathrm{Li}$ and ${ }^{40} \mathrm{~K} 34,105$.

In the classical regime, the atom loss rate during evaporation can be related to the temperature to trap depth ratio $\eta=k_{B} T / U_{0}$, according to [106]:

$$
\frac{\dot{N}}{N}=-2(\eta-4) e^{-\eta} \Gamma_{c},
$$

which gives $\eta \simeq 10$ for our experiment. In the limit of large $\eta$ values, the gain in phase space density is related to the atom number loss according to:

$$
n \lambda_{d B}^{3} \propto N^{\eta-3} \simeq N^{7},
$$

showing the excellent evaporation efficiency achieved with ${ }^{6} \mathrm{Li}$ mixtures in the unitary limit. As soon as the gas enters the Fermi degeneracy regime, the atom number decreases more sharply when the trap depth is lowered (see Fig 2.12. This is expected as Pauli blocking plays an increasing role 107. 


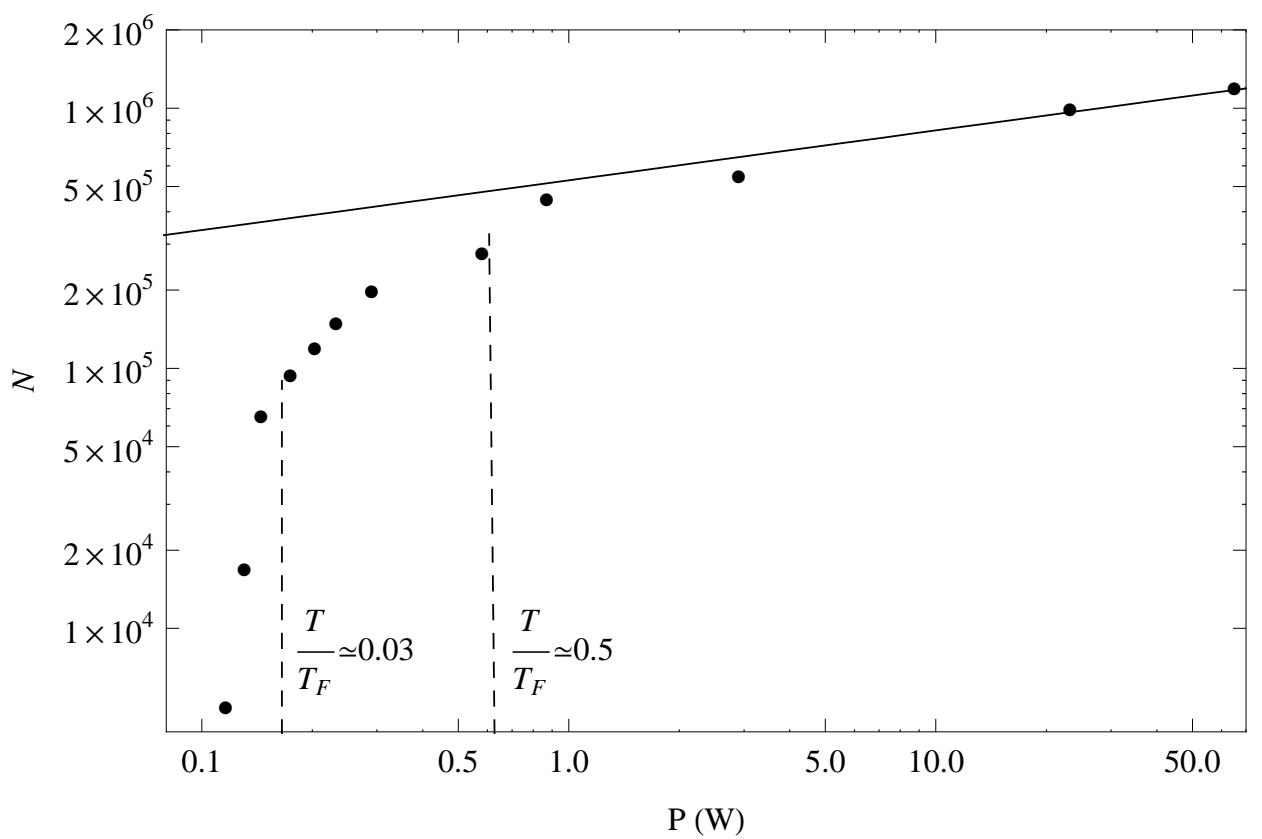

Figure 2.12: Evaporation trajectory for a ${ }^{6} \mathrm{Li}$ spin-balanced mixture with resonant interactions: atom number per spin state $N$ as a function of the dipole laser power $P$. The solid line is the evaporation trajectory in the classical regime, corresponding to $\eta=10$.

\section{Spin-Polarization Issues During Evaporation}

In Chapter 5 and 6 we study spin-imbalanced ${ }^{6} \mathrm{Li}$ mixtures at low temperature. Since in the degenerate regime it is not possible to remove atoms in one spin state without heating the gas, one needs to prepare a spin-imbalanced mixture before starting the evaporation. The performance of evaporation is not dramatically affected by spin polarization, since it was possible to produce extremely imbalanced mixtures $\left(N_{2}<0.02 N_{1}\right)$ with a temperature consistent with $T / T_{F}=0.03$ (see Chapter 5 ).

However, the final gas polarization is very sensitive to the initial condition, due to the combination of the following effects:

- In the classical regime, the velocity distribution is identical for both components. In particular collisions ejecting atoms from the trap act symmetrically on both spin states, and we expect $\mathrm{d} N_{1}=$ $\mathrm{d} N_{2}$. This behavior leads to a progressive increase of spin polarization, as shown in Fig $2.13 \mathrm{a}$.

- In the degenerate regime, we observe a strong decrease of spin polarization, the tendency becoming increasingly pronounced when evaporation is pushed further. A physical interpretation of this phenomenon can be given in the BEC and BCS regimes: in the BEC regime, atoms with opposite spins form deeply bound molecules, which have twice the atom polarizability and thus feel an optical trap twice deeper compared to atoms. This makes single atom evaporation much more likely. In the BCS regime, collisions mostly occur between majority atoms with a speed $v_{F 1}$ and minority atoms with a speed $v_{F 2}<v_{F 1}$. Majority atoms are thus more easily ejected from the trap. Many-body effects appearing in the degenerate regime are also expected to decrease the cloud polarization $[100$.

The large polarization variation during evaporation leads to a strong sensitivity to initial conditions, and makes the control of polarization for deeply degenerate gases difficult. In Fig $2.13 \mathrm{~b}$ we show the large shot-to-shot polarization fluctuations for gases prepared at the lowest temperatures. For some experimental runs all minority atoms are evaporated during the trap depth decrease. However, as long 
(a)

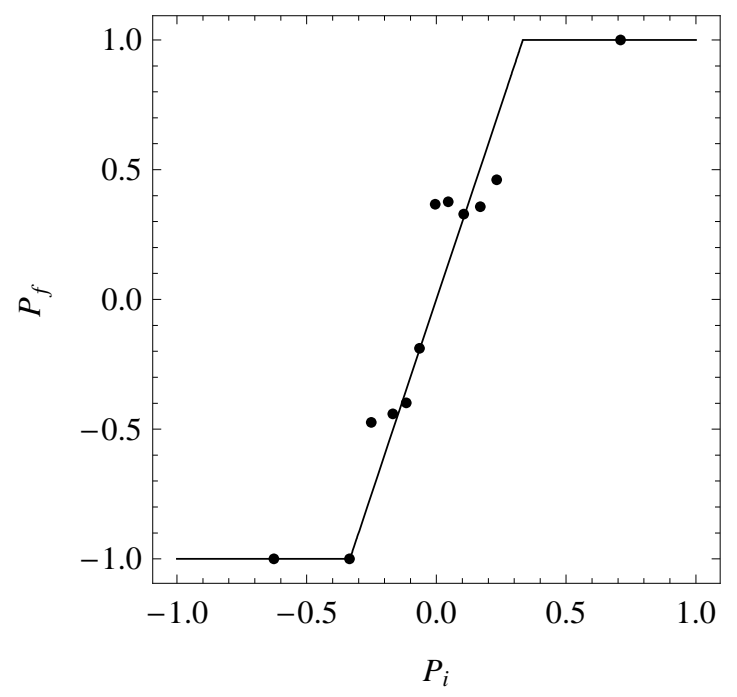

(b)

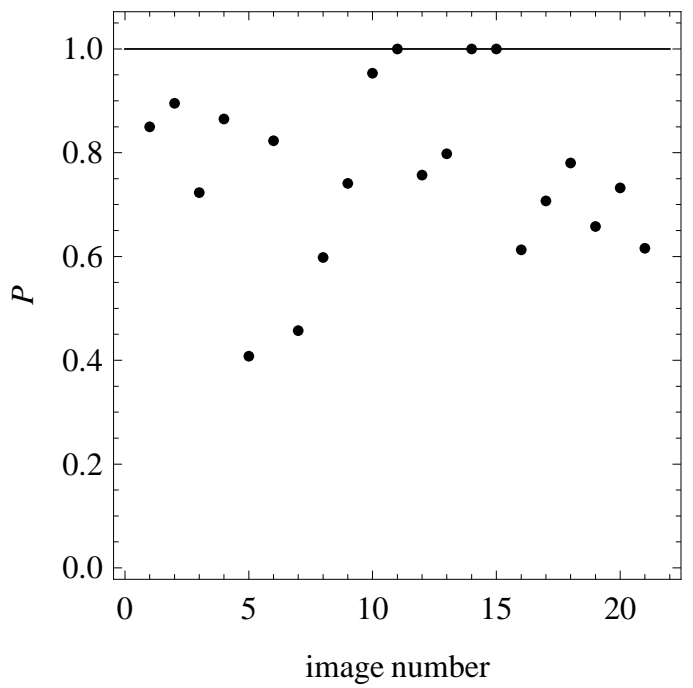

Figure 2.13: (a) Polarization $P_{f}$ after evaporation as a function of polarization before evaporation $P_{i}$, for an evaporation up to $T \sim T_{F}$. The solid line is a guide to the eye. (b) Evolution of polarization for consecutive experimental sequences preparing a deeply degenerate gas. For three sequences the gas becomes fully polarized during evaporation.

as $100-\%$ polarized samples have a low probability, this phenomenon is not really penalizing since it is possible to post-select images as a function of spin polarization.

\section{Trap Frequency Calibrations}

The trap frequencies $\omega_{r}$ and $\omega_{z}$ are calibrated by exciting the center-of-mass dipole mode along $x$ or $z$.

The magnetic confinement, that has a long-term stability, is calibrated with a very good precision. We use the set of data taken for the study of collective modes described in Chapter 6. In addition to the excitation of axial compression modes, we observe an oscillation of the gas center-of-mass along $z$. According to Kohn's theorem, the center of mass always oscillates at the trap frequency $\omega_{z} / 2 \pi$, whatever interactions. For very cold samples, the optical dipole trap power is so small that its contribution to the axial trapping frequency is less than $1 \%$. The axial confinement is thus completely characterized by the magnetic field curvature, provided by the curvature coils, plus a small contribution from the bias coils:

$$
\begin{aligned}
\left(\frac{\omega_{z}}{2 \pi}\right)^{2} & =\alpha_{\text {bias }} I_{\text {bias }}+\alpha_{c u} I_{c u} \\
B_{0} & =\beta\left(I_{\text {bias }}-I_{c u}\right), \quad \text { where } \beta=2.28 \mathrm{G} / \mathrm{A}
\end{aligned}
$$

The coefficients $\alpha_{\text {bias }}$ and $\alpha_{c u}$ are extracted from frequency calibrations using gases prepared at $B_{0}=$ $834 \mathrm{G}$, hence $I_{b i a s}-I_{c u}$ remains equal to $366 \mathrm{~A}$. From the frequency values measured for $I_{c u}=$ $50 \mathrm{~A}, 70 \mathrm{~A}, 100 \mathrm{~A}$ (see Fig 2.14, we deduce the coefficients $\alpha_{\text {bias }}=-2.06 \mathrm{~Hz}^{2} / \mathrm{A}$ and $\alpha_{c u}=24.8 \mathrm{~Hz}^{2} / \mathrm{A}$

By measuring the oscillation frequency of the center-of-mass motion along $x$, we obtain the following calibration of $\omega_{r}$ :

$$
\frac{\omega_{r}}{2 \pi} \simeq \alpha \sqrt{P} \quad \text { where } \quad \alpha=2.0(2) \mathrm{kHz} / \sqrt{\mathrm{W}},
$$

$P$ being the dipole laser power. 
(a)

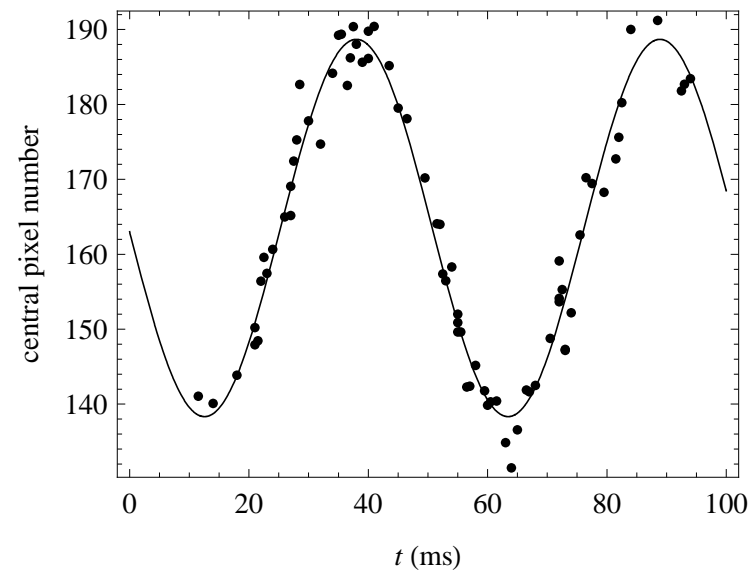

(b)

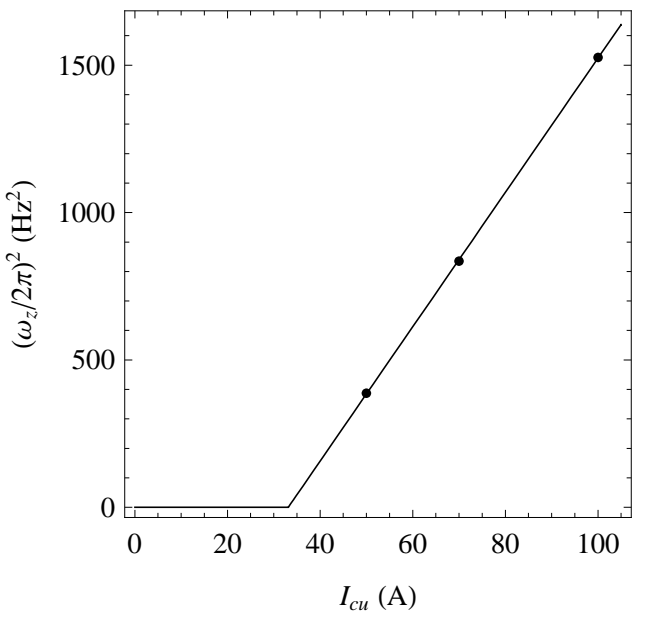

Figure 2.14: (a) Center-of-mass oscillation for $I_{b i a s}=416 \mathrm{~A}$ and $I_{c u}=50 \mathrm{~A}$, and fitted with a cosine, providing $\omega_{z} / 2 \pi=19.7(2) \mathrm{Hz}$. (b) Axial frequency as a function of curvature coil current, plotted as $\left(\omega_{z} / 2 \pi\right)^{2}$ versus $I_{c u}$, and fitted with a straight line.

\subsection{Absorption Imaging}

We use in situ absorption imaging for the study of ${ }^{6} \mathrm{Li}$ gases described in the next chapters, and also use ${ }^{7} \mathrm{Li}$ images for ${ }^{6} \mathrm{Li}$ thermometry in Chapter 4 . In this section we first explain the probe light production process for imaging atom clouds at high magnetic fields. We then describe the imaging optical system.

\subsubsection{Generation of High-Field Resonant Probes}

The ultracold gases studied in our experiment are produced around the Feshbach resonance center $B_{0}=834 \mathrm{G}$. Optical transitions used for absorption imaging are thus strongly detuned from zero-field transitions, and the preparation of the corresponding probe beams requires a specific setup.

At these large magnetic field values, the hyperfine coupling is smaller than the Zeeman shift and atoms are in the Paschen-Back regime, where electronic and nuclear spins are decoupled (see Fig 2.15. The two lowest internal states of ${ }^{6} \mathrm{Li}$ can thus be labeled as:

$$
\begin{aligned}
|1\rangle & =\left|J=1 / 2, m_{J}=-1 / 2, I=1, m_{I}=+1\right\rangle, \\
|2\rangle & =\left|J=1 / 2, m_{J}=-1 / 2, I=1, m_{I}=0\right\rangle,
\end{aligned}
$$

where $J, m_{J}$ are the electronic quantum numbers and $I, m_{I}$ are the nuclear spin quantum numbers. In the Paschen-Back regime, the nuclear spin is much less coupled to the electric dipole than the electronic spin; therefore optical transitions $\left|m_{J}=-1 / 2, m_{I}\right\rangle \rightarrow\left|m_{J}^{\prime}=-3 / 2, m_{I}^{\prime}=m_{I}-1\right\rangle$ towards $2^{2} P_{3 / 2}$ levels are almost closed. As we image ${ }^{6} \mathrm{Li}$ atoms along a direction perpendicular to the magnetic field, the transition strength is maximum for a linear polarization perpendicular to the magnetic field, with an effective Clebsch-Gordan coefficient equal to $1 / 2$. In the Feshbach resonance center $B_{0}=834 \mathrm{G}$, the transition frequency is detuned by $1.2 \mathrm{GHz}$ to the red from the zero-field $D_{2}$ transitions. We use a high-frequency acousto-optic frequency shifter operating in the range $200-1000 \mathrm{MHz}$, in a double-pass configuration, in order to generate the high-field imaging probe beams. The $\simeq 7 \%$ diffraction efficiency in double-pass configuration requires to use of an additional slave laser in order to obtain large enough laser power for imaging.

\footnotetext{
" Model BRI-GPF-650-225-670 from Brimrose Corp.
} 
The situation is much simpler for ${ }^{7} \mathrm{Li}$ high-field imaging. Indeed, the transition from the ground state:

$$
|7\rangle=\left|J=1 / 2, m_{J}=-1 / 2, I=3 / 2, m_{I}=+3 / 2\right\rangle,
$$

to the state $\left|m_{J}^{\prime}=3 / 2, m_{I}^{\prime}=-3 / 2\right\rangle$ is detuned to the red of the transition $|F=1\rangle \rightarrow\left|F^{\prime}=2\right\rangle$ at zero-field, by about $1.3 \mathrm{GHz}$. This corresponds to a detuning of $-500 \mathrm{MHz}$ with respect to the $|F=2\rangle \rightarrow\left|F^{\prime}=3\right\rangle$ transition at zero field, already used for laser cooling (see Fig 2.15). Using the powerful laser light already prepared for the MOT stage, and a more usual and efficient $200 \mathrm{MHz}$ AOM in double-pass configuration, we obtain a probe beam for ${ }^{7} \mathrm{Li}$ imaging at $B_{0}=834 \mathrm{G}$ with large enough power. For the experiments described in Chapter 4 the ${ }^{7} \mathrm{Li}$ component is imaged along the magnetic field direction. We thus use a circularly polarized $\sigma_{-}$probe, and the Clebsch-Gordan coefficient is equal to 1.
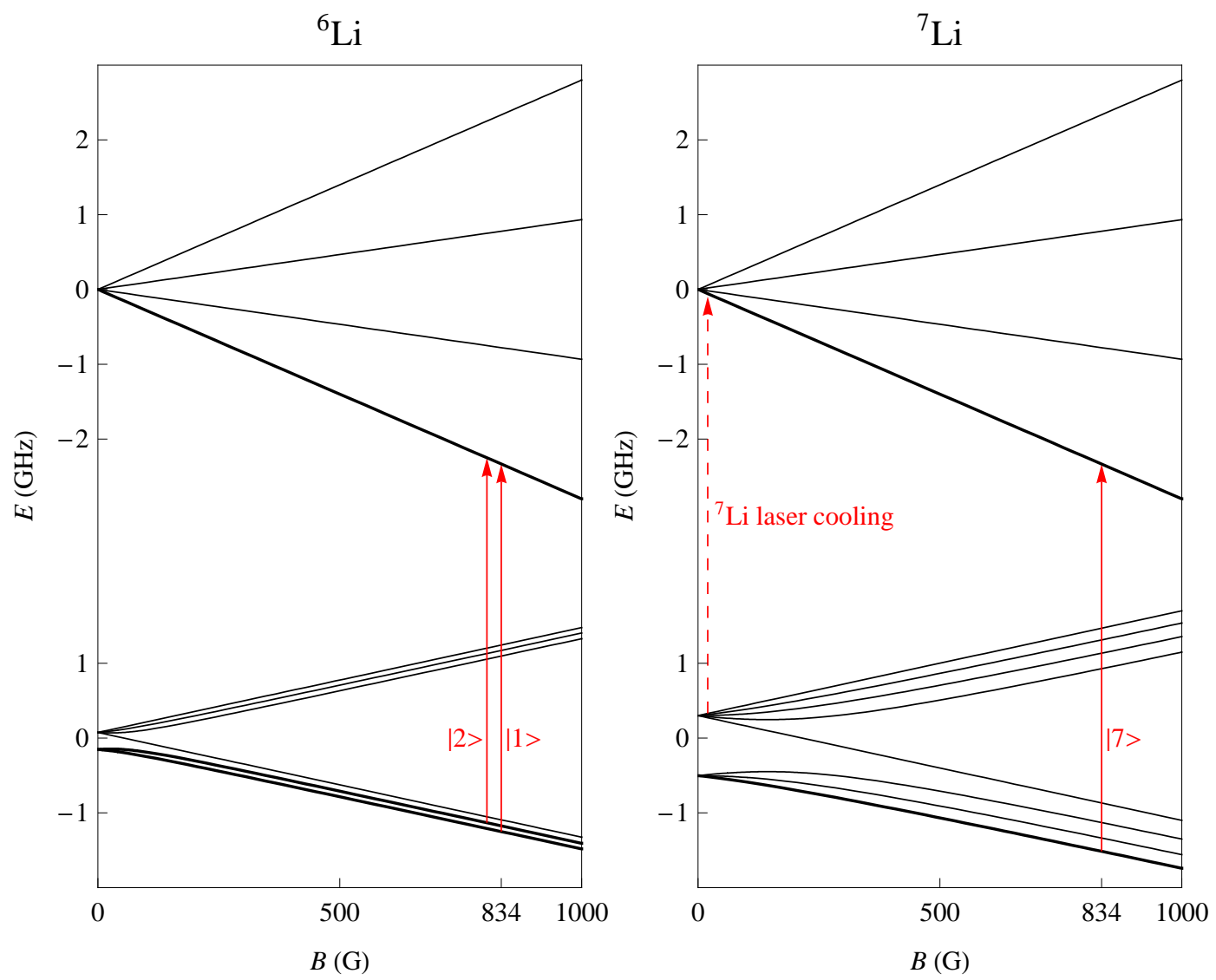

Figure 2.15: Energies of the $2^{2} S_{1 / 2}$ and $2^{2} P_{3 / 2}$ levels for ${ }^{6} \mathrm{Li}$ and ${ }^{7} \mathrm{Li}$ versus magnetic field. The thick lines represent the states $|1\rangle$ and $|2\rangle$ for ${ }^{6} \mathrm{Li}$ and $|7\rangle$ for ${ }^{7} \mathrm{Li}$ used in our experiments. The degeneracy due to the nuclear spin is invisible for the $2^{2} P_{3 / 2}$ levels $(\sim 1 \mathrm{MHz})$. Red arrows: imaging transitions at high field $\left|m_{J}=-1 / 2, m_{I}\right\rangle \underset{\sigma_{-}}{\rightarrow}\left|m_{J}^{\prime}=-3 / 2, m_{I}^{\prime}=m_{I}-1\right\rangle$. The dashed arrow is the laser cooling transition $|F=2\rangle \rightarrow\left|F^{\prime}=3\right\rangle$ at low magnetic field.

\subsubsection{Imaging Optical System}

The imaging optical system for ${ }^{6} \mathrm{Li}$ is represented in Fig 2.16. It is made of two parts: first a $4-f$ optical setup makes an intermediate image of the atom cloud, with a $\times(-1)$ magnification. We then use a pairs of doublets to conjugate the intermediate image onto a CCD camera. The overall optical system magnification, calibrated by monitoring the free fall under gravity of a cloud released from the Ioffe trap, is equal to $M=1.7(1)$. 


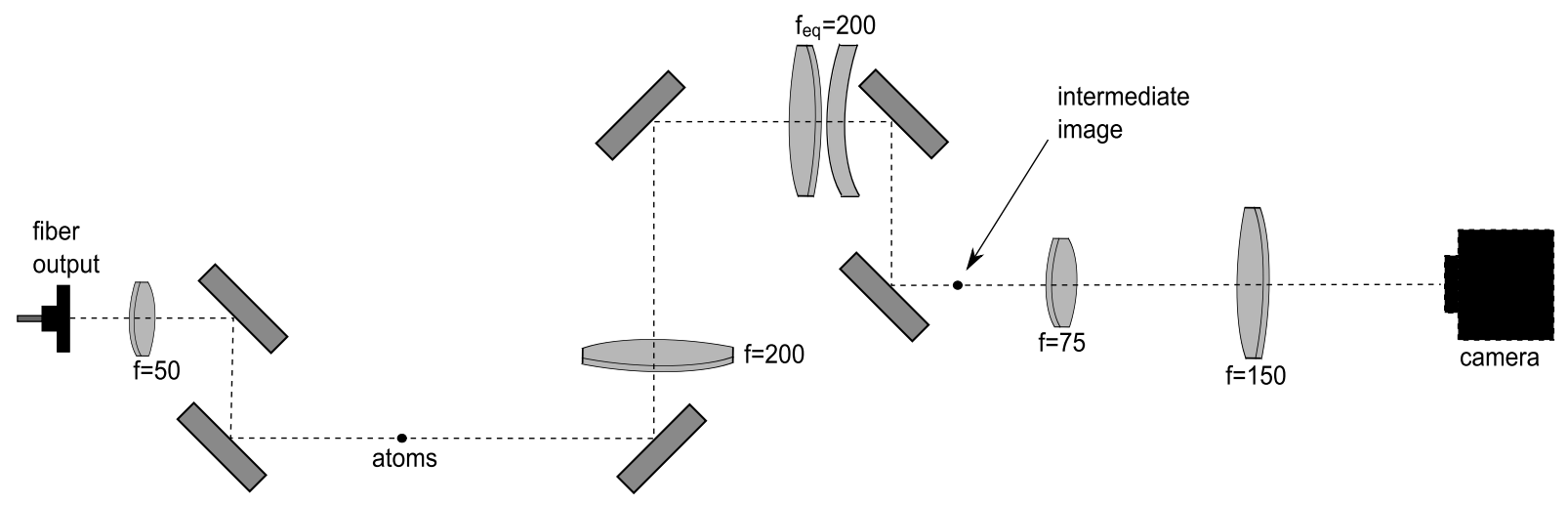

Figure 2.16: Scheme of the imaging system: the probe light exits from an optical fiber and is collimated with a doublet. The atom cloud is first imaged using a $4-f$ setup made of a $f=200 \mathrm{~mm}$ doublet and a combination of a $350 \mathrm{~mm}$ doublet and a $500 \mathrm{~mm}$ meniscus, essentially equivalent to a $200 \mathrm{~mm}$ lens. The intermediate image is then imaged onto the camera using $75 \mathrm{~mm}$ and $150 \mathrm{~mm}$ doublets.

Another imaging system along $z$ is used in Chapter 4 for ${ }^{7} \mathrm{Li}$ thermometry. After taking the ${ }^{6} \mathrm{Li}$ in situ image along $y$, we switch off the optical dipole trap, let the cloud expand for 1 to $4 \mathrm{~ms}$, and take an absorption image of the ${ }^{7} \mathrm{Li}$ component. By imaging along the axial direction $z$, we take advantage of the cloud ellipticity to increase the optical depth and hence the signal-to-noise ratio. We can then reliably deduce the cloud temperature from the size of a $\sim 3000$-atom ${ }^{7} \mathrm{Li}$ gas. Since the imaging beam passes through the Ioffe bars, the solid angle for atom imaging is small, resulting in a relatively poor resolution of $9 \mu \mathrm{m}$. However, it is still much smaller than the typical ${ }^{7} \mathrm{Li}$ cloud's radial size after free flight $\sigma>40 \mu \mathrm{m}$.

\subsubsection{Imaging System Resolution}

The theoretical imaging system resolution is evaluated to $4.1 \mu \mathrm{m}$ (Airy disc radius). Ultracold gases held in the optical dipole trap have a radial size $\sigma<10 \mu \mathrm{m}$ ( $\sigma$ of a gaussian fit), therefore details in the transverse direction are not resolved by our imaging system (see Fig 2.17). The measured cloud radial sizes are consistent with a resolution blurring acting as a convolution with a gaussian of size $\sigma_{r}=4 \mu \mathrm{m}$. The consequences of the finite imaging resolution on the analysis of $i n$ situ images is discussed in section 3.5.4. The magnification was chosen so that $M \sigma_{r}=7 \mu \mathrm{m}$ is comparable to the camera pixel size, equal to $6.45 \mu \mathrm{m}$. The atom signal per pixel is thus maximized without having the pixel discretization decrease the imaging resolution.

\subsubsection{Double in Situ Images}

In Chapter 5 we measure the pressure in spin-imbalanced gases using in situ absorption images of both spin components, taken in the same experimental run. In order to have a pixel-to-pixel correspondence, we use the same camera for both images. The probe beams are also emitted from the same optical fiber in order to share a common intensity pattern. Both images have to be sequentially taken, with special care so that the first absorption image does not degrade the second image.

We first studied the effect of pulse duration on the absorption image of a spin-balanced Fermi gas (see Fig 2.18 ). In that case we take a single absorption image, and maximize the atom signal by sending both probe beams simultaneously. The radial cloud size deduced from the absorption image depends on the probe pulse duration, while keeping the absorbed photon number per atom at $N \simeq 10$ per atom, by adjusting the probe beam power. In Fig 2.18 , we compare the images of a cloud prepared in the same 

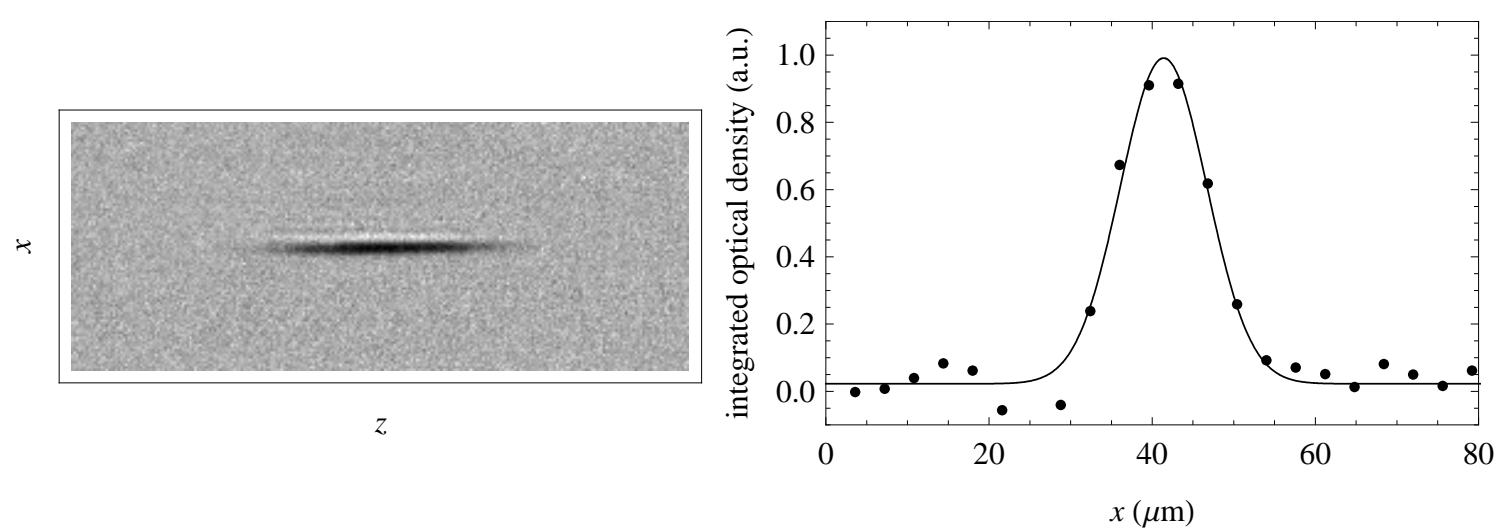

Figure 2.17: Typical absorption image of a spin-balanced Fermi gas used for the work presented in Chapter 4 The optical density integrated along the weak direction $z$ is fitted with a gaussian of width $\sigma=5.3 \mu \mathrm{m}$.

conditions, with pulse durations $10 \mu \mathrm{s}$ and $100 \mu \mathrm{s}$. The measured radial cloud size is equal to $\sigma=6.3 \mu \mathrm{m}$ for the short pulse and $\sigma=8.5 \mu \mathrm{m}$ for the long pulse, showing the effect of photon scattering on a $100-\mu$ s time scale. This has to be compared with the mean velocity $\sqrt{N} / 3 v_{r}$ induced along $x$ by photon scattering ( $v_{r}$ is the recoil velocity):

$$
v_{r} \frac{\sqrt{N}}{3} \frac{\tau}{2} \simeq 5 \mu \mathrm{m} \quad \text { for } \quad \tau=100 \mu \mathrm{s} .
$$

For the study of spin-imbalanced Fermi gases, it is crucial that both spin states are imaged in the same conditions. We use a PixelFly QE camera allowing us to take two images separated by $10 \mu \mathrm{s}$. Using $10-\mu$ s probe pulses separated by $10 \mu$ s (see Fig 2.18 ), we observe no significant perturbation on the second image. The minority spin state is imaged first in order to minimize the number of scattered photons during the first image. Using a saturation parameter $s=I / I_{s} \simeq 0.04$, absorption occurs in the linear regime, with $\simeq 8$ scattered photons per atom. With these parameters, typically 600 photons hit each pixel camera, i.e. 250 photon counts per pixels given the $40 \%$ quantum efficiency of the camera. This number has to be compared with the dark background level of 18 counts per pixel, showing the necessity to reduce the magnification as much as possible to concentrate the atom signal.

The PixelFly camera is able to take a series of two absorption images in a short time interval. Reference images for the computation of the optical densities:

$$
\operatorname{od}_{|i\rangle}=-\log \frac{I_{|i\rangle}}{I^{\text {ref }}}
$$

are thus taken separately, in practice at the end of an experimental run where atoms are absent. Since the reference intensity pattern slowly drifts with time, we take a series of reference images every 10 minutes. We mention that the reference image is not used for the computation of the optical density difference, which is an interesting observable for the identification of full $|1\rangle-|2\rangle$ pairing in the superfluid phase (see Chapter 5):

$$
\begin{aligned}
o d_{|1\rangle}-o d_{|2\rangle} & =-\log \frac{I_{|1\rangle}}{I^{\mathrm{ref}}}+\log \frac{I_{|2\rangle}}{I^{\mathrm{ref}}} \\
& =-\log \frac{I_{|1\rangle}}{I_{|2\rangle}} .
\end{aligned}
$$

The optical density difference thus does not suffer from reference imperfections due to the long delays between absorption and reference images. 
(a)

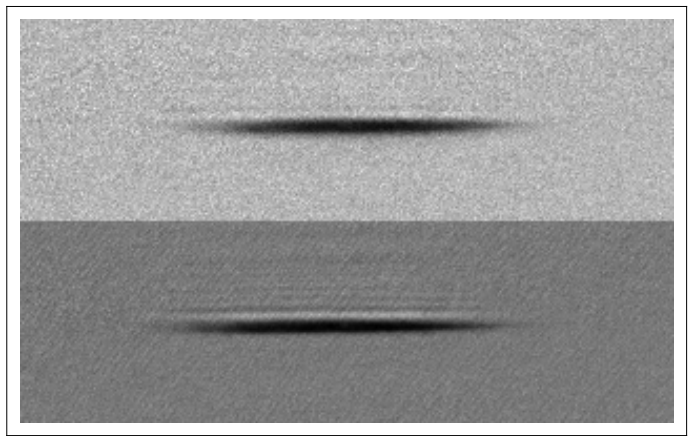

(b)

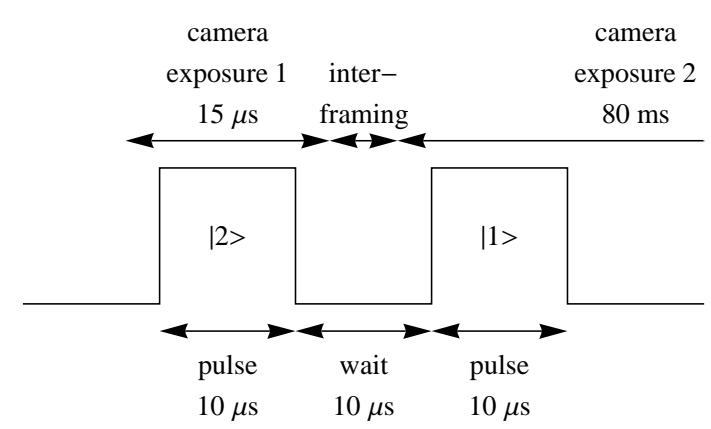

Figure 2.18: (a) Absorption image of a degenerate spin-balanced Fermi gas, using a $100 \mu$ s pulse (upper panel) and a $10 \mu$ s pulse (lower panel). The probe intensity is increased by a factor $\simeq 10$ for the short pulse. The radial sizes are $\sigma=2.3$ pixels and $\sigma=1.7$ pixels, respectively. (b) Scheme of the imaging sequence for spin-imbalanced gases.

To conclude this chapter, we described the experimental setup and the procedure used for producing quantum degenerate Fermi gases in the strongly-interacting regime. The performances of our setup in terms of final atom number and temperature are very good. The specificities of our setup allows us to use ${ }^{7} \mathrm{Li}$ for thermometry and investigate the physics of spin-imbalanced Fermi gases. 


\section{Chapter 3}

\section{Measuring the Equation of State of a Homogeneous Ultracold Gas}

The nearly complete purity and extreme diluteness of ultracold atoms makes them valuable tools for a precision investigation of model Hamiltonians from condensed matter physics. However, the density inhomogeneity induced by the trapping potential makes the comparison between observations in the laboratory and theoretical predictions for homogeneous gases indirect. However, in the local density framework, an ultracold gas can be considered as locally homogeneous. It is thus possible to measure the properties of homogeneous gases using trapped ones, by probing local properties inside the gas. However, ultracold gases are usually probed by measuring the atom induced absorption or phase shift of a laser beam. These techniques give access to a two-dimensional profile, proportional to the atomic density $n$ integrated along the probe beam direction (labeled $y$ ):

$$
\widetilde{n}(x, z)=\int \mathrm{d} y n(x, y, z) .
$$

Deducing local quantities from $\widetilde{n}(x, z)$ is a priori not simple. An important exception is the realization of two-dimensional ultracold gases, where the motion is frozen in one direction (here the direction $y$ ) using very tight confinement $26,108,109,110$. In that case the optical density is proportional to the surface density $n_{s}(x, z)$ and has a direct physical interpretation (see section 3.1.2.

In the first part of this chapter we describe previous works on the thermodynamics of Fermi gases. The first kind of approaches is based on the determination of an equation of state of the entire trapped gas, through the measurement of the gas energy using the virial theorem 62, 63, or the study of collective modes [64]. Since local properties are not probed, the comparison with theoretical results on homogeneous gases is indirect and requires an integration of the latter over the trap. We then describe the thermodynamic study performed at MIT, which relies on the measurement of the local density inside the gas using an Abel transform of $\widetilde{n}(x, z)[49$. This procedure dramatically decreases the signal-to-noise ratio and leads to a noisy equation of state of the homogeneous gas 68 .

We developed a new method for extracting from $\widetilde{n}(x, z)$ the local pressure $P$ inside the gas in a very simple manner [48. It can be used to provide, from a set of several absorption images, a complete equation of state $P(\mu, T)$ of the homogeneous gas, with a small statistical noise. After describing the general principle of the method, we illustrate its power by measuring the equation of state of an ultracold Bose gas in simple situations: from a single image of a Bose gas trapped in three dimensions [31, we deduce the equation of state of a weakly-interacting Bose gas from the classical regime to Bose-Einstein condensation. We also apply it to Bose gases in an optical lattice, in the regime of large lattice depth. The equation of state deduced from the data from the Mainz group 72 reveals characteristic features of 
a Mott insulator and can be used to investigate finite-temperature effects.

\subsection{Equation of State of an Ultracold Gas: State of the Art}

In that section we describe previous studies of the thermodynamics of an ultracold gas.

\subsubsection{Equation of State of a Trapped Gas}

A first approach for measuring thermodynamic properties of an ultracold gas is to extract from an absorption image $\widetilde{n}(x, z)$ physical quantities characterizing the entire gas. Repeating this measurement on a series of gases prepared with different total atom numbers or temperatures, one obtains the equation of state of the trapped gas.

As a first example, an equation of state of the Fermi-Hubbard model was recently measured in the temperature range $T \simeq 0.15 T_{F}$, by measuring the variation of the size $R^{2}=\int \mathrm{d} x \mathrm{~d} z\left(x^{2}+z^{2}\right) \widetilde{n}(x, z)$ of a Fermi gas held in an optical lattice, versus the trap frequency $\omega[21$. The quantity $\partial R / \partial \omega$ can be interpreted as the compressibility of the trapped gas and its variation as a function of confinement strength can be viewed as an equation of state of the trapped gas. This physical picture was helpful for identifying the Mott insulator phase, characterized by a small compressibility [21].

In the rest of this section we focus on techniques developed in the context of bulk Fermi gases since the associated physical results will be compared in the next sections with our measurements.

\section{Equation of State of a Trapped Fermi Gas with Resonant Interactions}

In 2005 the groups at JILA and Duke University studied the thermodynamics of a spin-balanced Fermi gas with resonant interactions 62,63 . They elegantly measured two simple quantities characterizing a trapped gas, its total energy $E_{t}$ and entropy $S_{t}$. The relationship $E_{t}\left(S_{t}\right)$ constitutes an equation of state of a trapped unitary gas.

The total energy of a trapped gas is directly deduced from an in situ absorption image using the virial theorem 111,112: assuming a harmonic confinement and resonant interactions, the total energy of a trapped gas is related to its total potential energy $E_{t}^{p}$ through:

$$
E_{t}=2 E_{t}^{p}
$$

where:

$$
E_{t}^{p}=\int \mathrm{d} x \mathrm{~d} y \mathrm{~d} z\left(\frac{1}{2} m \omega_{x}^{2} x^{2}+\frac{1}{2} m \omega_{y}^{2} y^{2}+\frac{1}{2} m \omega_{z}^{2} z^{2}\right) n(x, y, z)
$$

and $\omega_{i}$ is the trap frequency along the direction $i$. Provided that local density approximation is satisfied, all directions contribute equally to the potential energy, leading to:

$$
E_{t}=3 N \omega_{z}^{2}\left\langle z^{2}\right\rangle
$$

$E_{t}$ is thus easily extracted from the doubly-integrated density profile $\bar{n}(z)=\int \mathrm{d} x \widetilde{n}(x, z)$, using:

$$
\left\langle z^{2}\right\rangle=\frac{\int \mathrm{d} z z^{2} \bar{n}(z)}{\int \mathrm{d} z \bar{n}(z)} .
$$

The entropy $S_{t}$ is measured in another experimental run. A gas is prepared in the same conditions, and before imaging the magnetic field is slowly ramped to the BCS side of the resonance, where the scattering length is small and negative. Assuming that the scattering length change is adiabatic, the entropy after the ramp is equal to the entropy on resonance. On the BCS side of the resonance, interaction effects are small and one knows how to relate the density profile to the entropy $S_{t}$. 
(a)

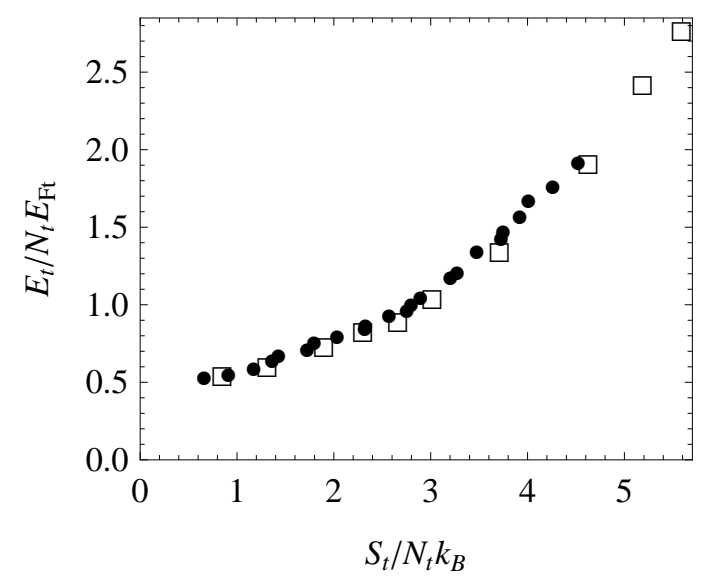

(b)

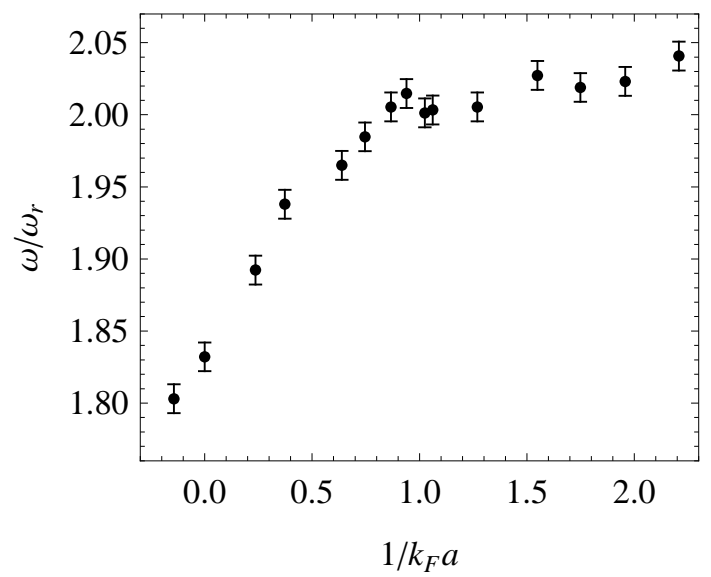

Figure 3.1: (a) Equation of state of a trapped Fermi gas in the unitary limit measured by the JILA group (open squares, from [62]) and at Duke University (black dots, from [63]). (b) Radial breathing mode frequency $\omega$, normalized to the radial trapping frequency $\omega_{r}$, as a function of interaction strength $1 / k_{F} a$ (from $[64])$.

This procedure provides an experimental determination of the equation of state of a trapped unitary gas, shown in Fig 3.1 , with local density approximation as the only assumption. The good agreement between the results obtained in the two groups, using two different atoms $\left({ }^{40} \mathrm{~K}\right.$ in $[62]$ and ${ }^{6} \mathrm{Li}$ in $[63$, constituted the first evidence of the universal character of the thermodynamics of unitary Fermi gases with short-range interactions.

However, it does not provide a direct comparison with many-body theories, which rather calculate the equation of state of a homogeneous gas. In order to make the comparison, one has to model the effect of trap inhomogeneity. Assuming the validity of local density approximation, the equation of state of a trapped gas is calculated by integration over space of the theoretical energy and entropy densities $e$ and $s:$

$$
\begin{aligned}
E_{t} & =\int \mathrm{d} \mathbf{r} e\left(\mu^{0}-V(\mathbf{r}), T\right), \\
S_{t} & =\int \mathrm{d} \mathbf{r} s\left(\mu^{0}-V(\mathbf{r}), T\right) .
\end{aligned}
$$

The equation of state $E_{t}$ as a function of $S_{t}$ is obtained as a parametric curve by varying the values of $\left(\mu^{0}, T\right)$. The calculation of a single point $\left(S_{t}, E_{t}\right)$ thus requires to know the equation of state for all values of $(\mu, T)$ with $\mu<\mu^{0}$. Hence the comparison with discrete numerical results is possible only when the data is dense enough to make a precise interpolation. This makes the comparison with time-consuming diagrammatic Monte Carlo calculations from [113 tricky. Moreover, the comparison with theory remains indirect and the trap averaging blurs the possibly small discrepancies between theories. We will see in section 4.3 that, by contrast, a direct measurement of the equation of state of a homogeneous gas provides a clear identification of the validity domain of theoretical results. Finally, the equation of state of the trapped gas does not easily reveal sharp features such as phase transitions, again because of the trap averaging. This is for instance illustrated by the different values of the critical entropy at the superfluid phase transition, given in [63 46], depending on the procedure used to extract it from the data.

\section{Superfluid Equation of State in the BEC-BCS Crossover}

Another tool for probing thermodynamic properties of a trapped gas is to study its response to a small perturbation. When excited out of equilibrium, the gas profile oscillates around the equilibrium profile. 
When the collision rate is much larger than the oscillation frequency, or if the gas is superfluid, the gas dynamics is well described by hydrodynamic equations [114]:

$$
\begin{aligned}
\frac{\partial}{\partial t} n & =-\nabla \cdot(n \mathbf{v}) \\
m \frac{\partial}{\partial t} v & =-\nabla\left(\frac{1}{2} m v^{2}+\mu(n)+V\right) .
\end{aligned}
$$

Linearizing the hydrodynamic equations around equilibrium then provides the value of the collective oscillation frequencies.

We consider here the experiment performed in the Innsbruck group [64], directly connected to the work described in section 5.5. In that experiment, a spin-balanced gas, prepared at a temperature $T \ll T_{F}$, is trapped in an elongated cylindrical trap, with $\omega_{x}=\omega_{y}=\omega_{r} \gg \omega_{z}$, and is excited in the radial breathing mode. As shown in Fig $3.1 \mathrm{p}$ the frequency measurement is particularly precise. Hydrodynamics predicts that the oscillation frequency is related, with an excellent approximation, to the equation of state $n(\mu)$ through 115:

$$
\frac{\omega^{2}}{\omega_{r}^{2}}=3 \int \mathrm{d} \mathbf{r} n^{2} \frac{\partial \mu}{\partial n}\left(\mu^{0}-V(\mathbf{r})\right) / \int \mathrm{d} \mathbf{r} n\left(\mu^{0}-V(\mathbf{r})\right) V(\mathbf{r}) .
$$

Equation 3.1 enables one to compare these experimental results with theoretical equations of state.

\section{Equation of State of a Trapped Spin-Imbalanced Fermi Gas}

The last example of equation of state of a trapped gas deals with the physics of spin imbalance in a twocomponent Fermi gas, a topic addressed in Chapter 5. In short, when imposing different atom numbers in the two spin states, a phase separation occurs in a trapped gas [54,55, 99]: one observes a fully paired superfluid core of radius $R_{S}$ at the center, then a partially polarized shell where the minority spin state is present up to the radius $R_{2}$, and finally a fully polarized part of radius $R_{1}$ t. The measurement of the radii $R_{S}, R_{2}$ and $R_{1}$ as a function of spin polarization $P=\left(N_{1}-N_{2}\right) /\left(N_{1}+N_{2}\right)$, where $N_{i}$ is the total atom number of species $i$, characterizes macroscopic properties of the ground state of a trapped two-component Fermi gas with resonant interactions 116 (see Fig 3.2 a). In particular one observes a critical polarization $P_{c} \simeq 0.75$ beyond which the superfluid core disappears. The effect of interaction strength on the critical polarization was also addressed using a Feshbach resonance [54] (see Fig 3.2p).

Similarly to the equation of state of a balanced unitary gas at finite temperature, the comparison between theory and experiment in the last two examples, namely the study of collective mode frequencies or the study of spin component radii, requires to integrate the equation of state of a homogeneous gas over the trap. In Chapter 5 we apply our method to these systems, and obtain the equation of state of a homogeneous gas, yielding a direct and much more discriminating comparison with theory.

\subsubsection{Direct Measurement of the Equation of State of a Homogeneous Gas}

In the previous examples, local density approximation was used to calculate characteristic quantities of a trapped gas from a theoretical equation of state of a homogeneous gas. It is possible to reverse the procedure, and to infer the equation of state of a homogeneous gas from an analysis of local properties inside a trapped gas.

\section{Equation of State of a Two-Dimensional Bose Gas}

As mentioned above, two-dimensional ultracold gases are directly suited for this approach, since $\widetilde{n}(x, z)$ is equal to the surface density $n_{S}(x, z)$ (the motion is frozen along $y$ ) 26, 108, 109, 110. In the quasi-2D

\footnotetext{
${ }^{*}$ The Rice group did not observe the intermediate shell, a difference which remains unexplained.
} 
(a)

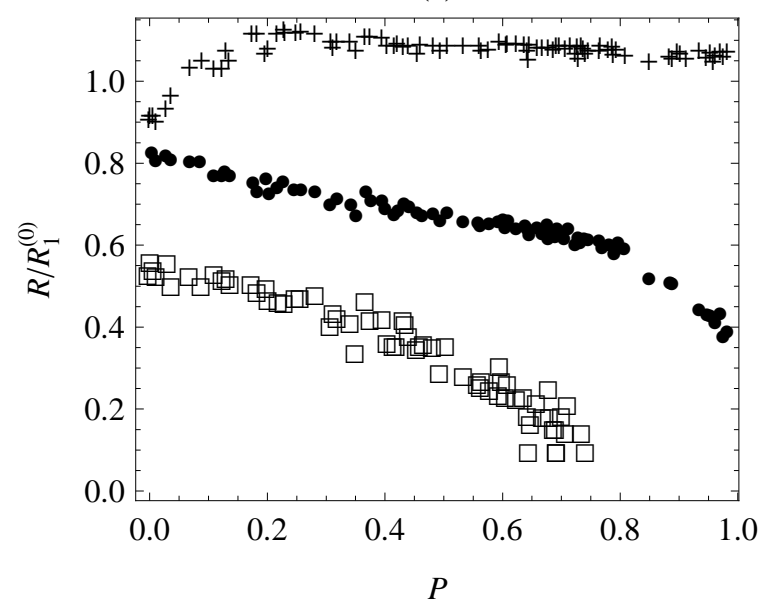

(b)

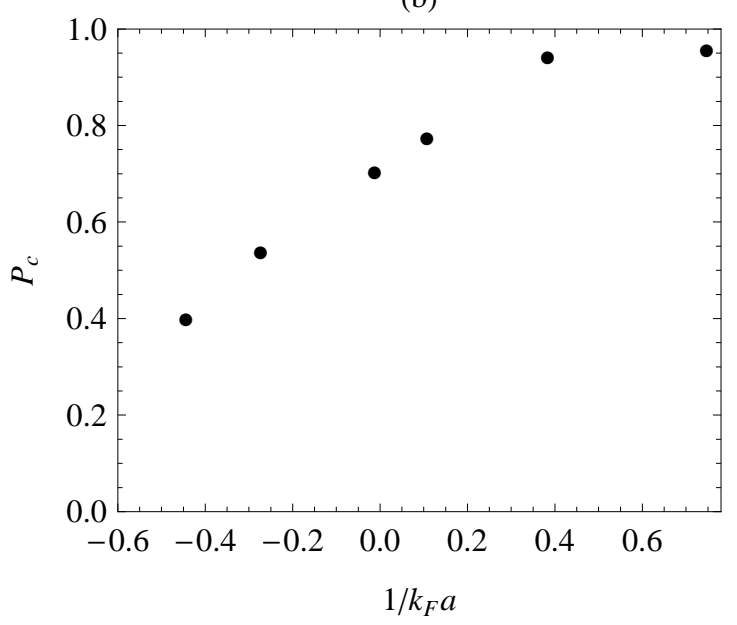

Figure 3.2: (a) Superfluid core radius $R_{S}$ (open squares), minority radius $R_{2}$ (dots) and majority radius $R_{1}$ (crosses) as a function of polarization $P$, from [116. The radii are normalized to the Thomas-Fermi radius of a balanced and non-interacting Fermi gas with same total atom number. We note in particular the disappearance of the superfluid core for $P>P_{c} \simeq 0.75$ (b) Critical polarization $P_{c}$ as a function of the interaction strength $1 / k_{F} a$, from [54].

regime, the equation of state of a homogeneous Bose gas can we written as 117]:

$$
n_{S} \lambda_{d B}^{-2}(T)=G\left(\alpha=\frac{\mu}{k_{B} T}, \widetilde{g}\right)
$$

where $\lambda_{d B}(T)$ is the thermal de Broglie wavelength, and $\widetilde{g}$ is a dimensionless number characterizing the interaction strength and which is independent of $\mu$ and $T$. This equation of state was measured in [110] by analyzing absorption images of trapped gases of ${ }^{87} \mathrm{Rb}$, using the following procedure: Assuming local density approximation, the chemical potential varies in a trapped gas according to $\mu(\mathbf{r})=\mu^{0}-V(\mathbf{r})$. The global chemical potential $\mu^{0}$ and temperature $T$ are extracted from the wings of the cloud, using a mean-field Hartree-Fock description. Each pixel $(x, z)$ then provides a measurement of the equation of state of a homogeneous gas:

$$
\begin{aligned}
\alpha & =\frac{\mu^{0}-V(x, z)}{k_{B} T}, \\
G(\alpha, \widetilde{g}) & =n_{S}(x, z) \lambda_{d B}^{-2}(T) .
\end{aligned}
$$

After averaging over several pixels, one obtains from a single image a low-noise equation of state.

\section{Local Density Measurement Using Abel Transform}

The situation is much more complicated for a three-dimensional gas since the column density $\widetilde{n}(x, z)=$ $\int \mathrm{d} y n(x, y, z)$ does not give a direct access to the local atom density. However, in the case of a cylindrically symmetric trap (the symmetry axis being $z$ ), the relation between the density and the column density can be inverted using an inverse Abel transform

$$
n(x, y, z)=-\frac{1}{\pi} \int_{\sqrt{x^{2}+y^{2}}}^{\infty} \mathrm{d} x^{\prime} \frac{1}{\sqrt{x^{\prime 2}-\left(x^{2}+y^{2}\right)}} \frac{\partial \widetilde{n}\left(x^{\prime}, z\right)}{\partial x^{\prime}} .
$$

\footnotetext{
$\dagger$ Interestingly, this formula does not assume that local density approximation is satisfied, and was used in 97 . where it is clearly violated.
} 


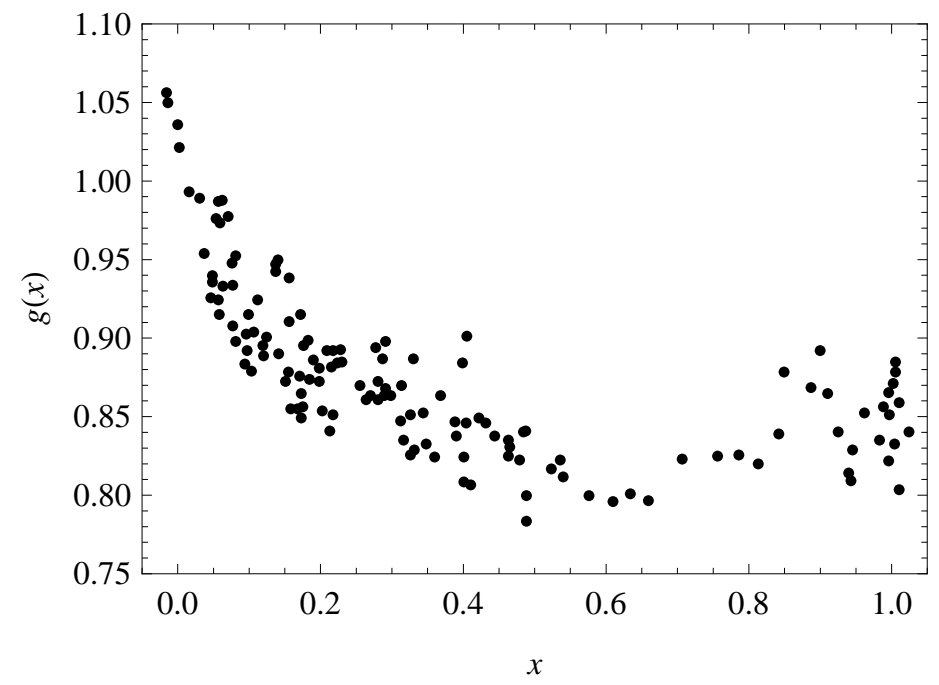

Figure 3.3: Equation of state $g(x)$ of a $T=0$ two-component Fermi gas in the unitary limit (from [68]).

In the MIT experiment, local density profiles $n_{i}(\mathbf{r})(i=1,2)$ of a two-component Fermi gas were computed using [3.2] [49], and used to determine the equation of state the homogeneous gas [68. At $T=0$ and for resonant interactions, the canonical equation of state of a two-component Fermi gas reads 81 :

$$
E\left(n_{1}, n_{2}\right)=\frac{3}{5} \frac{\hbar^{2}}{2 m}\left(6 \pi^{2}\right)^{2 / 3} n_{1}^{5 / 3} g\left(x=\frac{n_{2}}{n_{1}}\right) .
$$

A simple calculation leads to the expression $g=\left(\mu_{1} n_{1}+\mu_{2} n_{2}\right) /\left(\hbar^{2} / 2 m\left(6 \pi^{2}\right)^{2 / 3} n_{1}^{5 / 3}\right)$. Therefore, once the chemical potentials $\mu_{1}^{0}$ and $\mu_{2}^{0}$ are determined, each data point $\left(\mathbf{r}, n_{1}, n_{2}\right)$ provides one measurement of the equation of state:

$$
\begin{aligned}
x & =\frac{n_{2}}{n_{1}}, \\
g(x) & =\frac{2 m}{\hbar^{2}\left(6 \pi^{2}\right)^{2 / 3} n_{1}^{5 / 3}}\left(\left(\mu_{1}^{0}-V(\mathbf{r})\right) n_{1}+\left(\mu_{2}^{0}-V(\mathbf{r})\right) n_{2}\right) .
\end{aligned}
$$

However, the inverse Abel transform dramatically decreases the signal-to-noise ratio, and despite very large atom numbers, the noise of their equation of state is large (see Fig 3.3 ).

\subsection{Description of our Method}

We implemented a new method providing the equation of state of a homogeneous ultracold gas with a good precision. It relies on a simple relationship allowing us to measure the local pressure inside the gas by a simple integration of the column density $\widetilde{n}(x, z)$.

\subsubsection{Measurement of the Local Pressure inside a Trapped Gas}

We discuss here the case of a two-component gas, held in a cylindrically symmetric harmonic trap of frequencies $\omega_{r}$ along $x$ and $y$, and $\omega_{z}$ along $z$. A simple formula then relates the local gas pressure $P$ along the $z$ axis to the integrated density profiles $\bar{n}_{i}(z)=\int \mathrm{d} x \mathrm{~d} y n_{i}(x, y, z)=\int \mathrm{d} x \widetilde{n}_{i}(x, z)$, provided local density approximation is satisfied. We recall that $n_{i}$ is the atom density for species $i$. This relationship was first derived in [70] and more recently used in [69, and I found it independently during my PhD.

The derivation of the formula is straightforward. Gibbs-Duhem identity:

$$
\mathrm{d} P=-S \mathrm{~d} T+n_{1} \mathrm{~d} \mu_{1}+n_{2} \mathrm{~d} \mu_{2}
$$


reads, along $x\left(\mathrm{~d} T=0, \mathrm{~d} \mu_{i}=-m \omega_{r}^{2} x \mathrm{~d} x\right)$ :

$$
\mathrm{d} P=-m \omega_{r}^{2} x\left(n_{1}+n_{2}\right) \mathrm{d} x
$$

Integrating between $x=0$ and $x=+\infty$ gives the pressure along the $z$ axis, i.e. at the chemical potentials $\mu_{i z}=\mu_{i}^{0}-\frac{1}{2} m \omega_{z}^{2} z^{2}:$

$$
\begin{aligned}
P\left(\mu_{1 z}, \mu_{2 z}, T\right) & =m \omega_{r}^{2} \int_{0}^{\infty}\left(n_{1}+n_{2}\right) x \mathrm{~d} x \\
& =\frac{m \omega_{r}^{2}}{2 \pi} \int_{0}^{\infty}\left(n_{1}+n_{2}\right) 2 \pi r \mathrm{~d} r \\
& =\frac{m \omega_{r}^{2}}{2 \pi} \int_{0}^{\infty}\left(n_{1}+n_{2}\right) \mathrm{d} x \mathrm{~d} y
\end{aligned}
$$

which leads to the formula:

$$
P\left(\mu_{1 z}, \mu_{2 z}, T\right)=\frac{m \omega_{r}^{2}}{2 \pi}\left(\bar{n}_{1}(z)+\bar{n}_{2}(z)\right)
$$

This relation can easily be extended to any multi-component ultracold gas at equilibrium, provided each component is harmonically trapped and that local density approximation applies:

$$
P\left(\mu_{i}, T\right)=\frac{1}{2 \pi} \sum_{i} m_{i} \omega_{r i}^{2} \bar{n}_{i}(z)
$$

where $m_{i}$ is the atom mass of species $i$ and $\omega_{r i}, \omega_{z i}$ are the corresponding trapping frequencies. The generalization to non-axially symmetric situations is also straightforward.

Contrary to the inverse Abel transform, integrating $\widetilde{n}(x, z)$ over $x$ to obtain the pressure increases the signal-to-noise ratio. In the next section we describe how to deduce from the pressure profile the equation state of a homogeneous ultracold gas.

\subsubsection{Determination of a Grand-Canonical Equation of State}

The grand canonical equation of state $P\left(\mu_{i}, T\right)$ is particularly suited for the experimental study of the thermodynamics of an ultracold gas. Indeed, the local gas pressure is directly given by $(3.3)$, with a good signal-to-noise ratio. In the local density framework, the arguments $\left(\mu_{i}, T\right)$ of the equation of state are completely characterized by the global chemical potentials $\mu_{i}^{0}$ and the temperature $T$. If these numbers can be appropriately determined, each pixel line at position $z$ provides one point for the equation of state. From a single image one then obtains the equation of state $P\left(\mu_{i}, T\right)$ for a large set of values of $\left(\mu_{i}, T\right)$.

The determination of the temperature and of the global chemical potentials depends on the system considered. Let us describe briefly how they are determined in our study of Fermi gases, presented in the next Chapters. We did not characterize the complete equation of state $P\left(\mu_{1}, \mu_{2}, T, a\right)$ of a twocomponent Fermi gas due to the large number of degrees of freedom. We rather focused on particular physical situations, encompassing most of the physical phenomena:

1. In Chapter 4 we study the equation of state of a spin-balanced gas in the unitary limit $P(\mu, \mu, T, a=$ $\infty)$. The temperature $T$ is measured on a ${ }^{7} \mathrm{Li}$ cloud at thermal equilibrium with the ${ }^{6} \mathrm{Li}$ atoms. $\mu^{0}$ is measured using the wings of the clouds which are in the classical high-temperature regime.

2. In Chapter 5 , the gas is fully evaporated and the temperature $T$ is considered equal to 0 . We then measure the ground state pressure $P\left(\mu_{1}, \mu_{2}, T=0, a\right)$, and $\mu_{i}^{0}$ is determined by fitting the position at which the density $n_{i}$ vanishes. 


\subsection{Equation of State of a Weakly-Interacting Bose Gas}

In this section we apply equation (3.3) to the measurement of the equation of state of a weakly-interacting Bose gas. We use an in situ absorption image of a ${ }^{7} \mathrm{Li}$ cloud prepared in the $\left|F=1, m_{F}=-1\right\rangle$ state, and held in an elongated magnetic trap with a small bias field [31] (see Fig 3.4). The trap frequencies are $\omega_{r} / 2 \pi=4970 \mathrm{~Hz}$ and $\omega_{z} / 2 \pi=83 \mathrm{~Hz}$. As shown in Fig. 3.4 the integrated density profile $\bar{n}(z)$ directly reveals the presence of a Bose-Einstein condensate in the middle of the cloud. Our method will directly provide the grand-canonical equation of state, in both the thermal and condensed regimes.

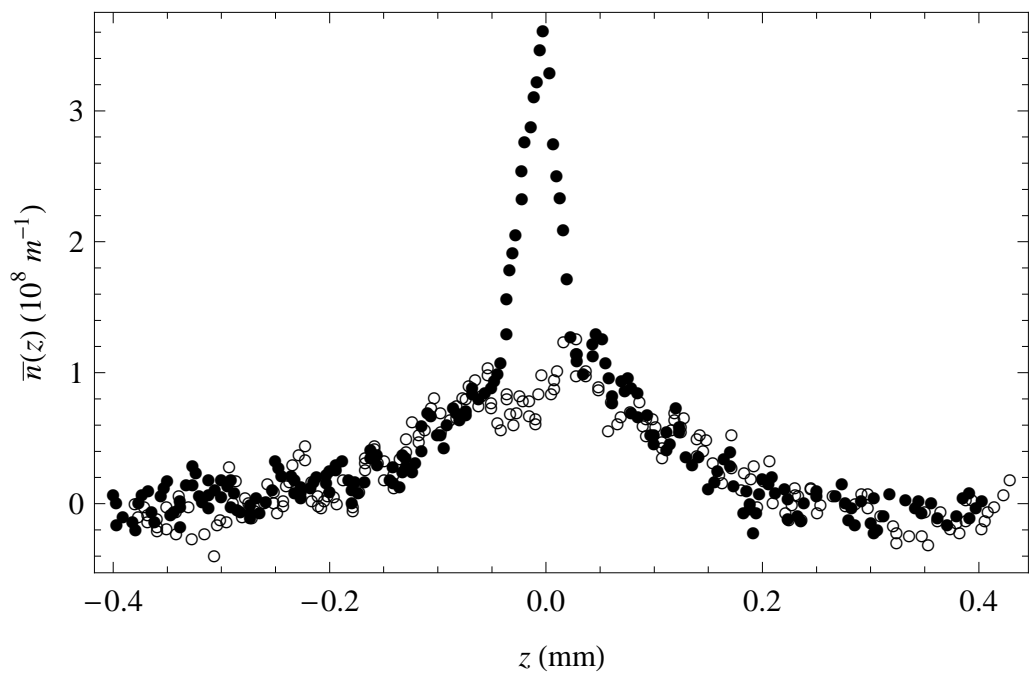

Figure 3.4: Doubly-integrated density profiles of ${ }^{7} \mathrm{Li}$ (dots) and ${ }^{6} \mathrm{Li}$ (open circles). The gas contains $3.5 \times 10^{4}{ }^{7} \mathrm{Li}$ atoms and $2.5 \times 10^{4}{ }^{6} \mathrm{Li}$ atoms.

Dimensional analysis allows us to write the equation of state as:

$$
P(\mu, T)=\frac{k_{B} T}{\lambda_{d B}^{3}(T)} g(\zeta), \quad \text { where } \quad \zeta=e^{-\mu / k_{B} T} .
$$

The variable $\zeta$ is the inverse of the fugacity, and is an increasing function of temperature, at fixed chemical potential. $\lambda_{d B}(T)$ is the thermal de Broglie wavelength. Our goal is to extract from the image the function $g(\zeta)$, which contains all the information on the gas thermodynamics.

The pressure along the $z$ axis is directly given by equation $(3.3)$. In order to obtain the value of $g$, we also need to know the cloud temperature. We use a spin-polarized ${ }^{6} \mathrm{Li}$ gas at thermal equilibrium with the ${ }^{7} \mathrm{Li}$ cloud for thermometry (see Fig 3.4): as the inter-species scattering length $a_{67}=40 a_{0}$ is small, the effect of the ${ }^{7} \mathrm{Li}$ component on the ${ }^{6} \mathrm{Li}$ profile is negligible. The latter is thus fitted with a non-interacting Fermi gas profile, providing the cloud temperature $T=1.6(1) \mu \mathrm{K}$. At this level, we can plot the data as:

$$
g(\zeta)=\frac{P \lambda_{d B}^{3}(T)}{k_{B} T} \quad \text { versus } \quad \exp \left(\frac{m \omega_{z}^{2} z^{2}}{2 k_{B} T}\right)=\frac{\zeta}{\zeta^{0}},
$$

where $\zeta^{0}=e^{-\mu^{0} / k_{B} T}$ is the global inverse fugacity. We thus already know the equation of state $g(\zeta)$ up to an unknown multiplication factor $\zeta^{0}$ in abscissa. We determination of $\zeta^{0}$ requires some information on the equation of state. We fit the data where no Bose-Einstein condensate is present, $\bar{n}(z)<10^{8} \mathrm{~m}^{-1}$ (see Fig 3.4), with the equation of state of an ideal Bose gas above the condensation threshold:

$$
g(\zeta)=g_{5 / 2}(\zeta), \quad \text { where } \quad g_{5 / 2}(z)=\sum_{k=1}^{\infty} \frac{z^{-k}}{k^{5 / 2}}
$$

and obtain the value $\zeta^{0}=0.904$ 
We then deduce the equation of state $g(\zeta)$, shown in Fig. 3.5 . Each pixel row $z$ provides one point $g(\zeta)$, and a single image thus gives the equation of state in the whole relevant temperature range, from the condensed regime to the classical regime. In the non-condensed region, our data is in close agreement with the equation of a weakly-interacting Bose gas $g(\zeta)=g_{5 / 2}(\zeta)$, from the classical regime $\zeta \gg 1$ to $\zeta \simeq 1$. The deviation from the one of a Boltzmann gas $g(\zeta)=\zeta^{-1}$ is a manifestation of the bosonic bunching effect A sharp transition occurs at $\zeta=\zeta_{c} \simeq 1$, indicating the Bose-Einstein condensation transition. While interactions essentially play no role in the description of the thermal part $\zeta>1$, the Bose-Einstein condensate pressure is dominated by the mean-field interaction:

$$
\mu=\frac{4 \pi \hbar^{2} a_{77}}{m_{7}} n
$$

where $a_{77}$ is the scattering length describing collisions between ${ }^{7} \mathrm{Li}$ atoms and $m_{7}$ is the ${ }^{7} \mathrm{Li}$ atom mass. The pressure is then obtained by integrating Gibbs-Duhem relation at fixed temperature $\mathrm{d} P=n \mathrm{~d} \mu$, providing:

$$
g(\zeta)=g_{5 / 2}\left(\zeta_{c}\right)+\frac{\lambda_{d B}(T)}{4 a_{77}}\left(\log ^{2} \zeta-\log ^{2} \zeta_{c}\right)
$$

Fitting our data with equation $(3.6)$ for $\zeta<\zeta_{c}$ and 3.4 for $\zeta>\zeta_{c}$, with $\zeta_{c}$ and $a_{77}$ as free parameters, we obtain the $\zeta_{c}=1.0(1)$ and $a_{77}=11(2) a_{0}=0.6(1) \mathrm{nm}$. The uncertainties take into account the fit uncertainty and the uncertainty related to the temperature determination. The condensation threshold is in agreement with the value $\zeta_{c}=1$ expected for a weakly-interacting Bose gas $\$$. The scattering length value is also in agreement with the calculation $a_{77}=11.5 a_{0}$ in 118 .

Extending this measurement to stronger interactions, on Bose gases prepared close to Feshbach resonances (for example using ${ }^{7} \mathrm{Li}$ clouds in the $|1,1\rangle$ or $|1,0\rangle$ states, held in an optical dipole trap 119 120 121), could reveal more complex beyond-mean-field phenomena.

\subsection{Mott Insulator Behavior of a Bose Gas in an Optical Lattice}

In this section we present an application of our image analysis technique to the measurement of the equation of state of a bosonic Mott insulator using experimental data from [72.

\subsubsection{Realization of a Bose-Hubbard Model}

A ${ }^{87} \mathrm{Rb}$ Bose-Einstein condensate is loaded in a trap consisting of the superposition of a harmonic trap $V_{h}(\mathbf{r})=\frac{1}{2} m\left(\omega_{r}^{2}\left(x^{2}+y^{2}\right)+\omega_{z}^{2} z^{2}\right)$ and a $3 \mathrm{D}$ optical lattice 72 :

$$
V(\mathbf{r})=V_{h}(\mathbf{r})+V_{0}\left(\sin ^{2}(k x)+\sin ^{2}(k y)+\sin ^{2}(k z)\right) .
$$

The optical lattice is created by three orthogonal standing waves of red-detuned laser light of wavelength $\lambda=2 \pi / k=843 \mathrm{~nm}$. Atoms occupy the lowest Bloch band and realize the Bose-Hubbard model [19]:

$$
\hat{H}=-J \sum_{\langle i, j\rangle} \hat{a}_{i}^{\dagger} \hat{a}_{j}+\frac{U}{2} \sum_{i}\left(\hat{n}_{i}-1\right) \hat{n}_{i}+\sum_{i} V_{h}\left(\mathbf{r}_{i}\right) \hat{n}_{i}
$$

\footnotetext{
$\ddagger$ The determination of $\zeta^{0}$ actually makes the overall agreement of the data with $g_{5 / 2}(\zeta)$, rather than $g(\zeta)=\zeta^{-1}$, automatic. However, the fact that some data points in the thermal component satisfy $g>1$, which is independent of the choice of $\zeta^{0}$, is a clear manifestation of the bosonic bunching effect.

$\S$ The chemical potential value at the Bose-Einstein condensation threshold is shifted, to lowest order, by the mean-field interaction with thermal atoms:

$$
\zeta_{c}-1 \simeq\left(\frac{\mu}{k_{B} T}\right)_{c}=\left(\frac{1}{k_{B} T} 2 \frac{4 \pi \hbar^{2} a_{77}}{m_{7}} n\right)_{c}=4\left(n \lambda_{d B}^{3}\right)_{c} \frac{a_{77}}{\lambda_{d B}}=4 \zeta(3 / 2) \frac{a_{77}}{\lambda_{d B}} \simeq 1 \%
$$
}

in our experiment. This value is much smaller than the $10 \%$ uncertainty on the determination of $\zeta_{c}$ from our data. 


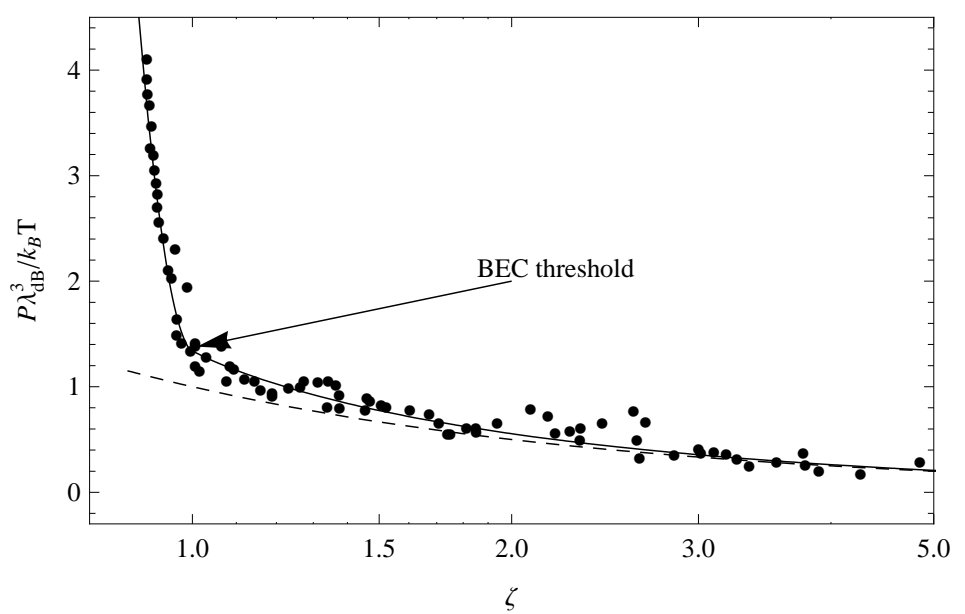

Figure 3.5: Grand-canonical equation of state $g(\zeta)$ of a weakly-interacting Bose gas (black dots). The solid line is a fit of the data with a Bose function $g_{5 / 2}(\zeta)$ in the thermal region and a Thomas-Fermi equation of state in the condensed region (see text). The dashed line is the classical equation of state $g(\zeta)=\zeta^{-1}$.

The index $i$ refers to a potential well at position $\mathbf{r}_{i} . J$ is the tunneling amplitude between nearest neighbors and $U$ is the onsite interaction, both of them being a function of lattice depth [14]. In this section $n$ no longer denotes the atom density bur rather the occupation number per site. The slow variation of $V_{h}\left(\mathbf{r}_{i}\right)$ compared with the lattice period $\lambda / 2$ ensures that local density approximation is satisfied. The system is thus locally described by a homogeneous Hubbard model, given by (3.7) without the last term, and with a local chemical potential $\mu(\mathbf{r})=\mu^{0}-V_{h}(\mathbf{r})$.

\subsubsection{The Mott-Insulator Regime}

The phase diagram of the Bose-Hubbard model was established in 122 and is shown in Fig. 3.6a. At low temperature and for large tunneling values $J \gg U$ the gas forms a Bose-Einstein condensate in the state $|\mathbf{q}=\mathbf{0}\rangle^{N}=\left(N_{l}^{-1 / 2} \sum_{i}|i\rangle\right)^{N}$, where $N_{l}=V(2 / \lambda)^{3}$ is the number of lattice sites.

The condensate depletion becomes large when $U \sim J$ and a quantum phase transition occurs towards an insulator state. We focus here on the limit $U \gg J$. In that situation lattice sites are essentially independent, with a Hamiltonian per site:

$$
\hat{H}_{0}=\frac{U}{2}(\hat{n}-1) \hat{n}
$$

where $\hat{n}$ is the occupation number. The grand-partition function then reads:

$$
\Xi_{0}=\operatorname{Tr} e^{-\beta\left(\hat{H}_{0}-\mu \hat{n}\right)}=\sum_{n=0}^{\infty} e^{-\beta(U n(n-1) / 2-\mu n)}, \quad \beta=1 / k_{B} T,
$$

and the pressure is given by 123,124 Tा

$$
\begin{aligned}
P(\mu, T) & =\frac{k_{B} T}{(\lambda / 2)^{3}} \log \Xi_{0} \\
& \simeq \frac{k_{B} T}{(\lambda / 2)^{3}} \sum_{n=1}^{\infty} \log \left(1+\exp \frac{\mu-(n-1) U}{k_{B} T}\right) .
\end{aligned}
$$

T The last equality in 3.9 is a convenient approximate that introduces an exponentially small error at low temperature $\left(k_{B} T \ll U\right)[123,124$. 
This provides the occupation number (see Fig 3.6 p):

$$
n(\mu, T)=\sum_{n=1}^{\infty} \frac{1}{1+\exp \frac{\mu-(n-1) U}{k_{B} T}} .
$$

In the limit $k_{B} T \ll U$, these Fermi-Dirac-type terms tend to $H(\mu-(n-1) U)$, where $H$ is the Heaviside function, and

$$
n(\mu, T=0)=\left\lceil\frac{\mu}{U}\right\rceil,
$$

i.e. equal to the first integer larger than $\mu / U$ (see Fig 3.6p). At zero temperature the occupation number is thus constant upon varying the chemical potential (except around $\mu=n U$ ), and the compressibility $\kappa=$ $\partial n / \partial \mu$ is equal to zero. This is the characteristic feature of a Mott insulating phase. At low temperature $k_{B} T \ll U$, the occupation number remains flat, until the Mott insulator melts at a temperature $k_{B} T^{*} \simeq$ $0.2 U$ (see Fig 3.6 ).

The grand-canonical equation of state at $T=0$ is calculated by integrating Gibbs-Duhem relation:

$$
\begin{aligned}
P(\mu, T=0) & =\int_{0}^{\mu}\left(\frac{2}{\lambda}\right)^{3} n\left(\mu^{\prime}, T=0\right) \mathrm{d} \mu^{\prime} \\
& =\left(\frac{2}{\lambda}\right)^{3}\left(\mu-\frac{n-1}{2} U\right) n \quad \text { where } n=\left\lceil\frac{\mu}{U}\right\rceil .
\end{aligned}
$$

(a)

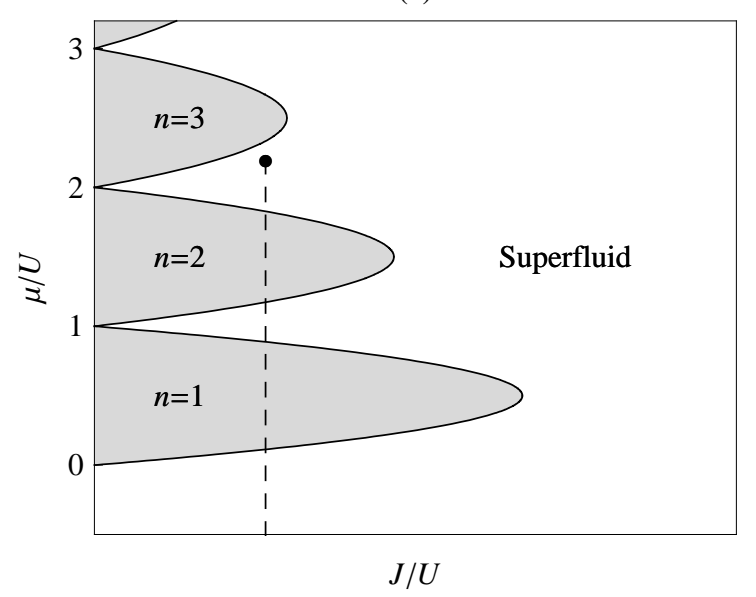

(b)

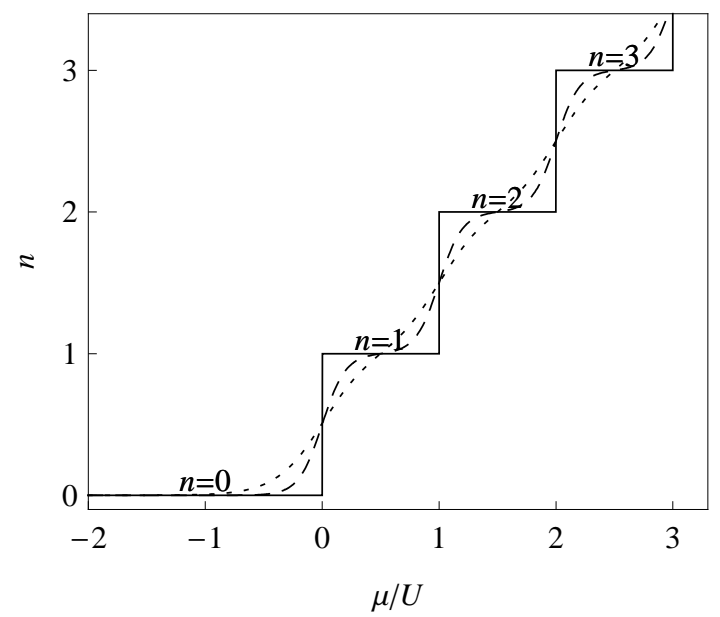

Figure 3.6: (a) Phase diagram of the Bose Hubbard model at zero temperature. The gray regions are the Mott insulating phases with an integer occupation number $n$. In a trapped gas, the chemical potential varies from the value $\mu^{0}$ at the bottom of the trap to $-\infty$ and our method thus provides the equation of state of the Bose Hubbard model along a line (dashed line). (b) Mean occupation number $n$ in the Mott regime $J \ll U$, for $k_{B} T=0$ (solid line), $k_{B} T=0.1 U$ (dashed line), and $k_{B} T=0.2 U$ (dotted line).

\subsubsection{Extraction of the Equation of State}

We illustrate our method by extracting the equation of state of the Bose-Hubbard model in the regime $U \gg J$, from doubly-integrated density profiles from the Mainz group [72].

\section{Local Pressure Measurement}

Resolving details of in situ images of a $\sim 30 \mu \mathrm{m}$ cloud requires a resolution on the order of $1 \mu \mathrm{m}$. As the experiment was not designed for implementing a high-resolution optical imaging system, the gas 
was rather probed using a tomographic technique [72]. In the trap bottom, atoms experience a linear gradient of $3.4 \mathrm{G} / \mathrm{cm}$ along $z$. By applying a radio-frequency pulse, atoms are transfered into another internal state, with a spatial selectivity $\mathrm{d} z \simeq 1 \mu \mathrm{m}$ determined by the pulse characteristics [125]. The number $\mathrm{d} N=\bar{n}(z) \mathrm{d} z$ of transfered atoms is then counted using absorption imaging after a time-of-flight, thus giving access to the doubly-integrated density profile $\bar{n}(z)$ and hence, using equation (3.3), to the gas pressure. Repeating this measurement for different probe frequencies provides the complete pressure profile. In Fig $3.7 \mathrm{a}$ we show four pressure profiles $a, b, c, d$ corresponding to different total atom numbers $N_{a}=0.6 \times 10^{5}, N_{b}=10^{5}, N_{c}=2 \times 10^{5}$ and $N_{d}=3.5 \times 10^{5} 72125$.

\section{Construction of the Equation of State}

The chemical potential varies along $z$ according to $\mu_{z}=\mu^{0}-\frac{1}{2} m \omega_{z}^{2} z^{2}$. Therefore, the data plotted as:

$$
\frac{P}{U(\lambda / 2)^{-3}} \text { versus } \quad-\frac{1}{2} m \omega_{z}^{2} z^{2},
$$

is equal to the equation of state $P / U(\lambda / 2)^{-3}$ versus $\mu$, up to a translation in abscissa by the global (unknown) chemical potential $\mu^{0}$. Since all images correspond to the same equation of state (we assume a common temperature for all images), the sets of data from the each image can all be superimposed by translating all images onto one of them, let us say image $c$ (see Fig $3.7 \mathrm{p}$ ). We thus determine the differences $\mu_{\alpha}^{0}-\mu_{c}^{0}$ between global chemical potentials, and gather all data points in a single equation of state (still translated from the actual equation of state by the unknown quantity $\mu_{c}^{0}$ ).

(a)

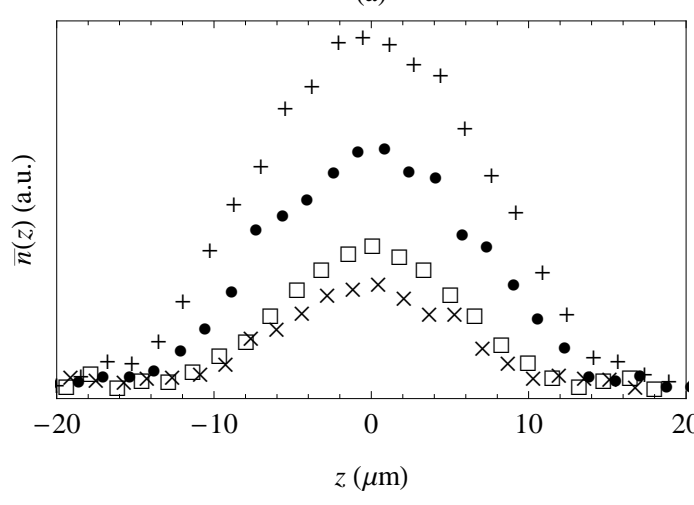

(b)

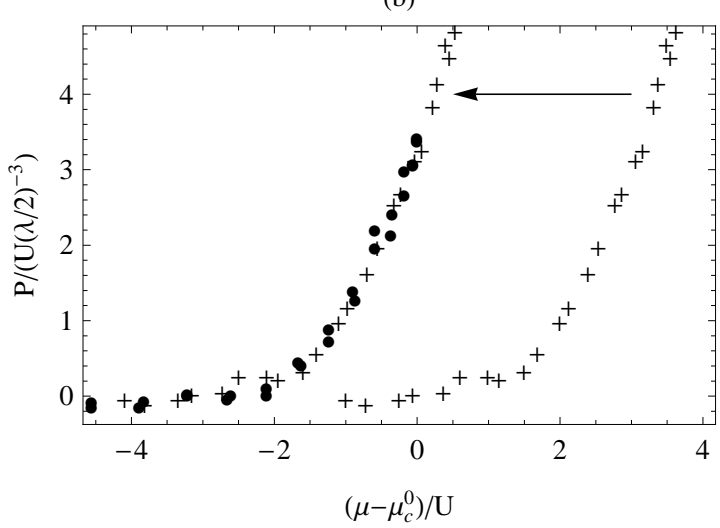

Figure 3.7: (a) Doubly-integrated density profiles for a cloud prepared with $N_{a}=0.6 \times 10^{5}$ (crosses), $N_{b}=10^{5}$ (open squares), $N_{c}=2 \times 10^{5}$ (black dots) and $N_{d}=3.5 \times 10^{5}$ (plus). (b) Superposition of the data from image $d$ on the one from image $c$. (3.11).

\subsubsection{Observation of a Mott-Insulator Behavior}

In order to observe a Mott-Insulator behavior, we fit the data with piecewise linear function consistent with equation (3.11):

$$
\begin{aligned}
\frac{P}{U(\lambda / 2)^{-3}} & =0 \text { for } \mu<0 \\
& =n_{1} \frac{\mu}{U} \text { for } 0<\mu<\delta \mu_{1} \\
& =n_{1} \frac{\delta \mu_{1}}{U}+n_{2} \frac{\mu-\delta \mu_{1}}{U} \text { for } \delta \mu_{1}<\mu<\delta \mu_{1}+\delta \mu_{2} \\
& =n_{1} \frac{\delta \mu_{1}}{U}+n_{2} \frac{\delta \mu_{2}}{U}+n_{3} \frac{\mu-\delta \mu_{1}-\delta \mu_{2}}{U} \text { for } \delta \mu_{1}+\delta \mu_{2}<\mu,
\end{aligned}
$$


with $\delta \mu_{1}, \delta \mu_{2}, n_{1}, n_{2}$ and $n_{3}$ as free parameters. We also allow a free translation in abscissa by a quantity $\mu_{c}^{0}$. The determination from the fit of $\mu_{c}^{0}$ provides the unknown quantity that was missing to obtain the equation of state of the Bose-Hubbard model in the Mott regime, plotted in Fig.3.8.

The other fit parameters exhibit the characteristic features of the incompressible Mott phases. The size of the first Mott region is $\delta \mu_{1}=0.9(1) U$, with an occupation number $n_{1}=1.0(1)$ atom per site. The size of the second Mott region size is $\delta \mu_{2}=1.1(1) U$, with an occupation number $n_{2}=2.0(1)$. Finally, the occupation number in the third Mott region is $n_{3}=3.1(1)$. These values agree with the theoretical values $\delta \mu_{i}=U$ and $n_{i}=i$.

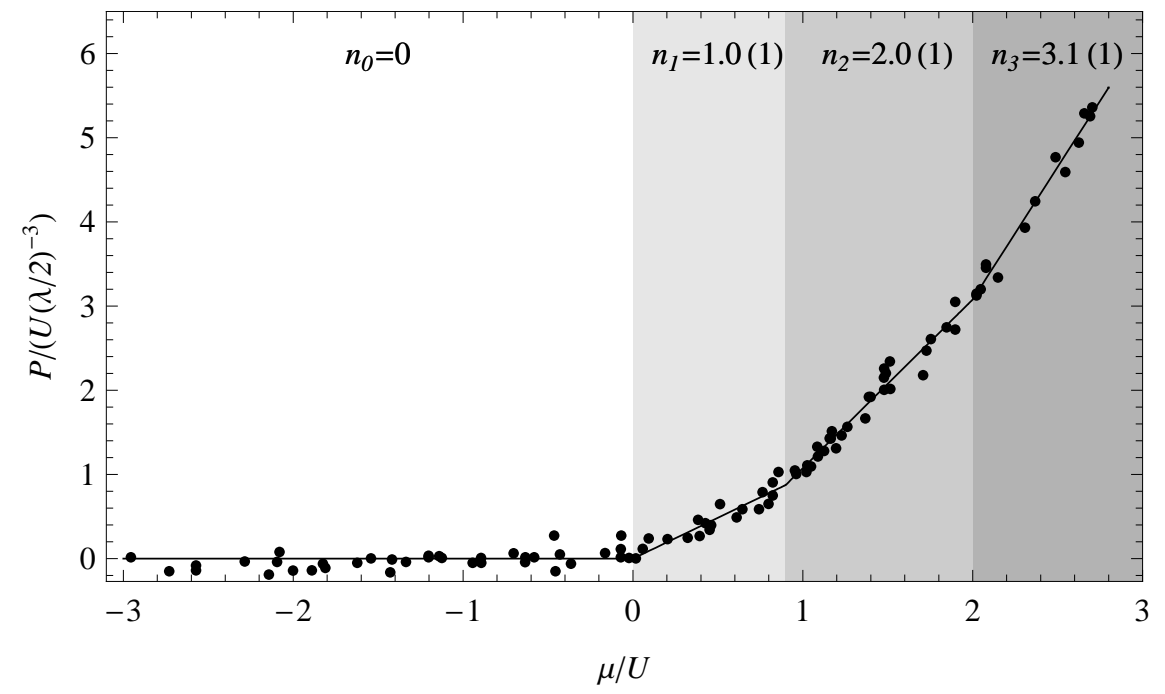

Figure 3.8: Equation of state of the Bose-Hubbard model in the Mott regime $U \gg J$ (black dots). The solid line is a fit of the equation of state by a piecewise linear function, revealing the Mott phases.

\subsubsection{Estimation of Finite-Temperature Effects}

Finite-temperature effects tend to smear the occupation plateaus of a Mott insulator, until a their disappearance at the melting temperature $k_{B} T^{*} \simeq 0.2 U[123$ (see Fig 3.6 b). Fitting the experimental equation of state with the finite-temperature equation of state $(3.9)$, we obtain:

$$
k_{B} T=0.09_{-0.1}^{+0.04} U
$$

i.e. a value significantly lower than the melting temperature.

In order to estimate the effect of the pressure profile smearing due to the finite resolution of the tomographic technique, we convolved a theoretical $T=0$ profile with a point-spread function associated with the finite probe resolution (see 125$)$. The simulated profile is then fitted with $(3.9)$, and we obtain $k_{B} T=0.08(3) U$. This shows that the investigation of lower temperature regimes would require a better resolution.

\subsection{Validity of the Pressure Measurement}

In this section we discuss the validity of equation (3.3) used for measuring the local gas pressure. We show how to calibrate the pressure measurement in order to minimize systematic errors. We also estimate the magnitude of the deviation from local density approximation and the effect of trap anharmonicity or finite imaging system resolution. 


\subsubsection{Calibration of the Pressure}

The systematic error on the pressure measured using equation $(3.3)$ is a priori defined by the uncertainty of atom counting and of the radial frequency. Since it is very difficult to achieve an atom counting precision better than $\sim 10 \%$, we rather directly calibrate the pressure using a reference pressure profile.

We use the pressure profile of a deeply evaporated spin-balanced Fermi gas, in the unitary limit, as a reference (see Fig 3.9. Indeed the equation of state of such a gas is well known:

$$
P(\mu)=\xi_{s}^{-3 / 2} \frac{2}{15 \pi^{2}}\left(\frac{2 m}{\hbar^{2}}\right)^{3 / 2} \mu^{5 / 2} .
$$

The parameter $\xi_{s}=0.415(20)$ is measured in Chapter 5 without using this calibration, and this value is in agreement with most previous experimental and theoretical results. Fitting an experimental pressure profile $P\left(\mu_{z}\right)$ with 3.12 provides a precise calibration of the pressure. Finally, we estimate the error due to a shot-to-shot drift of the probe laser frequency or radial trapping frequency to be less than $5 \%$.

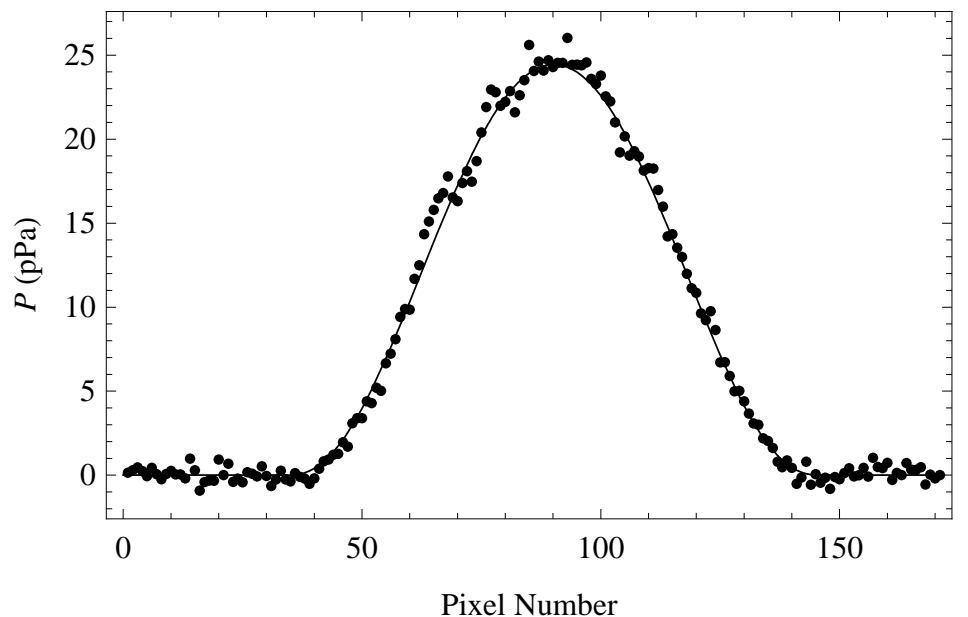

Figure 3.9: Pressure profile of a balanced Fermi gas at unitarity and very low temperature. The solid line is a fit with a Thomas-Fermi profile multiplied by $\xi_{s}^{-3 / 2}$, providing a calibration of the pressure.

\subsubsection{Deviation from Local Density Approximation}

The pressure measurement is based on local density approximation. In the case of strong interactions there is no prediction for the amplitude of deviations from local density approximation. We thus consider the simpler situation of a two-component balanced Fermi gas in the BEC limit $1 / k_{F} a \gg 1$. The gas is then a molecular Bose-Einstein condensate, described by the Gross-Pitaevskii equation:

$$
\left(-\frac{\hbar^{2}}{4 m} \Delta+V+\frac{4 \pi \hbar^{2} a_{d d}}{2 m} n\right) \sqrt{n}=\mu^{0} \sqrt{n}
$$

where $n$ is the molecule density and $a_{d d} \simeq 0.6 a$ is the dimer-dimer scattering length 16 . For simplicity the trap is assumed isotropic, with a frequency $\omega$. In the local density approximation framework, the first term is neglected, leading to the Thomas-Fermi density profile:

$$
\begin{aligned}
n(\mathbf{r}) & =\frac{2 m}{4 \pi \hbar^{2} a_{d d}}\left(\mu^{0}-V(\mathbf{r})\right), \\
& =n_{0}\left(1-\frac{r^{2}}{R_{T F}^{2}}\right), \quad \text { where } \quad R_{T F}=\sqrt{\frac{2 \mu^{0}}{m \omega^{2}}} \quad \text { and } \quad n_{0}=\frac{2 m}{4 \pi \hbar^{2} a_{d d}} \mu^{0},
\end{aligned}
$$


plotted in Fig 3.10 as a dashed line. Writing the Gross-Pitaevskii equation using dimensionless variables $\widetilde{r}=r / R_{T F}$ and $\widetilde{n}=n / n_{0}$ :

$$
\widetilde{n}=1-\widetilde{r}^{2}-\epsilon \frac{\Delta(\sqrt{\widetilde{n}})}{\sqrt{\tilde{n}}}, \quad \text { where } \quad \epsilon=\left(\frac{\hbar \omega}{2 \mu^{0}}\right)^{2},
$$

quantifies the gradient term in terms of a dimensionless number $\epsilon$. For a typical molecular condensate with $N=5 \times 10^{4}$ molecules, prepared at $750 \mathrm{G}$ where $a \simeq 2000 a_{0}$, and held in a trap with a mean frequency $\omega=2 \pi \times 330 \mathrm{~Hz}$, the parameter $\epsilon$ is on the order of $0.5 \%$. A numerical resolution of the Gross Pitaevskii equation, shown in Fig 3.10 . confirms the very small deviation from the Thomas-Fermi density profile 126 . The size of the region where local density approximation is incorrect is on the order of the healing length $\xi=1 / \sqrt{8 \pi n a_{d d}}$, which is much smaller than the cloud size. As a conclusion, local density approximation accurately describes the density profile of a trapped Fermi gas in the BEC limit. In more complex situations such as the unitary limit, the exact calculation is not possible, but we expect the conclusion to remain the same because the correlation length $\sim k_{F}$ is much smaller than the cloud size.

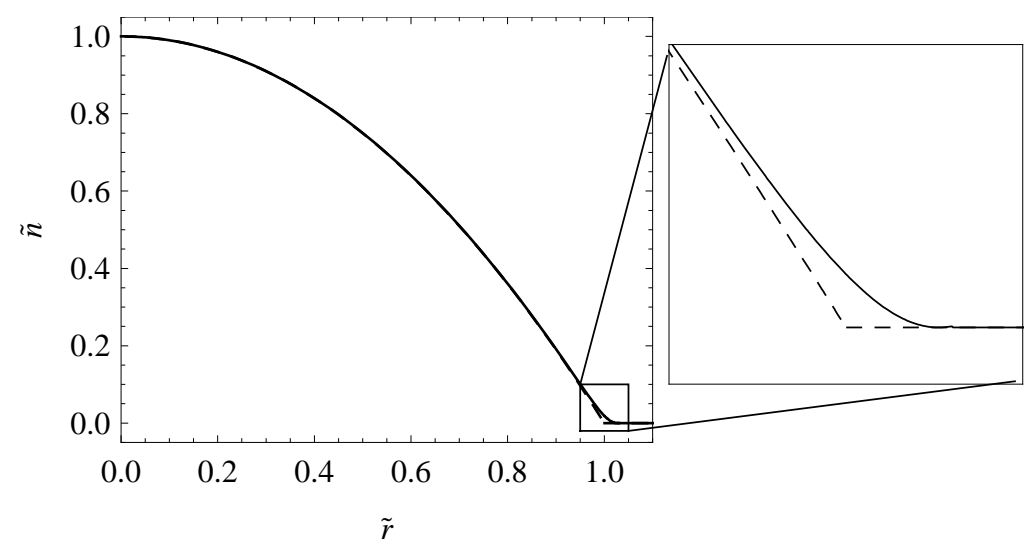

Figure 3.10: Density profile $\widetilde{n}(\widetilde{r})$ of a molecular Bose-Einstein condensate calculated using the GrossPitaevskii equation (solid line) and the Thomas-Fermi approximation (dashed line). The total molecule number is $N=5 \times 10^{4}$, the trap frequency is $\omega=2 \pi \times 330 \mathrm{~Hz}$, and the scattering length is $a=2000 a_{0}$ (corresponding to a magnetic field $B \simeq 750 \mathrm{G}$ ).

However, the effect of the gradient term can be much more pronounced if the density profile abruptly changes. This typically occurs around a density jump associated with a first-order phase transition, a situation encountered in Chapter 5 when considering spin-imbalanced trapped gases. When the populations of the two spin states differ, a phase separation occurs between a central superfluid core and an external normal shell. At the interface between the two phases, local density approximation predicts an abrupt density jump. Gradient terms beyond local density approximation then play an essential role in the description of the interface. In a first approximation, they might be captured by adding a surface tension term to the force balance at the interface between the superfluid $(\mathrm{S})$ and normal $(\mathrm{N})$ phases $[127,128,129$ :

$$
P_{N}-P_{S}=\frac{\sigma}{2 R}
$$

where $\sigma$ is the surface tension coefficient and $R$ is the mean curvature radius of the interface. In particular, in the case of an anisotropic confinement, surface tension tends to deform the interface from a trap equipotential. In Appendix B.4 we show that, in our experiment, the surface tension effect is very small and that local density approximation applies well for the description of Fermi gases.

Local density approximation is also expected to fail in the critical region of a second-order phase transition, due to the divergence of the correlation length. This situation will be encountered in section 4.7 when considering the superfluid transition of a spin-balanced Fermi gas at unitarity. However, we 
show in section 4.7 .4 that in practice the size over which local density approximation is incorrect is very small, and that $(3.3)$ provides the pressure of a homogeneous gas with a good precision.

\subsubsection{Effect of the Trap Anharmonicity}

\section{Correction to the Pressure Formula due to the Trap Anharmonicity}

Besides local density approximation, the other key assumption for obtaining equation (3.3) is the trap harmonicity. However, the optical dipole trap potential is actually gaussian in the transverse directions:

$$
V_{r}(x, y)=U_{0}\left(1-\exp \left(-\frac{r^{2}}{w_{0}^{2}}\right)\right)
$$

Let us estimate the error introduced by the anharmonicity of this potential. For simplicity we consider a spin-balanced mixture of total density $n(\mu, T)$. In the local density approximation framework, the local chemical potential is given by:

$$
\mu(x, y, z)=\mu^{0}-V_{r}(x, y)-\frac{1}{2} m \omega_{z}^{2} z^{2}
$$

and the integrated density reads:

$$
\bar{n}(z)=\int 2 \pi r \mathrm{~d} r n\left(\mu^{0}-V_{r}(x, y)-\frac{1}{2} m \omega_{z}^{2} z^{2}, T\right) .
$$

Using the variable $v=V_{r}(r)$ and $n=\partial P / \partial \mu$ leads to:

$$
\frac{m \omega_{r}^{2}}{2 \pi} \bar{n}(z)=\int_{0}^{U_{0}} \frac{1}{1-v / U_{0}} \frac{-\partial P}{\partial \mu}\left(\mu_{z}-v, T\right) \mathrm{d} v,
$$

where $\omega_{r}=\sqrt{2 U_{0} / m w_{0}^{2}}$ is the radial frequency at the trap bottom. Expanding this expression for large trap depths $U_{0}$, and integrating by parts, we obtain:

$$
\frac{m \omega_{r}^{2}}{2 \pi} \bar{n}(z)=P\left(\mu_{z}, T\right)+\int_{0}^{\infty} \frac{\mathrm{d} v}{U_{0}} P\left(\mu_{z}-v, T\right) .
$$

In the limit of a very large depth $U_{0} \rightarrow \infty$, the first term in 3.13 dominates and one recovers equation (3.3). The second term in 3.13 is the first correction to 3.3 due to the trap anharmonicity.

\section{Amplitude of the Anharmonic Correction}

Let us now evaluate the amplitude of this correction for typical situations encountered in our experiment.

We first consider the case of a high-temperature Fermi gas in the classical regime, a situation encountered in the measurement of the equation of state of the unitary gas at finite temperature (see Chapter 4. We recall the expression of the pressure for a classical gas:

$$
P(\mu, T)=2 k_{B} T \lambda_{\mathrm{dB}}^{-3}(T) \exp \left(\mu / k_{B} T\right)
$$

The integration over the chemical potential in $(3.13)$ is then straightforward, leading to:

$$
\frac{m \omega_{r}^{2}}{2 \pi} \bar{n}(z)=P\left(\mu_{z}, T\right)\left(1+\frac{k_{B} T}{U_{0}}\right) .
$$

The relative error is equal to $k_{B} T / U_{0}$, typically equal to 0.1 when we do not compress the optical trap at the end of evaporation (see section 2.4.4. It does not depend on the position in the trap, hence the pressure profile is not distorted but globally shifted. Therefore, the systematic error could be corrected by modifying the calibration of the pressure. 
The error introduced by the trap anharmonicity is also simple to estimate for a single-component Fermi gas at zero temperature. The pressure is then given by:

$$
P(\mu, T=0)=\frac{1}{15 \pi^{2}}\left(\frac{2 m}{\hbar^{2}}\right)^{3 / 2} \mu^{5 / 2}
$$

and integrating over the chemical potential in $(3.13)$ gives:

$$
\frac{m \omega_{r}^{2}}{2 \pi} \bar{n}(z)=P\left(\mu_{z}, 0\right)\left(1+\frac{2}{7} \frac{\mu_{z}}{U_{0}}\right) .
$$

In that case, the correction is inhomogeneous, i.e. the doubly-integrated profile, compared with the pressure profile, is distorted. The larger distortion amplitude occurs at the trap center and is equal to $2 \mu^{0} / 7 U_{0}$. For typical trap parameters considered in our study of zero-temperature Fermi gases in Chapter 5. $\mu^{0} / U_{0} \simeq 0.1$ and the maximal distortion is $3 \%$, below the statistical noise of our data.

\subsubsection{Effect of the Imaging System Resolution}

The finite resolution of the imaging system has to be considered for a precise understanding of in situ images. As shown in section 2.5.3. the effect of the resolution of our imaging system can be captured by a convolution of the probe intensity profile with a gaussian of size $\sigma_{r}=4 \mu \mathrm{m}$. The Thomas-Fermi radius of a trapped gas is typically equal to $15 \mu \mathrm{m}$ in the transverse direction, and details of the profile are not resolved. However, the axial Thomas-Fermi radius being on the order of $300 \mu \mathrm{m}$, fine details are well resolved in the axial direction. We show here that the pressure $P\left(\mu_{z}\right) \propto \bar{n}(z)$, obtained by integration of the column density $\widetilde{n}(x, z)$, is insensitive to the finite resolution of the imaging system, at least for small optical densities. We also address the combined effect of finite resolution and large optical densities.

\section{Insensitivity to the Resolution for Low-Density Clouds}

We consider here the case of clouds with a small optical density, for which the column density is proportional to the probe intensity absorption:

$$
\widetilde{n}(x, z) \propto \frac{I_{0}-I(x, z)}{I_{0}},
$$

where $I_{0}$ is the incoming light intensity and $I(x, z)$ is the light intensity after passing through the atom cloud. The intensity profile actually measured is obtained by convoluting the real profile with a gaussian of size $\sigma_{r}$ :

$$
I_{\text {measured }}(x, z)=(I * f)(x, z)=\int \mathrm{d} x^{\prime} I\left(x^{\prime}, z\right) f\left(x-x^{\prime}\right), \quad \text { where } \quad f(x)=\frac{1}{\sqrt{2 \pi} \sigma_{r}} \exp \left(-\frac{x^{2}}{2 \sigma_{r}^{2}}\right) .
$$

Since the cloud profile varies along $z$ on a much larger length scale than $\sigma_{r}$, the convolution along $z$ has a negligible effect on the intensity profile and has not been included. In the linear regime, the proportionality between the column density and the intensity absorption leads to:

$$
\widetilde{n}_{\text {measured }}(x, z)=\int \mathrm{d} x^{\prime} \widetilde{n}\left(x^{\prime}, z\right) f\left(x-x^{\prime}\right) .
$$

The integrated density profile is then given by:

$$
\begin{aligned}
\bar{n}_{\text {measured }}(z) & =\int \mathrm{d} x \widetilde{n}_{\text {measured }}(x, z) \\
& =\int \mathrm{d} x^{\prime} \widetilde{n}\left(x^{\prime}, z\right) \int \mathrm{d} x f\left(x-x^{\prime}\right) \\
& =\bar{n}(z) .
\end{aligned}
$$

Therefore, when light absorption is in the linear regime, doubly-integrated density profiles are insensitive to the finite resolution of the imaging system [55]. 


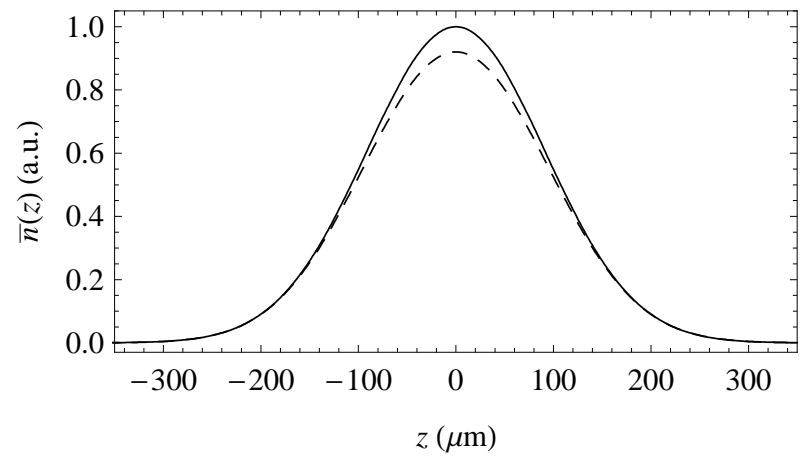

Figure 3.11: Theoretical integrated profile $\bar{n}(z)$ of a spin-balanced gas with a total atom number $N=10^{5}$, at a temperature $T=180 \mathrm{nK}$, and held in a trap of frequencies $\omega_{r} / 2 \pi=1.1 \mathrm{kHz}$ and $\omega_{z} / 2 \pi=37 \mathrm{~Hz}$. The solid line (dashed line) is the theoretical profile with $\sigma_{r}=0\left(\sigma_{r}=4 \mu \mathrm{m}\right)$.

\section{Large Optical Density Effects}

In the case of large optical densities, the relationship between intensity absorption and column density is no longer linear:

$$
I(x, z)=I_{0} \exp \left(-\sigma_{0} \widetilde{n}(x, z)\right)
$$

where $\sigma_{0}$ is the light scattering cross section. The measured intensity profile then reads:

$$
I_{\text {measured }}(x, z)=\int \mathrm{d} x^{\prime} I_{0} \exp \left(-\sigma_{0} \widetilde{n}\left(x^{\prime}, z\right)\right) f\left(x-x^{\prime}\right)
$$

The measured column density is thus given by:

$$
\begin{aligned}
\widetilde{n}_{\text {measured }}(x, z) & =\frac{1}{\sigma_{0}} \log \frac{I_{0}}{I_{\text {measured }}(x, z)} \\
& =\frac{1}{\sigma_{0}} \log \frac{I_{0}}{(I * f)(x, z)} \\
& \neq(\widetilde{n} * f)(x, z),
\end{aligned}
$$

and in particular $\bar{n}_{\text {measured }}(z) \neq \bar{n}(z)$.

In Fig 3.11 we simulate the effect of the finite resolution of our imaging system for a cloud with typical parameters used in Chapter 4. The maximum optical density is then 1.2. The pressure deduced from the integrated density profile is $9 \%$ less than the actual pressure at the trap center. As a result, in the determination of the equation of state of a spin-balanced unitary gas, we do not use the data of optical density larger than $\simeq 1$, and the accuracy of the pressure measurement is then better than $5 \%$. For the study of the ground state of a spin-imbalanced Fermi gas described in Chapter 5 , the axial trapping frequency is reduced to $\omega_{z} / 2 \pi=20 \mathrm{~Hz}$ in order to reduce the cloud's optical density and minimize non-linear effects. 


\section{Chapter 4}

\section{Thermodynamics of a Strongly-Interacting Fermi gas}

The first application of our method to Fermi gases is the measurement of the equation of state of a two-component Fermi gas with an equal number of atoms in each spin state, and prepared in the unitary limit $a=\infty$. As described in section 3.1.1 the equation of state of a trapped unitary gas was measured in 62 63. Despite its importance as the first measurement of a 'model-independent' equation of state, it cannot be directly compared with many-body theories, which rather deal with homogeneous gases. The comparison requires to integrate the theoretical equations of state over the trap, making use of local density approximation. However, some advanced theories, such as the Diagrammatic Monte Carlo calculations from the Amherst group [113], only provide several points for the equation of state, and the integration over the trap can not be performed. These theories have thus remained untested by experiments up to now. The aim of our study is to provide the equation of state of a homogeneous gas in order to make a direct comparison with theory. We will see that this comparison reveals unexpected features.

Moreover, it is clear that sharp features such as phase transitions are expected to be smeared out by the trap averaging. As an example, the critical temperature for superfluidity is almost invisible on the equation of state of a trapped gas 63, 46. We will show that the equation of state of the homogeneous gas is more suited for measuring the critical temperature, as well as other physical quantities such as virial coefficients.

\section{Universal Thermodynamics of a Fermi Gas in the Unitary Limit}

In this chapter we consider a gas prepared at $834 \mathrm{G}$, where the scattering length $a$ is infinite, and with equal atom numbers in each spin state. In that situation, the two chemical potentials $\mu_{1}$ and $\mu_{2}$ are equal and we will use the notation $\mu=\mu_{1}=\mu_{2}$ in the rest of the chapter. The equation of state is then reduced to a relation between the pressure $P$ and the intensive variables $\mu$ and $T$. At unitarity, the scattering length drops from the equation of state and the only way to construct a dimensionless number is the combination $\mu / k_{B} T$. Therefore the equation of state can be written as the product of a reference pressure, taken as the pressure $P_{0}(\mu, T)$ of a single-component ideal gas, multiplied by a dimensionless function $h_{T}(\zeta)$, where $\zeta=\exp \left(-\mu / k_{B} T\right)$ is the inverse of the fugacity:

$$
P(\mu, T)=2 P_{0}(\mu, T) h_{T}(\zeta)
$$

$\zeta$ is a convenient grand-canonical equivalent of $T / T_{F}$, and is an increasing function of temperature. 
All informations of the thermodynamics of a Fermi gas with resonant interactions are included in the function $h_{T}(\zeta)$. In this Chapter, we present the measurement of $h_{T}(\zeta)$ using the method described in Chapter 3, as well as a physical interpretation of our data.

\section{1 ${ }^{7} \mathrm{Li}$ Thermometry}

In Chapter 3 we showed how to extract from in situ images the pressure $P\left(\mu_{z}, T\right)$ along the $z$ axis. Deducing the equation of state from the pressure profile of a trapped gas also requires to know the temperature $T$ and the global chemical potential $\mu^{0}$. The temperature cannot be extracted from the cloud image without invoking a model, since the relation between density profile and temperature is precisely given by the equation of state we want to measure. Inspired by the Innsbruck group [73], we developed a new thermometry method, using ${ }^{7} \mathrm{Li}$ atoms immersed in the ${ }^{6} \mathrm{Li}$ gas, and at thermal equilibrium with it. The temperature is measured on the ${ }^{7} \mathrm{Li}$ component through its size after time-of-flight, a technique specific to weakly-interacting gases.

\subsubsection{Preparation of a Three-Component ${ }^{6} \mathrm{Li}^{-} \mathrm{Li}$ Mixture}

Adding a small amount of ${ }^{7} \mathrm{Li}$ atoms in the optical trap is particularly simple. We control the amount of ${ }^{7} \mathrm{Li}$ at the end of sympathetic cooling by appropriately choosing the final frequency of the RF knife expelling ${ }^{7} \mathrm{Li}$ atoms from the magnetic trap. As we do not need a large number of ${ }^{7} \mathrm{Li}$ atoms, we keep a smaller amount of ${ }^{7} \mathrm{Li}$ than ${ }^{6} \mathrm{Li}$, hence the final ${ }^{6} \mathrm{Li}$ temperature and transfer efficiency to the optical trap are essentially unchanged. However this procedure leads to a larger sensitivity to atom number drifts. Indeed, a larger ${ }^{6} \mathrm{Li}$ atom number leads to a higher temperature at the end of sympathetic cooling, and requires an adjustment of the final knife frequency to maintain a constant ${ }^{7} \mathrm{Li}$ atom number.

Once loaded into the optical dipole trap, atoms are transfered into the final internal states. Under a 10$\mathrm{G}$ bias magnetic field, they are simultaneously transfered from $\left|F=3 / 2, m_{F}=3 / 2\right\rangle$ to $\left|F=1 / 2, m_{F}=1 / 2\right\rangle=|1\rangle$ for ${ }^{6} \mathrm{Li}$ and from $\left|F=2, m_{F}=2\right\rangle$ to $\left|F=1, m_{F}=1\right\rangle=|7\rangle$ for ${ }^{7} \mathrm{Li}$ using an adiabatic passage around the hyperfine transitions, respectively around $245 \mathrm{MHz}$ and $825 \mathrm{MHz}$. The collision rate being especially large in the optical trap at the highest power, it is crucial to make the transfer as fast as possible to avoid spin-exchange inelastic collisions between low-field and high-field seeking states. Using high-power amplifiers (50 W and $30 \mathrm{~W}$ for the ${ }^{6} \mathrm{Li}$ and ${ }^{7} \mathrm{Li}$ frequencies, respectively), we are able to make the transfer with essentially no atom loss in $100 \mathrm{~ms}$. The magnetic field is then ramped to $834 \mathrm{G}$ and we prepare a balanced $|1\rangle-|2\rangle$ mixture using a series of non-adiabatic Landau-Zener passages around the $|1\rangle-|2\rangle$ transition. We do not observe significant losses during this phase.

\section{Stability of the Three-Component ${ }^{6} \mathrm{Li}-{ }^{7} \mathrm{Li}$ Mixture}

Inelastic losses constitute the main limit to the realization of arbitrary ultracold mixtures. We discuss here the stability of the ${ }^{6} \mathrm{Li}-{ }^{7} \mathrm{Li}$ mixture of the two lowest states of ${ }^{6} \mathrm{Li}$ and of the ground state of ${ }^{7} \mathrm{Li}$ with respect to inelastic collisions. The hyperfine structure of the electronic ground state of ${ }^{6} \mathrm{Li}$ and ${ }^{7} \mathrm{Li}$ is shown in Fig, 4.1. The splitting between $|1\rangle$ and $|2\rangle$ is $76 \mathrm{MHz}$ at high field, while the splitting between the two lowest states of ${ }^{7} \mathrm{Li},|7\rangle$ and $|8\rangle$, is $177 \mathrm{MHz}$. The inelastic spin-exchange collision:

$$
{ }^{6} \mathrm{Li}_{|2\rangle}+{ }^{7} \mathrm{Li}_{|7\rangle} \rightarrow{ }^{6} \mathrm{Li}_{|1\rangle}+{ }^{7} \mathrm{Li}_{|8\rangle}
$$

is therefore endothermic and cannot occur. Moreover, two-body dipolar losses were estimated by S. Kokkelmans to be very small, on the order of $2 \times 10^{-18} \mathrm{~cm}^{3} / \mathrm{s}$. Before starting the evaporation, no appreciable atom loss is observed after waiting in the optical trap at full power, thereby confirming the very good stability of the mixture. 

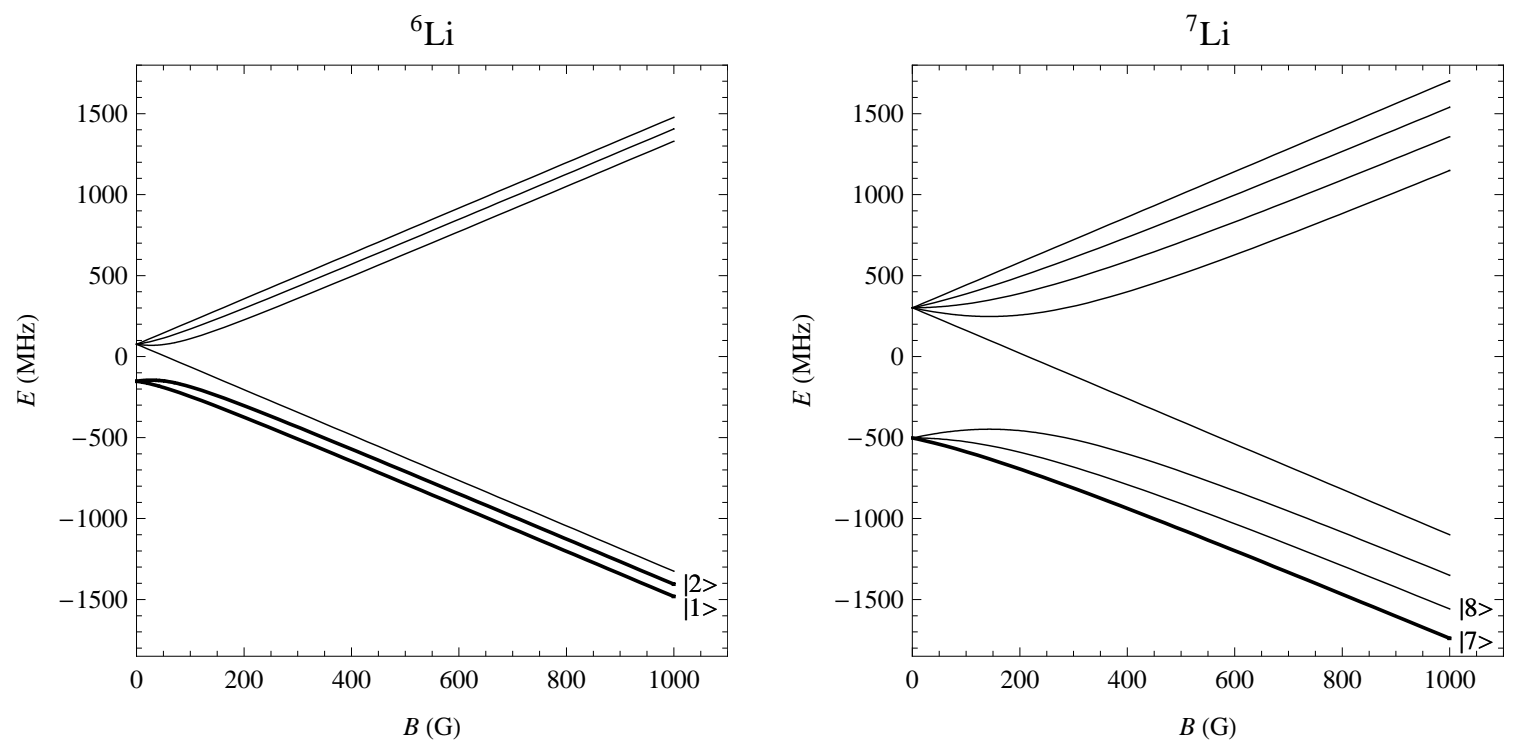

Figure 4.1: Energy levels of the electronic ground state of ${ }^{6} \mathrm{Li}$ (left) and ${ }^{7} \mathrm{Li}$ (right). The gas is prepared in a mixture of the two lowest states of ${ }^{6} \mathrm{Li}$, labeled $|1\rangle$ and $|2\rangle$, and of the ground state of ${ }^{7} \mathrm{Li}$, labeled $|7\rangle$.

\subsubsection{Two-Species Evaporation and Thermalization}

The scattering length describing collisions between ${ }^{6} \mathrm{Li}$ (in $|1\rangle$ or $|2\rangle$ ) and ${ }^{7} \mathrm{Li}$ atoms is equal to $a_{67}=41 a_{0}$, with essentially no variation with magnetic field. On the other hand, the scattering length $a_{77}$ between ${ }^{7} \mathrm{Li}$ atoms strongly depends on the magnetic field value because of the existence of a 200-G-wide Feshbach resonance centered at $737 \mathrm{G} 119120130$. At the magnetic field $B=834 \mathrm{G}$ corresponding to the center of the ${ }^{6} \mathrm{Li}$ Feshbach resonance, the scattering length value is $a_{77}=-73 a_{0}$.

At the beginning of evaporation, the optical dipole trap typically holds $N_{6}=2 \times 10^{6}{ }^{6} \mathrm{Li}$ atoms in each spin state and $N_{7}=10^{6}{ }^{7} \mathrm{Li}$ atoms, at a temperature $T \simeq 250 \mu \mathrm{K}$, and with a mean trapping frequency $\bar{\omega} / 2 \pi \simeq 2.5 \mathrm{kHz}$. The collision rate between ${ }^{6} \mathrm{Li}$ atoms, interacting with resonant interactions, is very large [104:

$$
\Gamma_{66}=\frac{2 N_{6} \hbar^{2} \bar{\omega}^{3}}{\pi\left(k_{B} T\right)^{2}} \simeq 4000 \mathrm{~s}^{-1},
$$

while the collision rate between ${ }^{6} \mathrm{Li}$ and ${ }^{7} \mathrm{Li}$ atoms and between ${ }^{7} \mathrm{Li}$ atoms are respectively:

$$
\Gamma_{76}=\frac{2 N_{6} m \bar{\omega}^{3} a_{67}^{2}}{\pi k_{B} T} \simeq 150 \mathrm{~s}^{-1}, \quad \text { and } \quad \Gamma_{77}=\frac{4 N_{7} m \bar{\omega}^{3} a_{77}^{2}}{\pi k_{B} T} \simeq 200 \mathrm{~s}^{-1} .
$$

These large collision rates ensure an efficient thermalization during evaporative cooling. In the classical regime $\left(T>0.3 T_{F}\right)$ where the ${ }^{6} \mathrm{Li}$ temperature can directly be measured, we indeed observe identical temperatures for both species. It is also important to be convinced of a good thermalization in the degenerate regime, where the ${ }^{6} \mathrm{Li}$ temperature can no longer be measured. At the end of evaporation, the trap typically contains $5 \times 10^{4}{ }^{6} \mathrm{Li}$ atoms in each spin state and $10^{4}{ }^{7} \mathrm{Li}$ atoms, the mean trapping frequency being $\bar{\omega} / 2 \pi \simeq 300 \mathrm{~Hz}$. The classical collision rate is then on the order of $30 \mathrm{~s}^{-1}$, but collisions between ${ }^{7} \mathrm{Li}$ and ${ }^{6} \mathrm{Li}$ atoms may be strongly inhibited by Pauli exclusion principle in the degenerate ${ }^{6} \mathrm{Li}$ cloud. Indeed, a ${ }^{6} \mathrm{Li}$ atom undergoing a collision needs to be scattered in an empty state, which restricts the allowed final states above the Fermi level, and thus decreases the collision probability. In Chapter 5. we show that ${ }^{6} \mathrm{Li}$ gases can be evaporated up to very low temperatures $T=0.03(3) T_{F}$, showing that collisions still occur in the deeply degenerate regime. In our experiment, we observed that the ${ }^{7} \mathrm{Li}$ 


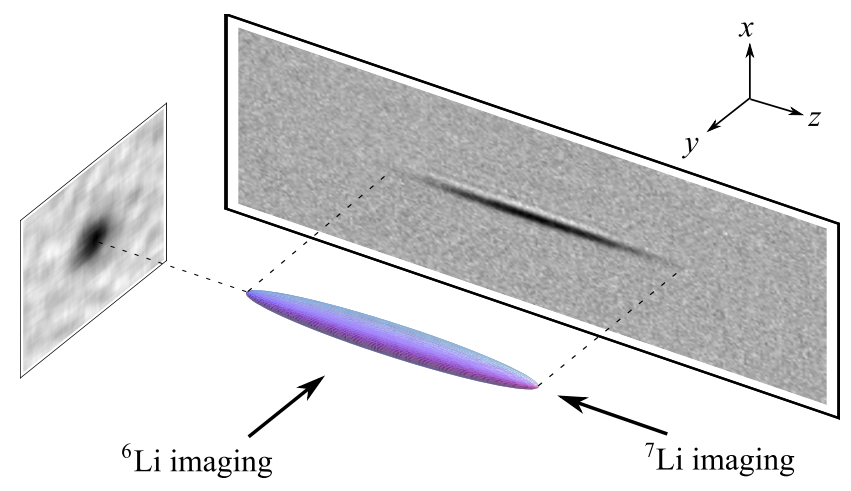

Figure 4.2: ${ }^{6} \mathrm{Li}$ atoms are imaged in situ along $y$, and ${ }^{7} \mathrm{Li}$ atoms are imaged along $z$, after a time of flight. Typical absorption images are shown, corresponding to a high-temperature cloud at $T=1.5 \mu \mathrm{K}$ containing $1.5 \times 10^{5}{ }^{6} \mathrm{Li}$ atoms in each spin state and $10^{4}{ }^{7} \mathrm{Li}$ atoms.

temperature no longer varies after evaporation down to a given trap depth, as if the temperature reaches a steady state in less than $100 \mathrm{~ms}$. This indicates a good thermalization efficiency even at the lowest temperatures. This has to be compared with the experimental results from 73 obtained on a mixture of ${ }^{6} \mathrm{Li}$ and ${ }^{40} \mathrm{~K}$. A steady state is reached after more than $3 \mathrm{~s}$, and the final temperatures for the ${ }^{6} \mathrm{Li}$ cloud and the ${ }^{40} \mathrm{~K}$ cloud differ by $\simeq 30 \%$, probably due to different heating rates for the two species. In our case, ${ }^{6} \mathrm{Li}$ and ${ }^{7} \mathrm{Li}$ atoms experiencing exactly the same trapping potential, we expect trap-induced heating to be almost identical for the two species and therefore the final ${ }^{6} \mathrm{Li}$ and ${ }^{7} \mathrm{Li}$ temperatures to be identical.

\subsubsection{Temperature Measurement}

At the end of evaporative cooling, the ${ }^{6} \mathrm{Li}$ component is imaged in situ using absorption imaging along the transverse direction $y$ (see Fig 4.2. The trap is switched off during the pulse, and ${ }^{7} \mathrm{Li}$ atoms are imaged along $z$ after a time of flight chosen between 1 and $4 \mathrm{~ms}$, depending on the radial trapping frequency. Imaging along $z$ increases the optical depth, and the size of the cloud can reliably be fitted for ${ }^{7} \mathrm{Li}$ atom numbers down to $\sim 3000$. Essentially no collision occur between the two species during the time-of-flight, and we checked that, indeed, ${ }^{6} \mathrm{Li}$ imaging has no significant effect on the ${ }^{7} \mathrm{Li}$ profile. The imaging system magnification along $z$ is calibrated by comparison of the size of a high-temperature cloud imaged simultaneously along $z$ and $y$. We then use the calibration of the imaging system magnification along $y$ (see section 2.5.2 to deduce the one along $z$. As the measured temperatures can be close to the Bose-Einstein condensation threshold for ${ }^{7} \mathrm{Li}$, we fit the ${ }^{7} \mathrm{Li}$ density profile with a Bose distribution 131 .

\subsubsection{Limitation of our Thermometer at Low Temperature}

The scattering length $a_{77}=-73 a_{0}$ being negative, ${ }^{7} \mathrm{Li}$ Bose-Einstein condensates are unstable above a critical atom number 132,133 :

$$
N_{c} \simeq 0.37 \sqrt{\frac{\hbar}{m_{7} \bar{\omega}_{7}}} \frac{1}{\left|a_{77}\right|} \simeq 230
$$

where we have taken into account the effect of trap ellipticity [134. When evaporated down to the lowest trap depths, we observed that the temperature saturates at the critical temperature for condensation:

$$
k_{B} T_{\mathrm{BEC}}=\hbar \bar{\omega}_{7}\left(\frac{N_{7}}{g_{3}(1)}\right)^{1 / 3}, \quad \text { where } \quad g_{3}(1) \simeq 1.202 .
$$


For a typical mean ${ }^{7} \mathrm{Li}$ trapping frequency $\bar{\omega}_{7} / 2 \pi=270 \mathrm{~Hz}$, and a typical ${ }^{7} \mathrm{Li}$ atom number $N_{7}=3000$, the critical temperature is $T_{\mathrm{BEC}}=175 \mathrm{nK}$. We did not study in detail the physics around $T=T_{\mathrm{BEC}}$. As we never observed a sharp feature in the density profile, we assume the cloud to be at thermal equilibrium, with no condensed fraction and at $T \simeq T_{\mathrm{BEC}}$.

The temperature lower bound imposed by the nature of our thermometer has to be compared with the ${ }^{6} \mathrm{Li}$ Fermi temperature $T_{F}=\hbar \bar{\omega}_{6}\left(6 N_{6}\right)^{1 / 3}$ :

$$
T \geq T_{\mathrm{BEC}}=\sqrt{\frac{m_{6}}{m_{7}}}\left(\frac{1}{6 g_{3}(1)} \frac{N_{7}}{N_{6}}\right)^{1 / 3} T_{F} .
$$

This limit is pushed to its lowest values by reducing the ${ }^{7} \mathrm{Li}$ atom number to the minimum value $N_{7} \simeq 2500$ allowing us to make a reliable temperature measurement. With $N_{6}=5 \times 10^{4}{ }^{6} \mathrm{Li}$ atoms in each spin state, this yields the following numerical value for the temperature limit:

$$
T \geq 0.18 T_{F} .
$$

This lower bound nearly coincides with the temperature at which a part of the gas becomes superfluid 43, 46, 39, 47. Therefore this thermometer is not suited for investigating low-temperature effects in the superfluid phase. However, we will see in section 4.7 that we still managed to observe and characterize the onset of superfluidity.

\subsection{Extraction of $h_{T}(\zeta)$ from In Situ Images}

Let us remind the information we have at our disposal at this stage of the data analysis. The pressure profile $P\left(\mu_{z}, T\right)$ is determined from an in situ image using (3.3) (see Chapter 3), and the temperature is determined using the ${ }^{7} \mathrm{Li}$ thermometer (see section 4.1). We can calculate for each position $z$ along the $z$ axis the quantity:

$$
\frac{P\left(\mu_{z}, T\right)}{k_{B} T \lambda_{d B}^{-3}(T)}=f_{5 / 2}\left(\zeta_{z}^{-1}\right) h_{T}\left(\zeta_{z}\right),
$$

which is a function of the local inverse fugacity:

$$
\zeta_{z}=\zeta^{0} \exp \left(\frac{m \omega_{z}^{2} z^{2}}{2 k_{B} T}\right)
$$

where $\zeta^{0}=e^{-\mu^{0} / k_{B} T}$ is the global inverse fugacity. $\zeta^{0}$ is the remaining unknown parameter.

\subsubsection{Direct Measurement of the High-Temperature Equation of State}

In the wings of a high-temperature cloud, $\zeta_{z}$ is much larger than 1 , i.e. the gas is in the classical regime and $f_{5 / 2}\left(\zeta_{z}^{-1}\right) h_{T}\left(\zeta_{z}\right) \simeq \zeta_{z}^{-1}$. As pictured in Fig, 4.3 , we obtain the value of $\zeta^{0}$ as the only value consistent with this high-temperature equation of state.

Actually, interaction effects are not negligible even for the largest values $\zeta_{z} \simeq 5$ reached in our data. A better estimate of the equation of state is given by the second-order virial expansion, i.e. the first correction to the equation of state of an ideal and classical gas, due to interactions and quantum statistics. The second-order virial correction is known exactly for a gas in the unitary limit [135] (see section 4.5):

$$
f_{5 / 2}\left(\zeta^{-1}\right) h(\zeta)=\zeta^{-1}+\left(-2^{-5 / 2}+b_{2}\right) \zeta^{-2}+\ldots, \quad \text { where } \quad b_{2}=\frac{1}{\sqrt{2}} .
$$

$\zeta^{0}$ is chosen on each image so that the high-temperature data corresponding to $\zeta_{z}>2.5$ matches the second-order virial expansion*

${ }^{*}$ The next correction to the second-order virial expansion, discussed in section 4.5 is less than $2 \%$ for $\zeta>2.5$, justifying the use of 4.2 in that region. 
(a)

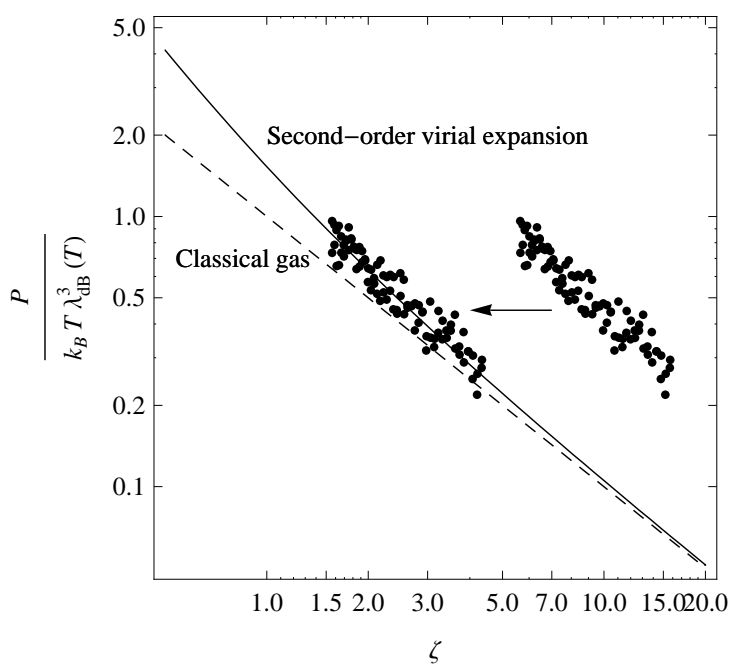

(b)

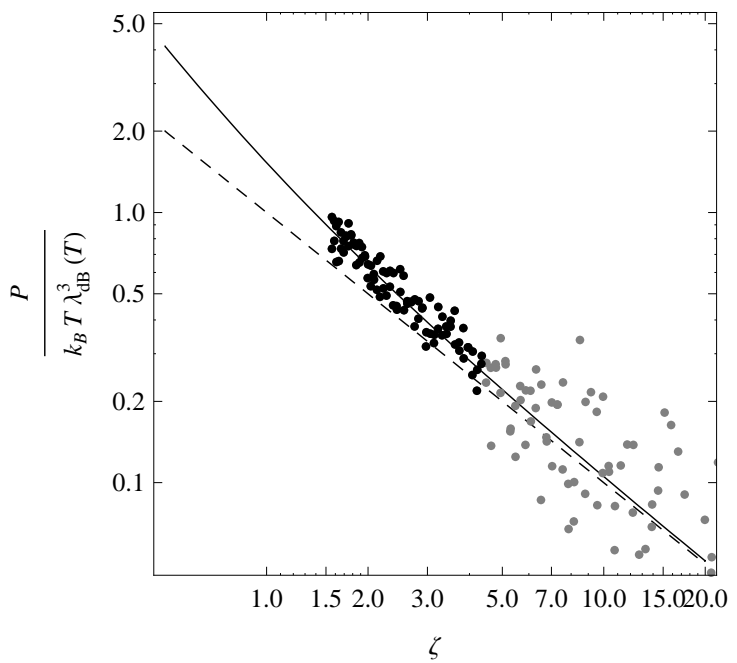

Figure 4.3: (a) The procedure used for the determination of $\zeta^{0}$ at high temperature is well illustrated by plotting $P\left(\mu_{z}, T\right) / 2 k_{B} T \lambda_{d B}^{-3}(T)$ as a function of $\zeta$ in $\log$ scale. Indeed, as $\log \zeta=\log \zeta^{0}+\log \frac{m \omega_{z}^{2} z^{2}}{2 k_{B} T}$, the degree of freedom $\zeta^{0}$ corresponds in that representation to a translation of the data in abscissa. We choose the value of $\zeta^{0}$ so that the data corresponding to $\zeta_{z}>2.5$ matches the second-order virial expansion (solid line). The dashed line is the equation of state of a classical ideal gas. (b) Equation of state given by all the pixel columns along the $z$ axis from a high-temperature cloud. For $\zeta_{z} \gtrsim 4$ (in gray), the signal-to-noise decreases because of the small optical density in the cloud's wings. We exclude these points for the rest of the data analysis.

After averaging over the equations of state given by 7 high-temperature clouds prepared in the same conditions, we obtain a low-noise equation of state, displayed in Fig.4.4. For a given image, each pixel located in a region of high enough signal-to-noise ratio gives a point of the equation of state $h(\zeta) .7$ images thus provide $\sim 1000$ points, leading after averaging to an equation of state with a very low statistical noise. As shown in Fig.4.4, at the highest $\zeta$ values the equation of state agrees with the second-order virial expansion (4.2), while at the lowest $\zeta$ values it clearly deviates from 4.2 . This procedure therefore provides an equation of state in a temperature range well below the validity of the second-order virial expansion. However, the lowest $\zeta$ values obtained with these images, $\zeta \simeq 1$, are too large to reveal the low-temperature physics corresponding to $\zeta \ll 1$ (the superfluid transition is expected to occur at $\zeta=\zeta_{c} \simeq 5 \times 10^{-2}$ ). Preparing a low-temperature gas, for example with $\zeta^{0} \simeq \zeta_{c}$, i.e. with a small superfluid core at the center, would not allow us to use such a procedure because the signal from classical wings of the cloud $\zeta_{z}>2.5$ would be far below the noise. Obtaining the equation of state at lower temperatures thus requires an additional step.

\subsubsection{Construction of the Low-Temperature Equation of State}

For colder clouds, we cannot use the second-order virial expansion as a reference for fitting $\zeta^{0}$. As the low-noise equation of state measured using the hottest clouds is valid up to $\zeta \gtrsim 1$, i.e. in a much broader range than the second-order virial expansion, we use it as a reference for fitting $\zeta^{0}$ in the wings of colder clouds. This procedure can be used for clouds prepared at an intermediate temperature, so that the signal-to-noise ratio in the region $\zeta>1$ is good enough. We then iterate this procedure: these clouds provide a precise equation of state for $\zeta>0.2$ and are used to fit $\zeta^{0}$ for colder clouds, and so on 


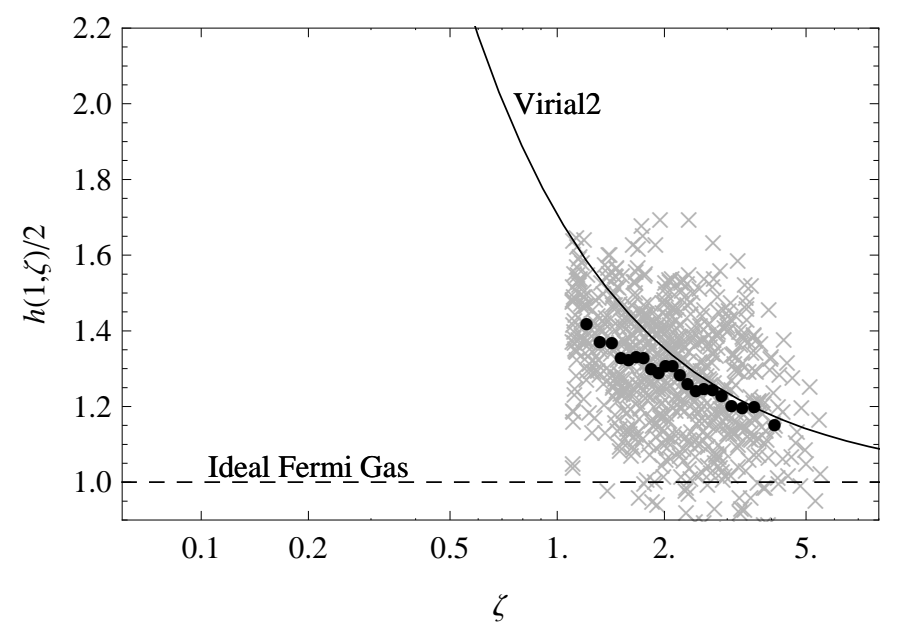

Figure 4.4: Equation of state given by 7 images prepared at the highest temperatures. The gray crosses are the raw data, each corresponding to one pixel row of a single image. The black points result of an averaging of 60 consecutive gray points.

(see Fig 4.5). We finally obtain a reference equation of state that can be used to fit $\zeta^{0}$ on the coldest samples, at a temperature limited by the ${ }^{7} \mathrm{Li}$ thermometer (see section 4.1), corresponding to $\zeta \simeq 0.02$. By gathering the data from all images, we obtain $\sim 2500$ points in a temperature range $0.02<\zeta<5$, i.e. from the classical to the degenerate regime. After averaging over consecutive points, we obtain a low-noise equation of state with 58 points (see Fig 4.6 ).
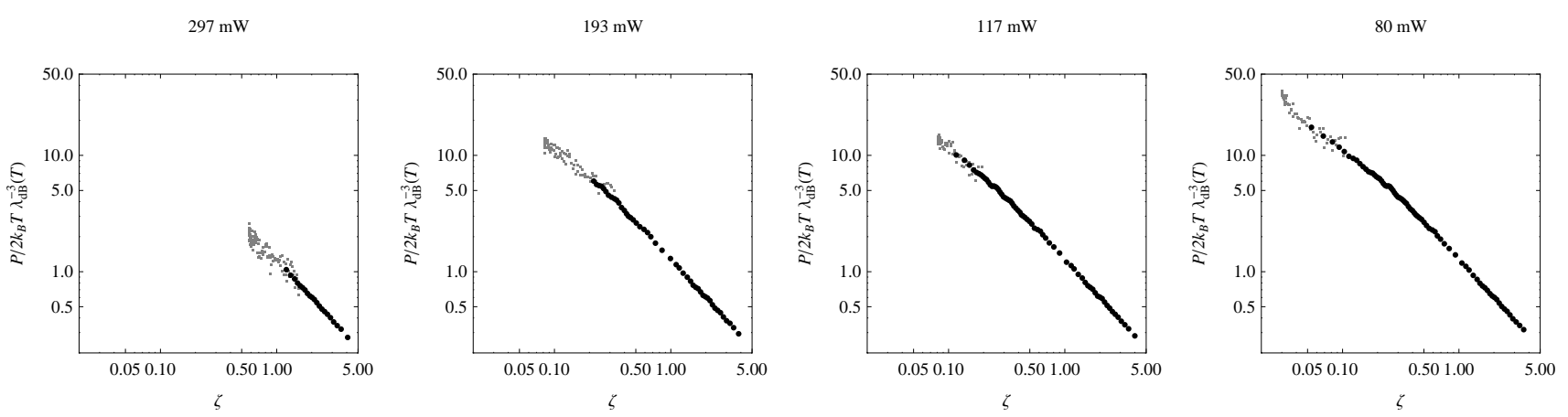

Figure 4.5: Step-by-step construction of the equation of state. For a given image (gray points), the determination of $\zeta^{0}$ makes use of the equation of state (black circles) determined from colder clouds. We show here 4 such steps corresponding to different final optical trap depths.

\subsubsection{Systematic Error on the Equation of State Determined from our Data}

After averaging, the statistical noise of our data is less than 5\% (see Fig 4.6). We evaluate in this section the systematic error introduced by our procedure.

\section{Absolute Error on the Pressure}

As explained in section 3.5 .1 the pressure $P\left(\mu_{z}, T\right)$ is calibrated using a reference profile whose equation of state is well known, namely a spin-balanced Fermi gas in the unitary limit, and at a temperature well below the superfluid threshold. This procedure leads to a $5 \%$ systematic uncertainty. 


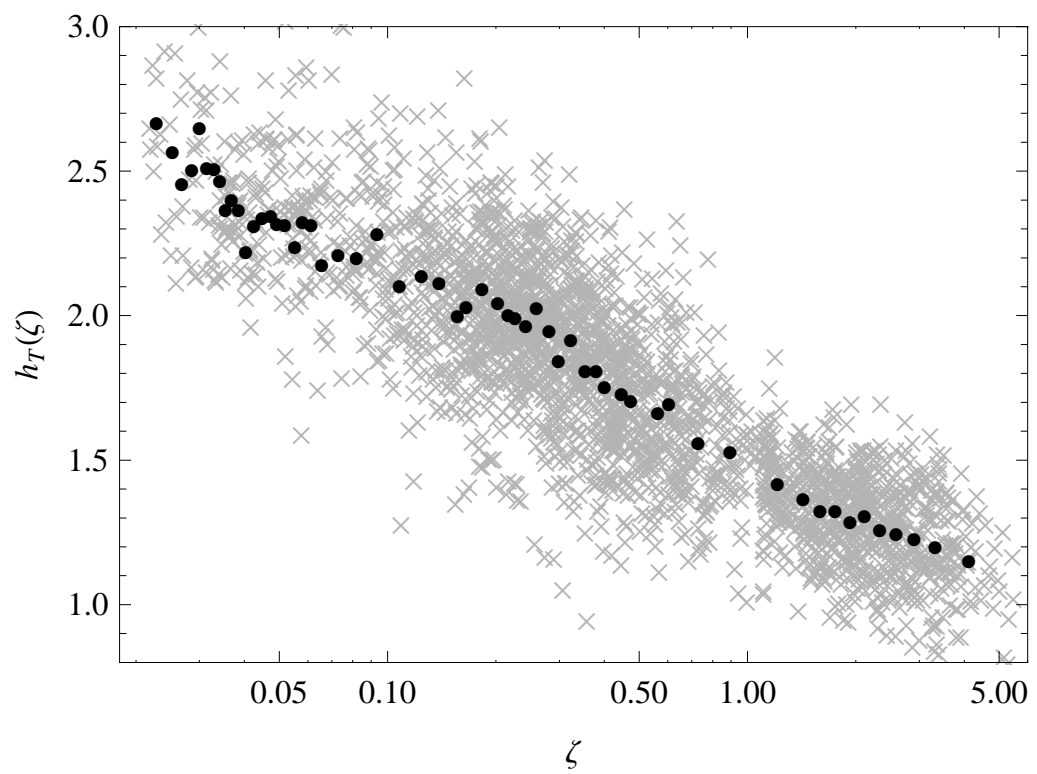

Figure 4.6: Thermodynamic function $h_{T}(\zeta)$ of a homogeneous Fermi gas with resonant interactions. The complete data are the gray crosses. The black points are averages of raw data points.

\section{Noise Induced by the Determination of $\zeta^{0}$}

During the step-by-step construction of the equation of state, an error on the determination of $\zeta^{0}$ for a given image induces an error on the reference equation of state used for colder images. The noise of our pressure data leads to a statistical uncertainty on the determination of $\zeta^{0}$. Typically 100 pixels with a signal-to-noise ratio $>3$ are used for the determination of $\zeta^{0}$, leading to a $0.3 / \sqrt{100}=3 \%$ uncertainty on $\zeta^{0}$. The data from a single image is overlaped with typically 10 other images to form the low-noise equation of state used as a reference for colder clouds. The error in $\zeta$ on the reference equation is thus reduced to $0.03 / \sqrt{10}=1 \%$. The $1 \%$ statistical error induced by each image results in a random walk of the noise during the construction of the complete equation of state, leading to a final error of $0.01 \sqrt{40} \simeq 5 \%$.

In the next sections, we discuss the content of this equation of state. We make a direct comparison of our data with theory. We then extract the high-temperature and low-temperature asymptotic behaviors, and the critical temperature for superfluidity.

\subsection{Direct Comparison with Theory}

Our data provides the equation of state of a homogeneous Fermi gas in the unitary limit. It can thus directly be compared with theory, contrary to the equations of state of a trapped gas 62 63] which require to integrate the theoretical equations of state over the trap. In particular our data allow us to compare for the first time the several points given by time-consuming diagrammatic Monte-Carlo calculations with experiment 113. Moreover, the trap averaging smears the possibly small differences between theoretical equations of state, so that up to now trapped equations of state have not be used to clearly discriminate between theories.

The comparison is made in Fig 4.7 with the following theories:

- double-dot-dashed line: BCS mean field theory 


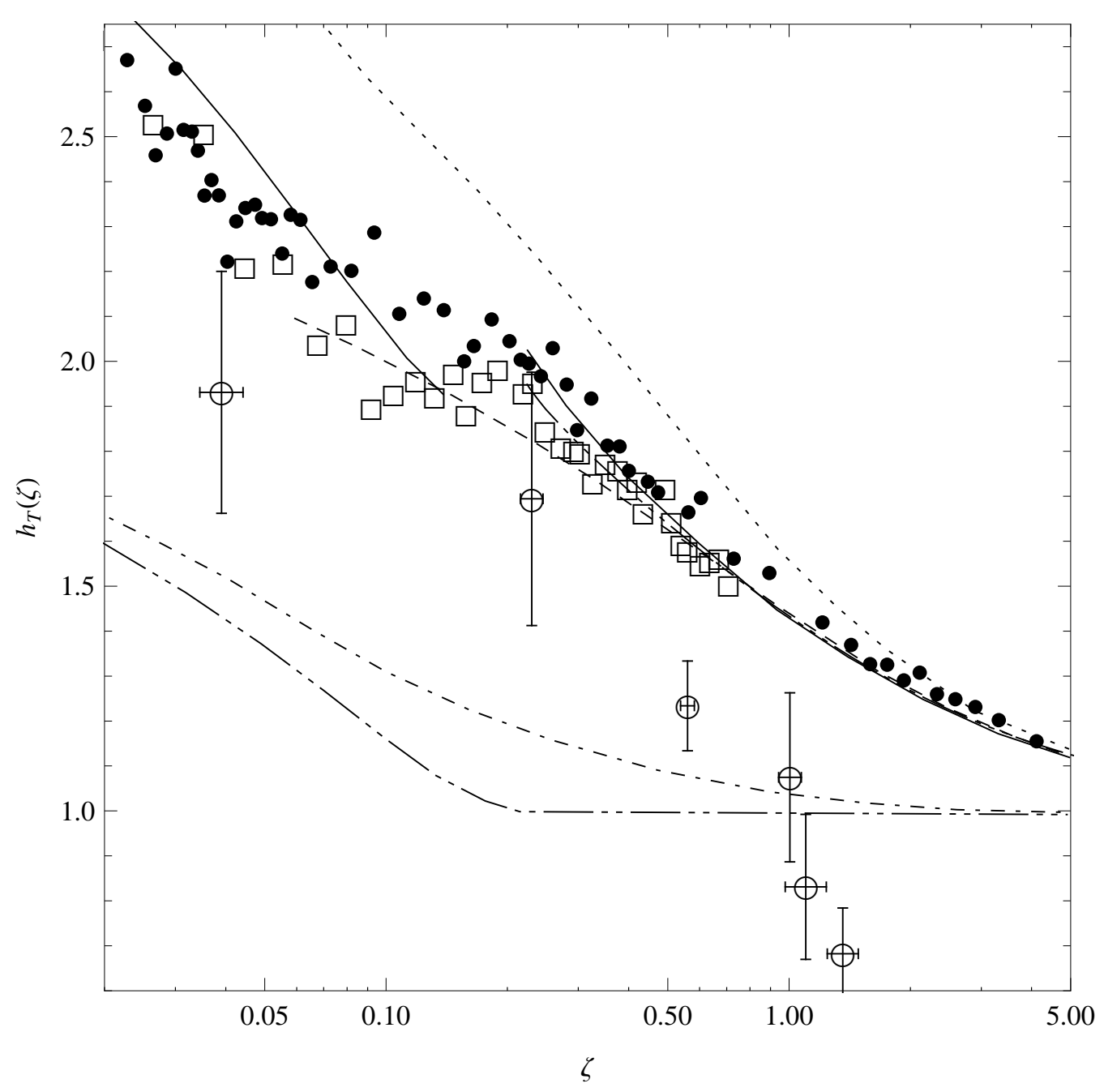

Figure 4.7: Comparison of our data with theory (see text for the legend).

- open circles: Diagrammatic Monte Carlo calculations 113

- open squares: Quantum Monte Carlo calculations 136

- dotted line: $G G$ perturbation theory 137,138

- dashed line: $G G_{0}$ perturbation theory [139

- triple-dot-dashed line: Ladder diagrams approximation 140

- dot-dashed line: Pseudogap theory [139

- solid line: Gaussian pair fluctuation/Nozières-Schmitt Rink theory [141

Our data clearly discriminates between these theories. We observe that none of them account for our data over their full range. In particular our observations are not consistent with the diagrammatic Monte Carlo calculations from [113]. Their highest-temperature data exhibits a clear inconsistency: they violate the exact constraint on the equation of state $h_{T}(\zeta) \geq 1$, valid for an attractive gas such as a Fermi gas in the unitary limit [48]. Finally our data agrees well with the Quantum Monte Carlo calculations from [136], except in the region $0.05<\zeta<0.2$. 


\subsection{Comparison with the Tokyo Group Measurements}

The thermodynamics of a homogeneous unitary Fermi gas was experimentally studied, simultaneously to our work, by the Tokyo group 47. Using the equation of state of a trapped unitary gas measured in the Duke group [63] as a reference for thermometry, they obtain a canonical equation of state for the homogeneous gas, from the analysis of density profiles after a hydrodynamic expansion (see Fig 4.8 a). This equation of state is written as:

$$
E=\frac{3}{5} N E_{F} g\left(\theta=\frac{T}{T_{F}}\right),
$$

where $E$ is the energy, $N$ is the total atom number, and $E_{F}\left(T_{F}\right)$ is the Fermi energy (temperature). The function $g(\theta)$ is the canonical equivalent of $h_{T}(\zeta)$.

(a)

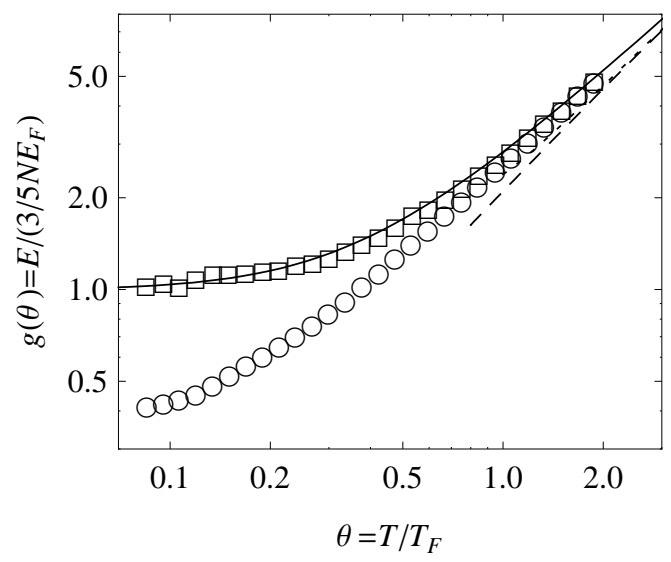

(b)

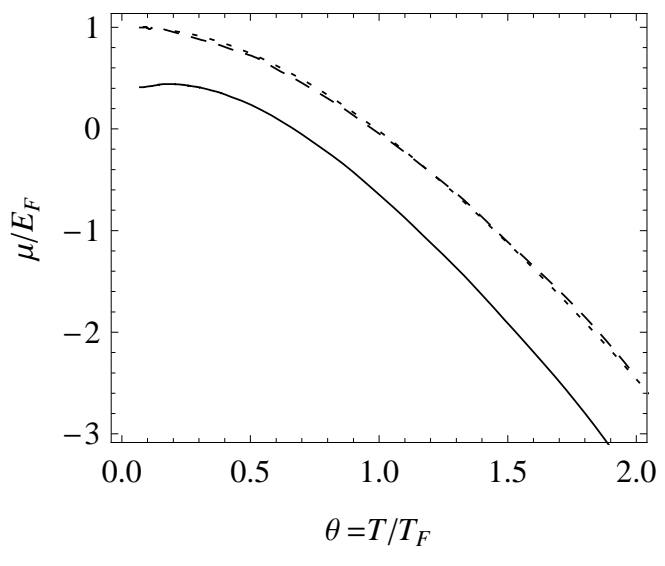

Figure 4.8: (a) Open circles (open squares): canonical equation of state of a homogeneous unitary (ideal) gas measured in the Tokyo group [47. Solid line: equation of state of an ideal gas. Dashed line: secondorder virial expansion. (b) Chemical potential computed from the experimental data for a unitary gas (solid line) and an ideal gas (dashed line). The dotted line is the exact chemical potential for an ideal gas.

In order to compare with our data, we have to make the correspondence between the canonical equation of state $g(\theta)$ and the grand-canonical equation of state $h_{T}(\zeta)$. We first express the data from the Tokyo group in the variables $(\zeta, h)$. This requires to compute the chemical potential from the experimental data (see Appendix A.2 for a detailed calculation):

$$
\frac{\mu}{E_{F}}=g_{\mu}(\theta)=g(\theta)-\frac{3}{5} \theta \int_{0}^{\theta} \frac{g^{\prime}\left(\theta^{\prime}\right) \mathrm{d} \theta^{\prime}}{\theta^{\prime}} .
$$

The integral in 4.3 is calculated using a function interpolating the experimental data. In order to test the robustness of this procedure, we calculated the chemical potential of an ideal gas using the experimental equation of state of an ideal gas also measured in [47] (see Fig 4.8a). As shown in Fig.4.8p, the chemical potential deduced from the experimental data agrees with the theoretical chemical potential of an ideal gas. This validates our calculation of $g_{\mu}(\theta)$ from the experimental data.

There is then a one-to-one correspondence between the data $(\theta, g)$ and the data $\left(\zeta, h_{T}\right)$ (see Appendix A.2):

$$
\begin{aligned}
\zeta & =\exp \left(-\frac{g_{\mu}(\theta)}{\theta}\right) \\
h_{T} & =\frac{8}{15 \sqrt{\pi}} \frac{g(\theta)}{\theta^{5 / 2} f_{5 / 2}\left(\zeta^{-1}\right)} .
\end{aligned}
$$




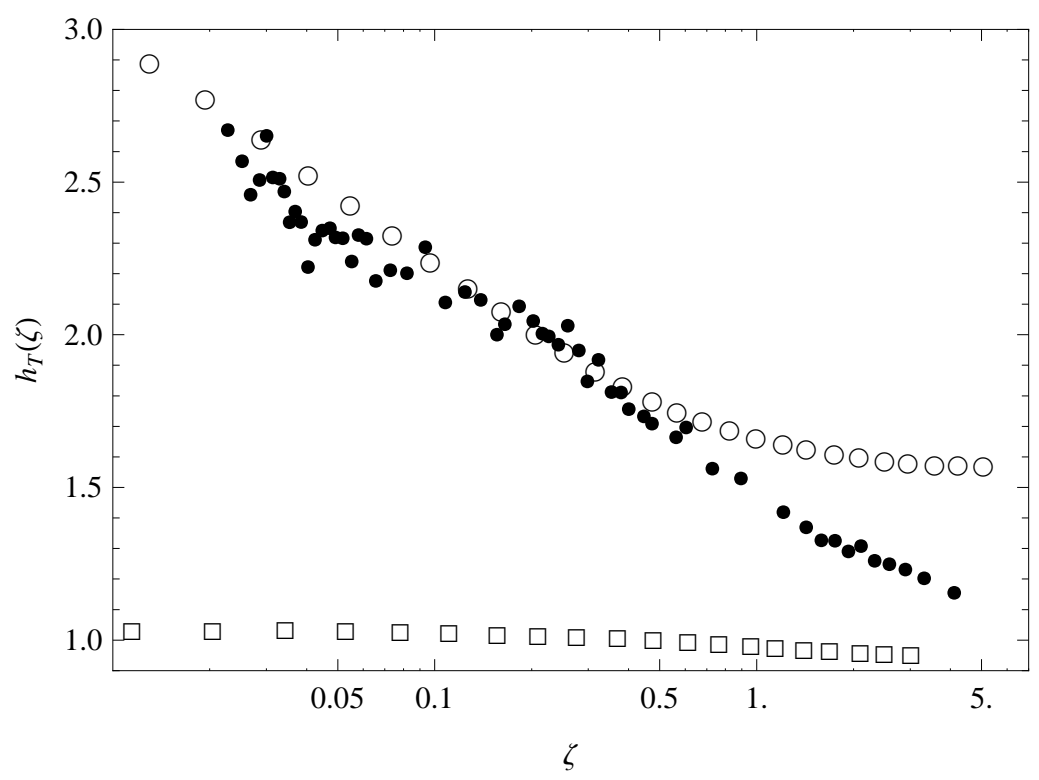

Figure 4.9: Comparison between the grand-canonical equation of state $h_{T}(\zeta)$ deduced from our data (black dots) and from the one measured in [47. (open circles). The open squares are calculated with the ideal gas data from [47], using the same procedure. The good agreement with the exact equation of state $h_{T}(\zeta)=1$ for an ideal gas shows the robustness of the correspondence canonical/grand-canonical.

In Fig 4.9 we make the comparison in the variables $\left(\zeta, h_{T}\right)$ between our data and the one from 47 . They are in good agreement for $\zeta<0.5$ but strongly differ in the high-temperature regime.

Alternatively, we express our data in the canonical ensemble to make the comparison without having to transform the data from [4]. The canonical equation of state $g(\theta)$ is calculated from our data $h_{T}(\zeta)$ according to (see Appendix A.2.

$$
\begin{aligned}
& \theta=\left(\frac{16}{9 \pi}\right)^{1 / 3}\left(-\zeta \frac{\mathrm{d} f_{5 / 2}\left(\zeta^{-1}\right) h_{T}(\zeta)}{\mathrm{d} \zeta}\right)^{-2 / 3} \\
& g=5\left(\frac{2}{9 \pi}\right)^{1 / 3}\left(-\zeta \frac{\mathrm{d} f_{5 / 2}\left(\zeta^{-1}\right) h_{T}(\zeta)}{\mathrm{d} \zeta}\right)^{-5 / 3} f_{5 / 2}\left(\zeta^{-1}\right) h_{T}(\zeta)
\end{aligned}
$$

Calculating $g(\theta)$ thus requires to take the derivative of our experimental data, which decreases the signalto-noise ratio. In order to highlight the differences between the two sets of data, we plot $g(\theta) / g^{(0)}(\theta)$ as a function of $\theta$, where $g^{(0)}(\theta)$ is the equation of state for an ideal gas. As shown in Fig 4.10 our data agree within our signal-to-noise ratio with the one from 47 for $\theta<0.5$. In the high-temperature regime the two measurements significantly differ. In the high-temperature regime, our data is in excellent agreement with the exact high-temperature asymptotic behavior given by the third-order virial expansion [75] (see section 4.5. Therefore we believe that the data from 47 have a systematic error at high temperature, possibly due a deviation from hydrodynamics during the time-of-flight.

\subsection{High-Temperature Virial Expansion}

As described in section 4.3 , our data can be used as a benchmark for many-body theories, from the lowtemperature to the high-temperature regimes. It is also important to get a more physical picture of the equation of state. In this section we extract several virial coefficients of the high-temperature expansion of the equation of state in a series of $\zeta^{-1}$. We also give a physical interpretation of these numbers in terms of few-body physics. 


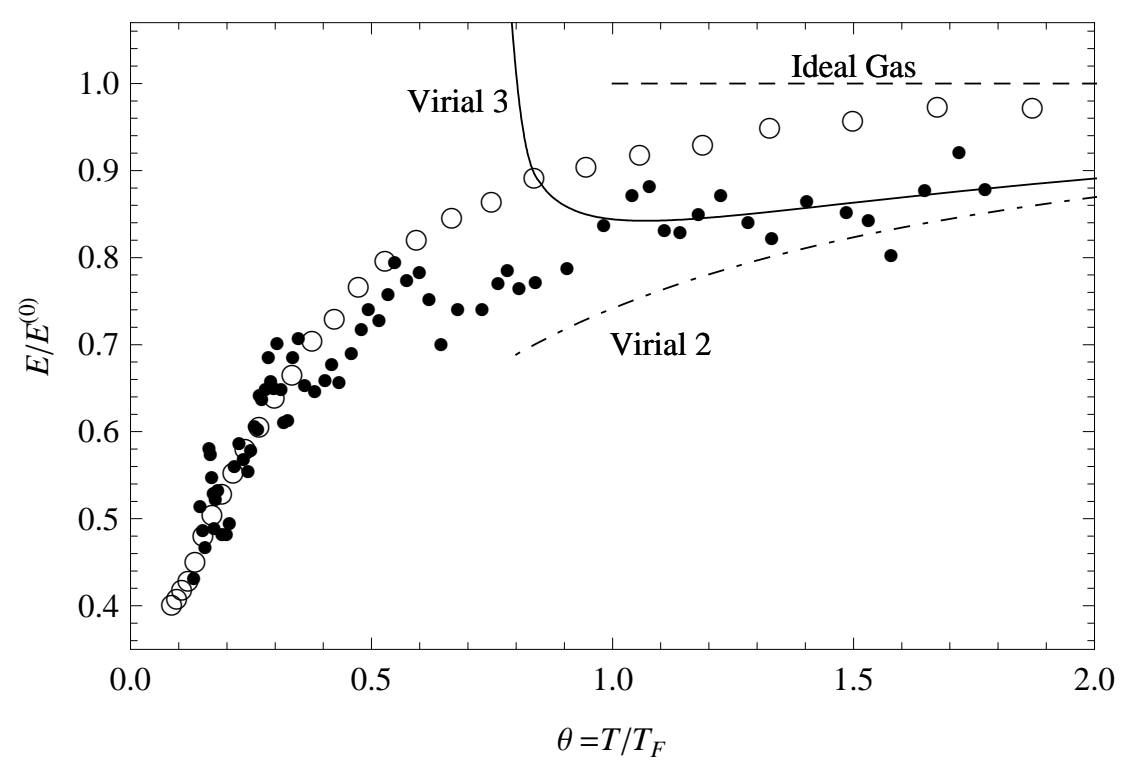

Figure 4.10: Comparison between the canonical equation of state deduced from our data (black dots) and the one measured in [47] (open circles), and expressed as $E(\theta) / E^{(0)}(\theta)$, where $E^{(0)}(\theta)$ is the energy of an ideal gas with same atom number. The lines are the successive theoretical virial expansions of the equation of state at high temperature.

\subsubsection{Virial Expansion of a Unitary Fermi Gas: Generalities}

\section{Connection of the Virial Expansion to Few-Body Physics}

The grand-canonical partition function $\Xi$ can be decomposed by gathering all terms with given total atom numbers $N_{1}, N_{2}$ :

$$
\Xi(\mu, T)=\sum_{N_{1}, N_{2}} Z\left(N_{1}, N_{2}, T\right) \zeta^{-\left(N_{1}+N_{2}\right)}, \quad \text { where } Z\left(N_{1}, N_{2}, T\right)=\sum_{\text {state } \alpha\left(N_{1}, N_{2} \text { fixed }\right)} e^{-E_{\alpha} / k_{B} T}
$$

is the canonical partition functions for fixed atom numbers $N_{1}, N_{2}$. Calculating $Z\left(N_{1}, N_{2}, T\right)$ requires to know the complete energy spectrum of a system with $N_{1}$ particles of species 1 , and $N_{2}$ particles of species 2. The grand potential $\Omega=-P V=-k_{B} T \log \Xi$ (related to the pressure by $\Omega=-P V$ ) can then be expressed as a high-temperature series of $\zeta^{-1}$ :

$$
\Omega(\mu, T)=-\frac{2 k_{B} T V}{\lambda_{d B}^{3}(T)}\left[\zeta^{-1}+\left(-2^{-5 / 2}+b_{2}\right) \zeta^{-2}+\left(3^{-5 / 2}+b_{3}\right) \zeta^{-3}+\left(-4^{-5 / 2}+b_{4}\right) \zeta^{-4}+\ldots\right] .
$$

The coefficients $b_{k}$ are the so-called virial coefficients. The coefficient $b_{k}$ is obtained by expanding log $\Xi$ in powers of $\zeta^{-1}$ up to $k^{\text {th }}$ order, and thus involves the values of the partition functions $Z\left(N_{1}, N_{2}, T\right)$ for $N_{1}+N_{2} \leq k$. As an example, it is simple to show that the second-order virial coefficient reads $b_{2}=\left(Z_{1,1}-Z_{1,1}^{0}\right) / 2 Z_{1,0}$, where the superscript ${ }^{0}$ refers to partition functions for a non-interacting gas. Our convention for the definition of the virial coefficients is chosen so that a non-interacting twocomponent Fermi gas corresponds to $b_{k}=0$ for all values of $k$. As a conclusion $b_{k}$ can be interpreted as a coefficient quantifying the effect of interactions in the high-temperature expansion up to $k^{\text {th }}$ order, and is given by the eigen-spectrum of the $k$-body problem.

\section{Virial Coefficients for Uniform and Trapped Gases}

A simple relationship can be established between the virial coefficients for a uniform gas and for a harmonically-trapped gas 75$]$. For a trapped gas, the virial expansion is defined as an expansion of the 
grand potential $\Omega_{t}$ in powers of $\left(\zeta^{0}\right)^{-1}$, where $\zeta^{0}=e^{-\mu^{0} / k_{B} T}$ is the global inverse fugacity. In the local density approximation framework, the grand potential $\Omega_{t}$ is obtained by integrating the grand potential of a uniform gas over the trap:

$$
\begin{aligned}
\Omega_{t}\left(\mu^{0}, T\right) & =\int \frac{\mathrm{d} \mathbf{r}}{V} \Omega\left(\mu^{0}-V(\mathbf{r}), T\right) \\
& =\frac{2 k_{B} T}{\lambda_{d B}^{3}(T)}\left[\left(\zeta^{0}\right)^{-1} \int \mathrm{d} \mathbf{r} e^{-V(\mathbf{r}) / k_{B} T}+\left(-2^{-5 / 2}+b_{2}\right)\left(\zeta^{0}\right)^{-2} \int \mathrm{d} \mathbf{r} e^{-2 V(\mathbf{r}) / k_{B} T}+\ldots\right] .
\end{aligned}
$$

Integration over space is straightforward:

$$
\int \mathrm{d} \mathbf{r} e^{-k V(\mathbf{r}) / k_{B} T}=\left(\frac{2 \pi k_{B} T}{m \bar{\omega}^{2}}\right)^{3 / 2} \frac{1}{k^{3 / 2}}
$$

where $\bar{\omega}$ is the geometrical mean trap frequency. The virial expansion for a trapped gas then reads:

$\Omega_{t}\left(\mu^{0}, T\right)=-2 k_{B} T\left(\frac{k_{B} T}{\hbar \bar{\omega}}\right)^{3}\left[\left(\zeta^{0}\right)^{-1}+\left(-2^{-4}+b_{t 2}\right)\left(\zeta^{0}\right)^{-2}+\left(3^{-4}+b_{t 3}\right)\left(\zeta^{0}\right)^{-3}+\ldots\right], \quad$ where $\quad b_{t k}=b_{k} / k^{3 / 2}$.

Compared with the virial coefficients for a uniform gas, the virial coefficients of a trapped gas are suppressed by a factor $b_{t k} / b_{k}=k^{3 / 2}$. Therefore we can already feel that the equation of state of a homogeneous gas is more suited to extract virial coefficients than the equation of state of a trapped gas (see section 4.8.

\section{Theoretical Values for the Second- and Third-Order Virial Coefficients}

The calculation of the second- and third-order virial coefficients can be performed exactly, making use of the exact resolution of the two-body problem in $[142$ and of the three-body problem in 74 .

The calculation of the second-order virial coefficient was first made in [135]:

$$
b_{2}=1 / \sqrt{2} .
$$

The derivation of this result is simple and can be found in Appendix A

The three-body problem at unitarity and in a harmonic trap was solved recently in [74]. The calculation of the third-order virial coefficient using this energy spectrum was performed in $[75]$ :

$$
b_{3}=-0.3551 \text {. }
$$

This coefficient was also calculated in [143] using an effective field theory, and surprisingly the result $b_{3}=1.05$ is very different. Our data will unambiguously show which of the two calculations is correct.

The next virial coefficient has not been calculated yet since the four-body problem has not been treated yet.

\subsubsection{Virial Coefficients Extracted from our Data}

As we use in our procedure the second-order virial expansion 4.2 for the determination of the global chemical potential $\mu^{0}$, our high-temperature data cannot serve as a measurement of $b_{2}$. However the deviation of our data from 4.2 provides the value of the next-order coefficients.

\section{Third-Order Virial Coefficient}

At high temperature, the deviation from the second-order virial expansion reads:

$$
f_{5 / 2}\left(\zeta^{-1}\right)\left(h_{T}(\zeta)-1\right)-\zeta^{-1}-b_{2} \zeta^{-2} \simeq b_{3} \zeta^{-3}
$$


In Fig 4.11 , we show that the deviation of our data from the second-order virial expansion agrees with the asymptotic behavior (4.12) using the theoretical value $b_{3}=-0.3551$ from 75$]$. Our observations clearly exclude the other theoretical value $b_{3}=1.05$ from [143]. In order to extract an experimental value of $b_{3}$, we fit our high-temperature data $\zeta>\zeta_{\text {cutoff }}$ in 4.11 a with the asymptotic behavior (4.12). In Fig $4.11 \mathrm{~b}$ we plot the fit result as a function of $\zeta_{\text {cutoff }}$ defining the number of data points used for the fit. This shows the robustness of the determination of $b_{3}$, and provides the value of $b_{3}$ consistent with our data: $b_{3}=-0.345(25)$.

(a)

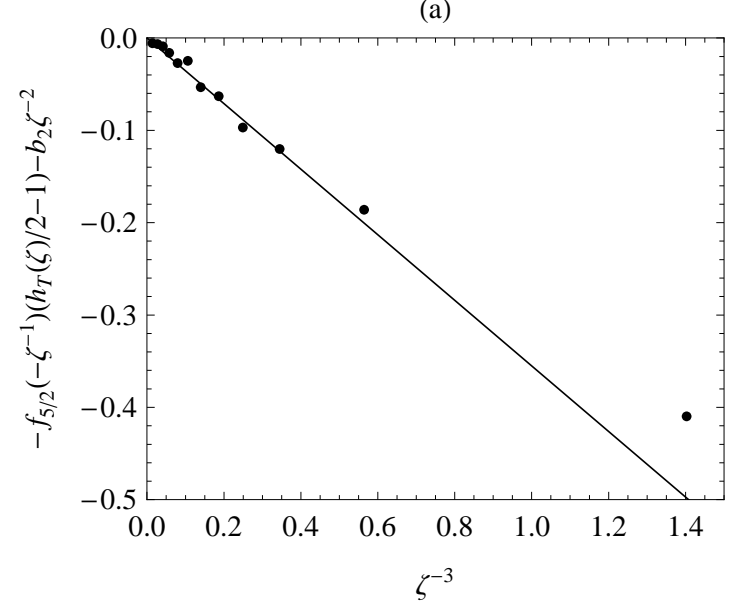

(c)

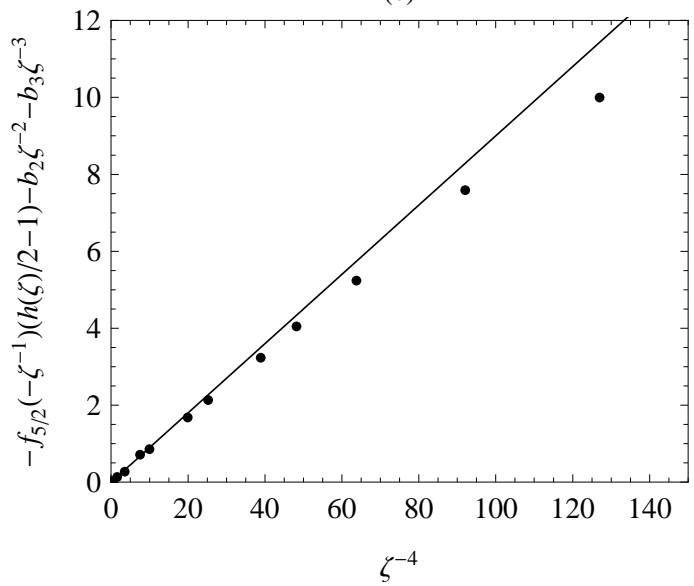

(b)

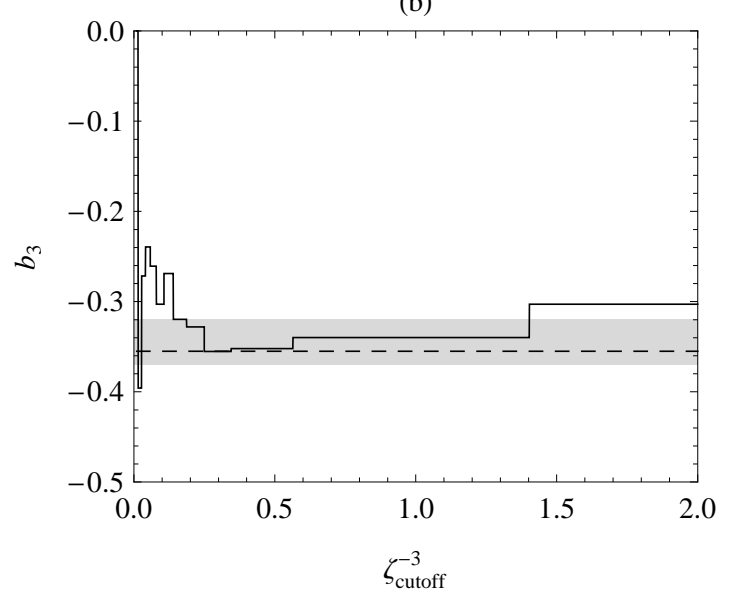

(d)

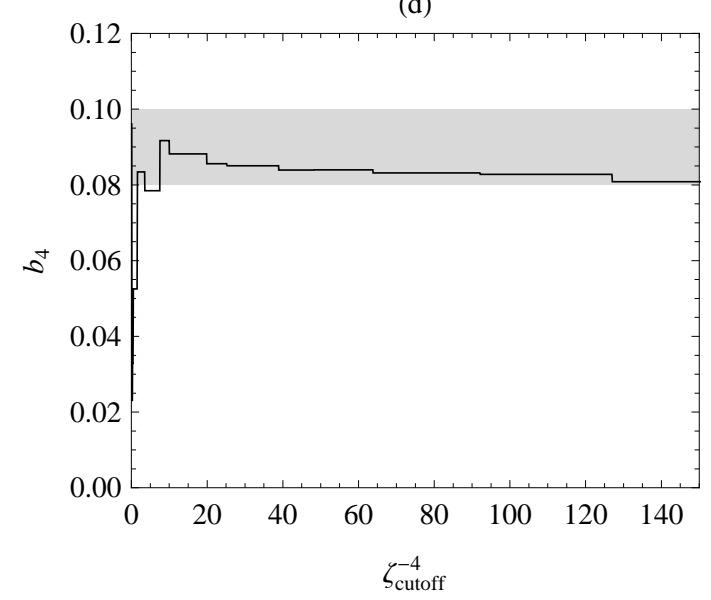

Figure 4.11: (a) Deviation of our data from the second-order virial expansion, compared with the behavior of the third-order virial expansion 4.12 with $b_{3}=-0.3551$ (solid line). (b) Result of the fit 4.12 using the data $\zeta>\zeta_{\text {cutoff }}$, as a function of $\zeta_{\text {cutoff }}$. The gray rectangle represents the values of $b_{3}$ consistent with our data. (c) Deviation of our data from the third-order virial expansion, compared with the behavior of the third-order virial expansion (4.13) with $b_{4}=0.09$ (solid line). (b) Result of the fit 4.13 ) using the data $\zeta>\zeta_{\text {cutoff }}$, as a function of $\zeta_{\text {cutoff }}$.

\section{Fourth-Order Virial Coefficient}

Our measurement of the third-order virial coefficient agrees with a very good precision with the calculation $b_{3}=-0.3551$. Having checked this result, we can go one step beyond and extract the next term of the virial expansion. The high-temperature deviation from the third-order virial expansion reads:

$$
f_{5 / 2}\left(\zeta^{-1}\right)\left(h_{T}(\zeta)-1\right)-\zeta^{-1}-b_{2} \zeta^{-2}-b_{3} \zeta^{-3} \simeq b_{4} \zeta^{-4} .
$$


We show in Fig 4.11, that our high-temperature data agree with the asymptotic behavior (4.13). Applying the same procedure than for the measurement of the coefficient $b_{3}$, we obtain the coefficient $b_{4}=0.09(1)$ (see Fig $4.11 \mathrm{~d}$ ). The calculation of $b_{4}$ would require to solve the four-body problem. As shown in [75, numerical calculations of the energy spectrum of three-body problem from [144] are precise enough to calculate the third-order coefficient $b_{3}$ with an excellent precision. A numerical calculation of the energy spectrum of the four-body problem was already carried out in [145], and could possibly be used to calculate the fourth-order virial coefficient $b_{4}$.

To conclude this section, we plot in Fig 4.12 the successive virial expansions up to fourth order, together with the data $h_{T}(\zeta)$. The fourth-order virial expansion accounts for our observations within $5 \%$ up to $\zeta=0.4$.

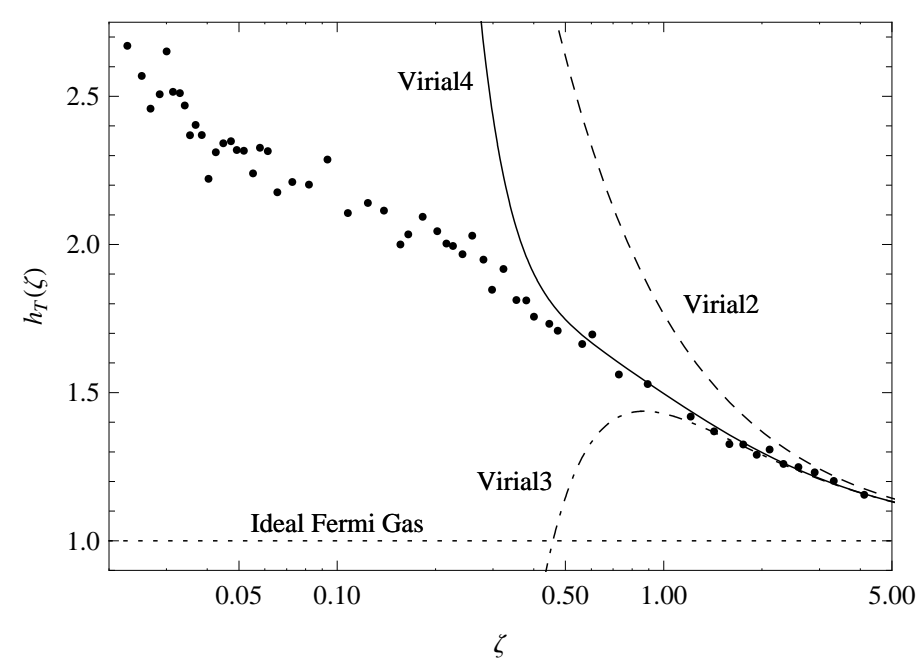

Figure 4.12: Grand-canonical equation of state of a Fermi gas in the unitary limit extracted from our data (black dots), compared with the successive virial expansions described in the text.

\subsection{Fermi-Liquid Behavior in the Normal Phase}

We now consider the low-temperature behavior of the equation of state determined from our data. At very low temperature a phase transition from a normal phase to a superfluid phase occurs 40 . We discuss the observation of this phase transition in section 4.7 and focus here on the low-temperature behavior in the normal phase.

Understanding the thermodynamic properties in the normal phase of strongly correlated materials such as high- $T_{c}$ copper oxides is a challenge for condensed matter physics. It is one of the key ingredients for modeling the superconducting phase transition, governed by the energy competition between normal and superconducting states.

\subsubsection{Low-Temperature Normal Phases in Strongly-Interacting Systems}

In this section we briefly present two families of normal states in strongly correlated materials: the family of Fermi liquids and the family of the pseudogap phase.

\section{The Fermi Liquid Family}

Fermi liquid theory, developed by Landau in [146, provides a phenomenological description of thermodynamic properties of most metals. According to this theory, the effect of interactions on electrons can be 

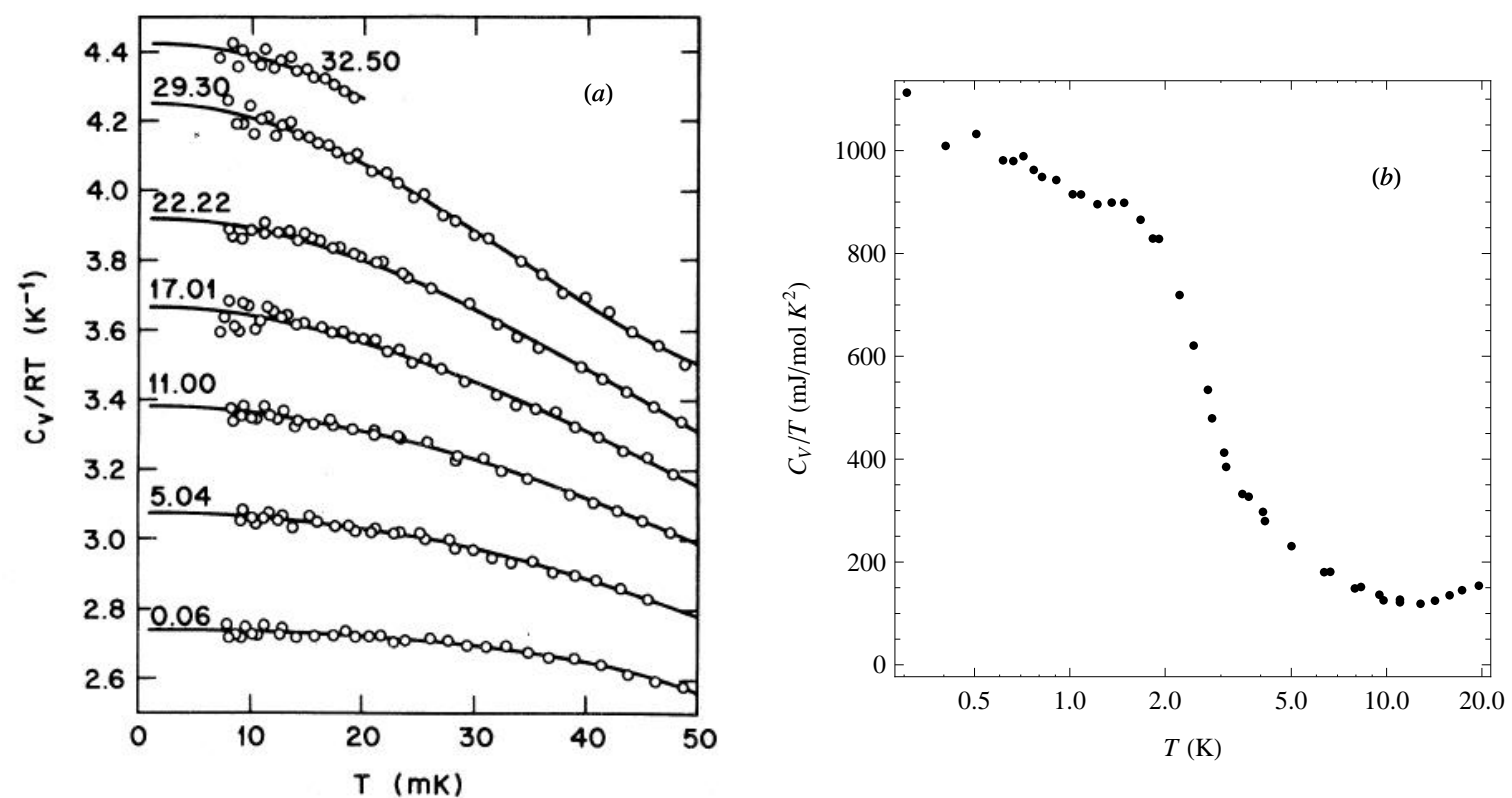

Figure 4.13: (a) Experimental values of ${ }^{3} \mathrm{He}$ specific heat, plotted as $C_{V} / R T$ as a function of $T$ (from [147]). The numbers indicate the pressure at which each set of measurements was performed. (b) Experimental values of $C_{V} / T$ as a function of $T$ for the heavy-fermion metal $\mathrm{CeCu}_{2} \mathrm{Si}_{2}$, taken from [148. The heavy-fermion regime is observed for $T \lesssim 2 \mathrm{~K}$.

reduced to a renormalization of the electron physical characteristics, such as its mass. The metal is then described as a non-interacting Fermi gas of renormalized electrons, the so-called Landau quasiparticles.

The structure of the dispersion relation for low-energy excitations around the Fermi surface remains identical to the one of non-interacting fermions:

$$
\epsilon_{k}=\mu+\frac{\hbar^{2} k_{F}}{m^{*}}\left|k-k_{F}\right|
$$

where $k_{F}=\left(3 \pi^{2} n\right)^{2 / 3}$ is the Fermi momentum and $n$ is the total electron density. For $k>k_{F}$ excitations correspond to the extra particles above the Fermi level, while for $k<k_{F}$ they corresponds to the removal of one particle under the Fermi level, and can been seen as the creation of a 'hole'. Due to interactions, the bare electron mass $m$ is replaced by the quasiparticle effective mass $m^{*}$, and $\mu$ may differ from $E_{F}=\hbar^{2} k_{F}^{2} / 2 m$. At finite temperature, quasi-particles are populated according to the Fermi-Dirac distribution $1 /\left(1+e^{\left(\epsilon_{k}-\mu\right) / k_{B} T}\right)$. The calculation of the specific heat at low temperature is then similar to the one of an ideal Fermi gas, and gives:

$$
C_{V}=\frac{m^{*}}{m} \frac{V m k_{B}^{2}\left(3 \pi^{2} n\right)^{1 / 3}}{3 \hbar^{2}} T .
$$

Therefore, the quasi-particle effective mass can directly be obtained from a measurement of the specific heat, by comparison with the one of an ideal Fermi gas with the same electron density.

As shown in Fig 4.13 this low-temperature linear dependence of the specific heat with temperature is observed in a large number of materials.

For simple metals, the effective mass value $m^{*}$ is comparable to the bare electron mass $m$. As an example, the effective mass in $\mathrm{Cu}$ is $m^{*}=1.3 m[149$. At low temperature (above the superfluid transition temperature), ${ }^{3} \mathrm{He}$ is a Fermi liquid whose quasiparticle effective mass $m^{*}$ is on the order of the bare ${ }^{3} \mathrm{He}$ mass $m$ (see Fig 4.13 ) $: m^{*} \simeq 3 m$ at ambient pressure 147 .

The most exotic Fermi liquids are heavy-fermion metals, which exhibit a Fermi liquid behavior with effective masses up to $\sim 1000 \mathrm{~m}$, where $m$ is the electron mass. Heavy-fermion metals contain a matrix 


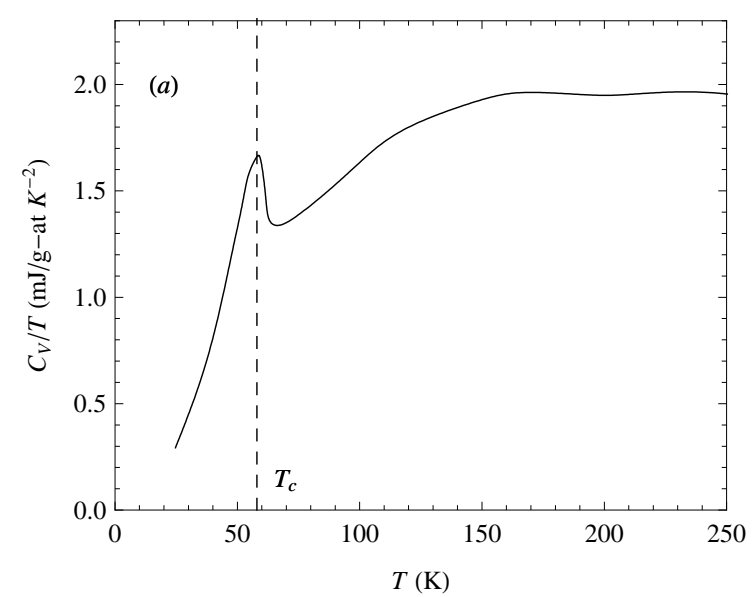

(b)

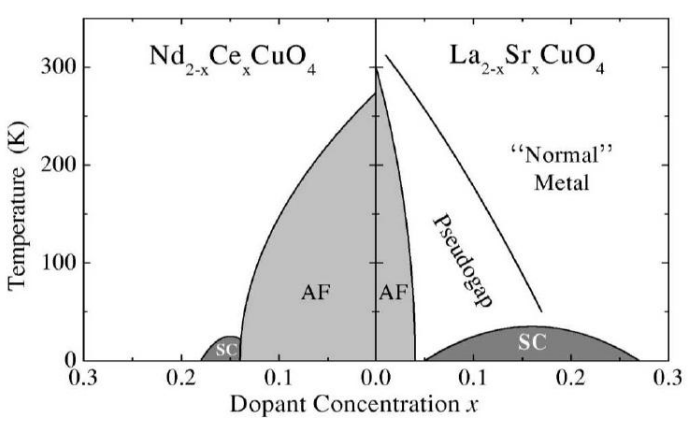

Figure 4.14: (a) Specific heat coefficient $C_{V} / T$ as a function of temperature for the high- $T_{c}$ material $\mathrm{YBa}_{2} \mathrm{Cu}_{3} \mathrm{O}_{6+y}$, here for a doping $y=0.57$ (from [150]). (b) Typical phase diagram for high- $T_{c}$ superconductors with electron doping (left side) or hole doping (right side) (from [152]), showing the large parameter range where the pseudo-gap phase, or 'strange metal' phase, is observed.

of rare-earth or actinide ions acting as magnetic impurities, coupled to a Fermi sea of mobile conduction electrons. Interactions between a single ion and the surrounding electron Fermi sea result in the so-called Kondo effect, i.e. a complete screening of the magnetic impurity by electrons at low temperature. In such materials, if the magnetic screening is stronger than the tendency to form an insulating antiferromagnet, impurities are dissolved into the mobile electron Fermi sea and form charged mobile quasi-particles with a large effective mass (see Fig 4.13 ).

The Landau Fermi liquid prescription does not predict which systems are Fermi liquids or how to calculate quasi-particle characteristics. Nevertheless, its relevance for describing most metals is remarkable.

\section{The Pseudogap Phase}

The normal state in high- $T_{c}$ cuprates does not seem to exhibit the characteristic features of a Fermi liquid for a wide range of temperatures and doping above the critical temperature for superfluidity. In Fig 4.14 we show the specific heat of the compound $\mathrm{YBa}_{2} \mathrm{Cu}_{3} \mathrm{O}_{6.57}$, measured in [150]. The sharp feature indicates the normal to superfluid phase transition at $T=T_{c}=57 \mathrm{~K}$. In the wide temperature range $T_{c}<T \lesssim T^{*}=150 \mathrm{~K}$, the specific heat is not linear with temperature, indicating the non-applicability of Fermi liquid theory. In Fig $4.14 \mathrm{~b}$ we show a typical phase diagram, illustrating the large width of this 'strange metal' phase as a function of impurity concentration.

The microscopic origin of this behavior is attributed to the existence of a gap in the single-particle excitations in the range $T_{c}<T \lesssim T^{*}=150 \mathrm{~K}$. Using angle-resolved photoemission, momentum-resolved single-particle excitation spectrum are measured and one observes an energy gap along certain momentum directions for $T_{c}<T \lesssim T^{*} 151$. Understanding this phenomenon and its connection to superconductivity is a major research topic in condensed matter physics.

\section{Normal State of a Two-Component Fermi Gas}

The investigation of a Fermi liquid or pseudogap behavior in unitary Fermi gases, which constitute model high- $T_{c}$ systems, could shed some light on the physics of high- $T_{c}$ superfluidity.

Before addressing the case of the unitary limit $a=\infty$, we briefly discuss the weak interaction limits of the BEC-BCS crossover. On the BEC side of the resonance $\left(1 / k_{F} a \gg 1\right)$, the normal gas above the critical temperature $T_{c} \simeq 0.22 E_{F}[153$ is a thermal gas of molecules, whose thermodynamics strongly 


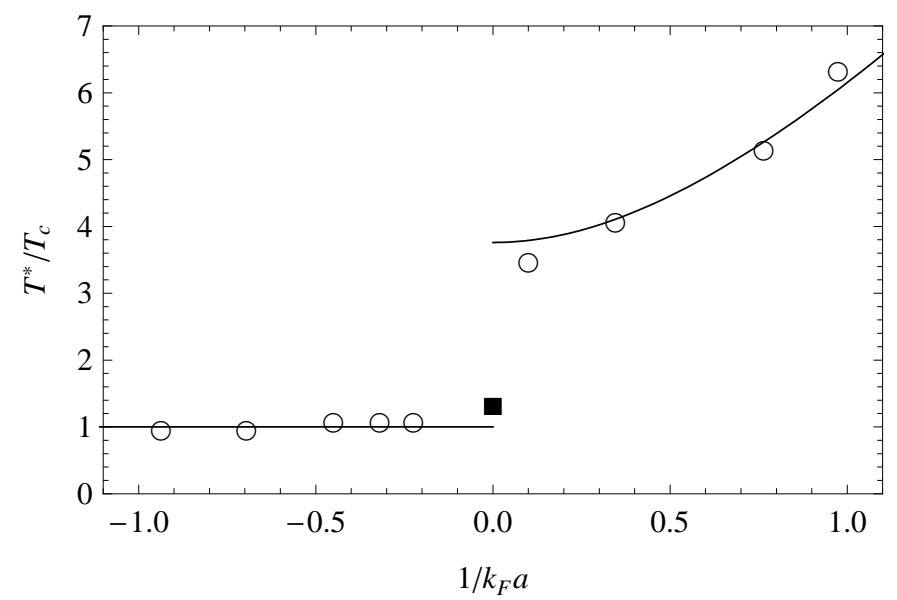

Figure 4.15: Temperature $T^{*}$ at which the single-particle excitation gap vanishes, as a function of the interaction strength $1 / k_{F} a$. The two solid lines are the predictions in the BCS limit, $T^{*}=T_{c}$, and in the BEC limit, $T^{*} \sim\left|E_{b}\right| / k_{B}$ (see 157 for details). The circles are the diagrammatic calculations from [154, and the square is the Quantum Monte Carlo prediction from 155 .

differs from a Fermi liquid, as long as the temperature is much smaller than the molecular binding energy. Therefore, the ideal Fermi gas picture is recovered only for $k_{B} T \gg k_{B} T^{*}=\left|E_{b}\right|=\hbar^{2} / m a^{2} \gg E_{F}$. Due to the molecular binding energy, single-particle excitations are gapped in the normal phase for $T \lesssim T^{*}$ (see Fig 4.15). On the other hand, in the BCS limit the gap is directly associated with the superfluid order parameter and therefore it vanishes at $T=T_{c}$, with no pseudogap region. In [154], the temperature $T^{*}$ at which the pseudogap vanishes was estimated, interpolating between the BEC regime and the BCS regime, but this study does not predict whether $T^{*}$ significantly differs from $T_{c}$ in the unitary limit. In a recent Monte Carlo calculation by P. Magierski et al [155], the single-particle spectral function of a finite-temperature unitary gas was calculated, and presents a pseudogap for $T_{c}<T \lesssim 1.3 T_{c}$, falling from $\Delta \simeq 0.2 E_{F}$ at $T \simeq T_{c}$ to $\Delta=0$ at $T=1.3 T c$. In $[156$ the single-particle spectral function was directly measured by the JILA group, for a unitary gas prepared at a temperature $T / T_{c}=0.9(1)$, i.e. right below the normal to superfluid transition. They deduce from their measurements the existence of a large gap of single-particle excitations $\Delta / \mu=0.75$. At present there is no experimental evidence for the existence of a pseudogap for $T>T_{c}$.

We will address these open questions by analyzing the low-temperature behavior of the equation of state extracted from our data.

\subsubsection{Observation of a Fermi Liquid Behavior}

\section{Grand-Canonical Fermi Liquid Equation of State}

In order to compare our data $h_{T}(\zeta)$ to a Fermi liquid behavior, we derive in this section the grandcanonical equation of state of a Fermi liquid. The starting assumption of Fermi liquid theory is the quasi-particle dispersion relation 4.14. The corresponding density of states reads, around the Fermi level:

$$
\begin{aligned}
\rho(\epsilon) & =2 \frac{V}{(2 \pi)^{3}} \int \mathrm{d} \mathbf{k} \delta\left(\epsilon-\mu-\frac{\hbar^{2} k_{F}}{m^{*}}\left|k-k_{F}\right|\right) \\
& \simeq V \frac{2}{\pi^{2}} \frac{m^{*} k_{F}}{\hbar^{2}} \quad \text { assuming } \quad \epsilon-\mu \ll \mu \\
& =\frac{m^{*}}{m} \xi_{n}^{-1 / 2} V \frac{2 \sqrt{2} m^{3 / 2} \sqrt{\mu}}{\pi^{2} \hbar^{3}} \quad \text { where we define } \mu=\xi_{n} \frac{\hbar^{2} k_{F}^{2}}{2 m} .
\end{aligned}
$$


Therefore the density of states is simply equal to the one of a two-component ideal Fermi gas with the same chemical potential, multiplied by $m^{*} / m \xi_{n}^{-1 / 2}$. The parameter $\xi_{n}$ defines the Fermi liquid equation of state extrapolated at zero temperature $\mu=\xi_{n} E_{F}$. The corresponding pressure reads:

$$
P(\mu, T=0)=\xi_{n}^{-3 / 2} 2 P_{0}(\mu, T=0),
$$

where $2 P_{0}(\mu, T=0)=2 / 15 \pi^{2}\left(2 m / \hbar^{2}\right)^{3 / 2} \mu^{5 / 2}$ is the pressure of a non-interacting two-component Fermi gas at $T=0$. Low-temperature effects are then calculated similarly to the ones of an ideal gas (see Appendix A.1):

$$
\begin{aligned}
P(\mu, T) & =P(\mu, 0)+\frac{k_{B} T}{V} \int_{\mu}^{\infty} \mathrm{d} \epsilon \rho(\epsilon) \log \left(1+e^{(\epsilon-\mu) / k_{B} T}\right) \\
& =P(\mu, 0)+k_{B} T \frac{m^{*}}{m} \xi_{n}^{-1 / 2} \frac{2 \sqrt{2} m^{3 / 2} \sqrt{\mu}}{\pi^{2} \hbar^{3}} \int_{\mu}^{\infty} \mathrm{d} \epsilon \log \left(1+e^{(\epsilon-\mu) / k_{B} T}\right) \\
& =2 P_{0}(\mu, 0)\left(\xi_{n}^{-3 / 2}+\frac{5 \pi^{2}}{8} \frac{m^{*}}{m} \xi_{n}^{-1 / 2}\left(\frac{k_{B} T}{\mu}\right)^{2}\right)
\end{aligned}
$$

The ideal gas equation of state A.8) derived in Appendix A.1 is recovered by taking $\xi_{n}=1$ and $m^{*}=m$.

\section{Fermi Liquid Behavior of the Equation of State Deduced from our Data}

In order to compare our data $h_{T}(\zeta)$ to 4.15 , we plot our data as $P(\mu, T) / 2 P_{0}(\mu, 0)$ versus $\left(k_{B} T / \mu\right)^{2}$. There is a single correspondence between the data $\left(\zeta, h_{T}\right)$ and $\left(\left(k_{B} T / \mu\right)^{2}, P(\mu, T) / 2 P_{0}(\mu, 0)\right)$ :

$$
\begin{aligned}
\left(\frac{k_{B} T}{\mu}\right)^{2} & =(\log \zeta)^{-2} \\
\frac{P(\mu, T)}{2 P_{0}(\mu, 0)} & =\frac{P_{0}(\mu, T)}{P_{0}(\mu, 0)} h_{T}=\frac{15 \sqrt{\pi}}{8} \frac{f_{5 / 2}\left(\zeta^{-1}\right)}{(-\log \zeta)^{5 / 2}} h_{T} .
\end{aligned}
$$

Therefore, each data point $h_{T}(\zeta)$ results in one point in this representation.

Our data for $k_{B} T \lesssim \mu$ is in very good agreement with a Fermi liquid equation of state 4.15 with $\xi_{n}=0.51(2)$ and $m^{*}=1.13(4) m$ (see Fig 4.16a and b). The relative deviation of our data with (4.15) is less than $3 \%$ for $0.1<\left(k_{B} T / \mu\right)^{2}<0.6$, and around $T=\mu$ is about $5 \%$,

Equivalently, the Fermi liquid characteristics can be expressed in terms of Landau parameters 146, $F_{0}^{s}=\xi_{n} m^{*} / m-1=-0.42$ and $F_{1}^{s}=3\left(m^{*} / m-1\right)=0.39$.

\section{Condensation Energy of a Fermi Gas with Resonant Interactions}

The extrapolation to $T=0, P(\mu, 0)=2 P_{0}(\mu) \xi_{n}^{-3 / 2}$, corresponds to a pressure lower than the actual ground state pressure $P(\mu, 0)=2 P_{0}(\mu) \xi_{s}^{-3 / 2}$, where $\xi_{s} \simeq 0.41$ is a characteristics of the $T=0$ superfluid measured in Chapter 5. This means that the normal state is thermodynamically unstable with respect to the superfluid state. The difference between the $T=0$ energy in the normal and superfluid states is referred to as the condensation energy:

$$
\begin{aligned}
E_{c} & \equiv E_{N}-E_{S} \\
& =\frac{3}{5} N E_{F}\left(\xi_{n}-\xi_{s}\right) \\
& =0.10(2) \frac{3}{5} N E_{F} .
\end{aligned}
$$

\footnotetext{
$\dagger$ As a comparison, the relative difference between the equation of state of an ideal gas and its Sommerfeld expansion up to second order in $k_{B} T / \mu$ is $7 \%$ at $k_{B} T=\mu$.
} 
(b)
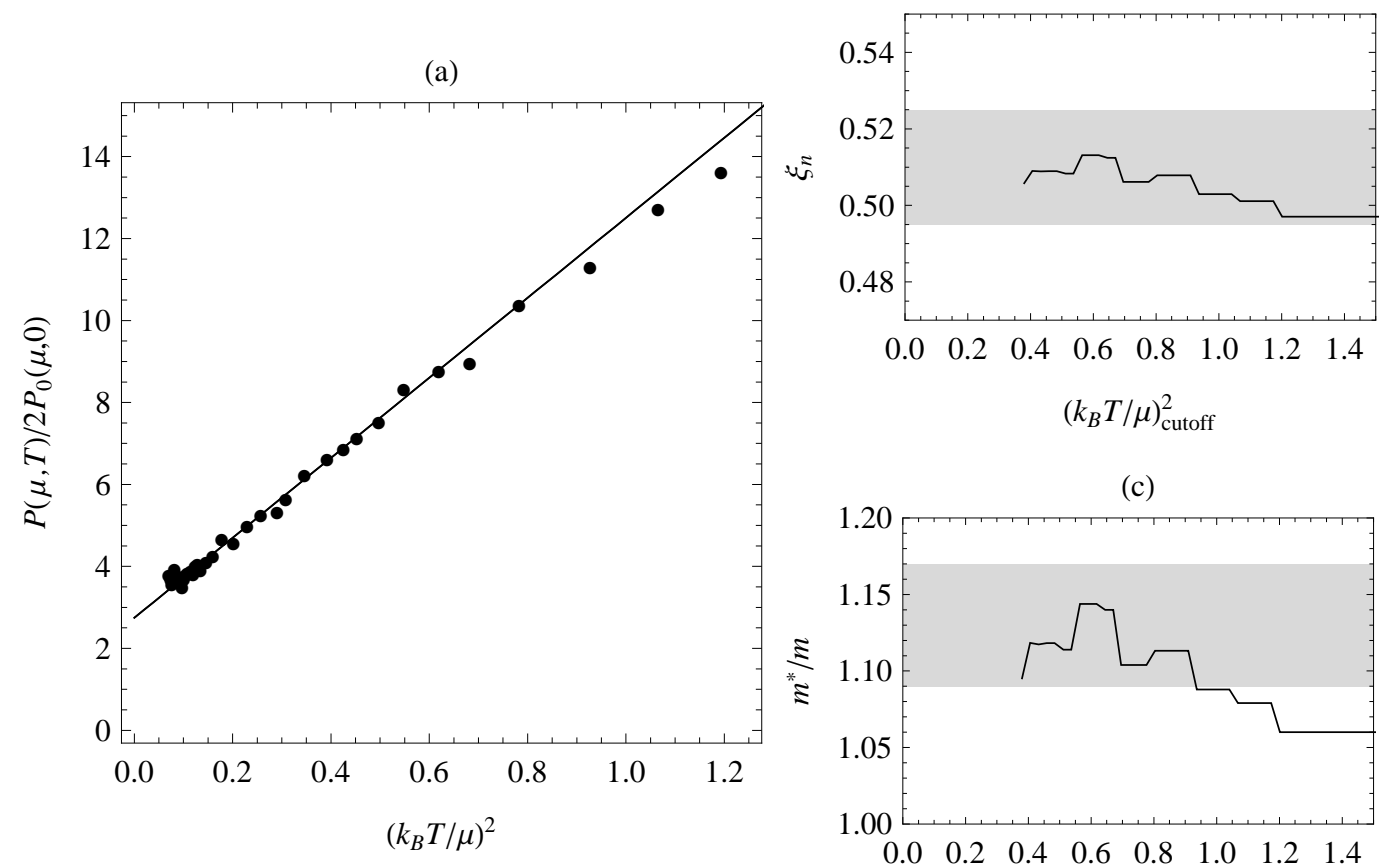

(c)

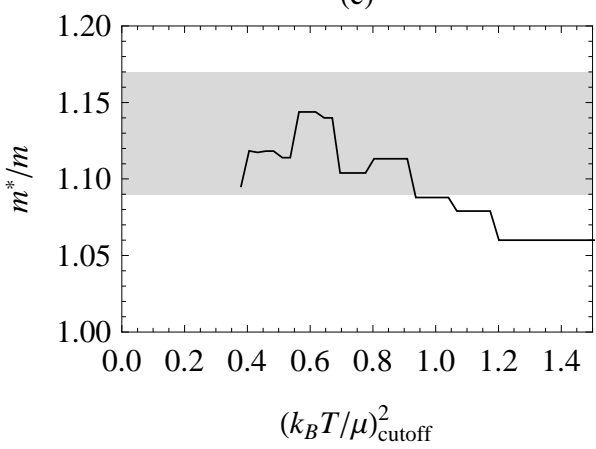

Figure 4.16: (a) Equation of state of a unitary Fermi gas, plotted as $P(\mu, T) / 2 P_{0}(\mu, 0)$ versus $\left(k_{B} T / \mu\right)^{2}$. For $k_{B} T \lesssim \mu$ our data are in very good agreement with a Fermi liquid equation of state, with $\xi_{n}=0.51$ and $m^{*}=1.13 m$ (solid line). (b) and (c) Results of the fit of our data for $\left(k_{B} T / \mu\right)<\left(k_{B} T / \mu\right)_{\text {cutoff }}$ with a Fermi liquid equation of state 4.15 . The gray regions correspond to the values of $\xi_{n}$ or $m^{*} / m$ compatible with our low-temperature data.

In the BCS limit of weak interactions, the condensation energy is directly related to the single-particle excitation gap $\Delta_{0}$ through:

$$
E_{c}=\frac{5}{8}\left(\frac{\Delta_{0}}{E_{F}}\right)^{2} \frac{3}{5} N E_{F} .
$$

Interestingly, using the experimental value $\Delta_{0}=0.44 E_{F}$ from $[51]$ (which agrees with a recent calculation using an unbiased Monte-Carlo computation $\mid 158)$, we obtain $5 / 8\left(\Delta_{0} / E_{F}\right)^{2} \simeq 0.12$, showing that equation 4.16 approximately remains valid even for resonant interactions. It would be interesting to investigate more deeply this behavior and how it depends on the interaction strength.

\subsubsection{Estimation of the Maximum Pseudogap Amplitude}

Our data thus agrees with a simple Fermi liquid picture, while it is generally believed that the normal gas above $T_{c}$ exhibits a pseudogap in the unitary limit $159,160,155,161,162,163$. In this section we estimate the maximum pseudogap values in agreement with our observations, assuming the existence of a well-defined dispersion relation $\epsilon_{k}$ (i.e. the spectral function $A(k, \omega)$ of the unitary gas is approximated by $\delta\left(\epsilon_{k}-\omega\right)$ ). Inspired by the quasi-particle dispersion relation in the BCS limit [157], we introduce a pseudogap $\Delta$ in the excitation spectrum in the following manner (see Fig 4.17):

$$
\epsilon_{k}=\mu+\frac{\hbar^{2} k_{F}}{m^{*}}\left|k-k_{F}\right| \quad \rightarrow \quad \epsilon_{k}=\mu+\sqrt{\left(\frac{\hbar^{2} k_{F}}{m^{*}}\left(k-k_{F}\right)\right)^{2}+\Delta^{2}} .
$$

$\mu+\Delta$ is then indeed the minimum single-particle excitation energy. From the density of states associated 


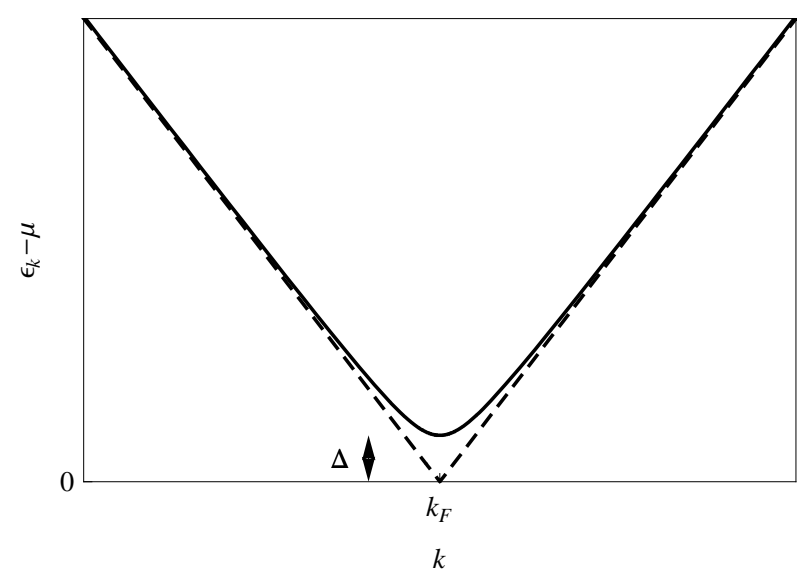

Figure 4.17: Quasi-particle dispersion for a Fermi liquid (dashed line) and with a pseudogap $\Delta$ (solid line)

with such a dispersion relation:

$$
\rho(\epsilon)=\frac{m^{*}}{m} \xi_{n}^{-1 / 2} V \frac{2 \sqrt{2} m^{3 / 2} \sqrt{\mu}}{\pi^{2} \hbar^{3}} \frac{\epsilon-\mu}{\sqrt{(\epsilon-\mu)^{2}-\Delta^{2}}},
$$

one calculates the gas pressure:

$$
\begin{aligned}
P(\mu, T) & =P(\mu, 0)+\frac{k_{B} T}{V} \int_{\mu+\Delta}^{\infty} \mathrm{d} \epsilon \rho(\epsilon) \log \left(1+e^{(\epsilon-\mu) / k_{B} T}\right) \\
& =P(\mu, 0)+k_{B} T \frac{m^{*}}{m} \xi_{n}^{-1 / 2} \frac{2 \sqrt{2} m^{3 / 2} \sqrt{\mu}}{\pi^{2} \hbar^{3}} \int_{\mu+\Delta}^{\infty} \mathrm{d} \epsilon \frac{\epsilon-\mu}{\sqrt{(\epsilon-\mu)^{2}-\Delta^{2}}} \log \left(1+e^{(\epsilon-\mu) / k_{B} T}\right) \\
& =2 P_{0}(\mu, 0)\left(\xi_{n}^{-3 / 2}+\frac{5 \pi^{2}}{8} \frac{m^{*}}{m} \xi_{n}^{-1 / 2}\left(\frac{k_{B} T}{\mu}\right)^{2} \mathcal{F}\left(\frac{\Delta}{k_{B} T}\right)\right)
\end{aligned}
$$

where the function $\mathcal{F}$, defined by:

$$
\mathcal{F}(y)=\frac{12}{\pi^{2}} \int_{y}^{\infty} \mathrm{d} x \frac{x}{\sqrt{x^{2}-y^{2}}} \log \left(1+e^{-x}\right),
$$

is plotted in Fig 4.18 a. $\mathcal{F}$ quantifies the deviation of the equation of state from the one of a Fermi liquid. As shown in Fig 4.16, the deviation of our data from a Fermi liquid equation of state is less than $5 \%$ in the range $0.1<\left(k_{B} T / \mu\right)^{2}<1$, i.e. $0.95<\mathcal{F}\left(\Delta / k_{B} T\right)<1.05$. This leads to an upper bound for the pseudogap values in agreement with our observations (Fig 4.18a):

$$
\Delta<0.25 k_{B} T
$$

As shown in Fig 4.18, this bound excludes the Monte Carlo pseudogap values from 155. Indeed, just above the critical temperature for superfluidity, our data are consistent with $\Delta \lesssim 0.25 T_{c} \simeq 0.05 E_{F}$. Our measurements also exclude the larger pseudogap values, $\Delta \sim 0.6 E_{F}$ at $T / T_{F}=0.24$, given by a finite- $T$ extended BCS-Leggett theory 160 or a Nozières Schmitt-Rink theory 164 .

To conclude, our measurement strongly supports an accurate description of the normal state of the unitary gas as a Fermi liquid. The pseudogap values $\Delta \lesssim 0.05 E_{F}$ consistent with our data are very small compared with the values measured in the superfluid state [51,156]. Developing a more complex model accounting for a finite width of the spectral function $A(k, \omega)$ could provide a more precise comparison with theories supporting a pseudogap. 
(a)

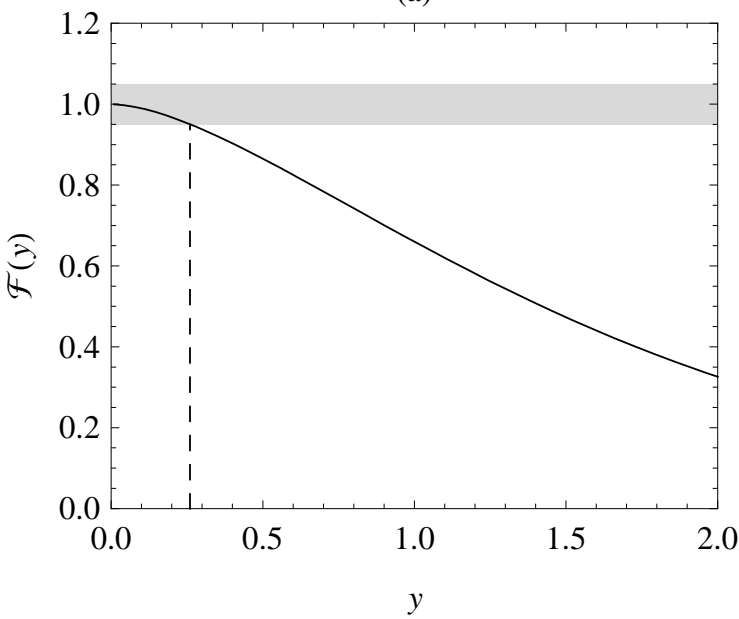

(b)

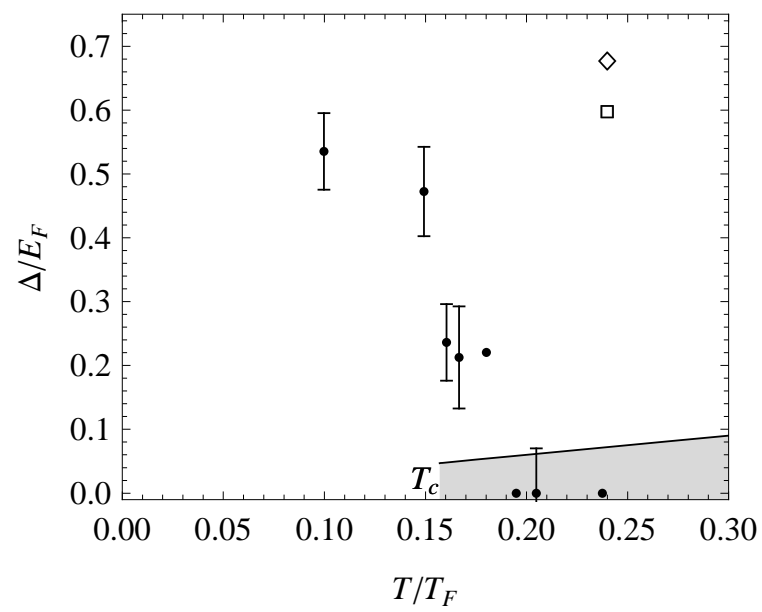

Figure 4.18: (a) Function $\mathcal{F}$, defined in equation 4.17, that quantifies the deviation of the equation of state from a Fermi liquid equation. The gray rectangle represent the possible values of $\mathcal{F}$ in agreement with our data. (b) Upper bound on the value of the pseudogap given by equation (4.18), compared with the pseudogap Monte Carlo values from [155] (black dots). Our data exclude the value $\Delta \simeq 0.2 E_{F}$ at $T=0.16 T_{F}$. We also show the pseudogap values given by a finite- $T$ extended BCS-Leggett theory 160 (square) or a Nozières Schmitt-Rink theory 164 (diamond).

\subsection{Superfluid Transition}

At lower temperature, we expect the gas to become superfluid. The superfluid character of a lowtemperature Fermi gas with resonant interactions was unambiguously identified through its response to a rotation of the trapping potential: at a low rotation speed the gas does not respond to rotation [39], while at a larger frequency the gas starts to rotate through the formation of a vortex lattice [40]. The measurement of the critical temperature for superfluidity attracted a large amount of work in the past few years [43, 46, 39, 47]. However, similarly to previous measurements of the equation of state, these studies determine the transition point for a trapped gas, expressed as $T_{c} / T_{F}$ where $k_{B} T_{F}=\hbar \bar{\omega}(3 N)^{1 / 3}$ is the Fermi energy of a trapped gas. The comparison with theories of the homogeneous gas then requires to integrate the equation of state over the trap, using values of the equation of state for $T>T_{c}$, a region especially difficult to handle. In this section we identify the transition point for a homogeneous gas on the equation of state deduced from our data, which allows us to make the first direct comparison with many-body theories.

\subsubsection{Deviation from the Fermi Liquid Equation of State}

In Fig 4.19 we focus on the low-temperature data, limited to $\left(k_{B} T / \mu\right)^{2}>0.07$ due to the instability of ${ }^{7} \mathrm{Li}$ at low temperature (see section 4.1). For $\left(k_{B} T / \mu\right)^{2}<0.1$ our data deviates from the Fermi liquid equation of state 4.15 and $P / 2 P_{0}$ seems $T$-independent. As the deviation is small, we add some arguments showing that this behavior is indeed expected in the superfluid state.

At $T=0$ the equation of state is well known and solely involves the parameter $\xi_{s}=\mu / E_{F}$, that has been extensively measured and calculated in the past [165. In section 5.5 we measure the pressure of the ground state in the BEC-BCS crossover and in particular we confirm the value $\xi_{s}=0.415(10)$. The pressure at $T=0$ is then given by:

$$
\frac{P(\mu, 0)}{2 P_{0}(\mu, 0)}=\xi_{s}^{-3 / 2}=3.8(1)
$$


which is equal to the values of $P(\mu, T) / 2 P_{0}(\mu, 0)$ that we measure at $\left(k_{B} T / \mu\right)^{2} \simeq 0.1$. Moreover, $P(\mu, T) / 2 P_{0}(\mu, 0)$ is an increasing function of $T$ since:

$$
\begin{aligned}
\left.\frac{\partial}{\partial T}\left(\frac{P(\mu, T)}{2 P_{0}(\mu, 0)}\right)\right|_{\mu} & =\frac{\partial P(\mu, T) /\left.\partial T\right|_{\mu}}{2 P_{0}(\mu, 0)} \\
& =\frac{S(\mu, T)}{2 V P_{0}(\mu, 0)} \geq 0 .
\end{aligned}
$$

This shows that, constrained by the values at $T=0$ and at $\left(k_{B} T / \mu\right)^{2} \simeq 0.1$, the value of $P(\mu, T) / 2 P_{0}(\mu, 0)$ necessarily remains almost equal to $\xi_{s}^{-3 / 2}$ in the whole range $0<\left(k_{B} T / \mu\right)^{2}<0.1$.

In the next section we show that this small temperature dependence is expected in the superfluid phase.

\subsubsection{Low-Temperature Excitations in the Superfluid Phase}

To first order, low-temperature effects in the superfluid are captured by the thermal population of its lowenergy excitations. Two kinds of excitations are considered here: the fermionic single-particle excitations and the Bogoliubov-Anderson collective excitations associated with the propagation of sound.

\section{Single-Particle Fermionic Excitations}

The dispersion relation of single-particle fermionic excitations was directly measured by the MIT group in [166, and is well accounted for by a BCS-type dispersion relation:

$$
\epsilon_{k}=\mu+\sqrt{\left(\frac{\hbar^{2} k^{2}}{2 m^{*}}+U-\mu\right)^{2}+\Delta^{2} .}
$$

$m^{*} \simeq m$ is the quasi-particle effective mass, $U=-0.43 E_{F}=-1.02 \mu$ is the Hartree energy shift, and $\Delta=0.44 E_{F}$ is the excitation gap. In addition, these measurements are in agreement with Quantum Monte-Carlo calculations 167, 155. The dispersion relation is thus essentially the same than the one considered for the estimation of the pseudogap amplitude in the normal phase (equation (4.17)). Using the calculations made in section 4.6 (and replacing $\xi_{n}$ by $\xi_{s}$ ), we obtain the pressure increase due to the thermal population of fermionic excitations:

$$
\begin{aligned}
P(\mu, T) & =2 P_{0}(\mu, 0)\left(\xi_{s}^{-3 / 2}+\frac{5 \pi^{2}}{8} \frac{m^{*}}{m} \xi_{s}^{-1 / 2}\left(\frac{k_{B} T}{\mu}\right)^{2} \mathcal{F}\left(\frac{\Delta}{k_{B} T}\right)\right) \\
& \simeq 2 P_{0}(\mu, 0)\left(\xi_{s}^{-3 / 2}+\frac{15 \sqrt{2 \pi}}{4} \frac{m^{*}}{m} \xi_{s}^{-1 / 2} \frac{\left(k_{B} T\right)^{3 / 2} \Delta^{1 / 2}}{\mu^{2}} \exp \left(-\frac{\Delta}{k_{B} T}\right)\right),
\end{aligned}
$$

in the low-temperature limit $k_{B} T \ll \Delta^{\text {f }}$. At low temperature these excitations are thus exponentially suppressed due to the gap, and the pressure increase at $T \simeq 0.3 \mu$ is only $3 \%$ (assuming a gap equal to its $T=0$ value, $\Delta=0.44 E_{F}[51$ ) (see Fig 4.19 a).

\section{Sound Excitations}

The lowest energy excitations are the collective excitations associated with the propagation of sound. The speed of sound is related to the equation of state through:

$$
c_{s}=\sqrt{\frac{n}{m} \frac{\partial \mu}{\partial n}}=\sqrt{\frac{2}{3} \frac{\mu}{m}}
$$

\footnotetext{
†The asymptotic behavior of $\mathcal{F}(x)$ for $x \gg 1$ is given by $\mathcal{F}(x) \simeq 12 / \sqrt{2} \pi^{3 / 2} x^{1 / 2} e^{-x}$.
} 
(a)

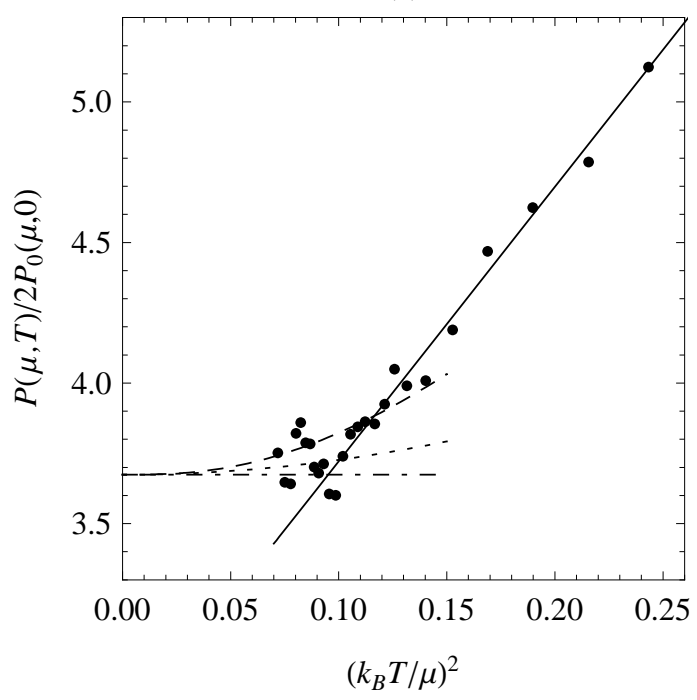

(b)

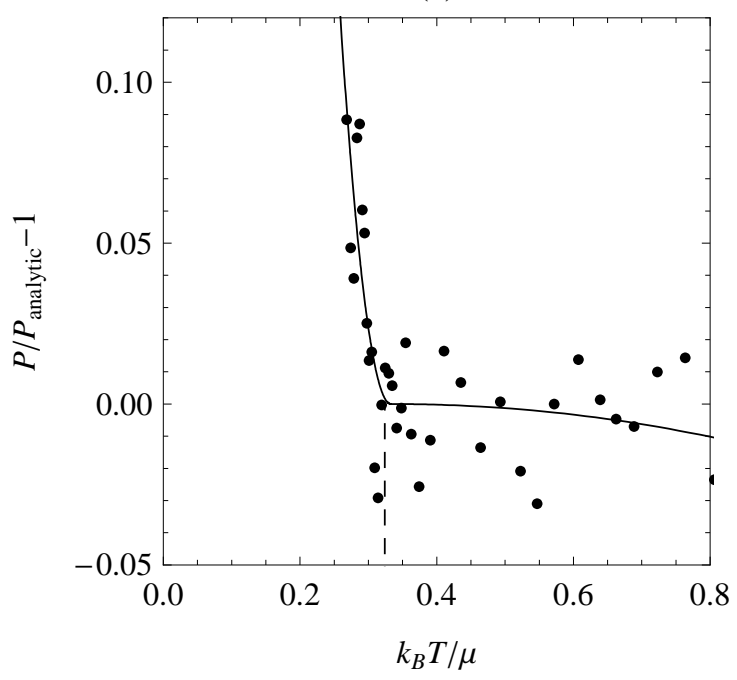

Figure 4.19: (a) Equation of state of a unitary Fermi gas, plotted as $P(\mu, T) / 2 P_{0}(\mu, 0)$ versus $\left(k_{B} T / \mu\right)^{2}$, and zoomed around $\left(k_{B} T / \mu\right)^{2}=0.1$. The solid line is the Fermi liquid equation of state (4.15), the dashed line is the $T=0$ value $\xi_{s}^{-3 / 2}$, the dotted line takes into account the phonon correction (4.20), and the dot-dashed line the phonon and fermionic-excitation corrections 4.20 and $(4.19)$. (b) Fit of our data around the critical point $\left(k_{B} T / \mu\right)_{c}=0.32$ with a function capturing the critical behavior (see text). The data are expressed as $P / P_{\text {analytic }}-1$ versus $k_{B} T / \mu$, where $P_{\text {analytic }}$ is the Fermi liquid pressure in the normal phase.

where we have used $\mu \propto n^{2 / 3}$ at $T=0$. Bosonic quasi-particles (phonons) associated with the propagation of sound have a dispersion relation $\epsilon_{k}=\hbar c_{s} k$. Their population induced by a non-zero temperature leads to a pressure correction given by the Stefan law of black body radiation:

$$
\begin{aligned}
\delta P_{\text {phonons }}(\mu, T) & =\frac{\pi^{2}}{90 \hbar^{3} c_{s}^{3}}\left(k_{B} T\right)^{4} \\
& =2 P_{0}(\mu, 0) \frac{\pi^{4} \sqrt{3}}{32}\left(\frac{k_{B} T}{\mu}\right)^{4} .
\end{aligned}
$$

While at very low temperature the effect of phonons dominates over the fermionic excitations (see Fig 4.19 a), it is less than $2 \%$ at $T \simeq 0.3 \mu$.

The overall pressure increase at $T \simeq 0.3 \mu$ is therefore expected to be less than $5 \%$, in agreement with the behavior $P(\mu, T) / 2 P_{0}(\mu, 0) \simeq$ cst consistent with our data.

\subsubsection{Critical Temperature for Superfluidity}

These arguments show that the deviation from the Fermi liquid equation of state indicates a phase transition from a normal to a superfluid state.

In [48] we proposed to extract the critical temperature $\left(k_{B} T / \mu\right)_{c}$ in a very simple manner. We fitted our data around $\left(k_{B} T / \mu\right)^{2}=0.3$ with a continuous and piecewise linear function, one part being equal to the Fermi liquid equation of state, the other part being a constant. The breaking point was let as a free parameter and was identified with $\left(k_{B} T / \mu\right)_{c}$. This procedure leads to:

$$
\left(\frac{k_{B} T}{\mu}\right)_{c}=0.316(7) .
$$


This fitting function, whose slope is discontinuous at the superfluid transition, could be suited for a first-order phase transition. However, the superfluid transition of a Fermi gas is expected to be of second order and to belong to the three-dimensional $X Y$ universality class. In the critical region, expected to be rather large for the unitary gas [168, the pressure variation is then given by:

$$
\begin{aligned}
P(\mu, T) & =P_{\text {analytic }}(\mu, T)\left(1+a_{+}\left(\frac{T-T_{c}}{T_{c}}\right)^{\alpha+2}\right) \quad \text { for } T>T_{c} \\
& =P_{\text {analytic }}(\mu, T)\left(1+a_{-}\left(\frac{T_{c}-T}{T_{c}}\right)^{\alpha+2}\right) \text { for } T<T_{c}
\end{aligned}
$$

where $P_{\text {analytic }}(\mu, T)$ is the analytic equation of state far from the critical region, here the Fermi liquid equation of state. For the three-dimensional $X Y$ universality class, the specific heat exponent $\alpha=$ $-0.012(3)$ is known with an excellent precision from experiments on ${ }^{4} \mathrm{He} 169$ or field theory calculations 170 171. Fitting our data with this function (see Fig $4.19 \mathrm{~b}$ ) leads to the coefficients $a_{-}=22(12$ ), $a_{+}=0.0(1)$, and:

$$
\left(\frac{k_{B} T}{\mu}\right)_{c}=0.33(1) .
$$

The two fit procedures lead to very similar values and differ by less than the $10 \%$ uncertainty due to the systematic error of our data. The critical temperature value extracted from our data is thus finally given by:

$$
\left(\frac{k_{B} T}{\mu}\right)_{c}=0.32(3),
$$

This constitutes the first measurement of the critical temperature of a homogeneous Fermi gas in the unitary limit. It is compared in Table 4.1 to several theoretical results. Our measurement is in very good agreement with the most robust numerical calculations 113,172,173. It is also interesting to extract from our data the value of $T / T_{F}$ at the phase transition, where $k_{B} T_{F}=\hbar^{2} / 2 m\left(3 \pi^{2} n\right)^{2 / 3}$ is the Fermi energy. The density is calculated from the pressure using $n=\partial P /\left.\partial \mu\right|_{T}$ and requires to compute the derivative of our data. It is safe to assume that the derivative of $P(\mu, T) / 2 P_{0}(\mu, T)$ at the phase transition is between the one given by the Fermi liquid equation of state 4.15 , and 0 . This leads to the value:

$$
0.13<\left(\frac{T}{T_{F}}\right)_{c}<0.16,
$$

also in good agreement with $113,172,173$. In addition, we deduce from this calculation the chemical potential value at the phase transition:

$$
0.41<\left(\frac{\mu}{E_{F}}\right)_{c}<0.5 .
$$

We also compare our value to other experimental values which are less direct than our method. In the MIT group, the superfluid transition of a spin-imbalanced Fermi gas was studied below the tri-critical point at $T=0.07 T_{F}$, i.e. in the temperature range where the phase transition is of first order 49 . Extrapolating the critical temperature to the spin-balanced situation, they obtained $\left(T / T_{F}\right)_{c} \simeq 0.15$, but this extrapolation is rather difficult to justify. In the Tokyo group, the condensate fraction was directly measured and the identification of the superfluid transition is straightforward [47. In addition the atomic density is obtained from a fit of the cloud absorption image after a hydrodynamic expansion. Using the equation of state of a trapped unitary gas measured in 63 as a reference for thermometry, they obtained $\left(T / T_{F}\right)_{c}=0.17(1)$, in agreement with our value. 


\begin{tabular}{|c|c|c|c|c|}
\hline & Our values & Amherst group & Seattle group & Utrecht group \\
\hline & & Diagram. MC 113,172 & Quantum MC 173 & Renorm. group 174 \\
\hline$\left(k_{B} T / \mu\right)_{c}$ & $0.32(3)$ & $0.32(2)$ & $0.35(3)$ & 0.24 \\
\hline \multirow[t]{3}{*}{$\left(T / T_{F}\right)_{c}$} & $0.145(20)$ & $0.152(7)$ & $0.15(1)$ & 0.13 \\
\hline & München group & Tokyo/Seattle group & Harvard group & Brisbane group \\
\hline & Diagrams 138 & $d=4-\epsilon, d=2+\epsilon 175$ & $1 / N$ expansion 176 & NSR theory 141 \\
\hline$\left(k_{B} T / \mu\right)_{c}$ & 0.41 & 1.38 & 0.23 & 0.49 \\
\hline$\left(T / T_{F}\right)_{c}$ & 0.16 & 0.25 & 0.136 & 0.22 \\
\hline
\end{tabular}

Table 4.1: Comparison between our measurement of the critical temperature for superfluidity with different theories.

\subsubsection{Validity of Local Density Approximation in the Critical Region}

In the critical region of the superfluid transition, the coherence length diverges according to:

$$
\xi \sim k_{F}^{-1}\left|\frac{T-T_{c}}{T}\right|^{-\nu}
$$

where $\nu \simeq 0.67$ is a critical exponent of the three-dimensional $X Y$ universality class. This may invalidate local density approximation in the critical region. Let us consider a trapped gas prepared below the superfluid transition, i.e. $k_{B} T / \mu^{0}<0.32$. For simplicity we consider an isotropic trap, the calculations for a more realistic trap essentially giving the same conclusions. In the critical region, the coherence length varies according to $\xi(r) \sim k_{F}^{-1}\left|\left(\mu(r)-\mu\left(r_{c}\right)\right) / \mu\left(r_{c}\right)\right|^{-\nu}$, where $r_{c}$ is the radius at which the phase transition occurs, defined by $k_{B} T / \mu\left(r_{c}\right)=0.32$. Local density approximation is expected to be incorrect in the region $r_{c}-\delta r<r<r_{c}+\delta r$, where [168:

$$
\begin{aligned}
\delta r & =\xi\left(r_{c}+\delta r\right) \\
& \sim k_{F}^{-1}\left(\frac{m \omega^{2} r_{c} \delta r}{\mu\left(r_{c}\right)}\right)^{-\nu}
\end{aligned}
$$

A simple calculation leads to the typical spatial extent of the breakdown of local density approximation, compared with the Thomas-Fermi radius $R_{\mathrm{TF}}$ defined as $\frac{1}{2} m \omega^{2} R_{\mathrm{TF}}^{2}=\mu^{0}$ :

$$
\begin{aligned}
\frac{\delta r}{R_{\mathrm{TF}}} & \sim\left(\frac{2 R_{\mathrm{TF}} r_{c}}{R_{\mathrm{TF}}^{2}-r_{c}^{2}}\right)^{-\frac{\nu}{1+\nu}}\left(k_{F} R_{\mathrm{TF}}\right)^{-\frac{1}{1+\nu}} \\
& =\left(\frac{2 R_{\mathrm{TF}} r_{c}}{R_{\mathrm{TF}}^{2}-r_{c}^{2}}\right)^{-\frac{\nu}{1+\nu}}(24 N)^{-\frac{1}{3(1+\nu)}} \\
& \simeq 5 \% \text { for } r_{c}=R_{\mathrm{TF}} / 2 \text { and } N=10^{5} \text { atoms. }
\end{aligned}
$$

Therefore the actual atom density may not be described by local density approximation is a small region around the superfluid transition. The impact on the pressure value is much smaller, due to the double integration:

$$
P\left(\mu_{z}, T\right)-P_{\text {measured }}\left(\mu_{z}, T\right)=\frac{m \omega_{r}^{2}}{2 \pi} \int \mathrm{d} x \mathrm{~d} y\left(n_{\mathrm{LDA}}(\mathbf{r})-n_{\text {real }}(\mathbf{r})\right)<0.05 P\left(\mu_{z}, T\right)
$$

since $n_{\mathrm{LDA}}(\mathbf{r})=n_{\text {real }}(\mathbf{r})$ on $95 \%$ of the integration domain. The equation of state extracted from our data thus coincides with the equation of state of a homogeneous gas, within the 5\%-noise of our data, even around the superfluid transition. The measurement of critical exponents would require a much larger signal-to-noise ratio, and a violation of local density approximation would then become visible. 


\subsection{Equation of State of a Trapped Gas}

Previous studies on the thermodynamics of a unitary Fermi gas, performed in JILA [62] and in Duke university 63 , 46 , were dealing with the equation of state of a trapped gas, relating the trapped gas total energy $E_{t}$ to its total entropy $S_{t}$ (see section 3.1.1). It can be written using dimensionless variables as a relationship between the entropy par particle $s=S_{t} / N_{t} k_{B}$ and the energy $E_{t}$ normalized by the energy $E_{t}^{(0)}$ of an ideal gas at same entropy $s$ :

$$
\frac{E_{t}}{E_{t}^{(0)}(s)}=g_{t}\left(s=\frac{S_{t}}{N_{t} k_{B}}\right) .
$$

In section 4.5 we calculated the virial expansion 4.9 of a trapped gas as a function of the virial expansion of a homogeneous gas. The equation of state of a trapped ideal gas is directly obtained from 4.9 by canceling all virial coefficients:

$$
\Omega_{t}^{(0)}\left(\mu^{0}, T\right)=2 k_{B} T\left(\frac{k_{B} T}{\hbar \bar{\omega}}\right)^{3} f_{4}\left(e^{\mu^{0} / k_{B} T}\right),
$$

where $f_{4}(z)=-\operatorname{PolyLog}(4,-z)=\sum_{k \geq 1}(-1)^{k+1} k^{-4}$. The ideal gas equation of state $E_{t}^{(0)}(s)$ is then calculated from 4.23 .

In order to obtain the equation of state 4.22 from our data $h_{T}(\zeta)$, we express the total atom number $N_{t}$, energy $E_{t}$, and entropy $S_{t}$ using local density approximation, as an integral over the trap of thermodynamic quantities of the homogeneous gas. As an example, the total atom number is given by

$$
N_{t}\left(\mu^{0}, T\right)=\int \mathrm{d} \mathbf{r} n\left(\mu^{0}-V(\mathbf{r}), T\right),
$$

where $n(\mu, T)=\partial P / \partial \mu$ is the density of a homogeneous gas, whose equation of state is $P(\mu, T)=$ $2 k_{B} T \lambda_{d B}^{-3}(T) f_{5 / 2}\left(e^{\mu / T}\right) h_{T}\left(e^{-\mu / T}\right)$. We then express the integral using the variable $\zeta$, leading after a straightforward calculation to:

$$
N_{t}\left(\mu^{0}, T\right)=\frac{4}{\sqrt{\pi}}\left(\frac{k_{B} T}{\hbar \omega}\right)^{3} \int_{\zeta^{0}}^{\infty} \frac{\mathrm{d} \log ^{1 / 2}\left(\zeta / \zeta^{0}\right)}{\mathrm{d} \zeta} f_{5 / 2}\left(\zeta^{-1}\right) h_{T}(\zeta) \mathrm{d} \zeta
$$

Similar expressions are obtained for $E_{t}$ and $S_{t}$ and can be found in 177 . Using a discretized version of these integrals taken on our data point:\$ we obtain the equation of state $g_{t}(s)$ plotted in Fig 4.20.

Our data are in good agreement with previous studies $62,63,46$ but has a much smaller statistical noise. Indeed, integrating over the trap significantly increases the signal-to-noise ratio. However, the $5 \%$ systematic error is unchanged. In Fig 4.20 we also make the comparison with successive virial expansions up to fourth order, using the exact relation $b_{t k}=b_{k} / k^{3 / 2}$ between the virial coefficients $b_{k}$ measured for a homogeneous gas and the ones of a trapped gas. Due to the coefficient $k^{-3 / 2}$, the effect of higher-order coefficients is much smaller on the equation of state of a trapped gas, and we see in Fig 4.20 that the signal-to-noise ratio required to extract $b_{3 t}$ and $b_{4 t}$ was not achieved in previous studies $62,63,46$.

Finally, we also compare our measurement with previous studies of the superfluid transition. A large amount of work $43,46,39,47$ focused on the characterization of the onset of superfluidity in a trapped gas: these works provided the ratio of the temperature $T$ over the Fermi temperature of the trapped gas $k_{B} T_{F t}=\hbar \bar{\omega}\left(3 N_{t}\right)^{1 / 3}$, for which the central part of the cloud becomes superfluid. In the local density approximation framework, this occurs when $k_{B} T / \mu$ is equal, at the bottom of the trap, to the critical value for superfluidity of a homogeneous gas: $k_{B} T / \mu^{0}=0.32(3)$. Using equation 4.24 to obtain the atom number and hence the Fermi temperature corresponding to the onset of superfluidity, we get:

$$
\left(\frac{T}{T_{F t}}\right)_{c}=0.19(2) \text {. }
$$

\footnotetext{
$\S$ In order to make the integral over $\zeta$ up to $+\infty$, we complete our data with theoretical values given by the second-order virial expansion for $\zeta>5$.
} 


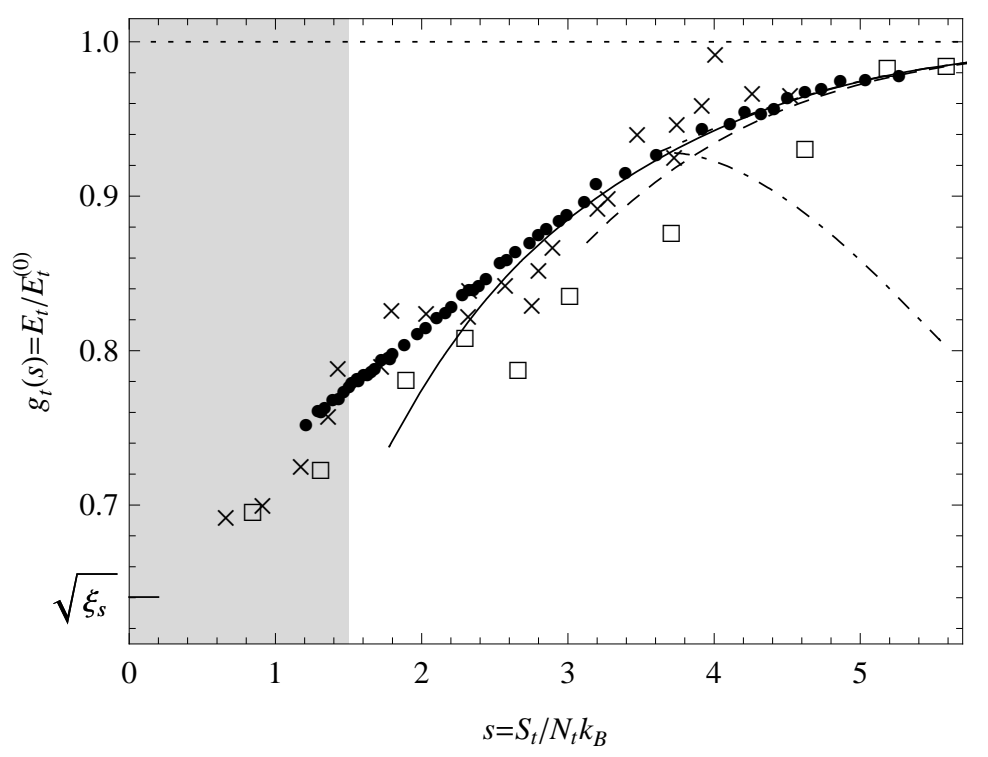

Figure 4.20: Equation of state $g(s)$ of a trapped unitary gas. The black dots are calculated from our experimental data. The crosses are data from Duke university [46], the open squares are data from the JILA group [62]. The dotted (dashed, dot-dashed, solid) line is the ideal gas (second-order virial, thirdorder virial, fourth-order virial) expansion. The third-order virial expansion has an unphysical cusp at $s \simeq 3.5$.

This value is in very good agreement with other experimental results listed in Table 4.8 .

\begin{tabular}{lcccc}
\hline \hline & Our value & \multicolumn{2}{c}{$\begin{array}{c}\text { Duke Univ. group } \\
\text { EOS trapped gas } 46\end{array}$} & \multicolumn{2}{c}{$\begin{array}{c}\text { Innsbruck group } \\
\text { Momentum of inertia }\end{array}$} & \multicolumn{2}{c}{$\begin{array}{c}\text { Tokyo group } \\
\text { Condensate fraction }\end{array}$} \\
$\left(T / T_{F t}\right)_{c}$ & $0.19(2)$ & $0.21(1)$ & $0.185(15)$ & $0.21(2)$ \\
\hline \hline
\end{tabular}

Table 4.2: Comparison between our measurement of the onset of superfluidity in a trapped gas to other previous measurements.

To conclude, we described the measurement of the equation of state of a spin-balanced Fermi gas with resonant interactions. We combined the use of ${ }^{7} \mathrm{Li}$ to measure the temperature of a strongly-interacting ${ }^{6} \mathrm{Li}$ mixture with the measurement of the local pressure inside a trapped gas, to obtain the equation of state of the homogeneous gas. Thanks to the low noise of our data, we made a strongly discriminating comparison with many-body theories, and extracted a series of characteristics of the unitary Fermi gas. The virial coefficients could be helpful for the resolution of the four-body problem. The Fermi liquid behavior of the normal phase remains to be understood, and related to single-particle excitation spectra. Finally this work could be extended to the BEC-BCS crossover. Among several motivations (see the conclusion), let us mention that on the BEC side of the resonance, the pseudogap should become apparent on the equation of state. 


\section{Chapter 5}

\section{Ground State of an Attractive Fermi Gas: Phase Diagram and Equation of State}

In this chapter we describe the measurement of the equation of state of a two-component attractive Fermi gas at low temperature [71. As described in the introduction, in the case of short-range interactions the equation of state of a two-component Fermi gas is universal, in the sense that interactions between atoms are completely characterized by the scattering length $a$ describing low-energy collisions between atoms with opposite spins. In this chapter we measure the pressure of a low-temperature Fermi gas for arbitrary values of interactions or spin imbalance. The physics associated with this system is very rich: it encompasses the BEC-BCS crossover of a spin-balanced superfluid, as well as the more recent topic of spin-imbalanced Fermi gases.

We first picture a qualitative phase diagram of this system, using simple mean-field or impurity models. We then describe the equation of state measurement scheme, give a physical interpretation our measurement, and compare it with previous works.

\subsection{Sketch of the Phase Diagram}

In this section we give a qualitative description of the phase diagram addressed in our study.

In this work we measure the grand-canonical equation of state $P\left(\mu_{1}, \mu_{2}, a\right)$ (we assume $T=0$ for the rest of this chapter, see Appendix B.2 for an estimate of finite-temperature effects). With the quantities $\mu_{1}, \mu_{2}$, and $a$, we can form two independent dimensionless numbers:

- By analogy with the interaction parameter $1 / k_{F} a$, where $k_{F}=\left(3 \pi^{2} n\right)^{1 / 3}$, defined for a balanced Fermi gas of given density $n$, we define a grand-canonical interaction parameter relative to species 1 :

$$
\delta_{1}=\frac{\hbar}{\sqrt{2 m \mu_{1}} a} .
$$

$\delta_{1}$ is equal to $1 / k_{F} a$ for a balanced and weakly-interacting Fermi gas.

- The other dimensionless parameter:

$$
\eta=\frac{\mu_{2}}{\mu_{1}}
$$

describes the chemical potential imbalance between the two spin states. By convention we assume $\mu_{2} \leq \mu_{1}$, i.e. the spin state labeled 1 is the majority spin state.

The ground-state pressure can then be written as:

$$
P\left(\mu_{1}, \mu_{2}, a\right)=P_{0}\left(\mu_{1}\right) h\left(\delta_{1}, \eta\right),
$$




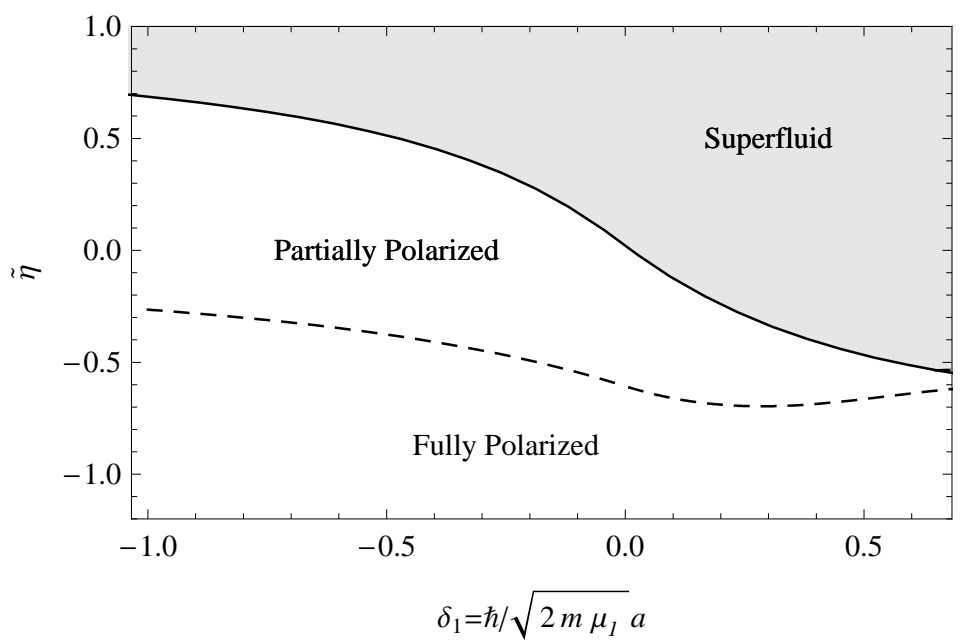

Figure 5.1: Phase diagram of a two-component Fermi gas in the plane $\left(\delta_{1}, \eta\right)$. The solid line is the superfluid/normal first order phase transition at $\eta=\eta_{c}\left(\delta_{1}\right)$. The dashed line $\eta=A\left(\delta_{1}\right)$ is the threshold beyond which minority atoms are absent. For clarity we have replaced $\eta$ by $\widetilde{\eta}=\eta+2 \delta_{1}^{2}$ on the BEC side of the resonance.

where $P_{0}(\mu)$ is the Fermi pressure of a single-component (ideal) gas. The function $h\left(\delta_{1}, \eta\right)$ characterizes the equation of state and can be used to calculate any other thermodynamic quantity. As an example, the minority density reads:

$$
n_{2}=\left.\frac{\partial P}{\partial \mu_{2}}\right|_{\mu_{1}, a}=\frac{P_{0}\left(\mu_{1}\right)}{\mu_{1}} \frac{\partial h}{\partial \eta} .
$$

The rest of this section describes the phase diagram in the $\left(\delta_{1}, \eta\right)$ plane drawn in Fig.5.1.

\subsubsection{Superfluid to Normal Quantum Phase Transition}

A spin-symmetric Fermi gas is superfluid at low temperature for all interaction strengths. By imposing a chemical potential imbalance $(\eta \neq 1)$, a competition between pairing and spin polarization occurs. This problem was first studied in the context of solid state superconductors by Clogston [52] and Chandrasekhar 53. Using a BCS approach, they found the superfluid state resists to a chemical potential imbalance* up to a critical value:

$$
\left(\mu_{1}-\mu_{2}\right)_{c}=\sqrt{2} \Delta_{0}
$$

where $\Delta_{0}$ is the BCS pairing gap. In the variables $\left(\delta_{1}, \eta\right)$, this criterion reads:

$$
\eta_{c}\left(\delta_{1}\right)=1-\sqrt{2} \frac{\Delta_{0}}{\mu_{1}}
$$

For $\eta_{c}<\eta<1$ the gas remains superfluid and is fully paired: $n_{1}=n_{2}$. When one varies $\eta$ across $\eta_{c}$, a first-order phase transition occurs towards a normal phase with $n_{2}<n_{1}$. A derivation of this result using a mean-field BCS ansatz is made in Appendix A.4

The mean-field approach is expected to be correct in the BCS limit $\left(\delta_{1} \rightarrow-\infty\right)$. In our work we rather address the strongly-interacting regime $-0.8<\delta_{1}<0.65$. Therefore the relation $(5.2)$ between the critical chemical potential ratio and the gap is not expected to be valid in our case. Nevertheless, the observations of the MIT group [79 and the ones described in the next sections [71 show that the phase diagram qualitatively remains the same: the superfluid remains unpolarized until a critical value $\eta_{c}$ at which a phase transition occurs towards a partially-polarized normal phase. The theoretical prediction

*induced by a magnetic field which lifts the degeneracy between the two electronic spin states 
of $\eta_{c}$ is challenging. Up to now the only quantitative prediction is provided by Fixed-Node Monte Carlo simulations in 178 In Fig 5.1 we drew a guess for $\eta_{c}\left(\delta_{1}\right)$.

\subsubsection{The Impurity Problem}

When the chemical potential imbalance is very large, the gas is fully polarized: $n_{2}=0$ and $P=P_{0}\left(\mu_{1}\right)$, i.e. $h\left(\delta_{1}, \eta\right)=1$. The transition towards the partially polarized normal phase corresponds to the value of $\eta$, denoted $A\left(\delta_{1}\right)$, at which minority atoms appear. $A\left(\delta_{1}\right)$ corresponds to the chemical potential of a single minority atom immersed in a Fermi sea of majority atoms. This 'impurity' problem is much simpler than the general problem with macroscopic atom numbers in both spin states. Up to now all theories give the same value for $A\left(\delta_{1}\right)$ within less than $1 \%$ [83, 85, 178, 84, 86], and are in agreement with the MIT measurement [82] (see Fig 5.1).

In the regime of weak interactions $\left(\delta_{1} \rightarrow-\infty\right)$, the ground state is essentially the ground state $\left|\psi_{0}\right\rangle$ with no interactions and the minority chemical potential is given by the mean-field energy shift:

$$
\mu_{2}=\frac{4 \pi \hbar^{2} a}{m} n_{1}, \quad \text { i.e. } \quad A\left(\delta_{1}\right)=\frac{4}{3 \pi \delta_{1}} .
$$

When interactions increase, the ground state substantially differs from the non-interacting ground state. The minority atom collides with majority particles and creates particle-hole pairs in the Fermi sea. It was shown in 84 that a good approximation of the ground state energy is obtained by solely taking into account the creation of a single particle-hole excitation. The ground state is then written as a linear combination of the non-interacting ground state $\left|\psi_{0}\right\rangle$ and states $\left|\psi_{\mathbf{k q}}\right\rangle$ with a single particle-hole excitation 80 (see Fig 5.2 a):

$$
|\psi\rangle=\phi_{0}\left|\psi_{0}\right\rangle+\sum_{\substack{q \leq k_{F} \\ k \geq k_{F}}} \phi_{\mathbf{k q}}\left|\psi_{\mathbf{k q}}\right\rangle .
$$

Minimizing the energy in this subspace 80 , 83 gives a good (and simple to calculate) approximation of the actual energy [84], even when interaction-induced particle-hole excitations are likely, i.e. when $|\psi\rangle$ should appreciably differ from $\left|\psi_{0}\right\rangle$, such as in the unitary limit.

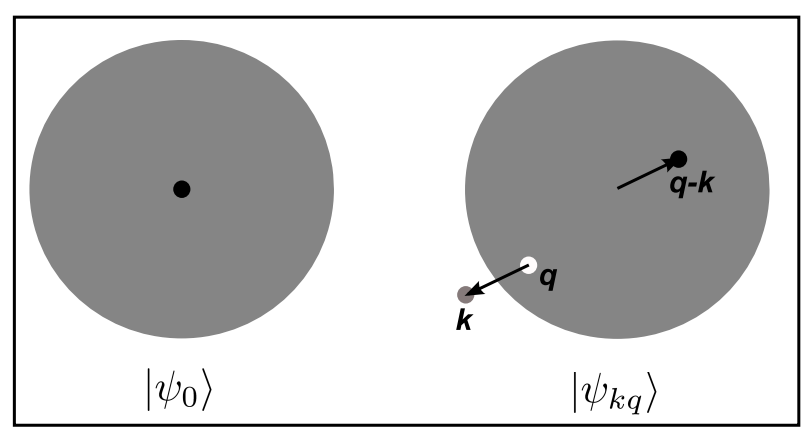

Figure 5.2: Representation of the ground state on the BCS side of the resonance. In the non-interacting ground state $\left|\psi_{0}\right\rangle$, the impurity momentum is $\mathbf{0}$ and the majority Fermi sea is fully occupied for $k<k_{F}$. In the excited state $\left|\psi_{\mathbf{k q}}\right\rangle$, the impurity has collided with a majority atom, bringing it from the initial momentum $\mathbf{q}, q<k_{F}$, towards $\mathbf{k}, k>k_{F}$.

${ }^{\dagger}$ The work in 178 actually does not directly provides $\eta_{c}$, the superfluid/normal phase transition being expressed in the canonical ensemble. 


\subsubsection{Beyond the Impurity Problem}

Understanding from theory the partially polarized normal phase $A<\eta<\eta_{c}$ a priori requires to solve the much more difficult problem of a macroscopic number of minority atoms among majority particles. However, a simple and powerful description can be proposed in terms of Landau Fermi liquid theory 81. In this approach, minority atoms are renormalized by interactions with majority atoms into fermionic quasi-particles, named Fermi polarons. The gas is then described as an ideal mixture of bare majority atoms and polarons. In particular the Fermi pressure associated with polarons is calculated from the single-polaron spectrum $E(p)$ as the sum of all energies below the Fermi level. Therefore we not only need to know the ground state but also the excited energies of the impurity problem. These eigenenergies, parametrized by the impurity momentum p, exhibit a quadratic dependence with momentum, encapsulated by a mass renormalization $81,83,85,178,86]$ :

$$
E(p)=A\left(\delta_{1}\right) \mu_{1}+\frac{p^{2}}{2 m^{*}\left(\delta_{1}\right)}
$$

It is then straightforward to calculate the gas pressure, as the sum of the majority component Fermi pressure and of the polaron Fermi pressure:

$$
P\left(\mu_{1}, \mu_{2}, a\right)=P_{0}\left(\mu_{1}\right)+\left(\frac{m^{*}\left(\delta_{1}\right)}{m}\right)^{3 / 2} P_{0}\left(\mu_{2}-A\left(\delta_{1}\right) \mu_{1}\right) .
$$

To conclude this section, we remind the state of the art concerning the phase diagram of spinimbalanced Fermi gases. The phase diagram was explored by the MIT group in 79,82 . In [82] the polaron chemical potential shift $A\left(\delta_{1}\right)$ was measured in the BEC-BCS crossover, in agreement with theory $83,85,178,86$. We are thus entitled to use $A\left(\delta_{1}\right)$ as a reference for extracting the equation of state from in situ images (see section 5.2. In [79, the maximum density ratio $n_{2} / n_{1}$ at the normal to superfluid phase transition was measured. However, the critical chemical potential ratio $\eta_{c}\left(\delta_{1}\right)$ remains unknown. Finally, the equations of state in the partially polarized and superfluid phase have never been measured for a homogeneous gas.

The phase diagram drawn at this stage is sufficient for the understanding of the work described in the next sections. We mention that in the BEC regime, further away from the parameter space addressed in this study, new phases are expected. We give an introduction to this still largely unexplored field at the end of this chapter (see section 5.7).

\subsection{Equation of State Measurement Scheme}

In this section we describe the procedure used to extract the equation of state of a two-component Fermi gas from in situ absorption images.

\subsubsection{Experimental Sequence}

Here we highlight the parts of the experimental sequence that are specific to this study. We prepare a spin-imbalanced mixture of ${ }^{6} \mathrm{Li}$ in the two lowest internal states $|1\rangle$ and $|2\rangle$, held in an optical dipole trap, a magnetic curvature being used for the axial confinement (along $z$ ). The gas is evaporated by lowering the trap depth down to $U_{0} \simeq 4 \mu \mathrm{K}$. The final trap frequencies are $\omega_{r} / 2 \pi \simeq 800 \mathrm{~Hz}$ and $\omega_{z} / 2 \pi \simeq 20 \mathrm{~Hz}$. During evaporation the bias magnetic field is ramped towards a value $755 \mathrm{G}<B_{0}<981 \mathrm{G}$ in order to address the entire BEC-BCS crossover. The final atom number is on the order of $5 \times 10^{4}$ atoms per spin state. 


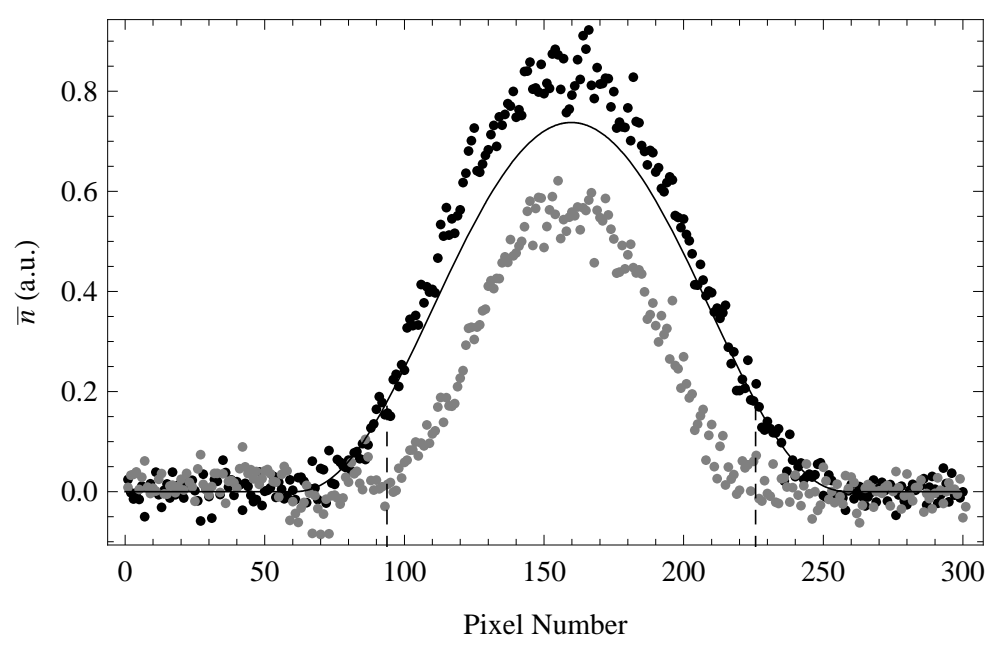

Figure 5.3: In situ absorption images of a spin-imbalanced Fermi gas, prepared at $B_{0}=834 \mathrm{G}$. The total atom numbers are $N_{1}=1.1 \times 10^{5}$ for the majority spin state (black dots) and $N_{2}=6 \times 10^{4}$ for the minority spin state (gray dots). The solid line is a fit of the majority density profile in the fully polarized region (outer wing of the atomic cloud) with a Thomas-Fermi profile. The increase of the majority profile $\bar{n}_{1}(z)$ above the Thomas-Fermi fit is a manifestation of the strong attraction with the minority component, when the two clouds overlap.

\subsubsection{In Situ Image Analysis}

In situ absorption images of both spin components are taken after evaporation (see section 2.5 for details on double in situ imaging). After integration of the column density $\widetilde{n}_{i}(x, z)$ over the transverse direction $x$, we obtain the doubly-integrated density profiles $\bar{n}_{i}(z)$ (see Fig 5.3 ).

Determination of $h$ and $\delta_{1 z}$

We observe that the minority atom density vanishes before the majority component. In the fully polarized region, majority atoms form a single-component (ideal) Fermi gas whose density profile is given by:

$$
\frac{m \omega_{r}^{2}}{2 \pi} \bar{n}_{1}(z)=P_{0}\left(\mu_{1 z}\right)=\frac{1}{15 \pi^{2}}\left(\frac{2 m}{\hbar^{2}}\right)^{3 / 2}\left(\mu_{1}^{0}-\frac{1}{2} m \omega_{z}^{2} z^{2}\right)^{5 / 2}
$$

Fitting in the fully polarized region the doubly-integrated density profile $\bar{n}_{1}(z)$ with a function $\bar{n}_{1}^{0}(z)=$ $\alpha\left(1-z^{2} / R_{1}^{2}\right)^{5 / 2}$, we obtain the majority Fermi radius $R_{1}=\sqrt{2 \mu_{1}^{0} / m \omega_{z}^{2}}$. The axial frequency being calibrated with a good precision (see section 2.4.4), we deduce from $R_{1}$ the majority chemical potential $\mu_{1}^{0}$ with a good accuracy. The interaction parameter at position $z$ is then given by $\delta_{1 z}=\hbar / \sqrt{2 m \mu_{1 z}} a$. Finally, we obtain the value of $h\left(\delta_{1}, \eta\right)$ through:

$$
h\left(\delta_{1 z}, \eta_{z}\right)=\frac{P\left(\mu_{1 z}, \mu_{2 z}, a\right)}{P_{0}\left(\mu_{1 z}\right)}=\frac{\bar{n}_{1}(z)+\bar{n}_{2}(z)}{\bar{n}_{1}^{0}(z)} .
$$

In the calculation of $h$, the normalization by the fit $\bar{n}_{1}^{0}(z)$ avoids using a calibration of the pressure and thus cancels the associated systematic error. In other words, the fit result $\alpha$ extracted from the cloud's wings serves as a pressure calibration for the rest of the cloud, as first shown in [49].

\section{Determination of $\eta_{z}$}

Finally, one has to determine the chemical potential $\eta_{z}=\mu_{2 z} / \mu_{1 z}$ along the $z$ axis. This requires to extract $\mu_{2}^{0}$ from the data. As the minority atoms strongly interact with the majority component, 
the relation between $\mu_{2}^{0}$ and the minority density profile calls upon an additional information on the equation of state. As described in section 5.1, the chemical potential ratio for a large density imbalance, $\eta=A\left(\delta_{1}\right)$, is known from the resolution of the impurity problem. Fitting the minority radius $R_{2}$ at which the minority density vanishes (see Appendix B.1.1 for more details) then provides $\mu_{2}^{0}$ according to:

$$
A\left(\delta_{1 R_{2}}\right)=\frac{\mu_{2}^{0}-\frac{1}{2} m \omega_{z}^{2} R_{2}^{2}}{\mu_{1}^{0}-\frac{1}{2} m \omega_{z}^{2} R_{2}^{2}} .
$$

The chemical potential ratio along the $z$ axis is then given by $\eta_{z}=\left(\mu_{2}^{0}-\frac{1}{2} m \omega_{z}^{2} z^{2}\right) /\left(\mu_{1}^{0}-\frac{1}{2} m \omega_{z}^{2} z^{2}\right)$.

\subsubsection{Equation of State Deduced from our Data}

By gathering the data from all images taken at a given magnetic field $800 \mathrm{G}<B<981 \mathrm{G}$, we obtain after averaging a series of low-noise equations of state shown in Fig.5.4. They provide a paving of the plane $\left(\delta_{1}, \eta\right)$ in a domain $-0.8<\delta_{1}<0.65$ and $-2<\eta<0.7$. The next sections are devoted to the physical interpretation of these data.

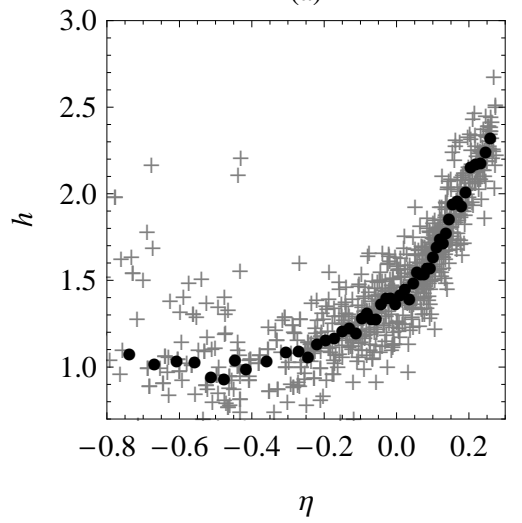

(b)

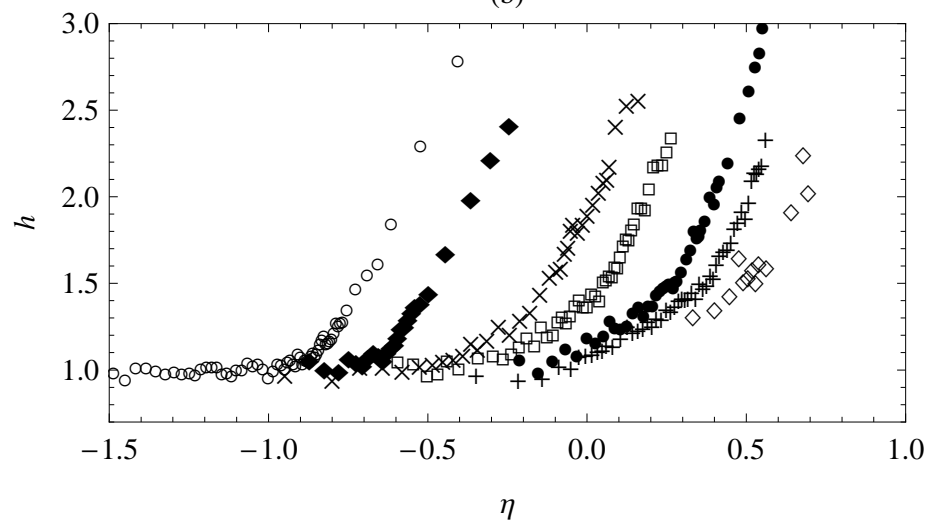

(c)

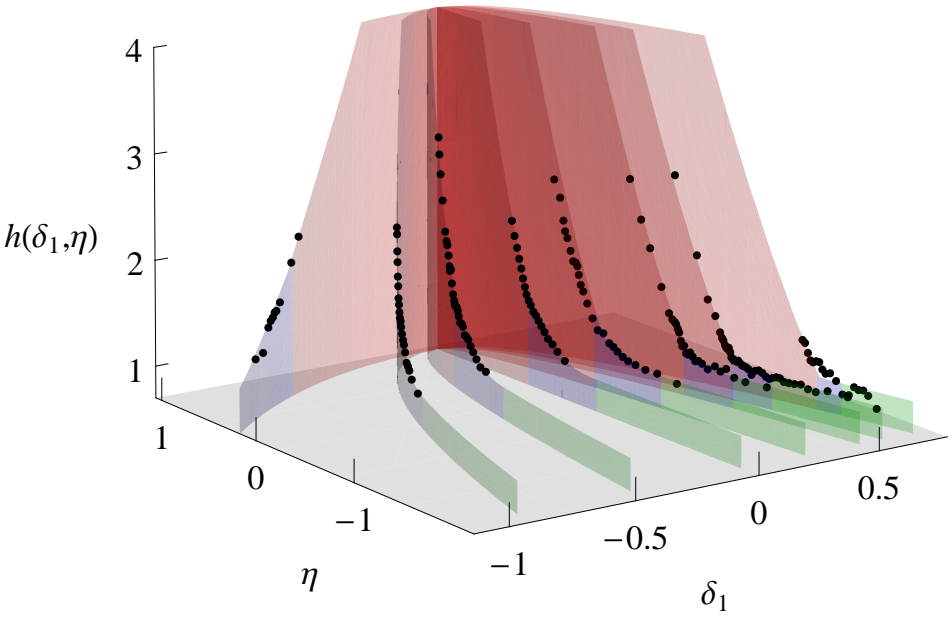

Figure 5.4: (a) Equation of state of a two-component Fermi gas at zero temperature and in the unitary limit, deduced from the analysis of 20 images. Crosses: raw data, black dots: average of 20 consecutive points. (b) Averaged equations of state corresponding to a given magnetic field. From left to right: 800 G, 811 G, 822 G, 834 G, 855 G, 871 G, 981 G. (c) Same equations of state in the space $\left(\delta_{1}, \eta, h\right)$. The regions in green (blue, red) are fully polarized (partially polarized, superfluid). 


\subsubsection{Systematic Error of our Data}

The chemical potential ratio $\eta_{z}=\mu_{2 z} / \mu_{1 z}$ and the interaction parameter $\delta_{1 z}=\hbar / \sqrt{2 m \mu_{1}} a$ vary simultaneously along $z$. By eliminating $z$ from these relations, we find that $\delta_{1 z}$ and $\eta_{z}$ are related by:

$$
\delta_{1 z}=\delta_{1}^{0} \sqrt{\frac{1-\eta_{z}}{1-\eta^{0}}} .
$$

Therefore a single image provides the equation of state $h\left(\delta_{1}, \eta\right)$ along a line in the $\left(\delta_{1}, \eta\right)$ plane. In the image averaging process, we superpose the equations of state obtained from clouds prepared at a given bias magnetic field $B_{0}$. These images thus correspond to the same scattering length value $a\left(B_{0}\right)$ but the parameter $\delta_{1}^{0} / \sqrt{1-\eta^{0}}$ may differ from one image to another, and the equations of state cannot strictly speaking be superimposed. In Appendix B.1.2 we show that the systematic error introduced by this issue is less than $4 \%$. We also mention that the superposition of images taken in the unitary limit is not affected by this problem since $\delta_{1 z}=0$.

In Appendix B.2 we estimate the temperature of our data, and deduce that the associated systematic error is less that $3 \%$.

As a conclusion, the total systematic error is $5 \%$.

\subsection{Superfluid to Normal Phase Transition}

In Fig 5.4 be plot the experimental equation of state $h\left(\delta_{1}, \eta\right)$ along several lines corresponding to different magnetic fields, and parametrized by equation (5.4). Except for the magnetic field $B_{0}=981 \mathrm{G}$ where the noise of our data is large, we observe on each experimental equation of state an abrupt change of slope. This jump in the derivative of $h\left(\delta_{1}(\eta), \eta\right)$ at $\eta=\eta_{c}\left(\delta_{1}\right)$ indicates a first-order phase transition. In the next paragraphs we describe how to extract the position of this phase transition from the data, and identify the nature of the phases corresponding to both sides of the phase transition. We also give a direct proof of the first-order nature of the transition through the observation of a jump of the minority concentration occurring at $\eta=\eta_{c}$.

\subsubsection{Critical Chemical Potential Ratio}

In order to extract the value of $\eta_{c}$, we fit the data inside an interval $\bar{\eta}-0.2<\eta<\bar{\eta}+0.2$ using a piecewise linear function with a change of slope occurring at $\eta=\eta_{0}$. The fit result $\eta_{0}$ is plotted as a function of $\bar{\eta}$ in Fig.5.5. The change of slope position $\eta_{0}$ is found to be insensitive to the value of $\bar{\eta}$ in a large range of parameters. We then identify this stable fit result as the actual change of slope position in the equation of state, at $\eta=\eta_{c}$.

Let us now confirm that the phase $\eta>\eta_{c}$ corresponds to a fully paired superfluid. In Fig 5.6 we plot the integrated minority density profile $\bar{n}_{2}(z)$, together with the integrated density difference $\bar{n}_{d}(z)=\bar{n}_{1}(z)-\bar{n}_{2}(z)$, for a gas prepared in the unitary limit. Fitting the pressure in the fully polarized region and the low-density minority profile with Thomas-Fermi profiles, we obtain the Thomas-Fermi radii $R_{1}$ and $R_{2}$ for each spin state. We infer the chemical potential ratio at the bottom of the trap $\eta^{0}=A\left(\delta_{1}=0\right)\left(1-R_{2}^{2} / R_{1}^{2}\right)+R_{2}^{2} / R_{1}^{2}$, as well as the radius at which the phase transition occurs:

$$
R_{s}=R_{1} \sqrt{\frac{\eta^{0}-\eta_{c}}{1-\eta_{c}}}
$$

We observe that $\bar{n}_{d}(z)$ increases from $|z|=R_{1}$ to $|z|=R_{s}$ and remains constant for $|z|<R_{s}$. A simple 

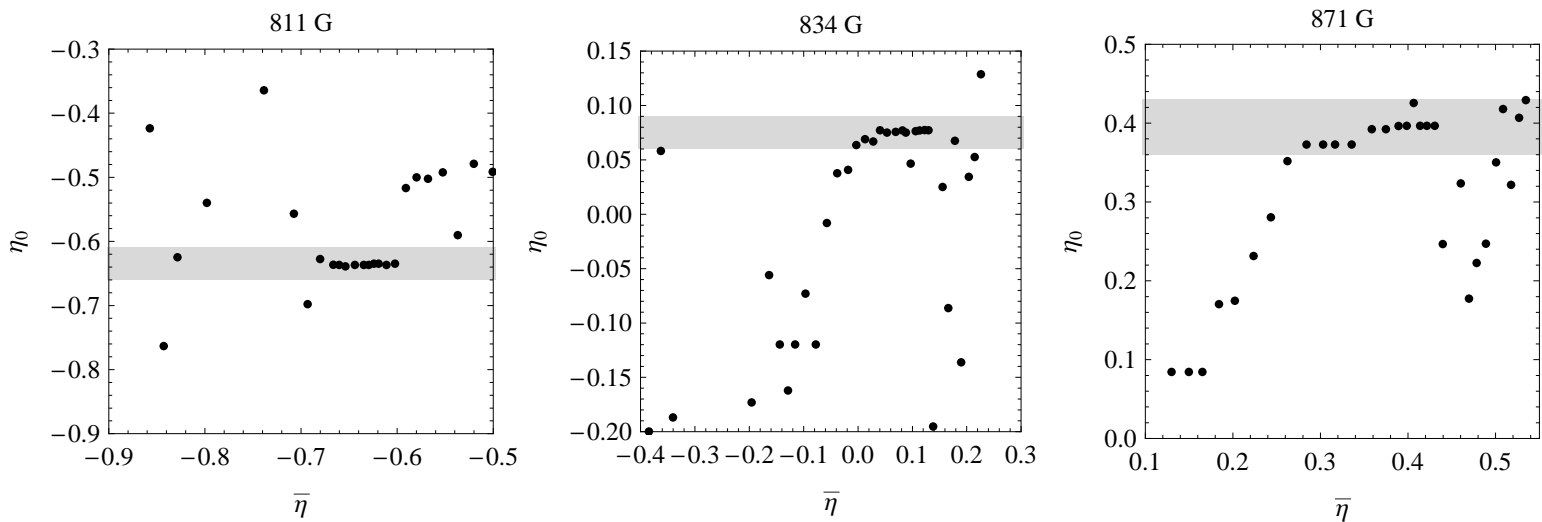

Figure 5.5: Determination of the critical chemical potential imbalance from the equations of states at $811 \mathrm{G}, 834 \mathrm{G}$ and $871 \mathrm{G}$. We fit the data $\bar{\eta}-0.2<\eta<\bar{\eta}+0.2$ using a piecewise linear function with a change of slope at $\eta_{0}$, and plot the fit result $\eta_{0}$ as a function of $\bar{\eta}$. For a large number of $\bar{\eta}$ values the fit result is identical, indicating the actual $\eta_{c}$ value.

physical interpretation is provided by an explicit calculation of the slope of $\bar{n}_{d}(z)$ [127]:

$$
\frac{\mathrm{d} \bar{n}_{d}(z)}{\mathrm{d} z}=-2 \pi \frac{\omega_{z}^{2}}{\omega_{r}^{2}} z\left(n_{1}(z)-n_{2}(z)\right) .
$$

The plateau on the density difference observed for $|z|<R_{s}$ thus reveals that the phase $\eta>\eta_{c}$ is fully paired: $n_{1}=n_{2}$ (despite $\mu_{1} \neq \mu_{2}$ ). This is a characteristic feature of superfluid pairing between atoms with opposite spins. In the MIT experiment the superfluidity of the fully paired phase was unambiguously identified through the observation of vortices [54]. As a conclusion, the observed phase transition at $\eta=\eta_{c}$ indeed corresponds to the superfluid/normal phase transition previously introduced in section 5.1.1.

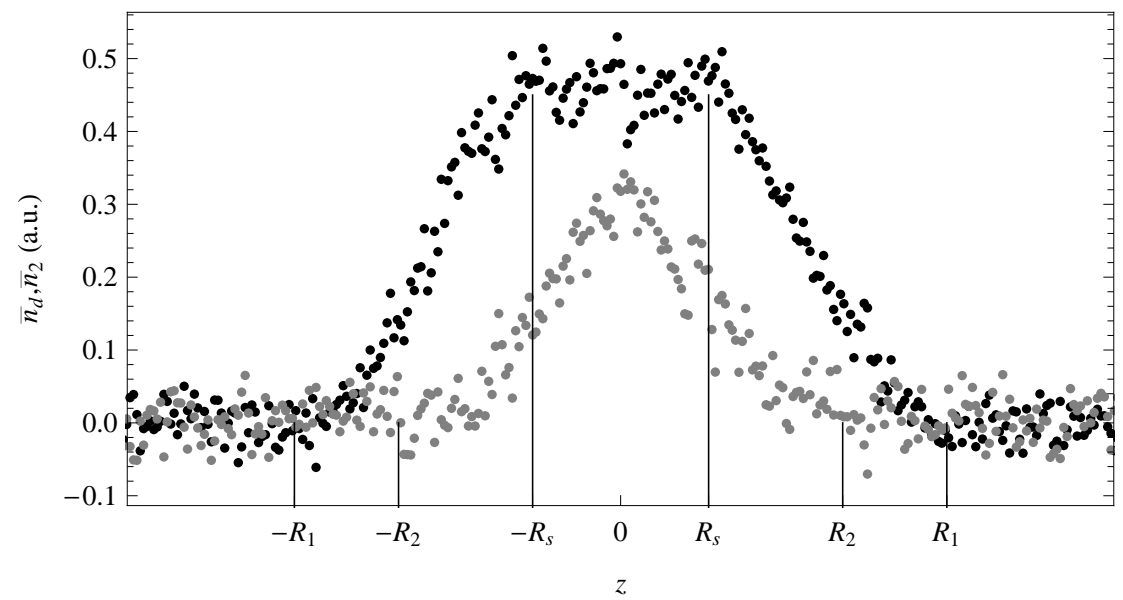

Figure 5.6: Integrated minority density $\bar{n}_{2}(z)$ (gray dots) and integrated difference density $\bar{n}_{d}(z)$ (black dots), for a cloud prepared in the unitary limit. The plateau on $\bar{n}_{d}(z)$ indicating full pairing coincides with the region $|z|<R_{s}$ for which $\eta>\eta_{c}$.

\subsubsection{Critical Impurity Concentration}

The first-order nature of the phase transition can directly be observed as a jump in the minority density occurring at the phase transition. The absorption images easily give access to the minority concentration 
$x=n_{2} / n_{1}$ in the cloud along the $z$ axis. Indeed, from $\mathrm{d} \bar{n}_{i}(z) / \mathrm{d} z=-2 \pi \omega_{z}^{2} / \omega_{r}^{2} z n_{i}$, we get the simple relation:

$$
x=\frac{\mathrm{d} \bar{n}_{2}}{\mathrm{~d} \bar{n}_{1}} .
$$

We thus obtain the concentration by computing the derivative of the data expressed as $\bar{n}_{2}$ as a function of $\bar{n}_{1}$. In Fig 5.7 we plot the concentration $x$ as a function of $\eta$ at different magnetic fields. For all magnetic fields the same behavior is observed:

- In the partially polarized phase the concentration smoothly increases with $\eta$, up to a maximum value $x_{c}$ at $\eta=\eta_{c}\left(\delta_{1}\right)$. The quantitative understanding of $x\left(\delta_{1}, \eta\right)$ in the partially polarized phase will be given in section 5.4 .4 .

- In the superfluid phase the concentration remains equal to 1 , indicating a full pairing.

- Around $\eta=\eta_{c}$ the concentration abruptly changes from $x=x_{c}$ to $x \simeq 1$. This behavior reveals a concentration jump occurring at the phase transition, thereby confirming the order of the transition.

Even on the BEC side of the resonance $\left(0<\delta_{1}<0.65\right)$, we found no evidence for a finite polarization in the superfluid phase. This is in agreement with the observations of the MIT group [79] or several theoretical predictions using Monte Carlo calculations [67] or excitation spectrum calculations [179, 180]: the superfluid can be polarized for $\delta_{1} \gtrsim 1$, which is beyond the parameter range addressed in this work.
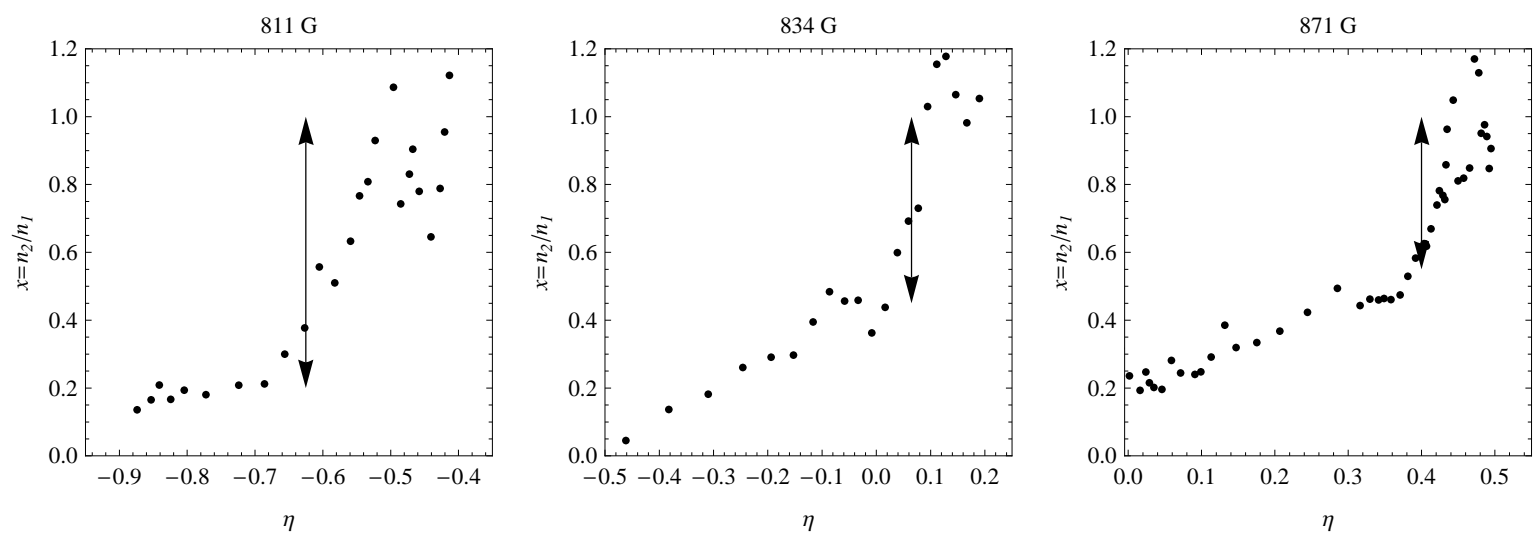

Figure 5.7: Concentration $x=n_{2} / n_{1}$ as a function of $\eta$ for bias magnetic fields $B_{0}=811,834,871 \mathrm{G}$. The arrows indicate the concentration jump from $x=x_{c}$ to $x=1$ occurring at the phase transition.

\subsubsection{Phase Diagram}

Gathering the values of $\eta_{c}$ and $x_{c}$ for all magnetic fields, we obtain the phase diagram plotted in Fig 5.8 , either in the plane $\left(\delta_{1}, \eta\right)$ (Fig $\left.5.8 \mathrm{a}\right)$ or in the plane $\left(\delta_{1}, x\right)$ (Fig $\left.5.8 \mathrm{p}\right)$.

Apart from BCS mean-field theory which is quantitatively incorrect, the only theoretical prediction for the equation of state is provided by Fixed-Node Monte Carlo calculations [67]. As the numerical data are expressed in canonical variables, the comparison with our results requires to use fits of the Monte Carlo data $\mathrm{f}^{7}$. The pressure and chemical potentials are calculated from the fit functions, and the phase transition position $\eta_{c}\left(\delta_{1}\right)$ is computed by imposing the condition of coexistence of the superfluid and normal phases at equilibrium, namely the equality of chemical potentials and pressure. As shown in

\footnotetext{
$\ddagger$ We use fits proposed by the authors of the Monte Carlo calculations in 181 . They are limited to the BEC side of the
} resonance. 
Fig 5.8 a, the transition line $\eta_{c}\left(\delta_{1}\right)$ computed from the Monte-Carlo data is in excellent agreement with our measurements.

The data $x_{c}\left(\delta_{1}\right)$ cannot be directly compared with the measurements of the MIT groups which rather provide $x_{c}\left(1 / k_{F 1} a\right) 79$.

(a)

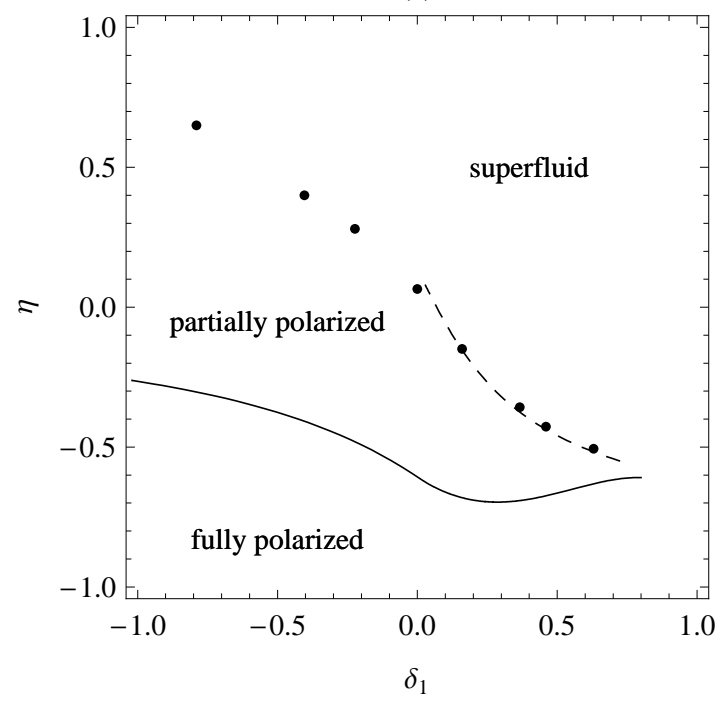

(b)

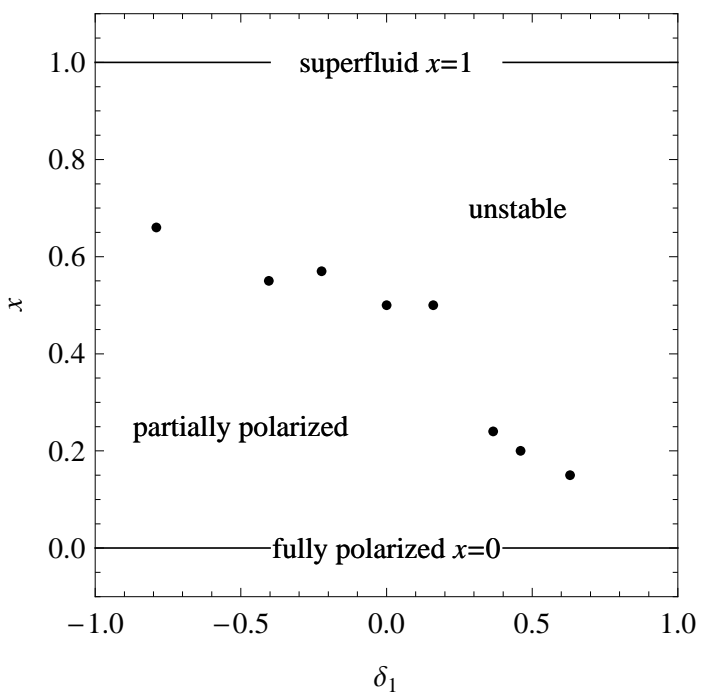

Figure 5.8: (a) Phase diagram established for $\delta_{1}<0.65$, plotted in the plane $\left(\delta_{1}, \eta\right.$ ) (we have added $2 \delta_{1}^{2}$ to $\eta$ for $\left.\delta_{1}>0\right)$. The solid line marks the appearance of minority atoms at $\eta=A\left(\delta_{1}\right)$. The black dots are the experimental values of $\eta_{c}\left(\delta_{1}\right)$ at which the superfluid/normal phase transition occurs. The dashed line is the transition line $\eta_{c}\left(\delta_{1}\right)$ calculated from fits of $T=0$ Monte Carlo data 67, 181. (b) Phase diagram plotted in the plane $\left(\delta_{1}, x=n_{2} / n_{1}\right)$. The fully polarized (superfluid) phase corresponds to $x=0(x=1)$, respectively. The black dots are the experimental values for the critical polarization $x_{c}$ at the superfluid/normal phase transition. The region $x_{c}<x<1$ is thermodynamically unstable.

\subsubsection{Comparison with the Single-Particle Excitation Gap}

In the BCS limit, the superfluid/normal phase transition position is directly related to the single-particle excitation gap $\Delta_{0}$ in the superfluid (see section 5.1.1):

$$
\eta_{c}=1-\sqrt{2} \frac{\Delta_{0}}{\mu_{1}}, \quad \text { for } \quad \delta_{1} \rightarrow-\infty .
$$

It is simple to show that the single-particle excitation gap also provides a lower bound on $\eta_{c}$ which is valid for all interaction strengths: when the majority chemical potential $\mu_{1}$ is larger than the superfluid chemical potential $\mu=\left(\mu_{1}+\mu_{2}\right) / 2$ plus the excitation gap $\Delta_{0}$, extra majority atoms are spontaneously populated and break the superfluid $\$$. This provides a lower bound on $\eta_{c}$ :

$$
\eta_{c}>1-2 \frac{\Delta_{0}}{\mu_{1}} .
$$

In Fig 5.9 we compare the values of $\eta_{c}$ extracted from our data to lower bounds $1-2 \Delta_{0} / \mu_{1}$, using values of the gap measured by the MIT group in [166, or calculated in $179,60,182,158$. Quite surprisingly, the

\footnotetext{
$\S$ In the BEC limit, it is possible to add extra majority atoms to a superfluid without breaking it. However this is expected to occur for $\delta_{1}>1$, i.e. outside the parameter range addressed in this work. We anyway checked that the superfluid is indeed not polarized in section 5.3 .1
} 


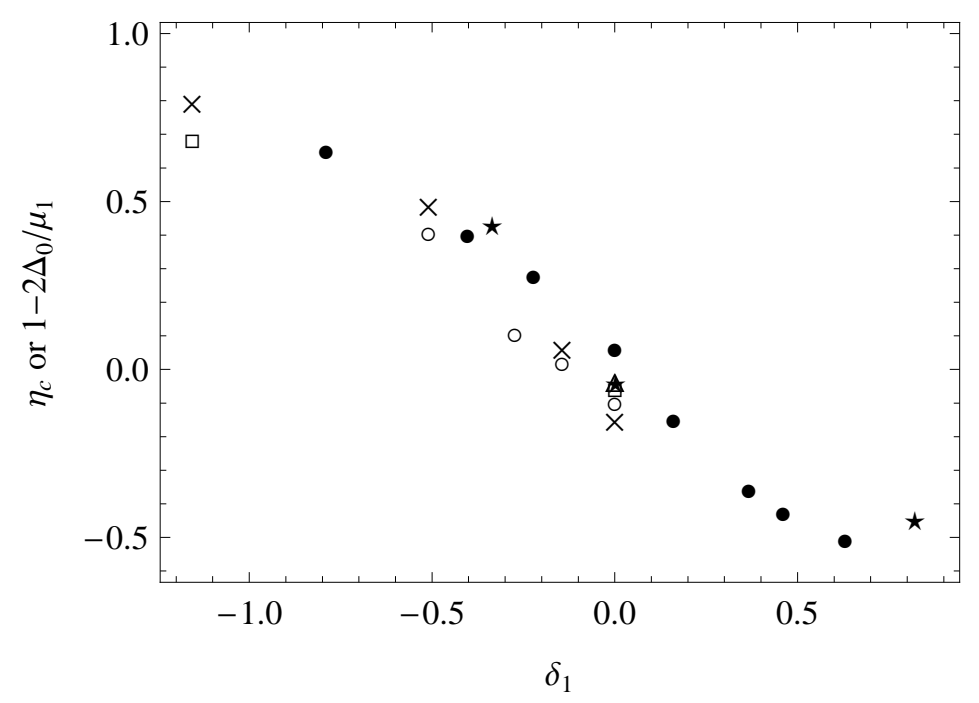

Figure 5.9: Comparison between the experimental $\eta_{c}$ values determined from our work (black dots) to the lower bounds $1-2 \Delta_{0} / \mu_{1}$ provided by the single-particle excitation gap. The stars are given by the experimental $\Delta_{0}$ values from the MIT group [166, the open squares from diagrammatic calculations [179, the open circles from the most recent Fixed Node Monte Carlo calculations [60], the crosses from a Quantum Monte Carlo calculation extrapolated at $T=0$ [182, and the open triangle from a zerotemperature Quantum Monte Carlo calculation 158.

values of $\eta_{c}$ are closer to the bounds $1-2 \Delta_{0} / \mu_{1}$ than to the Clogston limit $1-\sqrt{2} \Delta_{0} / \mu_{1}$, a phenomenon actually anticipated in 183 . However, a more quantitative estimate of the relationship between $\Delta_{0}$ and $\eta_{c}$ is difficult to make, due to the uncertainty on the gap illustrated by the scatter of theoretical predictions.

In the unitary limit, the single-particle gap $\Delta_{0}=0.44 E_{F}$ is known more precisely, from experiment 166 and unbiased Quantum Monte Carlo calculations 158. Using $\eta_{c}=0.065(2)$ and the relation $\mu=\xi_{s} E_{F}, \xi_{s}=0.41(1)$, we obtain:

$$
\eta_{c}=1-1.8(1) \frac{\Delta_{0}}{\mu_{1}}
$$

a value closer to the bound $1-2 \Delta_{0} / \mu_{1}$ than to the Clogston limit $1-\sqrt{2} \Delta_{0} / \mu_{1}$. This confirms more quantitatively the picture described above.

\subsection{Fermi Liquid Behavior in the Partially Polarized Phase}

In this section we focus on the partially polarized normal phase $A\left(\delta_{1}\right)<\eta<\eta_{c}\left(\delta_{1}\right)$.

\subsubsection{Observation of a Fermi Liquid Behavior}

Inspired by the Trento group [81], we proposed in section 5.1.2 a simple description of the partially polarized phase in terms of Landau's Fermi liquid theory. The impurity problem suggests that minority atoms are dressed by surrounding majority atoms into quasi-particles, the polarons. In the limit of low polaron density, we expect polarons to form a weakly-interacting Fermi sea. The gas pressure is then given by the sum of the Fermi pressures of bare majority atoms and of polarons:

$$
P\left(\mu_{1}, \mu_{2}, a\right)=\frac{1}{15 \pi^{2}}\left(\frac{2 m}{\hbar^{2}}\right)^{3 / 2} \mu_{1}^{5 / 2}+\frac{1}{15 \pi^{2}}\left(\frac{2 m^{*}\left(\delta_{1}\right)}{\hbar^{2}}\right)^{3 / 2}\left(\mu_{2}-A\left(\delta_{1}\right) \mu_{1}\right)^{5 / 2} .
$$


We remind that $A\left(\delta_{1}\right) \mu_{1}$ is the energy shift of a single polaron and $m^{*}\left(\delta_{1}\right)$ is the polaron effective mass. By dividing equation (5.5) by $P_{0}\left(\mu_{1}\right)$, we obtain the thermodynamic function $h\left(\delta_{1}, \eta\right)$ of this 'ideal Fermi liquid':

$$
h\left(\delta_{1}, \eta\right)=1+\left(\frac{m^{*}\left(\delta_{1}\right)}{m}\right)^{3 / 2}\left(\eta-A\left(\delta_{1}\right)\right)^{5 / 2} .
$$

For large minority concentrations, deviations to (5.6) may appear, either due to interactions between quasi-particles, or merely as a breakdown of Fermi liquid theory. As an interesting example, it was proposed in [184] that effective $p$-wave interactions between polarons could be induced by the interactions with majority atoms, and might lead to exotic $p$-wave superfluidity at very low temperature.

In order to reveal a Fermi liquid behavior, we plot our data as $h-1$ as a function of $(\eta-A)^{5 / 2}$ (see Fig 5.10). We observe a linear variation, characteristic of an ideal Fermi liquid behavior (see equation (5.6)), for a wide range of chemical potential imbalance. On the BEC side of the resonance and in the unitary limit, equation (5.6) gives an adequate description of our data for $A<\eta<\eta_{c}$, i.e. in the entire partially polarized normal phase. This results is all the more surprising since interactions are strong and minority concentrations can be large, up to $n_{2} / n_{1} \simeq 0.5$ in the unitary limit (see section 5.3.3). In the BCS limit, we observe a deviation from (5.6) close to the superfluid to normal transition. In that limit minority and majority densities are comparable and it is not surprising that a model constructed from the characteristics of the impurity problem is no longer adequate.
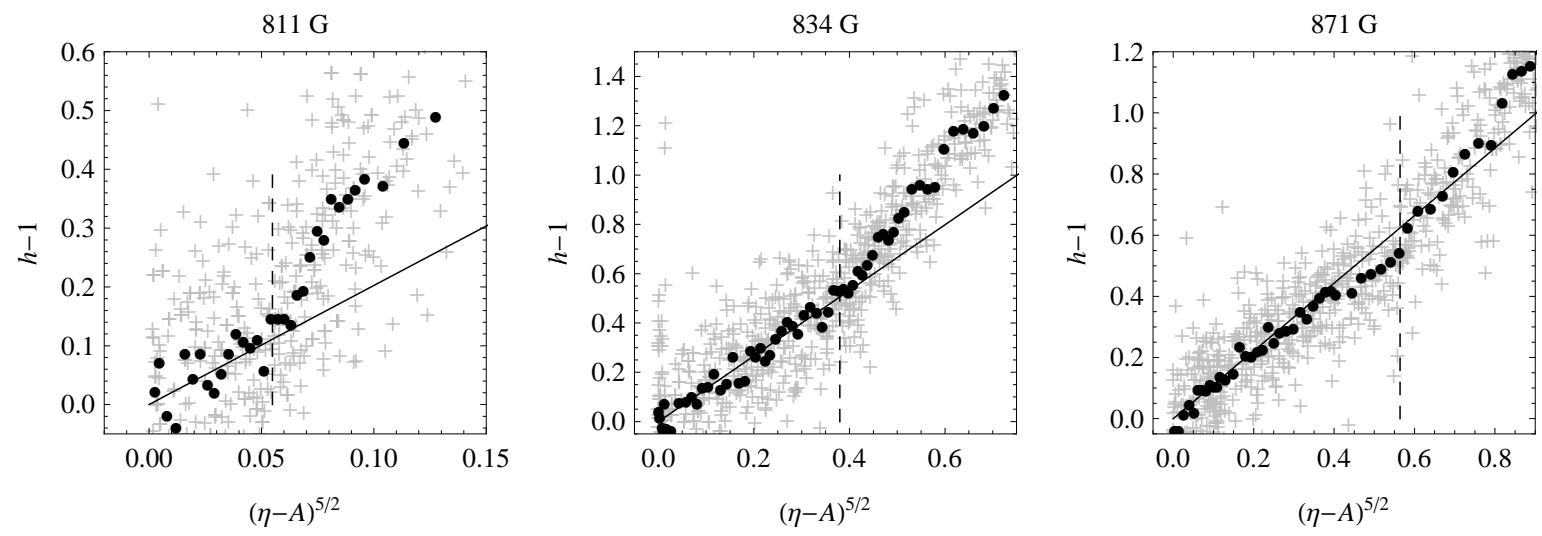

Figure 5.10: Equation of state plotted as $h-1$ as a function of $(\eta-A)^{5 / 2}$ for different bias magnetic fields. The dashed lines indicate the superfluid/normal transition and the solid lines are fits with a Fermi liquid equation of state.

\subsubsection{Measurement of the Polaron Effective Mass}

Fitting the data represented in Fig 5.10 with the ideal Fermi liquid equation of state 5.6. provides the effective mass value $m^{*}\left(\delta_{1}\right)$ (see Fig 5.11). In the BCS limit we do not use for the fit the data close to the superfluid transition where the polaron concentration is large. The effective mass extracted from our data is an increasing function of $\delta_{1}$. At unitarity, we obtain a very precise value $m^{*} / m=1.21(2)$, which is remarkably small for a strongly-interacting system. The effective mass values extracted from our data are in agreement with the most advanced calculations, namely diagrammatic Monte-Carlo calculations [85, and an analytic theory involving two particle-hole excitations [84, as well a simple variational calculation involving one particle-hole excitation 83 . The effective mass values predicted by Fixed-Node MonteCarlo calculations from [67] are systematically slightly lower than our measurements. 


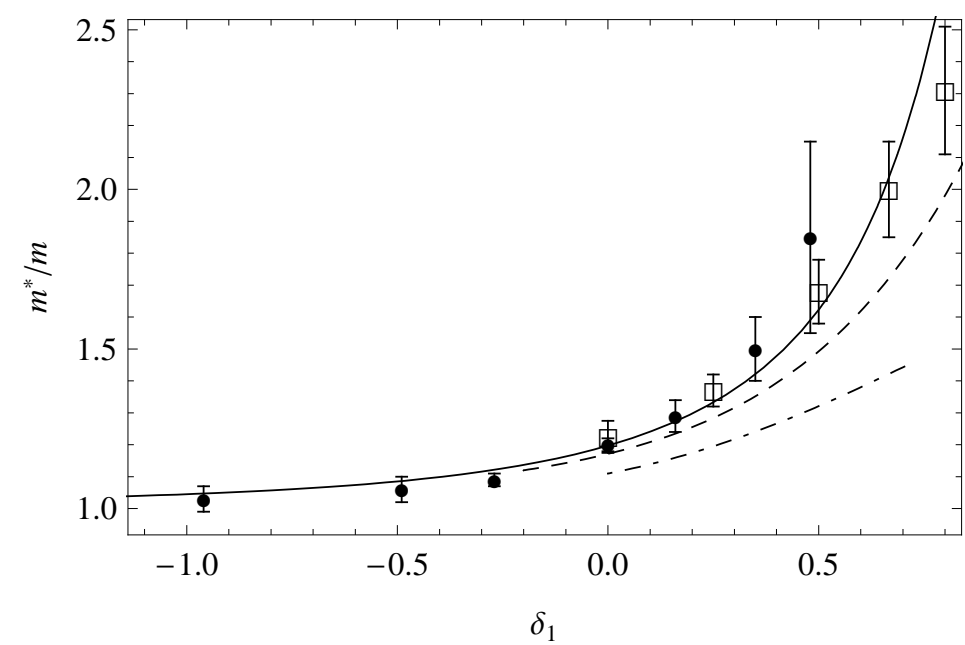

Figure 5.11: Polaron effective mass $m^{*} / m$ as a function of the interaction parameter $\delta_{1}$ (black dots). The open squares are diagrammatic Monte-Carlo data [85], the solid line is an analytic theory involving two particle-hole excitations [84], the dashed line a simple variational calculation involving one particle-hole excitation [83, and the dot-dashed line a Fixed-Node Monte-Carlo calculation from 67.

\subsubsection{Fermi Liquid Equation of State in the Unitary Limit}

We showed that the ideal Fermi liquid equation 5.5 accurately describes the partially polarized phase except for large minority concentrations in the BCS regime. In Fig 5.12 we also make a direct comparison with a Fixed-Node Monte Carlo calculation [81,67].The difference between the Monte-Carlo calculation and our data is quite small in the entire normal phase $A=-0.615<\eta<\eta_{c}=0.065$.

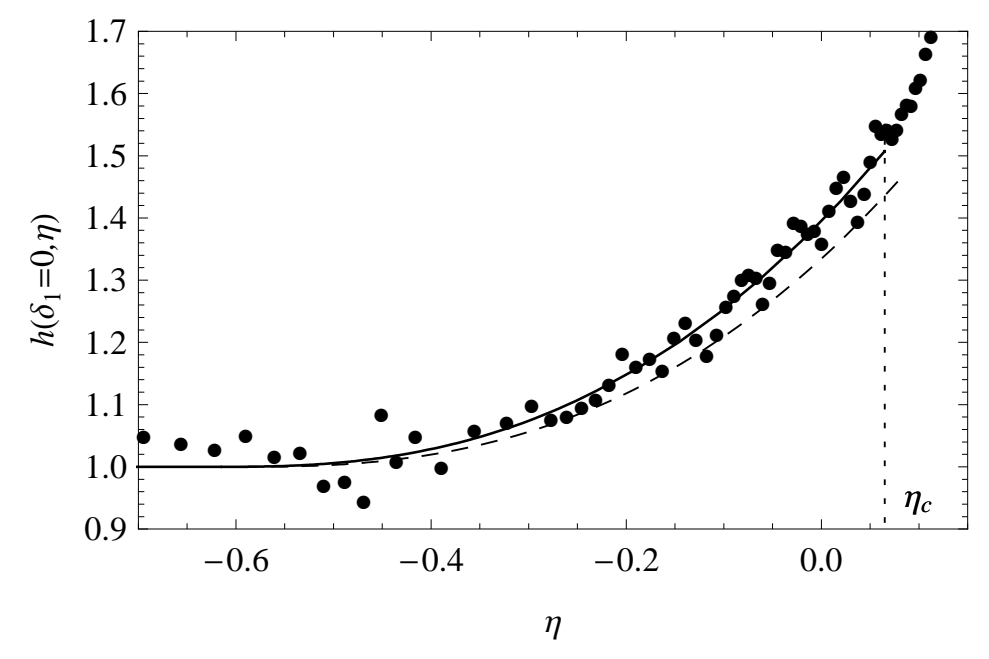

Figure 5.12: Equation of state in the partially polarized phase deduced from our data (black dots), compared with equation (5.6) (solid line) and with a Fixed-Node Monte-Carlo calculation [81 67] (dashed line).

We now give a more detailed physical interpretation of the Fermi liquid equation of state in the unitary limit:

$$
P\left(\mu_{1}, \mu_{2}, a\right)=\frac{1}{15 \pi^{2}}\left(\frac{2 m}{\hbar^{2}}\right)^{3 / 2} \mu_{1}^{5 / 2}+\frac{1}{15 \pi^{2}}\left(\frac{2 m_{0}^{*}}{\hbar^{2}}\right)^{3 / 2}\left(\mu_{2}-A_{0} \mu_{1}\right)^{5 / 2},
$$

where $A_{0}=-0.615$ and $m_{0}^{*} / m=1.21$ are the polaron energy shift and effective mass for $\delta_{1}=0$. Due to the polaron chemical potential shift, minority and majority densities are coupled. Indeed, differentiating 
the pressure 5.7 with respect to $\mu_{1}$ or $\mu_{2}$ gives the following relationship between the densities 9 .

$$
n_{1}=\frac{1}{6 \pi^{2}}\left(\frac{2 m}{\hbar^{2}}\right)^{3 / 2} \mu_{1}^{3 / 2}-A_{0} n_{2} .
$$

Therefore the majority density is enhanced by the presence of minority atoms, as if each minority atom was surrounded by $-A_{0} \simeq 0.6$ majority atom. Integrating this relation over the radial coordinates, we obtain a relationship between the doubly-integrated profiles:

$$
\bar{n}_{1}(z)=\bar{n}_{1}^{0}(z)-A_{0} \bar{n}_{2}(z) .
$$

We remind that $\bar{n}_{1}^{0}(z)$ is the Thomas Fermi profile deduced from the fully polarized region. In Fig 5.13 we plot $\bar{n}_{1}-\bar{n}_{1}^{0}$ as a function of $\bar{n}_{2}$. In the partially polarized phase we observe a linear dependence, whose slope gives $A_{0}=-0.58(5)$, in agreement with the exact value $A_{0} \simeq-0.615$. This constitutes an independent check of the value of $A_{0}$ that we use as a reference for the data analysis.

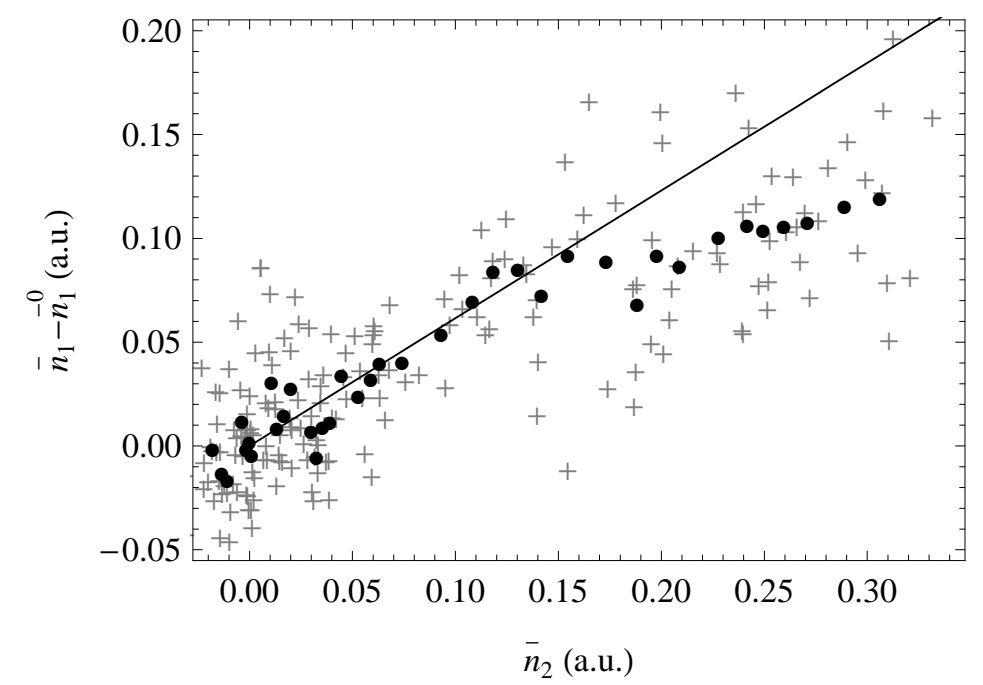

Figure 5.13: $\bar{n}_{1}-\bar{n}_{1}^{0}$ as a function of $\bar{n}_{2}$ for a gas prepared in the unitary limit. The data in the normal phase corresponds for this image to $\bar{n}_{2}<0.15$, and is in very good agreement with the Fermi liquid prediction 5.8 .

\subsubsection{Canonical Equation of State}

\section{Canonical Equation of State in the Unitary Limit}

In this section we make a direct comparison with the canonical equation of state of a spin-imbalanced Fermi gas in the unitary limit measured in the MIT group 49,68 and presented in section 3.1.2. The canonical equation of state is expressed as:

$$
E\left(n_{1}, n_{2}\right)=\frac{3}{5} \frac{\hbar^{2}}{2 m}\left(6 \pi^{2}\right)^{2 / 3} n_{1}^{5 / 3} g\left(x=\frac{n_{2}}{n_{1}}\right) .
$$

From the grand-canonical equation of state in the unitary limit $h_{0}(\eta)=h\left(\delta_{1}=0, \eta\right)$, we compute the canonical equation of state $g(x)$ :

$$
\begin{aligned}
x & =\frac{h_{0}^{\prime}(\eta)}{\frac{5}{2} h_{0}(\eta)-\eta h_{0}^{\prime}(\eta)}, \\
g(x) & =\frac{h_{0}(\eta)}{\left(h_{0}(\eta)-\frac{2}{5} \eta h_{0}^{\prime}(\eta)\right)^{5 / 3}} .
\end{aligned}
$$

I This relation can not be directly generalized out of the unitary limit. 
In Fig 5.14 we plot the equations of state $g(x)$ determined from the MIT group 68 and from our data, together with the following theoretical predictions:

- The most recent Fixed-Node Monte Carlo prediction, fitted with $g(x)=1+\frac{5}{3} A_{0} x+\frac{m}{m_{0}^{*}} x^{5 / 3}+F x^{2}$, with $A_{0}=-0.595, m_{0}^{*}=1.09 m$ and $F=0.1467$

- The canonical equation of state $g(x)=\left(1+A_{0} x\right)^{5 / 3}+\frac{m}{m_{0}^{*}} x^{5 / 3}$, with $A_{0}=-0.615, m_{0}^{*}=1.21 m$, which is the canonical equivalent of the ideal Fermi liquid equation (5.7) [48].

Our data shows significant differences with the MIT data and with the Monte-Carlo calculation.

As a remark, we mention that an expansion of the ideal Fermi liquid equation of state in a series of $x$ reads:

$$
g(x)=1+\frac{5}{3} A_{0} x+\frac{m}{m_{0}^{*}} x^{5 / 3}+F x^{2}+\ldots, \quad \text { with } \quad F=\frac{5 A_{0}^{2}}{9} \simeq 0.21
$$

and therefore contains a $x^{2}$ term, whose amplitude is close to the one extracted from the Monte-Carlo data. The term $F x^{2}$ can be interpreted an effective interaction between polarons. This connection between the coefficient $F$ and the single-polaron characteristics $A_{0}$ was established from theory in 185 .

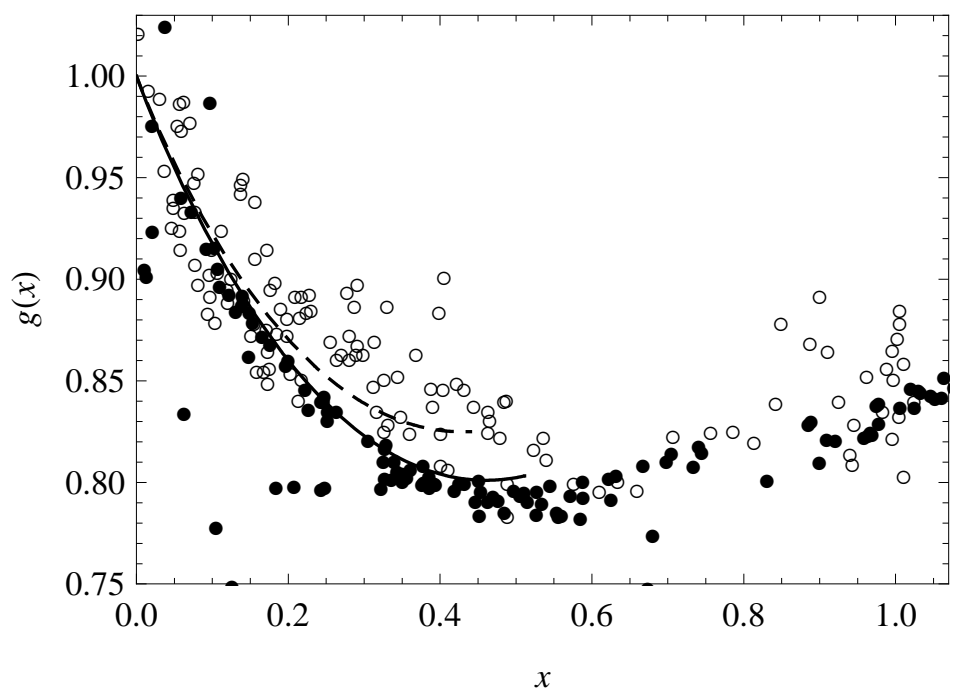

Figure 5.14: Canonical equation of state $g(x)$ extracted from our data (black dots) and from the MIT data [68] (open circles), compared with a Fixed-Node Monte Carlo calculation [81 (dashed line) and the ideal Fermi liquid equation (5.7) (solid line).

\section{Critical Concentration in the BEC-BCS Crossover}

In section 5.3 .2 we extracted from our data the concentration $x=n_{2} / n_{1}$ in the BEC-BCS crossover. We compare this data to the prediction given by the ideal Fermi liquid equation of state (5.6). Away from the unitary limit, the concentration is related to $h\left(\delta_{1}, \eta\right)$ by the formula:

$$
x\left(\delta_{1}, \eta\right)=\frac{\partial_{\eta} h}{\frac{5}{2} h-\eta \partial_{\eta} h-\delta_{1} / 2 \partial_{\delta_{1}} h} .
$$

Using equation (5.6), we calculate the concentration profiles in the partially polarized phase for different magnetic fields. In order to locate the position $\eta_{c}\left(\delta_{1}\right)$ of superfluid/normal phase transition, we solve the pressure equilibrium condition between the superfluid and normal phases:

$$
\mu_{1}^{5 / 2}\left(1+\left(\frac{m^{*}\left(\delta_{1}\right)}{m}\right)^{3 / 2}\left(\eta-A\left(\delta_{1}\right)\right)^{5 / 2}\right)=\widetilde{\mu}^{5 / 2} h_{S}(\widetilde{\delta}) \quad \text { at } \quad \eta=\eta_{c}\left(\delta_{1}\right) .
$$

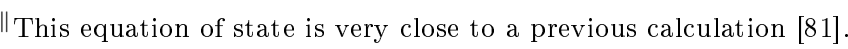


Here $\widetilde{\mu}^{5 / 2} h_{S}(\widetilde{\delta})$ is a parametrization of the superfluid equation of state that will be explained in the next section. In Fig 5.15 we plot the experimental data together with theoretical concentration profiles. We find a good agreement, with the exception of the value of $\eta_{c}$ on the BCS side of the resonance. This deviation is expected since we observed that equation (5.6) does not account for the experimental equation of state in this region.

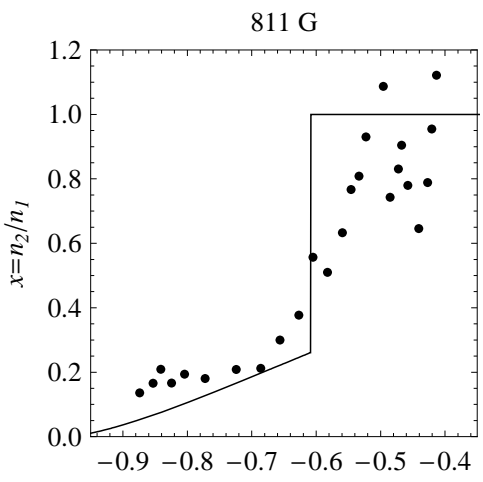

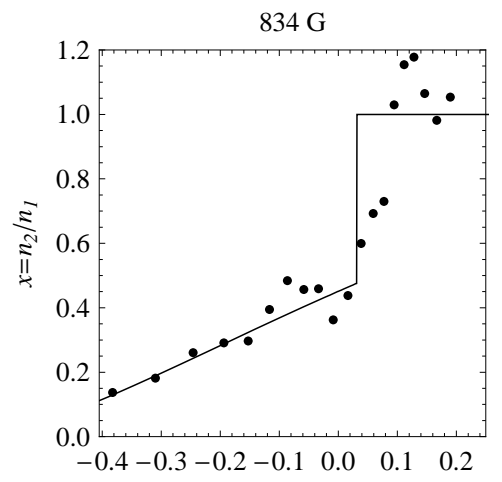

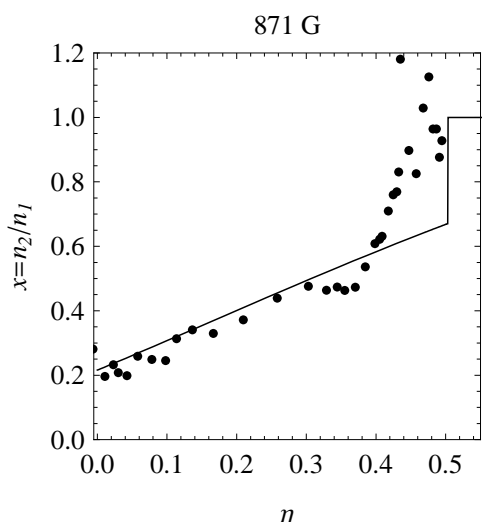

Figure 5.15: Concentration $x$ as a function of the chemical potential ratio $\eta$ for different magnetic fields, compared with the theoretical profiles (5.9) (solid lines).

The maximum concentration in the partially polarized phase $x_{c}\left(\delta_{1}\right)=x\left(\delta_{1}, \eta_{c}\left(\delta_{1}\right)\right)$ is also computed using the Fermi liquid equation of state and compared with the maximum concentration directly measured from the concentration profiles in Fig 5.16. We observe a good agreement between this calculation and the direct measurements.

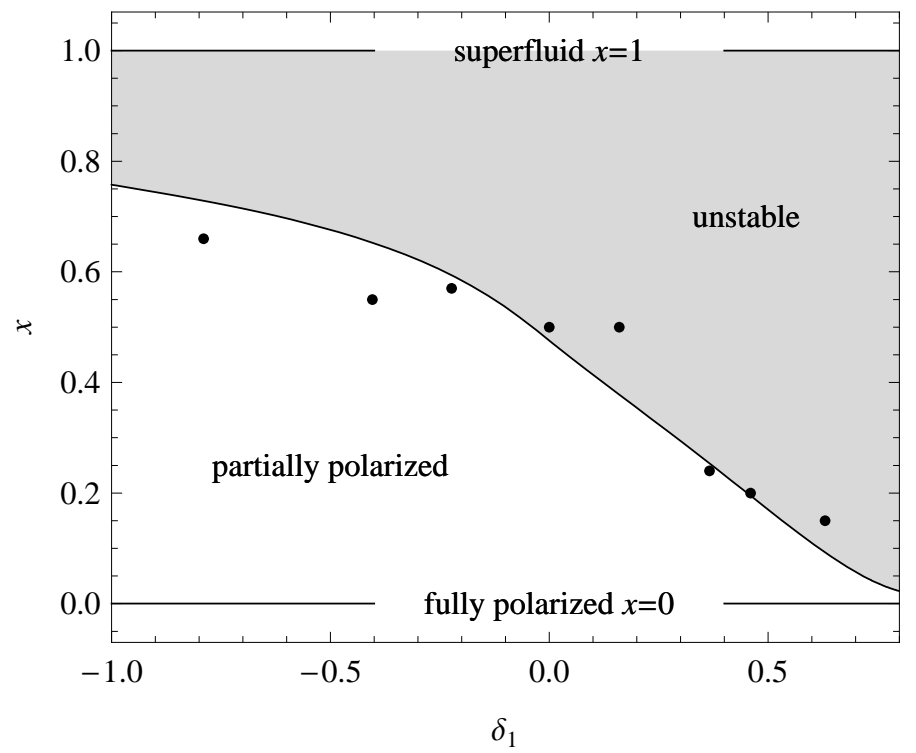

Figure 5.16: Phase diagram of a spin-imbalanced Fermi gas in the $\left(\delta_{1}, x\right)$ plane. The solid line is the maximum minority concentration allowed in the partially polarized phase. The region $x_{c}<x<1$ in gray is thermodynamically unstable.

\subsubsection{Magnetic Susceptibility of a Spin-Unpolarized Fermi Gas}

In the previous paragraphs we provided a description of the partially polarized normal phase in terms of a Fermi liquid of majority atoms and polarons, the latter being characterized by the resolution of the 
impurity problem. In this section we consider the opposite limit of small chemical potential imbalance. We no longer express the equation of state in terms of $\mu_{1}$ and $\eta=\mu_{2} / \mu_{1}$, but rather in terms of mean chemical potential $\mu=\left(\mu_{1}+\mu_{2}\right) / 2$ and 'effective magnetic field' $\delta \mu=\left(\mu_{1}-\mu_{2}\right) / 2$. The equation of state can then be expressed as:

$$
P\left(\mu_{1}, \mu_{2}, a\right)=P_{0}(\mu) h_{B}\left(\delta=\frac{\hbar}{\sqrt{2 m \mu} a}, b=\frac{\delta \mu}{\mu}\right) .
$$

In the next paragraphs we study the behavior of $h_{B}(\delta, b)$ at low values of $b$.

\section{Magnetic Susceptibility in the Superfluid and Normal Phases}

We have shown in section 5.3 .1 that in the superfluid phase the atom densities of both spin states are equal: $n_{1}=n_{2}$. The partial derivative of the pressure with respect to $\delta \mu$ reads:

$$
\left.\frac{\partial P}{\partial \delta \mu}\right|_{\mu, a}=\left.\left.\frac{\partial P}{\partial \mu_{1}}\right|_{\mu_{2}, a} \frac{\partial \mu_{1}}{\partial \delta \mu}\right|_{\mu, a}+\left.\left.\frac{\partial P}{\partial \mu_{2}}\right|_{\mu_{2}, a} \frac{\partial \mu_{2}}{\partial \delta \mu}\right|_{\mu, a}=\frac{1}{2}\left(n_{1}-n_{2}\right) .
$$

Therefore in the superfluid phase the pressure does not depend on $\delta \mu$, and $h_{B}(\delta, b)=h_{B}(\delta, b=0)$.

When one imposes a strong enough magnetic field $\delta \mu$, the superfluid breaks and one enters into a partially polarized normal phase. In dimensionless variables, the critical magnetic field is equal to $b_{c}=\left(1-\eta_{c}\right) /\left(1+\eta_{c}\right)$, where $\eta_{c}$ is the critical chemical potential ratio studied in section 5.3.1. If $b_{c}$ is not too large, $h_{B}(\delta, b)$ may be well described by the first terms of the expansion in series of $b$. Equation 5.10 taken in the spin-symmetric configuration $\delta \mu=0$ where it is clear that $n_{1}=n_{2}$, implies that $\partial h_{B} /\left.\partial b\right|_{\delta}(\delta, b=0)=0$. Hence the expansion of $h_{B}$ in series of $b$ reads:

$$
h_{B}(\delta, b)=h_{N}(\delta)+\frac{15}{8} \chi(\delta) b^{2}+\ldots
$$

The normalizations were chosen so that for an ideal gas $h_{N}=\chi=1 . h_{N}(\delta)$ is the grand-canonical equation of state of the normal phase extrapolated to the symmetric configuration $b=0 . \chi(\delta)$ is the magnetic susceptibility compared with the one of an ideal Fermi gas. In the Landau Fermi liquid framework, these parameters are related to the some Landau parameters:

$$
h_{N}^{2 / 3}=\frac{m^{*} / m}{1+F_{0}^{s}}, \quad \chi h_{N}^{1 / 3}=\frac{m^{*} / m}{1+F_{0}^{a}},
$$

where $m^{*}$ is the effective mass of the quasi-particles and $F_{0}^{s}$ and $F_{0}^{a}$ quantify interactions between quasiparticles 146 .

\section{Magnetic Susceptibility of a Spin-Unpolarized Fermi Gas in the Unitary Limit}

We first focus on the equation of state in the unitary limit, plotted in Fig 5.17 as $h_{B}(\delta=0, b)$ versus $b^{2}$. In the superfluid phase $b^{2}<0.75, h_{B}$ does not depend on $b$, a clear manifestation of the superfluid unpolarizability. In the normal phase one observes a clear $b^{2}$ dependence for $0.75<b^{2}<3$. This shows that the normal phase can be described as a Landau Fermi liquid, magnetized with respect to a spin-symmetric configuration.

The equation of state extrapolated to $b=0, h_{N}=2.55(5)$, is in agreement with the value obtained from the extrapolation to zero temperature of the equation of state of a balanced gas at finite temperature and in the normal phase (see section 4.6 in Chapter 4). This observation suggests that the two normal phases $\left(T=0, \delta \mu>\delta \mu_{c}\right)$ and $\left(T>T_{c}, \delta \mu=0\right)$ can be continuously connected in the plane $(T, \delta \mu)$, in agreement with the phase diagram established in the MIT group [49]. The equation of state of a spin-imbalanced Fermi gas at finite temperature will be the subject of future work.

We also obtain the parameter $\chi=0.73(3)$; using the effective mass value $m^{*} / m=1.13(3)$ determined in section 4.6 , we obtain the Landau parameter $F_{0}^{a}=1.1(1)$. 


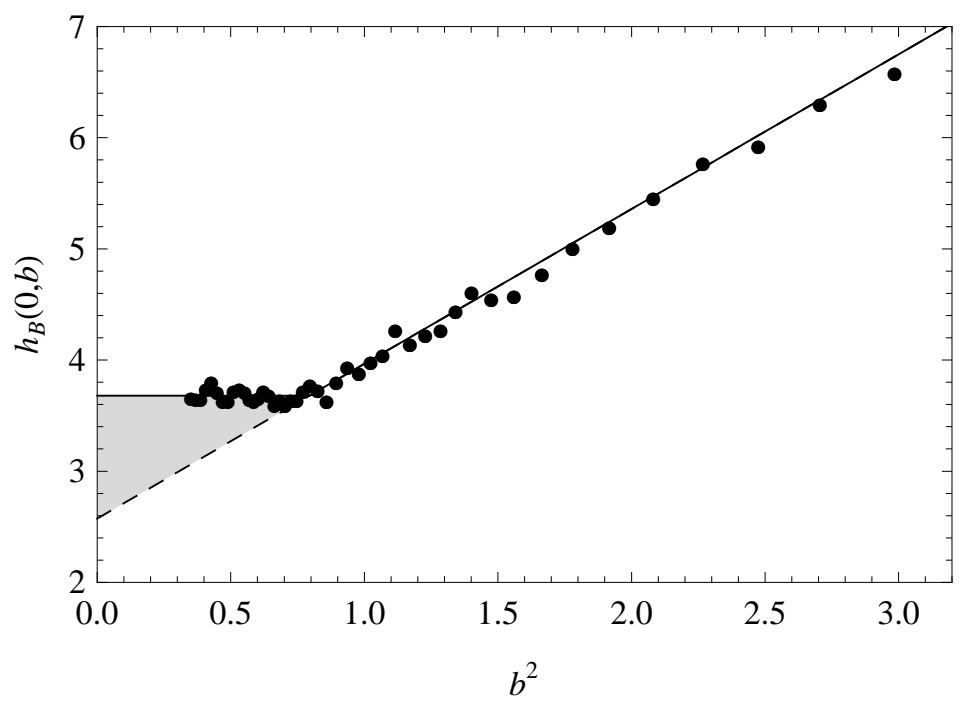

Figure 5.17: Equation of state $h_{B}(\delta=0, b)$ of a spin-imbalanced Fermi gas in the unitary limit, as a function of $b^{2}$. The superfluid phase corresponds to the region $b^{2}<0.75$ where $h_{B}$ is constant. In the normal phase $b^{2}>0.75$, the linear variation of $h_{B}$ with $b^{2}$ is a manifestation of a Fermi liquid behavior. The difference between the superfluid and normal equations of state is referred to as the condensation pressure (gray area).

\section{Fermi Liquid Parameters in the BEC-BCS Crossover}

A similar behavior $h_{B}=h_{N}+\chi b^{2}$ is observed on the BCS side of the resonance and at $B_{0}=822 \mathrm{G}$ (for the data deeper on the BEC side of the resonance, the critical field $b_{c}$ is much larger than one, casting doubt on the validity of an expansion in series of $b$ for $b>b_{c}$.). The parameters $h_{N}$ and $\chi$ are plotted in Fig 5.18 as a function of the interaction parameter $\delta$. The equation of state $h_{N}(\delta)$ of a balanced normal gas is in agreement with a Fixed-Node Monte Carlo calculation [178]. We also plot a fit of the superfluid equation of state $h_{S}(\delta)$ (see section 5.5); the difference between $h_{S}(\delta)$ and $h_{N}(\delta)$ represents the condensation pressure associated with superfluidity. The superfluid/normal transition, characterized by $h_{S}(\delta)=h_{B}\left(\delta, b_{c}\right)=h_{N}(\delta)+\frac{15}{8} \chi b_{c}^{2}$, leads to the following relationship between the condensation pressure and the critical field $b_{c}$ :

$$
h_{S}-h_{N}=\frac{15}{8} \chi b_{c}^{2},
$$

which is the analogous of the relationship between the condensation energy and critical magnetic field for superconductors 186 . It would also be interesting to study the effect of strong interactions on the relationship between the condensation energy and the gap that can be established in the BCS limit.

\subsection{Superfluid Equation of State in the BEC-BCS Crossover}

In this section we extract and interpret the superfluid equation of state from the data $\eta>\eta_{c}\left(\delta_{1}\right)$.

\subsubsection{Parametrization of the Superfluid Equation of State}

We have shown that the gas is fully paired in the superfluid phase:

$$
n_{1}\left(\mu_{1}, \mu_{2}, a\right)=n_{2}\left(\mu_{1}, \mu_{2}, a\right) \quad \text { for } \quad \eta>\eta_{c}\left(\delta_{1}\right) .
$$


(a)

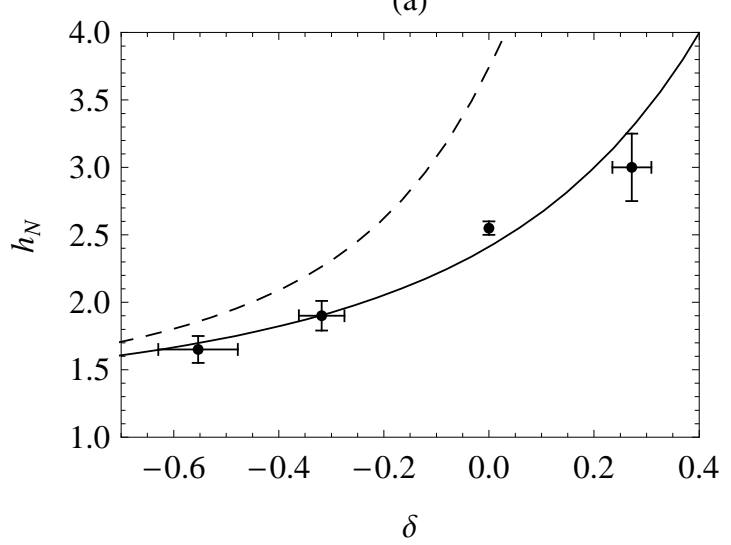

(b)

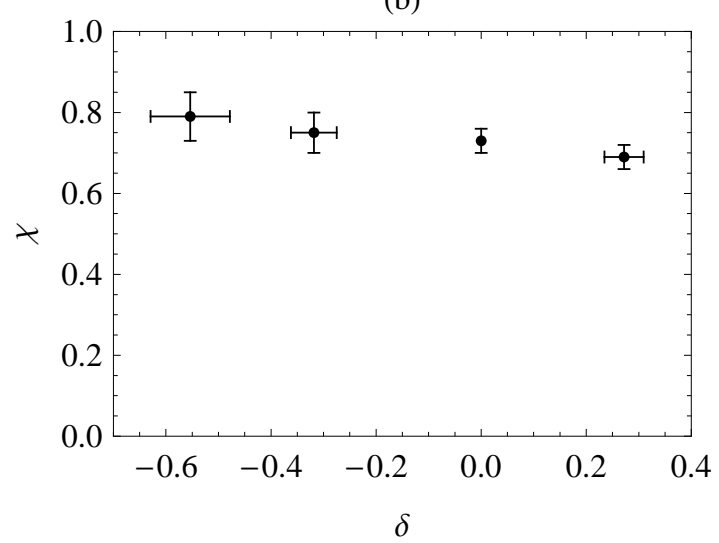

Figure 5.18: (a) Equation of state of the normal phase $h_{N}(\delta)$ extrapolated to a spin-symmetric configuration (black dots), compared with a fit of the Monte Carlo data from [178 (solid line), and with the superfluid equation of state $h_{S}(\delta)$ (dashed line). (b) Compressibility $\chi(\delta)$, normalized to the one of a non-interacting mixture.

This provides a constraint on the equation of state which can be expressed as:

$$
\begin{aligned}
\left.\frac{\partial P}{\partial(\delta \mu)}\right|_{\mu, a} & =\left.\frac{\partial P}{\partial \mu_{1}}\right|_{\mu_{2}, a}-\left.\frac{\partial P}{\partial \mu_{2}}\right|_{\mu_{1}, a} \\
& =n_{1}-n_{2} \\
& =0
\end{aligned}
$$

where $\delta \mu=\mu_{2}-\mu_{1}$ and $\mu=\left(\mu_{1}+\mu_{2}\right) / 2$. The pressure is thus a function of the mean chemical potential only. Instead of the parametrization $h\left(\delta_{1}, \eta\right)$ for the equation of state, we rather express it using the symmetric variable $\mu$ only:

$$
P\left(\mu_{1}, \mu_{2}, a\right)=2 P_{0}(\widetilde{\mu}) h_{S}(\widetilde{\delta}), \quad \text { where } \quad \widetilde{\mu}=\left\{\begin{array}{ll}
\mu, & a<0 \\
\mu+\frac{\hbar^{2}}{2 m a^{2}}, & a>0
\end{array} \quad \text { and } \quad \widetilde{\delta}=\frac{\hbar}{\sqrt{2 m \widetilde{\mu} a}}\right.
$$

We use $\widetilde{\mu}$ instead of $\mu$ in order to avoid handling negative chemical potentials on the BEC side of the resonance. We gather the data $h\left(\delta_{1}, \eta\right)$ in the superfluid phase and express it in term of $h_{S}(\widetilde{\delta})$ :

$$
\widetilde{\delta}=\left\{\begin{array}{ll}
((1+\eta) / 2)^{-1 / 2} \delta_{1}, & a<0 \\
\left((1+\eta) / 2+\delta_{1}^{2}\right)^{-1 / 2} \delta_{1}, & a>0
\end{array} \quad \text { and } \quad h_{S}(\widetilde{\delta})=\left\{\begin{array}{ll}
((1+\eta) / 2)^{-5 / 2} h\left(\delta_{1}, \eta\right), & a<0 \\
\left((1+\eta) / 2+\delta_{1}^{2}\right)^{-5 / 2} h\left(\delta_{1}, \eta\right), & a>0
\end{array} .\right.\right.
$$

Our experimental data, plotted in Fig 5.19 is the first experimental equation of state of a homogeneous superfluid Fermi gas. The cloud images taken at a given magnetic field contribute to the equation of state in a rather narrow region, typically in a region $\widetilde{\delta} \in[0.8\langle\widetilde{\delta}\rangle, 1.2\langle\widetilde{\delta}\rangle]$. The statistical noise of our data is about $10 \%$, and we recall that the systematic uncertainty is $5 \%$.

\subsubsection{Direct Comparison with Theory}

In this section we compare our data with several theories predicting the equation of state $h_{S}(\widetilde{\delta})$ of a homogeneous superfluid. Our data can directly be compared with numerical calculations providing discrete values for $h_{S}(\widetilde{\delta})$ :

- a Nozières-Schmitt-Rink approximation from 141,

- a quantum Monte-Carlo calculation from [173], extrapolated at $T=0$, 


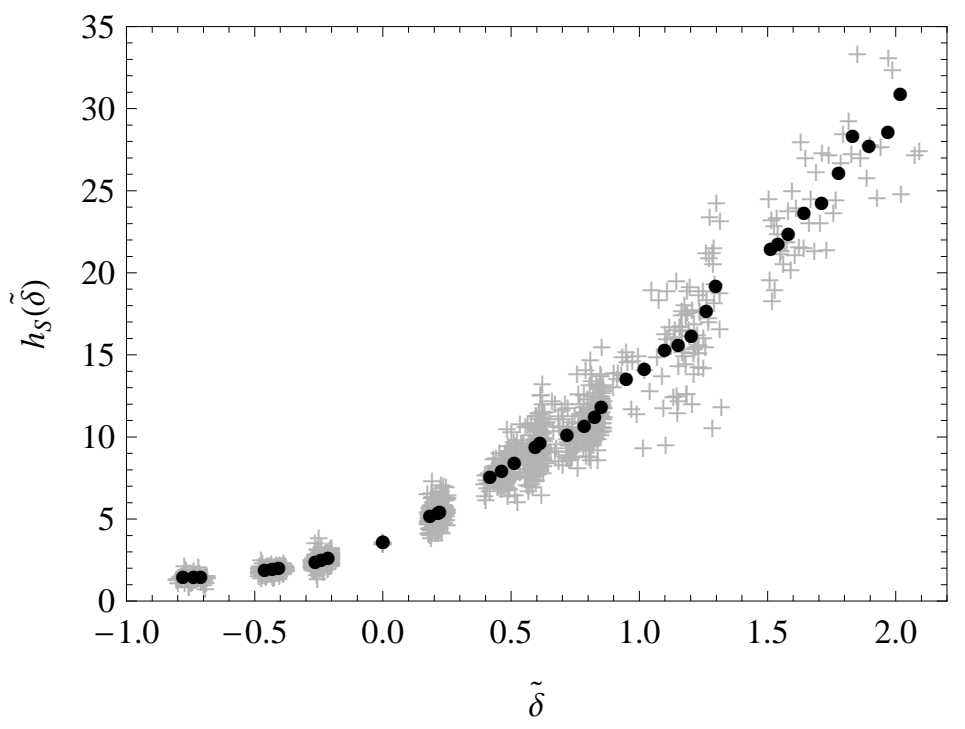

Figure 5.19: Superfluid equation of state in the BEC-BCS crossover $h_{S}(\widetilde{\delta})$ (gray crosses). The black dots are an average of successive points.

- a diagrammatic approach from 138.

As shown in Fig 5.20 , our data agrees with the first calculation but significantly differs from the other two predictions. The $\sim 100 \%$ inaccuracy of BCS mean-field theory shows that the equation of state $h_{S}(\widetilde{\delta})$ is a much more discriminating quantity than the collective mode frequency, for which BCS mean-field theory is wrong by $3 \%$ only 64 (see. section 3.1.1).

The comparison with Fixed-Node Monte Carlo calculations from 65, 66, 67 is less direct. Indeed, these data are expressed in the canonical ensemble as:

$$
E=\frac{N}{2} E_{b}+\frac{3}{5} N E_{F} \xi\left(\nu=\frac{1}{k_{F} a}\right), \quad \text { where } \quad E_{b}=\left\{\begin{array}{ll}
0, & a<0 \\
-\hbar^{2} / m a^{2}, & a>0
\end{array} .\right.
$$

Relating the equations of state $\xi(\nu)$ and $h_{S}(\widetilde{\delta})$ requires taking the derivative of the data:

$$
\widetilde{\delta}=\frac{\nu}{\left(\xi(\nu)-\nu \xi^{\prime}(\nu) / 5\right)^{1 / 2}}, \quad h_{S}(\widetilde{\delta})=\frac{\xi(\nu)-\nu \xi^{\prime}(\nu) / 2}{\left(\xi(\nu)-\nu \xi^{\prime}(\nu) / 5\right)^{5 / 2}} .
$$

In Fig 5.20 we calculate $h_{S}(\widetilde{\delta})$ using a fit of the most recent Monte-Carlo data [178, and find a good agreement with our data. The fitting function is proposed by the authors of the numerical calculations in [187], and differs from the discrete data by less than $1 \%$. It is important to mention that the FixedNode Monte Carlo method is a variational calculation of the ground state energy, and a priori gives an upper bound of the actual ground state energy, which corresponds to a lower bound for $h_{S}(\widetilde{\delta})$. This is in agreement with the slight positive difference between our data and the Fixed-Node Monte Carlo results (see Fig 5.20 ).

Alternatively, we calculate the canonical equation of state $\xi(\nu)$ using a fit of our data. The fitting function is described in detail in the next sections and in Appendix B.3. In Fig 5.21 we compare the equation of state given by the experimental data with Fixed-Node Monte Carlo calculations 65 66 67. We find a good agreement except with the data from $[66$ around $\nu=-0.4$, a region where the MonteCarlo method was expected to be less robust $[66$. The identification of a few inaccurate points in the

\footnotetext{
** In the unitary, BCS and BEC limits, the collective mode frequencies are identical for all theories due to the scalings $\mu \propto n^{2 / 3}$ or $\mu \propto n$ that are imposed by dimensional analysis. This intuitively explains why collective modes do not easily discriminate between theories.
} 

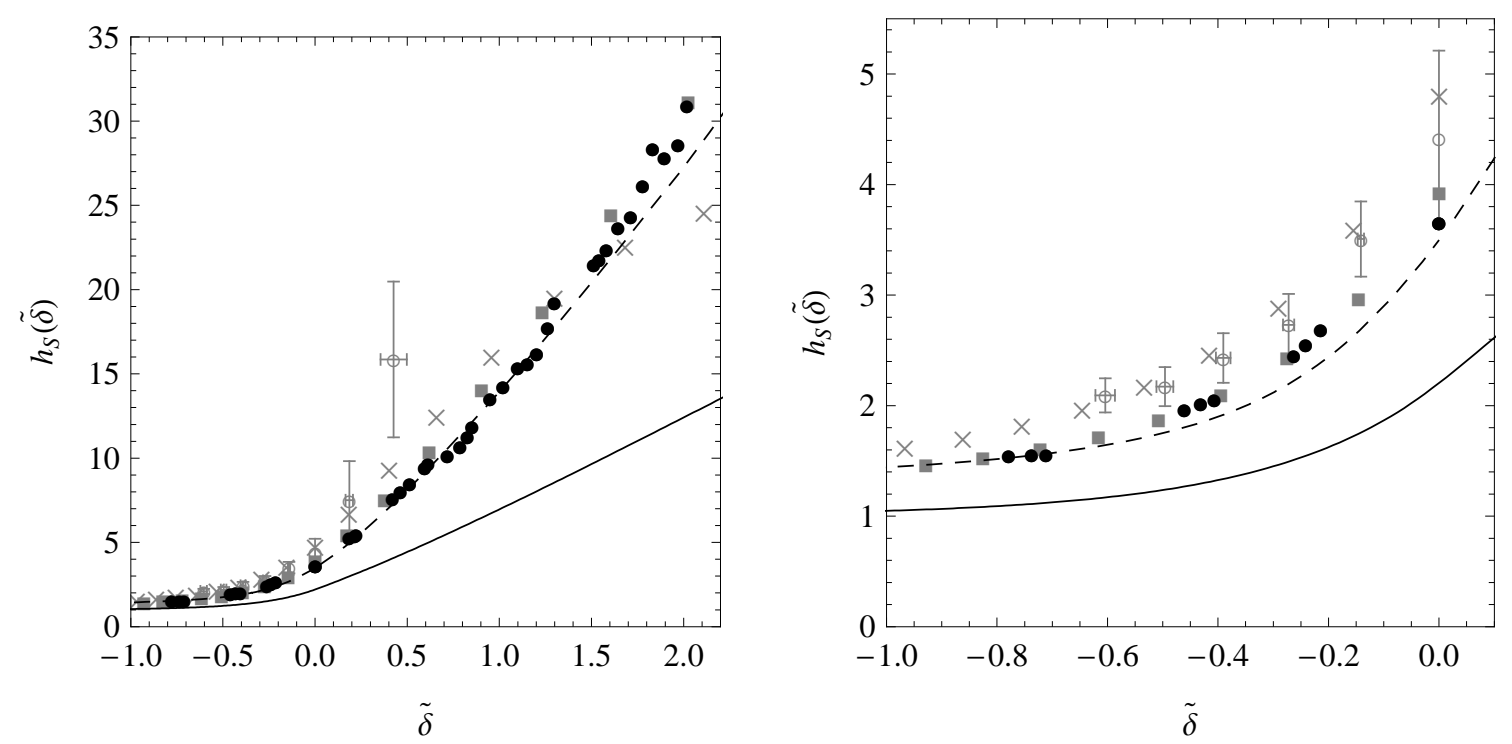

Figure 5.20: Comparison of the experimental equation of state (black dots) with BCS mean-field theory (solid line), a Nozières-Schmitt-Rink approximation from [141] (gray squares), a quantum Monte-Carlo calculation from 173] (gray open circles) and a diagrammatic approach from [138] (gray crosses). The dashed line is calculated from a fit of the Fixed-Node data 67 .

Monte-Carlo data from [66 is made possible by the direct measurement of the equation of state of a homogeneous Fermi gas. It would be much more difficult to establish using observables such as collective mode frequencies, since the comparison theory/experiment requires to integrate the theoretical prediction over the trap 115,64 .

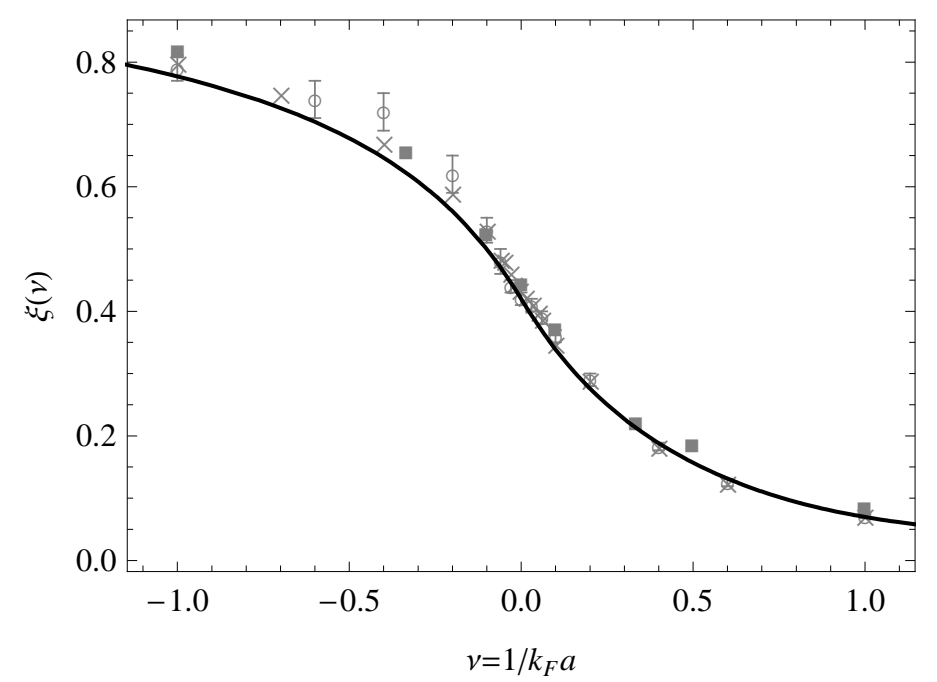

Figure 5.21: Comparison of the equation of state $\xi(\nu)$ deduced from a fit of our data (solid line) with Fixed-Node Monte Carlo calculations from [65] (squares), 66] (open circles) and [67 (crosses).

In the next sections we extract more physical quantities from our data by studying the asymptotic behaviors of the equation of state in the BCS limit, around the unitary limit, and in the BEC limit. 
(a)

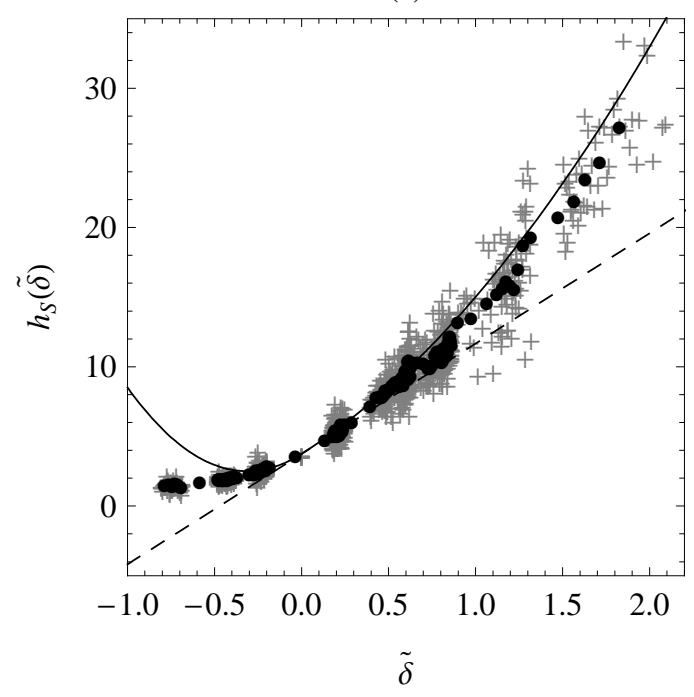

(b)

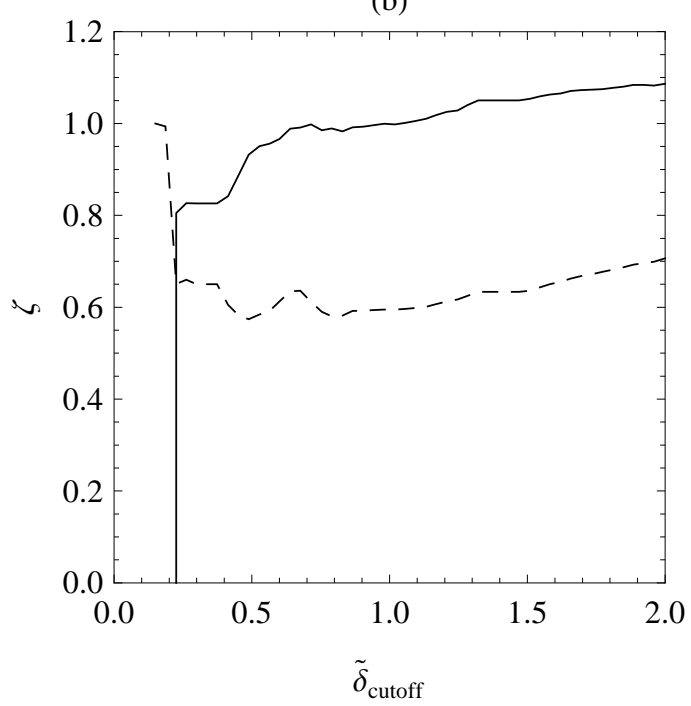

Figure 5.22: (a) Equation of state extracted from our data (crosses and dots), together with the linear and quadratic expansions in $\widetilde{\delta}$ around the unitary limit, with $\xi_{s}=0.415, \zeta=0.9, v=0.4$. (dashed and solid line, respectively) (b) Fit result $\zeta$ as a function of the cutoff $\widetilde{\delta}_{\text {cutoff }}$, for a linear fitting function (dashed line) and a quadratic fit function (solid line).

\subsubsection{Extracting Asymptotic Behaviors of the Equation of State}

Extracting asymptotic behaviors from a set of discrete data is not obvious. We illustrate this difficulty on the example of the unitary limit. We want to extract from our data the expansion of the equation of state around the unitary limit:

$$
E=\frac{3}{5} N E_{F}\left(\xi_{s}^{-3 / 2}-\zeta \nu-\frac{5 v}{3} \nu^{2}+\ldots\right), \quad \nu=\frac{1}{k_{F} a} \ll 1 .
$$

A physical interpretation of the expansion will be given in the next section, and we focus here on the problem of extracting the coefficients of the expansion from our data. The corresponding expansion in the grand-canonical ensemble reads:

$$
h_{S}(\widetilde{\delta})=\xi_{s}^{-3 / 2}+\frac{3 \zeta}{2 \xi_{s}^{2}} \widetilde{\delta}+\frac{12 \zeta^{2} \xi_{s}^{-1}+25 v}{10 \xi_{s}^{3 / 2}} \widetilde{\delta}^{2}+\left\{\begin{array}{ll}
0, & \widetilde{\delta}<0 \\
-5 \xi_{s}^{-3 / 2} \widetilde{\delta}^{2}, & \widetilde{\delta}>0
\end{array}+\ldots, \quad \widetilde{\delta} \ll 1 .\right.
$$

The discontinuity of the second-order derivative of $h_{S}(\widetilde{\delta})$ is due to the addition of the molecular binding energy to $\mu$ in $\tilde{\mu}$. This behavior does not play a role in this discussion.

In order to extract $\zeta$ from the data, we fit the data either with a linear expansion in $\widetilde{\delta}$ given by the first two terms in (5.11) or with a quadratic expansion given by (5.11). The points included in the fit are defined by $\widetilde{\delta} \in\left[-\widetilde{\delta}_{\text {cutoff }}, \widetilde{\delta}_{\text {cutoff }}\right]$. The fit result $\zeta$ as a function of the fitting function and of the cutoff

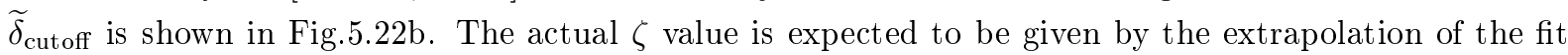

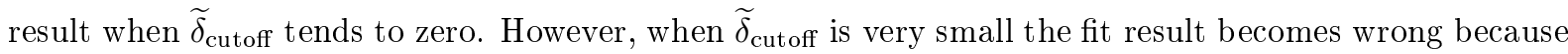
of the low number of data points involved in the fit. A good estimate is obtained if the fit result is stable in a wide region of cutoff values. However, this is not the case for the fits shown in Fig $5.22 \mathrm{p}$ and the fit results given by the two fitting functions always significantly differ. The inaccuracy of this procedure is due to the behavior of the fitting functions away from the unitary limit. In Fig $\sqrt[5.22]{5}$ we show the linear and quadratic fitting functions with the parameters $\xi_{s}=0.415, \zeta=0.9, v=0.4$ determined from a better fit procedure, described in the next sections. The divergence of the fitting functions in the $\mathrm{BCS}$ 
limit, while the data are expected to approach 1 , makes them inadequate for the description of the data except in a very narrow interval around the unitary limit.

This is an usual problem when one approximates a function with a Taylor expansion around a given point. A better estimate of the function is often given, instead of the polynomial Taylor expansion, by a rational function, a so-called Padé approximant, which remains finite at $\pm \infty$ [188. As an example, we consider the exponential function. The Padé approximant having the correct Taylor expansion up to fourth order around 0 is given by:

$$
\frac{1+x / 2+x^{2} / 12}{1-x / 2+x^{2} / 12}=1+x+x^{2} / 2+x^{3} / 6+x^{4} / 24+O\left(x^{5}\right),
$$

and gives an estimate of the exponential function within $5 \%$ in the interval $[-2,2]$, whereas the Taylor expansion is correct within $5 \%$ in a narrower interval $[-0.4,1.4]$.

We thus construct a set of fitting functions inspired by Padé approximants. As the limiting behaviors in the BEC and BCS limit differ, we use different functions for both sides of the resonance. On the BCS side of the resonance, we use the following function:

$$
h_{S}^{\mathrm{BCS}}(\widetilde{\delta})=\frac{\alpha_{1}+\alpha_{2} \widetilde{\delta}+\widetilde{\delta}^{2}}{\alpha_{3}+\alpha_{4} \widetilde{\delta}+\widetilde{\delta}^{2}},
$$

which is finite in the unitary limit and tends to 1 in the BCS limit. On the BEC side of the resonance, we use more complex fitting functions $h_{S}^{\mathrm{BEC}}(\widetilde{\delta})$ in order to capture a more subtle asymptotic behavior in the BEC limit (see section 5.5.6 and Appendix B.3. We finally connect both sides of the resonance by imposing a smooth connection in the unitary limit:

$$
h_{S}^{\mathrm{BCS}}(0)=h_{S}^{\mathrm{BEC}}(0), \quad\left(h_{S}^{\mathrm{BCS}}\right)^{\prime}(0)=\left(h_{S}^{\mathrm{BEC}}\right)^{\prime}(0), \quad\left(h_{S}^{\mathrm{BCS}}\right)^{\prime \prime}(0)=\left(h_{S}^{\mathrm{BEC}}\right)^{\prime \prime}(0)+5 h_{S}(0),
$$

the last condition being due to the convention $\widetilde{\mu}=\mu-E_{b} / 2$ for $\delta>0$.

We test the pertinence of these fitting functions on a simple example. We consider the equation of state $h_{S}^{\mathrm{BMF}}(\widetilde{\delta})$ given by the BCS mean-field model (see Fig 5.23 a). The parameter $\zeta$ discussed above is equal to 0.90 for this model. We fit $\zeta$ using the procedure described above, using either a linear fitting function, a quadratic fitting function, or a Padé-type fitting function. As shown in Fig 5.23 p, obtaining a good estimate for $\zeta$ does not require to extrapolate $\widetilde{\delta}_{\text {cutoff }}$ to very small values in the case of the Padé-type functions. This suggests that these functions are more suited for extracting asymptotic behaviors of the actual superfluid equation of state $h_{S}(\widetilde{\delta})$.

\subsubsection{Superfluid Equation of State Around the Unitary Limit}

We discuss in this section the asymptotic behavior around the unitary limit, written as:

$$
E=\frac{3}{5} N E_{F}\left(\xi_{s}^{-3 / 2}-\zeta \nu-\frac{5 v}{3} \nu^{2}+\ldots\right), \quad \nu=\frac{1}{k_{F} a} \ll 1 .
$$

We fit the data in the region $\left[-\widetilde{\delta}_{\text {cutoff }}, \widetilde{\delta}_{\text {cutoff }}\right]$ with the Padé-type approximant described in section 5.5 .3 and Appendix B.3. with $\xi_{s}, \zeta$ and $v$ as free parameters. As shown in Fig 5.24, the fit results $\xi_{s}$ and $\zeta$ do not vary depend much on $\widetilde{\delta}_{\text {cutoff }}$, except for small cutoff values for which the number of data points is small. The value of $v$ appears to be less robust. The gray regions indicate the actual values of the parameters in agreement with our data.

The coefficient $\xi_{s}$ was already introduced in Chapter 4. Its encapsulates all thermodynamic properties of a balanced unitary gas at zero temperature. We obtain:

$$
\xi_{s}=0.415(10)
$$


(a)

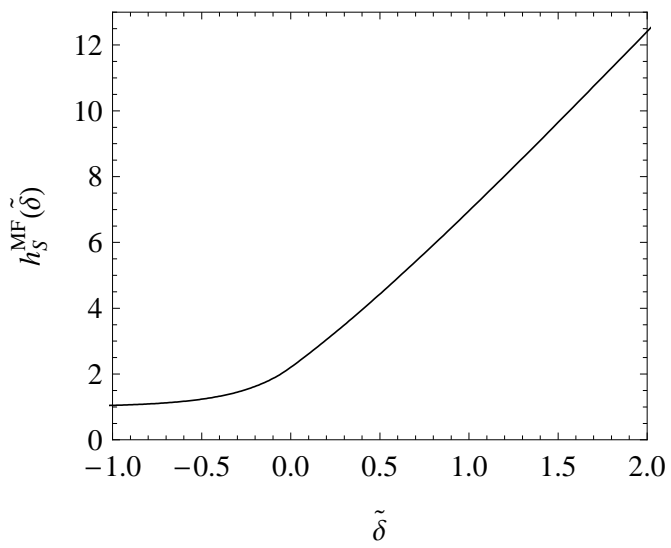

(b)

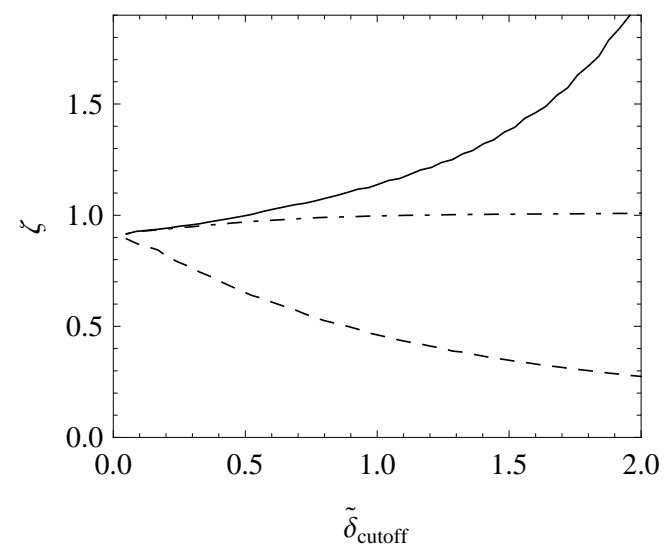

Figure 5.23: (a) Equation of state $h_{S}^{\mathrm{MF}}(\widetilde{\delta})$ given by the BCS mean-field model. (b) Fit result $\zeta$ as a function of $\widetilde{\delta}_{\text {cutoff }}$, for a linear fitting function (dashed line), a quadratic fitting function (solid line), and the more elaborate fitting function described in the text (dot-dashed line).

(a)

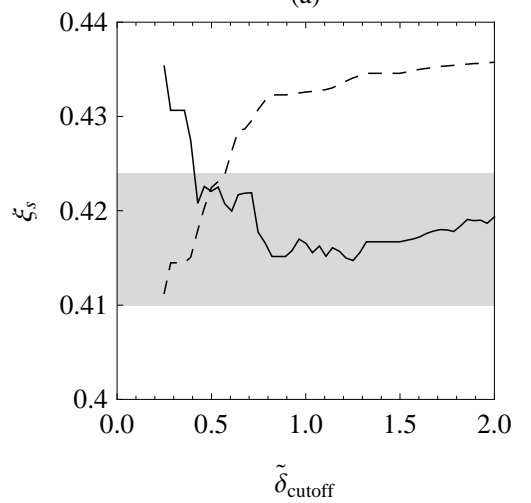

(b)

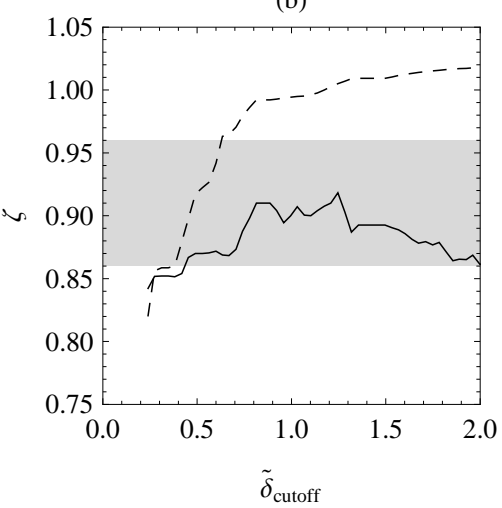

(c)

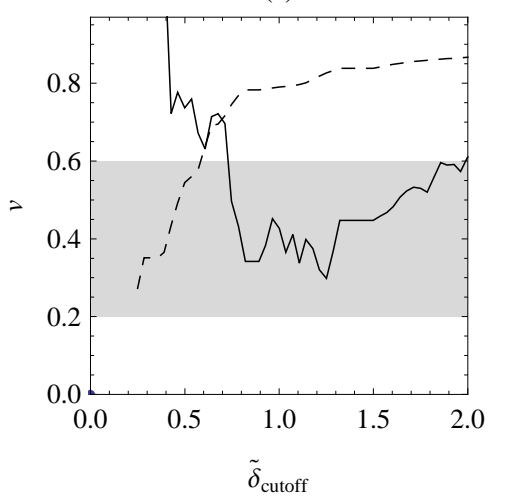

Figure 5.24: Fit results $\xi_{s}(\mathrm{a}), \zeta(\mathrm{b})$ and $v(\mathrm{c})$, as a function of $\widetilde{\delta}_{\text {cutoff. The gray regions indicate our }}$ estimation of the actual values of these parameters. The dashed lines are proportional to the number of points used for the fit. When $\widetilde{\delta}_{\text {cutoff }}$ is smaller than 0.5 , the number of data points becomes small and the fit results strongly depend on the noise of our data.

a value in agreement with previous measurements and numerical calculations [165.

A physical interpretation of $\zeta$ was first given by S. Tan in 189, 190, 191]. In [189], an exact relation between thermodynamic quantities and more microscopic observables is established for a gas with shortrange interactions: the derivative of the energy with respect to the scattering length $a$, encapsulated in the coefficient $\zeta$, is connected to the short-range behavior of the pair correlation function:

$$
g_{12}^{(2)}(r)=\frac{\left\langle\hat{\psi}_{1}^{\dagger}(\mathbf{r}) \hat{\psi}_{2}^{\dagger}(\mathbf{0}) \hat{\psi}_{2}(\mathbf{0}) \hat{\psi}_{1}(\mathbf{r})\right\rangle}{\left\langle\hat{\psi}_{1}^{\dagger}(\mathbf{r}) \hat{\psi}_{1}(\mathbf{r})\right\rangle\left\langle\hat{\psi}_{2}^{\dagger}(\mathbf{0}) \hat{\psi}_{2}(\mathbf{0})\right\rangle} \underset{r \rightarrow 0}{\sim} \frac{9 \pi \zeta}{10} \frac{1}{\left(k_{F} r\right)^{2}} .
$$

The scaling $g_{12}^{(2)}(r) \propto 1 /\left(k_{F} r\right)^{2}$ is expected for a gas with short-range interactions: when two particles (labeled $i$ and $j$ ) with opposite spins approach each other, the many-body wave function is proportional to the two-body scattering state between the particles $i$ and $j$ :

$$
\left.\Psi\left(\mathbf{r}_{1}, \ldots, \mathbf{r}_{\mathbf{N}}\right) \underset{\mathbf{r}_{\mathbf{i}} \rightarrow \mathbf{r}_{\mathbf{j}}}{\sim} A_{i j}\left(\frac{\mathbf{r}_{\mathbf{i}}+\mathbf{r}_{\mathbf{j}}}{2}, \mathbf{r}_{\mathbf{k}}\right)\right|_{k \neq i, j} \phi\left(\mathbf{r}_{\mathbf{j}}-\mathbf{r}_{\mathbf{i}}\right), \quad \text { where } \phi(\mathbf{r}) \underset{\mathbf{r} \rightarrow 0}{\sim} \frac{1}{r} .
$$

The coefficient $A_{i j}$ depends on the positions of all other particles. $g_{12}^{(2)}(r)$ is then equal the mean value 
of $|\Psi|^{2}$ for a fixed distance $\left|\mathbf{r}_{\mathbf{j}}-\mathbf{r}_{\mathbf{i}}\right|$, hence the $1 /\left(k_{F} r\right)^{2}$ behavior at low distance. The proportionality coefficient $9 \pi \zeta / 10$ between $g_{12}^{(2)}(r)$ and $1 /\left(k_{F} r\right)^{2}$ involves many-body physics. Since it quantifies the probability to find two atoms at short distances, $\zeta$ is often referred to as the contact coefficient.

Our experimental value $\zeta=0.91(5)$ is in agreement with a Fixed-Node Monte Carlo calculation of the pair correlation function giving $\zeta=0.95[192$ and with some recent experimental values. In 193 the static structure factor $S(q)$ was measured using inelastic Bragg scattering. $S(q)$ being the Fourier transform of the pair correlation function, $\zeta$ is given by the $S(q)$ behavior at high momentum:

$$
S_{t}(q) \underset{q \gg k_{F}}{\sim} \frac{128 \zeta}{175 \xi_{s}^{1 / 4}} \frac{k_{F}}{q},
$$

where we have written the formula for the trap-averaged structure factor $S_{t}(q)$ actually measured in the experiment [193]. The contact coefficient was also measured through other microscopic observables, such as the photo-association amplitude [38,194, the high- $k$ tail of the momentum distribution [189, 195], the high- $\nu$ tail of the radio-frequency spectrum 196 197, 195, 198, the photo-emission spectroscopy spectrum 156, and other macroscopic observables, namely the potential energy and the release energy 18,62 , 190, 191, 195]. These results are gathered in Table 5.1. While the agreement of our measurement with theory and with the dynamic structure factor measurement is excellent, the measurements from [195] are significantly different.

\begin{tabular}{|c|c|c|c|c|}
\hline \multicolumn{5}{|c|}{ Macroscopic observables } \\
\hline & Pressure & Energy & Energy & \\
\hline & Experiment 71 & Theory 66 & Experiment 195 & \\
\hline & $0.91(5)$ & 0.95 & $0.65(10)$ & \\
\hline \multicolumn{5}{|c|}{ Microscopic observables } \\
\hline Pair correlation & Structure factor & Momentum distrib. & RF spectrum & Photo-emission \\
\hline Theory 192 & Experiment 193 & Experiment 195 & Experiment 195 & Experiment 195 \\
\hline 0.95 & $0.92(3)$ & $0.91(5)$ & $0.67(7)$ & $0.63(7)$ \\
\hline
\end{tabular}

Table 5.1: List of $\zeta$ values determined from theory and experiment, and from macroscopic or microscopic observables. We only quote values performed in the unitary limit.

Finally, we obtain the second-order derivative coefficient $v=0.4(2)$. It was shown recently that $v$ must be positive [199], in agreement with our measurement.

\subsubsection{Equation of State of a Fermionic Superfluid from the Weakly-Interacting Regime}

In the BCS limit $\widetilde{\delta}, \nu \rightarrow-\infty$, interactions become weak and the gas pressure tends towards the ideal gas pressure corresponding to $h_{S}(\widetilde{\delta})=1$, or $\xi(\nu)=1$. The deviation from 1 is written as an expansion in powers of $\nu^{-1}$ :

$$
\xi(\nu)=1+\alpha_{\mathrm{MF}} \nu^{-1}+\alpha_{\mathrm{LY}} \nu^{-2}+\alpha_{\mathrm{BLY}} \nu^{-3} \ldots
$$

In order to extract these coefficients, we fit our data with the Padé-type functions, in the interval $\widetilde{\delta} \in\left[-\infty, \widetilde{\delta}_{\text {cutoff }}\right]$. Our best estimates of the coefficients $\alpha_{\mathrm{MF}}, \alpha_{\mathrm{LY}}$ and $\alpha_{\mathrm{BLY}}$ are given by extrapolating the fit results to $\widetilde{\delta}_{\text {cutoff }} \rightarrow-\infty$, knowing that for very small values of $\widetilde{\delta}_{\text {cutoff }}$ the result becomes wrong due to the small number of points used for the fit (see Fig 5.25).

The first coefficient $\alpha_{\mathrm{MF}}$ quantifies the mean-field interactions in a Fermi gas. We obtain an experimental value:

$$
\alpha_{\mathrm{MF}}=0.32(7),
$$


in agreement with the exact theoretical value 200 :

$$
\alpha_{\mathrm{MF}}=\frac{10}{9 \pi} \simeq 0.354
$$

The second coefficient $\alpha_{\mathrm{LY}}$ quantifies the first deviations to mean-field. It is also known exactly since the work of Lee and Yang in the 1950's [78]:

$$
\alpha_{\mathrm{LY}}=\frac{4(11-2 \log 2)}{21 \pi^{2}} \simeq 0.186
$$

and the value we extract from our data:

$$
\alpha_{\mathrm{LY}}=0.20(2)
$$

provides its first experimental verification.

Finally, our estimate of the coefficient beyond the Lee-Yang expansion:

$$
\alpha_{\mathrm{BLY}}=0.035(10),
$$

is also in agreement with more recent (non analytical) theoretical predictions $201,202,203 \mid 204$ :

$$
\alpha_{\mathrm{BLY}} \simeq 0.030
$$

(a)

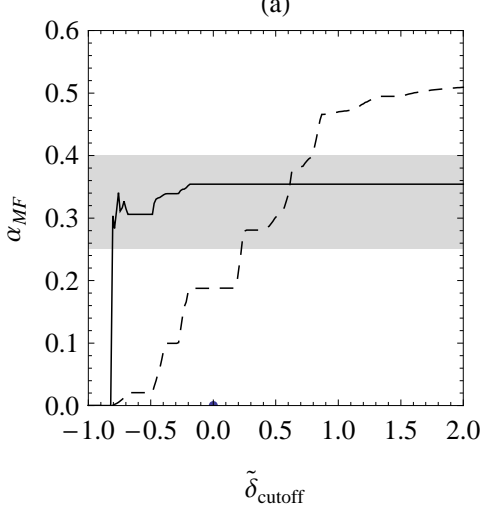

(b)

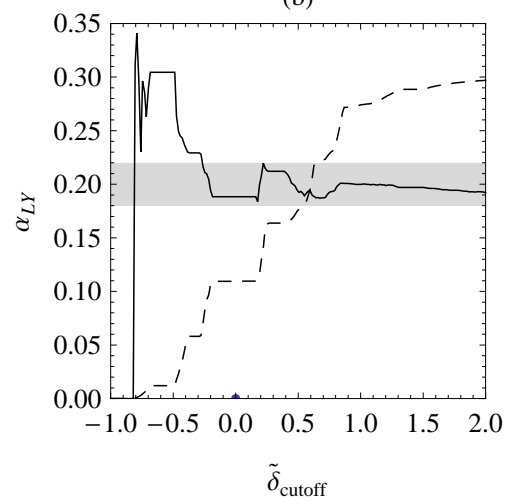

(c)

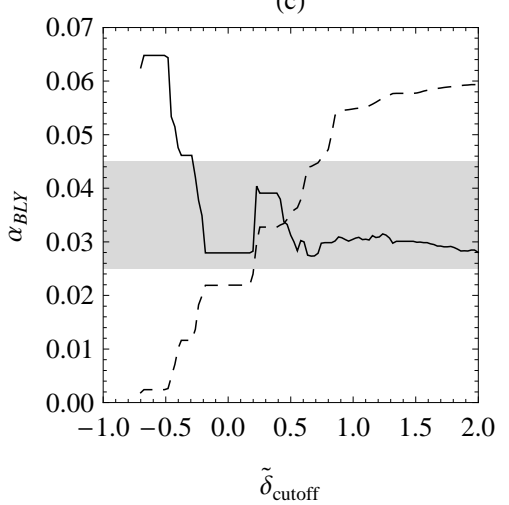

Figure 5.25: Fit results $\alpha_{\mathrm{MF}}(\mathrm{a}), \alpha_{\mathrm{LY}}$ (b) and $\alpha_{\mathrm{BLY}}(\mathrm{c})$, as a function of the cutoff $\widetilde{\delta}_{\text {cutoff }}$. The gray regions indicate our estimation of the actual values of these parameters. The dashed lines are proportional to the number of data points used for the fit. When $\widetilde{\delta}_{\text {cutoff }}$ is smaller than -0.3 a small number of points are used and the fit results become inaccurate.

Interestingly, the first non-universal corrections are also known exactly in the BCS limit and lead to a correction to $\xi(\nu)$ given by $201,202,203,204]$ :

$$
\delta \xi(\nu)=\frac{1}{6 \pi} \frac{r_{e}}{a} \nu^{-3}+\frac{1}{\pi}\left(\frac{a_{p}}{a}\right)^{3} \nu^{-3}+\ldots
$$

The effective range $r_{e}$ is a characteristics of the two-body $s$-wave scattering potential that quantifies the error made by replacing the true scattering potential by a pseudo-potential of scattering length $a$ [205]. Its value $r_{e} \sim 4.7 \mathrm{~nm}$ is very small for ${ }^{6} \mathrm{Li}$ gases $206 \mid 207$. The $p$-wave scattering length $a_{p}=1.8 \mathrm{~nm} 208$ describes low-energy $p$-wave interactions. Given the small values of these lengths compared with typical scattering length values $|a|>150 \mathrm{~nm}$, non-universal terms are completely negligible in our experiment. 


\subsubsection{Equation of State of a Bosonic Superfluid}

We now consider the BEC limit $\nu, \widetilde{\delta} \rightarrow+\infty$. In this regime atoms with opposite spins form deeply bound molecules, with a binding energy:

$$
E_{b}=-\frac{\hbar^{2}}{m a^{2}}, \quad\left|E_{b}\right| \gg E_{F}
$$

Composite molecules behave as point-like bosons and the gas is a molecular Bose-Einstein condensate. Interactions between dimers are described by a dimer-dimer scattering length $a_{d d} \simeq 0.60 a$, given by the exact resolution of the 4 -fermion scattering problem [16 209]. However, it is not clear to which extent the many-body ground state is merely equal to the equation of state of $n_{d}=n / 2$ bosons with short-range interactions described by a scattering length $a_{d d}$.

\section{Equation of State of a Gas of Point-Like Bosons}

Let us first remind the equation of state of a gas of $N=V n$ point-like bosons of mass $m$ and scattering length $a$. The equation of state, written as an expansion in powers of the interaction parameter $n a^{3}$, is universal up to the order $\sqrt{n a^{3}}$ :

$$
E / V=\frac{1}{2} \frac{4 \pi \hbar^{2} a}{m} n^{2}\left(1+\frac{128}{15 \sqrt{\pi}}\left(n a^{3}\right)^{1 / 2}+\ldots\right) .
$$

The first term is the mean-field interaction inside a Bose-Einstein condensate, and the second term is the Lee-Huang-Yang correction to mean-field, an exact result of many-body theory dating from the 1950's [76. Observing the Lee-Huang-Yang correction using ultracold Bose gases close to a Feshbach resonance is hindered by the enhancement of inelastic losses when approaching the strongly-interacting regime 15 . In 210 Bragg spectroscopy performed on ${ }^{85} \mathrm{Rb}$ demonstrated deviations to a mean-field behavior for large interaction strengths, but the connection with 5.14 remains indirect. Fermi gases, being stable in the entire BEC-BCS crossover, might be more suited for such studies.

The next order term is more complex. Its calculation requires the introduction of low- and highmomenta cutoffs [211]. A low $k$ values the natural length scale is the Bose-Einstein condensate's healing length $\xi=1 / \sqrt{8 \pi n a}$, while at high $k$ values one has to introduce non-universal length scales such as the potential's effective range. We end up with a correction [211]:

$$
E / V=\frac{1}{2} \frac{4 \pi \hbar^{2} a}{m} n^{2}\left(1+\frac{128}{15 \sqrt{\pi}}\left(n a^{3}\right)^{1 / 2}+8 \frac{4 \pi-3 \sqrt{3}}{3} n a^{3}\left(\log \left(n a^{3}\right)+B\left(a, r_{e}, D\right)\right)+\ldots\right),
$$

where the non-analytical log term is given by the low- $k$ divergence and is universal. The coefficient $B$ is not universal and depends on the precise shape of the interaction potential [212]. In [213] an explicit expression for $B$ is proposed, based on the study of a gas of hard spheres:

$$
B\left(a, r_{e}, D\right)=B_{0}+\frac{3}{8(4 \pi-3 \sqrt{3})}\left(\pi \frac{r_{e}}{a}+\frac{D}{12 \pi a^{4}}\right)
$$

involving the following parameters:

- the coefficient $B_{0} \simeq 6.03$ is expected to be universal, and was calculated in 214,213 ,

- the effective range $r_{e}$, introduced in section 5.5 .5 ,

- the three-body scattering hypervolume $D$, which is the equivalent of the scattering length $a$ for three-body interactions.

These parameters were calculated for a gas of hard spheres, giving $B \simeq 8.51$ [213]. For a gas with $r_{e} \ll a$, the three-boson problem exhibits a series of weakly bound states, the so-called Efimov states 215. The precise value of $D$ varies in a narrow range with the exact position of the Efimov spectrum: $7.11<B<7.18$ 214. 


\section{Equation of State of a Gas of Composite Bosons}

We now consider a two-component Fermi gas in the BEC limit, and the expansion of its equation of state in a powers of $n_{d} a_{d d}^{3}$. In $\mid 77$ it was shown that, up to order $\sqrt{n_{d} a_{d d}^{3}}$, the equation of state is accounted for by the Lee-Huang-Yang expansion (5.14) with the appropriate replacements:

$$
n \rightarrow n_{d}=n / 2, \quad a \rightarrow a_{d d}=0.60 a, \quad m \rightarrow m_{d}=2 m .
$$

For the next term, the effective range and scattering hypervolume relative to interactions between dimers are expected to be related to the scattering length $a$ by universal numbers, similarly to the effective scattering length between dimers $a_{d d}=0.60 a$. The dimer-dimer effective range can be viewed as the 'size' of the details of the effective dimer-dimer potential. This length scale being intuitively directly related to the dimer size $\sim a$, one expects $r_{e} \sim a$. In a recent paper, the energy spectrum of the four-fermion problem was computed and one infers from it the effective range between dimers $r_{d d}=0.13(2) a \simeq 0.2 a_{d d}$, i.e. a value comparable to $a_{d d}$ 216,217. The value of the effective three-dimer scattering hypervolume $D_{d d}$ is still unknown; its calculation would require the analysis of the six-fermion scattering problem. The dimer's composite nature is not expected to modify the coefficient in front of the $\log$ term in (5.15): the composite nature is invisible for large-wavelength phenomena $\lambda=2 \pi / k$ as soon as $\lambda$ is much larger than the dimer microscopic size $a$. On the other hand, it is not clear whether the coefficient $B$ is merely accounted for by equation 5.16 with the appropriate values for $r_{d d}$ and $D_{d d}$ given by the resolution of few-body scattering phenomena, or whether many-body effects modify this picture. Nevertheless, we expect the value of $B$ to be characteristic of Bose-Einstein condensates made of dimers of fermions interacting with short-range interactions, independently of the nature of the fermionic species.

\section{Measurement of the Equation of State of a Bosonic Superfluid}

We first consider the measurement of the Lee-Huang-Yang coefficient. In previous works [64 79], the Lee-Huang-Yang expansion (5.14) was shown to give a better description of the gas than the mean-field equation of state. Here we try to extract the expansion (5.14) more quantitatively . We fit our data for $\widetilde{\delta}>\widetilde{\delta}_{\text {cutoff }}$ with a Padé approximant whose asymptotic behavior in the BEC limit reads (see Appendix B.3 for an explicit expression):

$$
E / V=\frac{1}{2} \frac{4 \pi \hbar^{2} a_{d d}}{m_{d}} n_{d}^{2}\left(1+\alpha_{\mathrm{LHY}}\left(n_{d} a_{d d}^{3}\right)^{1 / 2}+\ldots\right) .
$$

We use the mean-field interaction in the BEC regime given by the first term in (5.14), and the values of $\xi_{s}$ and $\zeta$ previously determined, as constraints on the fitting functions. As shown in Fig $5.26 \mathrm{a}$, the fit result $\alpha_{\text {LHY }}$ depends on $\widetilde{\delta}_{\text {cutoff }}$ even for our data the most in the BEC regime. Our best estimate of the actual Lee-Huang-Yang coefficient is given by a linear extrapolation of the fit result $\alpha_{\text {LHY }}$ as a function of $\widetilde{\delta}_{\text {cutoff }}^{-1}$, towards the BEC limit $\widetilde{\delta}_{\text {cutoff }}^{-1} \rightarrow 0$ (see Fig $5.26 \mathrm{~b}$ ). We obtain:

$$
\alpha_{\mathrm{LHY}}=5.2(8) \text {, }
$$

in agreement with the exact value $\alpha_{\mathrm{LHY}}=128 / 15 \sqrt{\pi} \simeq 4.81$.

We then go one step beyond and extract from our data the unknown coefficient $B$. We use a more complex Padé approximant, described in Appendix B.3 and consistent with the expansion (5.15), to obtain (see Fig 5.26 ):

$$
B=7.2(8) \text {. }
$$

Interestingly, this value is close to the ones calculated for a gas of hard spheres [213 and for a gas with short-range interactions $[214], B \simeq 8.5$ and $B \simeq 7.1$, respectively.

To conclude this study of the superfluid equation of state, we show in Fig 5.27 the experimental data $h_{S}(\widetilde{\delta})$ together with the asymptotic behaviors extracted from it. 
(a)

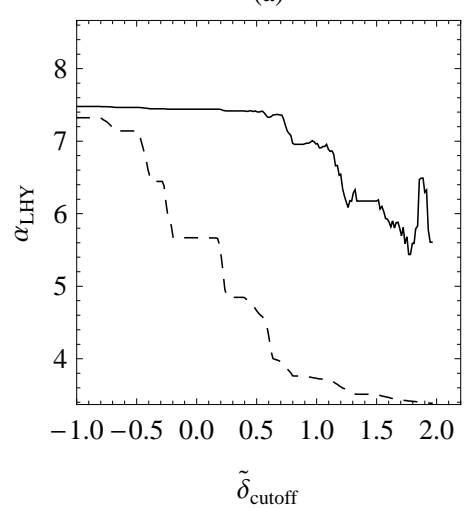

(b)

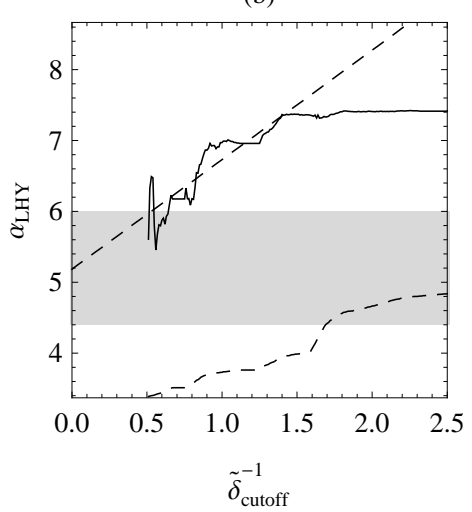

(c)

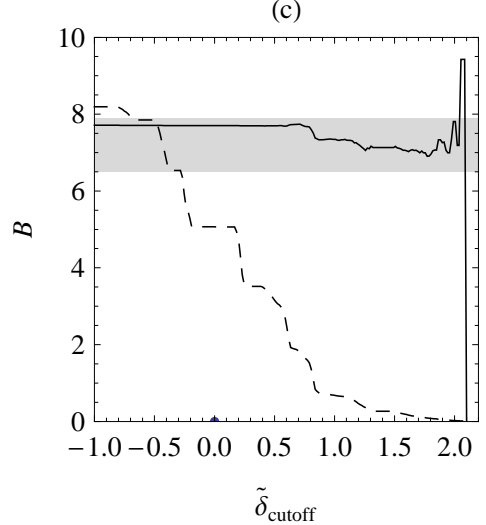

Figure 5.26: (a) Fit result $\alpha_{\text {LHY }}$ as a function of the cutoff $\widetilde{\delta}_{\text {cutoff }}$. The extrapolation to the BEC limit is more conveniently made by plotting $\alpha_{\mathrm{LHY}}$ as a function of $\widetilde{\delta}_{\text {cutoff }}^{-1}$, see (b). (c) Fit result $B$ as a function of $\widetilde{\delta}_{\text {cutoff }}$. The gray regions indicate our estimation of the actual values of these parameters, the dashed lines are proportional to the number of points used for the fit.
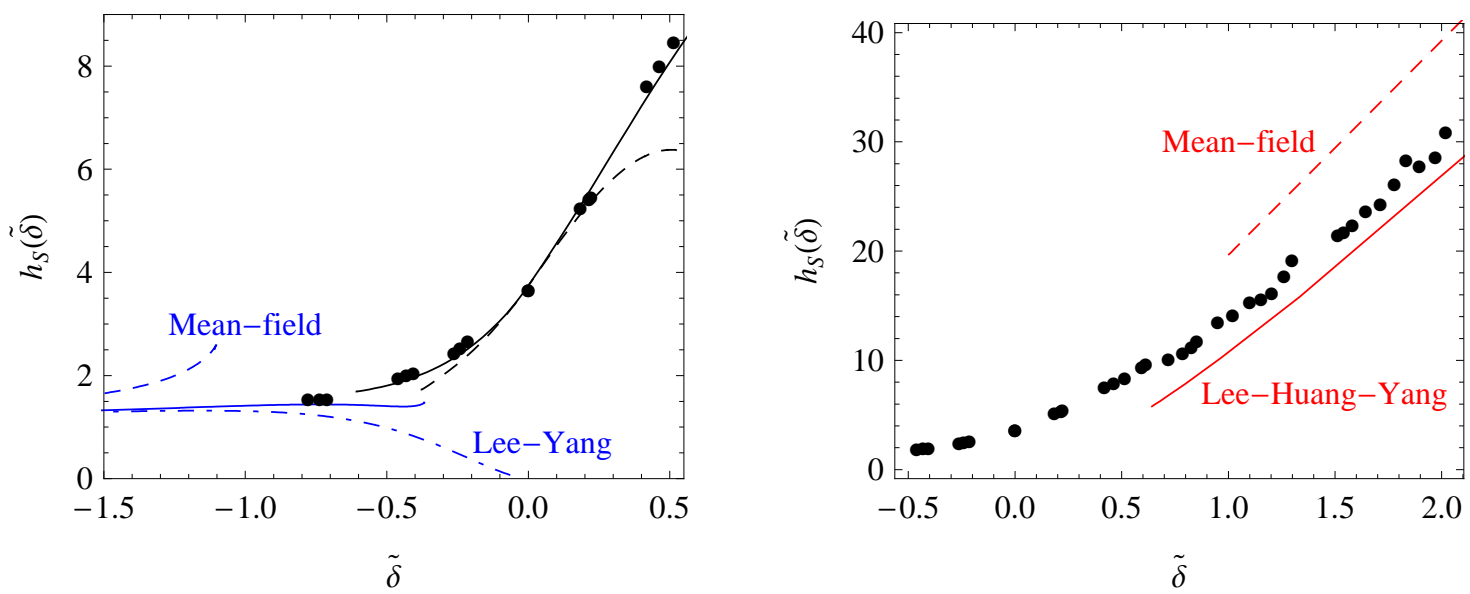

Figure 5.27: Equation of state $h_{S}(\widetilde{\delta})$ extracted from our data (black dots) compared with the following asymptotic behaviors: the blue lines are the expansions 5.13 in the BCS limit up to first order (dashed line), second order (dot-dashed line) and third order (solid line); the black lines are the expansions (5.12) around the unitary limit up to first order (dashed line) and second order (solid line); the red lines are the expansions (5.14) in the BEC limit up to first order (dashed line) and second order (solid line).

\subsection{Ground State of a Trapped Fermi Gas}

Here we make a comparison of our results with previous works discussed in section 3.1.1. In these works, the equation of state of the entire trapped gas is obtained through the measurement of the position of phase boundaries or the study of collective modes.

\subsubsection{Equation of State of a Trapped Spin-Imbalanced Fermi Gas}

\section{Equation of State in the Unitary Limit}

In the MIT experiment the radii $R_{1}, R_{2}, R_{S}$ of the different phases observed in a trapped spin-imbalanced Fermi gas are measured as a function of polarization $P[11649]$. We compare these measurements with the prediction given by the Fermi liquid equation of state 5.5 that accurately describes our measurements. 
In order to obtain the radii $R_{1}, R_{2}, R_{S}$ as a function of $P$, we calculate the atom numbers and radii as a function of the global chemical potential potentials $\mu_{i}^{0}$ :

$$
\begin{aligned}
N_{i} & =\int \mathrm{d} \mathbf{r} n_{i}\left(\mu_{1}, \mu_{2}\right), \quad \text { where } \mu_{j}=\mu_{j}^{0}-V(\mathbf{r}), \quad \text { and } n_{i}=\left.\frac{\partial P}{\partial \mu_{i}}\right|_{\mu_{j}}, \\
R_{1} & =\sqrt{\frac{2 \mu_{1}^{0}}{m \omega_{z}^{2}}}, \\
R_{2} & =R_{1} \sqrt{\frac{\eta^{0}-A_{0}}{1-A_{0}}}, \quad \text { where } A_{0}=-0.615, \\
R_{S} & =R_{1} \sqrt{\frac{\eta^{0}-\eta_{c}}{1-\eta_{c}}}, \quad \text { where } \quad \eta_{c}=0.065 .
\end{aligned}
$$

The curves $R_{i}(P)$ are then obtained as parametric curves by varying the global chemical potentials. As shown in Fig 5.28, they significant deviate from the data from [116], where the radii were measured after a time of flight, assuming a hydrodynamic expansion. This is probably due to the fact that the gas does not fully expands according to hydrodynamics, especially in the outer shell where the gas is fully polarized and no collision occurs. The radii measured from in situ density profiles [49] are in excellent agreement with the Fermi liquid equation of state.

(a)

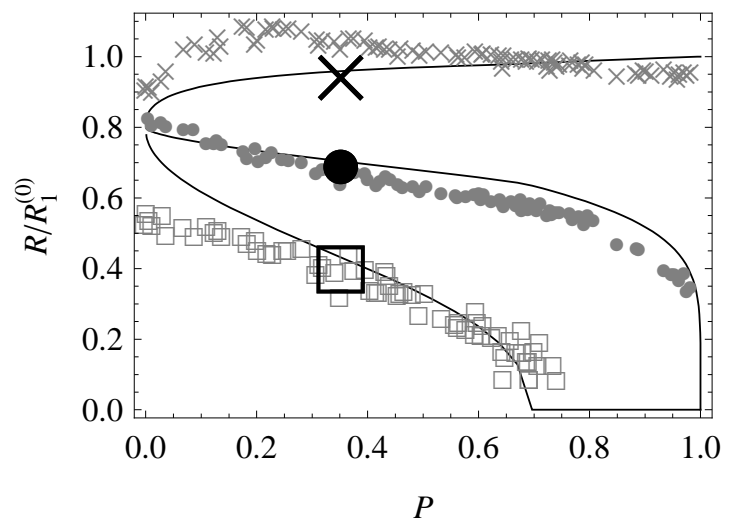

(b)

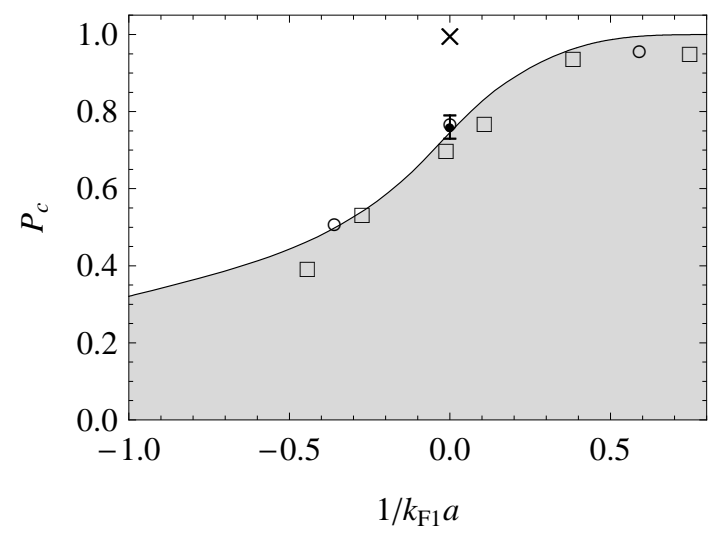

Figure 5.28: (a) Radii $R_{1}, R_{2}, R_{S}$ of the different phases observed in a trapped spin-imbalanced Fermi gas, in the unitary limit. The radii are normalized to the Thomas-Fermi radius $R_{1}^{(0)}$ of an ideal gas with $N_{1}$ atoms. The data in gray is inferred from the cloud profiles after a time of flight [116]. The large black symbols are obtained from in situ images [49]. The solid lines are calculated using the Fermi liquid equation of state 5.5 . (b) Critical polarization $P_{c}$ as a function of $1 / k_{F 1} a$, where $k_{F 1}$ is the Fermi momentum at the bottom of the trap of an ideal gas with $N_{1}$ atoms. The solid line is calculated from the theoretical profiles accounting for our data (see text). It agrees with the critical polarization values determined by the MIT group (open squares: [54], open circles: [218]) and by our group [99] (point with an error bar), but disagrees with the one of the Rice group [97] (cross).

\section{Critical Polarization in the BEC-BCS Crossover}

In Fig 5.28 , we see on the case of the unitary limit that the superfluid core disappears above a critical polarization $P_{c}$. The first characterization of the normal/superfluid phase transition was provided by the measurement of $P_{c}$, in the unitary limit [54, 97, 218, 99, and in the BEC-BCS crossover [54, 218]. We calculate the critical polarization in the BEC-BCS crossover using the Fermi liquid equation of state 5.5 
for the normal phase and a fit of the superfluid equation of state described in Appendix B.3. As shown in Fig 5.28 , the Fermi liquid equation of state accounts for most measurements, apart from the Rice university measurement $P_{c} \simeq 1$ in the unitary limit, and the data in the region $1 / k_{F 1} a>0.5$, where we did not check experimentally the validity of the Fermi liquid equation of state.

\subsubsection{Collective Modes of a Balanced Fermi Gas}

In this section we compare the equation of state of a balanced superfluid determined from our data with the collective mode frequency measurements from 64. In section 3.1.1 we discussed the relationship between the collective mode frequencies and the superfluid equation of state provided by a hydrodynamic description of the collective modes [115. Using fits of our data (see Appendix B.3), we calculate the radial breathing mode frequency $\omega$ given by equation (3.1), and make the comparison with the experimental data in Fig 5.29 . The agreement with the experiment is much better that the prediction using a BCS meanfield equation of state. Since our data are close to the Fixed-Node Monte Carlo calculations from 66, it is not surprising that the calculated frequencies are close to the ones previously calculated from the Monte Carlo data (see [64]).

As mentioned earlier, we see that discriminating between Fixed-Node Monte Carlo calculations and BCS mean-field theory required to reach a $\sim 0.5 \%$ accuracy on the frequency measurement. This has to be compared with the large difference between both theories in the variable $h_{S}(\widetilde{\delta})$ (see Fig 5.20. Similarly, the Lee-Huang-Yang correction, which leads to the following asymptotic behavior in the BEC limit $219,220,221$ :

$$
\omega=2 \omega_{r}\left(1+\alpha\left(k_{F} a\right)^{6 / 5}+\ldots\right), \quad \alpha=\frac{215^{6 / 5}}{2^{48 / 5}}\left(a_{d d} / a\right)^{6 / 5} \simeq 0.1
$$

where $\omega_{r}$ is the transverse trapping frequency, is difficult to observe from a collective mode study. The frequency upshift $\omega>2 \omega_{r}$ observed in the BEC limit is consistent with this behavior, but does not allow one to extract the value of the Lee-Huang-Yang term.

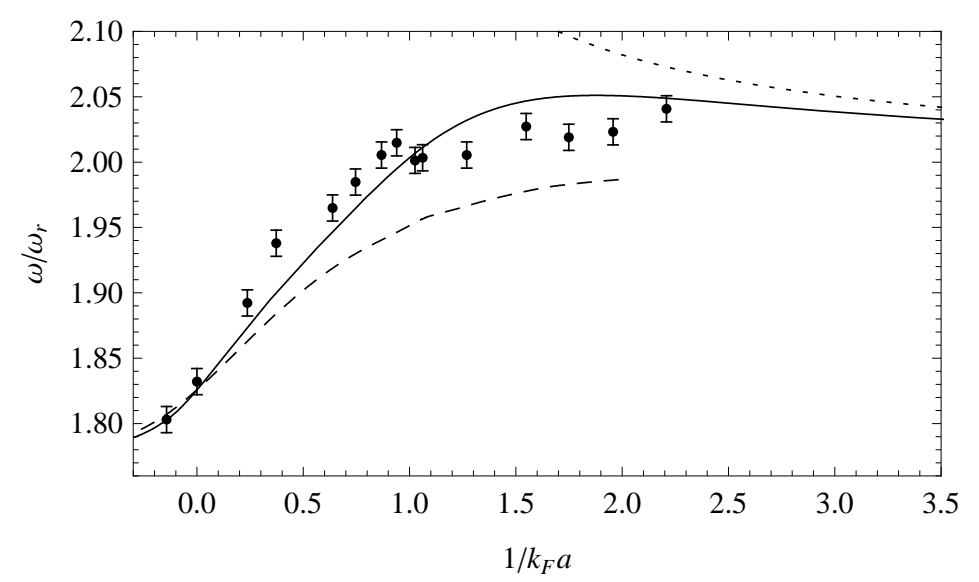

Figure 5.29: Radial breathing mode frequency from the Innsbruck experiment 64, compared with the hydrodynamic prediction using a mean-field BCS equation of state (dashed line), and the equation of state determined from our data (solid line). The dotted line is the Lee-Huang-Yang expansion (5.18).

To conclude, the equation of state of a homogeneous Fermi gas deduced from our study is consistent with most previous works on trapped gases. 


\subsection{Molecular Physics Beyond the Scope of this Work}

The parameter range addressed in our work is limited to $\delta_{1}<0.65$. Qualitatively new features in the phase diagram are expected for $\delta_{1} \gtrsim 1$. We give here a short review of this still largely unexplored physics, as a perspective for future work.

\subsubsection{Polarized Superfluid}

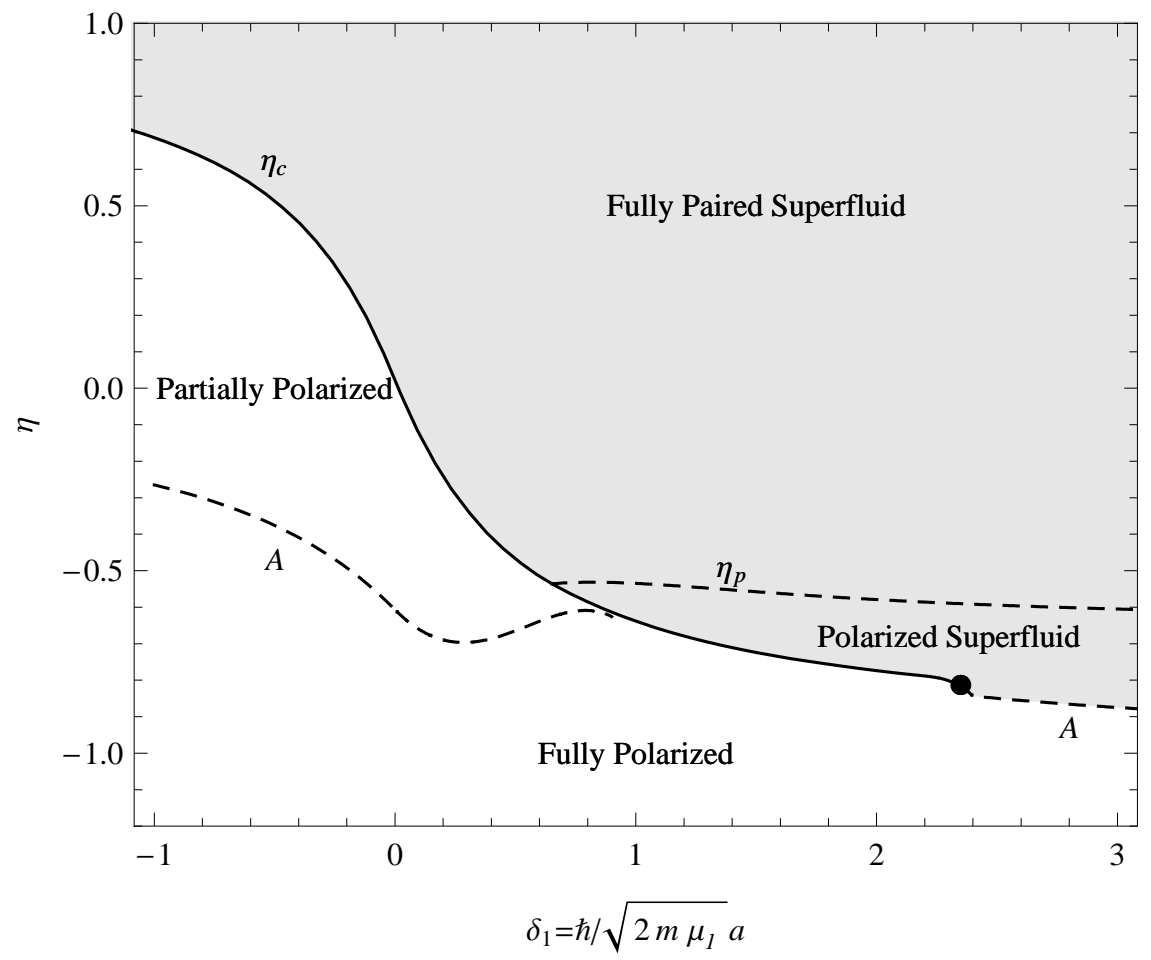

Figure 5.30: Phase diagram of a two-component Fermi gas in the plane $\left(\delta_{1}, \eta\right)$. The black dot is the tri-critical point. The gray region corresponds to a superfluid phase. We subtracted for clarity $-2 \delta_{1}^{2}$ to $\eta$ for $\delta_{1}>0$.

We have shown that, in the superfluid phase and for $\delta_{1}<0.65$, the gas remains unpolarized when one imposes a chemical potential difference. However, one expects that in the BEC regime a mixture of majority atoms and a fully paired molecular condensate can be stable (see Fig 5.30 ). This can easily be shown using a mean-field description, valid in the BEC limit. Let us consider a homogeneous mixture of molecules and unbound fermions, with respective densities $n_{b}$ and $n_{f}$. The system is thermodynamically stable if the compressibility matrix $\left(\partial^{2} e / \partial n_{i} \partial n_{j}\right)$, where $e$ is the energy density, is positive. Using the mean-field energy density [222]:

$$
e\left(n_{f}, n_{b}\right)=\frac{3}{5} n_{f} E_{f}+n_{b} E_{b}+\frac{1}{2} \frac{4 \pi \hbar^{2} a_{d d}}{2 m} n_{b}^{2}+\frac{4 \pi \hbar^{2} a_{a d}}{4 m / 3} n_{b} n_{f},
$$

we obtain the stability condition:

$$
n_{f}^{1 / 3} \leq \frac{\left(6 \pi^{2}\right)^{2 / 3}}{12 \pi} \frac{a_{d d}}{a_{a d}^{2}}
$$

This criterion is similar to the thermodynamic stability domain of ${ }^{3} \mathrm{He}-{ }^{4} \mathrm{He}$ mixtures at low temperature (see Fig 5.31). Therefore a molecular BEC can be polarized by imposing a chemical potential difference. It remains unpolarized as long as $\mu_{1}$ is smaller than the superfluid chemical potential $\mu=\left(\mu_{1}+\mu_{2}\right) / 2$ 
plus the single-particle excitation gap $\Delta_{0}$, i.e. for

$$
\eta \geq \eta_{p}=1-2 \frac{\Delta_{0}}{\mu_{1}} .
$$

When $\eta$ is decreased below $\eta_{p}$, the gas becomes polarized but remains superfluid (see Fig 5.30).

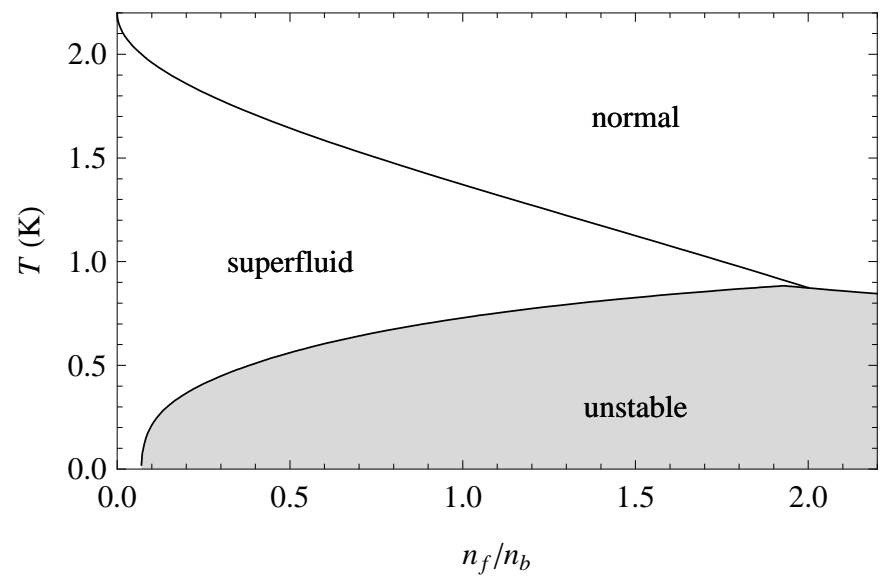

Figure 5.31: Phase diagram of a ${ }^{3} \mathrm{He}^{4} \mathrm{He}$ mixture at low temperature [224]. At $T=0$ the maximum ${ }^{3} \mathrm{He}$ density if $n_{f} \simeq 0.07 n_{b} 225$.

\subsubsection{Polaron to Molecule Transition in the Impurity Problem}

The large imbalance limit was addressed in section 5.1 .2 by considering the ground state of the impurity problem. It was expressed as the sum of the non-interacting ground state plus states with one particlehole excitation. This approach does not account for the ground state in the BEC limit, where we expect the minority atom to bind with one majority atom into a deeply bound molecule. This state is very different from the polaron state, in the sense that it cannot be expressed as the non-interacting ground state plus several particle-hole excitations.

In the mean-field approach, the chemical potential ratio of the impurity problem is obtained from (5.19) through [85]:

$$
A\left(\delta_{1}\right)=\lim _{n_{b} \rightarrow 0} \frac{\mu_{2}}{\mu_{1}}=-2 \delta_{1}^{2}-1+\frac{1}{\pi} \frac{a_{a d}}{a} \delta_{1}^{-1} .
$$

The molecular binding energy was calculated, beyond this mean-field approach, using a diagrammatic Monte Carlo calculations [85], an analytic theory involving up to two particle-holes excitations in the Fermi sea [86, and variational ansatz's 226 180, all results being in very good agreement (see Fig 5.30).

Polaronic and molecular impurities are expected to behave very differently when considering small but macroscopic minority atom numbers. Indeed, while polarons are fermionic quasi-particles (as clearly shown in section 5.4 , a set of molecules is expected to form a molecular Bose-Einstein condensate. In other words, these two kind of impurities obey different quantum statistics.

The transition between the two regimes occurs when the ground state of the impurity problem changes from a polaron-type to a molecular-type quantum state, at $\delta_{1}=0.91(2) 85$ (see Fig 5.30 .

${ }^{\dagger \dagger}$ In the BEC limit, one can use the mean-field energy 5.19 to calculate the asymptotic value of $\eta_{p}$ 223.:

$$
\eta_{p}=\lim _{n_{f} \rightarrow 0} \frac{\mu_{2}}{\mu_{1}}=-2 \delta_{1}^{2}-1+\frac{a_{d d}}{3 a_{a d}}
$$




\subsubsection{Thermodynamic Instability of the Impurity Problem}

Using a mean-field approach, we showed in section 5.7.1 that a spin-imbalanced gas is unstable for large extra majority atom densities. In the limit of vanishing minority atom density, which corresponds to the impurity problem, the stability condition can be written as 67 虾

$$
\delta_{1}>\delta_{1 c}=\frac{9}{4 \pi} \frac{a_{a d}^{2}}{a_{d d} a} \simeq 1.7 .
$$

In the region $\delta_{1}<\delta_{1 c}$, the transition between the fully and partially polarized regions thus becomes of first order (see Fig 5.30 ..$\delta_{1 c}$ is thus a tri-critical point. The instability of the impurity problem casts doubt on the possibility to directly observe the polaron/molecule transition.

To conclude this chapter, we measured the equation of state of the ground state of a two-component attractive Fermi gas with arbitrary spin-imbalance and interactions. The universal character of this equation of state makes it a relevant quantity for the description of other quantum many-body systems, such as the crust of neutron stars. We characterized the superfluid equation of state in the entire BECBCS crossover. Our observations are in very good agreement with Fixed-Node Monte-Carlo calculations and are used to extract several physical quantities such as the Lee-Yang and Lee-Huang-Yang corrections to mean-field. We showed that the partially polarized phase is well accounted for by a Landau Fermi liquid description. We obtain from our data the value of the effective mass of the associated Landau quasi-particles, the Fermi polaron.

In the last section we showed that qualitatively different physics is expected to occur in the deep BEC regime that is not addressed in this work. The measurement of the position of the tri-critical point, and of the polaron to molecule transition could be the subject of future work. It would also be interesting to investigate the existence of exotic phases such as the FFLO state, expected to be stable on the BCS side of the resonance (in a rather small window between the Fermi liquid and superfluid states). The signatures of these states on the equation of state are expected to be rather small [229, 230 and would require to improve the precision of our measurement or combine it with the measurement of other observables such as density correlations/fluctuations 231 230.

\footnotetext{
${ }^{\ddagger}$ Recently the first correction to 5.19 was obtained analytically 227 :

$$
e\left(n_{f}, n_{b}\right)=\frac{3}{5} n_{f} E_{f}+n_{b} E_{b}+\frac{1}{2} \frac{4 \pi \hbar^{2} a_{d d}}{2 m} n_{b}^{2}+\frac{4 \pi \hbar^{2} a_{a d}}{4 m / 3} n_{b} n_{f}\left(1+\frac{8 \log 2-3}{2 \pi} \delta_{1}^{-1}\right),
$$

which leads to

$$
\delta_{1 c}=\frac{9}{4 \pi} \frac{a_{a d}^{2}}{a_{d d} a}+\frac{4(8 \log 2-3)}{3 \pi} \frac{a_{a d}}{a} \simeq 2.9 .
$$

BCS mean-field theory predicts $\delta_{1 c}=2.4$ 228, 229] and Fixed-Node Monte Carlo calculations, expected to be the most
} precise result, give $\delta_{1 c}=1.7[67$. 


\section{Chapter 6}

\section{Axial Breathing Modes of a Spin-Imbalanced Fermi Gas}

In this chapter we describe a study of collective modes of a spin-imbalanced Fermi gas in the unitary limit $[99]$. Collective modes of trapped ultracold gases are the counterpart of sound waves in homogeneous systems. Their study provides a first-level understanding of the gas dynamics, and can be used to reveal for example characteristic features superfluidity through the observation of second sound 232 . The measurement of collective oscillations frequencies is a precision tool for the experimental study of trapped ultracold gases. In some situations the mode frequency can be related to physical quantities difficult to probe otherwise. An important example is the angular momentum measurement through a precession of a radial quadrupole excitation 233, 39. Moreover, as shown in section 3.1.1 the frequency of some collective modes can directly be related to the equation of state of the trapped gas and therefore its determination from experiment provides a benchmark for many-body theory [115 64].

The collective modes of a spin-imbalanced Fermi gas are rather complex. We saw in Chapter 5 that a phase separation occurs in a trapped gas: a superfluid core occupies the trap bottom (for a polarization $P<P_{c} \simeq 0.75$ ), and is surrounded by a partially polarized intermediate shell and a fully polarized outer rim. The dynamic behavior of these phases strongly differ. Indeed, the fully paired core is expected to evolve according to hydrodynamics as a consequence of superfluidity, while the fully polarized shell reacts as a collisionless ideal gas. Therefore, we expect the dominant collective mode frequencies to be typical of a hydrodynamic behavior when the superfluid core is large (i.e. for small polarizations), while collisionless frequencies are expected at the lowest minority concentrations, when the gas is essentially fully polarized. It is also tempting to think that the crossover between these two regimes, when one varies the spin polarization, may reveal the polarization threshold $P=P_{c}$ for the superfluid core disappearance.

In this work we consider the axial breathing modes of a gas held in an elongated trap. A twocomponent gas exhibits two axial breathing eigenmodes. The first collective mode corresponds to an in-phase oscillation of both spin components. It is the only low-lying collective mode encountered for a paired superfluid, since an out-of-phase excitation requires to break pairs and costs a large energy (on the order of the gap). We will see that, when the gas polarization is increased, this mode progressively evolves from a hydrodynamic to a collisionless behavior. The second mode corresponds to oscillations of both spin component with different phases. In the strong polarization limit, it corresponds to a minority component oscillation in an unperturbed Fermi sea, the oscillation being strongly affected by interactions. In particular interactions modify the minority atoms inertia through a mass renormalization, and we will thus extract the effective mass value from the frequency of this mode. 


\subsection{Hydrodynamic Behavior of a Balanced Fermi Gas}

Before addressing the effect of spin polarization, we first consider the dynamics of a spin-balanced Fermi gas in order to introduce the hydrodynamic equations that will be invoked in the rest of the chapter. We study the oscillation of a balanced Fermi gas which is deeply evaporated, up to temperatures much smaller than the superfluid transition temperature. The gas is thus a fully paired superfluid, whose dynamics is expected to be described by the laws of hydrodynamics, previously introduced in section 3.1.1:

$$
\begin{aligned}
\frac{\partial}{\partial t} n & =-\nabla \cdot(n \mathbf{v}) \\
m \frac{\partial}{\partial t} \mathbf{v} & =-\nabla\left(\frac{1}{2} m \mathbf{v}^{2}+\mu(n)+V\right)
\end{aligned}
$$

where $n$ is the total density and $\mu(n)=\xi_{s} \hbar^{2} / 2 m\left(3 \pi^{2} n\right)^{2 / 3}$ in the unitary limit.

\subsubsection{Scaling Ansatz Solution of the Hydrodynamic Equations}

We are going to study the gas dynamics in two situations:

- The optical trap is suddenly switched off and the cloud expands in the saddle potential created by the magnetic field curvature. The confining potential can be written as:

$$
\omega_{z}(t)=\omega_{z}^{0}, \quad \omega_{r}(t)=\left\{\begin{array}{ll}
\omega_{r}^{0}, & t<0 \\
-\omega_{z}^{0} / \sqrt{2}, & t>0
\end{array} .\right.
$$

- The gas is excited by switching the current in the curvature coils off during $\sim 1$ ms. The bias magnetic field is consequently brought to the BCS side of the resonance. After the gas is excited the confining potential and bias magnetic field are brought back to the values before excitation.

Both situations belong to the more general problem of solving the hydrodynamic equations in a harmonic trap with time-dependent frequencies. As first shown for the dynamics of Bose-Einstein condensates in 234,235], the scaling $\mu \propto n^{2 / 3}$ at equilibrium implies that an exact class of solutions of these equations is provided by a scaling ansatz $153{ }^{*}$

$$
n(x, y, z, t)=\frac{1}{b_{z}(t) b_{r}(t)^{2}} n^{0}\left(\frac{x}{b_{r}(t)}, \frac{y}{b_{r}(t)}, \frac{z}{b_{z}(t)}\right),
$$

where $n^{0}(x, y, z)$ is the equilibrium density profile. The density profile 6.1 is a solution of the hydrodynamic equations equations, provided the scaling factors $b_{i}(t)$ solve the differential equations [153]:

$$
\begin{aligned}
& \ddot{b}_{z}(t)=-\omega_{z}(t)^{2} b_{z}(t)+\frac{\omega_{z}(0)^{2}}{b_{z}(t)^{5 / 3} b_{r}(t)^{4 / 3}} \\
& \ddot{b}_{r}(t)=-\omega_{r}(t)^{2} b_{r}(t)+\frac{\omega_{r}(0)^{2}}{b_{z}(t)^{2 / 3} b_{r}(t)^{7 / 3}} .
\end{aligned}
$$

\subsubsection{Hydrodynamic Expansion}

In this section we study the expansion of the superfluid after the optical is suddenly switched off. The bias and curvature magnetic fields are left on, and the superfluid evolves in a saddle potential. The initial trapping frequencies are $\omega_{z}^{0} / 2 \pi=37 \mathrm{~Hz}$ and $\omega_{r}^{0} / 2 \pi=600 \mathrm{~Hz}$. In Fig 6.1 we plot experimental results for the time evolution of the cloud axial and radial sizes. A numerical resolution of equations $6.2,6.3$

*We mention that in the case of an isotropic harmonic trap, it was shown that such a scaling ansatz provides an exact solution of the gas dynamics 112 . 
accurately describes the superfluid dynamics even $5 \mathrm{~ms}$ after the optical trap switch off, despite a radial size increase by a factor $\simeq 25$. The decrease in axial size is an eloquent consequence of the hydrodynamic behavior, by opposition to the obvious solution $b_{z}(t)=1$ for a ballistic expansion into a potential whose axial confinement is unchanged. This behavior is consistent with previous observations of a hydrodynamic expansion for a unitary gas 17 236 (in which the axial confinement is switched off together with the optical trap).

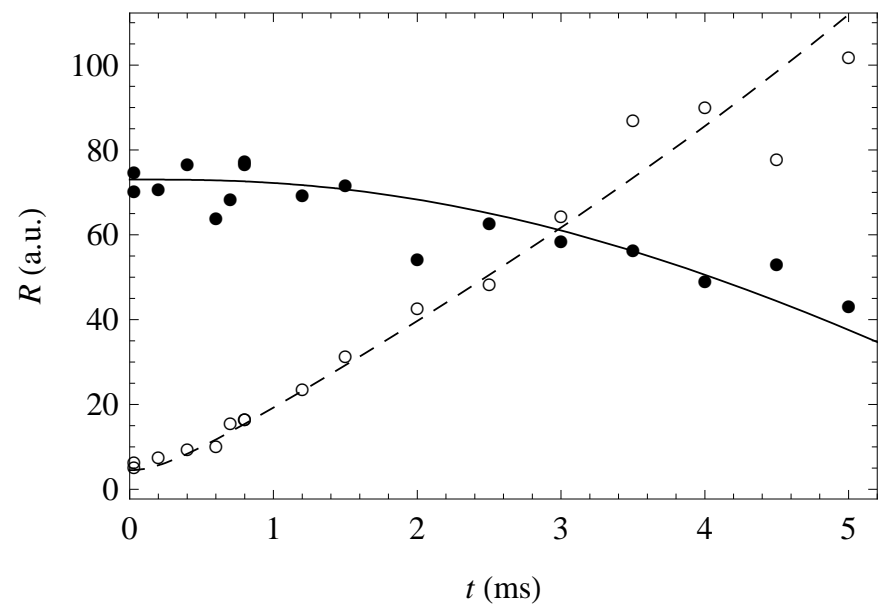

Figure 6.1: Time evolution of the axial (radial) cloud radius of a superfluid expanding in a saddle potential (black (open) dots). The solid (dashed) line is the hydrodynamic prediction.

\subsubsection{High- $Q$ Axial Breathing Mode}

In this section we present the response of a trapped superfluid to a weak perturbation. We switch off the curvature coils current during $0.5 \mathrm{~ms}$, come back to the initial current, and take an in situ image of the cloud after a variable wait time. In Fig 6.2 we plot the time evolution of cloud axial size over more than $600 \mathrm{~ms}$.

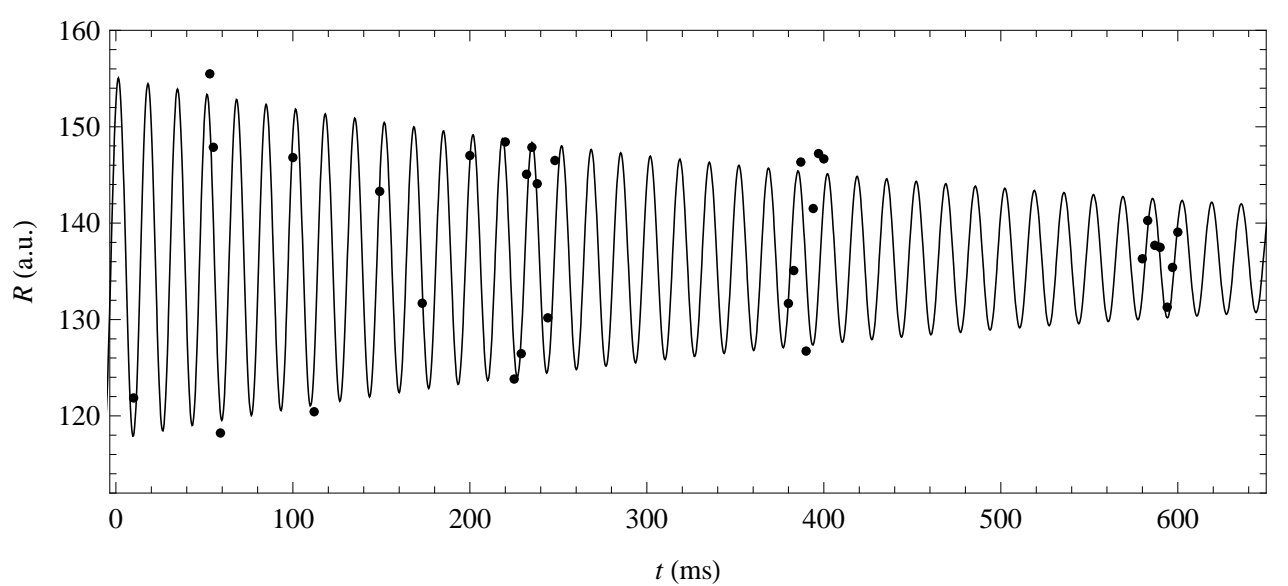

Figure 6.2: Cloud size time evolution (black dots). The solid line is a fit with a damped cosine.

The oscillation frequency of the axial radius is calculated by linearizing equations (6.2), 6.3) around the equilibrium positions $b_{i}=1$. Writing $b_{i}=1+a_{i} e^{i \omega t}, a_{i} \ll 1$, we obtain the linear system

$$
\left(\begin{array}{c}
-\omega^{2} a_{z} \\
-\omega^{2} a_{r}
\end{array}\right)=\left(\begin{array}{c}
-\omega_{z}^{2} a_{z}+\omega_{z}^{2}\left(5 / 3 a_{z}+4 / 3 a_{r}\right) \\
-\omega_{r}^{2} a_{r}+\omega_{r}^{2}\left(2 / 3 a_{z}+7 / 3 a_{r}\right)
\end{array}\right) .
$$


This systems admits two solutions, namely the axial (radial) breathing mode $\omega=\sqrt{12 / 5} \omega_{z}(\omega=$ $\left.\sqrt{10 / 3} \omega_{r}\right)$, for an elongated trap $\left(\omega_{r} \gg \omega_{z}\right)$.

Fitting our experimental data with a damped cosine, we obtain the axial breathing mode frequency $\omega=1.54(1) \omega_{z}$, in agreement with the theoretical value $(\sqrt{12 / 5} \simeq 1.549)$. The oscillation damping rate is very small, and corresponds to a quality factor $Q \simeq 200$. This value is comparable to the low damping rates previously reported in the Innsbruck experiment 33 .

The oscillation amplitude can easily be modeled. The excitation scheme chosen for this experiment has two effects:

- The axial frequency $\omega_{z}$ drops to 0 during the $0.5 \mathrm{~ms}$ excitation.

- The bias magnetic field becomes very large $B_{0} \simeq 1100 \mathrm{G}$. The gas, switched to the BCS side of the resonance, becomes weakly interacting and its equation of state reads $\mu=\hbar^{2} / 2 m\left(3 \pi^{2} n\right)^{2 / 3}$, i.e. the chemical potential is $\xi_{s}^{-1}$ times larger than the one of a unitary gas with the same density. However, the gas remains superfluid and its motion is still described by hydrodynamic equations.

Equations (6.2), (6.3) thus read, during the excitation:

$$
\begin{aligned}
& \ddot{b}_{z}(t)=\xi_{s}^{-1} \frac{\omega_{z}^{2}}{b_{z}(t)^{5 / 3} b_{r}(t)^{4 / 3}} \\
& \ddot{b}_{r}(t)=-\omega_{r}^{2} b_{r}(t)+\xi_{s}^{-1} \frac{\omega_{r}^{2}}{b_{z}(t)^{2 / 3} b_{r}(t)^{7 / 3}} .
\end{aligned}
$$

A numerical resolution of this system of differential equations leads to an oscillation amplitude equal to $\Delta R_{z} / R_{z}^{0}=0.26$ (peak-peak amplitude/mean value), in excellent agreement with our observations $\Delta R_{z} / R_{z}^{0}=0.28$. The change in trapping frequency and the change in bias field have a comparable contribution to the oscillation amplitude.

\subsection{In-Phase Axial Breathing Mode}

\subsubsection{Observation of the In-Phase Mode}

We now discuss the effect of polarization on the axial breathing mode. We evaporate a spin-imbalanced Fermi gas in the unitary limit up to a trap depth identical for all polarizations. The majority atom number $N_{1} \simeq 10^{5}$ does not depend much on polarization, while the minority atom number is scanned in a range $1000 \lesssim N_{2}<N_{1}$. The excitation procedure is identical to the one described above for a balanced gas. We fit the integrated density profiles for both spin components with Thomas-Fermi formulas:

$$
\bar{n}_{i}(z)=\alpha_{i}\left(R_{i}^{2}-z^{2}\right)^{5 / 2}, \quad i=1,2 .
$$

In order to study the gas dynamics at a given polarization $P$, we post-select the cloud images corresponding to polarizations in a window $[P-\Delta P / 2, P+\Delta P / 2]$, with $\Delta P$ chosen between 0.025 and 0.06 .

In Fig. 6.3 we plot $R_{2}$ as a function of $R_{1}$ in order to see whether both spin components oscillate in phase. Except for large polarizations $P \gtrsim 0.8$, the radii oscillations around the mean values $R_{i}^{0}$ are proportional:

$$
R_{2}-R_{2}^{0} \propto R_{1}-R_{1}^{0}
$$

This is a clear signature that both spin components oscillate in phase.

For $P>0.8$ we will see in section 6.3 that the weight of out-of-phase mode is more significant and strongly affects the time evolution of the minority radius. However, we expect the effect on the majority component to be much smaller. Therefore the time evolution of the majority radius is always dominated 

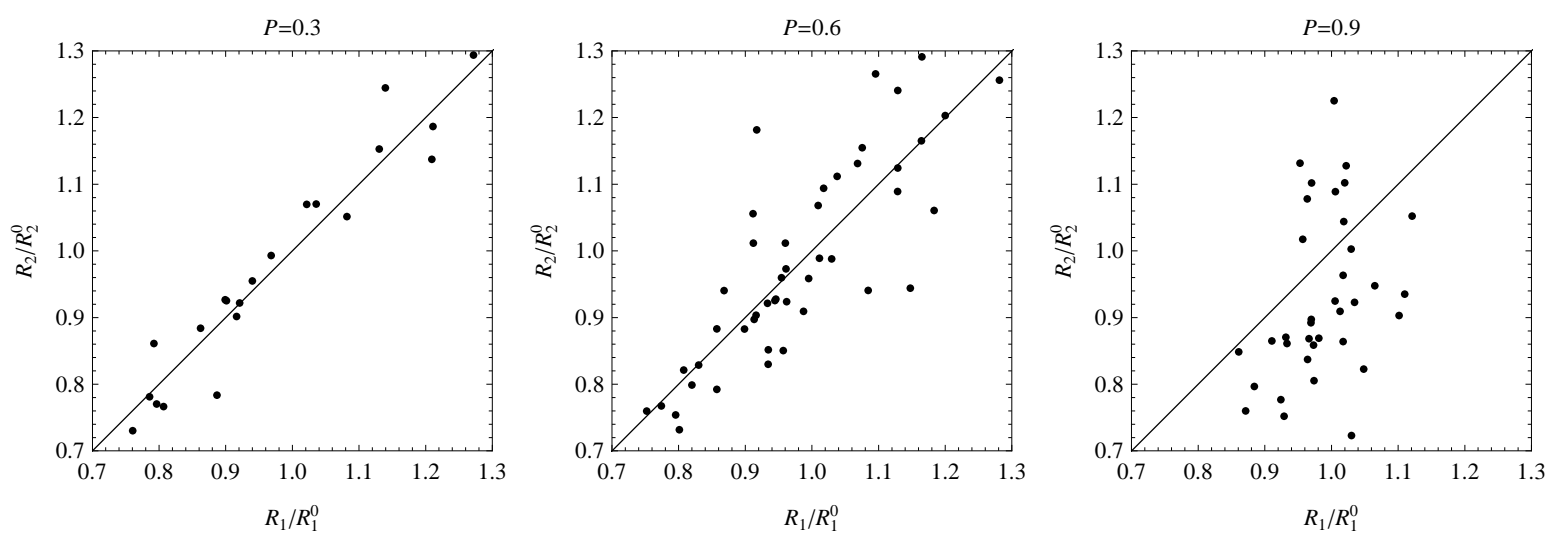

Figure 6.3: Radius $R_{2}$ as a function of the radius $R_{1}$, for three polarizations $P=0.3, P=0.6$ and $P=0.9$. The radii are normalized to the mean value $R_{i}^{0}, i=1,2$. The proportionality for $P=0.3$ and $P=0.6$ shows that both spin components oscillate in phase.

by the in-phase mode, even at large polarizations. We thus extract the in-phase mode characteristics on the majority component.

We fit the time evolution of the majority radius with a damped cosine (see Fig 6.4):

$$
R_{1}(t)=R_{1}^{0}\left(1+A_{1} e^{-\gamma_{1} t} \cos \left(\omega_{1} t+\phi_{1}\right)\right) .
$$

The constants $A_{1}, \omega_{1}, \phi_{1}$ and $\gamma_{1}$ are the in-phase mode amplitude, frequency, phase with respect to the excitation, and damping rate.
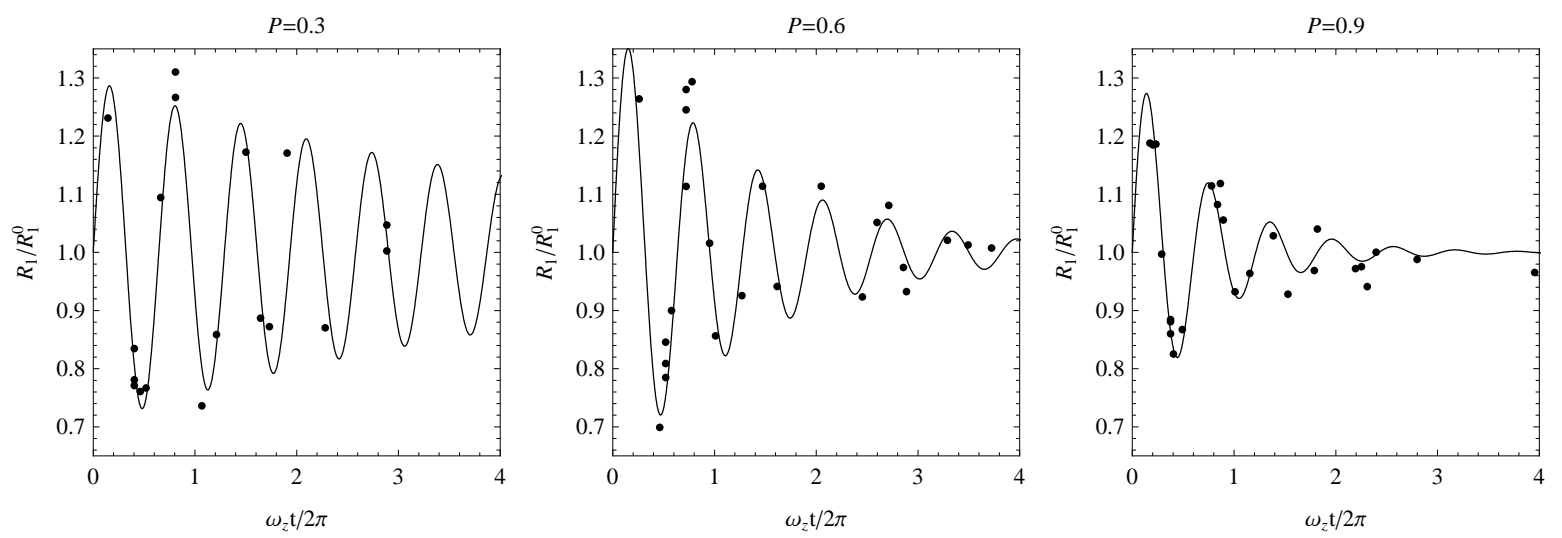

Figure 6.4: Time evolution of the majority radius, for $P=0.3, P=0.6$ and $P=0.9$. The solid lines are fits with a damped cosine 6.4 .

\subsubsection{Frequency in the Hydrodynamic and Collisionless Regimes}

The value of the in-phase mode frequency can be calculated in the collisionless and hydrodynamic regimes.

In the collisionless regime, particles freely oscillate at the trap frequency $\omega_{z}$. The oscillation frequency of the radius is then twice as large:

$$
\omega_{1}^{\mathrm{CL}}=2 \omega_{z} .
$$

In the hydrodynamic regime, an exact solution of the hydrodynamic equations is provided by a scaling 
ansatz with the same dilatation factors for both components:

$$
n_{i}(x, y, z, t)=\frac{1}{b_{z} b_{r}^{2}} n_{i}^{0}\left(\frac{x}{b_{r}}, \frac{y}{b_{r}}, \frac{z}{b_{z}}\right),
$$

where $n_{i}^{0}(x, y, z)$ are the equilibrium density profiles. The proof is rather simple: one inserts the ansatz (6.5) into the hydrodynamic equations. At unitarity, the equilibrium equation of state reads:

$$
\mu_{i}\left(n_{2}, n_{1}\right)=\frac{\hbar^{2}}{2 m}\left(6 \pi^{2}\right)^{2 / 3} n_{1}^{2 / 3} f_{i}\left(x=\frac{n_{2}}{n_{1}}\right) .
$$

When $n_{1}$ and $n_{2}$ are dilated by the same factor, $f_{i}\left(n_{2} / n_{1}\right)$ does not change, and one simply obtains:

$$
\mu_{i}\left(n_{2}, n_{1}\right)=\frac{1}{\left(b_{z} b_{r}^{2}\right)^{2 / 3}} \mu_{i}\left(n_{2}^{0}, n_{1}^{0}\right) .
$$

This shows that the hydrodynamic equations for each spin state are decoupled and identical to the ones of a spin-balanced unitary gas The axial breathing mode frequency, in the limit of a large aspect ratio, then reads:

$$
\omega_{1}^{\mathrm{HD}}=\sqrt{\frac{12}{5}} \omega_{z} \simeq 1.55 \omega_{z} .
$$

This result was also derived with different assumptions in [99, using a sum-rule approach.

\subsubsection{Crossover from a Hydrodynamic to a Collisionless Behavior}

In Fig 6.5 a we plot the in-phase mode frequency $\omega_{1} / \omega_{z}$ as a function of polarization $P$. At low polarization the frequency is close to the hydrodynamic value. This behavior is expected for a gas with a large superfluid core: the core itself is superfluid and therefore evolves according to hydrodynamics, and particles in the normal phase are likely to collide with the superfluid core during one axial oscillation period $T_{z}=2 \pi / \omega_{z}$. The mean free path is thus smaller than the cloud size, which is the condition for hydrodynamic behavior for a classical gas. As $P$ increases, the frequency slowly departs from the hydrodynamic value. For $P \gtrsim 0.75$ the frequency strongly increases towards the collisionless value. For extremely high polarizations, the collision rate per majority atom becomes smaller that the oscillation frequency and the gas freely oscillates at the collisionless value. This collisionless regime is approached but is not fully reached in our experiment because of the difficulty to sort the data by polarization when the minority atom number is very small $\left(N_{2} \simeq 1000\right)$. It is important to remark that no particular feature occurs at the critical polarization $P_{c} \simeq 0.75$ at which the superfluid phase disappears.

\subsubsection{Oscillation Amplitude and Phase}

For the calculation of the frequency in the hydrodynamic regime presented in section 6.2.2, we assumed that the density profiles were scaled for both components with the same factor. Therefore we expect here the amplitudes $A_{i}$ to be identical. In Fig.6.6 we plot the amplitudes $A_{i}$ as a function of polarization, and indeed observe that $A_{1} \simeq A_{2}$ in the hydrodynamic regime $P<0.75$. We also note that the amplitude of the majority component $A_{1} \simeq 0.28$ is rather independent of the polarization value.

We also check the in-phase character of the mode by comparing the phases $\phi_{i}$ for both components. As shown in Fig 6.6p, the two components oscillate in phase in the hydrodynamic regime $P<0.75$. Moreover, the oscillation is shifted by $\pi / 2$ compared to the excitation, as expected for an excitation which sets the cloud in movement but is much shorter than the oscillation period.

\footnotetext{
†Since this property relies on the identity of the scaling factors for both two spin states, it is specific to the in-phase mode.
} 
(a)

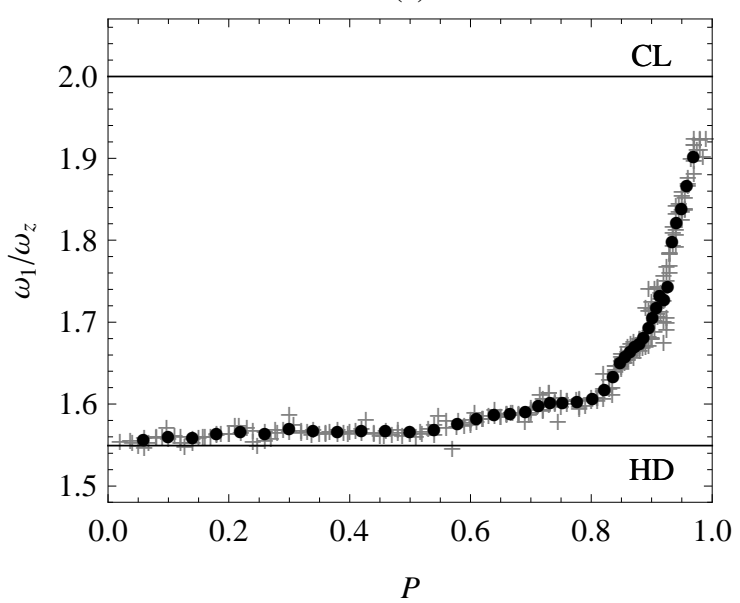

(b)

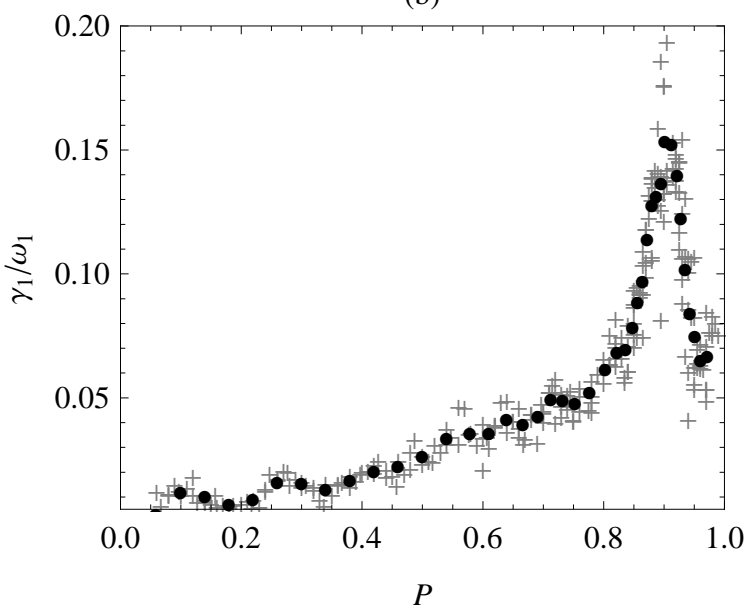

Figure 6.5: In-phase mode frequency (a) and damping rate (b) as a function of polarization. The gray crosses are the results of a fit of the data in a window $[P-\Delta P / 2, P+\Delta P / 2]$ results with a damped cosine (6.4). We vary the central polarization $0<P<1$ and the binning $0.02<\Delta P<0.07$ to obtain all gray data points. Black dots are averages of ten consecutive gray points. In (a), the solid lines are the hydrodynamic and collisionless frequencies. We mention that the data fitting procedure slightly differs from the one used in [99], leading to a better signal-to-noise ratio.

(a)

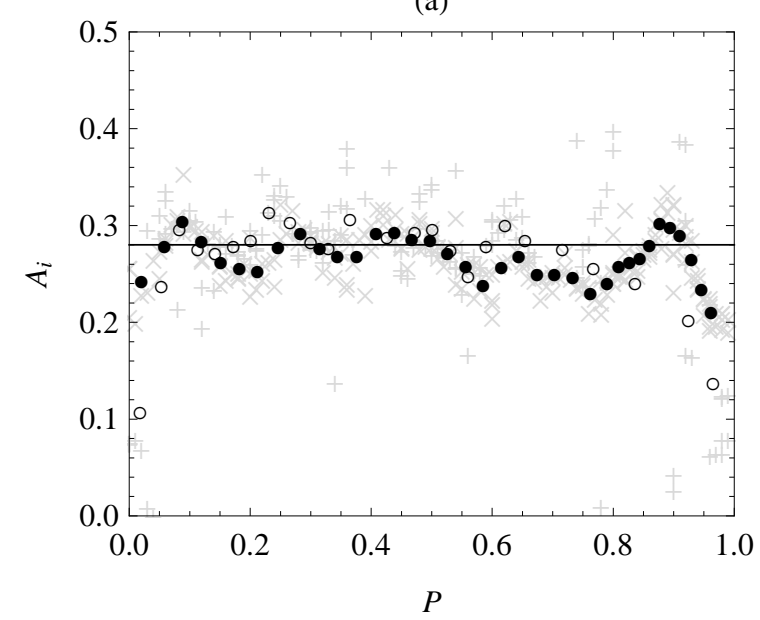

(b)

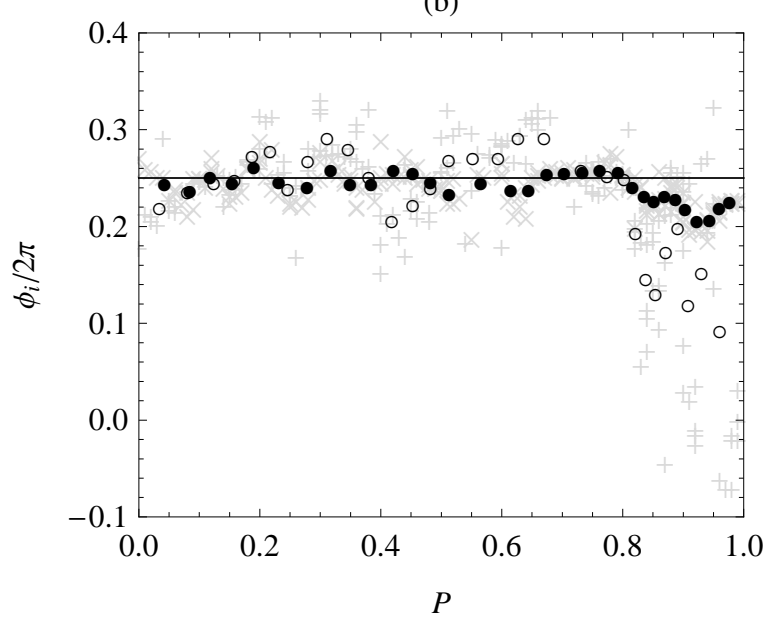

Figure 6.6: (a) Amplitude of the in-phase mode for both spin components, as a function of polarization. $\times(+)$ : raw data for species $1(2), \bullet(\circ)$ : average of ten raw data points for species 1 (2). (b) Phase of the in-phase mode as a function of polarization, with the same notations than (a). The solid line corresponds to an oscillation shifted by $\pi / 2$ compared to the excitation.

\subsubsection{Relaxation of a Two-Component Fermi Gas}

In Fig 6.5 we plot the damping rate $\gamma_{1} / \omega_{1}$ as a function of polarization. When the gas is either in the hydrodynamic or collisionless regimes the damping rate is small. It is maximum for $P \simeq 0.9$, i.e. in the middle of the crossover between the two limit regimes. We give in this section a physical explanation of this behavior. 


\section{Sound Propagation in Gases and Relaxation Phenomena}

Damping of sound waves in fluids is driven by the spontaneous relaxation towards equilibrium. We present here a simplified model for which the relaxation can be captured using a single relaxation time scale $\tau$ - model first introduced by Kneser in the 1930's [237. For a classical gas $\tau^{-1}$ is on the order of the collision rate per particle, and a typical value at ambient temperature and pressure is $\tau \sim 10^{-10} \mathrm{~s}$. In the acoustic domain, sound wave frequencies are much smaller than typical relaxation rates, therefore the gas locally remains almost at equilibrium. The speed of sound is then given by:

$$
c^{2}=\left.\frac{\partial P}{\partial n}\right|_{\text {equilibrium }} \equiv c_{0}^{2},
$$

and the oscillation quality factor is large. Using high-frequency ultrasonic waves $\omega / 2 \pi>1 \mathrm{GHz}$, it is possible to address the regime $\omega \tau \gtrsim 1$. For very large frequencies $\omega \tau \gg 1$, the gas equilibrium does not take place and the speed of sound reads:

$$
c^{2}=\left.\frac{\partial P}{\partial n}\right|_{\text {instantaneous }} \equiv c_{\infty}^{2} .
$$

In the case of a single relaxation time scale $\tau$, the speed of sound $c$ smoothly interpolates between $c_{0}$ and $c_{\infty}$ when $\omega$ is varied, according to 237238 :

$$
c^{2}=c_{\infty}^{2}+\frac{c_{\infty}^{2}-c_{0}^{2}}{1+i \omega \tau} .
$$

The imaginary part of the speed of sound accounts for the sound wave damping. In Fig 6.7 we plot the variation of the speed of sound and damping rate as a function of frequency. As expected, the speed of sound smoothly varies from $c \simeq c_{0}$ for $\omega \tau \ll 1$ to $c \simeq c_{\infty}$ for $\omega \tau \gg 1$. The oscillation qualify factor is large in the two limit regimes and is minimum in the middle of the crossover.

(a)

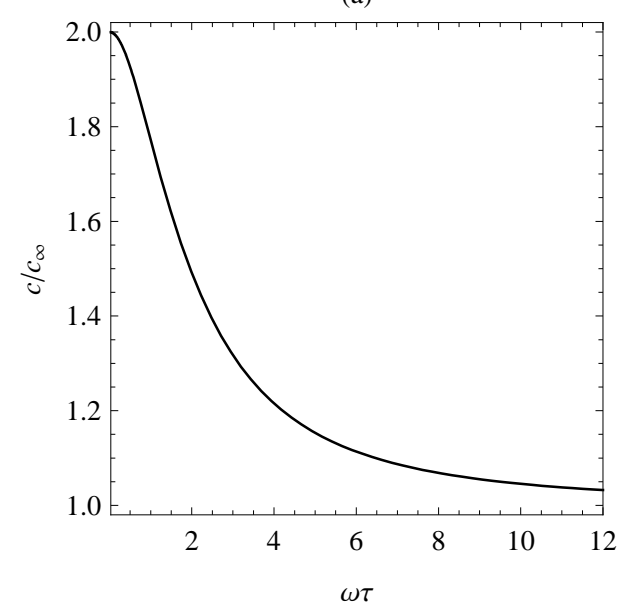

(b)

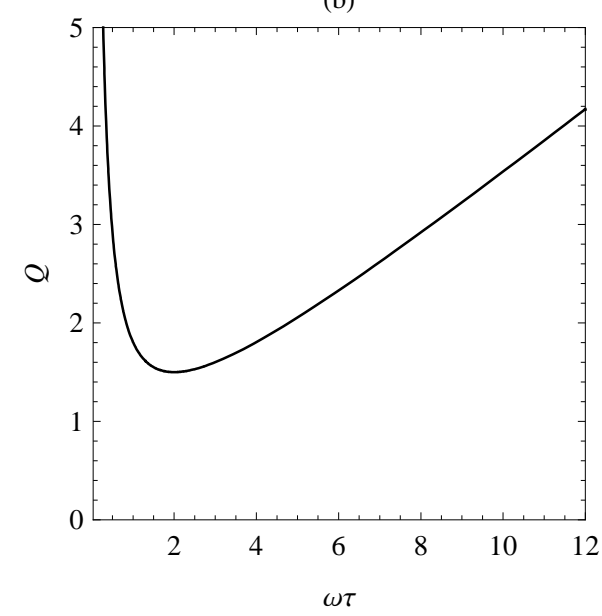

Figure 6.7: Speed of sound $c$ and sound wave quality factor $Q$ as a function of $\omega \tau$ for a Kneser fluid with $c_{0}=2 c_{\infty}$.

\section{Comparison of our Data to a Kneser Relaxation Model}

In the case of trapped gases, collective modes frequencies are quantized, with typical values on the order of $c / L$, where $c$ is the speed of sound and $L$ is the cloud size (along $z$ for the axial breathing modes). The transposition of equation 6.6 is then given by 239 :

$$
\omega^{2}=\left(\omega_{1}^{\mathrm{HD}}\right)^{2}+\frac{\left(\omega_{1}^{\mathrm{CL}}\right)^{2}-\left(\omega_{1}^{\mathrm{HD}}\right)^{2}}{1+i \omega \tau}, \quad \omega=\omega_{1}+i \gamma_{1} .
$$


The hydrodynamic frequency value $\omega_{1}^{\mathrm{HD}}$ is reached in the limit of a relaxation time much smaller than the oscillation period. The collisionless frequency value $\omega_{1}^{\mathrm{HD}}$ is reached in the opposite limit.

Taking the real part and imaginary part of 6.7), we obtain 2 equations for three variables $\omega_{z} \tau, \omega_{1} / \omega_{z}$ and $\gamma_{1} / \omega_{1}$. We can eliminate $\omega_{z} \tau$ and directly relate the damping rate to the frequency:

$$
\frac{\gamma_{1}}{\omega_{1}}=\sqrt{\frac{4 \sqrt{\left.15\left(\omega_{1} / \omega_{z}\right)^{2}-11\right)}-8-5\left(\omega_{1} / \omega_{z}\right)^{2}}{5\left(\omega_{1} / \omega_{z}\right)^{4}}} .
$$

A crossover between a hydrodynamic and a collisionless behavior in qualitative agreement with 6.8 was observed in 240 with balanced unitary gases, by playing with the gas temperature. Here the crossover is induced by the spin imbalance. In Fig 6.8 we plot our data in the plane $\left(\omega_{1} / \omega_{z}, \gamma_{1} / \omega_{1}\right)$. In the high polarization limit, our data are consistent with (6.8), given the relatively large noise of our data for low minority atom numbers. At low polarization, our data seem to significantly differ from 6.8). In between, the maximum damping rate $\gamma_{1} \simeq 0.15 \omega_{1}$ is equal to the maximum damping rate allowed by 6.8 .

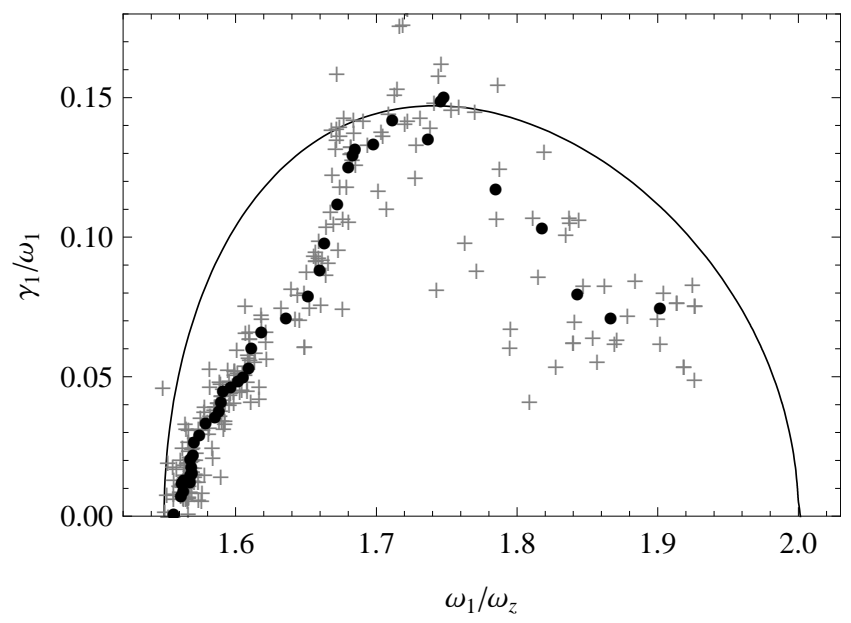

Figure 6.8: In-phase collective mode data plotted in the plane $\left(\omega_{1} / \omega_{z}, \gamma_{1} / \omega_{1}\right)$ (gray crosses). The black dots are averages of 10 raw data points. The solid line is the prediction given by equation 6.8.

\section{Relaxation Rate Value}

Assuming that equation (6.7) is valid, we fit our data with a damped cosine, where $\omega_{1} / \omega_{z}$ and $\gamma_{1} / \omega_{1}$ are expressed using a single parameter $\omega_{z} \tau$. In Fig 6.9 we plot the relaxation rate $1 /\left(\omega_{z} \tau\right)$ as a function of $N_{2} / N_{1}=(1-P) /(1+P)$. We observe a surprising linear dependence valid for all polarizations:

$$
\frac{1}{\omega_{z} \tau} \simeq 50 \frac{N_{2}}{N_{1}} .
$$

In particular the presence of a superfluid core does not seem to affect the variation of $\tau$ with polarization.

Understanding this behavior is rather difficult, especially when both a superfluid core and a fully polarized rim oscillate together. Above the Clogston limit $P>P_{c} \simeq 0.75$, it may be possible to model the system more easily. As shown in Chapter 5 the gas can then be described, at equilibrium, as a mixture of majority atoms and minority particles renormalized by the interaction with majority atoms. Within this Fermi liquid picture, the gas is merely a mixture of two Fermi seas with different atom numbers. Collective oscillations have already been modeled in this context using Boltzmann equation, but for slightly different situations, namely for spin-balanced gases 241,242, 243 or for the spin dipole mode in spin-imbalanced Fermi gases [244]. In Appendix C we adapt these calculations to the case relevant to our experiment, the axial breathing mode of a two-component Fermi gas with different atom numbers. 


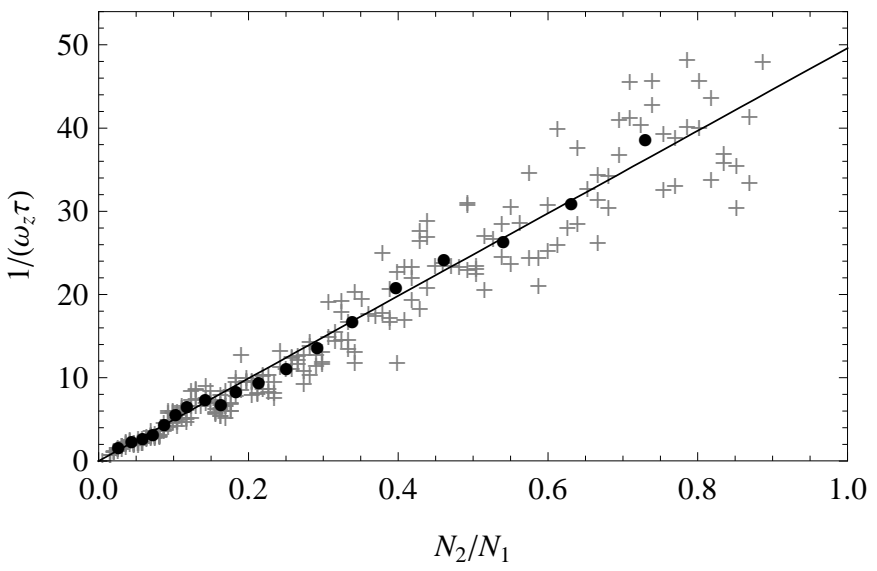

Figure 6.9: Relaxation rate $1 /\left(\omega_{z} \tau\right)$ as a function of $N_{2} / N_{1}$. The solid line is a linear fit of the complete data. The Clogston limit $P_{c} \simeq 0.75$ corresponds to $N_{2} / N_{1} \simeq 0.2$.

It is clear that Pauli blocking plays an essential role in the collision processes, the classical collision rate for a gas with such atom densities being $\sim 100$ times larger than the inverse relaxation time $1 / \tau$ that we observe. We mention that the axial breathing mode of a gas prepared at a higher temperature $\left(T=0.12 T_{F 1}\right)$ is always hydrodynamic in the range $0<P<0.95$ addressed in our experiment, probably due to the diminution of Pauli blocking effects. Here the proportionality of $\tau$ with $N_{2}$ suggests that Pauli blocking does not significantly affect the scattering of minority atoms. Otherwise, the collision rate would rather be proportional to the minority atom number available for scattering $\sim N_{2}\left(T / T_{F 2}\right)$, which scales differently with $N_{2}$. At zero temperature and at equilibrium, no scattering occurs because energy conservation prevents scattering towards unoccupied states above the Fermi levels. Therefore the relaxation is induced:

- either by a Fermi surface deformation associated with the gas oscillation itself (see Fig, 6.10a),

- or by a non-zero temperature allowing collisions to occur in a region $k_{B} T$ around the Fermi energies (see Fig.6.10p).

The deformation induced by our excitation, or a temperature $T=0.03(3) T_{F 1}$, provide the good order of magnitude for the relaxation rate (see Appendix C). However, a more precise understanding would require to know more precisely the gas temperature in order to isolate the two effects.

\subsection{Polaron Axial Breathing Mode}

In the hydrodynamic regime, out-of-phase oscillations are expected to be over-damped, as shown in 241 on the example of the spin dipole mode. However, the gas dynamics approaches a collisionless behavior at large polarization, and we expect the out-of-phase mode to become observable. In that limit of low minority atom number, the two axial breathing eigenmodes are easily identified: as the collision rate with the minority component is small, the majority component freely oscillates at the frequency $\omega_{1}^{\mathrm{CL}}=2 \omega_{z}$. The second mode can be pictured as the free oscillation of minority atoms inside an unperturbed Fermi sea. The coupling with the inhomogeneous majority component leads to an effective potential

$$
\begin{aligned}
V_{\mathrm{eff}}(\mathbf{r}) & =V(\mathbf{r})+A_{0} \mu_{1}(\mathbf{r}) \\
& =A_{0} \mu_{1}^{0}+\left(1-A_{0}\right) V(\mathbf{r}),
\end{aligned}
$$


(a)

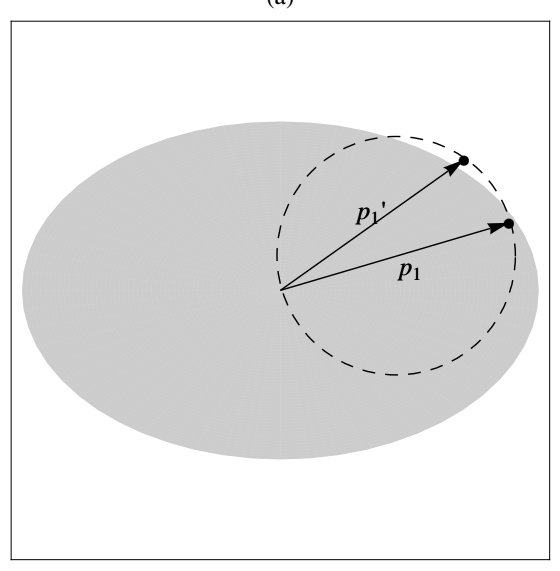

(b)

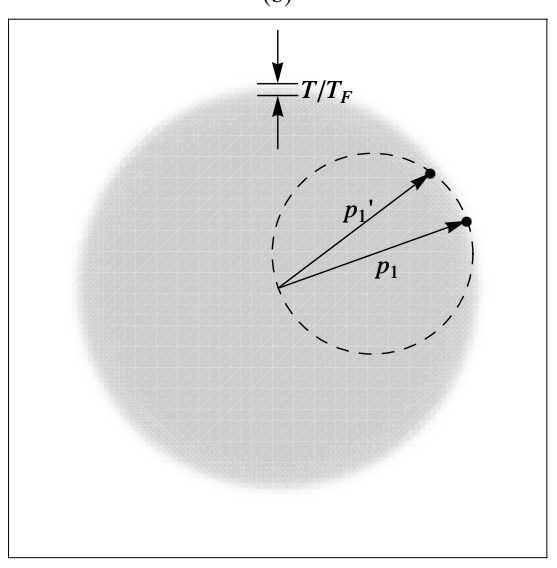

Figure 6.10: (a) Collision of a majority atom with an immobile impurity for a zero-temperature and deformed Fermi distribution. Energy conservation imposes the outcome momentum to belong to the dashed circle, and Pauli exclusion principle to be outside the deformed Fermi sea. (b) Collision of a majority atom with an immobile impurity for a finite-temperature Fermi distribution. The probability to make a collision is significant for atoms in a region of size $\sim T / T_{F}$.

where $A_{0} \mu_{1}=-0.615 \mu_{1}$ is the energy shift for one minority atom (see Chapter 5). In addition, interactions lead to a mass renormalization entering into second Newton law:

$$
m_{0}^{*} \frac{\mathrm{d} \mathbf{v}}{\mathrm{d} t}=-\nabla V_{\mathrm{eff}}(\mathbf{r})
$$

where $m_{0}^{*}$ is the polaron effective mass. The polaron axial oscillation frequency therefore reads:

$$
\omega_{z}^{*}=\sqrt{\frac{1-A_{0}}{m_{0}^{*} / m}} \omega_{z} .
$$

Measuring the out-of-phase mode frequency at large polarizations would thus provide the effective mass value (assuming that $A_{0}$ is exactly known).

The time evolution of the minority radius is expected to be described as the sum of two damped cosines, one at the frequency $\omega_{1} \simeq 2 \omega_{z}$, and one at the frequency $\omega_{2} \simeq 2 \omega_{z}^{*}$. However, the uncertainty on the minority radius becomes large for small minority atom numbers and the oscillation amplitude is smaller than noise of our data. In order to unambiguously reveal the appearance of the out-of-phase mode, we calculate the Fourier spectrum $\mathcal{P}(\omega)$ of the data $R_{2}(t)$ corresponding to a polarization in the window $[P-\Delta P / 2, P+\Delta P / 2]$. The Fourier spectrum calculation is adapted to an unevenly spaced set of data, as described in 245,246 . In Fig 6.11 we plot the Fourier spectrum as a function of polarization. For $P \gtrsim 0.75$ we observe the appearance of a second peak besides the peak corresponding to $\omega_{1}^{\mathrm{HD}}<\omega_{1}<\omega_{1}^{\mathrm{CL}}$, of frequency $\omega_{2}>\omega_{1}^{\mathrm{CL}}$, and which is identified to the out-of-phase mode.

By varying the mean polarization $P$ and the width $\Delta P$ defining the set of points $R_{2}(t)$ used for the frequency spectrum calculation, we obtain a series of peak positions $\omega_{2}$, in a window $0.75<P<0.9$ (see Fig 6.12. We observe that $\omega_{2}$ rapidly varies with polarization, from $\omega_{2} \simeq 3 \omega_{z}$ at $P=0.75$ to $\omega_{2} \simeq 2.6 \omega_{z}$ at $P=0.9$. We are interested in the high-polarization limit $P \rightarrow 1$. As our data $\omega_{2}(P)$ is well fitted by a straight line $\omega_{2}(P)=2.3(1)+2.9(3)(1-P)$ in the range $0.75<P<0.9$, our best estimate of the polaron oscillation frequency is given by:

$$
\omega_{z}^{*}=\frac{\omega_{2}(P \rightarrow 1)}{2}=1.15(5) \omega_{z} .
$$



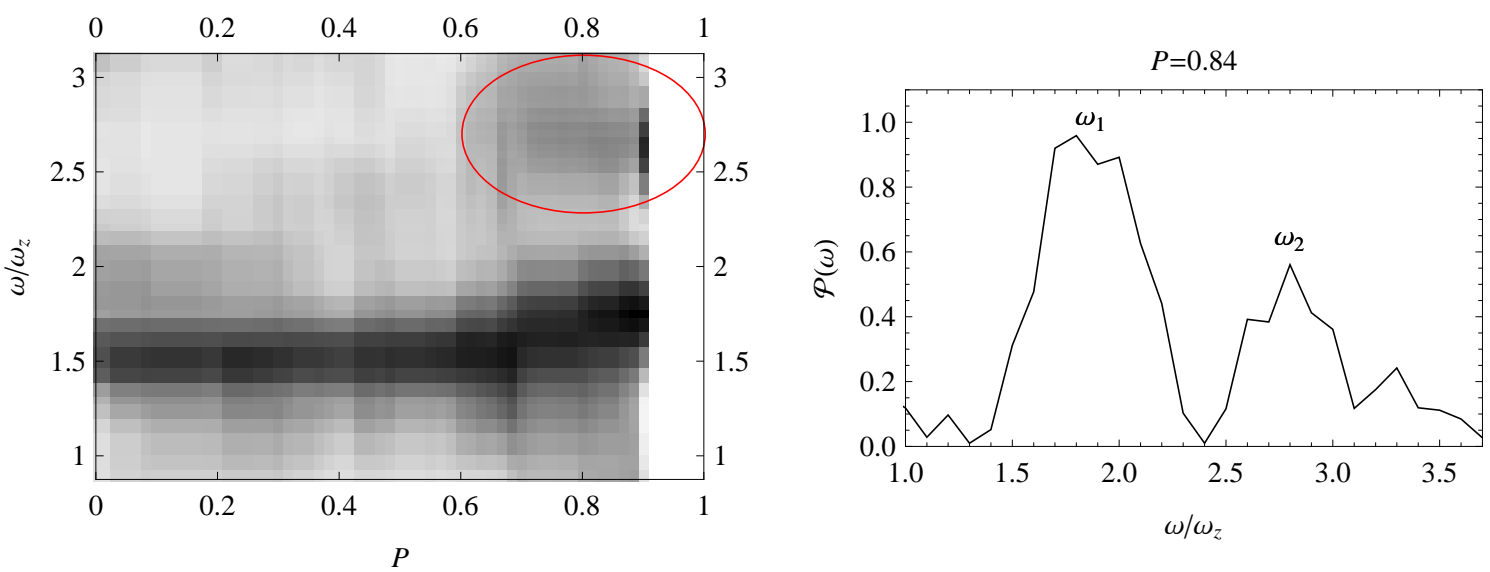

Figure 6.11: Left: Fourier spectrum $\mathcal{P}(\omega)$ of the data $R_{2}(t)$ as a function of polarization. The red ellipse indicates the frequency domain of the out-of-phase mode. Right: Fourier spectrum $\mathcal{P}(\omega)$ for $P=0.84$.

Using the exact result $A_{0}=-0.615$, we obtain the effective mass value:

$$
m_{0}^{*}=1.2(1) m,
$$

in agreement with theory 85,84 and with our determination from the equation of state in the unitary limit $m_{0}^{*}=1.21(2) m$ (see section 5.4.2.

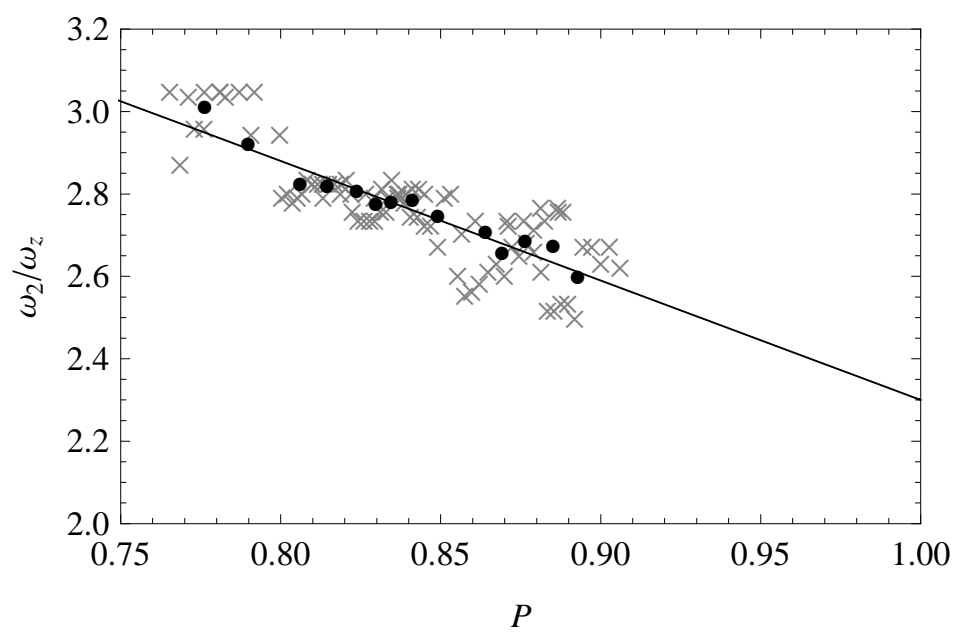

Figure 6.12: Out-of-phase mode frequency $\omega_{2}$, as a function of polarization $P$, and calculated for polarization windows $0.02<\Delta P<0.05$. The black dots are an average of 10 consecutive raw data points (gray crosses). The solid line is a linear fit of the complete data.

To conclude this chapter, we showed that the in-phase axial breathing mode frequency smoothly evolves from a hydrodynamic to a collisionless value. The out-of-phase mode appears at the largest frequencies and allows us to measure the polaron effective mass. Its large frequency variation may be due to a crossover towards a hydrodynamic behavior. In order to reach more easily the collisionless value, it would be interesting to study the radial breathing mode. Indeed, a collisionless behavior is expected when the atomic mean free path is larger than the cloud dimension relative to the mode considered, namely the radial (axial) cloud size for the radial (axial) breathing mode. Therefore it is clear that the radial mode is more favorable. This would allow a more simple comparison with theories of collective modes. 


\section{Conclusion}

In this thesis we studied experimentally the thermodynamics of ultracold Fermi gases. We implemented a new method for measuring the universal equation of state of a two-component Fermi gas with short-range interactions. The local pressure inside a trapped gas is extracted from its in situ absorption image. In the local density framework, this local probe provides the equation of state of the homogeneous gas. We combined this work with a new thermometry of strongly-interacting Fermi gases, using a small quantity of weakly-interacting ${ }^{7} \mathrm{Li}$ atoms as a thermometer.

We explored the phase diagram of a two-component Fermi gases under two angles of attack, namely the equation of state of a spin-balanced unitary Fermi gas at finite temperature, and of the one of a low-temperature Fermi gas with arbitrary interactions and spin imbalance.

The equations of state deduced from our work allowed us to make the first direct comparison between experiments and many-body theories. We validate Fixed-Node Monte-Carlo simulations at zero temperature, and show that, up to now, none of the existing many-body theories accounts for our observations at finite temperature over their full range.

For large enough temperature or chemical potential asymmetry, the gas is in a normal state. Surprisingly, in all the parameter domain (temperature, spin imbalance or interaction strength) addressed in this work, the understanding of its macroscopic properties can be unified using a Fermi liquid picture. This contradicts several theoretical predictions for which pair correlations lead to a different behavior. We extracted from our data the associated Landau parameters, such as the compressibility or the quasi-particle effective mass, for arbitrary interactions. For resonant interactions, we observed that the effective mass values are close to the bare mass, despite strong many-body correlations. A study of collective modes makes the link between the effective mass extracted from the equation of state and the inertia of oscillating quasi-particles.

We also characterized the superfluid threshold in terms of critical chemical potential imbalance and critical temperature, quantitatively confirming the exceptional robustness of the superfluid state in the strongly-interacting regime. We obtained the superfluid equation of state in the whole BEC-BCS crossover, a quantity directly relevant to describe the crust of neutron stars. We measured for the first time the corrections to mean field in low-density fermionic and bosonic superfluids, predicted by Lee, Huang and Yang in the 1950's.

A first extension of this work could be investigating other domains of the universal equation of state $P\left(\mu_{1}, \mu_{2}, T, a\right)$ of a two-component Fermi gas. Measuring the finite-temperature equation of state of a spin-balanced Fermi gas in the BEC-BCS crossover could bring new physical phenomena to light. On the BEC side of the resonance and right above the critical temperature, a two-component Fermi gas is made of molecules and is expected to behave as a thermal molecular Bose gas. Therefore the Fermi liquid picture should break down [154 79] (see Fig 6.13). On the BCS side of the resonance, the normal phase should remain described as a Fermi liquid. Extrapolating the Fermi liquid equation of state down to $T=0$, compared with the actual $T=0$ superfluid equation of state, would provide the condensation energy of the superfluid state. Finally, we showed that the critical temperature for superfluidity can be 
extracted from the equation of state in the unitary limit. By extending this measurement to the BECBCS crossover, we could measure the critical temperature curve, which has remained largely unexplored up to now 116 .

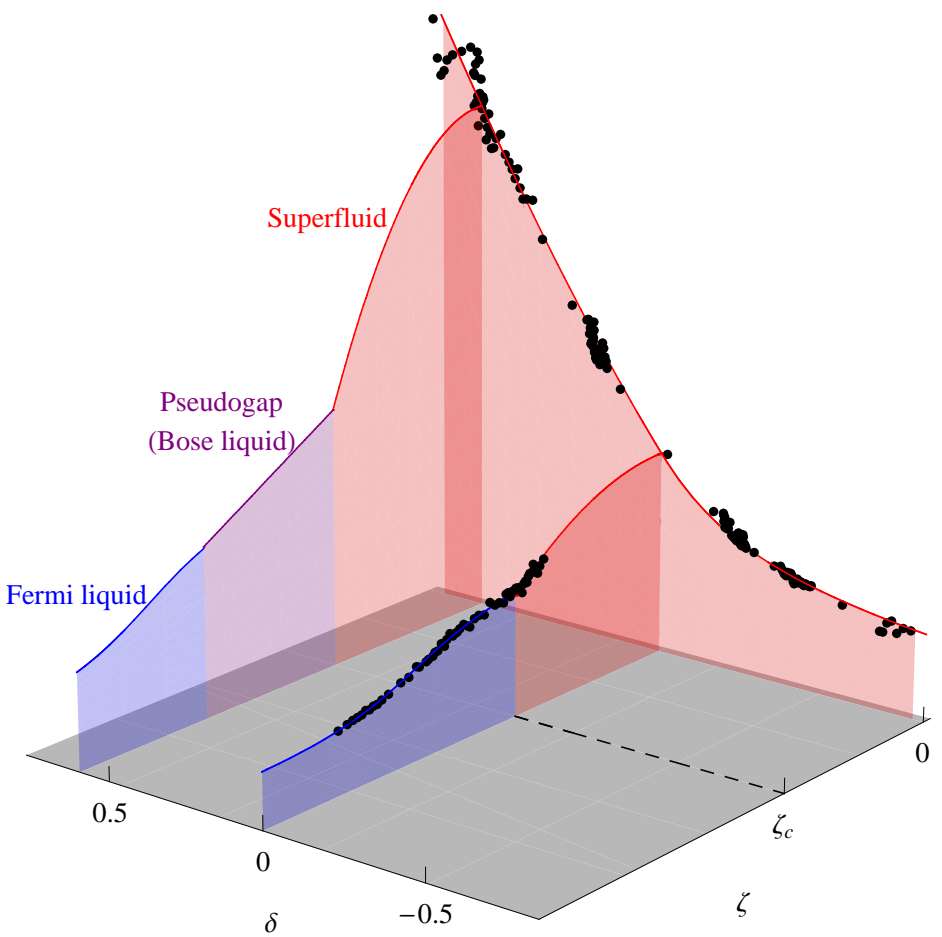

Figure 6.13: Grand-canonical equation of state of a balanced Fermi gas in the plane $(\delta, \zeta)$. The black dots are the experimental data corresponding to the equation of state of a unitary gas with resonant interactions (see Chapter 4) and to the equation of state of the superfluid at zero temperature, and in the BEC-BCS crossover (see Chapter 5). An experimental determination of the equation of state of a Fermi gas in the molecular regime, at finite temperature, would reveal thermodynamic signatures of a pseudogap phase.

We could also explore the ground-state properties in the far BEC regime. The ground state of the impurity problem is no longer a (fermionic) polaron but rather a (bosonic) molecule $85,86 \mid 226,180$. The change of quantum statistics should appear clearly on the grand-canonical equation of state given by our method. Strongly polarized gases in the BEC regime are also expected to be thermodynamically unstable for an interaction strength larger than a value associated with a tri-critical point [67]. This domain is still largely unexplored experimentally $\mid 79$. Investigating finite-temperature effects could also bring new phases of matter, such as the Sarma phase, to light [247]. Measuring the tri-critical line in the BEC-BCS crossover [229] would extend the work of the MIT group in the unitary limit [49] (see Fig,6.14).

The method developed during my $\mathrm{PhD}$ is general and could be used for the investigation of other systems. An extension of this work could be the measurement of the universal equation of state $P\left(\mu_{1}, \mu_{2}, T, a, m_{2} / m_{1}\right)$ describing two-component Fermi gases with different atomic species for each spin state. The mass difference degree of freedom is expected to enrich the phase diagram with new states of matter $248,249,250$. Recent experiments exploring the physics of ${ }^{6} \mathrm{Li}$ and ${ }^{40} \mathrm{~K}$ mixtures could address this subject in a near future 251,252. As an illustration of our method, we determined the equation of state of a weakly-interacting Bose gas. It would be very interesting to address Bose gases prepared close to a Feshbach resonance, hoping to reveal beyond-mean-field effects for large interaction strengths. ${ }^{7} \mathrm{Li}$ gases would be particularly adapted, thanks to the wide Feshbach resonances in the states 


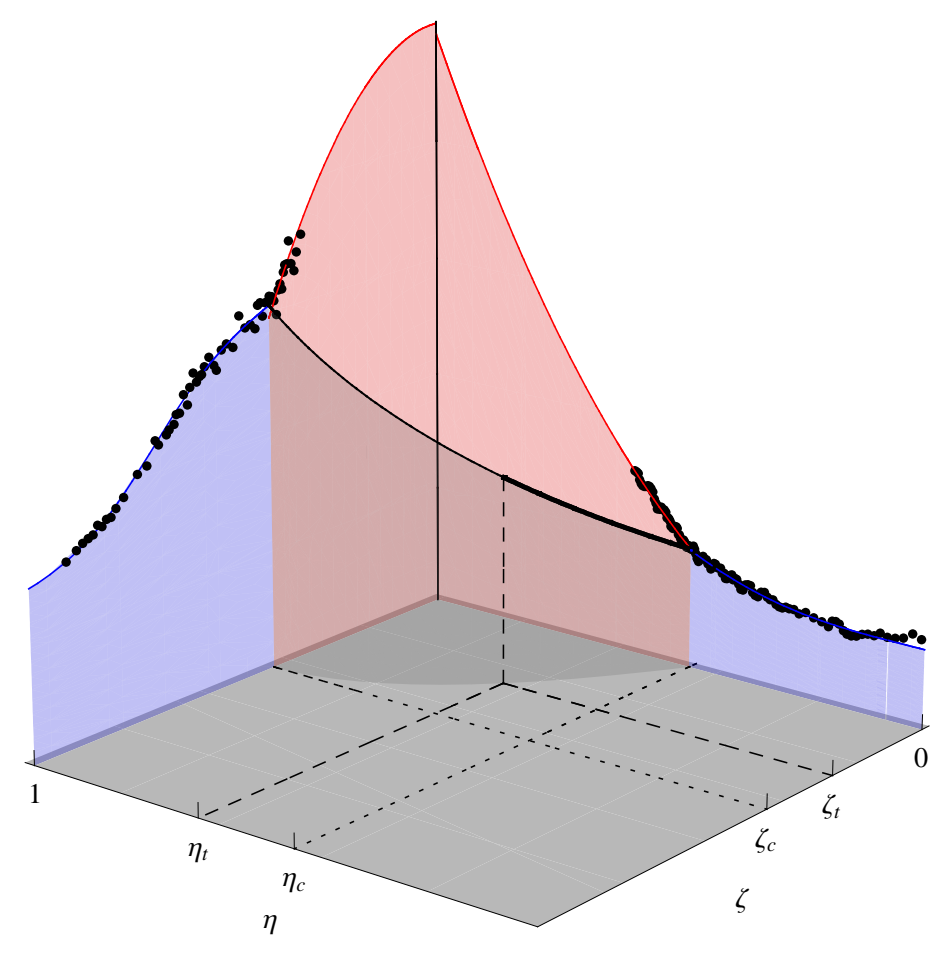

Figure 6.14: Grand-canonical equation of state of an two-component Fermi gas with resonant interactions, in the plane $(\eta, \zeta)$. The black dots are the experimental data corresponding to the equation of state of a spin-balanced gas at finite temperature (see Chapter 4) and to the equation of state of a spinimbalanced gas at zero temperature (see Chapter 5). The equation of state of a spin-imbalanced gas at finite temperature would reveal a superfluid to normal transition line. A tri-critical point $\left(\eta_{t}, \zeta_{t}\right)$ would set the separation between a first- and a second-order transition 49 .

$\left|F=1, m_{F}=1\right\rangle \quad 119,120$ and $|1,0\rangle[121$, and this work could be done with our experimental setup. We also showed the pertinence of our method for ultracold gases in optical lattices. The equation of state of a Bose gas held in a deep lattice that we deduce from in situ profiles directly reveals the Mottinsulator physics and is adapted to the investigation of finite-temperature effects. Its implementation on Fermi gases in an optical lattice could be helpful for characterizing the solution of the three-dimensional Fermi-Hubbard model from experiment 21,22 .

The universal equation of state explored in this thesis is relevant for the description of neutron matter at low density. We could go one step further in the simulation of matter encountered in nature. One direction could be to investigate the first correction to universality introduced by a finite size of the interaction potential. By using a different fermionic species, or using a recent proposal for tuning the potential range using an electric field [253], we could simulate neutron matter in the crust of neutron stars up to larger densities [60]. Simulating quantum chromodynamics models, such as color superconductivity models, could also be investigated using Bose-Fermi mixtures 61] or three-component Fermi gases 254 , 255 , the realization of the latter being the subject or current active research 256 257. Finally, problems of quantum magnetism 258,14 could be addressed using ultracold atoms in optical lattices. Among them, a great challenge is the investigation of the repulsive Fermi-Hubbard model in dimension 2, with tunable filling factor and interactions. The observation of $d$-wave superfluidity within this model is an important open issue, connected to the understanding of high- $T_{c}$ superconductivity. 


\section{Appendix A}

\section{Simple Thermodynamic Relations}

\section{A.1 Thermodynamics of an Ideal Fermi Gas}

\section{A.1.1 Equation of State of an Ideal Fermi Gas}

In this section we briefly derive the equation of state of an ideal Fermi gas $P_{0}(\mu, T)$, which serves as a reference for the expression (4.1) of the equation of state of the unitary gas. We consider here an ideal single-component gas in a box of volume $V$. In the grand-canonical ensemble, the grand-partition function $\Xi(V, \mu, T)$ factorizes over the eigenstates $\alpha[259$ :

$$
\Xi(V, \mu, T)=\prod_{\alpha}\left(1+\exp \left(-\frac{\epsilon_{\alpha}-\mu}{k_{B} T}\right)\right) .
$$

The grand-potential $\Omega=-k_{B} T \log \Xi=E-T S-\mu N=-P_{0} V$ thus reads:

$$
\begin{aligned}
\Omega & =-k_{B} T \sum_{\alpha} \log \left(1+\exp \left(-\frac{\epsilon_{\alpha}-\mu}{k_{B} T}\right)\right) \\
& =k_{B} T \int_{0}^{\infty} \mathrm{d} \epsilon \rho(\epsilon) \log \left(\frac{1}{1+\exp \left(-\frac{\epsilon-\mu}{k_{B} T}\right)}\right),
\end{aligned}
$$

where $\rho(\epsilon)=V m^{3 / 2} /\left(\sqrt{2} \pi^{2} \hbar^{3}\right) \sqrt{\epsilon}$ is the density of states for a single spinless particle. Equation A.1 also applies for a multicomponent ideal gas or for a trapped ideal gas, with the appropriate density of states. After integration by parts, one obtains the equation of state of the ideal Fermi gas:

$$
\begin{aligned}
P_{0}(\mu, T) & =\frac{1}{6 \pi^{2}}\left(\frac{2 m}{\hbar^{2}}\right)^{3 / 2} \int_{0}^{\infty} \mathrm{d} \epsilon \frac{\epsilon^{3 / 2}}{1+\exp \left(\frac{\epsilon-\mu}{k_{B} T}\right)} \\
& =\frac{k_{B} T}{\lambda_{d B}^{3}(T)} \frac{4}{3 \sqrt{\pi}} \int_{0}^{\infty} \mathrm{d} u \frac{u^{3 / 2}}{1+\exp \left(\frac{-\mu}{k_{B} T}\right) e^{u}} \\
& =\frac{k_{B} T}{\lambda_{d B}^{3}(T)} f_{5 / 2}\left(e^{\mu / k_{B} T}\right)
\end{aligned}
$$

after introducing the variable $u=\epsilon / k_{B} T$, the thermal De Broglie wavelength $\lambda_{d B}(T)=\sqrt{2 \pi \hbar^{2} / m k_{B} T}$. Expanding (A.3) in a series of $z, f_{5 / 2}$ can be expressed using the Polylogarithm function of order $5 / 2$ (see Fig A.1.19):

$$
f_{5 / 2}(z)=-\operatorname{PolyLog}\left(\frac{5}{2},-z\right)=\sum_{n=1}^{\infty}(-1)^{n+1} \frac{z^{n}}{n^{5 / 2}}
$$


(a)

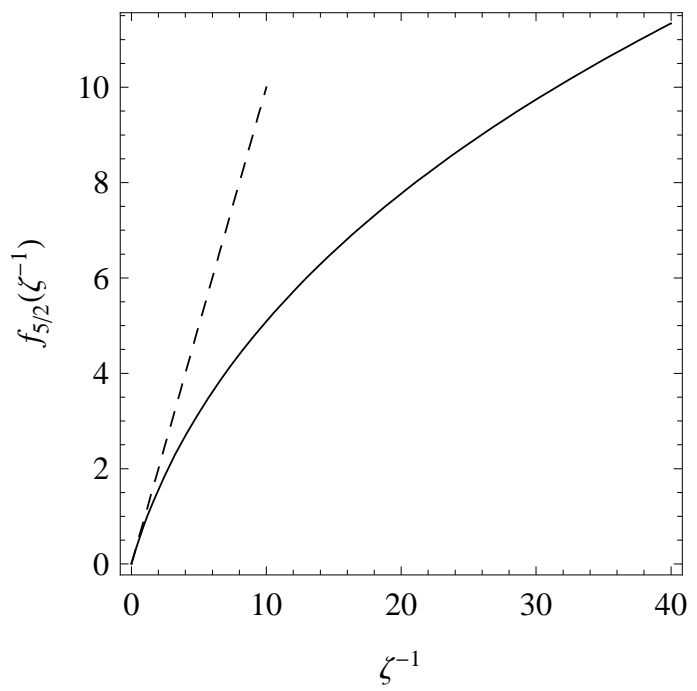

(b)

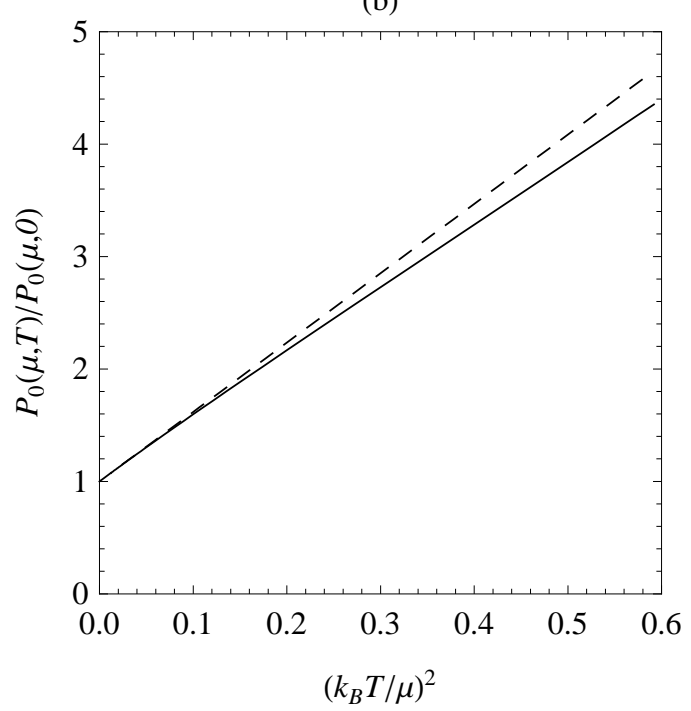

Figure A.1: (a) Solid line: equation of an ideal Fermi gas $f_{5 / 2}\left(\zeta^{-1}\right)=P_{0}(\mu, T) / k_{B} T \lambda_{d B}^{-3}(T)$, as a function of $\zeta^{-1}$. The dashed straight line is the equation of state of a Boltzmann gas $P_{B}(\mu, T) / k_{B} T \lambda_{d B}^{-3}(T)=\zeta^{-1}$. (b) Solid line: Equation of state of an ideal Fermi gas expressed as $P_{0}(\mu, T) / P_{0}(\mu, 0)$. The dashed line is the low-temperature quadratic temperature dependence, given by the first term of the Sommerfeld expansion A.8.

In our study of the thermodynamics of the unitary gas we rather use the inverse fugacity $\zeta=e^{-\mu / k_{B} T}$. Using this variable, the equation of state of an ideal gas reads:

$$
P_{0}(\mu, T)=\frac{k_{B} T}{\lambda_{d B}^{3}(T)} f_{5 / 2}\left(\zeta^{-1}\right)
$$

\section{A.1.2 High- and Low-Temperature Expansions}

Several physical quantities, such as the virial coefficients, will be extracted from our data by fitting the high- and low-temperature asymptotic behaviors of the equation of state, and by comparison with the case of an ideal gas. We thus remind in this section the asymptotic behaviors of the equation of state of an ideal gas.

The high-temperature expansion is explicitly given by equation A.5, as an expansion of $P_{0}$ in powers of $\zeta^{-1} \ll 1$. The equation of state of a classical Boltzmann gas is equal to (see Fig A.1.1):

$$
P_{B}(\mu, T)=\frac{k_{B} T}{\lambda_{d B}^{3}(T)} \zeta^{-1}
$$

Therefore the terms $n>1$ in the series account for the Fermi pressure due to the quantum statistics.

At low temperature, the pressure of a Fermi gas tends to a non-zero value, the so-called Fermi Pressure. Replacing in A.2 the Fermi-Dirac distribution by a Heaviside function $\Theta(\mu-\epsilon)$, we obtain:

$$
P_{0}(\mu, 0)=\frac{1}{15 \pi^{2}}\left(\frac{2 m}{\hbar^{2}}\right)^{3 / 2} \mu^{5 / 2} .
$$

Finite-temperature deviations are given by the Sommerfeld expansion. Using the variable $v=(\epsilon-\mu) / k_{B} T$ in A.2, we obtain:

$$
P_{0}(\mu, T)-P_{0}(\mu, 0)=\frac{1}{6 \pi^{2}}\left(\frac{2 m}{\hbar^{2}}\right)^{3 / 2} k_{B} T \mu^{3 / 2} \int_{-\infty}^{\infty} \mathrm{d} v\left(1+\frac{k_{B} T}{\mu} v\right)^{3 / 2}\left(\frac{1}{1+e^{v}}-\Theta(-v)\right),
$$


where we have extended the integral to $-\infty$, introducing an exponentially small error. Remarking that $1 /\left(1+e^{v}\right)-1=-1 /\left(1+e^{-v}\right)$, we can write A.6 as:

$$
P_{0}(\mu, T)-P_{0}(\mu, 0)=\frac{1}{6 \pi^{2}}\left(\frac{2 m}{\hbar^{2}}\right)^{3 / 2} k_{B} T \mu^{3 / 2} \int_{0}^{\infty} \mathrm{d} v \frac{1}{1+e^{v}}\left[\left(1+\frac{k_{B} T}{\mu} v\right)^{3 / 2}-\left(1-\frac{k_{B} T}{\mu} v\right)^{3 / 2}\right] .
$$

The last term in A.7 can then be expanded in power of $k_{B} T / \mu$ :

$$
P_{0}(\mu, T)-P_{0}(\mu, 0)=P_{0}(\mu, 0) \sum_{n=1}^{\infty} \alpha_{n}\left(\frac{k_{B} T}{\mu}\right)^{2 n}, \quad \text { where } \quad \alpha_{n}=5\left(\begin{array}{c}
3 / 2 \\
2 n-1
\end{array}\right) \int \mathrm{d} v \frac{v^{2 n-1}}{1+e^{v}} .
$$

Up to fourth order, this expansion reads (see Fig A.1.1p):

$$
P_{0}(\mu, T)=P_{0}(\mu, 0)\left(1+\frac{5 \pi^{2}}{8}\left(\frac{k_{B} T}{\mu}\right)^{2}-\frac{7 \pi^{4}}{384}\left(\frac{k_{B} T}{\mu}\right)^{4} \ldots\right) .
$$

Similarly to the high-temperature expansion, it is clear that measuring a few coefficients $\alpha_{i}$ of the lowtemperature expansion does not give any information on the high-temperature physics, since $\mu$ vanishes at some intermediate temperature and the expansion is no longer relevant.

\section{A.2 Conversion between the Canonical and Grand-Canonical Equa- tions of State of a Unitary Gas}

In this section we make the correspondence between the canonical equation of state:

$$
E(V, N, T)=\frac{3}{5} N E_{F} g(\theta), \quad \theta=\frac{T}{T_{F}},
$$

measured in 47 and the grand-canonical equation of state:

$$
P(\mu, T)=2 P_{0}(\mu) h(\zeta), \quad \zeta=e^{-\mu / k_{B} T},
$$

measured in this work.

\section{A.2.1 Conversion $g(\theta) \rightarrow h(\zeta)$}

In order to calculate $h(\zeta)$ from the data $g(\theta)$, we need to calculate the chemical potential:

$$
\mu=\left.\frac{\partial F}{\partial N}\right|_{V, T},
$$

where $F=E-T S$ is the free energy. Therefore we first have to calculate the entropy:

$$
\begin{aligned}
S(V, N, T) & =\left.\int_{0}^{T} \frac{\mathrm{d} T^{\prime}}{T^{\prime}} \frac{\partial E}{\partial T}\right|_{V, N}\left(V, N, T^{\prime}\right) \\
& =N k_{B} g_{S}(\theta) \text { where } g_{S}(\theta)=\int_{0}^{\theta} \frac{g^{\prime}\left(\theta^{\prime}\right) \mathrm{d} \theta^{\prime}}{\theta^{\prime}} .
\end{aligned}
$$

The chemical potential then reads:

$$
\frac{\mu}{E_{F}}=g_{\mu}(\theta)=g(\theta)-\frac{3}{5} \theta \int_{0}^{\theta} \frac{g^{\prime}\left(\theta^{\prime}\right) \mathrm{d} \theta^{\prime}}{\theta^{\prime}}
$$

leading to equation 4.4 in section 4.4. Finally, the pressure of a unitary gas is simply given by the exact relation $P=2 E / 3 V$, and we obtain 4.5. 


\section{A.2.2 Conversion $h(\zeta) \rightarrow g(\theta)$}

Expressing $g(\theta)$ in terms of $h(\zeta)$ is simpler since the grand-canonical equation of state $P(\mu, T)$ involves the natural variables of the grand-canonical ensemble. The energy, density and Fermi temperature are directly given by:

$$
E=\frac{3}{2} P V, \quad n=\left.\frac{\partial P}{\partial \mu}\right|_{T}, \quad k_{B} T_{F}=\frac{\hbar^{2}}{2 m}\left(3 \pi^{2} n\right)^{2 / 3},
$$

leading to equations 4.6 and $(4.7)$ in section 4.4 .

\section{A.3 Calculation of the Second-Order Virial Coefficient}

In this section we calculate the second-order virial coefficient of a spin-balanced Fermi gas with resonant interactions. Calculating of the partition function for a trapped gas involves a summation over discrete eigenenergies $[75]$ and is therefore more simple to write than the direct calculation of the partition function for a homogeneous gas [135]. Using local density approximation we can show that the virial coefficients are expected to be independent of the trap ellipticity. We thus consider for simplicity the case of an isotropic harmonic confinement. The Hamiltonian of the two-fermion problem in an isotropic trap was solved exactly in 142 . The center-of-mass motion can be separated from the relative motion and is insensitive to interactions. Its eigenstates are products of Fock states $\left|n_{x}, n_{y}, n_{z}\right\rangle$ along $x, y$ and $z$, of energies $E_{n_{x}, n_{y}, n_{z}}=\hbar \omega\left(n_{x}+n_{y}+n_{z}+\frac{3}{2}\right)$. The eigenstates $E_{n, l, m}$ of the relative motion are parametrized by the angular momentum numbers $l, m$ and by an additional quantum number $n$ relative to the radial degree of freedom. $s$-wave interactions only affect the eigenstates corresponding to zero angular momentum $l=m=0$. In the unitary limit $a=\infty$, the eigenenergies read 142 :

$$
\begin{aligned}
E_{n_{x}, n_{y}, n_{z}, n, l, m} & =E_{n_{x}, n_{y}, n_{z}}+E_{n, l, m} \\
& =E_{n_{x}, n_{y}, n_{z}, n, l, m}^{0}-\hbar \omega \delta_{l, 0},
\end{aligned}
$$

where the $E_{n_{x}, n_{y}, n_{z}, n, l, m}^{0}$ are the eigenstates for $a=0$ and $\delta_{l, 0}$ is the Kronecker symbol. Calculating the partition functions involved in the second-order virial coefficient $b_{t 2}=\left(Z_{1,1}-Z_{1,1}^{0}\right) / 2 Z_{1,0}$ is then straightforward: the center-of-mass degrees of freedom factorize in the partition function of a single particle, which simplifies with the denominator $Z_{1,0}$. Then, in the calculation of $Z_{1,1}-Z_{1,1}^{0}$, the terms with $l \geq 1$ cancel, which leads to a simple expression:

$$
\begin{aligned}
b_{t 2} & =\frac{1}{2} \sum_{n \in \mathbb{N}} e^{-E_{n, 0,0} / k_{B} T}-e^{-E_{n, 0,0}^{0} / k_{B} T} \\
& =\frac{1}{2} \sum_{n \in \mathbb{N}} e^{-2 \hbar \omega(n+1) / k_{B} T}-e^{-2 \hbar \omega(n+3 / 2) / k_{B} T} \\
& =\frac{1}{2} \frac{e^{-2 \hbar \omega / k_{B} T}}{1+e^{-\hbar \omega / k_{B} T}} \\
& \rightarrow \frac{1}{4} \text { for } \hbar \omega \ll k_{B} T .
\end{aligned}
$$

Using $b_{2}=b_{t 2} / 2^{3 / 2}$, we obtain the value of the second-order virial coefficient for a uniform gas:

$$
b_{2}=1 / \sqrt{2}
$$

\section{A.4 Clogston-Chandrasekhar Limit in a BCS Mean-Field Model}

In this section we use a BCS mean-field model to describe a two-component Fermi gas with arbitrary interaction strength and spin imbalance [260,261], providing a simple qualitative picture of this system. 
In this approach, the grand potential of this system:

$$
\begin{aligned}
\hat{\Omega} & =\hat{H}-\mu_{1} \hat{N}_{1}-\mu_{2} \hat{N}_{2} \\
& =\sum_{\mathbf{k}, i=1,2}\left(\epsilon_{\mathbf{k}}-\mu_{i}\right) \hat{a}_{i, \mathbf{k}}^{\dagger} \hat{a}_{i, \mathbf{k}}+\frac{g}{V} \sum_{\mathbf{k}, \mathbf{k}^{\prime}, \mathbf{q}} \hat{a}_{1, \mathbf{k}+\mathbf{q}}^{\dagger} \hat{a}_{2, \mathbf{k}^{\prime}-\mathbf{q}}^{\dagger} \hat{a}_{2, \mathbf{k}^{\prime}} \hat{a}_{1, \mathbf{k}},
\end{aligned}
$$

where $\epsilon_{k}=\hbar^{2} k^{2} / 2 m$ and $1 / g=m /\left(4 \pi \hbar^{2} a\right)-1 / V \sum_{k} 1 /\left(2 \epsilon_{k}\right)$ is the bare coupling constant, is replaced by a mean-field grand potential in which interactions occur only between atoms with opposite momentum $\left(\mathbf{k}=-\mathbf{k}^{\prime}\right)$, and interaction quartic terms are replaced with adequate mean-field quadratic terms:

$$
\hat{a}_{1, \mathbf{p}}^{\dagger} \hat{a}_{2,-\mathbf{p}}^{\dagger} \hat{a}_{2,-\mathbf{k}} \hat{a}_{1, \mathbf{k}} \rightarrow\left\langle\hat{a}_{1, \mathbf{p}}^{\dagger} \hat{a}_{2,-\mathbf{p}}^{\dagger}\right\rangle \hat{a}_{2,-\mathbf{k}} \hat{a}_{1, \mathbf{k}}+\hat{a}_{1, \mathbf{p}}^{\dagger} \hat{a}_{2,-\mathbf{p}}^{\dagger}\left\langle\hat{a}_{2,-\mathbf{k}} \hat{a}_{1, \mathbf{k}}\right\rangle-\left\langle\hat{a}_{1, \mathbf{p}}^{\dagger} \hat{a}_{2,-\mathbf{p}}^{\dagger}\right\rangle\left\langle\hat{a}_{2,-\mathbf{k}} \hat{a}_{1, \mathbf{k}}\right\rangle
$$

The grand potential then reads:

$\hat{\Omega}=-V \frac{\Delta^{2}}{g}+\sum_{\mathbf{k}, i=1,2}\left(\epsilon_{\mathbf{k}}-\mu_{i}\right) \hat{a}_{i, \mathbf{k}}^{\dagger} \hat{a}_{i, \mathbf{k}}-\Delta\left(\hat{a}_{2,-\mathbf{k}} \hat{a}_{1, \mathbf{k}}+\hat{a}_{1, \mathbf{k}}^{\dagger} \hat{a}_{2,-\mathbf{k}}^{\dagger}\right), \quad$ where $\quad \Delta=\Delta^{*}=-\frac{g}{V} \sum_{\mathbf{k}}\left\langle\hat{a}_{2,-\mathbf{k}} \hat{a}_{1, \mathbf{k}}\right\rangle$.

This quadratic grand potential can be diagonalized using a Bogoliubov transformation:

$$
\hat{\Omega}=-V \frac{\Delta^{2}}{g}+2 \sum_{\mathbf{k}}\left(\epsilon_{\mathbf{k}}-E_{\mathbf{k}}\right)+\sum_{\mathbf{k}, i=1,2}\left(E_{k}-\mu_{i}\right) \hat{b}_{i, \mathbf{k}}^{\dagger} \hat{b}_{i, \mathbf{k}}
$$

where $\hat{b}_{i, \mathbf{k}}$ is the quasi-particle creation operator for species $i$ and momentum $\mathbf{k}$ * and $E_{k}=\mu+$ $\sqrt{\left(\epsilon_{k}-\mu\right)^{2}+\Delta^{2}}$ the associated energy $\left(\mu=\left(\mu_{1}+\mu_{2}\right) / 2\right) . \Delta$ is the BCS pairing gap.

For given pairing gap $\Delta$ and chemical potentials $\mu_{i}$, the ground state is a product of empty states for $E_{k}>\mu_{i}$ and filled states for $E_{k}<\mu_{i}$, and the corresponding grand potential reads:

$$
\Omega\left(\mu_{1}, \mu_{2}, \Delta\right)=\Omega(\mu)+\sum_{\mathbf{k}, i=1,2}\left(E_{\mathbf{k}}-\mu_{i}\right) \theta\left(\mu_{i}-E_{\mathbf{k}}\right),
$$

where $\Omega(\mu)$ is the grand potential of the spin-symmetric configuration. Since $\mu_{2}<\mu_{1}$, one has $\mu_{2}<\mu<$ $E_{\mathbf{k}}$ for all $\mathbf{k}$, i.e. quasiparticles of species 2 are never populated in the ground state.

We finally obtain the actual ground state by minimizing $\Omega\left(\mu_{1}, \mu_{2}, \Delta\right)$ with respect to $\Delta$. If the minimum is obtained for $\Delta \neq 0$, the system is superfluid. In addition, if $\mu_{1}$ is larger than the singleparticle excitation gap $\mu+\Delta_{0}=\operatorname{Min}_{\mathbf{k}} E_{\mathbf{k}}$, extra majority atoms spontaneously appear, and the atom density for the two species differ, i.e. the system is partially polarized.

As shown in Fig A.2, we can show that the gas is superfluid in the BCS limit as long as

$$
\delta \mu=\mu_{1}-\mu_{2}<\delta \mu_{c}=\sqrt{2} \Delta
$$

where $\Delta$ is the gap for $\delta \mu=0$. This bound is the Chandrasekhar-Clogston limit 553,52 , originally expressed as the maximum magnetic field supported by a superconductor. It can also be written as a critical chemical potential ratio $\eta_{c}$ at the superfluid to normal transition:

$$
\eta>\eta_{c}=1-\sqrt{2} \frac{\Delta}{\mu_{1}} .
$$

As $\mu>0$ in the BCS regime, the minimum of the excitation energy spectrum $E_{\mathbf{k}}=\mu+\sqrt{\left(\epsilon_{k}-\mu\right)^{2}+\Delta^{2}}$ occurs at $k=\sqrt{2 m \mu} / \hbar$ and its value $\Delta_{0}$ is equal to the order parameter $\Delta$. The gas becomes partially

*The explicit expression of the Bogoliubov transform is the following:

$\left(\begin{array}{c}\hat{b}_{1, \mathbf{k}} \\ \hat{b}_{2,-\mathbf{k}}\end{array}\right)=\left(\begin{array}{cc}u_{k} & -v_{k} \\ v_{k} & u_{k}\end{array}\right)\left(\begin{array}{c}\hat{a}_{1, \mathbf{k}} \\ \hat{a}_{2,-\mathbf{k}}\end{array}\right)$, where $u_{k}=\frac{1}{2}\left(1+\frac{\epsilon_{k}-\mu}{\sqrt{\left(\epsilon_{k}-\mu\right)^{2}+\Delta^{2}}}\right) \quad$ and $\quad v_{k}=\frac{1}{2}\left(1-\frac{\epsilon_{k}-\mu}{\sqrt{\left(\epsilon_{k}-\mu\right)^{2}+\Delta^{2}}}\right)$. 

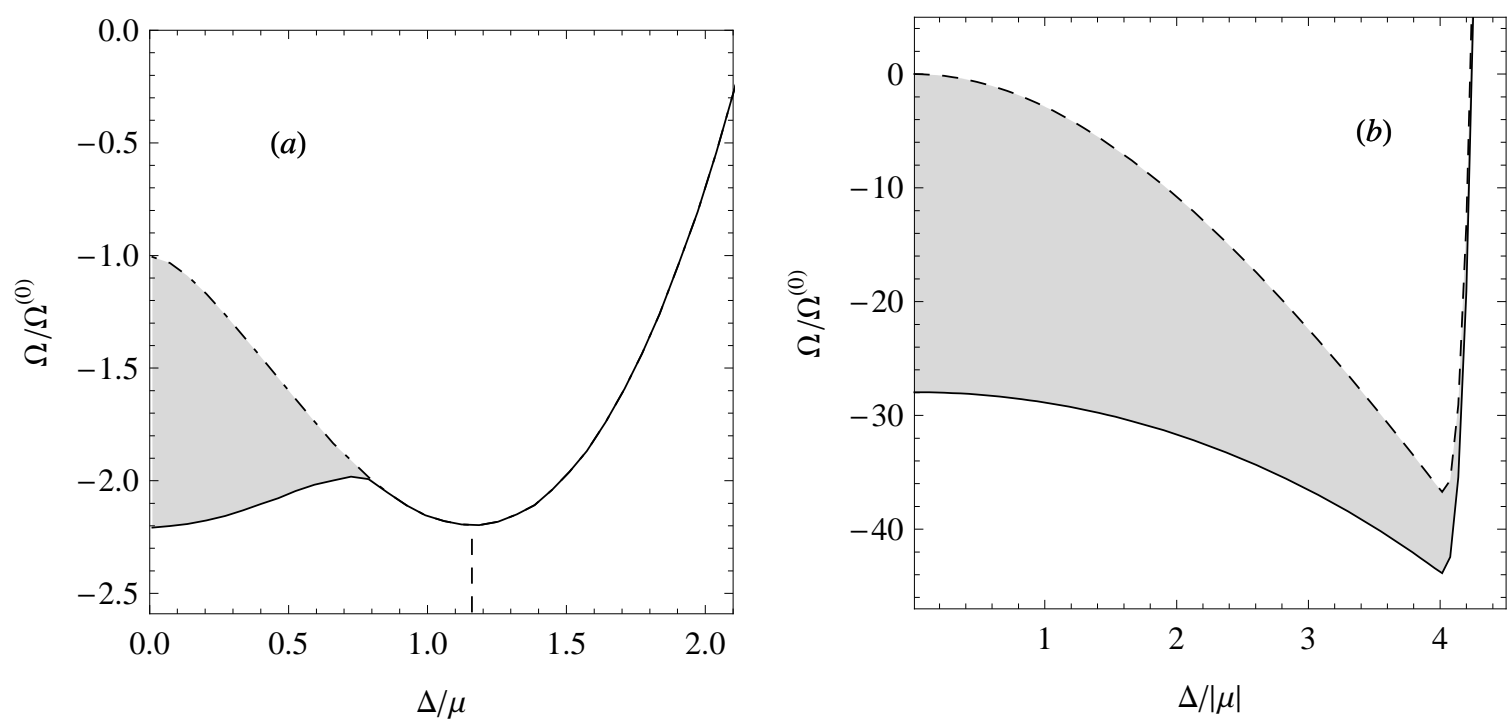

Figure A.2: (a) Grand potential $\Omega$, normalized to the non-interacting value, as a function of $\Delta / \mu$, in the unitary limit $a=\infty$, for $\delta \mu=0$ (dashed line) and $\delta \mu=1.6 \mu$ (solid line). For $\delta \mu<1.6 \mu$ the minimum of $\Omega$ is obtained for $\Delta>0$ and the gas is superfluid (and spin-balanced). For $\Delta>1.6 \mu$, the minimum of $\Omega$ is obtained for $\Delta=0$ and the gas becomes normal. (b) Grand potential as a function of $\Delta /|\mu|$, on the BEC side of the resonance $(\mu<0)$, for $\delta \mu=0$ (dashed line) and $\delta \mu=10 \mu$ (solid line). For $\delta \mu=0$ the gas is superfluid and $\Delta=4|\mu|$. For $\delta \mu=10 \mu$ the minimum is still reached for $\Delta \neq 0$. Therefore the gas is superfluid, but as $\delta \mu>2 \Delta_{0}$ it is partially polarized.

polarized for $\mu_{1}>\mu+\Delta$ or $\delta \mu>2 \Delta>\delta \mu_{c}$, i.e. for a chemical potential imbalance larger than the Clogston limit. Therefore, in the BCS regime the superfluid is never polarized.

On the BEC side of the resonance, as shown in Fig A.2 , the spin imbalance condition can be reached before superfluidity is lost. Indeed, in the $\mathrm{BEC}$ regime the chemical potential $\mu$ is negative, hence the minimum of the excitation energy spectrum $E_{\mathbf{k}}=\mu+\sqrt{\left(\epsilon_{k}-\mu\right)^{2}+\Delta^{2}}$ occurs for $k=0$ and strongly differs from the pairing gap: $\Delta_{0} \simeq \Delta^{2} / 2|\mu|$. It is then possible to polarize the gas by imposing $\delta \mu>2 \Delta_{0}$ and at the same time have a grand potential minimum for $\Delta \neq 0$. Therefore on the BEC side of the resonance the gas can be partially polarized and superfluid. 


\section{Appendix B}

\section{Technical Details in Chapter 5}

\section{B.1 Construction of the Equation of State}

\section{B.1.1 Determination of $\mu_{2}^{0}$}

The minority global chemical potential $\mu_{2}^{0}$ is extracted from in situ absorption images by fitting the radius $R_{2}$ at which minority atoms disappear, according to:

$$
A\left(\delta_{1 R_{2}}\right)=\frac{\mu_{2}^{0}-\frac{1}{2} m \omega_{z}^{2} R_{2}^{2}}{\mu_{1}^{0}-\frac{1}{2} m \omega_{z}^{2} R_{2}^{2}} .
$$

In practice this requires to know the behavior of $\bar{n}_{2}(z)$ when $z \rightarrow R_{2}$. As we expect the pressure of minority atoms to be nothing but the Fermi pressure of fermionic quasi-particles (see section 5.1.3), we are entitled to use a Thomas-Fermi profile $\bar{n}_{2}(z) \propto\left(1-z^{2} / R_{2}^{2}\right)^{5 / 2}$ around $z=R_{2}$ in the partially polarized normal phase. While the size of this phase is expected to be large on the BCS side of the resonance, it becomes very small on the BEC side of the resonance (see Fig,5.1), and the determination of $\mu_{2}^{0}$ is then rather difficult.

We tackled the issue of the determination of $R_{2}$ by averaging the equation of state given by several images without having to determine $\mu_{2}^{0}$ for each image. The determination of $\mu_{2}^{0}$ is then performed on the low-noise data obtained after averaging. For simplicity reasons we first describe the case of a gas in the unitary limit, i.e. the interaction parameter $\delta_{1 z}$ is always equal to 0 . Let us consider the equation of state $h\left(\eta_{z}\right)$ corresponding to a given image. The chemical potential imbalance varies along $z$ according to

$$
\eta_{z}=\frac{\mu_{2}^{0}-\frac{1}{2} m \omega_{z}^{2} z^{2}}{\mu_{1}^{0}-\frac{1}{2} m \omega_{z}^{2} z^{2}}
$$

which leads to:

$$
\log \left(1-\eta_{z}\right)=\log \left(1-\eta_{0}\right)-\log \left(1-\frac{z^{2}}{R_{1}^{2}}\right)
$$

where $\eta^{0}=\mu_{2}^{0} / \mu_{1}^{0}$ is the chemical potential imbalance at the bottom of the trap. In Fig B.1 we plot the data from a single image as $h$ as a function of $-\log \left(1-z^{2} / R_{1}^{2}\right)$. These data are equal to the equation of state expressed as $h$ as a function of $\log (1-\eta)$, translated in abscissa by the unknown quantity $\log \left(1-\eta_{0}\right)$. The data from all images corresponding to the same equation of state, we expect the data from two different images to be identical, in that representation, up to a translation in abscissa. We use this property to construct a low-noise equation of state without having to determine the value of $\eta^{0}$.

We start with a reference image labeled $a$ and express the data as $h$ as a function of $-\log \left(1-z^{2} / R_{1}^{2}\right)$. Then the data from another image coincide with the one of image $a$ after a translation of the quantity $\Delta \log \left(1-\eta^{0}\right)=\log \left(1-\eta^{0}\right)-\log \left(1-\eta_{a}^{0}\right)$. After translation, we obtain two sets of data equivalent to 
image $a$. They constitute a new reference data with a larger signal-to-noise ratio. We then iterate this procedure (see Fig.1 b): we compare the data from a given image with the high signal-to-noise data given by all previous images in order to fit $\Delta \log \left(1-\eta^{0}\right)$, and then add this data to the low-noise data.

(a)

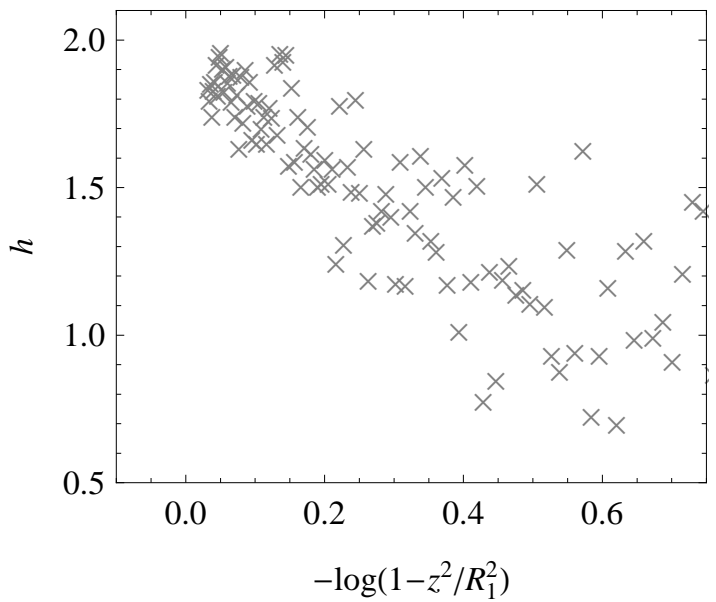

(b)

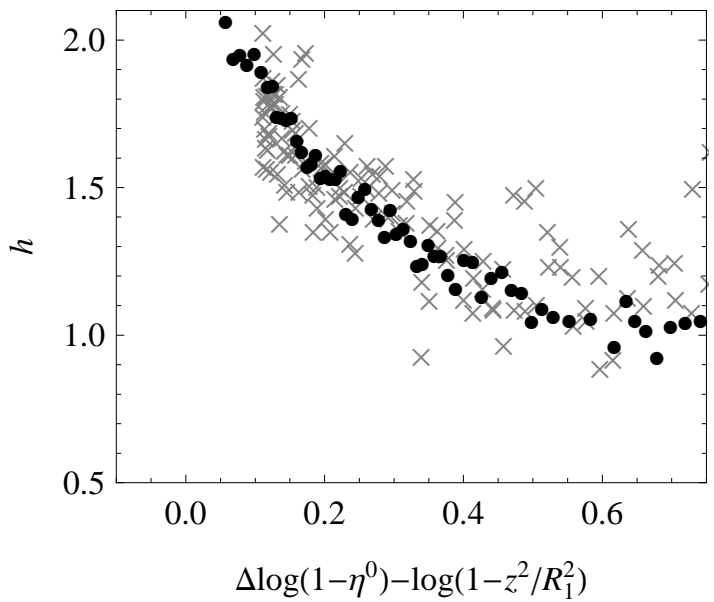

Figure B.1: (a) Data from a single image labeled $a$, plotted as $h$ as a function of $-\log \left(1-z^{2} / R_{1}^{2}\right)$ (gray crosses). (b) Data from a single image (gray crosses), plotted as $h$ as a function of $\Delta \log (1-$ $\left.\eta^{0}\right)-\log \left(1-z^{2} / R_{1}^{2}\right)$, where $\Delta \log \left(1-\eta^{0}\right)$ is chosen so that the data agree with the low noise data from previous images (black dots).

We end up with a low-noise data equivalent to image $a$. In Fig. B.2 a we plot the pressure increase $P-P_{0} \propto(h-1) \bar{n}_{1}^{0}(z)$ due to the minority component as a function of $z$. We fit the pressure increase in the high imbalance limit with a Thomas-Fermi profile $\beta\left(1-z^{2} / R_{2}^{2}\right)^{5 / 2}$, and obtain the minority radius $R_{2}=0.734 R_{1}$. The validity of the fit for $z$ close to $R_{2}$ is manifest in Fig B.2 b, where $P-P_{0}$ is plotted as a function of $\left(1-z^{2} / R_{2}^{2}\right)^{5 / 2}$. Using the solution of the single impurity problem at unitarity $A\left(\delta_{1}=0\right)=-0.615$ and equation $(\mathrm{B} .1)$, we obtain

$$
\eta_{a}^{0}=\frac{R_{2}^{2}}{R_{1}^{2}}+A\left(1-\frac{R_{2}^{2}}{R_{1}^{2}}\right)=0.255 .
$$

The chemical potential ratio along the $z$ axis $\eta_{z}$ is then calculated using $(\mathrm{B} .2)$, and we obtain the equation of state $h\left(\delta_{1}=0, \eta\right)$ plotted in Fig 5.4 .

\section{B.1.2 Equation of State in the BEC-BCS Crossover}

A similar procedure is used for the equation of state in the BEC-BCS crossover. Outside the unitary limit, the chemical potential ratio $\eta_{z}=\mu_{2 z} / \mu_{1 z}$ and the interaction parameter $\delta_{1 z}=\hbar / \sqrt{2 m \mu_{1}} a$ vary simultaneously along the $z$ axis, and are related by:

$$
\delta_{1 z}=\delta_{1}^{0} \sqrt{\frac{1-\eta_{z}}{1-\eta^{0}}} .
$$

Therefore a single image provides the equation of state $h\left(\delta_{1}, \eta\right)$ along a line in the $\left(\delta_{1}, \eta\right)$ plane. In the image averaging process, we superpose the equations of state obtained from clouds prepared at a given bias magnetic field $B_{0}$. These images thus correspond to the same scattering length value $a\left(B_{0}\right)$ but the parameter $\delta_{1}^{0} / \sqrt{1-\eta^{0}}$ may differ from one image to another, and the equations of state cannot strictly speaking be superimposed. At $800 \mathrm{G}$ the variation of $\delta_{1}^{0} / \sqrt{1-\eta^{0}}$ between the different images is the largest, about 0.1 around a mean value of 0.6 . We modeled the effect of the averaging procedure 
(a)

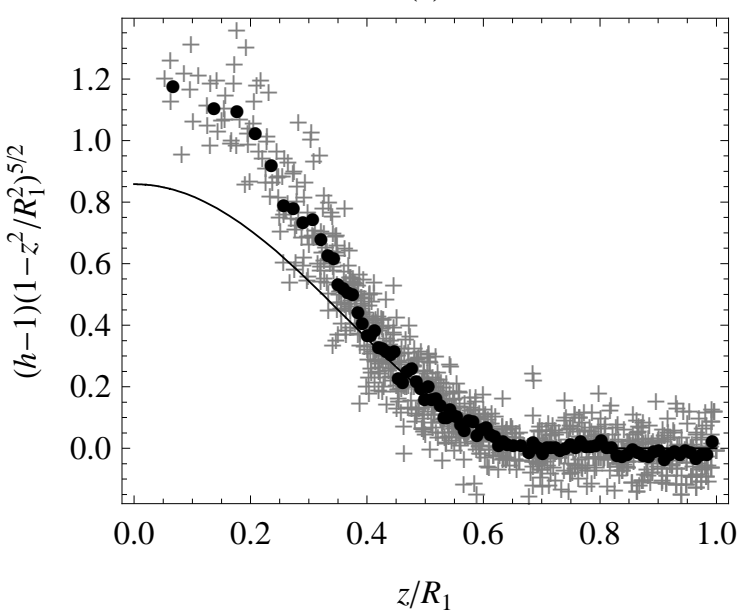

(b)

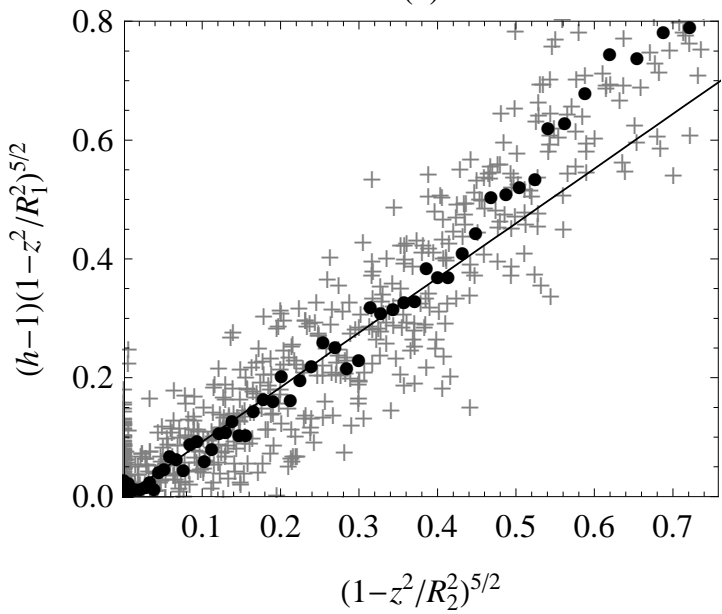

Figure B.2: (a) Pressure increase $P-P_{0} \propto(h-1)\left(1-z^{2} / R_{1}^{2}\right)^{5 / 2}$, as a function of $z / R_{1}$. The crosses are the raw data from 20 images superimposed to the image $a$ pressure profile, and the black dots are an average of 15 consecutive data. The solid line is a fit of the highly polarized region with a ThomasFermi profile. (b) Pressure increase $P-P_{0}$ as a function of $\left(1-z^{2} / R_{2}^{2}\right)^{5 / 2}$, showing the validity of the Thomas-Fermi fit for $0<\left(1-z^{2} / R_{2}^{2}\right)^{5 / 2}<0.4$.

using theoretical profiles corresponding to values of $\delta_{1}^{0}$ and $\eta^{0}$ similar to our data. After performing the averaging procedure described for the unitary gas, we obtain an equation of state which differs by less than $4 \%$ from the equation of state corresponding to the mean value of $\delta_{1}^{0} / \sqrt{1-\eta^{0}}$. Therefore we are entitled to average over all images of clouds prepared at the same bias magnetic field, paying the price of a $4 \%$ systematic error.

\section{B.2 Amplitude of Finite-Temperature Effects}

Here estimate the amplitude of the systematic error induced by a non-zero temperature in Chapter 5 .

\section{B.2.1 Upper Bound on the Cloud Temperature}

As first shown in [49], the fully polarized outer rim of a spin-imbalanced Fermi gas can be used for thermometry. Indeed, in this region the majority component forms an ideal Fermi gas, whose density profile is given by the Thomas-Fermi formula (see Appendix A):

$$
\begin{aligned}
\bar{n}_{1}(z) & =\frac{2 \pi}{m \omega_{r}^{2}} P_{0}\left(\mu_{1 z}, T\right) \\
& =\frac{2 \pi}{m \omega_{r}^{2}} \frac{k_{B} T}{\lambda_{d B}^{3}(T)} f_{5 / 2}\left(\exp \frac{\mu_{1}^{0}-\frac{1}{2} m \omega_{z}^{2} z^{2}}{k_{B} T}\right) .
\end{aligned}
$$

In Fig B.3 we fit the majority profile of a highly polarized gas (in the fully polarized region) with finitetemperature profiles. The difference between the profiles corresponding to different temperatures is revealed in the wings of the cloud. While the temperature clearly appears to be smaller than $0.1 \mu_{1}^{0}$, it is difficult to discriminate between lower temperatures. If $k_{B} T / \mu_{1}^{0}$ is let as a free parameter in the fit of the outer region, we obtain most of the time $T=0$ (and sometimes $k_{B} T \simeq 0.05$ to $0.1 \mu_{1}^{0}$, the result depending on very fine details such as the reference image used to calculate the absorption image profile or the number of pixels summed for the calculation of $\bar{n}_{1}(z)$ ). In order to estimate more quantitatively the uncertainty of this measurement, we fit a zero-temperature theoretical profile where a white noise is 
added on each pixel, with an amplitude corresponding to the signal-to-noise of our experimental data. Depending on the noise value, the fit results vary, with a mean value $k_{B} T / \mu_{1}^{0}=0.03$, and a standard deviation $\Delta\left(k_{B} T / \mu_{1}^{0}\right)=0.03$. We thus give the following estimate for the cloud temperature:

$$
\frac{k_{B} T}{\mu_{1}^{0}}=0.03(3) .
$$

On the BCS side of the resonance, the size of the fully polarized region is smaller than in the unitary limit or on the BEC side of the resonance. As less points are used for the fit, the uncertainty is larger. For the data taken at a bias magnetic field $B_{0}=981 \mathrm{G}$ and $B_{0}=871 \mathrm{G}$, the fit of the fully polarized shell is consistent with $k_{B} T / \mu_{1}<0.13$, i.e. a large upper bound which is not sufficient for our $T=0$ assumption. However, as described in section 5.3.2, we observe a jump in the minority concentration at the superfluid/normal phase transition which indicates that the temperature is smaller than the tricritical point temperature $T_{t r i}$. As $T_{t r i}$ exponentially decreases in the BCS limit, this bound becomes smaller that the one given by the fit of the fully polarized shell in the BCS regime.
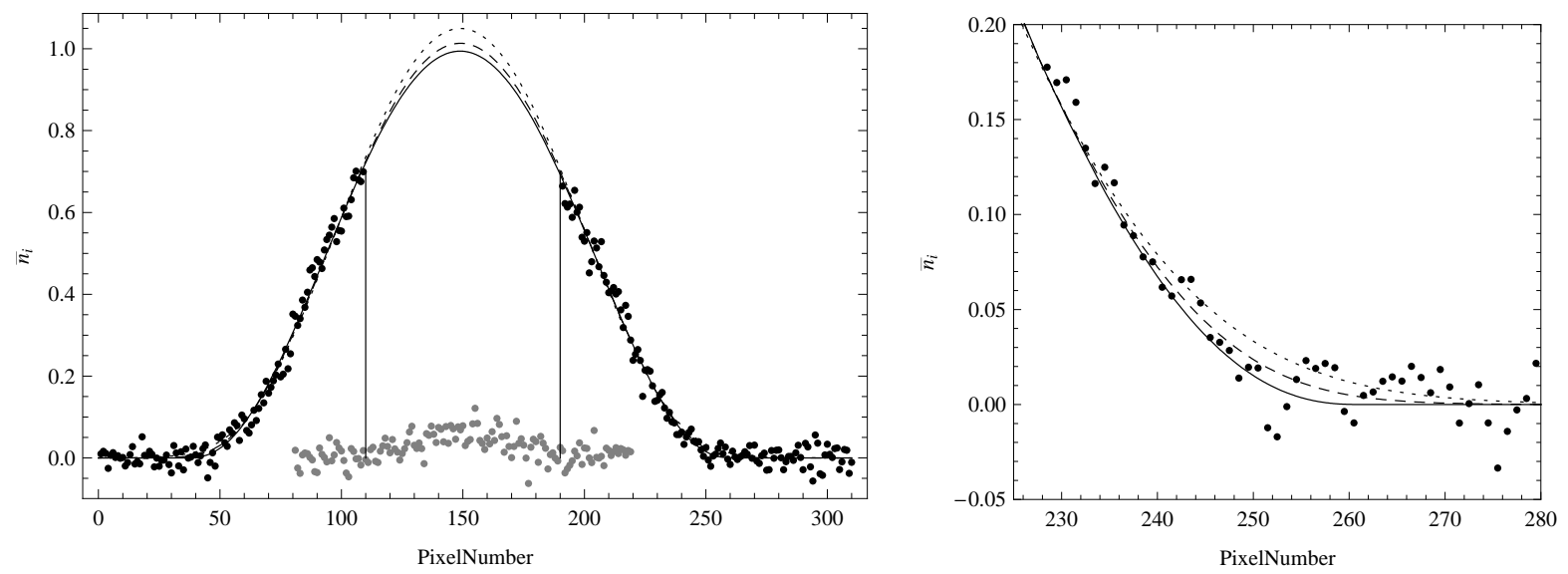

Figure B.3: Integrated density profile for the majority spin state $\bar{n}_{1}(z)$ in the fully polarized region of a very polarized gas $P=0.96$ (black dots). The minority profile is shown in gray. The lines are fits of the data in black with finite-temperature Thomas-Fermi profiles: solid line: $T / \mu_{1}^{0}=0$, dashed line: $T / \mu_{1}^{0}=0.1$, dotted line: $T / \mu_{1}^{0}=0.2$.

\section{B.2.2 Systematic Error in the Normal Phase}

The effect of non-zero temperature can be estimated in the normal phase by extending the Fermi liquid picture to a finite temperature. We showed that the pressure is well accounted for, at zero temperature, by the sum of the Fermi pressure of bare atoms with a chemical potential $\mu_{1}$ and the Fermi pressure of polarons with an effective mass $m^{*}$ and chemical potential $\mu_{2}-A \mu_{1}$. Replacing zero-temperature Fermi pressures by finite-temperature Sommerfeld expansions lead to the following estimate for the equation of state at $T \neq 0$ :

$$
\begin{aligned}
P\left(\mu_{1}, \mu_{2}, a\right) & \simeq \frac{1}{15 \pi^{2}}\left(\frac{2 m}{\hbar^{2}}\right)^{3 / 2} \mu_{1}^{5 / 2}\left(1+\frac{5 \pi^{2}}{8}\left(\frac{k_{B} T}{\mu_{1}}\right)^{2}\right)+ \\
& +\frac{1}{15 \pi^{2}}\left(\frac{2 m^{*}\left(\delta_{1}\right)}{\hbar^{2}}\right)^{3 / 2}\left(\mu_{2}-A\left(\delta_{1}\right) \mu_{1}\right)^{5 / 2}\left(1+\frac{5 \pi^{2}}{8}\left(\frac{k_{B} T}{\mu_{2}-A\left(\delta_{1}\right) \mu_{1}}\right)^{2}\right) .
\end{aligned}
$$

Using the temperature estimate $k_{B} T / \mu_{1}^{0}=0.03$ on the BEC side of the resonance, we estimate the relative systematic error to be less than $2 \%$, a value smaller than the systematic error induced by the image 
analysis procedure (see section 5.27 . On the BCS side of the resonance, we use the upper bound provided by the tri-critical point temperature. In the BCS limit, the tri-critical temperature is approximately equal to half the critical temperature for superfluidity of a balanced mixture, given by the mean-field BCS result with the Gorkov correction. This asymptotic result also gives a good estimate of the tri-critical point temperature $T_{t r i} \simeq 0.07 T_{F 1}$ measured in the MIT group in the unitary limit [4], and is therefore expected to be a good estimate of $T_{t r i}$ on the whole BCS side of the resonance. This temperature upper bound inserted in B.5 leads to a 3\% systematic error on the zero-temperature pressure on the BCS side of the resonance.

\section{B.2.3 Systematic Error in the Superfluid Phase}

In the superfluid phase temperature effects are expected to be much smaller. Indeed, as explained in section 4.7 .2 on the special case of a unitary gas, low-temperature excitations in the superfluid phase are either fermionic excitations, exponentially suppressed by the gap, or Bogoliubov-Anderson phonons. Let us estimate the amplitude of phonons black-body radiation, which is the dominant effect at the lowest temperatures. The speed of sound $c=\sqrt{n / m \partial \mu / \partial n}$ is calculated in the BEC-BCS crossover from the zero-temperature superfluid equation of state. The phonon contribution to the pressure (see section 4.7 .2 :

$$
\delta P_{\text {phonons }}(\mu, T)=2 P_{0}(\mu, T=0) \frac{\pi^{4} \sqrt{3}}{32}\left(\frac{\sqrt{2 \mu / 3 m}}{c}\right)^{3}\left(\frac{k_{B} T}{\mu}\right)^{4},
$$

evaluated at $T=T_{t r i}$, is always smaller that $2 \%$ of the zero-temperature pressure.

As a conclusion, the systematic error due to a non-zero temperature is at most $3 \%$. Together with the $4 \%$ error introduced by the data analysis procedure, we estimate the total systematic error to be $5 \%$.

\section{B.3 Padé Approximants}

We give in this appendix the explicit expression of the Padé approximants used to fit the experimental data in the superfluid phase.

\section{B.3.1 Padé Approximant on the BCS side of the Resonance}

On the BCS side of the resonance, rational fractions:

$$
h_{S}^{\mathrm{BCS}}(\widetilde{\delta})=\frac{\alpha_{1}+\alpha_{2} \widetilde{\delta}+\widetilde{\delta}^{2}}{\alpha_{3}+\alpha_{4} \widetilde{\delta}+\widetilde{\delta}^{2}}
$$

form a set of fitting functions which tend to 1 in the BCS limit and remain finite in the unitary limit.

\section{B.3.2 Padé Approximants on the BEC side of the Resonance}

On the BEC side of the resonance, the asymptotic behavior is analytic in the BEC limit up to the Lee-Huang-Yang expansion (see section 5.5.6). Therefore the latter is captured using a rational function:

$$
h_{S}^{\mathrm{BEC}}(\widetilde{\delta})=\frac{\beta_{1}+\beta_{2} \widetilde{\delta}+\beta_{3} \widetilde{\delta}^{2}}{1+\beta_{4} \widetilde{\delta}},
$$

which remains finite in the unitary limit and is asymptotic to a straight line in the BEC limit, in agreement with the mean-field equation of state:

$$
h_{S}(\widetilde{\delta}) \simeq \frac{15 \pi}{4} \frac{a}{a_{d d}}, \quad \widetilde{\delta} \ll 1 .
$$


The next order term is no longer analytical (see section 5.5.6), and is captured using a more complex Padé-type approximant:

$$
h_{S}^{\mathrm{BEC}}(\widetilde{\delta})=\frac{\beta_{1}^{\prime}+\beta_{2}^{\prime} \widetilde{\delta}+\beta_{3}^{\prime} \widetilde{\delta} \log (1+\widetilde{\delta})+\beta_{4}^{\prime} \widetilde{\delta}^{2}+\beta_{5}^{\prime} \widetilde{\delta}^{3}}{1+\beta_{6}^{\prime} \widetilde{\delta}^{2}},
$$

which remains analytical around the unitary limit and accounts for the log term in the BEC limit (see section 5.5.6.

\section{B.3.3 Fit Function of the Complete Data}

The best estimate of the complete experimental data is provided by the coefficients listed in Table B.1. and is used for the calculation of other quantities, such as the canonical equation of state for the comparison with Fixed-Node Monte Carlo data (see section 5.5.2. In Fig. B.4 we compare the experimental data with this best fit.

\begin{tabular}{cccccc}
\hline \hline & $\alpha_{1}$ & $\alpha_{2}$ & $\alpha_{3}$ & $\alpha_{4}$ & \\
& -1.065 & 0.441 & -0.535 & 0.1418 & \\
\hline$\beta_{1}^{\prime}$ & $\beta_{2}^{\prime}$ & $\beta_{3}^{\prime}$ & $\beta_{4}^{\prime}$ & $\beta_{5}^{\prime}$ & $\beta_{6}^{\prime}$ \\
3.74 & 7.92 & 8.29 & -4.24 & 3.67 & 0.187 \\
\hline \hline
\end{tabular}

Table B.1: Padé-type approximants coefficients $\alpha_{i}$ and $\beta_{i}^{\prime}$ fitted from our data.

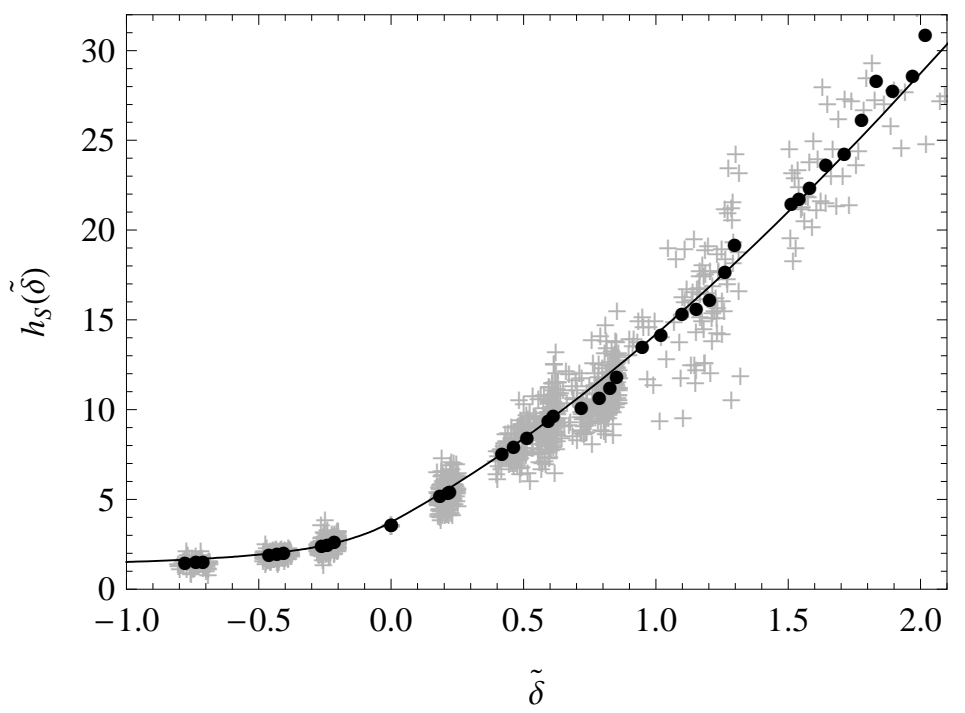

Figure B.4: Equation of state $h_{S}(\widetilde{\delta})$ extracted from our data, compared with the best fit (solid line).

\section{B.4 Surface Tension at the Superfluid/Normal Boundary of a Trapped Spin-Imbalanced Fermi Gas}

Our work relies on the validity of local density approximation. While it is expected to be valid with a very good accuracy in most situations (see section 3.5.2), it becomes inaccurate for the description of the density profile of a spin-imbalanced Fermi gas around the superfluid to normal boundary. Indeed, local density approximation predicts an unphysical density discontinuity. In the unitary limit, we expect the 


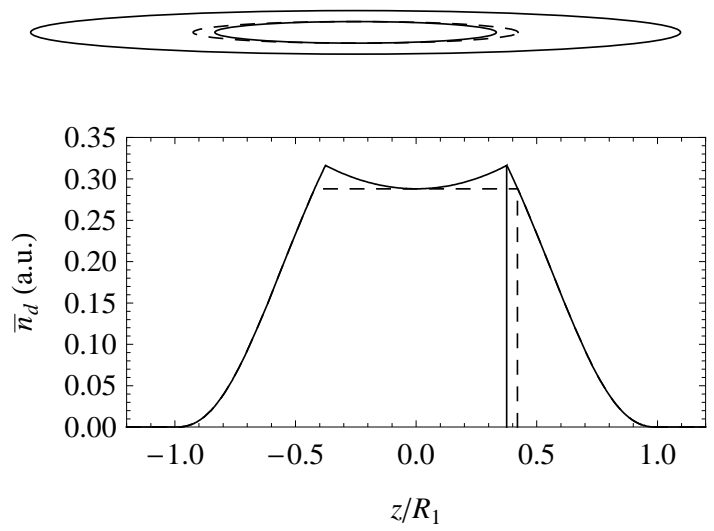

Figure B.5: Upper panel: Scheme of the fully polarized phase and superfluid phase boundaries, without deformation (dashed lines) and with a deformation $\lambda_{S} / \lambda=0.9$ (solid lines). Lower panel: Doublyintegrated density difference $\bar{n}_{d}(z)$ without deformation (dashed lines) and with a deformation $\lambda_{S} / \lambda=0.9$ (solid lines).

density jump to be smeared out over a distance on the order of the correlation length $\sim k_{F}^{-1}$. As $k_{F}^{-1}$ is much smaller than the cloud size, the violation of local density approximation may occur in a very small region.

However, a strong violation of local density approximation was observed at Rice university [97: the superfluid core aspect ratio strongly differs from the trap aspect ratio $\lambda \simeq 50$. The density profiles are consistent with a model in which the deviation from local density approximation is encapsulated by a surface tension associated with the superfluid/normal interface [127, 128, 129]. In this model, the grand potential reads:

$$
\Omega\left(\mu_{1}^{0}, \mu_{2}^{0}, \mathcal{S}\right)=-\int_{\mathbf{r} \in V_{\mathcal{S}}} \mathrm{d} \mathbf{r} P_{S}\left(\mu_{1}, \mu_{2}\right)-\int_{\mathbf{r} \notin V_{\mathcal{S}}} \mathrm{d} \mathbf{r} P_{N}\left(\mu_{1}, \mu_{2}\right)+\int_{\mathcal{S}} \sigma\left(\mu_{1}, \mu_{2}\right) \mathrm{d} S,
$$

where $\mathcal{S}$ is the normal/superfluid boundary, $V_{\mathcal{S}}$ is the superfluid core defined as the interior of $\mathcal{S}$, and $\sigma\left(\mu_{1}, \mu_{2}\right)$ is the surface tension coefficient associated with the normal/superfluid interface. In the unitary limit, dimensional analysis requires the surface tension coefficient to be written as ${ }^{*}$

$$
\sigma\left(\mu_{1}, \mu_{2}\right)=\alpha \frac{2 m}{\hbar^{2}} \mu_{1}^{2}
$$

where $\alpha$ is a dimensionless number. The equilibrium surface minimizes the grand potential value, a condition equivalent to the Laplace law:

$$
P_{S}-P_{N}=\sigma\left(\frac{1}{R_{1}}+\frac{1}{R_{2}}\right)
$$

where $R_{1}$ and $R_{2}$ are the surface principal curvature radii. The pressure drop occurring at the phase transition boundary shrinks the superfluid core, especially in the regions of small curvature radii. In an elongated trap, the curvature radius is smallest along the weak direction $z$, therefore the superfluid core aspect ratio $\lambda_{S}$ is smaller than the trapping potential aspect ratio $\lambda$ (see Fig.B.5).

The doubly-integrated density difference $\bar{n}_{d}(z)=\bar{n}_{1}(z)-\bar{n}_{2}(z)$ is particularly suited for the observation of surface tension effects. Indeed, as shown in Fig. B.5. $\bar{n}_{d}(z)$ is constant in the region $-R_{S}<z<R_{S}$ if the superfluid core is not deformed, while it increases with $|z|$ when $\lambda_{S}<\lambda$. Fitting an experimental profile $\bar{n}_{d}(z)$, such as the one shown in Fig 5.6 with a set of theoretical profiles calculated with $\lambda_{S} \neq \lambda$, we obtain an estimate of the deformation:

$$
0.95<\lambda_{S} / \lambda<1 .
$$

\footnotetext{
${ }^{*} \mu_{2}$ is equal to $\eta_{c} \mu_{1}$ at the normal/superfluid transition, therefore $\sigma$ is a function of $\mu_{1}$ only.
} 
The deformation is much smaller than the one observed in Rice experiment [97], despite very similar aspect ratio and atom number values. The origin of this disagreement remains an open question. A superfluid core deformation invalidates formula (3.3) relating the doubly-integrated density profiles to the gas pressure; the maximum deformation $\lambda_{S} / \lambda=0.95$ consistent with our data induces a maximum $5 \%$ systematic error on the gas pressure, the error being concentrated around $z=R_{S}$. The shrinking of the superfluid/normal interface also shiftes the $\eta_{c}$ value away from the value for a homogeneous gas. From the maximum deformation $\lambda_{S} / \lambda=0.95$, we estimate the maximum shift to be equal to:

$$
\Delta \eta_{c}=+0.02
$$

This value could explain the difference between our measurement $\eta_{c}=0.065(20)$ and the measurement $\eta_{c}=0.03(2)$ from the MIT group [49], where surface tension effects are expected to be much smaller due the larger atom number and smaller aspect ratio. 


\section{Appendix C}

\section{Relaxation of the Axial Breathing Mode}

In section 6.2.5 we showed that the frequency $\omega_{1}$ and damping $\gamma_{1}$ of the in-phase axial breathing mode are qualitatively related by a Kneser relaxation model. Both quantities can be expressed using a single parameter, namely the relaxation time $\tau$, according to:

$$
\omega^{2}=\left(\omega_{1}^{\mathrm{HD}}\right)^{2}+\frac{\left(\omega_{1}^{\mathrm{CL}}\right)^{2}-\left(\omega_{1}^{\mathrm{HD}}\right)^{2}}{1+i \omega \tau}, \quad \omega=\omega_{1}+i \gamma_{1} .
$$

Fitting our data with a damped cosine whose frequency and damping values are given by (C.1), we obtain the relaxation time $\tau$ as a function of polarization $P$, and observe that (see Fig 6.9):

$$
\frac{1}{\omega_{z} \tau} \simeq 50 \frac{N_{2}}{N_{1}}
$$

The relaxation time can be calculated above the Clogston limit using Boltzmann equation, modeling the gas as a mixture of two ideal Fermi gases, a Fermi sea of majority atoms and a Fermi sea of polarons. Closely related problems were studied in previous works, namely the axial breathing mode relaxation of a spin-balanced Fermi gas 241, 242 243 and the spin dipole mode of an imbalanced Fermi gas 244. We adapt here these calculations to the specific case of the axial breathing mode of a spin-imbalanced Fermi gas.

At equilibrium, the distribution function for both spin component are given by the Fermi-Dirac distribution function:

$$
f_{i}^{0}(\mathbf{r}, \mathbf{p})=1 /\left(1+\exp \left(\frac{\mu_{i}^{0}-p^{2} / 2 m-V(\mathbf{r})}{k_{B} T}\right)\right),
$$

where $T$ is the gas temperature. We forget for simplicity the renormalization effects on the minority atoms due to interactions with majority atoms. The axial breathing mode is accounted for by a dilatation in $z$ by a factor $(1+\beta)$, in $x$ and $y$ by a factor $(1-\beta / 2)$, in $p_{z}$ by a factor $(1+\alpha)$, and in $p_{x}$ and $p_{y}$ by a factor $(1-\alpha / 2)$ :

$$
f_{i}(\mathbf{r}, \mathbf{p}, t)=f_{i}^{0}\left(\frac{x}{1-\beta(t) / 2}, \frac{y}{1-\beta(t) / 2}, \frac{z}{1+\beta(t)}, \frac{p_{x}}{1-\alpha(t) / 2}, \frac{p_{y}}{1-\alpha(t) / 2}, \frac{p_{z}}{1+\alpha(t)}\right) .
$$

$f_{i}(\mathbf{r}, \mathbf{p}, t)$ remains normalized up to second order in $\alpha, \beta$. The quadrupole moment for the majority component is given by:

$$
q(t)=\int \frac{\mathrm{d} \mathbf{r} \mathrm{d} \mathbf{p}}{h^{3}}\left(2 p_{z}^{2}-p_{x}^{2}-p_{y}^{2}\right) f_{1}(\mathbf{r}, \mathbf{p}, t)=N_{1} \frac{p_{F 1}^{2}}{3} \alpha(t),
$$

and oscillates at the axial breathing mode frequency $\omega_{1}$. 
The relaxation for the quantity $q(t)$ is calculated in the Boltzmann equation framework according to:

$$
\begin{aligned}
-\frac{q(t)}{\tau}= & -\frac{1}{64 \pi^{4} \hbar^{4} m^{2} p_{F 1}^{2}} \int \mathrm{d} \mathbf{r} \mathrm{d} \mathbf{p}_{\mathbf{1}} \mathrm{d} \mathbf{p}_{\mathbf{2}} \mathrm{d} \mathbf{q}\left(2 p_{1 z}^{2}-p_{1 x}^{2}-p_{1 y}^{2}\right) \delta\left(\frac{p_{1}^{2}}{2 m}+\frac{p_{2}^{2}}{2 m}-\frac{p_{1}^{\prime 2}}{2 m}-\frac{p_{2}^{\prime 2}}{2 m}\right) \times \ldots \\
& \ldots \times\left(f_{1}\left(\mathbf{r}, \mathbf{p}_{\mathbf{1}}, t\right) f_{2}\left(\mathbf{r}, \mathbf{p}_{\mathbf{2}}, t\right)\left(1-f_{1}\left(\mathbf{r}, \mathbf{p}_{\mathbf{1}}^{\prime}, t\right)\right)\left(1-f_{2}\left(\mathbf{r}, \mathbf{p}_{\mathbf{2}}^{\prime}, t\right)\right)-\right. \\
\left.f_{1}\left(\mathbf{r}, \mathbf{p}_{\mathbf{1}}^{\prime}, t\right) f_{2}\left(\mathbf{r}, \mathbf{p}_{\mathbf{2}}^{\prime}, t\right)\left(1-f_{1}\left(\mathbf{r}, \mathbf{p}_{\mathbf{1}}, t\right)\right)\left(1-f_{2}\left(\mathbf{r}, \mathbf{p}_{\mathbf{2}}, t\right)\right)\right) &
\end{aligned}
$$

where $\mathbf{p}_{\mathbf{1}}^{\prime}=\mathbf{p}_{\mathbf{1}}+\mathbf{q}$ and $\mathbf{p}_{\mathbf{2}}^{\prime}=\mathbf{p}_{\mathbf{2}}-\mathbf{q}$ are the outcomes of a collision between a particle of species 1 and initial momentum $\mathbf{p}_{\mathbf{1}}$ and a particle of species 2 and initial momentum $\mathbf{p}_{\mathbf{2}}$. The collision amplitude is given by the unitary-limited cross section $\sigma=4 \pi / k_{F 1}^{2}$ at the Fermi level for species 1 [244. The terms in $\left(1-f_{i}\right)$ account for the Pauli principle which forbids scattering towards occupied states.

As we consider the limit $N_{2} \ll N_{1}$, we can assume that minority atoms are localized at the bottom of the trap, with momenta $p_{2} \ll p_{1}$. We thus consider in a first approach that minority atoms constitute a set of immobile impurities at $\mathbf{r}=\mathbf{0}$. It is then more convenient to write the collision outcome as:

$$
\mathbf{p}_{1}^{\prime}=\mathbf{p} / 2+p / 2 \mathbf{u}
$$

where $\mathbf{u}$ is a vector of modulus 1 . If we neglect the effect of Pauli exclusion principle on minority atoms, the integral over $\mathbf{p}_{2}$ and $\mathbf{r}$ is straightforward and gives $N_{2}$ :

$-\frac{q(t)}{\tau}=-N_{2} \frac{1}{4 \hbar m^{2} p_{F 1}^{2}} \int \mathrm{d} \mathbf{p}_{\mathbf{1}}\left(2 p_{1 z}^{2}-p_{1 x}^{2}-p_{1 y}^{2}\right) f_{1}\left(\mathbf{r}, \mathbf{p}_{\mathbf{1}}, t\right) p_{1} \mathcal{F}\left(\mathbf{p}_{\mathbf{1}}\right), \quad \mathcal{F}\left(\mathbf{p}_{\mathbf{1}}\right)=\int \frac{\mathrm{d} \mathbf{u}}{4 \pi}\left(1-f_{1}\left(\mathbf{r}=\mathbf{0}, \mathbf{p}_{\mathbf{1}}^{\prime}, t\right)\right)$.

$\mathcal{F}\left(\mathbf{p}_{\mathbf{1}}\right)$ represents the fraction of collisions allowed by Pauli exclusion principle for a particle initially at momentum $\mathbf{p}_{\mathbf{1}}$.

If we forget Pauli exclusion principle also for majority atoms, and calculate the integral, we obtain a very large relaxation rate:

$$
\frac{1}{\omega_{z} \tau} \simeq 5000 \frac{N_{2}}{N_{1}}
$$

The much smaller value measured in our experiment shows the importance of Pauli blocking at such low temperatures. Moreover, it is clear that for a zero-temperature distribution at equilibrium $f_{1}=f_{1}^{0}(T=$ $0), \mathcal{F}\left(\mathbf{p}_{1}\right)$ vanishes for $p<p_{F 1}$, therefore $1 / \tau=0$. We consider two contributions leading to a finite collision probability:

- The majority Fermi surface is deformed into a non-isotropic Fermi surface due to the quadrupole excitation. We calculate the collision integrals numerically and obtain the following law:

$$
\frac{\hbar}{\tau} \simeq 2.1 \frac{N_{2}}{N_{1}} \alpha^{3} E_{F}, \quad \text { i.e. } \quad \frac{1}{\omega_{z} \tau} \simeq 2.1 \alpha^{3} \lambda^{-2 / 3}\left(6 N_{1}\right)^{1 / 3} \frac{N_{2}}{N_{1}}
$$

where $\lambda=\omega_{z} / \omega_{r} \simeq 1 / 20$ is the trap aspect ratio, and $N_{1}=10^{5}$ is the majority atom number. The $\alpha^{3}$ dependence extracted from our calculations was derived analytically in the case of spin-balanced gases in 243. For the deformation amplitude $\alpha=0.3$ corresponding to our experiment, we obtain $1 / \omega_{z} \tau=30 N_{2} / N_{1}$, which is the correct order of magnitude.

- Finite temperature effects also make collisions possible (see Fig 6.10b). We calculate numerically the relaxation rate for an undeformed and finite-temperature distribution, and obtain:

$$
\frac{1}{\omega_{z} \tau} \simeq 36\left(\frac{T}{T_{F 1}}\right)^{2} \lambda^{-2 / 3}\left(6 N_{1}\right)^{1 / 3} \frac{N_{2}}{N_{1}}
$$

The gas temperature is estimated to be $T=0.03(3) T_{F 1}$, leading to $1 / \omega_{z} \tau=20(50) N_{2} / N_{1}$. 
While the amplitude $\alpha$ of the Fermi surface deformation is easily measured (and is found not to vary much with polarization), the cloud's temperature is not measured and may vary with polarization, as we expect evaporation to be more efficient for small polarizations. Therefore the effect of temperature may not lead to a law $1 / \omega_{z} \tau \propto N_{2} / N_{1}$ if $T$ varies with $P$. In order to have a more precise understanding of the relaxation process, it is thus necessary to isolate the effect of deformation and temperature.

Our model is based on a small effect of Pauli blocking for minority atoms. The effect of Pauli blocking on minority would be the freezing of motion for $p_{2}<p_{F 2}-\Delta p$, where $\Delta p$ is proportional to temperature or related to the amplitude of the deformation $\alpha$. Therefore we would expect the number of atoms involved in a collision to be reduced by a factor $\Delta p / p_{F 2}$, and thus $1 / \omega_{z} \tau \propto N_{2}^{2 / 3}$, contrary to what is observed. Minority atoms thus appear to behave as classical immobile particles. This is probably due to the small Fermi energy value for minority atoms.

We have shown that collisions induced by a Fermi surface deformation or a non-zero temperature may account for the relaxation time values extracted from our data. Understanding of the dependence $1 / \omega_{z} \tau \propto N_{2} / N_{1}$ for $P<P_{c}$, i.e. when a superfluid core is present, is clearly beyond the scope of this appendix and remains an open question. 
Appendix D

Publications 


\title{
Collective Oscillations of an Imbalanced Fermi Gas: Axial Compression Modes and Polaron Effective Mass
}

\author{
S. Nascimbène, N. Navon, K. J. Jiang, L. Tarruell, ${ }^{*}$ M. Teichmann, ${ }^{\dagger}$ J. McKeever, ${ }^{\ddagger}$ F. Chevy, and C. Salomon \\ Laboratoire Kastler Brossel, CNRS, UPMC, École Normale Supérieure, 24 rue Lhomond, 75231 Paris, France \\ (Received 15 July 2009; revised manuscript received 2 October 2009; published 20 October 2009)
}

\begin{abstract}
We investigate the low-lying compression modes of a unitary Fermi gas with imbalanced spin populations. For low polarization, the strong coupling between the two spin components leads to a hydrodynamic behavior of the cloud. For large population imbalance we observe a decoupling of the oscillations of the two spin components, giving access to the effective mass of the Fermi polaron, a quasiparticle composed of an impurity dressed by particle-hole pair excitations in a surrounding Fermi sea. We find $m^{*} / m=1.17(10)$, in agreement with the most recent theoretical predictions.
\end{abstract}

The study of the low-lying excitation modes of a complex system can be a powerful tool for investigation of its physical properties. For instance, Earth's structure has been probed using the propagation of seismic waves in the mantle, and the ripples in space-time propagated by gravitational waves can be used as probes of extreme cosmic phenomena. In ultracold atomic gases, the measurement of low energy modes of bosonic or fermionic systems has been used to probe superfluidity effects [1], to measure the angular momentum of vortex lattices [2], and to characterize the equation of state of fermionic superfluids $[3,4]$.

In this Letter, we study the excitation spectrum of an ultracold Fermi gas with imbalanced spin populations. This topic was initiated in the 1960 s by the seminal works of Clogston and Chandrasekhar [5,6] and only recently found experimental confirmation thanks to the latest developments in ultracold Fermi gases [7,8]. These dramatic experiments have observed that when a fermionic superfluid is polarized through imbalance of spin populations, the trapped atomic cloud forms a shell structure. The energy gap associated with pairing maintains a superfluid core where the two spin densities are equal, while the outer shell is composed by a normal gas with imbalanced spin densities (see Fig. 1). Here, we extend this work to the unexplored dynamical properties of these systems and we focus on the regime of strong interactions, where the scattering length $a$ is infinite. We show, in particular, that the study of the axial breathing mode provides valuable insight on the dynamical properties of a quasiparticle, the Fermi polaron, that was introduced recently to describe the normal component occupying the outer shell of the cloud [9-14]. The Fermi polaron is composed of an impurity (labeled 2) immersed in a noninteracting Fermi sea (labeled 1), and is analogous to the polaron of condensed matter physics, i.e., an electron immersed in a bath of noninteracting (bosonic) phonons. Understanding the static and dynamic properties of impurities immersed in an external bath is a paradigm of many-body systems. In addi- tion to polaron physics, famous examples include the Kondo effect, Higgs mechanism, or the dressed atom. Despite its apparent simplicity, this problem remains today very challenging in the limit of strong interactions.

According to the Landau theory of the Fermi liquid, the low energy spectrum of the polaron is similar to that of a free particle and can, in the local density approximation (LDA), be recast as

$$
E_{2}(\boldsymbol{r}, \boldsymbol{p})=A E_{F 1}(\boldsymbol{r})+V(\boldsymbol{r})+\frac{p^{2}}{2 m^{*}}+\ldots
$$

where $V$ is the trapping potential, $E_{F 1}(\boldsymbol{r})=E_{F 1}(0)-V(\boldsymbol{r})$

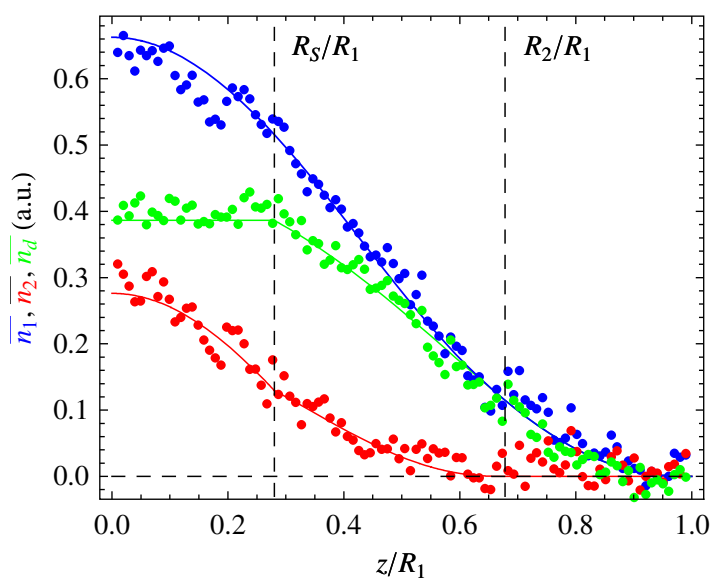

FIG. 1 (color online). Integrated density profiles of an imbalanced Fermi gas. Blue (dark gray): majority atoms $\bar{n}_{1}(z)$; Red (medium gray): minority atoms $\bar{n}_{2}(z)$; Green (light gray): difference $\bar{n}_{d}=\bar{n}_{1}-\bar{n}_{2}$. In this latter case, the flat-top feature signals a cancellation of the density difference at the center of the trap, characteristic of the existence of a fully paired superfluid core. The superfluid (resp. minority) radius $R_{S}$ (resp. $R_{2}$ ) are marked by vertical dashed lines. The solid color lines correspond to the prediction of Monte Carlo theories [20], the only fit parameters being the number of atoms in each spin state, $N_{1}=8.0 \times 10^{4}, N_{2}=2.4 \times 10^{4}$ for this image. The axial (radial) trap frequency is $18.6 \mathrm{~Hz}(420 \mathrm{~Hz})$. 
is the local Fermi energy of the majority species, $A$ is a dimensionless quantity characterizing the attraction of the impurity by the majority atoms, and $m^{*}$ is the effective mass of the Fermi polaron. For $a=\infty, A=-0.61$ has been determined both experimentally [14] and theoretically [9-13], while slight disagreements still exist on the value of the effective mass. Fixed node Monte Carlo suggests $m^{*} / m=1.09$ (2) [15], systematic diagrammatic expansion yields $m^{*} / m=1.20$ [11], and analysis of density profiles (such as Fig. 1) gives $m^{*} / m=1.06$ [16].

From Eq. (1), the quasiparticle evolves in an effective potential $V^{*}(\boldsymbol{r})=(1-A) V(\boldsymbol{r})$. Assuming $V(\boldsymbol{r})$ to be harmonic with frequency $\omega$, the polaron is trapped in an effective potential of frequency $\omega^{*}[9]$ :

$$
\frac{\omega^{*}}{\omega}=\sqrt{\frac{1-A}{m^{*} / m}} .
$$

In this Letter we explore the compression mode properties and determine the effective mass through the measurement of the oscillation frequency $\omega^{*}$ in the axial direction (labeled $z$ ) of a cylindrically symmetric trap.

Our experimental setup is an upgraded version of the one presented in [17]. $7 \times 10^{6}{ }^{6} \mathrm{Li}$ atoms in the hyperfine state $\left|F=3 / 2, m_{F}=+3 / 2\right\rangle$ are loaded into a mixed magnetic or optical trap at $100 \mu \mathrm{K}$. The optical trap uses a single beam of waist $w_{0}=35 \mu \mathrm{m}$ and maximum power $P=60 \mathrm{~W}$ operating at a wavelength $\lambda=1073 \mathrm{~nm}$. The atoms are transferred into the hyperfine ground state $|1 / 2,1 / 2\rangle$, and a spin mixture is created by a radiofrequency sweep across the hyperfine transition $|1 / 2,1 / 2\rangle \rightarrow|1 / 2,-1 / 2\rangle$. By varying the rate of this sweep, we control the sample's degree of polarization $P \equiv$ $\left(N_{1}-N_{2}\right) /\left(N_{1}+N_{2}\right)$, where $N_{1}$ (resp. $\left.N_{2}\right)$ is the atom number of the majority (resp. minority) spin species. The mixture is then evaporatively cooled in $6 \mathrm{~s}$ by reducing the laser power to $70 \mathrm{~mW}$. This is done at a magnetic field $B=$ $834 \mathrm{G}$, which corresponds to the position of the broad Feshbach resonance in ${ }^{6} \mathrm{Li}$ where the scattering length is infinite and where further experiments are performed. Typical radial frequencies are $\omega_{x}=\omega_{y} \sim 2 \pi \times 400 \mathrm{~Hz}$. The axial confinement of the dipole trap is enhanced by the addition of a magnetic curvature, leading to an axial frequency $\omega_{z} \sim 2 \pi \times 30 \mathrm{~Hz}$. Our samples contain $\sim 8 \times 10^{4}$ atoms in the majority spin state at a temperature $T \lesssim$ $0.09 T_{F}$. The temperature is evaluated by fitting the wings of the majority density profile outside the minority radius. In this region, the gas is noninteracting, allowing unambiguous thermometry of the inner, strongly interacting part of the cloud [18]. Here, $T_{F}$ is defined as the Fermi temperature of an ideal gas whose density profile overlaps the majority one in the fully polarized rim.

The two spin states are imaged sequentially using in situ absorption imaging. To prevent heating from the scattered photons and the strong interactions between the two species, the duration of the two imaging pulses as well as their separation must be short (10 $\mu$ s each). By reversing the order in which we image the two spin components, we checked that imaging of the first species did not significantly influence the second. Typical integrated density profiles of the atom cloud $\bar{n}(z)=\int d x d y n(x, y, z)$, where $n(x, y, z)$ is the $3 \mathrm{D}$ atom density, are presented in Fig. 1. These profiles display the characteristic features already observed by the MIT group [18]: a flat-top structure in the superfluid region confirming the existence of a fully paired core satisfying the LDA [19], an intermediate phase where the two spin species are present with unequal densities, and an outer rim containing only majority atoms. Following [20], we compare our density profiles to the prediction for the equation of state of the different phases and find fairly good agreement. In particular, we observe that the superfluid core disappears for polarizations $P>0.76(3)$. This limit agrees well with the measurement of the MIT group [7] but differs from the Rice group value [8]. Our data also show no evidence for surface tension effects $[8,21]$.

We excite the axial breathing mode by switching off the axial magnetic trapping field for $1 \mathrm{~ms}$. The effect of this excitation is twofold: in addition to nearly suppressing the axial confinement, the bias field is increased up to $1050 \mathrm{G}$, where $k_{F} a \sim-1$, so that the gas is no longer strongly interacting. This scheme provides a spatially selective excitation of the cloud. Indeed, while the reduction of the trapping frequency perturbs the whole cloud, the modification of the scattering length only acts in the region where the two spin components overlap. In the regime of strong polarization, these two regions are well separated, leading to a differential excitation of the two spin components.

Let us first focus on the oscillations of the majority spin species presented in Fig. 2. Typical dynamics of the outer radius $R_{1}(t)$ of the majority component are exemplified by Fig. 2(a). For each polarization, this time evolution is fitted using an exponentially damped sinusoid, with $R_{1}(t)=R_{1}^{(0)}\left[1+A_{1} \cos \left(\omega_{1} t+\varphi\right) e^{-\gamma_{1} t}\right]$, and the variations of $\omega_{1}$ and $\gamma_{1}$ as a function of $P$ are displayed in Figs. 2(b) and 2(c). One remarkable feature of this graph is the frequency plateau for polarizations $P \lessgtr 0.7$, corresponding approximately to the domain where a superfluid core is present in the cloud. Although in this range of parameters, the dynamics of the system is fairly complex due to the strong coupling between the superfluid and normal components, a simple argument based on a sum rule approach generalizing the result of [22] allows us to understand this property.

We describe the system by the Hamiltonian $H=$ $\sum_{i} p_{i}^{2} / 2 m+U\left(\boldsymbol{r}_{1}, \boldsymbol{r}_{2}, \ldots\right)$, where $\boldsymbol{r}_{i}$ (resp. $\left.\boldsymbol{p}_{i}\right)$ is the position (resp. momentum of particle $i$ ), $m$ is the mass of the atoms and $U$ includes both trapping potential and interatomic interaction. The compression of the trapping frequency in the $z$ direction is associated with the operator $F=\sum_{i} z_{i}^{2}$. Let us introduce the moments of the spectral distribution associated with $F$ and defined by

$$
m_{k}=\sum_{n \neq 0}\left(E_{n}-E_{0}\right)^{k}|\langle 0|F| n\rangle|^{2},
$$



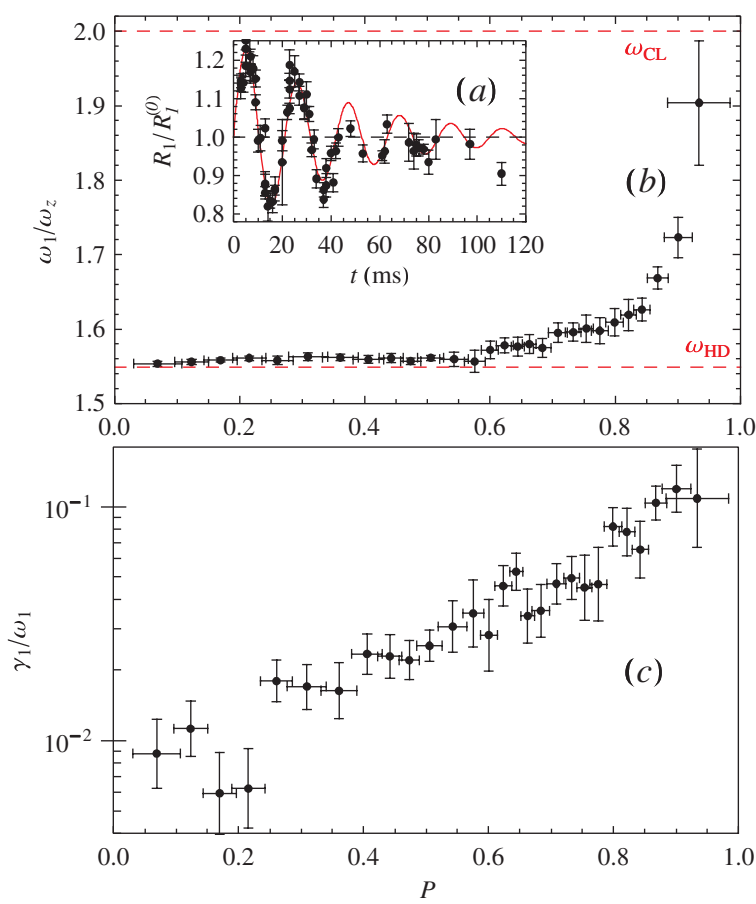

FIG. 2 (color online). (a) Oscillations of the axial radius of the majority component, for a population imbalance $P=0.85(2)$, beyond the Clogston limit. The solid line corresponds to a fit by an exponentially damped sinusoid. (b) Frequency of the breathing mode $\omega_{1}$ normalized to the axial trapping frequency $\omega_{z}$ versus polarization. The superfluid (resp. collisionless) limits $\omega_{1}=\sqrt{12 / 5} \omega_{z}\left(\right.$ resp. $\left.2 \omega_{z}\right)$ are indicated by the dashed red lines. The axial (radial) trap frequency is $28.9(1) \mathrm{Hz}(420 \mathrm{~Hz})$. (c) Damping rate $\gamma_{1}$ versus polarization (in log scale). Note that our data are limited to $P<0.95$ due to the small minority atom number $\left(N_{2} \lesssim 2 \times 10^{3}\right)$ at such high polarizations.

where the $|n\rangle$ are the eigenstates of $H$ associated with the eigenvalue $E_{n}$, and $|0\rangle$ is the many-body ground state. We assume that the operator $F$ mainly couples $|0\rangle$ to one excited state $|1\rangle$. In this case, the frequency of the breathing mode excited by the axial compression of the trap is given by $\omega_{1}=\left(E_{1}-E_{0}\right) / \hbar \simeq \sqrt{m_{1} / m_{-1}} / \hbar$. An explicit calculation of these two moments leads to the following expression:

$$
\omega_{1}^{2} \simeq-2\left\langle z^{2}\right\rangle / \frac{\partial\left\langle z^{2}\right\rangle}{\partial \omega_{z}^{2}} .
$$

For a unitary gas, LDA imposes that the mean radius of the cloud is given by $\left\langle z^{2}\right\rangle=R_{\mathrm{TF}}^{2} f(P)$, where $R_{\mathrm{TF}}$ is the radius of an ideal Fermi gas in the same trap and with the same atom number and $f$ is some universal function of the polarization [23]. Using this assumption, the calculation of the oscillation frequency is straightforward and yields $\sqrt{\frac{12}{5}} \omega_{z}=1.55 \omega_{z}$, i.e., the hydrodynamic prediction $[3,24]$ for $P=0$, regardless of the polarization of the sample. This argument is in good agreement with our experimental findings [Fig. 2(b)].
At larger polarizations the frequency sharply increases towards the collisionless value. The damping rate, very small in the balanced superfluid, increases by a factor $\sim 20$ for higher imbalances [25]. Interestingly, as seen in Fig. 3, this behavior is consistent with a general argument about relaxation processes in fluid dynamics [26]. Indeed, one can relate $\omega_{1}$ and $\gamma_{1}$ through

$$
\omega^{2}=\omega_{\mathrm{CL}}^{2}+\frac{\omega_{\mathrm{HD}}^{2}-\omega_{\mathrm{CL}}^{2}}{1+i \omega \tau},
$$

where $\omega=\omega_{1}+i \gamma_{1}, \quad \omega_{\mathrm{HD}}=\sqrt{12 / 5} \omega_{z}$ (resp. $\omega_{\mathrm{CL}}=$ $2 \omega_{z}$ ) is the hydrodynamic (resp. collisionless) frequency and $\tau$ is an effective relaxation rate.

Measurements of $\omega_{1} / \omega_{z}$ in three different traps of aspect ratios 8.2, 9.0, and 14.5 give identical results (within $3 \%$ ) for all polarizations. By contrast, the effect of temperature is more pronounced: for instance at $0.12(1) T_{F}$, $\omega_{1}(P)$ remains equal to the hydrodynamic prediction at all attainable polarizations with $P_{\max }=0.95$, for a cloud of $N_{1} \sim 2 \times 10^{5}$ majority atoms held in a trap of aspect ratio 22. This illustrates the role of Pauli blocking at the lowest temperatures which favors collisionless behavior. This is in contrast with the balanced gas case where the collisionless regime was observed at higher temperature $\left(T \gtrsim T_{F}\right)$ [27].

Let us now consider the dynamics of the minority cloud (we recall that subscript 2 refers to the impurity atoms). We observe that for polarizations smaller than $P \sim 0.75$, the oscillation frequencies and damping rates of the two spin species are equal, indicating a strong coupling between them. By contrast, for $P>0.75$, a Fourier spectrum of $R_{2}(t)$ reveals two frequencies [Fig. 4(a)], a generic feature of systems with multiple phases $[28,29]$. The lower frequency $\omega_{2 a}$ is equal to the majority oscillation frequency $\omega_{1}$. We interpret the higher frequency $\omega_{2 b}$, whose weight increases with polarization, as the axial breathing of the minority atoms out of phase with the majority cloud. A linear extrapolation of this frequency to $P=1$ gives the oscillation frequency of a dilute gas of weakly interacting polarons inside a Fermi sea at rest, $\omega_{2 b}(P \rightarrow 1)=$ 2.35(10) $\omega_{z}$ [Fig. 4(b)]. The uncertainty represents the standard deviation of a linear fit taking into account the

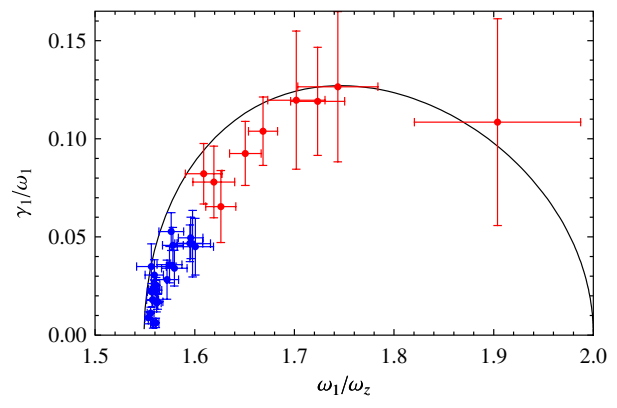

FIG. 3 (color online). Comparison of our experimental results with the parametric curve $\left(\omega_{1}(\tau) / \omega_{z}, \gamma_{1}(\tau) / \omega_{1}(\tau)\right)$ deduced from prediction (4). The data in blue (dark gray) [red (medium gray)] correspond to polarizations $P<0.8[P>0.8]$. 


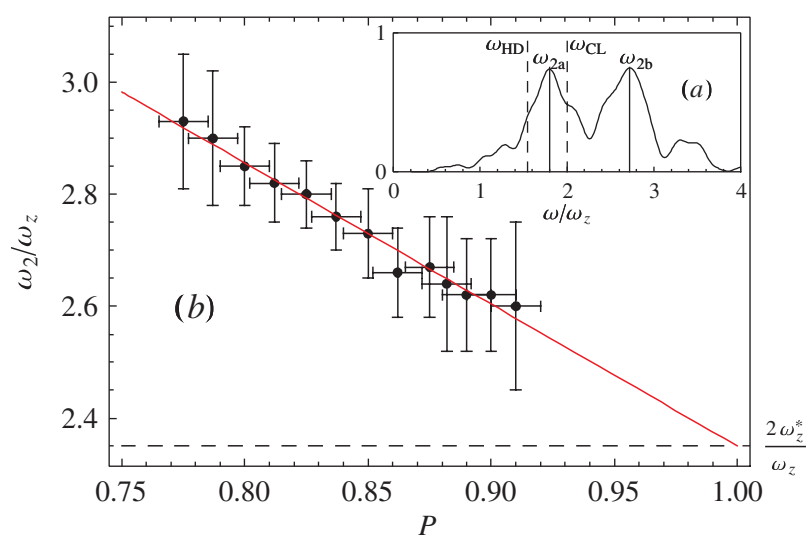

FIG. 4 (color online). (a) Frequency power spectrum of the minority spin state for $P=0.90(2)$. The peak between $\omega_{\mathrm{HD}}$ and $\omega_{\mathrm{CL}}$ corresponds to the oscillation in phase with the majority, the other one to the polaron oscillation. (b) Frequency of the polaron component as a function of polarization. All frequencies are normalized to $\omega_{z}$.

statistical uncertainties of the $\omega_{2 b}$ measurements for each polarization.

By identifying the breathing mode frequency $\omega_{2 b}$ as $2 \omega_{z}^{*}$ and using (2), we deduce the mass of the quasiparticle: $m^{*} / m=1.17(10)$. This is the first dynamic measurement of the polaron effective mass, in good agreement with the most recent theoretical predictions $[11,15]$. The previous measurement of $m^{*}$ through analysis of density profiles required an approximate equation of state for the polaron gas, with uncontrolled accuracy [16]. Extrapolating $\omega_{2 b}(P)$ to $P=1$ allows us to overcome this issue. $m^{*}$ is close to $m$ (albeit different), a surprising feature for a system at unitarity.

In conclusion, we have studied the low frequency breathing modes of an elongated Fermi gas with imbalanced spin populations. In the presence of a superfluid core, the majority and minority components oscillate in phase with a frequency that is largely independent of the spin polarization. At strong polarizations, the minority atom oscillation reveals a second frequency, that we interpret as the Fermi polaron breathing mode. Further investigations will extend our work to all values of the scattering length. In particular, they should provide a clear signature of the polaron-molecule transition $[14,30]$. The role of interactions between polarons and damping phenomena should also be clarified [31].

We are grateful to S. Stringari, A. Recati, C. Lobo, M. Zwierlein, J. Dalibard, and Y. Castin for fruitful discussions as well as K. Magalhães and G. Duffy for experimental support. We acknowledge support from ESF (FerMix), SCALA, ANR FABIOLA, Région Ile de France (IFRAF), ERC and Institut Universitaire de France.

*Present address: Institute for Quantum Electronics, ETH Zurich, 8093 Zurich, Switzerland.
${ }^{\dagger}$ Present address: Max-Born-Institut, Max-Born-Strasse 2 A, 12489 Berlin, Germany.

${ }^{\star}$ Present address: IOS, CQIQC and Department of Physics, University of Toronto, Canada.

[1] S. Giorgini, L. Pitaevskii, and S. Stringari, Rev. Mod. Phys. 80, 1215 (2008).

[2] F. Chevy, K. W. Madison, and J. Dalibard, Phys. Rev. Lett. 85, 2223 (2000).

[3] M. Bartenstein, A. Altmeyer, S. Riedl, S. Jochim, C. Chin, J. Denschlag, and R. Grimm, Phys. Rev. Lett. 92, 203201 (2004).

[4] J. Kinast, S. Hemmer, M. Gehm, A. Turlapov, and J. Thomas, Phys. Rev. Lett. 92, 150402 (2004).

[5] A. Clogston, Phys. Rev. Lett. 9, 266 (1962).

[6] B. S. Chandrasekhar, Appl. Phys. Lett. 1, 7 (1962).

[7] M. Zwierlein, A. Schirotzek, C. Schunck, and W. Ketterle, Science 311, 492 (2006).

[8] G. Partridge, W. Li, R. Kamar, Y. Liao, and R. Hulet, Science 311, 503 (2006).

[9] C. Lobo, A. Recati, S. Giorgini, and S. Stringari, Phys. Rev. Lett. 97, 200403 (2006).

[10] F. Chevy, Phys. Rev. A 74, 063628 (2006).

[11] R. Combescot and S. Giraud, Phys. Rev. Lett. 101, 050404 (2008).

[12] R. Combescot, A. Recati, C. Lobo, and F. Chevy, Phys. Rev. Lett. 98, 180402 (2007).

[13] N. Prokof'ev and B. Svistunov, Phys. Rev. B 77, 125101 (2008).

[14] A. Schirotzek, C.-H. Wu, A. Sommer, and M.W. Zwierlein, Phys. Rev. Lett. 102, 230402 (2009).

[15] S. Pilati and S. Giorgini, Phys. Rev. Lett. 100, 030401 (2008).

[16] Y. Shin, Phys. Rev. A 77, 041603 (2008).

[17] T. Bourdel, L. Khaykovich, J. Cubizolles, J. Zhang, F. Chevy, M. Teichmann, L. Tarruell, S. Kokkelmans, and C. Salomon, Phys. Rev. Lett. 93, 050401 (2004).

[18] Y. Shin, C. Schunck, A. Schirotzek, and W. Ketterle, Nature (London) 451, 689 (2008).

[19] M. Haque and H. Stoof, Phys. Rev. A 74, 011602 (2006).

[20] A. Recati, C. Lobo, and S. Stringari, Phys. Rev. A 78, 023633 (2008).

[21] T. De Silva and E. Mueller, Phys. Rev. Lett. 97, 070402 (2006).

[22] L. Vichi and S. Stringari, Phys. Rev. A 60, 4734 (1999).

[23] F. Chevy, Phys. Rev. Lett. 96, 130401 (2006).

[24] M. Amoruso, I. Meccoli, A. Minguzzi, and M. Tosi, Eur. Phys. J. D 7, 441 (1999).

[25] The damping rate is expected to vanish in the truly collisionless limit, a regime difficult to access experimentally.

[26] L. Landau and E. Lifshitz, Fluid Mechanics, Course of Theoretical Physics Vol. 6 (Butterworth-Heinemann, Oxford, 1987).

[27] S. Riedl, E. Sánchez Guajardo, C. Kohstall, A. Altmeyer, M. Wright, J. Denschlag, R. Grimm, G. Bruun, and H. Smith, Phys. Rev. A 78, 053609 (2008).

[28] M. Urban, Phys. Rev. A 78, 053619 (2008).

[29] G. Bruun and B. Mottelson, Phys. Rev. Lett. 87, 270403 (2001).

[30] N. Prokof'ev and B. Svistunov, Phys. Rev. B 77, 020408 (2008).

[31] G. Bruun, A. Recati, C. Pethick, H. Smith, and S. Stringari, Phys. Rev. Lett. 100, 240406 (2008). 


\title{
Exploring the thermodynamics of a universal Fermi gas
}

\author{
S. Nascimbène ${ }^{1}$, N. Navon ${ }^{1}$, K. J. Jiang ${ }^{1}$, F. Chevy ${ }^{1} \&$ C. Salomon ${ }^{1}$
}

One of the greatest challenges in modern physics is to understand the behaviour of an ensemble of strongly interacting particles. A class of quantum many-body systems (such as neutron star matter and cold Fermi gases) share the same universal thermodynamic properties when interactions reach the maximum effective value allowed by quantum mechanics, the so-called unitary limit ${ }^{1,2}$. This makes it possible in principle to simulate some astrophysical phenomena inside the highly controlled environment of an atomic physics laboratory. Previous work on the thermodynamics of a two-component Fermi gas led to thermodynamic quantities averaged over the trap ${ }^{3-5}$, making comparisons with many-body theories developed for uniform gases difficult. Here we develop a general experimental method that yields the equation of state of a uniform gas, as well as enabling a detailed comparison with existing theories ${ }^{6-15}$. The precision of our equation of state leads to new physical insights into the unitary gas. For the unpolarized gas, we show that the low-temperature thermodynamics of the strongly interacting normal phase is well described by Fermi liquid theory, and we localize the superfluid transition. For a spin-polarized system $^{16-18}$, our equation of state at zero temperature has a 2 per cent accuracy and extends work ${ }^{19,20}$ on the phase diagram to a new regime of precision. We show in particular that, despite strong interactions, the normal phase behaves as a mixture of two ideal gases: a Fermi gas of bare majority atoms and a non-interacting gas of dressed quasi-particles, the fermionic polarons ${ }^{10,18,20-22}$.

In this Letter we study the thermodynamics of a mixture of the two lowest spin states $(i=1,2)$ of ${ }^{6} \mathrm{Li}$ prepared at a magnetic field $B=834 \mathrm{G}$ (see Methods), where the dimensionless number $1 / k_{\mathrm{F}} a$ characterizing the $s$-wave interaction is equal to zero, the unitary limit. (Here $k_{\mathrm{F}}$ is the Fermi momentum and $a$ the scattering length.) Understanding the universal thermodynamics at unitarity is a challenge for many-body theories because of the strong interactions between particles. Despite this complexity at the microscopic scale, all the macroscopic properties of an homogeneous system are encapsulated within a single equation of state (EOS), $P\left(\mu_{1}, \mu_{2}, T\right)$, that relates the pressure $P$ of the gas to the chemical potentials $\mu_{i}$ of the species $i$ and to the temperature $T$. In the unitary limit, this relationship can be expressed as ${ }^{1}$ :

$$
P\left(\mu_{1}, \mu_{2}, T\right)=P_{1}\left(\mu_{1}, T\right) h\left(\eta=\frac{\mu_{2}}{\mu_{1}}, \zeta=\exp \left(\frac{-\mu_{1}}{k_{\mathrm{B}} T}\right)\right)
$$

where $P_{1}\left(\mu_{1}, T\right)=-k_{\mathrm{B}} T \lambda_{\mathrm{dB}}^{-3}(T) f_{5 / 2}\left(-\zeta^{-1}\right)$ is the pressure of a single component non-interacting Fermi gas. Here $k_{\mathrm{B}}$ is the Boltzmann constant, $\lambda_{\mathrm{dB}}(T)$ is the de Broglie wavelength and $f_{5 / 2}(z)=\sum_{n=1}^{\infty} z^{n} / n^{5 / 2}$. $h(\eta, \zeta)$ is a universal function which contains all the thermodynamic information of the unitary gas (Fig. 1). In cold atomic systems, the inhomogeneity due to the trapping potential makes the measurement of $h(\eta, \zeta)$ challenging. However, this inhomogeneity of the trap can be turned into an advantage, as shown in refs 20 and 23.

We directly probe the local pressure of the trapped gas using in situ images, following a recent proposa ${ }^{23}$. In the local density approximation, the gas is locally homogeneous with local chemical potentials:

$$
\mu_{i}(\mathbf{r})=\mu_{i}^{0}-V(\mathbf{r})
$$

here $\mu_{i}^{0}$ is the chemical potential at the bottom of the trap for species $i$ and $V(\mathbf{r})$ is the trapping potential. Then a simple formula relates the pressure $P$ to the doubly-integrated density profiles $^{23}$ :

$$
P\left(\mu_{1 z}, \mu_{2 z}, T\right)=\frac{m \omega_{\mathrm{r}}^{2}}{2 \pi}\left(\bar{n}_{1}(z)+\bar{n}_{2}(z)\right)
$$

where $\bar{n}_{i}(z)=\int n_{i}(x, y, z) \mathrm{d} x \mathrm{~d} y, n_{i}$ being the atomic density. $\omega_{\mathrm{r}}$ and $\omega_{z}$ are respectively the transverse and axial angular frequency of a cylindrically symmetric trap (see Fig. 2 ), $m$ is the ${ }^{6} \mathrm{Li}$ mass, and $\mu_{i z}=\mu_{i}(0,0, z)$ is the local chemical potential along the $z$ axis. From a single image, we thus measure the EOS, equation (1), along the parametric line $(\eta, \zeta)=\left(\mu_{2 z} / \mu_{1 z}, \exp \left(-\mu_{1 z} / k_{\mathrm{B}} T\right)\right)$; see below.

The interest of this method is straightforward. First, one directly measures the EOS of the uniform gas. Second, each pixel row $z_{i}$ gives a point $h\left(\eta\left(z_{i}\right), \zeta\left(z_{i}\right)\right)$ whose signal to noise ratio is essentially given by that of $\bar{n}_{1}(z)+\bar{n}_{2}(z)$; typically one experimental run leads to $\sim 100$ points with a signal to noise ratio between 3 and 10 . With about 40 images one gets $\sim 4,000$ points $h(\eta, \zeta)$, which after averaging provides a low-noise EOS of standard deviation $\sigma=2 \%$. In the following we illustrate the efficiency of our method on two important sectors of the parameter space $(\eta, \zeta)$ in Fig. 1: the balanced gas at finite temperature $(1, \zeta)$ and the zero-temperature imbalanced gas $(\eta, 0)$.

We first measure the EOS of the unpolarized unitary gas at finite temperature, $P\left(\mu_{1}, \mu_{2}, T\right)=P(\mu, T)$. The measurement of $h(1, \zeta)$ through the local pressure, equation (3), can be done provided one knows the temperature $T$ of the cloud and its central chemical potential $\mu^{0}$.

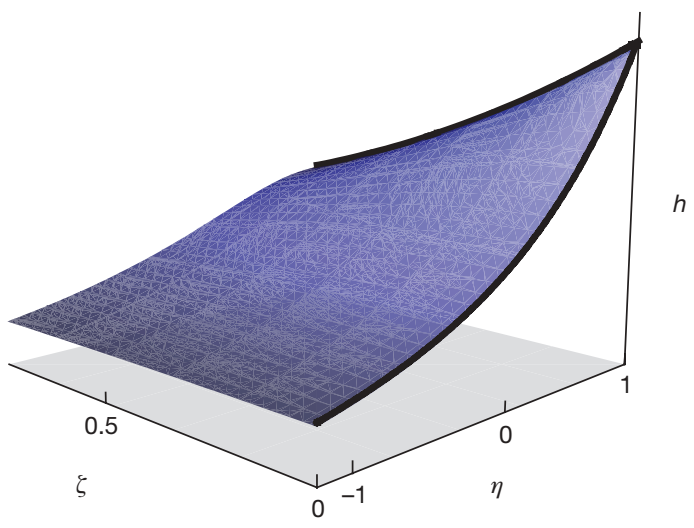

Figure 1 | Schematic representation of the universal function $h(\eta, \zeta)$. It fully describes the thermodynamics of the unitary gas as a function of chemical potential imbalance $\eta=\mu_{2} / \mu_{1}$ and of the inverse of the fugacity $\zeta=\exp \left(-\mu_{1} / k_{\mathrm{B}} T\right)$. In this paper we measure the function $h$ over the black lines $(1, \zeta)$ and $(\eta, 0)$, which correspond to the balanced unitary gas at finite temperature and to the spin-imbalanced gas at zero temperature, respectively. 


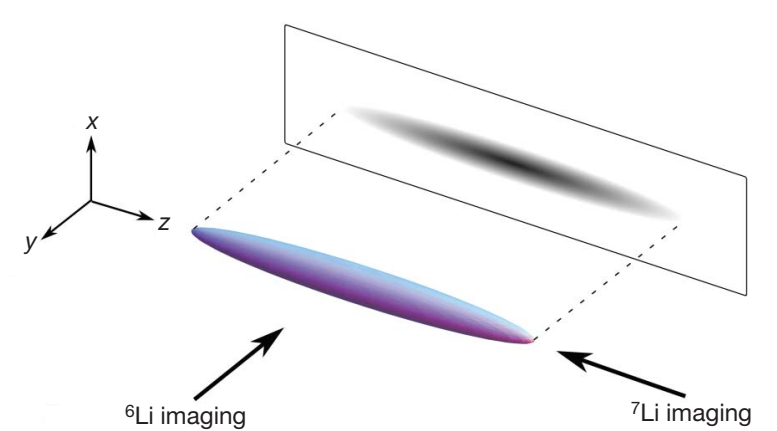

Figure $\mathbf{2}$ | Schematic representation of our atomic sample. The ${ }^{6} \mathrm{Li}$ atomic cloud is imaged in the direction $y$; the column density is then integrated along the direction $x$ to give $\bar{n}(z)$. The ${ }^{7} \mathrm{Li}$ atoms are imaged after a time of flight along the $z$ direction.

In the balanced case, model-independent thermometry is notoriously difficult because of the strong interactions. Inspired by ref. 24, we overcome this issue by measuring the temperature of a ${ }^{7} \mathrm{Li}$ cloud in thermal equilibrium with the ${ }^{6} \mathrm{Li}$ mixture (see Methods).

The central chemical potential $\mu^{0}$ is fitted on the hottest clouds so that the EOS agrees in the classical regime $\zeta \gg 1$ with the secondorder virial expansion $h(1, \zeta) \approx 2\left(1+\zeta^{-1} / \sqrt{2}\right.$ ) (ref. 25). For colder clouds we proceed recursively. The EOS of an image recorded at temperature $T$ has some overlap with the previously determined EOS from all images with $T^{\prime}>T$. In this overlap region, $\mu^{0}$ is fitted to minimize the distance between the two EOSs. This provides a new portion of the EOS at lower temperature. Using 40 images of clouds prepared at different temperatures, we thus reconstruct a low-noise EOS from the classical part down to the degenerate regime, as shown in Fig. 3a.

We now comment on the main features of the EOS. At high temperature, the EOS can be expanded in powers of $\zeta^{-1}$ as a virial expansion $^{11}$ :

$$
\frac{h(1, \zeta)}{2}=\frac{\sum_{k=1}^{\infty}\left((-1)^{k+1} k^{-5 / 2}+b_{k}\right) \zeta^{-k}}{\sum_{k=1}^{\infty}(-1)^{k+1} k^{-5 / 2} \zeta^{-k}}
$$

where $b_{k}$ is the $k^{\text {th }}$ virial coefficient. As we have $b_{2}=1 / \sqrt{2}$ in the measurement scheme described above, our data provide for the first time the experimental values of $b_{3}$ and $b_{4} . b_{3}=-0.35(2)$ is in excellent agreement with the recent calculation $b_{3}=-0.291-3^{-5 / 2}=-0.355$ from ref. 11, but not with $b_{3}=1.05$ from ref. 12 . $b_{4}=0.096(15)$ involves the four-fermion problem at unitarity and could interestingly be computed along the lines of ref. 11 .

Let us now focus on the low-temperature regime of the normal phase $\zeta \ll 1$. As shown in Fig. 3b, we observe a $T^{2}$ dependence of the pressure with temperature. This behaviour is reminiscent of a Fermi liquid, and indicates that pseudogap effects expected for strongly interacting Fermi superfluids ${ }^{26}$ do not show up at the thermodynamic level within our experimental precision. In analogy with ${ }^{3} \mathrm{He}$ or heavy-fermion metals, we fit our data with the EOS:

$$
P(\mu, T)=2 P_{1}(\mu, 0)\left(\xi_{\mathrm{n}}^{-3 / 2}+\frac{5 \pi^{2}}{8} \xi_{\mathrm{n}}^{-1 / 2} \frac{m^{*}}{m}\left(\frac{k_{\mathrm{B}} T}{\mu}\right)^{2}\right)
$$

Here $P_{1}(\mu, 0)=1 / 15 \pi^{2}\left(2 m / \hbar^{2}\right)^{3 / 2} \mu^{5 / 2}$ is the pressure of a singlecomponent Fermi gas at zero temperature, $m^{*}$ is the quasi-particle mass, and $\xi_{\mathrm{n}}^{-1}$ is the compressibility of the normal gas extrapolated to zero temperature, and normalized to that of an ideal gas of same density. We deduce two new parameters $m^{*} / m=1.13(3)$ and $\xi_{\mathrm{n}}=0.51(2)$. Despite the strong interactions, $m^{*}$ is close to $m$, unlike the weakly interacting ${ }^{3} \mathrm{He}$ liquid for which $2.7<m^{*} / m<5.8$, depending on pressure. Our $\xi_{\mathrm{n}}$ value is in agreement with the variational fixed-node Monte Carlo calculations $\xi_{\mathrm{n}}=0.54$ in ref. 27 and
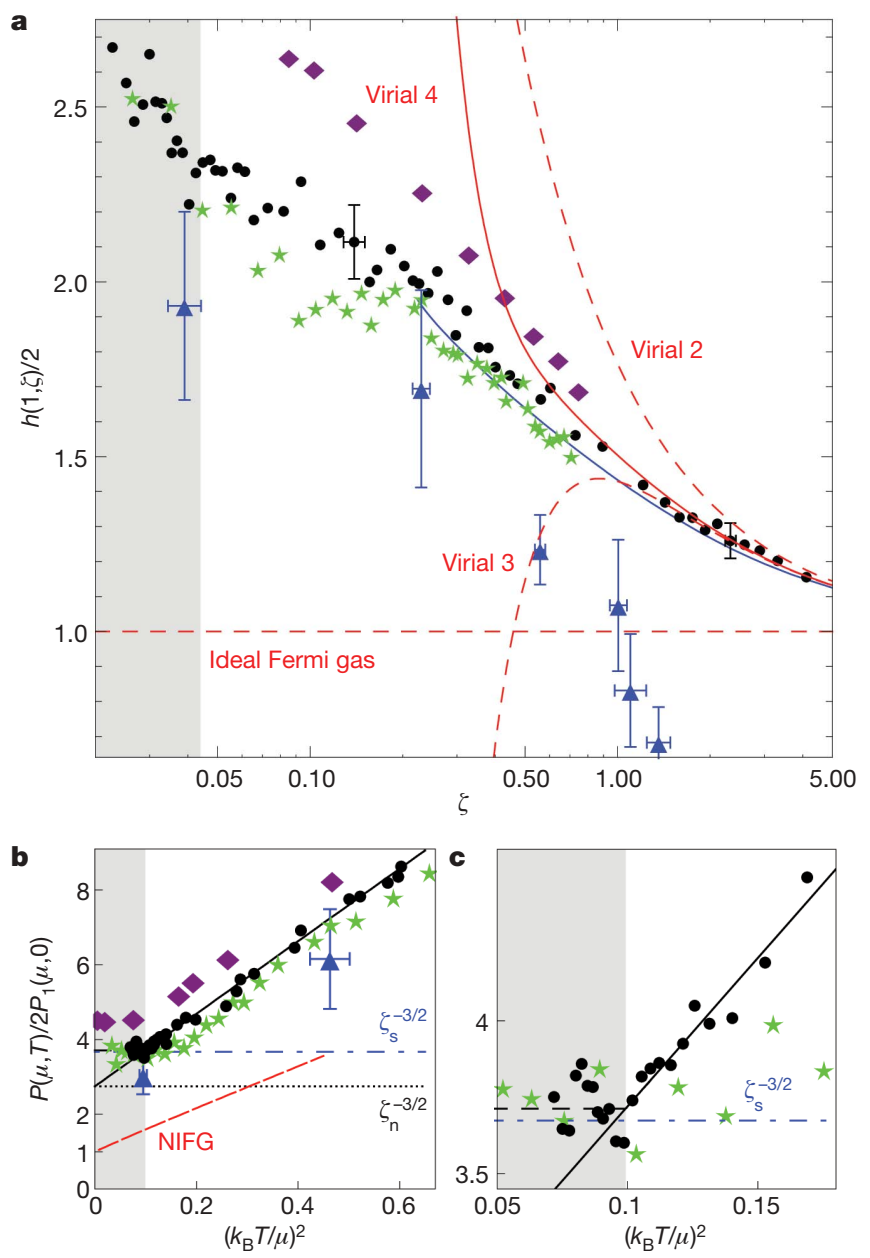

Figure 3 | Equation of state of a spin-balanced unitary Fermi gas. a, Finitetemperature equation of state (EOS) $h(1, \zeta)$ (black dots). The error bars represented at $\zeta=0.14$ and $\zeta=2.3$ indicate the $6 \%$ accuracy in $\zeta$ and $h$ of our EOS. The red curves are the successive virial expansions up to fourth order. The blue triangles are from ref. 6, the green stars from ref. 7, the purple diamonds from ref. 8 , and the blue solid line from ref. 9. The grey region indicates the superfluid phase. $\mathbf{b}, \operatorname{EOS} P(\mu, T) / 2 P_{1}(\mu, 0)$ as a function of $\left(k_{\mathrm{B}} T / \mu\right)^{2}$, fitted by the Fermi liquid EOS, equation (4). The red dashed line is the non-interacting Fermi gas (NIFG). The horizontal dot-dashed and dotted lines indicate respectively the zero-temperature pressure of the superfluid phase $\propto \xi_{s}^{-3 / 2}$ and that of the normal phase $\propto \xi_{n}^{-3 / 2}$.c, Expanded view of $\mathbf{b}$ near $T_{\mathrm{c}}$. The sudden deviation of the data from the fit occurs at $\left(k_{\mathrm{B}} T / \mu\right)_{\mathrm{c}}=0.32(3)$ that we interpret as the superfluid transition. The black dashed line indicates the mean value of the data points below $T_{\mathrm{c}}$.

$\xi_{\mathrm{n}}=0.56$ in ref. 10 , and with the quantum Monte Carlo calculation $\xi_{\mathrm{n}}=0.52$ in ref. 28. This yields the Landau parameters $F_{0}^{\mathrm{s}}=\xi_{\mathrm{n}} m^{*} / m-1=-0.42$ and $F_{1}^{\mathrm{s}}=3\left(\mathrm{~m}^{*} / \mathrm{m}-1\right)=0.39$.

In the lowest temperature points (Fig. $3 c$ ) we observe a sudden deviation of the data from the fitted equation $(4)$ at $\left(k_{\mathrm{B}} T / \mu\right)_{\mathrm{c}}=$ 0.32 (3) (see Supplementary Information). We interpret this behaviour as the transition from the normal phase to the superfluid phase. This critical ratio has been extensively calculated in recent years. Our value is in close agreement with the diagrammatic Monte Carlo calculation $\left(k_{\mathrm{B}} T / \mu\right)_{\mathrm{c}}=0.32(2)$ of ref. 6 and with the quantum Monte Carlo calculation $\left(k_{\mathrm{B}} T / \mu\right)_{\mathrm{c}}=0.35$ (3) of ref. 28; but it differs from the self-consistent approach in ref. 8 that gives $\left(k_{\mathrm{B}} T / \mu\right)_{\mathrm{c}}=0.41$, from the renormalization group prediction 0.24 in ref. 29 , and from several other less precise theories. From equation (4) we deduce the total density $n=n_{1}+n_{2}=\partial P\left(\mu_{i}=\mu, T\right) / \partial \mu$ and the Fermi energy $E_{\mathrm{F}}=k_{\mathrm{B}} T_{\mathrm{F}}=\hbar^{2} / 2 m\left(3 \pi^{2} n\right)^{2 / 3}$ at the transition point. We obtain $(\mu /$ $\left.E_{\mathrm{F}}\right)_{\mathrm{c}}=0.49(2)$ and $\left(T / T_{\mathrm{F}}\right)_{\mathrm{c}}=0.157(15)$, in very good agreement with ref. 6. Our measurement is the first direct determination of $\left(\mu / E_{\mathrm{F}}\right)_{\mathrm{c}}$ 
and $\left(T / T_{\mathrm{F}}\right)_{\mathrm{c}}$ in the homogeneous gas. It agrees with the extrapolated value of the MIT measurement ${ }^{19}$.

Below $T_{\mathrm{c}}$, advanced theories ${ }^{7,8}$ predict that $P(\mu, T) / 2 P_{1}(\mu, 0)$ is nearly constant (Fig. 3b). Therefore at $T=T_{\mathrm{c}}, P / 2 P_{1} \approx \xi_{\mathrm{s}}^{-3 / 2} \approx 3.7$, and is consistent with our data. Here $\xi_{s}=0.42(1)$ is the fundamental parameter characterizing the EOS of the balanced superfluid at zero temperature, a quantity extensively measured and computed in recent years ${ }^{2}$.

Our data are compared at all temperatures with the calculations from refs $6-9$ in Fig. 3a. The agreement with ref. 7 is very good for a large range of temperatures. Concerning ref. 6 , the deviation from our data is about one error bar of the Monte Carlo method below $\zeta=0.2$, and the deviation increases with temperature (Fig. 3a). Furthermore, we show in the Supplementary Information that $h(1$, $\zeta) / 2$ must be greater than 1 , an inequality violated by the two hottest Monte Carlo points of ref. 6 .

From our homogeneous EOS we can deduce the EOS of the harmonically trapped unitary gas by integrating $h(1, \zeta)$ over the trap (see Supplementary Information). In particular, we find a critical temperature for the trapped gas $\left(T / T_{\mathrm{F}}\right)_{\mathrm{c}}=0.19(2)$, where $T_{\mathrm{F}}=\hbar\left(3 \omega_{\mathrm{r}}^{2} \omega_{z} N\right)^{1 / 3}$ and $N$ is the total atom number. This value agrees very well with the recent measurement of ref. 30 , and with less precise measurements ${ }^{5,31,32}$.

Let us now explore a second line in the universal diagram $h(\eta, \zeta)$ (Fig. 1) by considering the case of the $T=0$ spin-imbalanced mixture $\mu_{2} \neq \mu_{1}$, that is, $\eta \neq 1$. Previous work ${ }^{16-18}$ has shown that phase separation occurs in a trap. Below a critical population imbalance a fully paired superfluid occupies the centre of the trap. It is surrounded by a normal mixed phase and an outer rim consisting of an ideal gas of the majority component. In two out of the three previous experiments including ours ${ }^{16,18}$, the local density approximation has been carefully checked. We are therefore justified in using equation (3) to analyse our data.

As in the previous case, the relationship between the pressure and the EOS requires the knowledge of the chemical potentials $\mu_{1}^{0}$ and $\mu_{2}^{0}$ at the centre of the trap. $\mu_{1}^{0}$ is determined using the outer shell of the majority spin component $(i=1)$. The pressure profile $P\left(\mu_{1 z}, \mu_{2 z}, 0\right)$ corresponds to the Fermi-Dirac distribution and is fitted with the Thomas-Fermi formula $P_{1}=\alpha\left(1-z^{2} / R_{1}^{2}\right)^{5 / 2}$, providing $\mu_{1}^{0}=\frac{1}{2} m \omega_{z}^{2} R_{1}^{2}$. Using $P_{1}$ for the calculation of $h=P / P_{1}$ cancels many systematic effects on the absolute value of the pressure. Moreover, fitting the outer shell using a finite-temperature Thomas-Fermi profile ${ }^{19}$, we measure a temperature $k_{\mathrm{B}} T=0.03(3) \mu_{1}^{0}$. $\mu_{2}^{0}$ is fitted by comparison in the superfluid region with the superfluid EOS at zero temperature ${ }^{21}$ :

$$
h(\eta, 0)=(1+\eta)^{5 / 2} /\left(2 \xi_{\mathrm{s}}\right)^{3 / 2}
$$

Our measured EOS $h(\eta, 0)$ is displayed in Fig. 4. By construction our data agree for $\eta \gtrsim 0.1$ with equation (5). In Fig. 4 the slope of $h(\eta, 0)$ displays an obvious discontinuity for $\eta=\eta_{c}=0.065(20)$. This is a signature of a first-order quantum phase transition to the partially polarized normal phase. The error bar is dominated by the uncertainty on $\xi_{s}$. This value is slightly higher than the prediction $\eta_{c}=0.02$ given by the fixed-node Monte Carlo ${ }^{10}$ and than the value $\eta_{c}=0.03(2)$ measured in ref. 19.

From the relations $n_{i}=\partial P / \partial \mu_{i}$, we deduce from $h(\eta, 0)$ the density ratio $n_{2} / n_{1}$ (Fig. 4 inset). This ratio is discontinuous at the phase transition, from a maximum value in the normal phase $\left(n_{2} / n_{1}\right)_{\mathrm{c}}=$ 0.5 (1) to $n_{2}=n_{1}$ in the superfluid phase. Our value is close to the zero-temperature calculation 0.44 (ref. 10) and agrees with the coldest MIT samples ${ }^{19,20}$. It confirms that the temperature is much smaller than the tricritical point temperature $T=0.07 T_{\mathrm{F}}$ (ref. 19) where the discontinuity vanishes, justifying our $T=0$ assumption made above.

For $\eta<\eta_{\mathrm{c}}$ our data display a good agreement with a simple polaron model, based on the pioneering work in ref. 10. A polaron is a quasi-particle describing a single minority atom immersed in the majority Fermi sea ${ }^{15,18,21,22}$. It is characterized ${ }^{10}$ by a renormalized

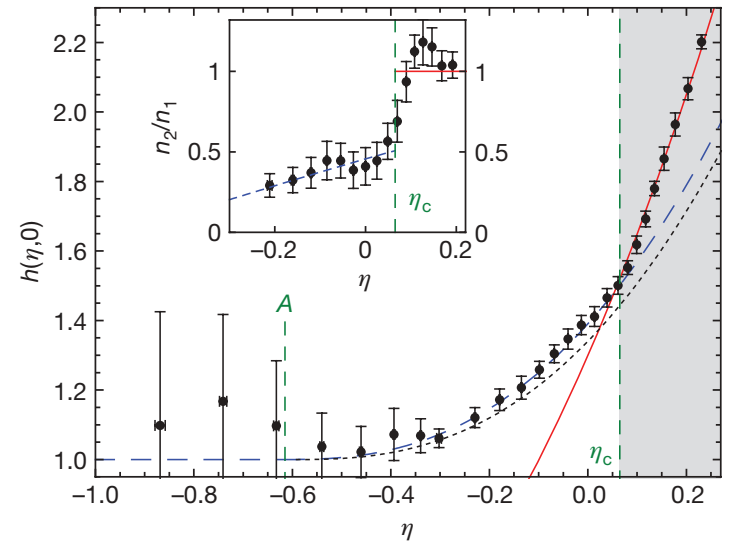

Figure 4 | Equation of state of the zero-temperature spin-imbalanced unitary gas $h(\eta, 0)$. The EOS is shown as filled black circles; error bars are equal to one standard error. The red solid line is the superfluid EOS, the blue dashed line is the ideal Fermi liquid, equation (7), with $A=-0.615$, $m^{*}=1.20 m$ and the black dotted line is the Monte Carlo calculation from ref. 10. Inset, local density ratio $n_{2} / n_{1}$ as a function of $\eta$. The red solid line $n_{2} /$ $n_{1}=1$ corresponds to the fully paired superfluid and blue dashed line to the model, equation (7)

chemical potential $\mu_{2}-A \mu_{1}$ and an effective mass $m_{\mathrm{p}}^{*}$. Following this picture, we write the pressure as the sum of the Fermi pressures of ideal gases of majority atoms and of polarons:

$$
P=\frac{1}{15 \pi^{2}}\left(\frac{2 m}{\hbar^{2}}\right)^{3 / 2}\left(\mu_{1}^{5 / 2}+\left(\frac{m_{\mathrm{p}}^{*}}{m}\right)^{3 / 2}\left(\mu_{2}-A \mu_{1}\right)^{5 / 2}\right)
$$

which can be written as:

$$
h(\eta, 0)=1+\left(\frac{m_{\mathrm{p}}^{*}}{m}\right)^{3 / 2}(\eta-A)^{5 / 2}
$$

$A$ and $m_{\mathrm{p}}^{*}$ have recently been calculated exactly ${ }^{14,15}: A=-0.615$, $m_{\mathrm{p}}^{*} / m=1.20(2)$, and with these values inserted in equation (7) the agreement with our data is perfect. Note that our data lie slightly above the variational fixed-node Monte Carlo calculation ${ }^{10}$. We therefore conclude that interactions between polarons are not visible at this level of precision.

Alternatively, we can fit our data with $m_{\mathrm{p}}^{*} / m$ as a free parameter in equation (7). We obtain $m_{\mathrm{p}}^{*} / m=1.20(2)$. The uncertainty combines the standard error of the fit and the uncertainty on $\xi_{s}$. This value agrees with our previous measurement ${ }^{18} m_{\mathrm{p}}^{*} / m=1.17(10)$ (with a fivefold improvement in precision), with the theoretical value ${ }^{14,15}$ $m_{\mathrm{p}}^{*} / m=1.20(2)$, and with the variational calculation ${ }^{13}$. It differs from the values $1.09(2)$ in ref. 33, 1.04(3) in ref. 10, and from the experimental value 1.06 in ref. 20.

We arrive at a simple physical picture of the $T=0$ spin-polarized gas: the fully paired superfluid is described by an ideal gas EOS renormalized by a single coefficient $\xi_{s}$; the normal phase is nothing but two ideal gases, one of bare majority particles and one of polaronic quasiparticles.

In conclusion, we have introduced a powerful method for the measurement of the EOS of the unitary and homogeneous Fermi gas that enables direct comparison with theoretical models and provides a set of new parameters shown in Table 1. The method

Table 1 | Table of quantities measured in this work

\begin{tabular}{llllll}
\hline Parameter & $b_{3}$ & $b_{4}$ & $\left(k_{\mathrm{B}} T / \mu\right)_{\mathrm{c}}$ & $\left(\mu / E_{\mathrm{F}}\right)_{\mathrm{c}}$ & $\left(T / T_{\mathrm{F}}\right)_{\mathrm{c}}$ \\
Value & $-0.35(2)$ & $0.096(15)$ & $0.32(3)$ & $0.49(2)$ & $0.157(15)$ \\
Parameter & $\xi_{\mathrm{n}}$ & $m^{*} / m$ & $\eta_{\mathrm{c}}$ & $\left(n_{2} / n_{1}\right)_{\mathrm{c}}$ & $m_{\mathrm{p}}^{*} / m$ \\
Value & $0.51(2)$ & $1.13(3)$ & $0.065(20)$ & $0.5(1)$ & $1.20(2)$ \\
\hline
\end{tabular}


can readily be extended to any multi-component cold atom gas in three dimensions that fulfils the local density approximation (see Supplementary Discussion). We have shown that the normal phase of the unitary Fermi gas is a strongly correlated system whose thermodynamic properties are well described by Fermi liquid theory, unlike high- $T_{\mathrm{c}}$ copper oxides.

Note added in proof: Since this paper was accepted for publication, we have become aware of the measurement of a similar equation of state for the balanced unitary Fermi gas at finite temperature by different methods ${ }^{34}$

\section{METHODS SUMMARY}

Our experimental set-up is presented elsewhere ${ }^{18}$. We load into an optical dipole trap and evaporate a mixture of ${ }^{6} \mathrm{Li}$ in the $|1 / 2, \pm 1 / 2\rangle$ states and of ${ }^{7} \mathrm{Li}$ in the $|1,1\rangle$ state at $834 \mathrm{G}$. The cloud typically contains $N_{6}=(5-10) \times 10^{4}$ atoms of ${ }^{6} \mathrm{Li}$ in each spin state and $N_{7}=(3-20) \times 10^{3}$ atoms of ${ }^{7} \mathrm{Li}$ at a temperature from $T=150 \mathrm{nK}$ to $1.3 \mu \mathrm{K}$. The ${ }^{6} \mathrm{Li}$ trap frequencies are $\omega_{z} / 2 \pi=37 \mathrm{~Hz}, \omega_{\mathrm{r}} / 2 \pi$ varying from $830 \mathrm{~Hz}$ to $2.20 \mathrm{kHz}$, and the trap depth is $25 \mu \mathrm{K}$ for our hottest samples, with $T \approx 2 T_{\mathrm{F}} .{ }^{6} \mathrm{Li}$ atoms are imaged in situ using absorption imaging, while ${ }^{7} \mathrm{Li}$ atoms are imaged after time of flight, providing the temperature in the same experimental run (Fig. 4). As the scattering length describing the interaction between ${ }^{7} \mathrm{Li}$ and ${ }^{6} \mathrm{Li}$ atoms, $a_{67}=2 \mathrm{~nm}$, is much smaller than $k_{\mathrm{F}}^{-1}$, the ${ }^{7} \mathrm{Li}$ thermometer has no influence on the ${ }^{6} \mathrm{Li}$ density profiles. The ${ }^{7} \mathrm{Li}-{ }^{6} \mathrm{Li}$ collision rate, $\Gamma_{67}=10 \mathrm{~s}^{-1}$, is large enough to ensure thermal equilibrium between the two species.

Full Methods and any associated references are available in the online version of the paper at www.nature.com/nature.

\section{Received 2 November 2009; accepted 6 January 2010.}

1. Ho, T.-L. Universal thermodynamics of degenerate quantum gases in the unitarity limit. Phys. Rev. Lett. 92, 090402 (2004).

2. Inguscio, M., Ketterle, W. \& Salomon, C. (eds) Proc. Int. School of Physics Enrico Fermi (Course CLXIV, IOS Press, Amsterdam, 2006).

3. Stewart, J., Gaebler, J., Regal, C. \& Jin, D. Potential energy of a ${ }^{40} \mathrm{~K}$ Fermi gas in the BCS-BEC crossover. Phys. Rev. Lett. 97, 220406 (2006).

4. Luo, L., Clancy, B., Joseph, J., Kinast, J. \& Thomas, J. Measurement of the entropy and critical temperature of a strongly interacting Fermi gas. Phys. Rev. Lett. 98, 080402 (2007)

5. Luo, L. \& Thomas, J. Thermodynamic measurements in a strongly interacting Fermi gas. J. Low Temp. Phys. 154, 1-29 (2009).

6. Burovski, E., Prokofev, N., Svistunov, B. \& Troyer, M. Critical temperature and thermodynamics of attractive fermions at unitarity. Phys. Rev. Lett. 96, 160402 (2006).

7. Bulgac, A., Drut, J. \& Magierski, P. Spin $1 / 2$ fermions in the unitary regime: a superfluid of a new type. Phys. Rev. Lett. 96, 090404 (2006).

8. Haussmann, R., Rantner, W., Cerrito, S. \& Zwerger, W. Thermodynamics of the BCS-BEC crossover. Phys. Rev. A 75, 023610 (2007)

9. Combescot, R., Alzetto, F. \& Leyronas, X. Particle distribution tail and related energy formula. Phys. Rev. A 79, 053640 (2009).

10. Lobo, C., Recati, A., Giorgini, S. \& Stringari, S. Normal state of a polarized Fermi gas at unitarity. Phys. Rev. Lett. 97, 200403 (2006).

11. Liu, X., Hu, H. \& Drummond, P. Virial expansion for a strongly correlated Fermi gas. Phys. Rev. Lett. 102, 160401 (2009).

12. Rupak, G. Universality in a 2-component Fermi system at finite temperature. Phys Rev. Lett. 98, 090403 (2007).

13. Combescot, R., Recati, A., Lobo, C. \& Chevy, F. Normal state of highly polarized Fermi gases: simple many-body approaches. Phys. Rev. Lett. 98, 180402 (2007)
14. Combescot, R. \& Giraud, S. Normal state of highly polarized Fermi gases: full many-body treatment. Phys. Rev. Lett. 101, 050404 (2008)

15. Prokof'ev, N. \& Svistunov, B. Fermi-polaron problem: diagrammatic Monte Carlo method for divergent sign-alternating series. Phys. Rev. B 77, 020408 (2008).

16. Shin, Y Zwierlein, M. Schunck, C Schirotzek, A. \& Ketterle, W Observation of phase separation in a strongly interacting imbalanced Fermi gas. Phys. Rev. Lett. 97, 030401 (2006).

17. Partridge, G., Li, W., Kamar, R., Liao, Y. \& Hulet, R. Pairing and phase separation in a polarized Fermi gas. Science 311, 503-505 (2006).

18. Nascimbene, S. et al. Collective oscillations of an imbalanced Fermi gas: axial compression modes and polaron effective mass. Phys. Rev. Lett. 103, 170402 (2009).

19. Shin, Y., Schunck, C., Schirotzek, A. \& Ketterle, W. Phase diagram of a twocomponent Fermi gas with resonant interactions. Nature 451, 689-693 (2008).

20. Shin, Y. Determination of the equation of state of a polarized Fermi gas at unitarity. Phys. Rev. A 77, 041603 (2008)

21. Chevy, F. Universal phase diagram of a strongly interacting Fermi gas with unbalanced spin populations. Phys. Rev. A 74, 063628 (2006).

22. Schirotzek, A., Wu, C.-H., Sommer, A. \& Zwierlein, M. W. Observation of Fermi polarons in a tunable Fermi liquid of ultracold atoms. Phys. Rev. Lett. 102, 230402 (2009).

23. Ho, T.-L. \& Zhou, Q. Obtaining phase diagram and thermodynamic quantities of bulk systems from the densities of trapped gases. Nature Phys. 6, 131-134 (2010).

24. Spiegelhalder, F. et al. Collisional stability of ${ }^{40} \mathrm{~K}$ immersed in a strongly interacting Fermi gas of ${ }^{6}$ Li. Phys. Rev. Lett. 103, 223203 (2009).

25. Ho, T.-L. \& Mueller, E. High temperature expansion applied to fermions near Feshbach resonance. Phys. Rev. Lett. 92, 160404 (2004).

26. Chen, Q., Stajic, J., Tan, S. \& Levin, K. BCS BEC crossover: from high temperature superconductors to ultracold superfluids. Phys. Rep. 412, 1-88 (2005).

27. Carlson, J., Chang, S., Pandharipande, V. \& Schmidt, K. Superfluid Fermi gases with large scattering length. Phys. Rev. Lett. 91, 050401 (2003).

28. Bulgac, A., Drut, J. \& Magierski, P. Quantum Monte Carlo simulations of the BCSBEC crossover at finite temperature. Phys. Rev. A 78, 023625 (2008).

29. Gubbels, K. \& Stoof, H. Renormalization group theory for the imbalanced Fermi gas. Phys. Rev. Lett. 100, 140407 (2008).

30. Riedl, S., Guajardo, E., Kohstall, C., Denschlag, J. \& Grimm, R. Superfluid quenching of the moment of inertia in a strongly interacting Fermi gas. Preprint at $\langle\mathrm{http}: / /$ arXiv.org/abs/0907.3814) (2009).

31. Greiner, M., Regal, C. \& Jin, D. Emergence of a molecular Bose-Einstein condensate from a Fermi gas. Nature 426, 537-540 (2003).

32. Inada, Y. et al. Critical temperature and condensate fraction of a fermion pair condensate. Phys. Rev. Lett. 101, 180406 (2008).

33. Pilati, S. \& Giorgini, S. Phase separation in a polarized Fermi gas at zero temperature. Phys. Rev. Lett. 100, 030401 (2008).

34. Horikoshi, M., Nakajima, S., Ueda, M. \& Mukaiyama, T. Measurement of universal thermodynamic functions for a unitary Fermi gas. Science 327, 442-445 (2010).

Supplementary Information is linked to the online version of the paper at www.nature.com/nature.

Acknowledgements We are grateful to R. Combescot, X. Leyronas, Y. Castin, A. Recati, S. Stringari, S. Giorgini, M. Zwierlein and T. Giamarchi for discussions and to C. Cohen-Tannoudji, J. Dalibard, F. Gerbier and G. Shlyapnikov for critical reading of the manuscript. We acknowledge support from ESF (Euroquam), SCALA, ANR FABIOLA, Région lle de France (IFRAF), ERC and Institut Universitaire de France.

Author Contributions S.N. and N.N. contributed equally to this work. S.N., N.N. and K.J.J. took the experimental data, and all authors contributed to the data analysis and writing of the manuscript.

Author Information Reprints and permissions information is available at www.nature.com/reprints. The authors declare no competing financial interests. Correspondence and requests for materials should be addressed to S.N. (sylvain.nascimbene@ens.fr). 


\section{METHODS}

Construction of the EOS by successive patches. A typical image at high temperature provides about 100 pixels corresponding to $\zeta$ values varying from 2 at the trap centre to 6 at the edges, with a signal-to-noise from 3 to 10 . Seven such images are fitted in the wings using the second-order virial expansion and averaged to obtain a low-noise EOS up to $\zeta=2$. Then images of clouds where the evaporation has been pushed to a slightly lower temperature are recorded. They show about $75 \%$ overlap in $\zeta$ with the previous EOS. After minimization of the distance between a new image and the previously determined EOS in the overlap region, we obtain the value of $\mu^{0}$ for a single image with $3 \%$ statistical uncertainty. This process is repeated for six successive trap depths. When averaging one image with typically 10 previous images, we obtain a new EOS with an error on $\zeta$ of about $0.03 / \sqrt{10} \approx 1 \%$. The EOS experiences a random walk error on the 40 images of $0.01 \times \sqrt{40} \approx 5 \%$ for the coldest data. An independent check of the maximum error is provided by the good agreement with the superfluid EOS for temperatures lower than $T_{\mathrm{c}}$ (refs 7,8 ).

Evaluation of the systematic uncertainties. For the measurement of $h(1, \zeta)$, the combined uncertainties on the radial frequency of the trap, trap anharmonicity, magnification of our imaging system, and atom counting affect the pressure measurement given in equation (3) at $20 \%$ level. However, two measurements, one at relatively high temperature and one at very low temperature, enable us to show that the overall error does not exceed $6 \%$. In the temperature range $\zeta>0.5$, the agreement between the experimental value $b_{3}=-0.35(2)$ and the theoretical value $b_{3}=-0.355$ of the third virial coefficient indicates that the global systematic error is smaller than $6 \%$. Second, at very low temperature, theory ${ }^{7,8}$ predicts that the variation of $P / 2 P_{1}$ as a function of $k_{\mathrm{B}} T / \mu$ in the superfluid phase remains smaller than $5 \%$. Our value of $P / 2 P_{1}=3.75$ below the critical point is within $5 \%$ of the $T=0$ prediction $\xi_{\mathrm{s}}^{-3 / 2}=3.7(2)$. This confirms that systematic errors for our coldest samples are also smaller than $6 \%$.

For the determination of the critical transition to superfluidity we fit the lowtemperature data $P(\mu, T) / 2 P_{1}(\mu, 0)$ with a variable horizontal line for $T<T_{\mathrm{c}}$ and with the Fermi-liquid equation (4) for $T>T_{\mathrm{c}}$. The result of the fit is the dashed black line in Fig. $3 c$, which intersects equation $(4)$ at $\left(k_{\mathrm{B}} T / \mu\right)_{\mathrm{c}}=0.315(8)$. This statistical error is negligible compared to the error induced by the $6 \%$ systematic uncertainty discussed above, justifying our very simplified fit procedure. Indeed a $6 \%$ error on the pressure induces a $10 \%$ error on $\mu$ for images recorded in the vicinity of the critical temperature, leading to $\left(k_{\mathrm{B}} T / \mu\right)_{\mathrm{c}}=0.32(3)$.

For the measurement of $h(\eta, 0)$, the fit of the fully polarized wings of the cloud serves as a pressure calibration for the rest of the cloud, cancelling many systematic effects.

In order to estimate temperature effects in the polarized gas, let us first remark that in the superfluid phase corrections scale as $T^{4}$ for the bosonic excitations and are exponentially suppressed by the gap for the fermionic ones ${ }^{7}$. So in our temperature range $k_{\mathrm{B}} T=0.03 \mu_{1}^{0}$ their contributions will be very small. On the other hand, in the partially polarized normal phase, we expect a typical Fermi liquid $T^{2}$ scaling. In order to obtain an estimate of the error on the EOS, we develop the following simple model. In equation (6) which describes a mixture of zero-temperature ideal gases, we replace the Fermi pressures by the finitetemperature pressures of ideal gases (see equation (1)):

$$
P\left(\mu_{1}, \mu_{2}, T\right)=P_{1}\left(\mu_{1}, T\right)+\left(\frac{m_{\mathrm{p}}^{*}}{m}\right)^{3 / 2} P_{1}\left(\mu_{2}-A \mu_{1}, T\right)
$$

and run the analysis described in the main text. At $T=0.05 \mu_{1}^{0}$, the correction on $h$ is less than $1 \%$, half of our current error bar.

Limit of ${ }^{7} \mathrm{Li}$ thermometry. As the scattering length between the ${ }^{7} \mathrm{Li}$ atoms, $a_{77}=-3 \mathrm{~nm}$ is negative, the ${ }^{7} \mathrm{Li}$ cloud becomes unstable when a BEC forms. This occurs at $T \approx 150 \mathrm{nK}$ with typically 3,500 atoms. Precise thermometry with lower atom numbers becomes difficult. For the measurement of the zerotemperature EOS of the imbalanced gas, we do not use ${ }^{7} \mathrm{Li}$ thermometry but rather the fit of the wings of the majority spin component. 
43210, USA. ${ }^{12}$ Istituto Nazionale di Fisica Nucleare, Sezione di Perugia, I-06123 Perugia, Italy. ${ }^{13}$ Dipartimento di Fisica, Università degli Studi di Perugia, I-06123 Perugia, Italy. ${ }^{14}$ Dipartimento di Fisica "M. Merlin" dell'Università e del Politecnico di Bari, I-70126 Bari, Italy. ${ }^{15}$ Istituto Nazionale di Fisica Nucleare, Sezione di Bari, 70126 Bari, Italy. ${ }^{16}$ Laboratoire Leprince-Ringuet, École polytechnique, CNRS/IN2P3, Palaiseau, France. ${ }^{17}$ Department of Physics, University of Washington, Seattle, WA 98195-1560, USA. ${ }^{18}$ Institut de Ciencies de l'Espai (IEEC-CSIC), Campus UAB, 08193 Barcelona, Spain. ${ }^{19}$ Istituto Nazionale di Astrofisica (INAF)-Istituto di Astrofisica Spaziale e Fisica Cosmica, I-20133 Milano, Italy. ${ }^{20}$ Agenzia Spaziale Italiana Science Data Center, I-00044 Frascati (Roma), Italy. ${ }^{21}$ NASA Goddard Space Flight Center, Greenbelt, MD 20771 , USA. ${ }^{22}$ Center for Research and Exploration in Space Science and Technology and NASA Goddard Space Flight Center, Greenbelt, MD 20771, USA. ${ }^{23}$ Department of Physics and Center for Space Sciences and Technology, University of Maryland Baltimore County, Baltimore, MD 21250, USA. ${ }^{24}$ George Mason University, Fairfax, VA 22030, USA. ${ }^{25}$ Laboratoire de Physique Théorique et Astroparticules, Université Montpellier 2, CNRS/IN2P3, Montpellier, France. ${ }^{26}$ Department of Physics and Astronomy, Sonoma State University, Rohnert Park, CA 94928-3609, USA.

${ }^{27}$ Department of Physics, Stockholm University, AlbaNova, SE-106 91 Stockholm, Sweden. ${ }^{28}$ The Oskar Klein Centre for Cosmoparticle Physics, AlbaNova, SE-106 91 Stockholm, Sweden.

${ }^{29}$ Dipartimento di Fisica, Università di Udine and Istituto Nazionale di Fisica Nucleare, Sezione di Trieste, Gruppo Col- legato di Udine, I-33100 Udine, Italy. ${ }^{30}$ Université de Bordeaux, Centre d'Études Nucléaires Bordeaux Gradignan, UMR 5797, Gradignan, 33175, France. ${ }^{31}$ CNRS/IN2P3, Centre d'Etudes Nucléaires Bordeaux Gradignan, UMR 5797, Gradignan, 33175, France. ${ }^{32}$ Department of Physical Sciences, Hiroshima University, Higashi-Hiroshima, Hiroshima 739-8526, Japan. ${ }^{33}$ Department of Astronomy and Astrophysics, Pennsylvania State University, University Park, PA 16802, USA. ${ }^{34}$ Department of Physics and Department of Astronomy, University of Maryland, College Park, MD 20742, USA ${ }^{35}$ INAF Istituto di Radioastronomia, 40129 Bologna, Italy. ${ }^{36}$ Max-Planck-Institut für Radioastronomie, Auf dem Hügel 69, 53121 Bonn, Germany. ${ }^{37}$ Center for Space Plasma and Aeronomic Research, University of Alabama in Huntsville, Huntsville, AL 35899, USA. ${ }^{38}$ Department of Physics, Royal Institute of Technology, AlbaNova, SE-106 91 Stockholm, Sweden. ${ }^{39}$ Waseda University, 1-104 Totsukamachi, Shinjukuku, Tokyo, 169-8050, Japan. ${ }^{40}$ Department of Physics, Tokyo Institute of Technology, Meguro City, Tokyo 152-8551, Japan. ${ }^{41}$ Cosmic Radiation Laboratory, Institute of Physical and Chemical Research (RIKEN), Wako, Saitama 351-0198, Japan. ${ }^{42}$ Centre d'Étude Spatiale des Rayonnements, CNRS/UPS, BP 44346, F-30128 Toulouse Cedex 4, France. ${ }^{43}$ Istituto Nazionale di Fisica Nucleare, Sezione di Roma "Tor Vergata," I-00133 Roma, Italy. ${ }^{44}$ Department of Physics and Astronomy, University of Denver, Denver, CO 80208 , USA. ${ }^{45}$ Max-Planck Institut für Extraterrestrische Physik, 85748 Garching, Germany. ${ }^{46}$ Institut für Astro- und Teilchenphysik and Institut für Theoretische Physik, Leopold-Franzens-Universität Innsbruck, A-6020 Innsbruck,
Austria. ${ }^{47}$ Space Sciences Division, NASA Ames Research Center, Moffett Field, CA 94035-1000, USA. ${ }^{48}$ NYCB Real-Time Computing, Lattingtown, NY 11560-1025, USA. ${ }^{49}$ Astronomical Observatory, Jagiellonian University, 30-244 Kraków, Poland. ${ }^{50}$ Department of Chemistry and Physics, Purdue University Calumet, Hammond, IN 46323-2094, USA ${ }^{51}$ Institute of Space and Astronautical Science, lapanese Aerospace Exploration Agency, 3-1-1 Yoshinodai, Sagamihara, Kanagawa 229-8510, Japan. ${ }^{52}$ Institució Catalana de Recerca i Estudis Avançats, Barcelona, Spain. ${ }^{53}$ Consorzio Interuniversitario per la Fisica Spaziale, I-10133 Torino, Italy. ${ }^{54}$ Dipartimento di Fisica, Università di Roma "Tor Vergata," I-00133 Roma, Italy. ${ }^{55}$ School of Pure and Applied Natural Sciences, University of Kalmar, SE-391 82 Kalmar, Sweden. ${ }^{56}$ Centre for Astrophysics Research, University of Hertfordshire, College Lane, Hatfield AL10 9AB, UK.

\section{Supporting Online Material}

www.sciencemag.org/cgi/content/full/science.1184656/DC1 Materials and Methods

SOM Text

Figs. $\mathrm{S} 1$ to $\mathrm{S3}$

Tables S1 to S3

References

13 November 2009; accepted 19 March 2010

Published online 1 April 2010;

10.1126/science. 1184656

Include this information when citing this paper.

\section{The Equation of State of a}

\section{Low-Temperature Fermi Gas with Tunable Interactions}

\section{N. Navon, ${ }^{*} \dagger$ S. Nascimbène, ${ }^{*}$ F. Chevy, C. Salomon}

Interacting fermions are ubiquitous in nature, and understanding their thermodynamics is an important problem. We measured the equation of state of a two-component ultracold Fermi gas for a wide range of interaction strengths at low temperature. A detailed comparison with theories including Monte-Carlo calculations and the Lee-Huang-Yang corrections for low-density bosonic and fermionic superfluids is presented. The low-temperature phase diagram of the spin-imbalanced gas reveals Fermi liquid behavior of the partially polarized normal phase for all but the weakest interactions. Our results provide a benchmark for many-body theories and are relevant to other fermionic systems such as the crust of neutron stars.

$\mathrm{R}$ ecently, ultracold atomic Fermi gases have become a tool of choice to study strongly correlated quantum systems because of their high controllability, purity, and tunability of interactions (1). In the zero-range limit, interactions in a degenerate Fermi system with two spin-components are completely characterized by a single parameter $1 / k_{F} a$, where $a$ is the $s$-wave scattering length and $k_{F}=\left(6 \pi^{2} n\right)^{1 / 3}$ is the Fermi momentum ( $n$ is the density per spin state). In cold atom gases, the value of $|a|$ can be tuned over several orders of magnitude using a Feshbach resonance; this offers an opportunity to entirely explore the so-called BCS-BEC crossover, that is, the smooth transition from BardeenCooper-Schrieffer (BCS) superfluidity at small

Laboratoire Kastler Brossel, CNRS, Université Pierre et Marie Curie, École Normale Supérieure, 24 rue Lhomond, 75231 Paris, France.

*These authors contributed equally to this work.

†To whom correspondence should be addressed. E-mail: navon@ens.fr negative values of $a$ to molecular Bose-Einstein Condensation (BEC) at small positive values of $a(1,2)$. Between these two well-understood limiting situations, $a$ diverges, leading to strong quantum correlations. The description of this system is a challenge for many-body theories, as testified by the large amount of work in recent years (1). The physics of the BEC-BCS crossover is relevant for very different systems, ranging from neutron stars to heavy nuclei and superconductors.

In the grand-canonical ensemble and at zero temperature, dimensional analysis shows that the Equation of State (EoS) of a two-component Fermi gas, relating the pressure $P$ to the chemical potentials $\mu_{1}$ and $\mu_{2}$ of the spin components can be written as

$$
\begin{aligned}
& P\left(\mu_{1}, \mu_{2}, a\right)= \\
& \quad P_{0}\left(\mu_{1}\right) h\left(\delta_{1} \equiv \frac{\hbar}{\sqrt{2 m \mu_{1}} a}, \eta \equiv \frac{\mu_{2}}{\mu_{1}}\right)
\end{aligned}
$$

where $P_{0}\left(\mu_{1}\right)=1 / 15 \pi^{2}\left(2 m / \hbar^{2}\right)^{3 / 2} \mu_{1}^{5 / 2}$ is the pressure of a single-component ideal Fermi gas, $m$ is the atom mass, $\hbar$ is the Planck constant divided by $2 \pi$, and $\delta_{1}$ is the grand-canonical analog of the dimensionless interaction parameter $1 / k_{F} a$. The indices 1 and 2 refer to the majority and minority spin components, respectively. From the dimensionless function $h\left(\delta_{1}, \eta\right)$, it is possible to deduce all the thermodynamic properties of the gas, such as the compressibility, the magnetization, or the existence of phase transitions. The aim of this paper is to measure $h\left(\delta_{1}, \eta\right)$ for a range of interactions $\left(\delta_{1}\right)$ and spin imbalances $(\eta)$ and discuss its physical content. Because it contains the same information as Eq. 1, the function $h$ will also be referred to as the EoS in the rest of the text.

In situ absorption images of harmonically trapped gases are particularly suited to investigate the EoS, as first demonstrated in (3) and (4). In the particular case of the grand-canonical ensemble, a simple formula relates the local pressure $P$ at a distance $z$ from the center of the trap along the $z$ axis to the doubly integrated density profiles $\bar{n}_{1}$ and $\bar{n}_{2}(5)$

$$
P\left(\mu_{1}(z), \mu_{2}(z), a\right)=\frac{m \omega_{r}^{2}}{2 \pi}\left(\bar{n}_{1}(z)+\bar{n}_{2}(z)\right)
$$

Here, we define the local chemical potentials $\mu_{i}(z)=\mu_{i}^{0}-\frac{1}{2} m \omega_{z}^{2} z^{2}$, where $\mu_{i}^{0}$ is the chemical potential of the component $i$ at the bottom of the trap, assuming local density approximation. $\omega_{r}$ and $\omega_{z}$ are the transverse and axial angular frequencies of a cylindrically symmetric trap, respectively, and $\bar{n}_{i}(z)=\int n_{i}(x, y, z) \mathrm{d} x \mathrm{~d} y$ is the atomic density $n_{i}$ of the component $i$, doubly integrated over the transverse $x$ and $y$ directions. In a single experimental run at a given magnetic field, two images are recorded, providing $\bar{n}_{1}(z)$ and $\bar{n}_{2}(z)$ (fig. S4); the $z$-dependence of the chemical potentials then enables the measurement of $P$ along a curve in the $\left(\delta_{1}, \eta\right)$ plane $(\emptyset)$. This method was validated in (4) for the particular case of the 
unitary limit $a=\infty$. Deducing the function $h$ from the doubly integrated profiles further requires a precise calibration of $\omega_{z}$ and the knowledge of the central chemical potentials $\mu_{i}^{0}($ ) .

Our experimental setup is presented in (7). We prepared an imbalanced mixture of ${ }^{6} \mathrm{Li}$ in the two lowest internal spin states, at the magnetic field of $834 G$ (where $a=\infty$ ), and trapped it in a hybrid magnetic-optical dipole trap. We then performed evaporative cooling by lowering the optical trap power, while the magnetic field was ramped to the final desired value for $a$. The cloud typically contained $N=2$ to $10 \times 10^{4}$ atoms in each spin state at a temperature of 0.03(3) $T_{F}$, justifying our $T=0$ assumption ( 6 ). The final trap frequencies are $\omega_{z} / 2 \pi \sim 30 \mathrm{~Hz}$ and $\omega_{r} / 2 \pi \sim 1 \mathrm{kHz}$. Below a critical spin population imbalance, our atomic sample consists of a fully paired superfluid occupying the center of the trap, surrounded by a normal mixed phase and an outer rim of an ideal gas of majority component atoms $(4,7,8)$.

For a given magnetic field, 10 to 20 images are taken, leading after averaging to a low-noise EoS along one line in the $\left(\delta_{1}, \eta\right)$ plane. Measurements at different magnetic fields chosen between $766 \mathrm{G}$ and $981 \mathrm{G}$ give a sampling of the surface $h\left(\delta_{1}, \eta\right)$ in the range $-1<\delta_{1}<0.6$ and $-2<\eta<0.7$ (Fig. 1). Let $A\left(\delta_{1}\right)$ be the limiting value of the ratio of chemical potentials $\mu_{1}(z) / \mu_{2}(z)$ below which the minority density vanishes. At fixed $\delta_{1}$ and $\eta<A\left(\delta_{1}\right), h\left(\delta_{1}, \eta\right)$ represents the EoS of an ideal Fermi gas of majority atoms and is equal to 1 . For $\eta>A\left(\delta_{1}\right)$, it slowly rises and corresponds to the normal mixed phase, where both spin components are present. At a critical value $\eta=$ $\eta_{c}\left(\delta_{1}\right)$, the slope of $h$ abruptly changes (6), the signature of a first-order phase transition from the normal phase (for $A<\eta<\eta_{c}$ ) to a superfluid phase with a lower chemical potential imbalance $\left(\eta>\eta_{c}\right)$. We notice that the discontinuity is present for all values of $\delta_{1}$ we investigated, and this feature is more pronounced on the BEC side.

Let us first consider the EoS of the superfluid phase, $\eta>\eta_{c}$. Each of our in situ images has, along the $z$ axis, values of the chemical potential ratio $\eta(z)=\mu_{2}(z) / \mu_{1}(z)$ both lower and greater than $\eta_{c}$. In the region where $\eta(z)>\eta_{c}$, the doubly integrated density difference $\bar{n}_{1}(z)-\bar{n}_{2}(z)$ is constant within our signal-to-noise ratio (fig. S4). This is the signature of equal densities of the two species in the superfluid core, that is, the superfluid is fully paired. Using Gibbs-Duhem relation $n_{i}=\frac{\partial P}{\partial \mu_{i}}$, equal densities $n_{1}=n_{2}$ imply that $P\left(\mu_{1}, \mu_{2}, a\right)$ is a function of $\mu$ and $a$ only, where $\mu \equiv\left(\mu_{1}+\mu_{2}\right) / 2$. For the balanced superfluid, we then write the EoS symmetrically.

$$
P\left(\mu_{1}, \mu_{2}, a\right)=2 P_{0}(\tilde{\mu}) h_{S}\left(\tilde{\delta} \equiv \frac{\hbar}{\sqrt{2 m \tilde{\mu} a}}\right)
$$

To avoid using negative chemical potentials, we define here $\tilde{\mu}=\mu-E_{b} / 2$, where $E_{b}$ is the molecular binding energy $E_{b}=-\hbar^{2} / m a^{2}$ for $a>0$ (and 0 for $a \leq 0) . h_{s}(\tilde{\delta})$ is then a single-variable function. It fully describes the ground-state
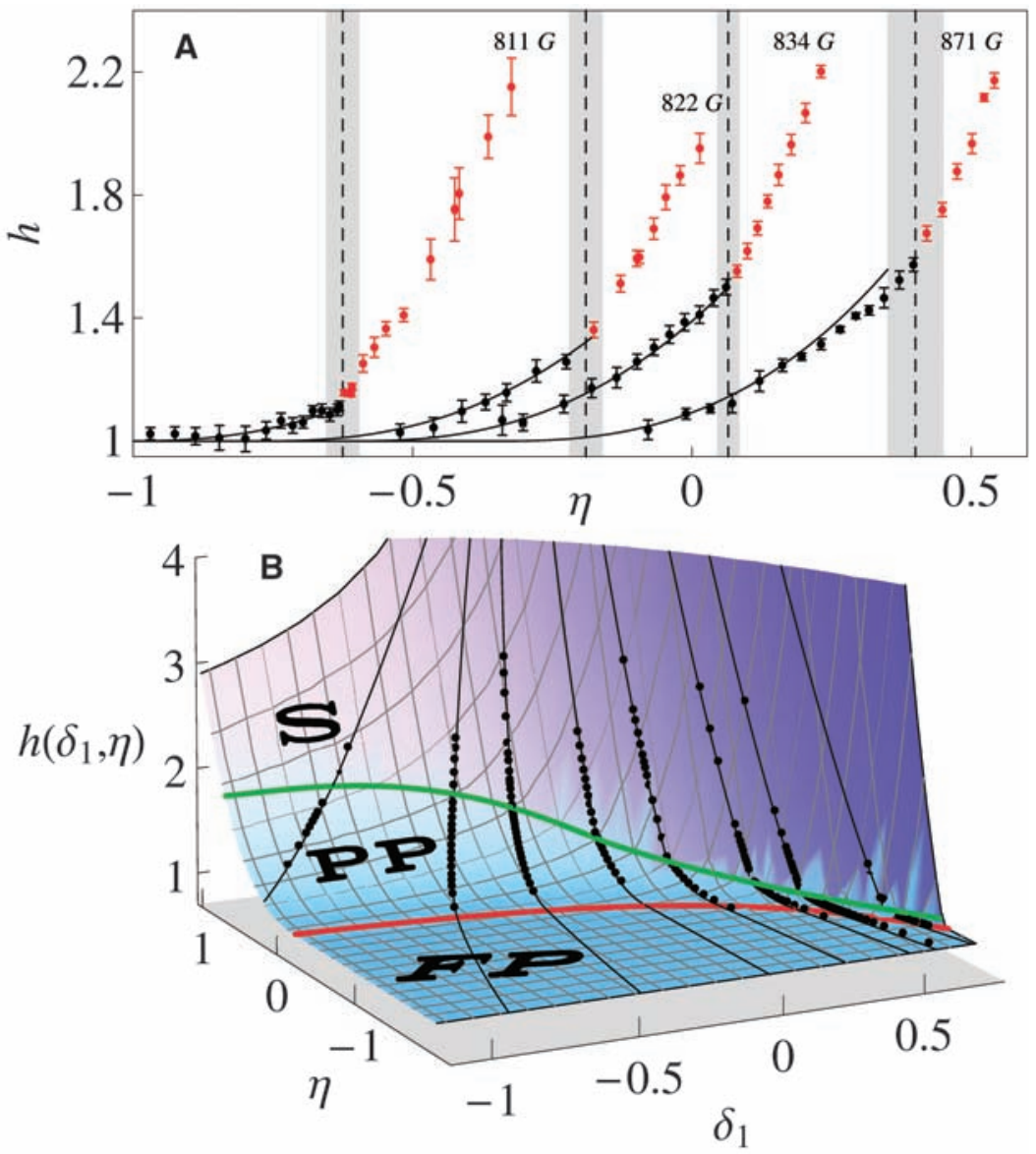

Fig. 1. $h\left(\delta_{1}, \eta\right)$ of a zero-temperature two-component Fermi gas in the BEC-BCS crossover. (A) Samples of the data for different magnetic fields. The black (red) data points correspond to the normal (superfluid) phase and are separated at $\eta_{c}\left(\delta_{1}\right)$ by a clear kink in the local slope of $h$. Solid black lines are the predictions of the polaron ideal gas model (Eq. 8). The scattering length corresponding to each curve is (from left to right): $(1.7,3.4, \infty$, and -1.3$)$ in units of $10^{4} a_{0}$, where $a_{0}$ is the Bohr radius. (B) $h\left(\delta_{1}, \eta\right)$. The black dots are data recorded for each magnetic field value (as in Fig. $1 \mathrm{~A}$ ). The black lines correspond to the parametric curves $\left[\delta_{1}(\eta), \eta\right]$ scanned by the density inhomogeneity in the harmonic trap (6). The red line is $A\left(\delta_{1}\right)$, the frontier between the fully polarized (FP) ideal gas $h=1$ and the normal partially polarized (PP) phase. The green line is $\eta_{c}\left(\delta_{1}\right)$, marking the phase transition between the normal and superfluid (S) phases. The surface is the parametrization of $h\left(\delta_{1}, \eta\right)$ given in the text.

Fig. 2. $h_{S}(\tilde{\delta})$ of the $T=0$ balanced superfluid in the BEC-BCS crossover (black dots). The blue solid line is the fit $h_{S}^{\mathrm{BCS}}(\tilde{\delta})$ on the BCS side of the resonance; the red solid line is the fit $h_{S}^{\mathrm{BEC}}(\tilde{\delta})$ on the BEC side. The dotted (dashed) red line is the mean-field (LHY) theory (32). (Inset) Zoom on the BCS side. The dotted and dashed blue lines are the EoS, including the mean-field and LHY terms, respectively. The systematic uncertainties on the $x$ and $y$ axes are about $5 \%$. The errors bars represent the standard deviation of the statistical uncertainty.

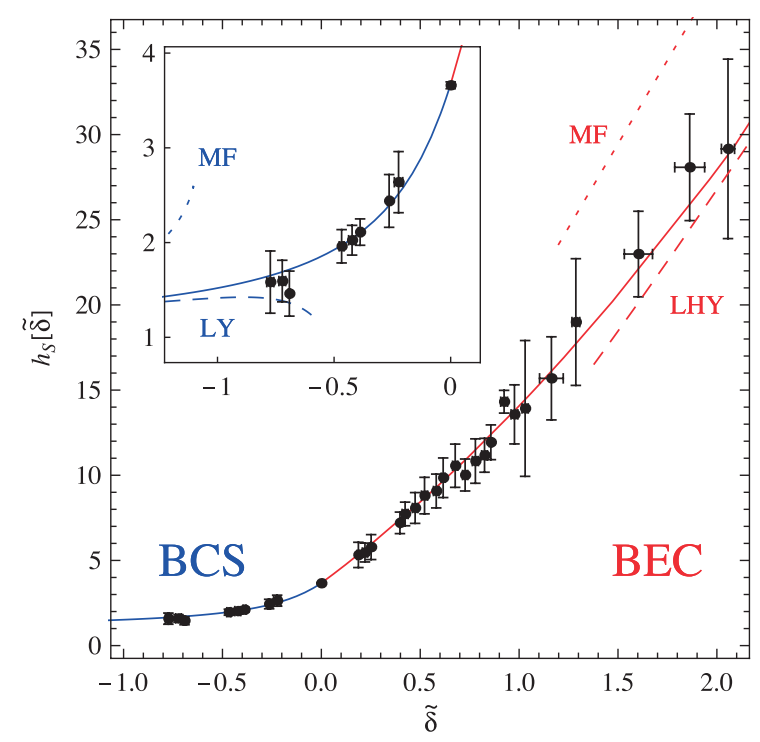


Fig. 3. Comparison with many-body theories. (A) Direct comparison of $h_{S}(\tilde{\delta})$ with a quantum Monte-Carlo calculation [red open circles (22)], a diagrammatic method [green open squares (23)], a NozièresSchmitt-Rink approximation [blue open triangles (21)], and the BCS mean-field theory (solid blue line). (Inset) Zoom on the $B C S$ side. (B) EoS in the canonical ensemble $\xi\left(1 / k_{F} a\right)$ (solid black line) deduced from the Padé-type approximants to the experimental data $h_{S}^{\mathrm{BCS}}$ and $h_{S}^{\mathrm{BEC}}$ plotted in Fig. 2. FixedNode Monte-Carlo theories: red squares (24), blue circles (25), and green triangles (26).
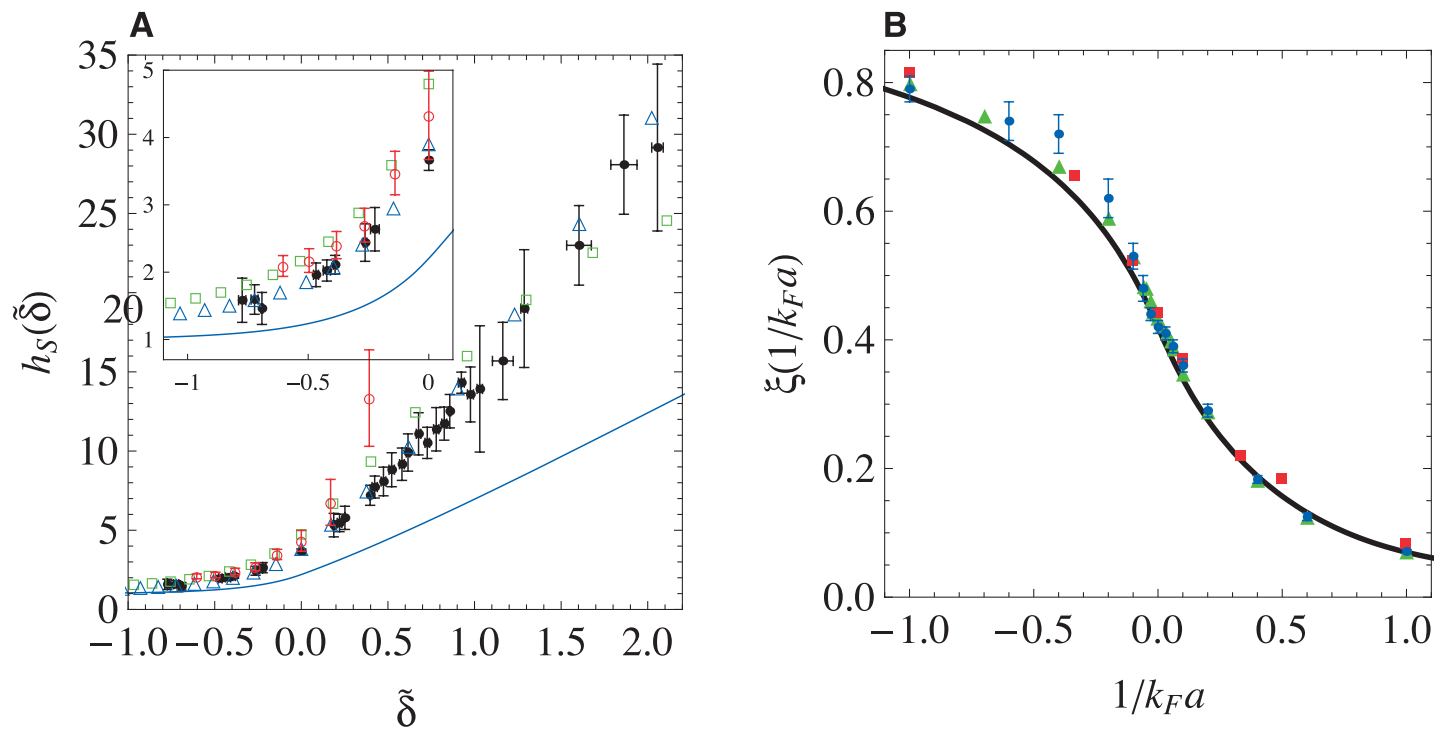

macroscopic properties of the balanced superfluid in the BEC-BCS crossover and is displayed in Fig. 2 as black dots.

To extract relevant physical quantities, such as beyond mean-field corrections, it is convenient to parametrize our data with analytic functions. In this pursuit, we use Padé-type approximants (6), interpolating between the EoS measured around unitarity and the well-known mean-field expansions on the BEC and BCS limits. The two analytic functions, $h_{S}^{\mathrm{BCS}}$ and $h_{S}^{\mathrm{BEC}}$, are respectively represented in blue and red solid lines in Fig. 2 and represent our best estimate of the EoS in the whole BEC-BCS crossover.

On the BCS side, $(\tilde{\delta})<0, h_{S}^{\mathrm{BCS}}$ yields the following perturbative expansion of the energy in series of $k_{F} a$

$$
\begin{gathered}
E=\frac{3}{5} N E_{F}\left(1+\frac{10}{9 \pi} k_{F} a+0.18(2)\left(k_{F} a\right)^{2}+\right. \\
\left.0.03(2)\left(k_{F} a\right)^{3}+\ldots\right)
\end{gathered}
$$

where $N$ is the total number of atoms, $E_{F}$ is the Fermi energy, and where by construction of $h_{S}^{\mathrm{BCS}}$, the mean-field term (proportional to $k_{F} a$ ) is fixed to its exact value $10 / 9 \pi$. We obtain beyond meanfield corrections up to the third order. The term proportional to $\left(k_{F} a\right)^{2}$ agrees with the Lee-Yang $(9,10)$ theoretical calculation $4(11-2 \log 2) / 21 \pi^{2} \cong 0.186$. The third-order coefficient also agrees with the value 0.030 computed in (11).

Around unitarity, the energy expansion yields

$$
E=\frac{3}{5} N E_{F}\left(\xi_{s}-\zeta \frac{1}{k_{F} a}+\ldots\right)
$$

We find the universal parameter of the unitary $T=0$ superfluid, $\xi_{s}=0.41(1)$ with $2 \%$ accuracy. This value is in agreement with recent calculations and measurements (1). Our thermodynamic measurement $\zeta=0.93(5)$ can be compared with a recent experimental value $\zeta=0.91(4)$ (12), as well as the theoretical value $\zeta=0.95$ (13), both of them

Fig. 4. Effective mass $m^{*} / m$ of the polaron in the BEC-BCS crossover (black dots). The blue dashed line is a calculation from (29), red open squares (30), green dot-dashed line (26), and blue solid line (31). Measurements at unitarity through density profile analysis [blue triangle (3)] and collective modes study [brown empty circle (7)] are also displayed. (Inset) Phase diagram of a zero-temperature imbalanced Fermi gas in the $B E C-B C S$ crossover. The blue line is the theoretical value of $A(26,29,30)$ that sets the separation between the partially polarized (PP) and the fully polarized (FP) phases. Black dots are the measured values

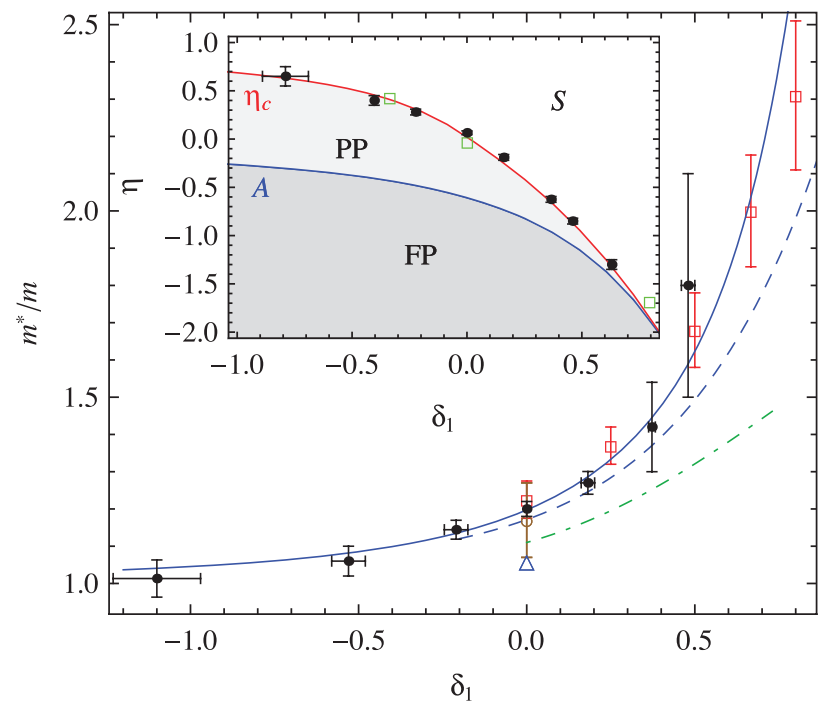

of $\eta_{c}$ (as in Fig. 1A), which set the separation between the superfluid (S) phase and the partially polarized phase. The red line is the calculation of $\eta_{c}$ using our EoS of the superfluid and the model (Eq. 8) for the normal phase. The green squares are lower bounds of $\eta_{c}$ given by the values of the gap measured in (33); see (6).

obtained through the study of the pair correlation function. This experimental agreement confirms the link between the macroscopic thermodynamic properties and the microscopic short-range pair correlations, as shown theoretically in (14).

In the BEC limit, the energy of the superfluid is that of a weakly interacting Bose-Einstein condensate of molecules $(9,15)$

$$
\begin{aligned}
E=\frac{N}{2} & E_{b}+N \frac{\pi \hbar^{2} a_{d d}}{2 m} \\
& \times n\left(1+\frac{128}{15 \sqrt{\pi}} \sqrt{n a_{d d}^{3}}+\ldots\right)
\end{aligned}
$$

where $a_{d d}=0.6 a$ is the dimer-dimer scattering length (1) and $n$ is the dimer density. The term in $\sqrt{n a_{d d}^{3}}$ is the well-known Lee-Huang-Yang (LHY) correction to the mean-field interaction between molecules $(9,15)$. Signatures of beyond mean-field effects were previously observed through a pioneering study of collective modes (16) and density profile analysis (17), but no quantitative comparison with Eq. 6 was made. Fitting our data in the deep BEC regime with Eq. 6, we measure the bosonic LHY coefficient 4.4(5), in agreement with the exact value $128 / 15 \sqrt{\pi} \simeq 4.81$ calculated for elementary bosons in $(9)$ and recently for composite bosons in (15).

Having checked this important beyond meanfield contribution, we can go one step further in the expansion. The analogy with point-like bosons suggests that the next term should be written as $\left[\frac{8}{3}(4 \pi-3 \sqrt{3}) n a_{d d}^{3}\left(\log \left(n a_{d d}^{3}\right)+B\right)\right]$ $(6,18,19)$. Using $h_{S}^{\mathrm{BEC}}(\tilde{\delta})$ (Fig. 2) (๑), we deduce the effective three-body parameter for composite bosons $B=7(1)$. Interestingly, this value is close 
to the bosonic hard-sphere calculation $B=8.5$ (20) and to the value $B \approx 7.2$ for point-like bosons with large scattering length (19).

Our measurements also allow direct comparison with advanced many-body theories developed for homogeneous gases in the strongly correlated regime. As displayed in Fig. 3A, our data are in agreement with a Nozières-SchmittRink approximation (21) but show significant differences from a quantum Monte-Carlo calculation (22) and a diagrammatic approach (23). The measured EoS strongly disfavors the prediction of BCS mean-field theory.

Comparison with Fixed-Node Monte-Carlo theories requires the calculation of the EoS $\xi\left(1 / k_{F} a\right)$ in the canonical ensemble

$$
\xi\left(\frac{1}{k_{F} a}\right) \equiv \frac{E-\frac{N}{2} E_{b}}{\frac{3}{5} N E_{F}}
$$

that is deduced from $h_{S}^{\mathrm{BCS}}(\tilde{\delta})$ and $h_{S}^{\mathrm{BEC}}(\tilde{\delta})($ ()) As shown in Fig. 3B, the agreement with theories $(24-26)$ is very good.

We now discuss the EoS of the partially polarized normal phase (black points in Fig. 1). At low concentrations, we expect the minority atoms to behave as noninteracting quasiparticles, the fermionic polarons (27). The polarons are dressed by the majority Fermi sea through a renormalized chemical potential $\mu_{2}-A\left(\delta_{1}\right) \mu_{1}(28)$ and an effective mass $m^{*}\left(\delta_{1}\right)(26,29,30)$. Following a Fermi liquid picture, we propose to express the gas pressure as the sum of the Fermi pressure of the bare majority atoms and of the polarons (4).

$$
h\left(\delta_{1}, \eta\right)=1+\left(\frac{m^{*}\left(\delta_{1}\right)}{m}\right)^{3 / 2}\left(\eta-A\left(\delta_{1}\right)\right)^{5 / 2}
$$

Our measured EoS agrees with this model at unitarity and on the BEC side of the resonance (Fig. 1), where for $m^{*}\left(\delta_{1}\right)$ we use the calculations from $(30,31)$. On the BCS side of the resonance, however, we observe at large minority concentrations an intriguing deviation to Eq. 8. In the $\mathrm{BCS}$ regime, the superfluid is less robust to spin imbalance. Consequently, the ratio of the two densities $n_{1} / n_{2}$ in the normal phase becomes close to unity near the superfluid/normal boundary $\eta_{c}$. The polaron ideal gas picture then fails.

Alternatively, we can let the effective mass $m^{*}$ be a free parameter in the model in Eq. 8 in the fit of our data around $\eta=A$. We obtain the value of the polaron effective mass in the BECBCS crossover (Fig. 4).

An important consistency check of our study is provided by the comparison between our direct measurements of $\eta_{c}\left(\delta_{1}\right)$ (from Fig. 1, black dots in the inset of Fig. 4) and a calculated $\eta_{c}\left(\delta_{1}\right)$ from Eq. 8 and the EoS of the superfluid $h_{S}$. Assuming negligible surface tension, the normal/ superfluid boundary is given by equating the pressure and chemical potential in the two phases. This procedure leads to the solid red line in the inset of Fig. 4, in excellent agreement with the direct measurements. In addition, by integrating our measured EoS of the homogeneous gas over the trap, one retrieves the critical polarization for superfluidity of a trapped gas, in agreement with most previous measurements (6).

We have measured the equation of state of a two-component Fermi gas at zero temperature in the BEC-BCS crossover. Extensions of our work include exploring the thermodynamics of the far $\mathrm{BEC}$ region of the phase diagram where a new phase associated with a polarized superfluid appears $(17,26)$, mapping the EoS as a function of temperature, and investigating the influence of finite interaction range, which is playing a key role in higher-density parts of neutron stars.

\section{References and Notes}

1. M. Inguscio, W. Ketterle, C. Salomon, Eds. Ultra-cold Fermi Gases: Proceedings of the International School of Physics "Enrico Fermi", Course CLXIV, Varenna, 20 to 30 June 2006 (IOS Press, Amsterdam, 2008).

2. A. J. Leggett, in Modern Trends in the Theory of Condensed Matter, A. Pekalski, R. Przystawa, Eds. (Springer-Verlag, Berlin, 1980), pp. 13-27.

3. Y. Shin, Phys. Rev. A 77, 041603 (2008).

4. S. Nascimbène, N. Navon, K. J. Jiang, F. Chevy, C. Salomon, Nature 463, 1057 (2010).

5. T. Ho, Q. Zhou, Nat. Phys. 6, 131 (2009).

6. Materials and methods are available as supporting material on Science Online.

7. S. Nascimbène et al., Phys. Rev. Lett. 103, 170402 (2009).

8. Y. Shin, M. Zwierlein, C. Schunck, A. Schirotzek, W. Ketterle, Phys. Rev. Lett. 97, 30401 (2006).

9. T. D. Lee, C. N. Yang, Phys. Rev. 105, 1119 (1957).

10. R. B. Diener, R. Sensarma, M. Randeria, Phys. Rev. A 77, 023626 (2008)

11. G. Baker Jr., Rev. Mod. Phys. 43, 479 (1971).

12. H. Hu et al., "Universal structure of a strongly interacting Fermi superfluid," http://arxiv.org/abs/1001.3200 (2010).

13. C. Lobo, I. Carusotto, S. Giorgini, A. Recati, S. Stringari, Phys. Rev. Lett. 97, 100405 (2006)

14. S. Tan, Ann. Phys. 323, 2971 (2008).

15. X. Leyronas, R. Combescot, Phys. Rev. Lett. 99, 170402 (2007).

16. A. Altmeyer et al., Phys. Rev. Lett. 98, 040401 (2007).

17. Y. I. Shin, A. Schirotzek, C. H. Schunck, W. Ketterle, Phys. Rev. Lett. 101, 070404 (2008).

18. T. Wu, Phys. Rev. 115, 1390 (1959).
19. E. Braaten, H. W. Hammer, T. Mehen, Phys. Rev. Lett. 88 040401 (2002)

20. S. Tan, Phys. Rev. A 78, 013636 (2008)

21. H. Hu, X. Liu, P. Drummond, Europhys. Lett. 74, 574 (2006)

22. A. Bulgac, ]. Drut, P. Magierski, Phys. Rev. A 78, 023625 (2008).

23. R. Haussmann, W. Rantner, S. Cerrito, W. Zwerger, Phys. Rev. A 75, 023610 (2007).

24. S. Chang, V. Pandharipande, J. Carlson, K. Schmidt, Phys. Rev. A 70, 043602 (2004).

25. G. E. Astrakharchik, ]. Boronat, ]. Casulleras, A. S. Giorgini, Phys. Rev. Lett. 93, 200404 (2004).

26. S. Pilati, S. Giorgini, Phys. Rev. Lett. 100, 030401 (2008).

27. C. Lobo, A. Recati, S. Giorgini, S. Stringari, Phys. Rev. Lett. 97, 200403 (2006).

28. A. Schirotzek, C.-H. Wu, A. Sommer, M. W. Zwierlein, Phys. Rev. Lett. 102, 230402 (2009).

29. R. Combescot, A. Recati, C. Lobo, F. Chevy, Phys. Rev. Lett. 98, 180402 (2007).

30. N. Prokof'ev, B. Svistunov, Phys. Rev. B 77, 020408 (2008).

31. R. Combescot, S. Giraud, X. Leyronas, Europhys. Lett. 88, 60007 (2009).

32. In the BEC limit, the grand-canonical EoS expands as $h_{s}(\tilde{\delta}) \cong 15 \pi a / 4 a_{d d} \tilde{\delta}-16 \sqrt{2}$; the first term is the mean-field interaction and the second is the LHY correction asymptotic behavior.

33. A. Schirotzek, Y. I. Shin, C. H. Schunck, W. Ketterle, Phys. Rev. Lett. 101, 140403 (2008).

34. We thank K. Jiang for participation in the early phase of the experimental work. We are grateful to X. Leyronas, C. Mora, Y. Castin, F. Werner, R. Combescot, ]. Dalibard, F. Gerbier, and G. Shlyapnikov for stimulating discussions and critical comments on the manuscript. We thank S. Giorgini, P. Drummond, J. Drut, R. Haussmann, and W. Zwerger for providing us with their data. We acknowledge support from European Research Council, European Science Foundation (Euroquam), SCALA (Scalable Quantum Computing with Light and Atoms), Agence Nationale de la Recherche FABIOLA (Fermions and Bosons in Optical Lattices), Région Ile de France Institut Francilien de Recherche sur les Atomes Froids, and Institut Universitaire de France.

Supporting Online Material

www.sciencemag.org/cgi/content/full/science.1187582/DC1 Materials and Methods

Figs. S1 to S4

References

27 January 2010; accepted 30 March 2010

Published online 15 April 2010;

10.1126/science. 1187582

Include this information when citing this paper.

\section{Nanoscale Three-Dimensional Patterning of Molecular Resists by Scanning Probes}

David Pires, ${ }^{1}$ James L. Hedrick, ${ }^{2}$ Anuja De Silva, ${ }^{3}$ ]ane Frommer, ${ }^{2}$ Bernd Gotsmann, ${ }^{1}$ Heiko Wolf, ${ }^{1}$ Michel Despont, ${ }^{1}$ Urs Duerig, ${ }^{1}$ Armin W. Knoll ${ }^{1_{*}}$

For patterning organic resists, optical and electron beam lithography are the most established methods; however, at resolutions below 30 nanometers, inherent problems result from unwanted exposure of the resist in nearby areas. We present a scanning probe lithography method based on the local desorption of a glassy organic resist by a heatable probe. We demonstrate patterning at a half pitch down to 15 nanometers without proximity corrections and with throughputs approaching those of Gaussian electron beam lithography at similar resolution. These patterns can be transferred to other substrates, and material can be removed in successive steps in order to fabricate complex three-dimensional structures.

7 date, a wide variety of techniques has been available for nanofabrication (1), including electron beam lithography (EBL) and scanning probe lithography (SPL) (2-4) as direct-write methods. Although EBL is used in critical applications such as the fabrication of 


\section{References}

[1] M. Anderson, J. Ensher, M. Matthews, C. Wieman, and E. Cornell, Observation of Bose-Einstein condensation in a dilute atomic vapor, Science 269(5221), 198-201 (1995).

[2] C. Bradley, C. Sackett, J. Tollett, and R. Hulet, Evidence of Bose-Einstein condensation in an atomic gas with attractive interactions, Phys. Rev. Lett. 75(9), 1687-1690 (1995).

[3] K. Davis, M. Mewes, M. Andrews, N. Van Druten, D. Durfee, D. Kurn, and W. Ketterle, BoseEinstein condensation in a gas of sodium atoms, Phys. Rev. Lett. 75(22), 3969-3973 (1995).

[4] P. Kapitza, Viscosity of liquid helium below the $\lambda$-point, Nature 141(3558), 74 (1938).

[5] F. London, The-phenomenon of liquid helium and the Bose-Einstein degeneracy, Nature 141, 643-644 (1938).

[6] I. Bloch, T. Hänsch, and T. Esslinger, Measurement of the spatial coherence of a trapped Bose gas at the phase transition, Nature 403, 166-170 (2000).

[7] J. Billy, V. Josse, Z. Zuo, A. Bernard, B. Hambrecht, P. Lugan, D. Clément, L. Sanchez-Palencia, P. Bouyer, and A. Aspect, Direct observation of Anderson localization of matter waves in a controlled disorder, Nature 453(7197), 891-894 (2008).

[8] G. Roati, C. D'Errico, L. Fallani, M. Fattori, C. Fort, M. Zaccanti, G. Modugno, M. Modugno, and M. Inguscio, Anderson localization of a non-interacting Bose-Einstein condensate, Nature 453(7197), 895-898 (2008).

[9] M. Matthews, B. Anderson, P. Haljan, D. Hall, C. Wieman, and E. Cornell, Vortices in a BoseEinstein condensate, Phys. Rev. Lett. 83(13), 2498-2501 (1999).

[10] K. Madison, F. Chevy, W. Wohlleben, and J. Dalibard, Vortex formation in a stirred Bose-Einstein condensate, Phys. Rev. Lett. 84(5), 806-809 (2000).

[11] J. Abo-Shaeer, C. Raman, J. Vogels, and W. Ketterle, Observation of vortex lattices in BoseEinstein condensates, Science 292(5516), 476 (2001).

[12] E. Gross, Structure of a quantized vortex in boson systems, Il Nuovo Cimento (1955-1965) 20(3), $454-477$ (1961).

[13] L. Pitaevskii, Vortex lines in an imperfect Bose gas, Sov. Phys. JETP 13(2), 451-454 (1961).

[14] I. Bloch, J. Dalibard, and W. Zwerger, Many-body physics with ultracold gases, Rev. Mod. Phys. 80(3), 885-964 (2008).

[15] S. Cornish, N. Claussen, J. Roberts, E. Cornell, and C. Wieman, Stable ${ }^{85}$ Rb Bose-Einstein Condensates with Widely Tunable Interactions, Phys. Rev. Lett. 85(9), 1795-1798 (2000). 
[16] D. Petrov, C. Salomon, and G. Shlyapnikov, Weakly bound dimers of fermionic atoms, Phys. Rev. Lett. 93(9), 90404 (2004).

[17] K. O'Hara, S. Hemmer, M. Gehm, S. Granade, and J. Thomas, Observation of a strongly interacting degenerate Fermi gas of atoms, Science 298(5601), 2179 (2002).

[18] T. Bourdel, J. Cubizolles, L. Khaykovich, K. Magalhaes, S. Kokkelmans, G. Shlyapnikov, and C. Salomon, Measurement of the Interaction Energy near a Feshbach Resonance in a ${ }^{6} \mathrm{Li}$ Fermi Gas, Phys. Rev. Lett. 91(2), 20402 (2003).

[19] D. Jaksch, C. Bruder, J. Cirac, C. Gardiner, and P. Zoller, Cold bosonic atoms in optical lattices, Phys. Rev. Lett. 81(15), 3108-3111 (1998).

[20] M. Greiner, O. Mandel, T. Esslinger, T. Hänsch, and I. Bloch, Quantum phase transition from a superfluid to a Mott insulator in a gas of ultracold atoms, Nature 415(6867), 39-44 (2002).

[21] U. Schneider, L. Hackermuller, S. Will, T. Best, I. Bloch, T. Costi, R. Helmes, D. Rasch, and A. Rosch, Metallic and Insulating Phases of Repulsively Interacting Fermions in a 3D Optical Lattice, Science 322(5907), 1520 (2008).

[22] R. Jördens, N. Strohmaier, K. Günter, H. Moritz, and T. Esslinger, A Mott insulator of fermionic atoms in an optical lattice, Nature 455(7210), 204-207 (2008).

[23] B. Paredes, A. Widera, V. Murg, O. Mandel, S. Fölling, I. Cirac, G. Shlyapnikov, T. Hänsch, and I. Bloch, Tonks-Girardeau gas of ultracold atoms in an optical lattice., Nature 429(6989), 277 (2004).

[24] T. Kinoshita, T. Wenger, and D. Weiss, Observation of a one-dimensional Tonks-Girardeau gas, Science 305(5687), 1125 (2004).

[25] Z. Hadzibabic, P. Kruger, M. Cheneau, B. Battelier, and J. Dalibard, Berezinskii-KosterlitzThouless crossover in a trapped atomic gas, Nature 441, 1118-1121 (2006).

[26] P. Cladé, C. Ryu, A. Ramanathan, K. Helmerson, and W. Phillips, Observation of a 2D Bose Gas: From Thermal to Quasicondensate to Superfluid, Phys. Rev. Lett. 102(17), 170401 (2009).

[27] Y. Liao, A. Rittner, T. Paprotta, W. Li, G. Partridge, R. Hulet, S. Baur, and E. Mueller, SpinImbalance in a One-Dimensional Fermi Gas, arXiv:0912.0092 (2009).

[28] R. Feynman, Simulating physics with computers, International journal of theoretical physics 21(6), 467-488 (1982).

[29] B. DeMarco and D. Jin, Onset of Fermi degeneracy in a trapped atomic gas, Science 285(5434), 1703 (1999).

[30] A. Truscott, K. Strecker, W. McAlexander, G. Partridge, and R. Hulet, Observation of Fermi pressure in a gas of trapped atoms, Science 291(5513), 2570 (2001).

[31] F. Schreck, L. Khaykovich, K. Corwin, G. Ferrari, T. Bourdel, J. Cubizolles, and C. Salomon, Quasipure Bose-Einstein condensate immersed in a Fermi sea, Phys. Rev. Lett. 87(8), 80403 (2001).

[32] H. Stoof, M. Houbiers, C. Sackett, and R. Hulet, Superfluidity of Spin-Polarized ${ }^{6}$ Li, Phys. Rev. Lett. 76(1), 10-13 (1996). 
[33] M. Bartenstein, A. Altmeyer, S. Riedl, S. Jochim, C. Chin, J. Denschlag, and R. Grimm, Collective excitations of a degenerate gas at the BEC-BCS crossover, Phys. Rev. Lett. 92(20), 203201 (2004).

[34] T. Bourdel, L. Khaykovich, J. Cubizolles, J. Zhang, F. Chevy, M. Teichmann, L. Tarruell, S. Kokkelmans, and C. Salomon, Experimental Study of the BEC-BCS Crossover Region in Lithium 6, Phys. Rev. Lett. 93(5), 050401 (2004).

[35] C. Regal, M. Greiner, and D. Jin, Observation of resonance condensation of fermionic atom pairs, Phys. Rev. Lett. 92(4), 40403 (2004).

[36] M. Zwierlein, C. Stan, C. Schunck, S. Raupach, S. Gupta, Z. Hadzibabic, and W. Ketterle, Observation of Bose-Einstein condensation of molecules, Phys. Rev. Lett. 91(25), 250401 (2003).

[37] J. Kinast, A. Turlapov, J. Thomas, Q. Chen, J. Stajic, and K. Levin, Heat capacity of a strongly interacting Fermi gas, Science 307(5713), 1296 (2005).

[38] G. Partridge, K. Strecker, R. Kamar, M. Jack, and R. Hulet, Molecular probe of pairing in the BEC-BCS crossover, Phys. Rev. Lett. 95(2), 20404 (2005).

[39] S. Riedl, E. Guajardo, C. Kohstall, J. Denschlag, and R. Grimm, Superfluid Quenching of the Moment of Inertia in a Strongly Interacting Fermi Gas, arXiv:0907.3814 (2009).

[40] M. Zwierlein, J. Abo-Shaeer, A. Schirotzek, C. Schunck, and W. Ketterle, Vortices and superfluidity in a strongly interacting Fermi gas, Nature 435, 1047-1051 (2005).

[41] D. Miller, J. Chin, C. Stan, Y. Liu, W. Setiawan, C. Sanner, and W. Ketterle, Critical velocity for superfluid flow across the BEC-BCS crossover, Phys. Rev. Lett. 99(7), 70402 (2007).

[42] S. Jochim, M. Bartenstein, A. Altmeyer, G. Hendl, S. Riedl, C. Chin, J. Hecker Denschlag, and R. Grimm, Bose-Einstein condensation of molecules, Science 302(5653), 2101 (2003).

[43] M. Greiner, C. Regal, and D. Jin, Emergence of a molecular Bose-Einstein condensate from a Fermi gas, Nature 426(6966), 537-540 (2003).

[44] L. Cooper, Bound electron pairs in a degenerate Fermi gas, Phys. Rev. 104(4), 1189-1190 (1956).

[45] J. Bardeen, L. Cooper, and J. Schrieffer, Theory of superconductivity, Phys. Rev. 108(5), 11751204 (1957).

[46] L. Luo and J. Thomas, Thermodynamic Measurements in a Strongly Interacting Fermi Gas, J. Low Temp. Phys. 154(1), 1-29 (2009).

[47] M. Horikoshi, S. Nakajima, M. Ueda, and T. Mukaiyama, Measurement of Universal Thermodynamic Functions for a Unitary Fermi Gas, Science 327(5964), 442 (2010).

[48] S. Nascimbène, N. Navon, K. Jiang, F. Chevy, and C. Salomon, Exploring the thermodynamics of a universal Fermi gas., Nature 463(7284), 1057 (2010).

[49] Y. Shin, C. Schunck, A. Schirotzek, and W. Ketterle, Phase diagram of a two-component Fermi gas with resonant interactions, Nature 451(4), 689-693 (2008).

[50] C. Chin, M. Bartenstein, A. Altmeyer, S. Riedl, S. Jochim, J. Denschlag, and R. Grimm, Observation of the pairing gap in a strongly interacting Fermi gas, Science 305(5687), 1128 (2004).

[51] A. Schirotzek, Y. Shin, C. Schunck, and W. Ketterle, Determination of the superfluid gap in atomic Fermi gases by quasiparticle spectroscopy, Phys. Rev. Lett. 101(14), 140403 (2008). 
[52] A. Clogston, Upper limit for the critical field in hard superconductors, Phys. Rev. Lett. 9(6), 266-267 (1962).

[53] B. Chandrasekhar, A note on the maximum critical field of high-field superconductors, Applied Physics Letters 1(1), 7 (1962).

[54] M. Zwierlein, A. Schirotzek, C. Schunck, and W. Ketterle, Fermionic superfluidity with imbalanced spin populations, Science 311(5760), 492 (2006).

[55] G. Partridge, W. Li, R. Kamar, Y. Liao, and R. Hulet, Pairing and phase separation in a polarized Fermi gas, Science 311(5760), 503-505 (2006).

[56] P. Fulde and R. Ferrell, Superconductivity in a strong spin-exchange field, Phys. Rev. 135, A550 (1964).

[57] A. Larkin and I. Ovchinnikov, Inhomogeneous state of superconductors, Soviet Phys.-JETP 20, $762-769$ (1965).

[58] W. Liu and F. Wilczek, Interior gap superfluidity, Phys. Rev. Lett. 90(4), 47002 (2003).

[59] H. Müther and A. Sedrakian, Spontaneous breaking of rotational symmetry in superconductors, Phys. Rev. Lett. 88(25), 252503 (2002).

[60] A. Gezerlis and J. Carlson, Strongly paired fermions: Cold atoms and neutron matter, Phys. Rev. C $77(3), 32801$ (2008).

[61] K. Maeda, G. Baym, and T. Hatsuda, Simulating dense QCD matter with ultracold atomic bosonfermion mixtures, Phys. Rev. Lett. 103(8), 85301 (2009).

[62] J. Stewart, J. Gaebler, C. Regal, and D. Jin, Potential Energy of a ${ }^{40}$ K Fermi Gas in the BCS-BEC Crossover, Phys. Rev. Lett. 97(22), 220406 (2006).

[63] L. Luo, B. Clancy, J. Joseph, J. Kinast, and J. Thomas, Measurement of the entropy and critical temperature of a strongly interacting Fermi gas, Phys. Rev. Lett. 98(8), 80402 (2007).

[64] A. Altmeyer, S. Riedl, C. Kohstall, M. Wright, R. Geursen, M. Bartenstein, C. Chin, J. Denschlag, and R. Grimm, Precision measurements of collective oscillations in the BEC-BCS crossover, Phys. Rev. Lett. 98(4), 40401 (2007).

[65] S. Chang, V. Pandharipande, J. Carlson, and K. Schmidt, Quantum Monte Carlo studies of superfluid Fermi gases, Phys. Rev. A 70(4), 43602 (2004).

[66] G. Astrakharchik, J. Boronat, J. Casulleras, and S. Giorgini, Equation of state of a Fermi gas in the BEC-BCS crossover: A quantum Monte Carlo study, Phys. Rev. Lett. 93(20), 200404 (2004).

[67] S. Pilati and S. Giorgini, Phase separation in a polarized Fermi gas at zero temperature, Phys. Rev. Lett. 100, 030401 (2008).

[68] Y. Shin, Determination of the equation of state of a polarized Fermi gas at unitarity, Phys. Rev. A $77(4), 041603$ (2008).

[69] T. Ho and Q. Zhou, Obtaining the phase diagram and thermodynamic quantities of bulk systems from the densities of trapped gases, Nature Phys. 6(2), 131-134 (2009).

[70] J. Fuchs, X. Leyronas, and R. Combescot, Hydrodynamic modes of a one-dimensional trapped Bose gas, Phys. Rev. A 68(4), 43610 (2003). 
[71] N. Navon, S. Nascimbène, F. Chevy, and C. Salomon, The Equation of State of a Low-Temperature Fermi Gas with Tunable Interactions, Science 328, 729 (2010).

[72] S. Fölling, A. Widera, T. Müller, F. Gerbier, and I. Bloch, Formation of spatial shell structure in the superfluid to Mott insulator transition, Phys. Rev. Lett. 97(6), 60403 (2006).

[73] F. Spiegelhalder, A. Trenkwalder, D. Naik, G. Hendl, F. Schreck, and R. Grimm, Collisional Stability of ${ }^{40} \mathrm{~K}$ Immersed in a Strongly Interacting Fermi Gas of ${ }^{6} \mathrm{Li}$, Phys. Rev. Lett. 103(22), 223203 (2009).

[74] F. Werner and Y. Castin, Unitary quantum three-body problem in a harmonic trap, Phys. Rev. Lett. 97(15), 150401 (2006).

[75] X. Liu, H. Hu, and P. Drummond, Virial expansion for a strongly correlated Fermi gas, Phys. Rev. Lett. 102(16), 160401 (2009).

[76] T. Lee, K. Huang, and C. Yang, Eigenvalues and eigenfunctions of a Bose system of hard spheres and its low-temperature properties, Phys. Rev. 106(6), 1135-1145 (1957).

[77] X. Leyronas and R. Combescot, Superfluid equation of state of dilute composite bosons, Phys. Rev. Lett. 99(17), 170402 (2007).

[78] T. Lee and C. Yang, Many-body problem in quantum mechanics and quantum statistical mechanics, Phys. Rev. 105(3), 1119-1120 (1957).

[79] Y. Shin, A. Schirotzek, C. Schunck, and W. Ketterle, Realization of a strongly interacting BoseFermi mixture from a two-component Fermi gas, Phys. Rev. Lett. 101(7), 070404 (2008).

[80] F. Chevy, Universal phase diagram of a strongly interacting Fermi gas with unbalanced spin populations, Phys. Rev. A 74(6), 063628 (2006).

[81] C. Lobo, A. Recati, S. Giorgini, and S. Stringari, Normal state of a polarized Fermi gas at unitarity, Phys. Rev. Lett. 97(20), 200403 (2006).

[82] A. Schirotzek, C.-H. Wu, A. Sommer, and M. W. Zwierlein, Observation of Fermi Polarons in a Tunable Fermi Liquid of Ultracold Atoms, Phys. Rev. Lett. 102(23), 230402 (2009).

[83] R. Combescot, A. Recati, C. Lobo, and F. Chevy, Normal state of highly polarized Fermi gases: simple many-body approaches, Phys. Rev. Lett. 98(18), 180402 (2007).

[84] R. Combescot and S. Giraud, Normal state of highly polarized Fermi gases: Full many-body treatment, Phys. Rev. Lett. 101(5), 50404 (2008).

[85] N. Prokof'ev and B. Svistunov, Fermi-polaron problem: Diagrammatic Monte Carlo method for divergent sign-alternating series, Phys. Rev. B 77(2), 020408 (2008).

[86] R. Combescot, S. Giraud, and X. Leyronas, Analytical theory of the dressed bound state in highly polarized Fermi gases, Europhys. Lett. 88, 60007 (2009).

[87] L. Tarruell, Superfluidité dans un gaz de fermions ultrafroids, Thèse de doctorat, Université Paris VI (2008).

[88] G. Ferrari, M. Mewes, F. Schreck, and C. Salomon, High-power multiple-frequency narrow-linewidth laser source based on a semiconductor tapered amplifier, Opt. Lett. 24(3), 151-153 (1999).

[89] C. Cohen-Tannoudji, Cours du college de france 1998/1999, Paris (unpublished) (1998). 
[90] F. Schreck, Mélanges de gaz ultrafroids : mer de Fermi et condensat de Bose-Einstein des isotopes du lithium, Thèse de doctorat, Université Paris VI (2002).

[91] P. Schmidt, S. Hensler, J. Werner, T. Binhammer, A. Görlitz, and T. Pfau, Doppler cooling of an optically dense cloud of magnetically trapped atoms, Journal of the Optical Society of America B 20(5), 960-967 (2003).

[92] P. Spoden, M. Zinner, N. Herschbach, W. Van Drunen, W. Ertmer, and G. Birkl, Collisional Properties of Cold Spin-Polarized Metastable Neon Atoms, Phys. Rev. Lett. 94(22), 223201 (2005).

[93] A. Tychkov, T. Jeltes, J. McNamara, P. Tol, N. Herschbach, W. Hogervorst, and W. Vassen, Metastable helium Bose-Einstein condensate with a large number of atoms, Phys. Rev. A $\mathbf{7 3}(3)$, 31603 (2006).

[94] S. Granade, M. Gehm, K. O'Hara, and J. Thomas, All-Optical Production of a Degenerate Fermi Gas, Phys. Rev. Lett. 88(12), 120405 (2002).

[95] R. Grimm, M. Weidemüller, and Y. Ovchinnikov, Optical dipole traps for neutral atoms, Adv. at. mol. opt. phys 42(95), 130 (2000).

[96] L. Baksmaty, H. Lu, C. Bolech, and H. Pu, Concomitant Modulated Superfluidity In Polarized Fermionic Gases, arXiv:1003.4488v1 (2010).

[97] G. Partridge, W. Li, Y. Liao, R. Hulet, M. Haque, and H. Stoof, Deformation of a trapped Fermi gas with unequal spin populations, Phys. Rev. Lett. 97(19), 190407 (2006).

[98] M. Zwierlein, A. Schirotzek, C. Schunck, and W. Ketterle, Fermionic superfluidity with imbalanced spin populations, Science $\mathbf{3 1 1}(5760)$, 492-496 (2006).

[99] S. Nascimbene, N. Navon, K. Jiang, L. Tarruell, M. Teichmann, J. Mckeever, F. Chevy, and C. Salomon, Collective Oscillations of an Imbalanced Fermi Gas: Axial Compression Modes and Polaron Effective Mass, Phys. Rev. Lett. 103(17), 170402 (2009).

[100] M. Parish and D. Huse, Evaporative depolarization and spin transport in a unitary trapped Fermi gas, Phys. Rev. A 80(6), 63605 (2009).

[101] M. Gehm, K. O Hara, T. Savard, and J. Thomas, Dynamics of noise-induced heating in atom traps, Phys. Rev. A 58(5), 3914-3921 (1998).

[102] J. Zhang, E. Van Kempen, T. Bourdel, L. Khaykovich, J. Cubizolles, F. Chevy, M. Teichmann, L. Tarruell, S. Kokkelmans, and C. Salomon, P-wave Feshbach resonances of ultracold ${ }^{6} \mathrm{Li}$, Phys. Rev. A 70(3), 30702 (2004).

[103] C. Schunck, M. Zwierlein, C. Stan, S. Raupach, W. Ketterle, A. Simoni, E. Tiesinga, C. Williams, and P. Julienne, Feshbach resonances in fermionic ${ }^{6} \mathrm{Li}$, Phys. Rev. A 71(4), 45601 (2005).

[104] M. Gehm, S. Hemmer, K. OHara, and J. Thomas, Unitarity-limited elastic collision rate in a harmonically trapped Fermi gas, Phys. Rev. A 68(1), 11603 (2003).

[105] D. Petrov, C. Salomon, and G. Shlyapnikov, Scattering properties of weakly bound dimers of fermionic atoms, Phys. Rev. A 71(1), 12708 (2005).

[106] L. Luo, B. Clancy, J. Joseph, J. Kinast, A. Turlapov, and J. Thomas, Evaporative cooling of unitary Fermi gas mixtures in optical traps, New Journal of Physics 8, 213 (2006). 
[107] L. Carr, T. Bourdel, and Y. Castin, Limits of sympathetic cooling of fermions by zero-temperature bosons due to particle losses, Phys. Rev. A 69(3), 33603 (2004).

[108] N. Gemelke, X. Zhang, C. Hung, and C. Chin, In situ observation of incompressible Mott-insulating domains in ultracold atomic gases, Nature 460(7258), 995-998 (2009).

[109] W. Bakr, J. Gillen, A. Peng, S. Fölling, and M. Greiner, A quantum gas microscope for detecting single atoms in a Hubbard-regime optical lattice, Nature 462(7269), 74-77 (2009).

[110] S. Rath, T. Yefsah, K. Günter, M. Cheneau, R. Desbuquois, M. Holzmann, W. Krauth, and J. Dalibard, The equilibrium state of a trapped two-dimensional Bose gas, arXiv:1003.4545 (2010).

[111] J. Thomas, J. Kinast, and A. Turlapov, Virial theorem and universality in a unitary Fermi gas, Phys. Rev. Lett. 95(12), 120402 (2005).

[112] F. Werner and Y. Castin, Unitary gas in an isotropic harmonic trap: Symmetry properties and applications, Phys. Rev. A 74(5), 53604 (2006).

[113] E. Burovski, N. Prokof'ev, B. Svistunov, and M. Troyer, Critical temperature and thermodynamics of attractive fermions at unitarity, Phys. Rev. Lett. 96(16), 160402 (2006).

[114] F. Dalfovo, S. Giorgini, L. Pitaevskii, and S. Stringari, Theory of Bose-Einstein condensation in trapped gases, Rev. Mod. Phys. 71(3), 463-512 (1999).

[115] G. Astrakharchik, R. Combescot, X. Leyronas, and S. Stringari, Equation of state and collective frequencies of a trapped Fermi gas along the BEC-unitarity crossover, Phys. Rev. Lett. 95(3), 30404 (2005).

[116] M. Zwierlein, C. Schunck, A. Schirotzek, and W. Ketterle, Direct observation of the superfluid phase transition in ultracold Fermi gases, Nature 442(7098), 54-58 (2006).

[117] Z. Hadzibabic and J. Dalibard, Two-dimensional Bose fluids: An atomic physics perspective, Proceedings of the International School of Physics 'Enrico Fermi', Course CLXXIII, Varenna (2009).

[118] A. Moerdijk and B. Verhaar, Prospects for Bose-Einstein Condensation in Atomic ${ }^{7} \mathrm{Li}$ and ${ }^{23} \mathrm{Na}$, Phys. Rev. Lett. 73(4), 518-521 (1994).

[119] L. Khaykovich, F. Schreck, G. Ferrari, T. Bourdel, J. Cubizolles, L. Carr, Y. Castin, and C. Salomon, Formation of a matter-wave bright soliton, Science 296(5571), 1290 (2002).

[120] K. Strecker, G. Partridge, A. Truscott, and R. Hulet, Formation and propagation of matter-wave soliton trains, Nature 417(6885), 150-153 (2002).

[121] N. Gross and L. Khaykovich, All-optical production of ${ }^{7}$ Li Bose-Einstein condensation using Feshbach resonances, Phys. Rev. A 77(2), 23604 (2008).

[122] M. Fisher, P. Weichman, G. Grinstein, and D. Fisher, Boson localization and the superfluidinsulator transition, Phys. Rev. B 40(1), 546-570 (1989).

[123] F. Gerbier, Boson Mott insulators at finite temperatures, Phys. Rev. Lett. 99(12), 120405 (2007).

[124] B. Capogrosso-Sansone, N. Prokof'ev, and B. Svistunov, Phase diagram and thermodynamics of the three-dimensional Bose-Hubbard model, Phys. Rev. B 75(13), 134302 (2007). 
[125] S. Fölling, Probing strongly correlated states of ultracold atoms in optical lattices, PhD thesis, Johannes Gutenberg-Universität, Mainz, Fachbereich 08: Physik, Mathematik und Informatik, 2008.

[126] F. Dalfovo and S. Stringari, Bosons in anisotropic traps: Ground state and vortices, Phys. Rev. A 53(4), 2477-2485 (1996).

[127] T. De Silva and E. Mueller, Surface tension in unitary Fermi gases with population imbalance, Phys. Rev. Lett. 97(7), 70402 (2006).

[128] M. Haque and H. Stoof, Trapped Fermionic clouds distorted from the trap shape due to many-body effects, Phys. Rev. Lett. 98(26), 260406 (2007).

[129] S. Baur, S. Basu, T. De Silva, and E. Mueller, Theory of the normal-superfluid interface in population-imbalanced Fermi gases, Phys. Rev. A 79(6), 63628 (2009).

[130] S. Pollack, D. Dries, M. Junker, Y. Chen, T. Corcovilos, and R. Hulet, Extreme Tunability of Interactions in a ${ }^{7}$ Li Bose-Einstein Condensate, Phys. Rev. Lett. 102(9), 90402 (2009).

[131] W. Ketterle, D. Durfee, and D. Stamper-Kurn, Making, probing and understanding Bose-Einstein condensates, in Proceedings of the International School of Physics-Enrico Fermi, page 67, 1999.

[132] P. Ruprecht, M. Holland, K. Burnett, and M. Edwards, Time-dependent solution of the nonlinear Schrödinger equation for Bose-condensed trapped neutral atoms, Phys. Rev. A 51(6), 4704-4711 (1995).

[133] J. Gerton, D. Strekalov, I. Prodan, and R. Hulet, Direct observation of growth and collapse of a Bose-Einstein condensate with attractive interactions, Nature 408(6813), 692-695 (2000).

[134] A. Gammal, T. Frederico, and L. Tomio, Critical number of atoms for attractive Bose-Einstein condensates with cylindrically symmetrical traps, Phys. Rev. A 64(5), 55602 (2001).

[135] T. Ho and E. Mueller, High temperature expansion applied to fermions near Feshbach resonance, Phys. Rev. Lett. 92(16), 160404 (2004).

[136] A. Bulgac, J. Drut, and P. Magierski, Spin 1/2 fermions in the unitary regime: A superfluid of a new type, Phys. Rev. Lett. 96(9), 90404 (2006).

[137] R. Haussmann, Properties of a Fermi liquid at the superfluid transition in the crossover region between BCS superconductivity and Bose-Einstein condensation, Phys. Rev. B 49(18), 1297512983 (1994).

[138] R. Haussmann, W. Rantner, S. Cerrito, and W. Zwerger, Thermodynamics of the BCS-BEC crossover, Phys. Rev. A 75(2), 23610 (2007).

[139] Q. Chen, J. Stajic, S. Tan, and K. Levin, BCS BEC crossover: From high temperature superconductors to ultracold superfluids, Physics Reports 412, 1-88 (2005).

[140] R. Combescot, F. Alzetto, and X. Leyronas, Particle distribution tail and related energy formula, Phys. Rev. A 79(5), 053640 (2009).

[141] H. Hu, X. Liu, and P. Drummond, Equation of state of a superfluid Fermi gas in the BCS-BEC crossover, Europhys. Lett. 74, 574-580 (2006).

[142] T. Busch, B. Englert, K. Rzażewski, and M. Wilkens, Two cold atoms in a harmonic trap, Foundations of Physics 28(4), 549-559 (1998). 
[143] G. Rupak, Universality in a 2-Component Fermi System at Finite Temperature, Phys. Rev. Lett. 98(9), 90403 (2007).

[144] J. Kestner and L. Duan, Level crossing in the three-body problem for strongly interacting fermions in a harmonic trap, Phys. Rev. A 76(3), 033611 (2007).

[145] I. Stetcu, B. Barrett, U. Van Kolck, and J. Vary, Effective theory for trapped few-fermion systems, Phys. Rev. A 76(6), 063613 (2007).

[146] L. Landau, The theory of a Fermi liquid, Sov. Phys. JETP 3(6), 920-925 (1957).

[147] D. S. Greywall, Specific heat of normal liquid ${ }^{3} \mathrm{He}$, Phys. Rev. B 27(5), 2747-2766 (Mar 1983).

[148] G. Stewart, Z. Fisk, and J. Willis, Characterization of single crystals of $\mathrm{CeCu}_{2} \mathrm{Si}_{2}$. A source of new perspectives, Phys. Rev. B 28(1), 172-177 (1983).

[149] N. Ashcroft and N. Mermin, Solid Sate Physics, Holt Rinehart and Winston, New York NY (1976).

[150] J. Loram, K. Mirza, J. Cooper, and W. Liang, Electronic specific heat of $\mathrm{YBa}_{2} \mathrm{Cu}_{3} \mathrm{O}_{6+x}$ from 1.8 to 300 K, Phys. Rev. Lett. 71(11), 1740-1743 (1993).

[151] P. Lee, N. Nagaosa, and X. Wen, Doping a Mott insulator: Physics of high-temperature superconductivity, Rev. Mod. Phys. 78(1), 17-85 (2006).

[152] A. Damascelli, Z. Hussain, and Z. Shen, Angle-resolved photoemission studies of the cuprate superconductors, Rev. Mod. Phys. 75(2), 473-541 (2003).

[153] S. Giorgini, L. Pitaevskii, and S. Stringari, Theory of ultracold atomic Fermi gases, Rev. Mod. Phys. 80(4), 1215-1274 (2008).

[154] A. Perali, P. Pieri, G. Strinati, and C. Castellani, Pseudogap and spectral function from superconducting fluctuations to the bosonic limit, Phys. Rev. B 66(2), 24510 (2002).

[155] P. Magierski, G. Wlazłowski, A. Bulgac, and J. Drut, Finite-Temperature Pairing Gap of a Unitary Fermi Gas by Quantum Monte Carlo Calculations, Phys. Rev. Lett. 103(21), 210403 (2009).

[156] J. Stewart, J. Gaebler, and D. Jin, Using photoemission spectroscopy to probe a strongly interacting Fermi gas, Nature 454(7205), 744-747 (2008).

[157] W. Ketterle and M. Zwierlein, Making, probing and understanding ultracold Fermi gases, Nuovo Cimento Rivista Serie 31, 247-422 (2008).

[158] O. Juillet, Sign-free stochastic mean-field approach to strongly correlated phases of ultracold fermions, New Journal of Physics 9, 163 (2007).

[159] Y. Ohashi and A. Griffin, BCS-BEC crossover in a gas of Fermi atoms with a Feshbach resonance, Phys. Rev. Lett. 89(13), 130402 (2002).

[160] J. Stajic, J. Milstein, Q. Chen, M. Chiofalo, M. Holland, and K. Levin, Nature of superfluidity in ultracold Fermi gases near Feshbach resonances, Phys. Rev. A 69(6), 63610 (2004).

[161] S. Tsuchiya, R. Watanabe, and Y. Ohashi, Single-particle properties and pseudogap effects in the BCS-BEC crossover regime of an ultracold Fermi gas above $T_{c}$, Phys. Rev. A 80(3), 33613 (2009).

[162] Q. Chen and K. Levin, Momentum Resolved Radio Frequency Spectroscopy in Trapped Fermi Gases, Phys. Rev. Lett. 102(19), 190402 (2009). 
[163] H. Hu, X. Liu, P. Drummond, and H. Dong, Pseudo-gap pairing in ultracold Fermi atoms, arXiv:1003.1538 (2010).

[164] C. Chien, H. Guo, Y. He, and K. Levin, Comparative Study of BCS-BEC Crossover Theories above $T_{c}$ : the Nature of the Pseudogap in Ultra-Cold Atomic Fermi Gases, arXiv:0910.3699 (2009).

[165] M. Inguscio, W. Ketterle, and C. Salomon, Ultracold Fermi Gases, Proceedings of the International School of Physics Enrico Fermi, Course CLXIV, Varenna (2006).

[166] A. Schirotzek, Y. Shin, C. Schunck, and W. Ketterle, Determination of the superfluid gap in atomic fermi gases by quasiparticle spectroscopy., Phys. Rev. Lett. 101(14), 140403 (2008).

[167] J. Carlson and S. Reddy, Superfluid pairing gap in strong coupling, Phys. Rev. Lett. 100(15), 150403 (2008).

[168] E. Taylor, Critical behavior in trapped strongly interacting Fermi gases, Phys. Rev. A 80(2), 23612 (2009).

[169] J. Lipa and T. Chui, Very high-resolution heat-capacity measurements near the lambda point of helium, Phys. Rev. Lett. 51(25), 2291-2294 (1983).

[170] J. Le Guillou and J. Zinn-Justin, Critical exponents from field theory, Phys. Rev. B 21(9), 39763998 (1980)

[171] J. Le Guillou and J. Zinn-Justin, Accurate critical exponents from the $\varepsilon$-expansion, Journal de Physique Lettres 46(4), 137-141 (1985).

[172] E. Burovski, E. Kozik, N. Prokof'ev, B. Svistunov, and M. Troyer, Critical Temperature Curve in BEC-BCS Crossover, Phys. Rev. Lett. 101(9), 90402 (2008).

[173] A. Bulgac, J. Drut, and P. Magierski, Quantum Monte Carlo simulations of the BCS-BEC crossover at finite temperature, Phys. Rev. A 78(2), 23625 (2008).

[174] K. Gubbels and H. Stoof, Renormalization group theory for the imbalanced Fermi gas, Phys. Rev. Lett. 100(14), 140407 (2008).

[175] Y. Nishida and D. Son, $\epsilon$ expansion for a Fermi gas at infinite scattering length, Phys. Rev. Lett. 97(5), 050403 (2006).

[176] P. Nikolić and S. Sachdev, Renormalization-group fixed points, universal phase diagram, and $1 / N$ expansion for quantum liquids with interactions near the unitarity limit, Phys. Rev. A 75(3), 33608 (2007).

[177] H. Hu, X. Liu, and P. Drummond, Universal thermodynamics of a strongly interacting Fermi gas: theory versus experiment, arXiv:1001.2085v1 (2010).

[178] S. Pilati and S. Giorgini, Phase separation in a polarized Fermi gas at zero temperature, Phys. Rev. Lett. 100(3), 30401-30401 (2008).

[179] R. Haussmann, M. Punk, and W. Zwerger, Spectral functions and rf response of ultracold fermionic atoms, Phys. Rev. A 80(6), 063612 (Dec 2009).

[180] M. Punk, P. T. Dumitrescu, and W. Zwerger, Polaron-to-molecule transition in a strongly imbalanced Fermi gas, Phys. Rev. A 80(5), 053605 (Nov 2009). 
[181] G. Bertaina and S. Giorgini, Density profiles of polarized Fermi gases confined in harmonic traps, Phys. Rev. A 79(1) (2009).

[182] A. Bulgac, J. Drut, and P. Magierski, Thermodynamics of a trapped unitary fermi gas, Phys. Rev. Lett. 99(12), 120401 (2007).

[183] J. Carlson and S. Reddy, Asymmetric two-component fermion systems in strong coupling, Phys. Rev. Lett. 95(6), 60401 (2005).

[184] A. Bulgac, M. Forbes, and A. Schwenk, Induced P-Wave Superfluidity in Asymmetric Fermi Gases, Phys. Rev. Lett. 97(2), 20402 (2006).

[185] C. Mora and F. Chevy, The normal phase of an imbalanced Fermi gas, arXiv:1003.0213v1 (2010).

[186] J. Schrieffer, Theory of superconductivity, Il Nuovo Cimento (1955-1965) 7, 377-385 (1958).

[187] G. Bertaina and S. Giorgini, Density profiles of polarized Fermi gases confined in harmonic traps, Phys. Rev. A 79(1), 13616 (2009).

[188] G. Baker and P. Graves-Morris, Padé approximants, Cambridge Univ Pr, 1996.

[189] S. Tan, Large momentum part of a strongly correlated Fermi gas, Annals of Physics 323(12), 2971-2986 (2008).

[190] S. Tan, Generalized virial theorem and pressure relation for a strongly correlated Fermi gas, Annals of Physics 323(12), 2987-2990 (2008).

[191] S. Tan, Energetics of a strongly correlated Fermi gas, Annals of Physics 323(12), 2952-2970 (2008).

[192] C. Lobo, I. Carusotto, S. Giorgini, A. Recati, and S. Stringari, Pair correlations of an expanding superfluid Fermi gas, Phys. Rev. Lett. 97(10), 100405 (2006).

[193] H. Hu, E. Kuhnle, X. Liu, P. Dyke, M. Mark, P. Drummond, P. Hannaford, and C. Vale, Universal structure of a strongly interacting Fermi superfluid, arXiv:1001.3200 (2010).

[194] F. Werner, L. Tarruell, and Y. Castin, Number of closed-channel molecules in the BEC-BCS crossover, The European Physical Journal B-Condensed Matter and Complex Systems 68(3), 401415 (2009).

[195] J. Stewart, J. Gaebler, T. Drake, and D. Jin, Verification of universal relations in a strongly interacting Fermi gas, arXiv:1002.1987 (2010).

[196] P. Pieri, A. Perali, and G. Strinati, Enhanced paraconductivity-like fluctuations in the radiofrequency spectra of ultracold Fermi atoms, Nature Phys. 5(10), 736-740 (2009).

[197] W. Schneider, V. Shenoy, and M. Randeria, Theory of Radio Frequency Spectroscopy of Polarized Fermi Gases, arXiv:0903.3006 (2009).

[198] E. Braaten, D. Kang, and L. Platter, The Role of the Contact in the rf Spectroscopy of a Stronglyinteracting Fermi Gas, arXiv:1001.4518 (2010).

[199] F. Werner and Y. Castin, Exact relations for quantum-mechanical few-body and many-body problems with short-range interactions in two and three dimensions, arXiv:1001.0774 (2010).

[200] W. Lenz, Die Wellenfunktion und Geschwindigkeitsverteilung des entarteten Gases, Zeitschrift fur Physik A Hadrons and Nuclei 56(11), 778-789 (1929). 
[201] A. Fetter and J. Walecka, Quantum theory of many-particle systems, (1971).

[202] V. Efimov and M. Amusya, Ground state of rarefied Fermi gas consisting of rigid spheres, Zh. Eksperim. i Teor. Fiz 47 (1964).

[203] G. Baker, Singularity Structure of the Perturbation Series for the Ground-State Energy of a ManyFermion System, Rev. Mod. Phys. 43(4), 479-531 (1971).

[204] R. Bishop, On the ground state of an impurity in a dilute fermi gas, Annals of Physics 78, 391-420 (1973).

[205] Y. Castin, Basic theory tools for degenerate Fermi gases, arXiv:0612613 (2006).

[206] S. Simonucci, P. Pieri, and G. Strinati, Broad vs. narrow Fano-Feshbach resonances in the BCS-BEC crossover with trapped Fermi atoms, EuroPhys. Lett. 69, 713-718 (2005).

[207] M. Bartenstein, A. Altmeyer, S. Riedl, R. Geursen, S. Jochim, C. Chin, J. Denschlag, R. Grimm, A. Simoni, E. Tiesinga, et al., Precise Determination of ${ }^{6} \mathrm{Li}$ Cold Collision Parameters by RadioFrequency Spectroscopy on Weakly Bound Molecules, Phys. Rev. Lett. 94(10), 103201 (2005).

[208] S. Gautam and D. Angom, Scattering length for fermionic alkali atoms, The European Physical Journal D 56(2), 173-179 (2009).

[209] I. Brodsky, M. Kagan, A. Klaptsov, R. Combescot, and X. Leyronas, Exact diagrammatic approach for dimer-dimer scattering and bound states of three and four resonantly interacting particles, Phys. Rev. A 73(3), 32724 (2006).

[210] S. Papp, J. Pino, R. Wild, S. Ronen, C. Wieman, D. Jin, and E. Cornell, Bragg Spectroscopy of a Strongly Interacting ${ }^{85}$ Rb Bose-Einstein Condensate, Phys. Rev. Lett. 101(13), 135301 (2008).

[211] T. Wu, Ground state of a Bose system of hard spheres, Phys. Rev. 115(6), 1390-1404 (1959).

[212] N. Hugenholtz and D. Pines, Ground-state energy and excitation spectrum of a system of interacting bosons, Phys. Rev. 116(3), 489-506 (1959).

[213] S. Tan, Three-boson problem at low energy and implications for dilute Bose-Einstein condensates, Phys. Rev. A 78(1), 13636 (2008).

[214] E. Braaten, H. Hammer, and T. Mehen, Dilute Bose-Einstein condensate with large scattering length, Phys. Rev. Lett. 88(4), 40401 (2002).

[215] V. Efimov, Weakly-bound states of three resonantly-interacting particles, Sov. J. Nucl. Phys 12, 589 (1971).

[216] J. von Stecher, C. Greene, and D. Blume, BEC-BCS crossover of a trapped two-component Fermi gas with unequal masses, Phys. Rev. A 76(5), 53613 (2007).

[217] J. von Stecher, C. Greene, and D. Blume, Energetics and structural properties of trapped twocomponent Fermi gases, Phys. Rev. A 77(4), 43619 (2008).

[218] Y. Shin, M. Zwierlein, C. Schunck, A. Schirotzek, and W. Ketterle, Observation of phase separation in a strongly interacting imbalanced Fermi gas, Phys. Rev. Lett. 97(3), 30401 (2006).

[219] L. Pitaevskii and S. Stringari, Elementary excitations in trapped Bose-Einstein condensed gases beyond the mean-field approximation, Phys. Rev. Lett. 81(21), 4541-4544 (1998). 
[220] E. Braaten and J. Pearson, Semiclassical Corrections to the Oscillation Frequencies of a Trapped Bose-Einstein Condensate, Phys. Rev. Lett. 82(2), 255-258 (1999).

[221] S. Stringari, Collective oscillations of a trapped superfluid Fermi gas near a Feshbach resonance, EuroPhys. Lett. 65, 749-752 (2004).

[222] L. Viverit, C. Pethick, and H. Smith, Zero-temperature phase diagram of binary boson-fermion mixtures, Phys. Rev. A 61(5), 53605 (2000).

[223] P. Ring and P. Schuck, The Nuclear many-body problem, (1980).

[224] M. Blume, V. Emery, and R. Griffiths, Ising Model for the $\lambda$ Transition and Phase Separation in ${ }^{3}$ He- ${ }^{4}$ He Mixtures, Phys. Rev. A 4(3), 1071-1077 (1971).

[225] R. Schermer, L. Passoll, and D. Rorer, Phase Separation in ${ }^{3} \mathrm{He}-{ }^{4} \mathrm{He}$ Mixtures Observed With Slow Neutrons, Phys. Rev. 173(1), 277-284 (1968).

[226] C. Mora and F. Chevy, Ground state of a tightly bound composite dimer immersed in a Fermi Sea, Phys. Rev. A 80(3), 33607 (2009).

[227] X. Leyronas and F. Alzetto, Equation of state of a polarized Fermi gas in the Bose-Einstein Condensate limit, arXiv:0911.3312 (2009).

[228] D. Sheehy and L. Radzihovsky, BEC-BCS Crossover in Magnetized Feshbach-Resonantly Paired Superfluids, Phys. Rev. Lett. 96(6), 60401 (2006).

[229] M. Parish, F. Marchetti, A. Lamacraft, and B. Simons, Finite-temperature phase diagram of a polarized Fermi condensate, Nature Phys. 3(2), 124-128 (2007).

[230] A. Bulgac and M. Forbes, Unitary Fermi Supersolid: The Larkin-Ovchinnikov Phase, Phys. Rev. Lett. 101(21), 215301 (2008).

[231] E. Altman, E. Demler, and M. Lukin, Probing many-body states of ultracold atoms via noise correlations, Phys. Rev. A 70(1), 13603 (2004).

[232] L. Landau, Theory of the superfluidity of helium II, Phys. Rev. 60(4), 356-358 (1941).

[233] F. Chevy, K. Madison, and J. Dalibard, Measurement of the angular momentum of a rotating Bose-Einstein condensate, Phys. Rev. Lett. 85(11), 2223-2227 (2000).

[234] Y. Castin and R. Dum, Bose-Einstein condensates in time dependent traps, Phys. Rev. Lett. 77(27), 5315-5319 (1996).

[235] Y. Kagan, E. Surkov, and G. Shlyapnikov, Evolution of a Bose-condensed gas under variations of the confining potential, Phys. Rev. A 54(3), 1753-1756 (1996).

[236] L. Tarruell, M. Teichmann, J. McKeever, T. Bourdel, J. Cubizolles, N. Navon, F. Chevy, C. Salomon, L. Khaykovich, and J. Zhang, Expansion of a lithium gas in the BEC-BCS crossover, in Proceedings of the International School of Physics Enrico Fermi, Course CLXIV, Varenna, volume 164, page 845, IOS Press; Ohmsha; 1999, 2007.

[237] H. Kneser, Schallabsorption und-dispersion in Flussigkeiten, Annalen der Physik 424(3) (1938).

[238] L. Landau and E. Lifshitz, Fluid mechanics, Course of Theoretical Physics 6 (1959).

[239] G. Kavoulakis, C. Pethick, and H. Smith, Damping of hydrodynamic modes in a trapped Bose gas above the Bose-Einstein transition temperature, Phys. Rev. A 57(4), 2938-2941 (1998). 
[240] S. Riedl, E. Sánchez Guajardo, C. Kohstall, A. Altmeyer, M. Wright, J. Denschlag, R. Grimm, G. Bruun, and H. Smith, Collective oscillations of a Fermi gas in the unitarity limit: Temperature effects and the role of pair correlations, Phys. Rev. A 78(5), 53609 (2008).

[241] L. Vichi and S. Stringari, Collective oscillations of an interacting trapped Fermi gas, Phys. Rev. A 60(6), 4734-4737 (1999).

[242] L. Vichi, Collisional damping of the collective oscillations of a trapped Fermi gas, Journal of Low Temperature Physics 121(3), 177-197 (2000).

[243] S. Gupta, Z. Hadzibabic, J. Anglin, and W. Ketterle, Collisions in zero temperature Fermi gases, Phys. Rev. Lett. 92(10), 100401 (2004).

[244] G. Bruun, A. Recati, C. Pethick, H. Smith, and S. Stringari, Collisional Properties of a Polarized Fermi Gas with Resonant Interactions, Phys. Rev. Lett. 100(24), 240406 (2008).

[245] J. Scargle, Studies in astronomical time series analysis. II- Statistical aspects of spectral analysis of unevenly spaced data, The Astrophysical Journal 263, 835 (1982).

[246] W. Press, S. Teukolsky, W. Vetterling, and B. Flannery, Numerical recipes in C, Cambridge Univ. Press Cambridge MA, USA:, 1992.

[247] K. Gubbels, M. Romans, and H. Stoof, Sarma phase in trapped unbalanced Fermi gases, Phys. Rev. Lett. 97(21), 210402 (2006).

[248] I. Bausmerth, A. Recati, and S. Stringari, Chandrasekhar-Clogston limit and phase separation in Fermi mixtures at unitarity, Phys. Rev. A 79(4), 43622 (2009).

[249] A. Gezerlis, S. Gandolfi, K. Schmidt, and J. Carlson, Heavy-light fermion mixtures at unitarity, Phys. Rev. Lett. 103(6), 60403 (2009).

[250] K. Gubbels, J. Baarsma, and H. Stoof, Lifshitz Point in the Phase Diagram of Resonantly Interacting ${ }^{6} \mathrm{Li}^{-}{ }^{40} \mathrm{~K}$ Mixtures, Phys. Rev. Lett. 103(19), 195301 (2009).

[251] E. Wille, F. Spiegelhalder, G. Kerner, D. Naik, A. Trenkwalder, G. Hendl, F. Schreck, R. Grimm, T. Tiecke, J. Walraven, et al., Exploring an Ultracold Fermi-Fermi Mixture: Interspecies Feshbach Resonances and Scattering Properties of ${ }^{6} \mathrm{Li}$ and ${ }^{40} \mathrm{~K}$, Phys. Rev. Lett. 100(5), 53201 (2008).

[252] M. Taglieber, A. Voigt, T. Aoki, T. Hänsch, and K. Dieckmann, Quantum degenerate two-species Fermi-Fermi mixture coexisting with a Bose-Einstein condensate, Phys. Rev. Lett. 100(1), 10401 (2008).

[253] B. Marcelis, B. Verhaar, and S. Kokkelmans, Total control over ultracold interactions via electric and magnetic fields, Phys. Rev. Lett. 100(15), 153201 (2008).

[254] A. Rapp, G. Zaránd, C. Honerkamp, and W. Hofstetter, Color superfluidity and 'baryon' formation in ultracold fermions, Phys. Rev. Lett. 98(16), 160405 (2007).

[255] R. Cherng, G. Refael, and E. Demler, Superfluidity and magnetism in multicomponent ultracold fermions, Phys. Rev. Lett. 99(13), 130406 (2007).

[256] T. Ottenstein, T. Lompe, M. Kohnen, A. Wenz, and S. Jochim, Collisional stability of a threecomponent degenerate Fermi gas, Phys. Rev. Lett. 101(20), 203202 (2008).

[257] J. Huckans, J. Williams, E. Hazlett, R. Stites, and K. O Hara, Three-body recombination in a three-state Fermi gas with widely tunable interactions, Phys. Rev. Lett. 102(16), 165302 (2009). 
[258] M. Lewenstein, A. Sanpera, V. Ahufinger, and B. Damski, Ultracold atomic gases in optical lattices: mimicking condensed matter physics and beyond, Advances in Physics 56(2), 243-379 (2007).

[259] L. Landau, E. Lifshitz, D. Lavis, A. Lerda, R. Liboff, L. Loeb, W. Louisell, S. Lundqvist, B. McCoy, D. MacDonald, et al., Statistical Physics, Pergamon Press, Oxford, UK, 1980.

[260] G. Bruun, Y. Castin, R. Dum, and K. Burnett, BCS theory for trapped ultracold fermions, The European Physical Journal D-Atomic, Molecular, Optical and Plasma Physics 7(3), 433-439 (1999).

[261] P. Bedaque, H. Caldas, and G. Rupak, Phase separation in asymmetrical fermion superfluids, Phys. Rev. Lett. 91(24), 247002-247002 (2003). 




\section{Résumé}

Les gaz ultrafroids permettent d'étudier sous un angle nouveau des hamiltoniens complexes issus de la matière condensée, tels le modèle de Fermi-Hubbard. Cette thèse présente une nouvelle méthode de mesure de l'équation d'état d'un gaz ultrafroid, autorisant une comparaison directe avec la théorie. Elle repose sur une mesure de la pression à l'intérieur d'un gaz à partir de l'analyse de son image in situ.

Nous appliquons cette méthode à l'étude d'un gaz de fermions en interaction résonnante, un gaz de ${ }^{7} \mathrm{Li}$ en interaction faible servant de thermomètre. De manière surprenante, aucune des théories à $N$ corps du gaz unitaire ne rend compte dans son intégralité de l'équation déduite de cette analyse. Le développement du viriel extrait des données à haute température est en accord avec la résolution du problème à trois corps. À basse température nous montrons, contrairement à un certain nombre d'études antérieures, que la phase normale se comporte comme un liquide de Fermi. Enfin, nous obtenons la température critique de superfluidité grâce à une signature claire sur l'équation d'état.

Nous avons aussi mesuré la pression de l'état fondamental en fonction du déséquilibre de spin et de la force des interactions - mesure directement utile à la description de la croûte des étoiles à neutrons. Nos données valident les simulations Monte-Carlo et sont en accord avec les corrections Lee-Huang-Yang au champ moyen pour un superfluide fermionique ou bosonique. Nous observons que, dans presque tous les cas, la phase partiellement polarisée peut être décrite comme un liquide de Fermi de polarons. La masse effective du polaron déduite de l'équation d'état est en accord avec une étude de modes collectifs.

Mots-clés: gaz ultrafroids - superfluidité - thermodynamique - crossover BEC-BCS liquide de Fermi - polaron

\section{Abstract}

Complex Hamiltonians from condensed matter, such as the Fermi-Hubbard model, can be experimentally studied using ultracold gases. This thesis describes a new method for determining the equation of state of an ultracold gas, making the comparison with many-body theories straightforward. It is based on the measurement of the local pressure inside a trapped gas from the analysis of its in situ image.

We first apply this method to the study of a Fermi gas with resonant interactions, a weakly-interacting ${ }^{7} \mathrm{Li}$ gas acting as a thermometer. Surprisingly, none of the existing many-body theories of the unitary gas accounts for the equation of state deduced from our study over its full range. The virial expansion extracted from the high-temperature data agrees with the resolution of the three-body problem. At low temperature, we observe, contrary to some previous studies, that the normal phase behaves as a Fermi liquid. Finally we obtain the critical temperature for superfluidity from a clear signature on the equation of state.

We also measure the pressure of the ground state as a function of spin imbalance and interaction strength - measure directly relevant to describe the crust of neutron stars. Our data validate MonteCarlo simulations and quantify the Lee-Huang-Yang corrections to mean-field interactions in low-density fermionic or bosonic superfluids. We show that, in most cases, the partially polarized normal phase can be described as a Fermi liquid of polarons. The polaron effective mass extracted from the equation of state is in agreement with a study of collective modes.

Keywords: ultracold gases - superfluidity - thermodynamics - BEC-BCS crossover Fermi liquid - polaron 